
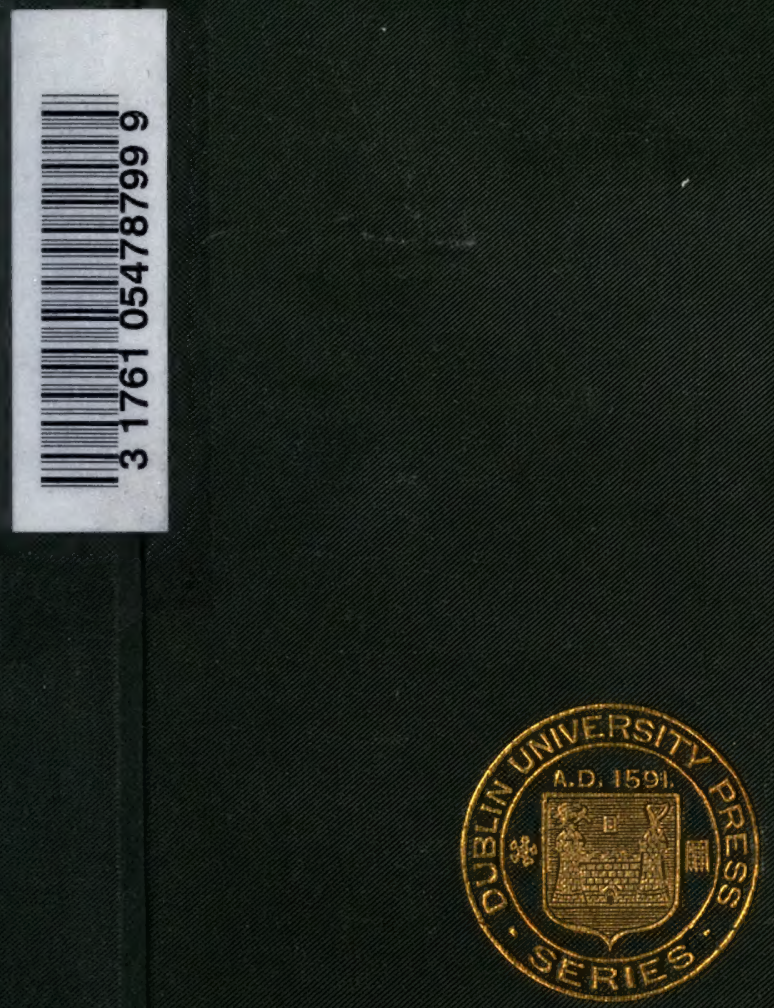


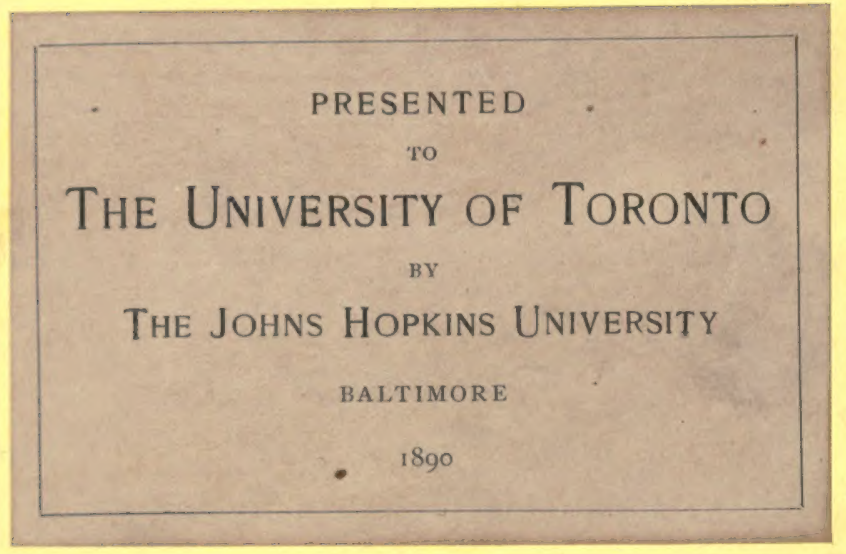




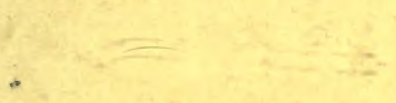


Digitized by the Internet Archive in 2007 with funding from Microsoft Corporation 
THE COLLECTED WORKS

JAMES MAC CULLAGH, LL.D. 

$M 1334$

DUBLIN UNIVERSITY PRESS SERIES.

\title{
THE COLLECTED WORKS
}

\author{
$\mathrm{OF}$
}

\section{JAMES MAC CULLAGH, LL.D.,}

FELLOW OF TRINITY COLLEGE, AND

PROFESSOR OF NATURAL PHILOSOPHY IN THE UNIVERSITY OF DUBLIN.

EDITED BY

JOHN H. JELLETT, B. D., AND

SAMUEL HAUGHTON, CLK., M.D.

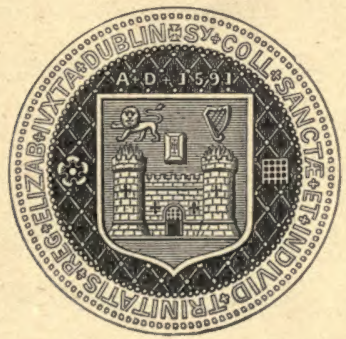

DUBLIN : HODGES, FIGGIS, \& CO., GRAFTON-STREET. LONDON : LONGMANS, GREEN, \& CO,, PATERNOSTER-ROW. I 880 . 


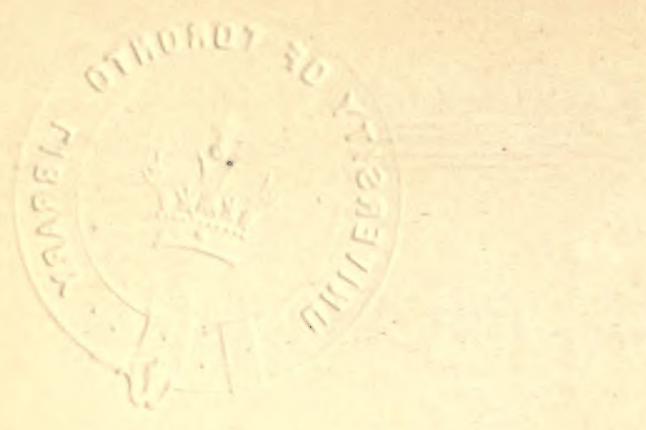

DUBLIN :

PRINTED AT THE UNIVERSITY PRESS.

$$
\frac{6044}{4+110100}
$$




\section{P R E F A C E.}

THE present volume contains a complete collection of the scientific works of the late Professor Mac Cullagh. They have been reprinted for the most part from the Proceedings and Transactions of the Royal Irish Academy, in which they originally appeared. Some few have been taken from the Philosophical Magazine.

Prof. Mac Cullagh's most important contributions to Science were made in the departments of Physical Optics and Geometry-the first class being very much the larger. The discrepancy is not, however, as great as might at first sight appear. A considerable part of his Optical researches, more especially those of earlier date, really belong to the domain of Geometry; and, if they were so classed, the inequality between the classes would be much reduced. Such a classification, however, involving the separation of the purely geometrical propositions from their physical applications, would be exceedingly-inconvenient, and these propositions have been allowed to remain in the connexion in which the author placed them. 
In his earlier Optical Memoirs, Prof. Mac Cullagh aimed chiefly at elucidating, by means of geometrical theorems, the physical theory of Fresnel. This is the principal object of the Memoirs I.-IV. in the present volume. In V. occurs the first notice of a problem which subsequently occupied so large a space in Prof. Mac Cullagh's researches, namely, the investigation of the laws according to which polarized light is reflected and refracted at the surface of a crystalline medium. This problem is discussed at length in XI. and XIV. In the former of these memoirs he deduces a solution of the problem from certain assumed physical principles. In the second he seeks to establish the theory upon a strictly mechanical basis by means of the general dynamical equation of Lagrange. These, which are the principal memoirs treating of the general question, are supplemented by Memoirs XVI.-XIX., in which the same problem is discussed. Two other important questions, namely, metallic reflexion and the double refraction of quart $z$, which required a peculiar mode of treatment, are considered in Memoirs VI., VII., XV., XVII., XXI.

Prof. Mac Cullagh's contributions to pure Geometry, excluding, as has been said, all those theorems which have been introduced by the author as auxiliary to his Optical researches, form the second Part of the present volume. The first of these is a Memoir on 
the Rectification of the Conic Sections, which (with his first Optical Memoir) was communicated to the Royal Irish Academy shortly after he obtained his B. A. degree. This was followed, many years afterwards, by an elaborate memoir, which may, indeed, be fairly called a treatise: "On Surfaces of the Second Order." In this memoir a new definition is given for this class of surfaces analogous to the well-known mode of defining curves of the second order by means of a focus and directrix.

The articles contained in the third and fourth parts of the present volume were not published during the lifetime of the author. They are records of Courses of Lectures on the subjects of Rotation and Attraction, given by Prof. Mac Cullagh. These records were preserved by Professors Haughton and Allman, and communicated by them to the Royal Irish Academy.

Two short Papers on Egyptian Chronology, which, like most of Prof. Mac Cullagh's writings, were originally communicated to the Royal Irish Academy, have been printed at the end of this volume. 



\section{C $0 \mathrm{~N}$ T E N T S.}

\section{PART I.}

\section{PHYSICAL OPTICS.}

PAGE

I. On the Double Refraction of Light in a Crystallized Medium, according to the principles of Fresnel, . . . . 1

II. On the Intensity of Light when the Vibrations are Elliptical, . 14

III. Note on the subject of Conical Refraction, . . . . 17

IV. Geometrical Propositions applied to the Wave Theory of Light, 20

V. A Short Account of some Recent Investigations Concerning the

Laws of Reflexion and Refraction at the Surface of Crystals, 55

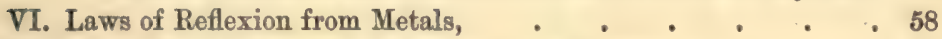

VII. On the Laws of the Double Refraction of Quartz, . . 63

VIII. On the Laws of Reflexion from Crystallized Surfaces, • . 75

IX. On the Probable Nature of the Light Transmitted by the Diamond and by Gold Leaf, . . . . . . . . 82

X. On the Laws of Crystalline Reflexion, . . . . . 83

XI. On the Laws of Crystalline Reflexion and Refraction, . . 37

XII. On a new Optioal Instrument, intended chiefly for the purpose of making Experiments on the Light Reflected from Metals, 138

XIII. Laws of Crystalline Reflexion.-Question of priority, . 140

XIV. An Essay towards a Dynamical Theory of Reflexion and Refraction, . . . . . . . . . . . 415

XV. On the Optical Laws of Rock-Crystals, . . . . . 185

XVI. On a Dynamical Theory of Crystalline Reflexion and Refraction:

(Supplement), . . . . . . . . , 194 
XVII. Notes on Some Points in the Theory of Light, . . . 187

XVIII. On the Problem of Total Reflexion, . . . . . 218

XIX. On the Dispersion of the Optic Axes, and of the Axes of Elastioity in Biaxal Crystals, . . . . . 221

XX. On the Law of Double Refraction, . . . . . . 227

XXI. On the Laws of Metallic Reflexion, and on the Mode of making Experiments upon Elliptic Polarization, . . 230

XXII. On the attempt lately made by M. Laurent to explain, on mechanical principles, the Phenomenon of Circular Polarization in Liquids, . . . . . . . 249

XXIII. On Total Reflexion, . . . . . . . . 250

\section{PART II.}

G EOM E R Y

I. Geometrical Theorems on the Rectification of the Conic Sections, . . . . . . . . . 255

II. On the Surfaces of the Second Order, . . . . 260

III. Note relative to the comparison of the Arcs of Curves, particu-

larly of Plane and Spherical Conies, . . . . . 318

IV. Note on Surfaces of the Second Order, . . . . . . 321

\section{PART III.}

ROTATION.

I. On the Rotation of a Solid Body round a Fixed Point; being an account of the late Professor Mac Cullagh's Lectures on that subject. Compiled by the Rev. Samuel Haughton, Fellow of Trinity College, Dublin, . . . . . . . . 3 


\section{PART IV.}

\section{A T T R A C T O N}

I. On a Difficulty in the Theory of the Attraction of Spheroids, . 349

II. On the Attraction of Ellipsoids, with a new demonstration of Clairaut's Theorem, being an account of the late Professor Mac Cullagh's Lectures on those subjects. Compiled by George Johnston Allman, LL.D., of Trinity College, Dublin, 352

\section{SUPPLEMENT.}

EGYPTIAN CHRONOLOGY.

I. On the Chronology of Egypt, . . . . . . . . . 373

II. On the Catalogue of Egyptian Kings, which is usually known by the name of the Laterculum of Eratosthenes, . . . . 376 . 

I.-ON THE DOUBLE REFRACTION OF LIGHT IN A CRYSTALLIZED MEDIUM, ACCORDING TO THE PRINCIPLES OF FRESNEL.

[Transactions of the Royal Irish Academy, Vou. xvi.-Read June 21, 1830.]

The mathematical difficulties under which the beautiful and interesting theory of Fresnel has hitherto laboured are well known, and have been regarded as almost insuperable. He tells us, in his Memoir (see the Memoirs of the Royal Academy of Sciences of Paris, tom. vii. p. 136), that the calculations, by which he assured himself of the truth of his construction for finding the surface of the wave, were so tedious and embarrassing, that he was obliged to omit them altogether. A direct demonstration has since been supplied by M. Ampere (Annales de Chimie et de Physique, tom. xxxix. p. 113) ; but his solution is excessively complicated and difficult.

Judging from the simplicity and eleganee of the results that there must be some simple method of arriving at them, I have been led to consider the subject with the attention which it merits, and have succeeded in discovering a method by which the whole may be explained with that simplicity which is characteristic of every theory that is founded in nature.

In the following Paper I shall give a brief view of this method, sufficient to enable those who are acquainted with the mechanical principles laid down in the original memoir of Fresnel, to trace, at a glance, the connexion between the several 
parts of his theory. For this purpose it will be convenient to premise the following Geometrical Lemmas :-

1. If $a, b, c$, be the semiaxes of an ellipsoid, and $a, \beta, \gamma$, the angles which they make with a perpendicular from the centre on a tangent plane, the square of the perpendicular will be equal to

$$
a^{2} \cos ^{2} \alpha+b^{2} \cos ^{2} \beta+c^{2} \cos ^{2} \gamma .
$$

Let a plane through the point of contact $Q$, and one of the semiaxes $O A$, intersect the ellipsoid in the ellipse $A O N$, and the tangent plane in the tangent $Q L$, and draw $Q M$ perpendicular to $O A$; then $O A$ is a semiaxis of the ellipse $A O N$, and therefore is a mean proportional between $O M$ and $O L$; whence $O L=\frac{a^{2}}{x}$, denoting $O M$ by

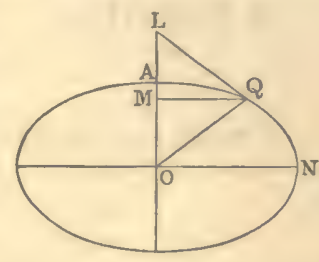

Fig. 1. $x$. But if $p$ denote the length of the perpendicular from the centre $O$ on the tangent plane at $Q$, the cosine of the angle which it makes with $O A$ will be equal to $\frac{p}{O L}$, and therefore

$$
\cos \alpha=\frac{p x}{a^{2}} \text {, and } a \cos a=\frac{p x}{a} \text {. }
$$

Similarly,

Hence

$$
b \cos \beta=\frac{p y}{b} \text {, and } c \cos \gamma=\frac{p z}{c} \text {. }
$$

$$
a^{2} \cos ^{2} \alpha+b^{2} \cos ^{2} \beta+c^{2} \cos ^{2} \gamma=p^{2}\left(\frac{x^{2}}{a^{2}}+\frac{y^{2}}{b^{2}}+\frac{z^{2}}{c^{2}}\right)=p^{2} .
$$

Cor.-Since $x, y, z$, are as the cosines of the angles which $O Q$ makes with the semiaxes, it appears from the demonstration that the cosines of the angles which the perpendicular to a tangent plane makes with the semiaxes are, with respect to each other, directly as the cosines of the angles which the semidiameter through the point of contact makes with the semiaxes, and inversely as the squares of the semiaxes themselves.

2. If the semiaxes $a, b, c$, and $a^{\prime}, b^{\prime}, c^{\prime}$, of two concentric 
ellipsoids coincide in direction, and be reciprocally proportional, so that $a a^{\prime}=b b^{\prime}=c c^{\prime}=k^{2}$; and if a semidiameter $O R$ of the one be out perpendicularly in $P$ by a plane which touches the other, then will $O R$ be inversely as $O P$, so that $O P \times O R$ will be always equal to $k^{2}$.

Let $O R$ be a semidiameter of the ellipsoid whose semiaxes are $a^{\prime}, b^{\prime}, c^{\prime}$; and let $a, \beta, \gamma$, be the angles which it makes with them ; then if $x, y, z$, be the co-ordinates of $R$, we have

$$
\frac{x^{2}}{a^{\prime 2}}+\frac{y^{2}}{b^{\prime 2}}+\frac{z^{2}}{c^{\prime 2}}=1,
$$

and therefore

$$
\begin{aligned}
\frac{1}{O R^{2}}=\frac{\cos ^{2} \alpha}{d^{\prime 2}} & +\frac{\cos ^{2} \beta}{b^{\prime 2}}+\frac{\cos ^{2} \gamma}{c^{\prime 2}} \\
& =\frac{1}{k^{4}}\left(a^{2} \cos ^{2} a+b^{2} \cos ^{2} \beta+c^{2} \cos ^{2} \gamma .\right)
\end{aligned}
$$

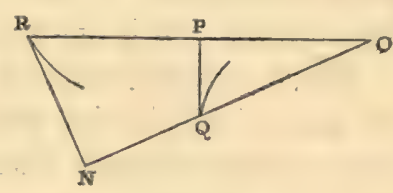

But by the preceding lemma, since $O P$ is perpendicular to the tangent plane at $Q$, we have

Hence

$$
O P^{2}=a^{2} \cos ^{2} \alpha+b^{2} \cos ^{2} \beta+c^{2} \cos ^{2} \gamma .
$$

and therefore

$$
\frac{1}{O R^{2}}=\frac{O P^{2}}{k^{4}},
$$

$$
O P \times O R=k^{2} .
$$

3. If through the point of contact $Q$ the straight line $O Q$ be drawn to meet in $N$ the tangent plane at $R$, it will meet it at right angles.

For the cosines of the angles made by $O P$ with the semiaxes are directly as the cosines of the angles made by $O Q$ with them, and inversely as the squares of $a, b, c$ (Lem. $1, C o r$.) ; and the cosines of the angles made with the semiaxes, by a perpendicular to the tangent plane at $R$, are directly as the cosines of the angles made with them by $O P$, and inversely as the squares of $a^{\prime}, b^{\prime}, c^{\prime}$, or as the same cosines and the squares of $a, b, c$, directly; that is, simply, as the cosines of the angles made by $O Q$ 
with the semiaxes. Hence $O Q$ coincides with the perpendicular to the tangent plane at $R$.

4. If a perpendicular at $O$ to the plane $P O Q$ meet the surface of the ellipsoid $a b c$ in $q$, then will $O Q$ and $O q$ be the semiaxes of the section $Q O q$ made by a plane passing through them.

For the tangent plane at $Q$ and the plane $Q O q$ are perpendicular to $P O Q$, and therefore the intersection of the two former, which is a tangent to the ellipse $Q O q$ at $Q$, is perpendicular to $O Q$; whence $O Q$ is one semiaxis, and $O q$ the other.

If the perpendicular $O q$ meet the other ellipsoid in $r$, then $O R$ and $O r$ will be the semiaxes of the section $R O r$ made by a plane passing through them; for (by lem. 3), the straight line $O Q N$ is perpendicular to the tangent plane at $R$.

5. In a straight line, at right angles to any diametral section $Q O q$ of the ellipsoid $a b c$, let $O T$ and $O V$ be taken equal to $O Q$ and $O q$, the semiaxes of the secticis, and imagine the double surface which is the locus of all the points $T$ and $V$; then if $O S$ be perpendicular to the plane which touches this surface in $T$, and $O P$ to that which touches the ellipsoid in $Q$, the lines $O P$ and $O S$ will be equal and perpendicular to each other, and the four, $O P, O Q, O S, O T$, will lie in the same plane, which will be at right angles to $O q$.

By the preceding lemma it is evident that $O q$ is perpendicular to the plane $P O Q$; and since $O T$ is perpendicular to the plane $Q O q$, it follows that $O P$, $O Q, O T$, are in the same plane at right angles to $O q$. In the surface which is the locus of $T$, and in the plane $T O q$, let a point $T$, be taken indefinitely near to $T$; then the plane of the section at right angles to $O T$, will pass through $O Q$, and will have one of its semiaxes (that to which $O T$, is equal) indefinitely near to $O Q$, and therefore differing from $O Q$ by an indefinitely small quan-

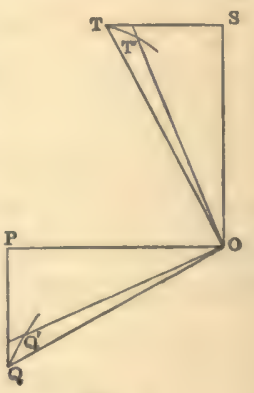

Fig. 3. tity of the second order, adopting, for brevity, the language of 
infinitesimals. To see this, it is only necessary to recollect that, by the property of maxima and minima, the semiaxis differs from any semidiameter indefinitely near to it, such as $O Q$, by an indefinitely small quantity of the second order. Hence, since $O T$ is equal to $O Q$, and $O T$, to the above-mentioned semiaxis, it follows that $O T$ and $O T$, differ by an indefinitely small quantity of the second order, and that therefore the angle $O T T$, is ultimately a right angle: consequently the tangent to the curve in which the plane $T O q$ intersects the locus of $T$ is perpendicular to the plane PQOT. But the tangent plane at $T$ passes through this tangent, and therefore the perpendicular $O S$ to the tangent plane must lie in the plane PQOT.

Again, let a point $T^{\prime}$ indefinitely near to $T$, and in the plane $P Q O T$, be taken in the surface which is the locus of $T$, and let the plane of the section which is perpendicular to $O T^{\prime}$ intersect the plane $P Q O T$ in the straight line $O Q^{\prime}$ which meets the ellipsoid in $Q^{\prime}$. Then that semiaxis of the section to which $O T^{\prime}$ is equal will be indefinitely near to $O Q^{\prime}$, and will therefore differ from it by an indefinitely small quantity of the second order. Hence, since $O T$ is equal to $O Q$, the angle $O T T^{\prime}$ will be ultimately equal to $O Q Q^{\prime}$; and therefore, $T S$ and $Q P$ being tangents, the angles $O T S$ and $O Q P$ are equal. But $O T=O Q$, and the angles $P$ and $S$ are right; therefore $O S=O P$, and the angle $S O T=P O Q$; whence $S O P=T O Q=$ a right angle .

Similarly, if one perpendicular be let fall from $O$ on a plane touching the locus in $V$, and another on the plane touching the ellipsoid in $q$, it may be proved that the two perpendiculars are equal and at right angles to each other, and that, with the lines $O V$ and $O q$, they lie in a plane at right angles to $O Q$.

6. An ellipsoid being cut by any plane through its centre, the difference between the squares of the reciprocals of the semiaxes of the section is proportional to the rectangle under the sines of the angles which the plane of the section makes with the planes of the two circular sections of the ellipsoid.-(See Fresnel's Memoir, p. 150.) 


\section{The Double Refraction of Light in a Crystallized}

Let the plane which cuts the ellipsoid intersect its circular sections in the lines $O R^{\prime}, O S^{\prime}$, and let the prineipal section $A O C$ of the ellipsoid cut the circular sections in $O R$ and $O S$; then $O R, O S, O R$, $O S^{\prime}$, will be all equal to the mean semiaxis $O B$, and hence the semiaxes $O A^{\prime}, O C^{\prime}$ of the section $A^{\prime} O C^{\prime}$ will bisect the acute and obtuse angles made by $O R^{\prime}$ and $O S^{\prime}$. Let a plane through $O B$ and $O A^{\prime}$ intersect the prineipal plane $A C O$ in the line $O T$. Then, by the nature of the ellipse, we have, in the ellipse $A^{\prime} O C^{\prime}$,
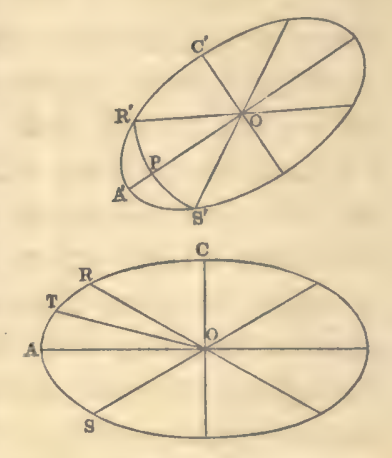

Fig. 4.

$$
\frac{1}{O R^{\prime 2}}-\frac{1}{O A^{\prime 2}}=\left(\frac{1}{O C^{12}}-\frac{1}{O A^{\prime 2}}\right) \sin ^{2} A^{\prime} O R^{\prime} ;
$$

and in the ellipse BOT,

$$
\frac{1}{O B^{2}}-\frac{1}{O A^{\prime 2}}=\left(\frac{1}{O B^{2}}-\frac{1}{O T^{2}}\right) \sin ^{2} B O A^{\prime} .
$$

Hence, observing that $O B$ and $O R^{\prime}$ are equal, we have

$$
\frac{1}{O C^{\prime 2}}-\frac{1}{O A^{\prime 2}}=\left(\frac{1}{O B^{2}}-\frac{1}{O T^{2}}\right) \frac{\sin ^{2} B O A^{\prime}}{\sin ^{2} A^{\prime} O R^{\prime \prime}}
$$

But the ellipse $O A C$ gives

Therefore

$$
\begin{array}{r}
\frac{1}{O R^{2}}-\frac{1}{O T^{2}}=\left(\frac{1}{O C^{2}}-\frac{1}{O A^{2}}\right)\left(\sin ^{2} A O R-\sin ^{2} A O T\right) \\
=\left(\frac{1}{O C^{2}}-\frac{1}{O A^{2}}\right) \times \sin R O T \sin S O T .
\end{array}
$$

$$
\frac{1}{O C^{\prime^{2}}}-\frac{1}{O A^{\prime 2}}=\left(\frac{1}{O C^{2}}-\frac{1}{O A^{2}}\right) \frac{\sin ^{2} B O A}{\sin ^{2} A^{\prime} O R^{\prime}} \sin R O T \sin S O T,
$$

because $O B$ and $O R$ are equal. 
Imagine a sphere described from the centre $O$ with a radius equal to $O B$, and passing through $R^{\prime}$ and $S^{\prime}$, and cutting $A^{\prime} O$ in $P$. The sides $B P$ and $P R^{\prime}$ of the spherical triangle $B P R^{\prime}$ subtend the angles $B O A^{\prime}$ and $A^{\prime} O R^{\prime}$ at the centre; and its angle $P B R^{\prime}$ is equal to the angle $R O T$; whence the sines of the sides being proportional to the sines of the opposite spherical angles, it follows that

$$
\frac{\sin B O A^{\prime}}{\sin A^{\prime} O R}, \sin R O T
$$

is equal to the sine of the spherical angle $B R^{\prime} P$, which is the angle made by the section $A^{\prime} O C^{\prime}$ with the plane of the circular section $B O R$. Similarly, by means of the spherical triangle $B P S^{\prime}$, it may be shown that

$$
\frac{\sin B O A^{\prime}}{\sin A^{\prime} O S^{\prime \prime}} \sin S O T
$$

is equal to the sine of the angle made by the plane $A^{\prime} O C^{\prime}$ with the plane of the circular section BOS. Therefore, since the angles $A^{\prime} O R^{\prime}$ and $A^{\prime} O S^{\prime}$ are equal, it follows that

$$
\frac{1}{O C^{\prime 2}}-\frac{1}{O A^{\prime 2}} \text { is equal to } \frac{1}{O C^{2}}-\frac{1}{O A^{2}}
$$

multiplied by the product of the sines of the angles which the plane $A^{\prime} O C^{\prime}$ makes with the planes of the two cireular sections.

I shall now demonstrate a geometrical construction for finding the magnitude and direction of the elastio foree arising from a displacement in any direction-a construotion which, with the help of the preceding lemmas, will lead us immediately to all the conclusions established by Fresnel.

Let $O$ be the position of a point in the medium when quiescent, and let three rectangular axes passing through it, and fixed in space, be taken for the axes of co-ordinates. For a small displacement in the direction of $x$, let the elastio forces, excited in the directions of $x, y, z$, be $a, b, c$; for an equal displacement in the direction of $y$ let the forces be $a^{\prime}, b^{\prime}, c^{\prime}$; and for the same 
in the direction of $z$ let them be $a^{\prime \prime}, b^{\prime \prime}, c^{\prime \prime}$. Then, if a point receive an equal displacement in a direction $O I$ making with $O X, O Y, O Z$, the angles $a, \beta, \gamma$, the forees in the direction of $x, y, z$ (denoting then by $X, Y, Z$, respectively), will be

$$
\begin{aligned}
& X=a \cos \alpha+a^{\prime} \cos \beta+a^{\prime \prime} \cos \gamma, \\
& \boldsymbol{Y}=b \cos a+b^{\prime} \cos \beta+b^{\prime \prime} \cos \gamma, \\
& Z=c \cos a+c^{\prime} \cos \beta+c^{\prime \prime} \cos \gamma,
\end{aligned}
$$

as follows from considering (see Fresnel's Memoir, p. 82) that the force arising from a displacement in any direction is the resultant of the forces arising from the three displacements in the directions of $x, y, z$, which are the statical components of that displacement. But since (p. 90) the force in the direction of one of the axes, arising from a displacement in the direction of another, is equal to the force in the direction of the latter, arising from an equal displacement in the direction of the former, it follows that $a^{\prime}=b, a^{\prime \prime}=c, b^{\prime \prime}=c^{\prime}$; and hence

$$
\begin{aligned}
& X=a \cos a+b \cos \beta+c \cos \gamma, \\
& Y=b \cos a+b^{\prime} \cos \beta+c^{\prime} \cos \gamma, \\
& Z=c \cos a+c^{\prime} \cos \beta+c^{\prime \prime} \cos \gamma .
\end{aligned}
$$

Let $a x^{2}+b^{\prime} y^{2}+c^{\prime \prime} z^{2}+2 c^{\prime} y z+2 c z x+2 b x y=1$ be the equation of a surface of the second order. Let $O I$ intersect it in $I$, the co-ordinates of $I$ being $x^{\prime}, y^{\prime}, z^{\prime}$; then the equation of the tangent plane at $I$ will be, by the known formulæ

$$
\left(a x^{\prime}+b y^{\prime}+c z^{\prime}\right) x+\left(b x^{\prime}+b^{\prime} y^{\prime}+c^{\prime} z^{\prime}\right) y+\left(c x^{\prime}+c^{\prime} y^{\prime}+c^{\prime \prime} z^{\prime}\right) z=1 \text {. }
$$

Put $O I=r$, and let the tangent plane intersect $O X, O Y, O Z$, in the points $P, Q, R$; then from this equation we have

$$
\begin{aligned}
& \frac{1}{O P}=a x^{\prime}+b y^{\prime}+c z^{\prime}=r(a \cos a+b \cos \beta+c \cos \gamma)=r \cdot X, \\
& \frac{1}{O Q}=b x^{\prime}+b^{\prime} y^{\prime}+c z^{\prime}=r\left(b \cos a+b^{\prime} \cos \beta+c^{\prime} \cos \gamma\right)=r Y, \\
& \frac{1}{O R}=c x^{\prime}+c^{\prime} y^{\prime}+c^{\prime \prime} z^{\prime}=r\left(c \cos a+c^{\prime} \cos \beta+c^{\prime \prime} \cos \gamma\right)=r Z .
\end{aligned}
$$


Now if $p$ be the length of the perpendicular let fall from $O$ on the tangent plane, the cosines of the angles which this perpendicular makes with the axes of co-ordinates will be equal to

$$
\frac{p}{O P}, \frac{p}{O Q}, \frac{p}{O R},
$$

respectively, that is, to $p r \bar{X}, p r \bar{Y}, \operatorname{pr} Z$; and since the sum of the squares of these cosines is equal to unity, we have

$$
p r \sqrt{X^{2}+\bar{Y}^{2}+Z^{2}}=1 .
$$

Hence it appears that the perpendicular let fall from $O$ on the tangent plane is parallel to the direction of the resultant elastic force, and that the magnitude of the resultant is expressed by $\frac{1}{p r}$.

From this conclusion we may, with the greatest facility, deduce several corollaries.

$1^{\circ}$. Since the elastic force is supposed finite, whatever be the direction of the displacement, it is manifest that the above-mentioned surface of the second order must be an ellipsoid, and that when the displacement is in the direction of any of the three axes of the ellipsoid, the elastic force excited will be in the direction of the same axis, because the tangent plane will be perpendieular to it. Hence the remarkable consequence, that there are ahways three axes of elasticity at right angles to each other.-(Memoir, p. 93.) Also the elasticities arising from equal displacements in the directions of the three axes are inversely as the squares of the axes; and hence, the positions of the axes and the elasticities in their respective directions being given, the ellipsoid may be constructed.

$2^{\circ}$. The ellipsoid being thus constructed, the direction of the elastic force, arising from a displacement in the direction of any of its semidiameters, will be parallel to the normal at the extremity of that semidiameter; and for equal displacements the magnitude of the foree will be inversely as the rectangle under the semidiameter and the perpendicular from the centre on the tangent plane at its extremity. If the displacements are pro- 


\section{IO \\ The Double Refraction of Light in a Crystallized}

portional to the semidiameters, the elastic forces will be both parallel and proportional to the normals; for the normal, terminated by any of the principal planes, is inversely as the perpendicular on the tangent plane.

$3^{\circ}$. If the elastic force be resolved into two, one parallel and the other perpendicular to the direction of the displacement, the former will be inversely as the square of the semidiameter in the direction of the displacement.

$4^{\circ}$. If the ellipsoid be cut by a plane through its centre, and if the elastic force arising from a displacement in the direction of either axis of the section be resolved parallel and perpendicular to that axis, the part perpendicular to the axis will also be perpendicular to the plane of the section. For (by Lem. 4) the plane passing through one axis and the perpendicular to the tangent plane at its extremity, is perpendicular to the other axis, and therefore to the plane of the section. But if the displacement be in the direction of any other diameter of the section, the elastic force, resolved perpendicularly to that diameter, will be oblique to the plane of the section.

To apply these things to the double refraction of light in a crystallized medium, imagine the ellipsoid to be described. as above, and let it be cut through its centre by a plane parallel to that of a plane wave of light incident on the crystal; then if the vibrations of the light be parallel to either of the axes of the section, the plane containing the direction of the vibrations and that of the elastic force arising from them will be perpendicular to the plane of the wave (No. 4, preceding); and therefore, according to Fresnel's theory, the direction of the vibrations will remain parallel to itself, while the wave is propagated. But if the light be common light, or if it be polarized, and the plane of polarization be not perpendicular to either of the axes of the section, the wave will be divided into two others having the directions of their vibrations parallel to the semiaxes of the elliptic section, and their planes of polarization perpendicular to them: their velocities of propagation-measured in a direction perpendicular to their plane-will be different, and 
will be in the sub-duplicate ratio of the elasticities in the direction of their respective vibrations, and therefore (by No. 3) inversely as the semiaxes of the section to which those vibrations are parallel.

If a wave be propagated in all directions from an origin $O$ within a crystal, its surface will at each instant be touched by the simultaneous position of a plane wave which passed through $O$ at the instant when the former began to be propagated (Memoir, p. 127). Hence, to find the surface of the double wave in a crystal, let the above-mentioned ellipsoid be cut by any plane through its centre $O$, and imagine two other planes parallel to this section, and at distances from it which are third proportionals to its semiaxes, $O R, O r$, and any given line $k$ : the double surface which touches these planes in all their positions will be the surface of the wave.

Now conceive another concentric ellipsoid, having the directions of its semiaxes the same, but their lengths $a, b, c$, inversely proportional to those of the former, so that the rectangle under any coinciding pair of semiaxes is equal to $k^{2}$ : then if a plane, touching this second ellipsoid in $Q$, cut $O R$ perpendicularly in $P$, the line $O P$ will be a third proportional to $O R$ and $k$ (Lem. 2); and if $\mathrm{Or}$ intersect the second ellipsoid in $q$, the semiaxes of the section $Q O q$ will be $O Q$ and $O q$ (Lem. 4). Draw $O T$ perpendicular to $Q O q$ and equal to $O Q$, and conceive the surface which is the locus of the point $T$ to be described, and a tan-

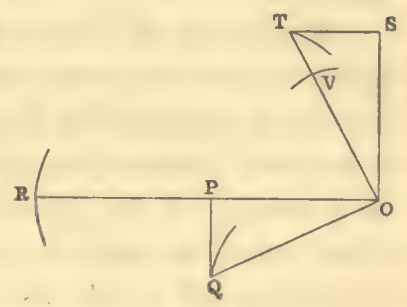

Fig. 5. gent plane, to which the line $O S$ is drawn perpendicular, to be applied at the point $T$. Then $O S$ will be perpendicular to the plane $R O r$ and equal to $O P$ (Lem. 5): and hence the point $T$ always lies in the surface of the wave. Similar things may be proved with respect to the other semiaxis $O r$ of the ellipse $R O r$. Hence we deduce the following construction for the surface of the wave :- 


\section{2 The Double Refraction of Light in a Crystallized}

"Describe an ellipsoid whose axes are in the directions of the axes of elasticity, the squares of their lengths being directly as the elasticities in their respective directions; cut the ellipsoid by a plane through its centre, as $Q O q$, and in a perpendicular to that plane take two portions $O T$ and $O V$ equal to the semiaxes $O Q$ and $O q$ of the section. The double surface which is the locus of the points $T$ and $V$ is the surface of the wave."

As to the planes of polarization of the rays belonging to the two parts of the wave, Fresnel has shown how to find them by means of a surface which he calls the surface of elasticity. But it is desirable to be able to find them by means of the same ellipsoid which serves to find the surface of the wave. Now TS, parallel to $O R$, is the direction of the vibrations of the ray $O T$, and the tangent plane at $Q$ is perpendicular to $O R$, and therefore parallel to the plane of polarization of the ray OT. In like manner the plane of polarization of the ray $O V$ is parallel to the tangent plane at $q$. Hence the planes of polarization of two different rays, having a common direction, are parallel to the planes which touch the ellipsoid at the extremities of the semiaxes of the diametral section perpendicular to their common direction.

When two of the axes are equal, the ellipsoid becomes a spheroid, and the crystal is said to be uniaxal, the double refraction being regulated by the third axis which is perpendicular to their plane. Let $A O B$ be a section of the spheroid through the third axis $O A$, which is its axis of revolution ; take $O B^{\prime}=O B$, and $O A^{\prime}=O A$, and let the ellipse $A^{\prime} O B^{\prime}$ and the circle $B O B^{\prime}$ revolve about $O B^{\prime}$ as an axis; they will describe a surface compounded of a sphe-

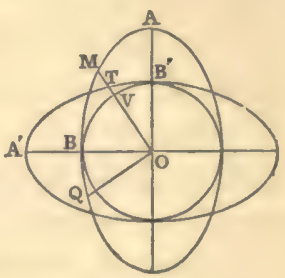

Fig. 6. roid and sphere, which will in this case be the surface of the double wave. For if $O M$ be the direction of a ray, and if a plane perpendicular to $O M$ cut the ellipse $A O B$ in $O Q$, and the equator of the spheroid in $O q$, the lines $O Q$ and $O q$, of which the latter is equal to $O B$, will be the semiaxes of the section 
QOq. Taking, therefore, $O T$ and $O V$ equal to $O Q$ and $O B$, the locus of $V$ will evidently be the circle $B O B^{\prime}$; and the locus of $T$ will be the ellipse $A^{\prime} O B^{\prime}$, since the angle $B^{\prime} O T^{\prime}$ is equal to the angle $B O Q$.

In the general case, $O T$ and $O V$, which are equal to $O Q$ and $O q$, represent the velocities of the two different sorts of rays having a common direction in the crystal ; and two right lines, which lie in the plane of the greatest and least axes of the ellipsoid, and are perpendicular to its two circular sections, are called the optio axes. It appears, therefore, by the 6th Lemma, that the difference of the squares of the reciprocals of the velocities of the two rays, having a common direction in the crystal, is proportional to the product of the sines of the angles which that direction makes with the optic axes. This is the celebrated law of M. Biot, to which he was led by analogy, and which he afterwards found to agree with that previously laid down by Dr. Brewster.

Such, in their simplest form, are the principal features of the Mechanical Theory of Double Refraction, invented by the late M. Fresnel - a theory which would do honour to the sagacity of Newton, and which gives us ample reason to regret that the life of its author was not longer spared, to enrich with further discoveries his favourite science. Of him it may be said, as Newton said of Cotes-and apparently with much greater reasonthat if he had lived longer, we should have known something at last of the laws of nature. 


\section{II.-ON THE INTENSITY OF LIGHT WHEN THE VIBRA- TIONS ARE ELLIPTICAL.}

[Edinburgh Journal of Science, April, 1831.]

According to the opinions commonly received, the intensity of light, in the undulatory hypothesis, is proportional to the vis viva, which again is proportional to the square of the greatest velocity. Now the greatest velocity will be the same in an ellipse and a right line which have the same period, if the greater axis of the former be equal to the whole extent of the latter; so that in elliptic vibrations the intensity would be independent of the minor axis, which is far from being true. I would propose the integral $S v^{2} d t$-so remarkable for its mechanical properties - as the measure of the intensity, the integral being extended to the whole time of a vibration. This gives precision to the notion of vis viva, and leads, moreover, to an elegant result; for if $a$ and $b$ denote the semiaxes of the ellipse, and $T$ the time of vibration, the integral, by an easy calculation, will be found equal to $\frac{2 \pi^{2}}{T}\left(a^{2}+b^{2}\right)$, showing that for the same colour the intensity is proportional to the sum of the squares of the semiaxes, and that for different colours it increases with the rapidity of the vibrations, as it would be natural to suppose a priori.

This theorem assigns very simply the reason why two portions of light polarized at right angles do not interfere; but to 
show this it will be necessary to lay down the following general rule for compounding rectilinear vibrations having the same period, whatever be the difference of their origin and direction :-

Let $A A^{\prime}$ and $B B^{\prime}$ (Fig. 7), bisecting each other at $O$, represent in extent and direction the vibrations to be compounded, and suppose $C$ and $D$ to be two simultaneous positions of the moving molecule, which it would have in virtue of each vibration singly. Complete the parallelograms $O E$ and $O P$, and through $P$ describe an ellipse having $O$ for its centre, and touching the sides of

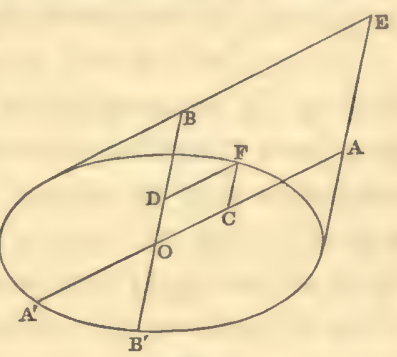

Fig. 7. the parallelogram $O E$; this ellipse will represent the resulting vibration; it will have the same period as the compound one, and equal areas will be described in equal times about its centre.

To apply this construction to the case proposed, it is necessary to show that when $O A$ and $O B$ are constant and at right angles to each other, the intensity of the elliptic vibration, or the sum of the squares of the semiaxes, is independent of the difference of origin, or of the position of the points $C$ and $D$.

Now in an ellipse, when a perpendicular from the centre on a tangent makes an angle $\phi$ with the major axis, the square of its length is equal to $a^{2} \cos ^{2} \phi+b^{2} \sin ^{2} \phi$. If $\phi$ be the angle which the major axis makes with $O A$, it will make its complement with $O B$, and we shall have

and therefore

$$
\begin{aligned}
& O A^{2}=a^{2} \cos ^{2} \phi+b^{2} \sin ^{2} \phi, \\
& O B^{2}=a^{2} \sin ^{2} \phi+b^{2} \cos ^{2} \phi,
\end{aligned}
$$

$$
O A^{2}+O B^{2}=a^{2}+b^{2} \text {. }
$$

Hence the intensity is independent of the difference of origin, and therefore the rays do not interfere.

This remarkable eircumstance is commonly accounted for by 
observing, that since the component velocities are at right angles to each other, the square of the actual velocity must be the sum of their squares; but this proves the proposition only when the greatest velocities are simultaneous, which happens only in the cases of a complete accordance or of a difference of a semiundulation in the interfering portions.

It may be observed also, that the method usually given for finding the intensity of the vibration resulting from two or more rectilinear vibrations proceeds upon no certain grounds. All the vibrations may be reduced to two in rectangular directions, the expression for the square of the velocity in each of their directions consisting of two parts, one of which is constant, and the other depends on the cosine of an are increasing proportionally to the time: the square of the resultunt velocity, therefore, consists also of a constant part, and a part depending similarly on the time; and it is assumed that the intensity of the resulting vibration is proportional to the former, which is true only in the very particular cases just mentioned, if the intensity be measured by the square of the greatest velocity.

This assumption, however, gives a correct result; and the reason that it does so is obvious from the principle laid down in the commencement; for if the expression for the square of the velocity be multiplied by the differential of the time and integrated, the variable parts will vanish when the integral is extended to the whole time of a vibration. 
III.-NOTE ON THE SUBJECT OF CONICAL REFRACTION.

[From the Philosophical Magazine, VoL. III., 1833.]

When Professor Hamilton announced his discovery of Conical Refraction, he did not seem to have been aware that it is an obvious and immediate consequence of the theorems published by me, three years ago, in the Transactions of the Royal Irish Academy, vol. xvi., pt. ii., p. 65, \&c. The indeterminate cases of my own theorems, which, optically interpreted, mean conical refraction, of course occurred to me at the time; but they had nothing to do with the subject of that Paper; and the full examination of them, along with the experiments they might suggest, was reserved for a subsequent essay, which I expressed my intention of writing. Business of a different nature, however, prevented me from following up the inquiry.

I shall suppose the reader to have studied the passage in pp. 75,76 , of the volume referred to. $\mathrm{He}_{\Theta}$ will see that when the section of either of the two ellipsoids employed there is a circle, the semiaxes-answering to $O R, O r$, and to $O Q, O q$, in the general statement*-are infinite in number, giving of course an infinite number of corresponding rays. And this is conical refraction. I shall add a few words on the two cases :-

1. When $R O r$ is a circle, any two of its rectangular radii may be taken for $O R$ and $O r$. The line $O S$ and the tangent plane perpendicular to it at $S$ are fixed; but the point of con-

* The right line Oqr is perpendicular to the plane of the figure, and intersects the two ellipsoids in $q$ and $r$. 
tact $T$ is variable, for the plane ROS in which it lies changes with $O R$. Thus we get a ourve of contact on the tangent plane of the wave surface, and a cone of rays $O T$ derived from the same incident ray. The vibrations of any ray $O T$ are in the line $T S$ passing through the fixed point $S$, as follows from a general remark in the place referred to.

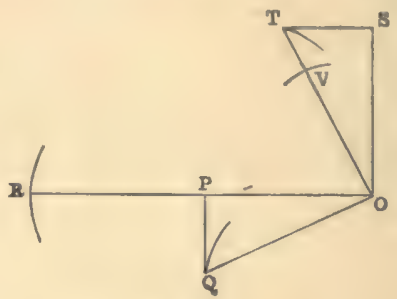

Fig. 8.

The three right lines $O Q, O r, O T$, are at right angles to each other, and a geometer will observe that the first two of them are confined to given planes. For $O r$ is always in the plane of the circle $R O r$; and the point $Q$ must be in a given plane, because the line $O P$, perpendicular to the plane that touches the ellipsoid in $Q$, is in a given plane $R O r$.

2. When $Q O q$ is a circle, the points $T$ and $V$ coincide in a nodal point $n$, where the two sheets of the wave surface cross each other. At this point there are an infinite number of tangent planes, for $O Q$ and $O q$ are now indeterminate. The same refracted ray $O n$ may therefore be derived from any one of an infinite number of incident rays, and its polarization will differ accordingly; for the vibrations are in the line $n S$ drawn from the node to the foot of the perpendicular $O S$ on the tangent plane. The ray $O n$, however, is always accompanied by another, but variable, refracted ray.

The lines, $O P, O q, O S$, are at right angles to each other, and the first two of them are confined, as before, to given planes. For $O q$ is in the plane of the circle $Q O q$; and $O P$, being perpendicular to the tangent plane at $Q$, must lie in a given plane. These given planes are parallel to two principal tangent planes passing through $n$, and touching the circle and ellipse that compose the wave section in the plane of the nodes: whence it is easy to see that every nodal tangent plane intersects the two principal tangent planes in lines that are constantly at right angles; for these lines are parallel to $O P$ and $O q$. 
The examination of both cases is completed by the following theorem :-

When three right lines at right angles to each other pass through a fixed point, in such a manner that two of them are confined to given planes, the plane of these two, in all its positions, touches the surface of a cone whose sections parallel to the given planes are parabolas ; while the third right line describes another cone, whose sections parallel to the same planes are circles.

The application is obvious. We see that the curve of contact in the first case is a circle. The points $S$ in the second case are also in a circle.

Note on the above, addressed by Professor Mac Cullagh to the Edtrors of the Philosophicat Magazine.-VoL, mi. 1833.

THE introductory part of my Note which appeared in your last Number was written in haste, and I have reason to think it may not be rightly understood. You will therefore allow me to add a few observations that seem to be wanting.

The principal thing pointed out in the Paper published some time ago in the Transactions of the Royal Irish Academy is a very simple relation between the tangent planes of Fresnel's Wave Surface and the sections of two reciprocal ellipsoids. Now this relation depends upon the axes of the sections, and therefore naturally suggested to me the peculiar cases of circular sections in which every diameter is an axis. Thus a new inquiry was opened to my mind. And accordingly, without caring just then to obtain final results, which seemed to be an easy matter at any time, I expressed in conversation my intention of returning to the subject of Fresnel's theory in a supplementary Paper. The design was interrupted, and I was prevented from attending to it again, until I was told that Professor Hamilton had discovered cusps and circles of contact on the wave surface. This reminded me of the cases of circular section, and the details given in my last note were immediately deduced. 


\section{IV.-GEOMETRICAL PROPOSITIONS APPLIED TO THE WAVE THEORY OF LIGHT.}

[Transuctions of the Royal Irish Academ! Vou. xvil--Read June 24, 1833.]

\section{Part I.-Geometrical Propositions.}

1. Theorem I.-Conceive a curved surface $B$ to be generated from a given eurved surface $A$ in the following manner : having assumed a fixed origin $O$, apply a tangent plane at any point $Q$ of the given surface, and perpendieular to this plane draw a right line $O P R$ eutting the plane in $P$, and terminated in $R$, so that $O P$ and $O R$ may be reciprocally proportional to each other, their rectangle being equal to a

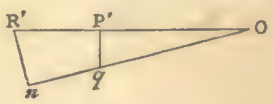

Fig. 9. constant quantity $k^{2}$, and let all the points $R$ taken according to this law generate the second surface $B$. Then the relation between these two surfaces, and between the points $Q$ and $R$, will be reciprocal; that is to say, if a tangent plane be applied at the point $R$ of the second surface, a perpendicular $O N$ to this plane will pass through the point $Q$ of the first surface, and $O N$ and $O Q$ will be reciprocally proportional to each other, the rectangle under them being also equal $k^{2}$.

2. To prove this theorem, take a point $q$, in the tangent plane of the surface $A$, and near the point of contact $Q$ (Fig. 9). Through $q$ let several other planes be drawn touching the surface $A$ in points $Q^{\prime}, Q^{\prime \prime}, Q^{\prime \prime \prime}, \&$ \&., and draw the perpendiculars $O P^{\prime} R^{\prime}, O P^{\prime \prime} R^{\prime \prime}, O P^{\prime \prime} R^{\prime \prime \prime}$, \&c., according to the same law as $O P R$. The points $R, R^{\prime}, R^{\prime \prime}, R^{\prime \prime}, \&$ \&., will thus be upon the second surface $B$, and they will moreover be all in the same 
plane ; for from any one of them $R^{\prime}$ let $R^{\prime} n$ be drawn perpendicular to the right line $O q$ and meeting $O q$ in $n$; then, on account of the similar right-angled triangles $O P^{\prime} q$ and $O n R^{\prime}$, the rectangle $n O q$ will be equal to the rectangle $R^{\prime} O P^{\prime}$, or to the constant quantity $k^{2}$, so that the point $n$, or the foot of the perpendicular let fall upon $O q$, will be the same for all the points $R, R^{\prime}, R^{\prime \prime}, R^{\prime \prime \prime}, \&$ \&., and consequently all these points will lie in a plane cutting the right line $O q n$ perpendicularly in $n$, so as to make the rectangle $n O q$ equal to $k^{2}$. Now, while the point $Q$ remains fixed, let the point $q$ approach to it without limit in the tangent plane at $Q$; and the points $R^{\prime}, R^{\prime \prime}, R^{\prime \prime \prime}$, \&c., will in like manner approach without limit to the fixed point $R$; the plane which contains all those neighbouring points having for its limiting position the tangent plane at $R$. Also the point $n$ will ultimately coincide with $N$. It follows, therefore, that the tangent plane at $R$ euts the right line $O Q$ perpendicularly in $N$, so as to make the rectangle $N O Q$ equal to $k^{2}$.

3. Corollary.-If any point $Q$ upon the surface $A$ should be a point of intersection, where the surface admits an infinite number of tangent planes, the perpendiculars from $O$ upon these planes will form a conical surface having $O$ for its vertex. In $O Q$ take, as before, a point $N$, so that $O N \times O Q=k^{2}$, and let a plane passing through $N$ at right angles to $O Q$ cut the conical surface. The interseetion will be a certain curve. From the preceding demonstration it is evident that every point of this curve belongs to the surface $B$, and that the plane which touches this surface at any point of the curve cuts $O Q$ perpendicularly in $N$; or, in other words, that the same plane touches the surface $B$ through the whole extent of the curve.

4. Two surfaces related to each other like $A$ and $B$ in the preceding theorem may be called reciprocal surfaces, and points like $Q$ and $R$ reciprocal points; the radii $O Q$ and $O R$ may likewise be termed reciprocal. A familiar example of such surfaces is afforded, as I have shown on a former oceasion, ${ }^{*}$ by two ellip-

* Transactions of the Rnyal Irish Academy, Vol. xvi., pt. ii., pp. 67,68.—supra, p. 3. 
soids having a common centre at the point $O$, and their semiaxes coincident in direction, and connected by the relation $a a^{\prime}=b b^{\prime}=c c^{\prime}=k^{2}$; where $a, b, c$, are semiaxes of one ellipsoid in the order of their magnitude, $a$ being the greatest ; and $a^{\prime}, b^{\prime}, c^{\prime}$, those of the other ellipsoid, $a^{\prime}$ being the least. The mean semiaxes $b$ and $b^{\prime}$ coincide, and the circular sections of both ellipsoids pass through the common direction of $b$ and $b^{\prime}$.

5. It has also been shown with regard to those ellipsoids, that if $Q$ and $R$ be reciprocal points on the surfaces of $a b c$ and $a^{\prime} b^{\prime} c^{\prime}$ respectively, and if a right line $O q r$, perpendicular to the plane $Q O R$, cut the first ellipsoid in $q$ and the second in $r$, the lines $O Q$ and $O q$ will be the semiaxes of the section made in the ellipsoid $a b c$ by a plane passing through them; and the lines $O R$ and $O r$, in like manner, will be the semiaxes of the section made in the other ellipsoid $d^{\prime} b^{\prime} c^{\prime}$ by the plane in which they lie.

6. It may further be remarked, that if the radius $O Q$ in one of the reciprocal ellipsoids describe a plane, the corresponding radius $O R$ will describe another plane: For the planes touching the ellipsoid $a b c$ in the points $Q$ will all be parallel to a certain right line, and therefore the perpendiculars $O R$ to these tangent planes will all lie in a plane perpendicular to that right line. These two planes, containing the reciprocal radii, may, for brevity, be called reciprocal planes.

When two reciprocal radii lie in a principal plane, at right angles to a semiaxis of the ellipsoids, it is evident that two planes intersecting in this semiaxis, and passing through the reciprocal radii, are reciprocal planes.

7. Theorem II.-If three right lines at right angles to each other pass through a fixed point $O$, so that two of them are confined to given planes, the plane of these two, in all its positions, touches the surface of a cone whose sections parallel to the given planes are parabolas; while the third right line deseribes another cone, whose sections parallel to the given planes are circles.

Let the plane of the figure (Fig. 10), supposed parallel to one of the given planes, be intersected by the other given plane in the right line $M N$; and let $O Q$ be perpendicular to the latter 
plane, while $O P$ is perpendicular to the former and to the plane of the figure, so that $P Q$ being joined will meet $M N$ at right angles in $R$. Let $O A, O B, O C$, be the three perpendicular lines, of which $O A$ is parallel to the plane of the figure; this plane will be intersected by the plane of $O A$ and $O B$ in a right line $B T$ parallel to $O A$, and therefore perpendicular to both $O B$ and $O P$, and to the plane $B O P$, and to the line $B P$. Thus the angle $P B T$ is always a right angle, and therefore $B T$ always touches the parabola whose focus is $P$ and vertex $R$; or, which

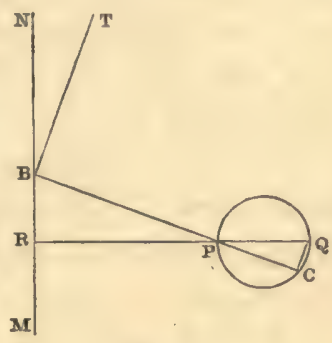

Fig. 10. comes to the same thing, the plane $A O B T$ always touches the cone which has $O$ for its vertex, and the parabola for its section.

Again, since $O B, O P, O C$, are all at right angles to $O A$, they are in the same plane, and therefore the points $B, P, C$, are in the same straight line; and as $B O C$ is a right angle, the rectangle under $B P$ and $P C$ is equal to the square of the perpendicular $O P$; but $Q O R$ is also a right angle, and therefore $Q P \times P R=O P^{2}$; whence $B P \times P C=Q P \times P R$, and therefore the points $B, R, C, Q$, are in the circumference of a circle, so that the angle at $C$ is a right angle, being in the same segment with the angle at $R$. Thus the point $C$ describes the circle whose diameter is $P Q$, and $O C$ describes the cone of which this circle is the section.

8. Of the two right lines $O P$ and $O Q$ perpendicular to the given planes, one is also perpendicular to the plane of the section. That one is $O P$. Its extremity $P$ is the foous of the parabola. The extremities of both are the extremities of the diameter $P Q$ of the circle. The vertex of the parabola is the point $R$, where the diameter of the circle intersects that given plane to which the plane of section is not parallel.

9. Theorem III.-In a straight line at right angles to any diametral section $Q O q$ of an ellipsoid $a b c$ whose centre is $O$, let $O T$ and $O V$ be taken respectively equal to $O Q$ and $O q$, the semiaxes of the section, and imagine the double surface which is the 
locus of all the points $T$ and $V$; then if $O S$ be perpendicular to the plane which touches the surface in $T$, and $O P$ to the plane which touches the ellipsoid in $Q$, the lines $O P$ and $O S$ will be equal and perpendicular to each other, and the four straight lines $O P, O Q, O S, O T$, will lie in the same plane at right angles to $O q$

10. This theorem is taken from a former communication to the Academy.* The surface to which it relates, being the wave surface of FresseL, is one of frequent occurrence in optical inquiries, and it is therefore desirable to give it a distinctive name not derived from any physical hypothesis. I shall call it a biaxal surface, from the circumstance implied in its construction, and adopted as the definition on which the preceding theorem is founded-namely, that any pair of its coincident diameters are equal to the two axes of a central section made in the generating ellipsoid abc, by a plane perpendicular to the common direction of the two diameters. The name, perhaps, may appear the more appropriate, as it reminds us of the place which the surface holds in the optical theory of biaxal crystals.

11. Theorem IV.-The biaxal surfaces generated by two reciprocal ellipsoids are themselves reciprocal.

For if $Q$ and $R$ (Fig. 11) be reciprocal points on the two ellipsoids, $a b c$ and $a^{\prime} b^{\prime} c^{\prime}$, a tangent plane at $Q$ will cut $O R$ penpendicularly in $P$; a tangent plane at $R$ will cut $O Q$ perpendicularly in $N$; and the rectangles $R O P$ and $N O Q$ will be equal to each other and to $k^{2}$ (Art. 4). Also if the straight line Oqr, at right angles to the plane of the figure, cut the first ellipsoid in $q$ and the second in $r$, then (5) the elliptio section $Q O q$ will have $O Q$ and $O q$ for its semiaxes, and the lines $O R$ and $O r$

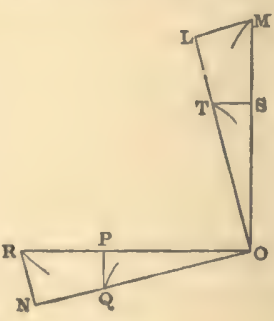

Fig. 11. will be the semiaxes of the other section $R O r$. Draw, therefore, in the plane of the figure, the right lines $O T L$ and $O S M$ penpendicular to the right lines $O Q N$ and $O P R$, making $O T, O L$,

\footnotetext{
* Transactions of the Royal Irish Academy, Vol. xvi., pt. ii., pp. 67, 68.-Supra, p. 4.
} 
$O S, O M$, equal to $O Q, O N, O P, O R$, respectively ; the angles. at $S$ and $L$ being of course right angles. Then it is evident that the point $T$ is on the biaxal surface generated by the ellipsoid $a b c$, because $O T$ is perpendicular to the plane of the ellipse $Q O q$ and equal to the semiaxis $O Q$; and by Theorem III. it appears that $O S$ is perpendicular to the tangent plane at $T$. In like manner, the point $M$ is on the biaxal surface generated by the other ellipsoid $a^{\prime} b^{\prime} c^{\prime}$, and $O L$ is perpendicular to the tangent plane at M. Moreover, the rectangles MOS and LOT, being equal to the rectangles $R O P$ and $N O Q$, are each equal to $k^{2}$. Hence the proposition is manifest.

12. As the ellipsoid whose semiaxes are $a, b, c$, may be called the ellipsoid $a b c$, so the biaxal surface generated by this ellipsoid may be called the biaxal $a b c$; and that which is generated by the ellipsoid $a^{\prime} b^{\prime} c^{\prime}$ may be called the biaxal $a^{\prime} b^{\prime} c^{\prime}$.

13. Proposition V.-To find what properties of biaxal surfaces are indicated by the cases wherein one of the two sections $Q O q, R O r$, in the preceding theorem, is a circle.

Case 1. - When $Q O q$ is a circular section of the ellipsoid $a b c$, the points $T$ and $V(9)$, in the description of the biaxal surface $a b c$, coincide in a single point $n$. At this point there are an infinite number of tangent planes; because the semiaxes of the circular section $Q O q$ being indeterminate, any two perpendicular radii of the circle may take the place of $O Q, O q$, in the general construction. The point $n$ is therefore a point of intersection (3), where the two biaxal sheets cross each other, and it may be called a nodal point, or simply a node. As $O Q$ always lies in the plane of the circle $Q O q$, the line $O R$, which is reciprocal to $O Q$, must lie (6) in a given plane reciprocal to the plane of the circle. And as $O q$ lies in the plane of the circle, we have three right lines $O R, O q, O S$, which are at right angles to each other, and of which the first two are confined to given planes. Therefore by Theorem II. the third line $O S$ describes a cone whose sections parallel to the given planes are circles. Now, $T S-$ or in the present case $n S$-is parallel to the fixed plane which contains $O R$, and therefore the point $S$ describes a circle; or, in other 
words, the feet of the perpendiculars $O S$, let fall from $O$ on the nodal tangent planes, occupy the circumference of a circle passing (8) through the nodal point.

14. Parallel to the plane of the circle and to its reciprocal plane, conceive two planes passing through the node, and call them the principal tangent planes at $n$. The plane of the circle and its reciprocal plane are intersected in the right lines $O q, O R$, by the plane $q O R$, which is parallel to a tangent plane at $n$. Consequently this tangent plane at $n$ intersects the two principal tangent planes in lines that are parallel to $O q, O R$; and as $O q$, $O R$ are perpendicular to each other, it follows that every nodal tangent plane intersects the two principal tangent planes in lines that are at right angles.

Hence again, the nodal tangent planes touch (7) the surface of a cone whose sections, parallel to the principal tangent planes, are parabolas. As this cone touches the biaxal surface all round the point $n$, it may be called the nodal tangent cone.

15. Case 2.-When ROr is a circular section of the ellipsoid $a^{\prime} b^{\prime} c^{\prime}$, any two perpendicular radii of the circle may be taken for $O R, O r$ : and because $O R=b^{\prime}$, and $O R \times O P=k^{2}=b b^{\prime}$, we have $O P$ or $O S$ equal to $b$, the mean semiaxis of the ellipsoid $a b c$. Hence OS is given both in position and length ; for it is perpendicular to the fixed plane ROr, and it is equal to $b$. Now, a plane cutting $O S$ perpendicularly at $S$ is a tangent plane to the biaxal $a b c$; and we have just seen that this tangent plane remains the same, whatever pair of rectangular radii are taken for $O R, O r$. But the point of contact $T$ is variable, for the plane $R O S$ in which it lies changes with $O R$. Therefore as $O R$ revolves, the point $T$ describes a curve of contact on the tangent plane of the biaxal $a b c$.

The lines $O R, O r$, are in the fixed plane $R O r$; and as $O Q$ is reciprocal to $O R$, it lies in a fixed plane reciprocal to the plane Ror (6). Therefore the first two of the three perpendicular right lines $O r, O Q, O T$, are confined to fixed planes. Hence the third line $O T$ describes a cone, whose sections parallel to these planes are circles. But the tangent plane is parallel to the fixed plane 


\section{to the Wave Theory of Light.}

ROr, and its intersection with $O T$ describes the curve of contact. Therefore the curve of contact is a circle passing (8) through the point $S$.

16. We have examined the two cases of circular section with reference only to the biaxal $a b c$. If we examine the same cases with regard to the second biaxal $a^{\prime} b^{\prime} c^{\prime}$, we shall find that their indications are reversed; the supposition which gives a node upon one biaxal, giving a circle of contact on the other : and that the node and the circle, thus corresponding, are so related, that a line drawn from $O$ to the node passes through the circumference of the circle, cutting the plane of the circle perpendicularly; whilst every line drawn from $O$ through the circumference of the circle is perpendicular to some nodal tangent plane.

These things are evident on looking at the figure. For when $R O r$ is a circle, it is plain that the point $M$ is a node of the biaxal $a^{\prime} b^{\prime} c^{\prime}$, since $O M$ is perpendicular to the plane of the circle $R O r$ and equal to its radius $O R$. But we have already seen (15) that when $R O r$ is a circle, the other biaxal $a b c$ has a circle of contact, whose plane is perpendicular to $O M$ at the point $S$ of its circumference. The line OTL is perpendicular, in general (11), to a tangent.plane at $M$, and therefore perpendicular, in the present case, to a nodal tangent plane; whilst the point $T$, through which it passes, is on the circle of contact. It is also evident that $O T \times O L=k^{2}$.

We have here an example of the general remark in the corollary of Theorem I.

17. The section made in the biaxal surface $a b c$, by any of the principal planes of its generating ellipsoid, consists of an ellipse and a circle.

For, let the plane $Q O q$ pass through one of the semiaxes, $a$, and let it revolve round this semiaxis, while the right line $O T V$ (9), perpendicular to the plane $Q O q$, revolves about $O$ in the plane of the semiaxes $b, c$. Then the semiaxis $a$ of the ellopsoid will always be one of the semiaxes of the ellipse $Q O q$; and if $O T$ be equal to this semiaxis, the point $T$ will describe a circle with the radius $a$ about the centre $O$. The other semiaxis of the 
ellipse $Q O q$ is that semidiameter of the principal ellipse $b c$ which lies in the intersection of the plane $b c$ with the plane $Q O q$; and as $O V$ is equal and perpendicular to this semidiameter, the point $V$ describes an ellipse equal to $b c$, but turned round through a right angle, so that the greater axis of the ellipse described by $V$ coincides in direction with the less axis of the ellipse $b c$. As the radius $a$ of the circle is greater (4) than both the semiaxes $b, c$, of the ellipse, the circle will lie wholly without the ellipse.

In like manner, the section made in the biaxal surface by the plane $a b$ consists of a circle with the radius $c$, and an ellipse with the semiaxes $a, b$; and as the radius of the circle is less than both the semiaxes of the ellipse, the circle lies wholly within the ellipse.

18. But when the section lies in the plane of the greatest and least semiaxes $a, c$, the circle and ellipse, of which it is composed, intersect each other. For the radius $b$ of the circle is less than one semiaxis of the ellipse $a c$ and greater than the other. Leaving the ellipse $a c$ in the position which it has as a section of the ellipsoid $a b c$, if we describe the circle $b$ with the centre $O$ and radius $b$, the ellipse and the circle will cut each other in four points at the extremities of two diameters; and planes, passing through these diameters and through the semiaxis $b$ of the ellipsoid, will evidently be the planes of the two circular sections of the ellipsoid. Now, turning the ellipse ac round through a right angle (17), the circle and the ellipse in its new position will constitute the section of the biaxal surface, and will cut each other (Fig. 12) in four points $n$ at the extremities of two diameters $n O n, n O n$, which are perpendicular to the two former diameters, and therefore perpendicular to the planes of the two circular sections. Consequently, the biaxal surface has four nodes at the four

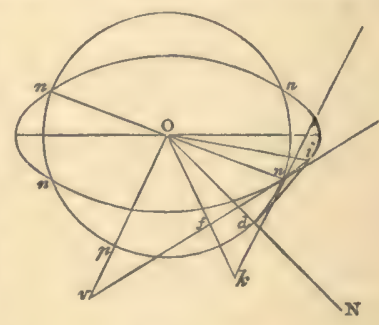

Fig. 12. points $n$. These nodes, it is manifest, are alike in all their properties; and they are the only points common to the two biaxal 
sheets, since the points $T$ and $V(9)$, in the description of the biaxal surface, cannot coincide unless the section $Q O q$, perpendicular to $O T V$, be a circle.

19. The plane of the greatest and least semiaxes, $a, c$, of the generating ellipsoid, may be called the plane of the nodes; and the two diameters $n O n, n O n$, passing through the nodes, may be called the nodal diameters.

At one of the nodes $n$ (Fig. 12) draw tangents $n f, n k$, to the ellipse and the circle that compose the biaxal section; and through $O$ draw $O p$ perpendicular to $O n$, cutting the circle in $p$. Then as $O n$ is perpendicular to the plane of a circular section of the ellipsoid $a b c$, this circular section will have $O p$ for its radius, and its circumference will cross that of the ellipse ac (belonging to the ellipsoid) in the point $p$. A line touching the ellipse ac at $p$ will be parallel to every plane that touches the ellipsoid in a point of the circular section, and will therefore (6) be perpendicular to the plane which is reciprocal to the plane of the circular section. But the tangent at $p$ is perpendicular to the tangent $n f$, since the two tangents would coincide if the ellipse $a c$ were turned round (18) through a right angle, the point $p$ then falling upon $n$. Hence the circular section and its reciprocal plane are parallel to the tangents $n k, n f$; and therefore two planes perpendicular to the plane of the figure, and passing through these tangents, are the planes that we have called (14) the principal tangent planes at $n$.

20. Produce $O p$ to meet $n f$ in $v$, and conceive a parabola having its focus at $O$, its vertex at $v(8)$, and its plane perpendicular to the plane of the figure. A cone, with its vertex at $n$ and this parabola for its section, is (14) the nodal tangent cone.

Draw $O f$ perpendicular to $n f$ at $f$, and meeting $n k$ in $k$. The perpendiculars let fall from $O$ upon the nodal tangent planes form a cone, of which the circles described in planes perpendicular to the figure upon the diameters $n f, n k$, are sections (8). On the other biaxal surface $a^{\prime} b^{\prime} c^{\prime}$ there is (16) a circle of contact whose plane is perpendicular to $O n$. This eircle of contact is (16) another section of the cone last mentioned. 
21. To the circle $b$ and to the principal section $a c$ of the ellipsoid $a b c$ conceive a common tangent $d^{\prime} i^{\prime}$ to be drawn, in a quadrant adjacent to that which contains the node $n$, and let it touch the circle in $d^{\prime}$ and the ellipse ac in $i^{\prime}$. A radius $O d^{\prime}$, drawn through the point $d^{\prime}$ to meet the ellipsoid $a^{\prime} b^{\prime} c^{\prime}$ in the point $d^{\prime \prime}$, will be reciprocal to the radius $O i^{\prime}$, because it is perpendicular to a tangent at $i^{\prime}$, and it will be equal in length to $b^{\prime}$, because $O d^{\prime \prime} \times O d^{\prime}=k^{2}=b b^{\prime}$, and $O d^{\prime}=b$; whence $O d^{\prime \prime}=b$. Therefore $O d^{\prime \prime}$ is in a circular section of the ellipsoid $a^{\prime} b^{\prime} c^{\prime}$. Two planes perpendicular to the plane of the figure, and passing through the reciprocal radii $O d^{\prime \prime}, O i^{\prime}$, are (6) reciprocal planes, and we have seen that the first of them makes a circular section in the ellipsoid $a^{\prime} b^{\prime} c^{\prime}$. They are therefore (15) the fixed planes in the second case of Prop. $\nabla$.

22. Now draw $d i$ a common tangent to the circle $b$ and ellipse ac composing the biaxal section, and let it touch the circle in $d$ and the ellipse in $i$. The lines $O d, O i$, are of course perpendicular to the lines $O d^{\prime}, O i^{\prime}$, and therefore perpendicular to the fixed planes just mentioned. Hence the line $O d$ and the point $d$ are the same as the fixed line $O S$ and the point $S$ in the second case of Prop. V. The plane of the circle of contact is therefore perpendicular to $O d$ at the point $d(15)$; and the points $d$ and $i$, where its plane intersects the right lines $O d, O i$, perpendicular to the fixed planes, are (8) the extremities of a diameter.

These things agree with the obvious remark, that the points of contact $d$ and $i$ must be points of the circle of contact; and that di must be a diameter, because the plane of the circle is perpendicular to the plane of the figure, and this latter plane divides the biaxal surface symmetrically.

As the circle and ellipse may have a common tangent opposite to each node, there are four circles of contact in planes perpendicular to the plane of the nodes.*

23. The biaxal surface belongs to a class that may be called.

* The curves of contact on biaxal surfaces, and the conical intersections or nodes, were lately discovered by Professor Hamilton, who deduced from these properties a theory of conical refraction, which has been confirmed by the experiments of 
apsidal surfaces, from the manner in which they are conceived to be generated.

Let $G$ be a given surface, and $O$ a fixed origin or pole. If a plane passing through $O$ cut the surface $G$, the curve of intersection will in general have several apsides $A, A^{\prime}, A^{\prime \prime}$, \&c., where the lines $O A, O A^{\prime}, O A^{\prime \prime}$, \&c., are perpendicular to the curve. Through the point $O$ conceive a right line perpendicular to the plane of the curve, and on this perpendicular take from $O$ the distances $O a, O a^{\prime}, O a^{\prime \prime}$, \&c., respectively equal to the apsidal distances $O A, O A^{\prime}, O A^{\prime \prime}$, \&o. Imagine a similar construction to be made in every possible position of the intersecting plane passing through $O$, and the points $a, a^{\prime}, a^{\prime \prime}$, \&e., will describe the different sheets of an apsidal surface.

The apsidal surface has a centre at the point $O$, because the lengths $\mathrm{Oa}, \mathrm{O} a^{\prime}, \mathrm{O} a^{\prime \prime}$, \&c., may be measured on the perpendicular at either side of the intersecting plane.

Referring* to the demonstration of Theorem III., it will be seen to depend only on the supposition that the point $Q$ is an apsis of the section made by the plane $Q O q$; or, which is the same thing, that $O Q$ is a position wherein the radius vector from $O$ to the curve of section is a maximum or a minimum. Hence we have the following general theorem :-

24. Prop. VI. Theorem.-If tangent planes be applied at corresponding points $A$, $a$, on the surface $G$ and the apsidal surface which it generates, these tangent planes will be perpendicular to each other and to the plane of the points $O, A, a$.

This is equivalent to saying that perpendiculars from $O$ on the tangent planes are equal to each other, and lie in the plane of the lines $O A, O a$.

25. If $Q$ and $R$ be reciprocal points on two reciprocal surfaces, of which $Q$ is the fixed origin or pole, the tangent plane at $Q$

Professor Lloyd. See Transactions of the Royal Irish Academp, Vol. xvII., part i., pp. 132, 145; and the present Paper, Art. 55-58.

The indeterminate cases of circular section-at least the case of the nodes-had occurred to me long ago; but having neglected to examine the matter attentively, I did not perceive the properties involved in it (13).

* Transactions of the Royal Irish Academy, Vol. xvi., part ii., p. 68. 
will be (1) perpendicular to $O R$ and to the plane QOR. Let a plane also perpendicular to the plane $Q O R$ pass through $O Q$, cutting the surface to which the point $Q$ belongs in a certain curve, and the tangent plane at $Q$ in a tangent to this curve. The tangent is evidently perpendicular to $O Q$, and therefore the point $Q$ is an apsis of the curve.

In like manner, the point $R$ is an apsis of the section made in the other surface by a plane passing through $O R$ and perpendicular to the plane $Q O R$.

26. From these observations, and from Prop. VI., it appears that if the points $Q, R$, in the figure of Theorem IV., be reciprocal points on any two reciprocal surfaces, and if the same construction be supposed to remain, the points $T$ and $M$ will be points on the apsidal surfaces generated by these reciprocal surfaces, and the tangent planes at $T$ and $M$ will be perpendicular to the lines $O M$ and $O T$ respectively. Also the rectangles $L O T$ and $M O S$ will be equal to $k^{2}$. Hence we have another general theorem:-

Prop. VII. Theorem.-The apsidal surfaces generated by two reciprocal surfaces are themselves reciprocal.

27. A very simple example of apsidal surfaces, with nodes and circles of contact, may be had by supposing the generatrix $G$ to be a sphere, and the pole $O$ to be within the sphere, between the surface and the centre $C$.

It is evident that the apsidal surface in this case will be one of revolution round the right line $O C$ as an axis. Therefore taking for the plane of the figure (Fig. 13) a plane passing through $O C$ and cutting the sphere in a great circle of which the radius is $C S$, let a plane at right angles to the figure revolve about $O$, cutting the circle $C S$ in the points $A, A^{\prime}$. The section of the sphere made by the revolving plane will have only two apsides $A, A^{\prime}$, with respect

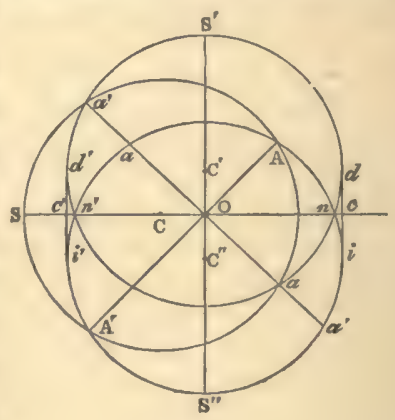

Fig. 13. to the point $O$, except when the plane is perpendicular to $O C$. 
Hence, if we draw the right line $O a a^{\prime}$ perpendicular to $A O A^{\prime}$, taking $O a, O a^{\prime}$, always equal to $O A, O A^{\prime}$, the points $a$, $a^{\prime}$, will describe a section of the apsidal surface. This section will evidently consist of two circles $C^{\prime} S^{\prime}, C^{\prime \prime} S^{\prime \prime}$, equal to the circle $C S$, and having their centres $C^{\prime}, C^{\prime \prime}$, on the opposite sides of $O$ in a right line $C^{\prime} O C^{\prime \prime}$ perpendicular to $O C$; the distances $O C, O C^{\prime}$, $O C^{\prime \prime}$ being equal. The circles $C^{\prime} S^{\prime}, C^{\prime \prime} S^{\prime \prime}$, intersect in two points $n, n^{\prime}$, on the line $O C$, and have two common tangents $d i$, $d^{\prime} i^{\prime}$, which are bisected at right angles by $O C$ in the points $c, c^{\prime}$.

28. Now let the circles $C^{\prime} S^{\prime}, C^{\prime \prime} S^{\prime \prime}$, with their common tangents, or only one of the circles with the half tangents, revolve about the axis $O C$, and we shall have the apsidal surface with nodes at $n, n^{\prime}$, and with circles of contact described by the radii $c d, c^{\prime} d^{\prime}$.

The section of the sphere, by a plane passing through $O$ at right angles to $O n$, is a circle of which $O$ is the centre. If therefore we suppose that the point $n$ answers to $a$ in Prop. VI., the apsis $A$ corresponding to $n$ will be indeterminate, and the position of the tangent plane at $n$ will also be indeterminate, which ought to be the case at a node.

The surface reciprocal to the sphere, the pole being at $O$, is evidently a surface of revolution about the axis $O C$ (it is easily shown to be a spheroid having a focus at $O$ ) ; and the section of this reciprocal surface, by a plane perpendicular to the axis at $O$, is a circle of which $O$ is the centre. This circumstance indicates (15) that on the apsidal surface there is a curve of contact, whose plane is parallel to the plane of circular section; which agrees with what we have already seen.

29. When the point $O$ is without the sphere, the axis $O C$ will pass between the circles $C^{\prime} S^{\prime}, C^{\prime \prime} S^{\prime \prime}$, without intersecting either of them. The apsidal surface, described by the revolution of one of these circles about $O C$, will be a circular ring. The nodes have disappeared; but the circles of contact still exist, as is evident: 


\section{Part II.-On the Wave Theory of Light.}

30. Some of the foregoing propositions lead to a simple transformation of the theory of light.

In this theory, the surface of waves, or the wave surface, is a geometrical surface used to determine the directions and velocities of refracted or reflected rays, being the surface of a sphere in a singly refracting medium; a double surface, or a surface of two sheets, in a doubly refracting medium; a surface of three sheets on the supposition of triple refraction; and having always a centre $O$ round which it is symmetrical. The radii of the wave surface, drawn from its centre $O$ in different directions, represent the velocities of rays to which they are parallel.

31. We shall consider particularly the case of a doubly refracting crystal, with two plane faces parallel to each other, and surrounded by a medium of the common kind wherein the constant velocity is $V$ : supposing, for the sake of clearness, that the crystal refracts more powerfully than the surrounding medium, so that the velocities in the crystal are less than the velocity $V$.

A ray $S^{\prime} O$, falling on the first surface of the crystal at the point $O$, is partly reflected according to the common law of reflection, and partly refracted. The two refracted rays pass on to the second surface, where each of them is divided by internal reflection into a pair, the two reflected pairs being parallel to each other; while the two emergent rays-one from each refracted ray-are parallel to each other and to the ineident ray $S^{\prime} O$. The directions of the rays within the crystals are usually found by the following construction:-

32. Deseribe a wave surface of the crystal, having its centre at $O$ the point of incidence. By the nature of the wave surface, a right line $O T U$, drawn from the point $O$, will in general cut this surface in two points $T, U$, on the same side of $O$; and a ray passing through the crystal in a direction parallel to $O T U$ will have one of the two velocities represented by the radii $O T$, 
$O U$, taking a line of a certain length $k$ to represent the uniform velocity $V$ in the external medium. With the centre $O$ and a radius $O S$ equal to this line $k$ describe a sphere. As the velocities in the crystal are supposed to be less than $V$, the wave surface will lie wholly within this sphere. Let the plane of the figure (Fig. 14) be the plane of incidence, perpendicular to the parallel faces of the crystal, and intersecting the first face in the right line $F A$. Through the point $S$, where the incident ray $S^{\prime} O$, produced through the crystal, cuts the surface of the sphere, draw $S I$ at right angles

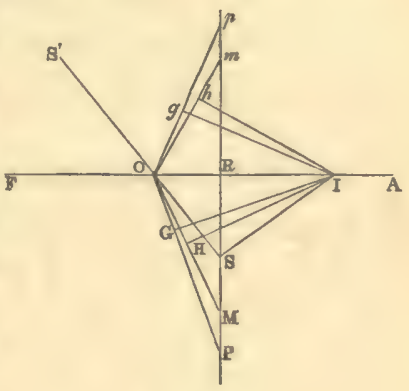

Fig. 14. to $O S$ and meeting $F A$ in the point $I$. A right line perpendicular to the plane of the figure, and passing through this point $I$, we shall call the right line $I$.

33. Through the right line $I$ draw two planes touching the two sheets of the wave surface, on the side remote from the incident light, in the points $T, T^{\prime}$, which will lie within the sphere (32); then the incident plane wave, perpendicular to OS, will be refracted into two plane waves parallel to these two tangent planes; and the lines $O T, O T^{\prime}$, will be the directions of the refracted rays along which the refracted waves are propagated. The lengths $O T, O T^{\prime}$, represent the velocities with which the light moves along the rays; and of course the normal velocities, which are the velocities of the refracted waves, are represented by the perpendiculars $O G, O H$, let fall from $O$ on the two tangent planes at $T, T^{\prime}$. These two perpendiculars $O G, O H$, evidently lie in the plane of the figure; but the points $T, T^{\prime}$, in general, do not lie in this plane.

34. Again, through the right line $I$ draw two other planes touching the wave surface, at the side of the incident light, in the points $t, t^{\prime}$. The rays $O T, O T^{\prime}$, arriving at the second surface of the crystal, will each be divided by internal reflection into two rays parallel to $O t, O t^{\prime}$; and these four reflected rays, 
arriving at the first surface, will each be divided, by a new reflection, into two rays parallel to $O T, O T^{\prime}$; and so on, for any number of reflections. Any of the rays emerging at the tirst surface, after internal reflections, is parallel to the ray $O s$ produced by ordinary reflection at the point of incidence; and any ray emerging at the second surface is parallel to the incident ray $S^{\prime} O S$.

35. This construction may be changed into another that will be found more convenient both in theory and practice.

Through $S$ draw $S R$ perpendicular to $O I$, and meeting $O G$, $O H$, produced, in the points $P, M$. Then as the angles at $G$ and $R$ are right angles, the points $I, R, G, P$, are in the circumference of a circle, and therefore $O P \times O G=O I \times O R=O S^{2}=k^{2}$; and similarly, $O M \times O H=k^{2}$. If then we take $O$ for the fixed origin, or pole, and $k^{2}$ for the constant rectangle ('Theorem I.), and describe the surface which is reciprocal to the wave surface, it is evident that the points $P$ and $M$ will be points of the surface so described, and that $O T, O T^{\prime}$, will coincide in direction with perpendiculars let fall from $O$ on planes touching the surface at $P$ and $M$, and will be inversely proportional to these perpendiculars. It follows in the very same manner, that if perpendiculars $O g, O h$, let fall from $O$ on the tangent planes at $t, t^{\prime}$, be produced to meet $S R$ in the points $p, m$, these points will also be on the surface reciprocal to the wave surface.

In the present case, it is manifest that this reciprocal surface lies wholly without the sphere $O S$.

36. The surface reciprocal to the wave surface, the pole being at $O$, we shall call the surface of refraction.

It is hardly necessary to observe that the surface of refracfion has a centre at the point $O$, round which it is symmetrical ; that it is a sphere in a singly refracting medium, a double surface in a doubly refracting medium, and a surface of three sheets If we suppose a case of triple refraction.

37. In the case that we are considering, let the figure Fig. 15) represent a section made in the double surface of refraction and its attendant sphere by the plane of incidence. 
Through the point $S$, where the incident ray $S^{\prime} O$, prolonged, cuts the circular section of the sphere, draw $S R$ perpendieular to the face of the crystal, or to $F A$; and let $S R$, produced, cut the circle again in the point $s$. Then $O s$ is the direction of the ray given by ordinary reflection at the first surface of the crystal.

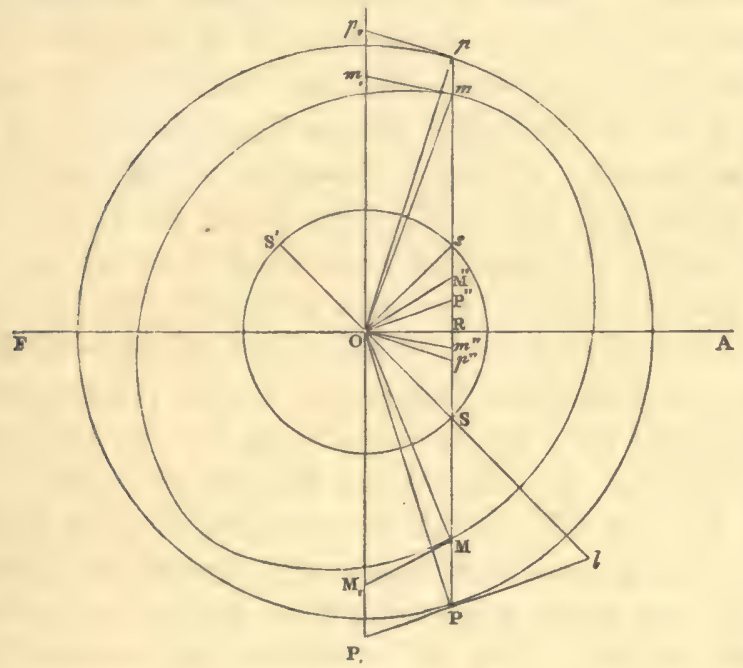

Fig. 15.

Produce the right line SRs both ways, to cut the surface of refraction in the points $P, M$, behind the crystal, and in the points $p, m$, before it ; and conceive planes to touch the surface of refraction at the points $P, M, p, m$. Suppose also that perpendiculars $O P^{\prime}, O M Y^{\prime}, O p^{\prime}, O m^{\prime}$, are let fall from $O$ upon these tangent planes, and that they intersect the planes in the points $P^{\prime}, \boldsymbol{M}^{\prime}$, $p^{\prime}, m^{\prime}$, respectively.

Then from the preceding observations $(33,34,35)$, it is manifest that $O P^{\prime}, O M^{\prime}$, are the directions of the rays into which $S^{\prime} O$ is divided by refraction; that each of these refracted rays, on arriving at the second surface of the crystal, is divided by internal reflection into two rays parallel to $O p^{\prime}, O m^{\prime}$; and that each of the four reflected rays, on arriving at the first surface, is again divided by reflection into two rays parallel to $O P^{\prime}, O Y^{\prime}$, 
and so on. In general, every ray going into the crystal from the first surface, whether after refraction or after any even number of internal reflections, is parallel either to $O P^{\prime}$ or to $O M \Gamma^{\prime}$; and every ray returning from the second surface of the crystal after any odd number of internal reflections, is parallel either to $O p^{\prime}$ or to $O m^{\prime}$. Thus the direction of every ray in the interior of the crystal is the same as the direction of some one of the four lines $O P^{\prime}, O \boldsymbol{M}^{\prime}, O p^{\prime}, O m^{\prime}$; and the velocity of the ray is inversely as the length of this line; so that the velocity of the ray $O M{ }^{\prime}$, for example, or of any ray parallel to $O M^{\prime}$, is to the velocity $V$ as $O S$ is to $O M^{\prime}$. The little plane waves that, keeping always parallel to themselves, move along these rays, are respectively perpendicular to the lines $O P, O M, O p, O m$; and the lengths of these lines are inversely as the velocities of the waves estimated in directions perpendicular to their planes; so that the velocity of the wave which moves along the ray $O M^{\prime}$, or along any parallel ray, is to the velocity $V$ as $O S$ is to $O M$.

38. The ray $O P^{\prime}$, and all the rays parallel to it, are perpendicular to the plane which touches at $P$ the surface of refraction; and the waves which move along these rays are perpendicular to the right line $O P$. Any ray of this set may be called a ray $P$, and any of the waves a wave $P$. In like manner, the rays $M, p, m$, are rays that are perpendicular to the tangent planes at the points $M, p, m$, respectively; and the waves $\boldsymbol{M}, p, m$, are the waves that belong to these rays, and that have their planes respectively perpendicular to the right lines $O M, O p, O m$. The rays $P, M$, all come from the first surface of the crystal; the rays $p, m$, from the second.

As the ordinates $R P, R p$, are greater than the ordinates $R M, R m$, so the rays $P, p$, are more refracted or more reflected than the rays $M, m$. The former rays may therefore be said to be plus refracted, or plus reflected, and the latter to be minus refracted, or minus reflected. Or-for the convenience of namingthe rays $P, p$, may be called plus rays; and the rays $M, m$, minus rays. The waves $P, p$, in like manner, may be termed plus waves, and the waves $M, m$, minus waves. 
For a medium of the eommon kind, or a singly refracting medium, we may use the letters $S$ and $s$. Thus the incident ray $S^{\prime} O S$, or any ray emerging parallel to $O S$ from the second surface of the crystal, may be marked by the letter $S$; while the ray $O_{s}$ produced by common reflection, or any ray emerging parallel to $O s$ from the first surface, may be denoted by the letter $s$.

39. The course of a ray through the crystal may now be easily expressed. A ray $S M p s$, for example, is a ray $(S)$ incident on the crystal, undergoing minus refraction $(\boldsymbol{M})$ at the first surface, plus reflection $(p)$ at the second, and emerging $(s)$ from the first surface in a direction parallel to $O s$. Of this ray the part within the crystal is $M p$. A ray SPS is a ray plus refracted, and then emerging in a direction parallel to that of incidence. A ray $S P p M S$ is a ray plus refracted at the first surface, then plus reflected at the second surface, then minus reflected at the first surface, and finally emerging from the second surface in a direction parallel to that of incidence. Its path within the crystal is $\boldsymbol{P p} \boldsymbol{M}$.

These examples indicate the general method of expressing the path of a ray.

Suppose light to be moving in the same direction and with the same velocity along two proximate parallel rays, so that it is at the point $A$ in one ray when it is at the point $B$ in the other ; and through the points $A$ and $B$ conceive two planes perpendicular to the common direction of the rays. These planes are either coincident, or maintain a constant distance. In the first case, the rays are said to be in complete accordance. In the second case, the constant distance between the planes is called the interval between the portions of light composing the rays, or the interval between the waves that move along the rays.

We proceed to find the lengths of these intervals in the case of rays emerging parallel to each other, at either side of the crystal that we have been hitherto considering.

41. Let the tangent planes at $P, M, p, m$, intersect the plane of the figure (Fig. 15) in the right lines $P P_{1}, M M I, p p, m m$, which of course are tangents to the section of the surface of re- 
fraction represented in the figure; let a perpendicnlar at $O$ to the face of the crystal cut these tangents in the points $P, M, p, m$; and let the lines $O P^{\prime \prime}, O M^{\prime \prime}, O p^{\prime \prime}, O m^{\prime \prime}$, respectively parallel to $P P, M M_{1}, p p$, $m m$, eut the line $S R s$ in the points $P^{\prime \prime}, N I^{\prime \prime}, p^{\prime \prime}, m^{\prime \prime}$.

The length of the path which a ray $P$ describes within the crystal is equal to the thickness $\Theta$ of the crystal divided by the cosine of the angle $P^{\prime} O P$, which the path of the ray makes with a perpendicular to the faces of the crystal; and the velocity of $P$ is equal to $V \times \frac{O S}{O P^{\prime}}(37)$ : dividing therefore the length of the path by the velocity, we find that the time in which a ray $P$ crosses the crystal is equal to

$$
\frac{\theta \times O P^{\prime}}{V \times O S \times \cos P^{\prime} O P}
$$

But as $O P^{\prime}$ is perpendicular to the tangent plane at $P$, we have

$$
\frac{O P^{\prime}}{\cos P^{\prime} O P}=O P_{,}=P P^{\prime \prime}
$$

Therefore the time is equal to $\frac{\theta \times P P^{\prime \prime}}{V \times O S}$. Similarly, the times in which rays $M, p, m$, pass from one surface of the crystal to the other are equal to

$$
\frac{\Theta \times M M^{\prime \prime}}{V \times O S}, \frac{\Theta \times p p^{\prime \prime}}{\bar{V} \times O S^{\prime}}, \frac{\Theta \times m m^{\prime \prime}}{V \times O S}, \text { respectively. }
$$

42. Now suppose the path of a ray $P$ to be projected perpendicularly on a right line having any proposed direction in space. Through $O$ conceive a right line $O L$ parallel to the proposed direction, and meeting in $L$ the tangent plane at $P$. The length of the projection is equal to the length of the path multiplied by the cosine of the angle $P^{\prime} O L$ which the ray $P$ makes with $O L$; that is, the projection is equal to $\theta \frac{\cos P^{\prime} O L}{\cos P^{\prime} O P}$. But because $O P^{\prime}$ is perpendicular to the tangent plane at $P$, we have

$$
\cos P^{\prime} O L=\frac{O P^{\prime \prime}}{O L}, \text { and } \cos P^{\prime} O P_{,}=\frac{O P^{\prime}}{O P}=\frac{O P^{\prime}}{P P^{\prime \prime}} ;
$$


therefore

$$
\frac{\cos P^{\prime} O L}{\cos P^{\prime} O P}=\frac{P P^{\prime \prime}}{O L}
$$

Hence the projection is equal to $\theta \frac{P P^{\prime \prime}}{O L}$.

If the path of a ray $P$ be projected on the incident ray $O S$, then producing $O S$ to meet $P P$, in $l$, we see, by what has just been proved, that the length of the projection is equal to

$$
\theta \frac{P P^{\prime \prime}}{O l}=\theta \frac{S P^{\prime \prime}}{O S}
$$

by similar triangles. In like manner, the projections of the paths of rays $M, p, m$, on the direction of the incident ray $O S$, are equal to

respectively.

$$
\Theta \frac{S M^{\prime \prime}}{O S}, \quad \Theta \frac{S p^{\prime \prime}}{O S}, \quad \Theta \frac{S m^{\prime \prime}}{O S},
$$

43. Let each rectilinear path be measured in the direction in which the light moves along it; and according as the direction so measured makes an acute or an obtuse angle with the direction $O S$, measured from $O$ to $S$, let the projection of the path on $O S$ be reckoned positive or negative. Then if $S P m M[M S$ be any ray entering the crystal at $O$, and emerging from its second surface at $E$, and if a perpendicular $E I$ be let fall from $E$ upon $O S$, meeting $O S$ in $I$, the distance $O I$, from $O$ to the foot of this perpendicular, will evidently be equal to the algebraic sum of the projections of the paths $P, m, M, p, M$, contained within the crystal, taking each projection with its proper sign. It is obvious that the projections of the $P$ and $M$ rays are always positive. And as the lines $O p^{\prime}, O m^{\prime}$ - the directions of the rays $p, m$-lie in planes which are respectively perpendicular to $p p, m m$, or to $O p^{\prime \prime}, O m^{\prime \prime}$, it is easy to see that these directions make acute or obtuse angles with $O S$, according as the points $p^{\prime \prime}, m^{\prime \prime}$, lie below the point $S$ or above it; that is, the projections are positive or negative according as the points $y^{\prime \prime}, m^{\prime \prime}$, lie without the circle $O S$ towards $P, M$, or within the 
circle. Therefore the distance $O I$, in the case of the figure, is equal to

$$
\frac{\theta}{O S}\left(S P^{\prime \prime}-S m^{\prime \prime}+S M^{\prime \prime}-S p^{\prime \prime}+S \boldsymbol{M}^{\prime \prime}\right)
$$

44. If the paths of rays $P, M, p, m$, be projected on the direotion $O s$ of the ordinarily reflected ray, the lengths of their projections will be

$$
\Theta \frac{s P^{\prime \prime}}{O S}, \quad \Theta \frac{s M^{\prime \prime}}{U S}, \quad \Theta \frac{s p^{\prime \prime}}{O S}, \quad \Theta \frac{s m^{\prime \prime}}{O S},
$$

respectively. The projections upon $O s$ of the rays $p, m$, will be always positive; and the projections of the rays $P, M$, will be positive or negative according as the points $P^{\prime \prime}, M^{\prime \prime}$, lie above the point $s$ or below it; that is, according as the points $P^{\prime \prime}, M \Gamma^{\prime \prime}$, lie without the circle $O S$ towards $p$ and $m$, or within the circle. So that if $S P m M p s$ be a ray entering the crystal at $O$ and emerging from the first surface at $e$, and if a perpendicular $e i$ be let fall from $e$ upon $O s$, the distance $O i$, from the point $O$ to the foot of this perpendicular, or the algebraic sum of the projections of the paths $P, m, M, p$, contained within the crystal, will be equal to

$$
\frac{\Theta}{O S}\left(-s P^{\prime \prime}+s m^{\prime \prime}-s M^{\prime \prime}+s p^{\prime \prime}\right)
$$

in the case of the figure.

45. Let us imagine that the light in the incident ray $S^{\prime} O$, instead of being interrupted at $O$ by the crystal, had continued to move with the same velocity $V$ in the same right line OS, leaving the point $O$ at the moment when the refracted light enters the crystal at $O$. Comparing the light in this imaginary ray with that in a ray emerging parallel to it from the second surface of the crystal, after an even number of internal reflections, we shall find that the emergent is behind the imaginary ray, and that the interval between them (40)-or the retardation of the former-may be derived very easily from the letters that designate that ray. Let $S P m \boldsymbol{M} \boldsymbol{M} \boldsymbol{M}$ be any such 
ray. The sum of the distances of the point $S$ from each of the points marked by the letters $(\boldsymbol{P} m \boldsymbol{M} p \boldsymbol{M})$ that denote (39) the part of the ray contained within the erystal, is proportional to the interval of retardation, that interval being equal to

$$
\frac{\theta}{O S}(S P+S m+S M+S p+S M) .
$$

For if from the point $E$, where the last internal ray $M$ emerges from the second surface of the erystal, a perpendicular $E I$ be let fall upon $O S$, meeting $O S$ in $I$, the time of describing $O I$ with the velocity $V$ would (43) be

$$
\frac{\theta}{V \times O S}\left(S P^{\prime \prime}-S m^{\prime \prime}+S M^{\prime \prime}-S p^{\prime \prime}+S M^{\prime \prime}\right) .
$$

But (41) the actual time of describing the broken path $P m M p M$ is

$$
\frac{\Theta}{V \times O S}\left(P P^{\prime \prime}+m m^{\prime \prime}+M M^{\prime \prime}+p p^{\prime \prime}+M M^{\prime \prime}\right) ;
$$

and, on inspecting the figure, this time is seen to be greater than the time of describing $O I$, by

$$
\frac{\Theta}{V \times O S}(S P+S m+S M+S p+S M),
$$

or by the time in which the line

$$
\frac{\theta}{O S}(S P+S m+S M+S p+S M)
$$

would be described with the velocity $V$. Consequently, at the moment when the light in the ray $S P m M p M S$ emerges at the point $E$ from the second surface of the crystal, the light in the imaginary uninterrupted ray $O S$ will have passed the point $I$ by an interval equal to the line just mentioned; and as the two rays afterwards have the same velocity and parallel directions, this interval is the retardation of the emergent ray.

46. The rays emerging from the first surface after any odd number of internal reflections are to be compared with the 
ordinarily reflected ray $O s$ to which they are parallel, the light in $O s$, which moves with the velocity $V$, being supposed to leave $O$ at the moment when the refracted light enters the crystal at $O$. The mode of proceeding in this case is exactly similar to that in the last, and the interval is determined in the same way, using $s$ in place of $S$; the retardation of the ray $S P m M_{p} s$, for example, of which the part $P m M P$ is contained within the crystal, being equal to

$$
\frac{\Theta}{O S}(s P+8 m+8 M+s p) .
$$

47. It is remarkable that the preceding demonstration in nowise depends upon the supposition that the planes perpendicular to the rays $P, M, p, m$, are tangent planes to the surface of refraction at the points $P, M, p, m$. If we had supposed any planes-different from the plane of the figure-to pass through the points $P, M, p, m$, and the rays to coincide in the direction with perpendiculars let fall from $O$ upon these planes, and to have velocities inversely proportional to the lengths of the perpendiculars, the intervals of retardation would have remained unchanged. Hence the retardations are the same as if the lines $O P, O M, O p, O m$, were the directions of the rays in passing through the crystal, as will appear by conceiving the planes that we have spoken of to be perpendicular to these lines.

If the incident ray $S^{\prime} O$ were refracted in the ordinary way with an index equal to $\frac{O P}{O S}$, it would take the direction $O P$; if it were refracted, in like manner, with the index $\frac{O M}{O S}$, it would take the direction $O M$; and if the two rays, thus ordinarily refracted, were to emerge from the second surface of the crystal in directions parallel to $O S$, it is evident, from what has been said, that they would be in complete accordance, respectively, with the rays SPS and SMS.

- The change of phase, which may take place at a surface of the crystal, is not here considered as affecting the intervals. 
If the surface of refraction should happen to have a node $N$, which is a point of intersection where it admits an infinite number of tangent planes (3), let the direetion of the incident ray $S^{\prime} O S$ be chosen, so that the right line $R S$ perpendicular to the face of the crystal, being produced below $S$, may pass through $N$, and we shall have a cone of refracted rays formed by the perpendiculars let fall from $O$ upon the tangent planes at $N$; all of which rays, on emerging parallel to $O S$ from the second surface of the crystal, will be in complete accordance with one another. For we have just seen that if the ray $S^{\prime} O S$ were supposed to emerge after being refracted in the ordinary way with an index equal to $\frac{O N}{O S}$, it would be in complete accordance with any ray of the cone.

48. The interval between any two rays emerging at the same side of the crystal is the difference of their retardations. In taking the difference, the letters that are common to the names of the two rays may be left out. Thus the ray SPmMS is behind the ray SPS by the interval

$$
\frac{\theta}{O S}(S m+S M)=\frac{\theta}{O S} M m .
$$

The line $\frac{\theta}{O S} P p$ is the interval between the rays $S M S$ and $S M p P S$, or between the reflected ray $O s$ and the ray $S P p s$, and so on.

49. The retardations of the two refracted rays SPS and SMS, emerging without internal reflection, are $\frac{\theta}{O S} S P$ and $\frac{\theta}{O S} S M$ respectively. The difference of these is $\theta \frac{P M}{O S}$. Consequently, when the two refracted rays have emerged from the second surface in directions parallel to the incident ray, the light in the plus emergent ray is behind the light in the minus emergent ray by an interval equal to $\frac{\Theta \times P M}{O S}$. Or, in other words, the incident plane wave, perpendicular to $O S$, produces 
two emergent waves parallel to each other and to the incident wave, moving along the emergent rays with equal velocities $V$, and preserving the distance $\frac{\Theta \times P M}{O S}$ between their planes, the minus wave being foremost. If $O S$, the radius of the sphere, be taken for unity, $P M$ will be a number-generally a very small fraction-and the interval will be the thickness of the crystal multiplied by this number.

50. Suppose the right line $P M R$, remaining always perpendicular to the face of the crystal, to describe a cylindrical surface, with the condition that the part $P M$, intercepted between the two sheets of the surface of refraction, shall remain of a constant length; the point $R$ will then describe, on the surface of the crystal, a curve whose radii $O R$ are the sines (to the radius $O S$ ) of the angles of incidence of a cone of rays ; and every ray $S^{\prime} O$ of this cone, when refracted by the crystal, will afford two emergent rays, or two waves, having the same given interval between them. Lines drawn from the eye parallel to the sides of this cone are the emergent rays belonging to a ring, when rings are made to appear, in any of the usual ways, on transmitting polarized light through the plate of crystal. In nominal conformity to this, we see that the line $P M$ describes a ring of constant breadth between the two sheets of the surface of refraction. The ring described by supposing $p m$ to remain constant corresponds to the interval between two rays $p$ and $m$ reflected at the same point of the second surface of the crystal, and then emerging at the first. The other intercepts $\boldsymbol{P}_{p}, \boldsymbol{M} m, \boldsymbol{P}_{m}, \boldsymbol{M}_{p}$, are proportional (48) to intervals like those in Newton's ringsto the intervals, namely, between the reflected ray $O s$ and the rays $S P p s, S M m s, S P m s, S M p s$, emerging at the first surface after one reflection within the crystal; or to the intervals between rays that are twice reflected in the crystal and the rays transmitted without reflection.

51. The general investigation of the figure of a geometrical ring does not distinguish between the different intercepts, and will therefore include all the rings $P \boldsymbol{M}, \mathrm{pm}, \boldsymbol{P} p, \boldsymbol{M} m, \boldsymbol{P} m, \boldsymbol{M p}$; 
so that it will be sufficient to contemplate any one of them, as $P M$, of which the breadth $P M$ is equal to a given line $I$.

The points $P$ and $M$ describe, in general, similar and equal curves of double curvature, which may be called ring-edges, as being the edges of the ring; and if we imagine the surface of refraction, carrying these curves along with it, to be shifted either way, in a direction parallel to $P M$, through a distance equal to $I$, it is clear that the new position of one of the ring-edges will exactly coincide with the first position of the other, and that therefore the curve of the latter ring-edge will be given by the intersection of the two equal surfaces in these two positions. Let $U=0$-where $U$ is a function of $x, y, z$, and given quantities - be the equation of the surface of refraction in its original position; and, the axes of co-ordinates being fixed, suppose that by the shifting of the surface the co-ordinates of a point assumed on it are diminished by the given lines $f, g, h$, which are the projections of the given line $I$ on the axes of $x, y, z$, respectively. Then the equation of the surface in its new position will be had by substituting $x+f, y+g, z+h$, for $x, y, z$, in the equation $U=0$, which will thus become $U+V=0$, where $V$ is the increment of $U$ produced by the substitution. These two equations combined are equivalent to the equations $U=0, V=0$, which are therefore the equations of one of the ring-edges. If the surface had been shifted the opposite way, in a direction parallel to $P M$, the intersection would have been the other ring-edge, whose equations are therefore deducible from those already found, by changing the signs of $f, g, h$.

52. If the equation of the surface of refraction be transformed, so that the plane of $x y$ may coincide with the face of the crystal, and the axis of $z$ be perpendicular to it, the origin of co-ordinates being at the centre $O$, no change will be produced in $x$ or in $y$ by the motion of the surface, because $P M$, the direction of the motion, is now parallel to the axis of $z$; but $z$ will be diminished or increased by $I$; and, accordingly, if $U^{\prime}=0$ be the equation of the surface in its first position, when the centre is at $O$, and if $U^{\prime}$ become $U^{\prime}+V^{\prime}$ when $z$ becomes $z+I$, the 
equation of the surface in its second position, when the centre has moved through a distance equal to $I$ along the axis of $z$, will be $U^{\prime}+V^{\prime}=0$; and these two equations combined will give $U^{\prime}=0, V^{\prime}=0$, for the equations of one of the ring-edges. The equations of the other ring-edge are deduced from these by changing the sign of $I$.

The projection of each of the ring-edges on the plane $x y$ is the curve traced by the point $R$ on the surface of the crystal (50). This curve may be called a ring-trace. Its equation is obtained by eliminating z between the equations of a ring-edge; and as the result must be the same whether $I$ be taken positive or negative, the equation of the ring-trace, when found by this general method, will contain only even powers of $I$. The radii drawn from $O$ to the points $R$ of the ring-trace are (50) the sines (to the radius $O S$ ) of the angles of incidence or emergence of the rays that form an optical ring, the rays that come from this ring to the eye being parallel to the sides of the cone described by the right line $S^{\prime} O S$, while the point $R$ describes the ring-trace.

53. It is evident that tangents to the ring-edges, at the points $P$ and $M$, are parallel to each other, and therefore parallel to the intersection of two planes touching the surface of refraction at $P$ and $M$, because these tangent planes pass through the tangents. But the directions $O P^{\prime}, O M^{\prime}$, are perpendicular to the tangent planes, and therefore the plane $P^{\prime} O M$, containing the two rays, is perpendicular to the intersection of the tangent planes, and of course perpendicular to the parallel tangents. Hence the plane $P^{\prime} O M^{\prime}$ intersects the face of the crystal in a right line perpendicular to the projection of the parallel tangents on the face of the crystal. As this projection is a tangent to the curre described by $R$, it follows that the normal to the ring-trace at the point $R$ is parallel to the line joining the points in which the two refracted rays cut the second surface of the crystal.

In like manner, taking any two consecutive rays $(\boldsymbol{P}$ and $m)$, having a common extremity on one surface of the crystal, the line joining the points where these rays cut the other surface is 
parallel to the normal at the point $R$ of the ring-trace which is described when the intercept $(\boldsymbol{P} m)$ between the letters that mark the rays is supposed to remain constant.

54. In all that precedes we have made no supposition about the surface of refraction except that it is a surface of two sheets; and if we supposed it to have three sheets, the conclusions would be easily extended to this hypothesis.

In the theory of FRESNEL, the wave surface is* a biaxal whose generating ellipsoid has its centre at the point $O$, and its semiaxes parallel to the three principal directions of the crystal, the length of each semiaxis being equal to $O S$ divided by one of the principal indices of refraction. The surface of refraction is reciprocal to the wave surface, and is (11) therefore another biaxal generated by an ellipsoid reciprocal to the former, having its centre at the same point $O$, and the directions of its semiaxes the same as before, the rectangle under each coincident pair of semiaxes being equal to $k^{2}$ or $O S^{2}$. Hence the semiaxes of the ellipsoid which generates the biaxal surface of refraction are equal in length to $O S$ multiplied by each of the three principal indices. This biaxal surface is of course to be substituted for the surface of refraction in the preceding observations.

55. When the line RS, produced below $S$, passes through a node $N$ of the biaxal surface of refraction, the points $P, \boldsymbol{M}$, coincide in the point $N$, and the interval $P M$ vanishes. At the point $N$ there are an infinite number of tangent planes, and the perpendiculars from $O$ on these tangent planes give a cone of refracted rays whose sections we have already shown how to determine (20). All the rays in this cone, on arriving at the second surface of the crystal, emerge parallel to the incident ray $O S$; and if the rays in the emergent cylinder be cut by a plane perpendicular to their common direction, they will all arrive at this plane at the same instant, because the interval $P \boldsymbol{M}$ vanishes. See Art. 47.

56. Suppose fig. 12 to be a section of the wave surface. The

- Transactions of the Royal Irish Academy, Vor. xvi., p. 76 (supra, p. 11). 
right line $O d$ will pass through $N$; and the circle of contact, described on the diameter $d i$ in a plane perpendicular to the right line $O d N$, will be a section of the refracted cone. Now it will be recollected* that, in general, the vibrations of a ray $O T$, which goes to any point $T$ of the wave surface, are parallel to the line which joins the point $T$ with the foot of the perpendicular let fall from $O$ on the tangent plane at $T$. In the present case, the perpendicular is the same for all the rays of the refracted cone, and its extremity coincides with the point $d$ : so that the line $d T$, drawn from $d$ to any point $T$ of the circle of contact, is parallel to the vibrations of the ray $O T$ which passes through $T$. Conceive, therefore, a plane perpendicular to $O N$ at the nodal point $N$. This plane will cut the refracted cone in a circle whose circumference will pass through $N$; and a line $N T^{\prime}$, drawn from the node to any other point $T^{\prime}$ of the circumference, will be the direction of the vibrations in a ray $O T^{\prime}$ which crosses the circle at this point. The plane of polarization is perpendicular to the direction of the vibrations.

57. The transverse section of the emergent cylinder is always a very small ellipse, affording a hollow pencil of parallel rays in complete accordance (55). If the crystal be thin, this ellipse will be of evanescent magnitude. Hence the line $O S$ will be the direction of a line drawn from the eye to the centre of the rings commonly observed $(50)$ with polarized light; or it will be what is called the apparent direction of one of the optic axes. The diameter passing through $N$ will be the direction of the optic axis within the crystal. There are therefore two optic axes, parallel to the two nodal diameters (19) of the surface of refraction.

As $O N$ is equal to the mean semiaxis of the generating ellipsoid, or to the mean index of refraction, when $O S$ is unity, it follows that the apparent direction of an optic axis is the direction of an incident ray, which, if refracted in the ordinary way, with an index equal to the mean index of refraction, would pass along a nodal diameter of the surface of refraction.

* Transactions of the Royal Irish Academy, You. xvi., p. 76 (supra, p. 12). 
58. We have seen (15) that there is a circle of contact on the biaxal surface of refraction. If an incident ray $S^{\prime} O S$ be taken, eutting the sphere in $S$, so that the line $R S$ produced may pass through the ciroumference of this circle, it is manifest that the direction of the refracted ray will be the same through whatever point $\Pi$ of the circumference the line $R S$ may pass, because that direction is perpendicular to the tangent plane at $\Pi$, which is in fact the plane of the circle itself. If, therefore, the line $R S$ move parallel to itself along the ciroumference of the circle, cutting the sphere in a series of points $S$, every incident ray $S^{\prime} O S$ which passes through a point $S$ so determined will be refracted into two rays, of which one will have a fixed direotion in the crystal, being perpendicular to the plane of the circle of contact, and therefore coinciding (16) with $n O n$, one of the nodal diameters of the wave surface. But though the direction $O n$ of the refracted ray is fixed, its polarization changes with the incident ray from which it is derived ; for if $\Pi$ be the point in which the line $R S$, corresponding to any position of the incident ray, crosses the circle of contact, the vibrations of the refracted ray $O n$ will be contained in the plane of the lines On, OП, and will be perpendicular to OП. Conceive a circle described on the diameter $n f$ in a plane perpendicular to the figure (Fig. 12). This circle, and the circle of contact on the surface of refraction, are (20) sections of the same cone. Let $\Pi^{\prime}$ therefore be the point at which $O \Pi$, in any position of the incident ray, crosses the circumference of the circle $n f$; and the line $\Pi n$, drawn to the node of the wave surface, will be the corresponding direction of the vibrations in the ray $O$ n.

59. With regard to the general law of polarization in the theory of Fresnel, it may be observed, that if the ellipsoid $a b c$ which generates the biaxal surface of refraction be cut by a plane perpendicular to $O P$, the vibrations of the ray $P$ will be parallel to the greater axis of the section, and therefore the plane of polarization will pass through $O P$ and the less axis; whence it is easy to show that the plane of polarization of a ray $P$ bisects one of the angles made by two planes intersecting in $O P$ and 
passing through the nodal diameters of the surface of refraction; the bisected angle being that which contains the least semiaxis $c$ of the generating ellipsoid. The plane of polarization of the ray $p$ is found in like manner. But for the rays $M, m$, the angle to be bisected is that which contains within it the greatest semiaxis $a$.

If $O P^{\prime}$ be perpendicular to a tangent plane at $P$, the vibrations of the ray $P$ will be perpendicular to $O P$, and will lie in the plane $P O P^{\prime}$. A similar remark applies to the rays $M, p, m$.

60. When two semiaxes $a, b$, of the ellipsoid $a b c$ become equal, it changes into a spheroid aac described by the revolution of the ellipse $a c$ about the semiaxis $c$; and the biaxal aac, generated by this spheroid, is* composed of a sphere whose radius is $a$, and a concentric spheroid acc described by the revolution of the ellipse $a c$ about the semiaxis $a$; so that, the diameter of the sphere being equal to the axis of revolution of the spheroid, the two surfaces touch at the extremities of the axis. This combination of a sphere and a spheroid is the surface of refraction for uniaxal crystals. In these crystals, therefore, the refracted ray whose direction is determined by the intersection of the right line $R S$ with the surface of the sphere follows the ordinary law of a constant ratio of the sines, and is called the ordinary ray; whilst the other, whose variable refraction is regulated by the intersection of $R S$ with the spheroid, is called the extraordinary ray. And hence uniaxal erystals are usually divided into the two classes of positive and negative, according to the character of the extraordinary ray; being called positive when it is the plus ray, and negative when it is the minus ray. The first case evidently happens when the spheroid is oblate, and therefore lies without the sphere described on its axis; the second, when the spheroid is prolate, and therefore lies within the sphere. The second case (which is that of Iceland spar) may be supposed to be represented in the figure (Fig. 15), where the elliptic section of the spheroid, made by a plane of incidence oblique to the axis, lies

- Transactions of the Royal Irish Academy, VoL. xvi., p. 77 (supra, p. 12). 
within the circular section of the sphere, and the minus ray is of course the extraordinary one.

61. Let $P M$, preserving a constant length $I$, move parallel to itself between the surfaces of the uniaxal sphere and spheroid, so as to form a ring (50). Then supposing the spheroid, with the ring-edge deseribed on it by the point $M$, to remain fixed, imagine the sphere, carrying the ring edge $P$ along with it, to move parallel to $P M$, from $P$ towards $M$, through a distance equal to $I$, and the two ring-edges will exactly coincide.

Hence the uniaxal ring-edge is the intersection of a sphere and a spheroid, the diameter of the sphere being equal to the axis of revolution of the spheroid, and the line joining their centres being perpendicular to the faces of the crystal and equal to the breadth $I$ of the ring. And the projection of this intersection, on a plane perpendicular to the line joining the centres of the sphere and the spheroid, is the uniaxal ring-trace.

62. The biaxal ring-edge is (51) the intersection of two equal biaxal surfaces similarly posited, the line joining their centres being perpendicular to the faces of the crystal and equal to the breadth of the ring. And the projection of this intersection, on a plane perpendicular to the line joining the centres of the surfaces, is the biaxal ring-trace.*

* In applying the general theory $(51,52)$ to biaxal rings, it is necessary to know the equation of a biaxal surface, which may be found in the following manner :-Let $r, r^{\prime}, r^{\prime \prime}$, be three rectangular radii of the generating ellipsoid $a b c$, the two latter being the semiaxes of the section made by a plane passing throngh them; so that if from the centre $O$ two distances $O T, O V$, equal to $r^{\prime}, r^{\prime \prime}$, be taken on the direction of $r$, the points $T$ and $V$ will belong $(9)$ to the biaxal surface; and let a plane parallel to the plane of $r^{\prime}, r^{\prime \prime}$, and touching the ellipsoid, cut the direction of $r$ at the distance $p$ from the centre. Then if $r$ make the angles $\alpha, \beta, \gamma$, with the semiaxes $a, b, c$, we shall have, by the nature of the ellipsoid,

$$
\begin{gathered}
\frac{1}{r^{2}}=\frac{\cos ^{2} a}{a^{2}}+\frac{\cos ^{2} \beta}{b^{2}}+\frac{\cos ^{2} \gamma}{c^{2}}, \\
p^{2}=a^{3} \cos ^{2} \alpha+b^{2} \cos ^{2} b+c^{2} \cos ^{2} \gamma .
\end{gathered}
$$

Now, since the sum of the squares of the reciprocals of three rectangular radii of 
an ellipsoid is constant, as well as the parallelopiped described on three conjugate semidiameters, we have the equations

or,

$$
\begin{aligned}
\frac{1}{r^{2}}+\frac{1}{r^{\prime 2}}+\frac{1}{r^{\prime 2}} & =\frac{1}{a^{2}}+\frac{1}{b^{2}}+\frac{1}{c^{2}}, \\
p^{2} r^{\prime 2} r^{\prime \prime 2} & =a^{2} b^{2} c^{2} ;
\end{aligned}
$$

$$
\begin{gathered}
\frac{1}{r^{2}}+\frac{1}{\gamma^{\prime \prime 2}}=\frac{1}{a^{2}}+\frac{1}{b^{2}}+\frac{1}{c^{2}}-\left(\frac{\cos ^{2} \alpha}{a^{2}}+\frac{\cos ^{2} \beta}{b^{2}}+\frac{\cos ^{2} \gamma}{c^{2}}\right)=M, \\
\frac{1}{r^{2} r^{\prime \prime 2}}=\frac{a^{2} \cos ^{2} \alpha+b^{2} \cos \beta+c^{2} \cos ^{2} \gamma}{a^{2} b^{2} c^{2}}=N .
\end{gathered}
$$

Whence it appears that $r^{\prime}, r^{\prime \prime}$, are the values of $\rho$ in the equation

$$
\frac{1}{\rho^{4}}-\frac{M}{\rho^{2}}+N=0
$$

in which $\rho$ denotes indifferently either semidiameter, $O T$ or $O V$, of the biaxal surface. Therefore putting for $M$ and $N$ their values, and writing $\frac{x}{\rho}, \frac{y}{\rho}, \frac{z}{\rho}$, instead of $\cos \alpha, \cos \beta, \cos \gamma$, and $x^{2}+y^{2}+z^{2}$ instead of $\rho^{2}$, we obtain, for the equation of the biaxal surface,

$$
\begin{aligned}
\left(x^{2}+y^{2}+z^{2}\right)\left(a^{2} x^{2}+b^{2} y^{2}+b^{2} z^{2}\right)-a^{2}\left(b^{2}+c^{2}\right) x^{2}-b^{2}\left(a^{2}+c^{2}\right) y^{2} & \\
& -a^{2}\left(a^{2}+b^{2}\right) z^{2}+a^{2} b^{2} c^{2}=0 .
\end{aligned}
$$

This is the equation of the surface of refraction for a biaxal crystal in which $a, b, c$, are (54) the three principal indices of refraction, taking $O S$ the radius of the sphere to be unity. The left-hand member of the equation is therefore the expression supplied by Fresnel for the function $U$ in Art. 51 .

When the faces of the crystal are parallel to any of the principal planes of the ellipsoid-to the plane of $x y$ for example-the nature of the ring-trace may be found very easily. For if the difference of the two values of $z$, deduced from the preceding equation of the surface of refraction, be put equal to a constant quantity $I$, the resclt, when cleared of radicals, will be an equation of the fourth degree in $x$ and $y$, which will be the equation of the corresponding ring-trace. This is a case that occurs frequently in practice; the crystal being often cut with its faces perpendicular to the axis of $x$ or of $z$, because these lines bisect the angles made by the optic axes. 


\section{V.-A SHORT ACCOUNT OF SOME RECENT INVESTIGA- TIONS CONCERNING THE LAWS OF REFLEXION AND REFRACTION AT THE SURFACE OF CRYSTALS.}

[Fifth Report of the British Association, 1835.]

To understand the nature of the general problem which a complete theory of double refraction requires to be solved, let it be supposed that a ray of light is reflected and refracted at the separating surface of an ordinary medium and a doubly refracting crystal, the light passing out of the former medium into the latter. This limited view of the subject is taken merely for the sake of clearness of conception; since we might suppose that both media are crystallized, without increasing the difficulty of the problem. The question, it is obvious, naturally divides into two distinct heads. The first relates to the laws of the propagation of light in the interior of either of the two media, before or after it has passed their separating surface; and this part of the subject has been fully treated, according to their different methods, by MM. Fresnel and Cauchy. The second division of the subject had been left completely untouched. It relates to the more complex consideration of what takes place at the separating surface of the media, the laws according to which the light is there divided between the reflected and refracted rays, including a determination of the attendant circumstances indicated by the wave theory, with regard to the vibrations in the reflected and refracted rays. In the case above mentioned, when the incident light is polarized, there are four things to be deter- 
mined, namely, the magnitude and direction of the reflected vibration, with the magnitudes of the two refracted vibrations. The four conditions necessary for this determination are furnished by two new laws, which could not be easily stated without entering too much into detail. The results applied to determine the polarizing angle of a crystal, in different azimuths of the plane of reflexion, agree very closely with the admirable experiments of Sir David Brewster on Iceland spar. In the course of these experiments it was observed that the polarizing angle remained the same when the crystal was turned half round (through an angle of $180^{\circ}$ ); although the inclination of the refracted rays to the axis of the crystal was thereby greatly changed. This remarkable fact is a consequence of theory. After some complicated substitutions in the primary equations, the value of the polarizing angle is found to contain only even powers of the sine or cosine of the azimuth of the plane of reflexion, and therefore a change of $180^{\circ}$ in the azimuth produces no change in the polarizing angle.

The two new laws above mentioned, on which the theory depends, occurred to the author in the beginning of last December; but, owing to an oversight in forming one of the equations, they were not fully verified until the beginning of June.

In this theory it is supposed that the vibrations are parallel to the plane of polarization, according to the opinion of M. Cauchy. This is contrary to the views of Fresnel, whose theory of double refraction obliged him to adopt the hypothesis that the vibrations are perpendicular to the plane of polarization. It is further supposed that the density of the vibrating ether is the same in both media; and the hypothesis of a constant density in different media, which was found necessary for the theory, seems to accord, better than the supposition of a varying density, with the phenomena of astronomical aberration.

If we conceive the three principal indices of refraction for the crystal to become equal, we shall obtain the solution of a very simple case of the general problem with which we have been occupied-the case of an ordinary refracting medium such 
as glass. This simple ease, it is well known, was solved by Fresnel. The foregoing theory leads to a simple law, expressing all the particulars of the case, but differing with regard to the magnitude of the refracted vibration from the formula of Fresnel. The law may be stated by saying that the refracted vibration is the resultant of the incident and reflected vibrations; the first vibration being the diagonal of a parallelogram, of which the other two vibrations are the sides, just as in the composition of forces. The plane of the parallelogram is the plane of polarization of the refracted ray. It is to be remembered, that the vibrations in each ray are perpendicular to the ray itself, and parallel to its plane of polarization.

This simple case has been considered by M. Cauchy in a short Paper inserted in the Bulletin Universel, tom. xiv.; but it does not seem to have been observed by anyone that his solution is erroneous. His formula for light polarized parallel to the plane of reflexion is that which belongs to light polarized perpendicular to the plane of reflexion, and vice versâ. 


\section{VI.-LAWS OF REFLEXION FROM METALS.}

[Proceedings of the Royal Irish Academy, Vou. I., p. 2.-Read Oct. 24, 1836.]

THE author observes that the theory of the action of metals upon light is among the desiderata of physical optios, whatever information we possess upon this subject being derived from the experiments of Sir David Brewster. But, in the absence of a real theory, it is important that we should be able to represent the phenomena by means of empirical formulæ; and, accordingly, the author has endeavoured to obtain such formulæ by a method analogous to that which Fresnel employed in the case of total reflexion at the surface of a rarer medium, and which, as is well known, depends on a peculiar interpretation of the sign $\sqrt{-1}$. For the ease of metallic reflexion, the author assumes that the velocity of propagation in the metal, or the reciprocal of the refractive index, is of the form

$$
m(\cos \chi+\sqrt{-1} \sin \chi) ;
$$

without attaching to this form any physical signification, but using it rather as a means of introducing two constants (for there must be two constants, $m$ and $\chi$, for each metal) into Fresnel's formulæ for ordinary reflexion, which contain only one constant, namely, the refractive index.

Then if $i$ be the angle of incidence on the metal, and $i^{\prime}$ the angle of refraction, we have

$$
\sin i^{\prime}=m(\cos \chi+\sqrt{-1} \sin \chi) \sin i
$$


and therefore we may put

if

$$
\cos i^{\prime}=m^{\prime}\left(\cos \chi^{\prime}-\sqrt{-1} \sin \chi^{\prime}\right) \cos i, .
$$

and

$$
m^{4} \cos ^{4} i=1-2 m^{2} \cos 2 \chi \sin ^{2} i+m^{4} \sin ^{4} i,
$$

$$
\tan 2 \chi^{\prime}=\frac{m^{2} \sin 2 \chi \sin ^{2} i}{1-m^{2} \cos 2 \chi \sin ^{2} i}
$$

Now, first, if the incident light be polarized in the plane of reflexion, and if the preceding values of $\sin i^{\prime}, \cos i^{\prime}$, be substituted in Fresnel's expression

$$
\frac{\sin \left(i-i^{\prime}\right)}{\sin \left(i+i^{\prime}\right)}
$$

for the amplitude of the reflected vibration, the result may be reduced to the form

if we put

$$
a(\cos \delta-\sqrt{-1} \sin \delta)
$$

$$
\begin{gathered}
\tan \psi=\frac{m}{m^{\prime \prime}} \\
\tan \delta=\tan 2 \psi \sin \left(\chi+\chi^{\prime}\right) \\
a^{2}=\frac{1-\sin 2 \psi \cos \left(\chi+\chi^{\prime}\right)}{1+\sin 2 \psi \cos \left(\chi+\chi^{\prime}\right)}
\end{gathered}
$$

Then, according to the interpretation, before alluded to, of $\sqrt{-1}$, the angle $\delta$ will denote the change of phase, or the retardation of the reflected light; and $a$ will be the amplitude of the reflected vibration, that of the incident vibration being unity. The values of $m^{\prime}, \chi^{\prime}$, for any angle of incidence, are found by formulæ (3), (4), the quantities $m, \chi$, being given for each metal. The angle $\chi^{\prime}$ is very small, and may in general be neglected. 
Secondly, when the incident light is polarized perpendicularly to the plane of reflexion, the expression

$$
\frac{\tan \left(i-i^{\prime}\right)}{\tan \left(i+i^{\prime}\right)}
$$

treated in the same manner, will become

if we make

$$
a^{\prime}\left(\cos \delta-\sqrt{-1} \sin \delta^{\prime}\right),
$$

$$
\begin{gathered}
\tan \psi^{\prime}=m m^{\prime}, \\
\tan \delta^{\prime}=\tan 2 \psi^{\prime} \sin \left(\chi-\chi^{\prime}\right), \\
a^{\prime 2}=\frac{1-\sin 2 \psi^{\prime} \cos \left(\chi-\chi^{\prime}\right)}{1+\sin 2 \psi^{\prime} \cos \left(\chi-\chi^{\prime}\right)}
\end{gathered}
$$

and here, as before, $\delta$ will be the retardation of the reflected light, and $a^{\prime}$ the amplitude of its vibration.

The number $M=\frac{1}{m}$ may be called the modulus, and the angle $\chi$ the characteristic of the metal. The modulus is something less than the tangent of the angle which Sir David Brewster has called the maximum polarizing angle. After two reflexions at this angle a ray originally polarized in a plane inclined $45^{\circ}$ to that of reflexion will again be plane-polarized in a plane inclined at a certain angle $\phi$ (which is $17^{\circ}$ for steel) to the plane of reflexion; and we must have

$$
\tan \phi=\frac{a^{\prime 2}}{a^{2}} .
$$

Also, at the maximum polarizing angle we must have

$$
\delta-\delta=90^{\circ} \text {. }
$$

And these two conditions will enable us to determine the constants $M$ and $\chi$ for any metal, when we know its maximum polarizing angle and the value of $\phi$; both of which have been 
found for a great number of metals by Sir David Brewster. The following Table is computed for steel, taking $\boldsymbol{M}=3 \frac{1}{2}$, $\dot{x}=54^{\circ}$.

\begin{tabular}{|c|c|c|c|c|c|}
\hline$i$ & 8 & $\overline{8}^{\prime}$ & $\alpha^{2}$ & $a^{2}$ & $\frac{1}{3}\left(a^{2}+a^{2}\right)$ \\
\hline $0^{\circ}$ & $27^{\circ}$ & $27^{\circ}$ & 0.526 & 0.526 & 0.526 \\
\hline 30 & 23 & 31 & 0.575 & 0.475 & 0.525 \\
\hline 45 & 19 & 38 & 0.638 & 0.407 & 0.522 \\
\hline 60 & 13 & 54 & 0.729 & 0.308 & $0 \cdot 5 ี 18$ \\
\hline 75 & 7 & 98 & 0.850 & 0.240 & 0.545 \\
\hline 85 & 2 & 152 & 0.947 & 0.491 & 0.719 \\
\hline 90 & 0 & 180 & 1.000 & 1.000 & $1 \cdot 000$ \\
\hline
\end{tabular}

The most remarkable thing in this Table is the last column, which gives the intensity of the light reflected when common light is incident. The intensity decreases very slowly up to a large angle of incidence (less than $75^{\circ}$ ), and then increases up to $90^{\circ}$, where there is total reflexion. This singular fact, that the intensity decreases with the obliquity of incidence, was discovered by Mr. Potter, whose experiments extend as far as an incidence of $70^{\circ}$. Whether the subsequent increase which appears from the Table indicates a real phenomenon, or arises from an error in the empirical formulæ, cannot be determined without more experiments. It should be observed, however, that in these very oblique incidences Fresnel's formulæ for transparent media do not represent the actual phenomena for such media, a great quantity of the light being stopped, when the formulæ give a reflexion very nearly total.

The value $\delta-\delta$, or the difference of phase, increases from $0^{\circ}$ to $180^{\circ}$. When a plane-polarized ray is twice reflected from a metal, it will still be plane-polarized if the sum of the values of $\delta-\delta$ for the two angles of incidence be equal to $180^{\circ}$.

It appears from the formulø that when the characteristic $\chi$ is very small, the value of $\delta^{\prime}$ will continue very small up to the 
neighbourhood of the polarizing angle. It will pass through $90^{\circ}$, when $m m^{\prime}=1$; after which the change will be very rapid, and the value of $\delta^{\gamma}$ will soon rise to nearly $180^{\circ}$. This is exactly the phenomenon which Mr. Airy observed in the diamond.

Another set of phenomena to which the author has applied his formulø are those of the coloured rings formed between a glass lens and a metallic reflector; and he has thus been enabled to account for the singular appearances described by M. Arago in the Memoires d'Arcueil, tom. iii., particularly the succession of changes which are observed when common light is incident, the intrusion of a new ring, \&c. But there is one curious appearance which he does not find described by any former author. It is this. Through the last twenty or thirty degrees of incidence the first dark ring, surrounding the central spot, which is comparatively bright, remains constantly of the same magnitude; although the other rings, like Newton's rings formed between two glass lenses, dilate greatly with the obliquity of incidence. This appearance was observed at the same time by Professor Lloyd. The explanation is easy. It depends simply on this circumstance (which is evident from the Table), that the angle $180^{\circ}-\delta^{\prime}$, at these oblique incidences, is nearly proportional to ${ }^{\circ} \cos i$.

As to the index of refraction in metals, the author conjectures that it is equal to $\frac{M}{\cos \chi}$. 
VII.-ON THE LAWS OF THE DOUBLE REFRACTION OF QUARTZ.

[Transactions of the Royal Irish Academy, VoL. xvir.-Read Feb. 22, 1836.]

THe singular laws of the double refraction of quartz, which have been discovered by the successive researches of Arago, Biot, Fresnel, and Airy, are known merely as so many independent facts; they have not been connected by a theory of any kind. I propose, therefore, to show how these laws may be explained hypothetically, by introducing differential coefficients of the third order into the equations of vibratory motion.

Suppose a plane wave of light to be propagated within a crystal of quartz. Let the co-ordinates $x, y, z$, of a vibrating molecule be rectangular, and take the axis of $z$ perpendicular to the plane of the wave, and the axis of $y$ perpendicular to the axis of the crystal. Let us admit that the vibrations are accurately in the plane of the wave, and of course parallel to the plane of $x y$. Then, using $\xi$ and $\eta$ to denote, at any time $t$, the displacements parallel to the axes of $x$ and $y$ respectively, we shall assume the two following equations for explaining the laws of quartz :-

$$
\begin{aligned}
& \frac{d^{2} \xi}{d t^{2}}=A \frac{d^{2} \xi}{d z^{2}}+C \frac{d^{3} \eta}{d z^{3}}, \\
& \frac{d^{2} \eta}{d t^{2}}=B \frac{d^{2} \eta}{d z^{2}}-C \frac{d^{3} \xi}{d z^{3}} .
\end{aligned}
$$

The peculiar properties of this crystal depend on the constant $C$. When $C=0$, the third differentials disappear, and 


\section{On the Laws of the Double Refraction of Quartz.}

the equations are reduced to the ordinary form, in which state they ought to agree with the common equations for uniaxal crystals. Henee, putting $a$ for the reciprocal of the ordinary index, $b$ for the reciprocal of the extraordinary, and $\phi$ for the angle made by the axis of $z$ with the axis of the crystal, we must have

$$
A=a^{2}, \quad B=a^{2}-\left(a^{2}-b^{2}\right) \sin ^{2} \phi,
$$

supposing the velocity of propagation in air to be unity.

Now, from the nature of equations (1) and (2), the vibrations must be elliptical. In fact, if we put

$$
\boldsymbol{\xi}=p \cos \left\{\frac{2 \pi}{l}(s t-z)\right\}, \quad \eta=q \sin \left\{\frac{2 \pi}{l}(s t-z)\right\}
$$

where $p, q, s, l$ are constant quantities, the differential equations will be satisfied by assigning proper values to $s$ and to the ratio $\frac{q}{p}$. For, after substituting in equations (1) and (2) the values of the partial differential coefficients obtained by differentiating formulæ (4), we shall find that every term of each equation will have the same sine or cosine for a factor ! omitting therefore, the common factors, and making $\frac{q}{p}=k$, we shall get the following equations of condition :-

$$
\begin{aligned}
& s^{2}=A-\frac{2 \pi}{l} C k, \\
& s^{2}=B-\frac{2 \pi}{l} \frac{C}{k} .
\end{aligned}
$$

Subtracting these, we have

$$
A-B+\frac{2 \pi C}{l}\left(\frac{1}{k}-k\right)=0,
$$

which, by formulæ (3), becomes

$$
k^{2}-\frac{l}{2 \pi C}\left(a^{2}-b^{2}\right) \sin ^{2} \phi \cdot k=1 .
$$

Let us now interpret these results. It is obvious, from for- 
mulæ (4), that 8 is the velocity of propagation for a wave whose length is $l$, and that each vibrating molecule describes a little ellipse whose semiaxes $p$ and $q$ are parallel to the directions of $x$ and $y$. But the number $k$, expressing the ratio of the semiaxes, has two values, one of which is the negative reciprocal of the other, as appears by equation (8) ; and each value of $k$ has a corresponding value of $s$ determined by equation (5) or (6). Hence there will be two waves elliptically polarized, and moving with different velocities, the ratio of the axes being the same in both ellipses; but the greater axis of the one will coincide with the less axis of the other. The difference of sign in the two values of $k$ shows that if the vibration be from left to right in one wave, it will be from right to left in the other. These laws were discovered by Mr. Airy.

The law by which the ellipticity of the vibrations depends on the inclination $\phi$, and on the colour of the light, is contained in equation (8). The value of the constant $C$ will be determined presently. In the mean time we may observe, that $C$ denotes a line, whose length is very small, compared with the length of a wave.

When $\phi=0$, the light passes along the axis of the crystal. In this case we have $k^{2}=1$, and $k= \pm 1$; which shows that there are two rays, circularly polarized in opposite directions. The value of $s$ for each ray may be had from equation (5) or (6), by putting +1 and -1 successively for $k$. Calling these values $s^{\prime}$ and $s^{\prime \prime}$, we find

$$
\begin{array}{ll}
8^{\prime 2}=a^{2}-2 \pi \frac{C}{l}, & s^{\prime}=a\left(1-\frac{\pi C}{a^{2} l}\right) ; \\
s^{\prime \prime 2}=a^{2}+2 \pi \frac{C}{l}, & s^{\prime \prime}=a\left(1+\frac{\pi C}{a^{2} l}\right) .
\end{array}
$$

Suppose a plate of quartz to have two parallel faces perpendicular to the axis; and conceive a ray of light, polarized in a given plane, to fall perpendicularly on it. The incident rectilinear vibration may be resolved into two opposite circular vibrations, 
which will pass through the crystal with different velocities; and which, after their emergence into air, will again compound a rectilinear vibration, whose direction will make a certain angle $\rho$ with that of the incident vibration: so that the plane of polarization will appear to have been turned round through an angle equal to $\rho$, called the angle of rotation. This angle may be determined by means of the preceding formulæ. Putting $\theta$ for the thickness of the crystalline plate, the circularly polarized wave whose velocity is $s^{\prime}$ will pass through it in the time

$$
\frac{\theta}{s^{\prime}}=\frac{\theta}{a}\left(1+\frac{\pi C}{a^{2} l}\right)
$$

and the wave whose velocity is $s^{\prime \prime}$ in the time

$$
\frac{\theta}{\delta^{\prime \prime}}=\frac{\theta}{a}\left(1-\frac{\pi C}{a^{2} l}\right) .
$$

Therefore, if $\delta$ be the difference of the times, we have

$$
\delta=\frac{2 \pi C \theta}{a^{3} l} .
$$

But, since the velocity of propagation in air is supposed to be unity, the time and the space described are represented by the same quantity; and therefore $\delta$, which is evidently a line, denotes the distance between the fronts of the two cireularly polarized waves, when they emerge into air. The waves heing at this distance from each other, if we conceive, at the same depth in each of them, a molecule performing its circular vibration, and carrying a radius of its circle along with it, the two radii will revolve in contrary directions, and will always cross each other in a position parallel to the incident rectilinear vibration. Now let two series of such waves be superposed, so as to agitate every molecule by their compound effect, and it is evident that, when the radius vector of one component vibration attains the position just mentioned, the radius vector of the other will be separated from it by an angle equal to $\frac{2 \pi \delta}{\lambda}$, where $\lambda$ is the length of 
a wave in air. The resultant rectilinear vibration will bisect this angle; and therefore $\rho$, the angle of rotation, will be equal to $\frac{\pi \delta}{\lambda}$. Hence, substituting for $\delta$ its value, and observing that $l$, the length of a wave in quartz, is equal to $a \lambda$, we find

$$
\rho=\frac{2 \pi^{2} C \theta}{a^{4} \lambda^{2}}
$$

which gives the experimental law of M. Biot, that the angle of rotation is directly as the thickness of the crystal, and inversely as the square of the length of a wave for any particular colour. By changing the sign of $C$, we should have an equal rotation in the opposite direction. And here we may remark, that $C$ may be made negative in all the preceding equations, its magnitude remaining. There are two kinds of quartz, the right-handed and left-handed, distinguished by the sign of $C$.

The angle of rotation, 'for a given colour and thickness, is known from M. Biot's experiments. We can therefore find the value of $C$ by means of the last formula; and substituting this value in equation (8), we shall be able to compute $k$ when $\phi$ and $l$ are given. Now it happens that Mr. Airy," by a very ingenious method of observation, has determined the values of $k$ in red light for two different values of $\phi$; and of course we must compare these observed values of $k$ with the independent results of theory. As Mr. Airy's experiments were made upon red light, we shall select, for the object of our calculations, the red ray which is marked by the letter $C$ in the spectrum of Fraunhofer. For this ray, Fraunhofer has given the length $\lambda$, which, expressed in parts of an English inch, is equal to 0000258 ; and M. Rudberg has found $a=\cdot 64859, b=64481$. Moreover, from the experiments of M. Biot, we may collect that the are of rotation, produced by the thickness of a millimetre, is something more than 19 degrees for the ray we have chosen; so that the fraction $\frac{1}{3}$ may be taken to express nearly the length of that

- Transactions of the Cambridge Philosophical Society, Vol, Iv., p. 205. 
68 On the Lawes of the Double Refraction of Quartz.

are in a circle whose radius is unity. We have, therefore, $\boldsymbol{\theta}=\cdot 03937$ inch, and $\rho=\cdot 333$. Substituting these values in the formula

$$
\frac{l}{2 \pi C}=\frac{\pi \theta}{a^{3} \lambda \rho}
$$

derived from (12), we find

$$
\frac{l}{2 \pi C}=52710 ;
$$

from which it appears that $C$ is about twenty thousand times less than the millionth part of an inch.

Again, since $a^{2}-b^{2}=\cdot 00489$, we have

$$
\frac{l}{2 \pi C}\left(a^{2}-b^{2}\right)=258,
$$

so that equation (8) becomes

$$
k^{2}-258 \sin ^{2} \phi \cdot k=1 .
$$

\begin{tabular}{|c|c|c|}
\hline \multirow{2}{*}{ Values of $\phi$. } & \multicolumn{2}{|c|}{ Values of $k$. } \\
\hline & Observed. & Calculated. \\
\hline $6^{\circ} \quad 15^{\prime}$ & $\tan 16^{\circ} 38^{\prime}=\cdot 2897$ & $\cdot 2980$ \\
\hline $8^{\circ} \quad 54^{\prime}$ & $\tan 8^{\circ} 56^{\prime}=\cdot 1582$ & $\cdot 1579$ \\
\hline
\end{tabular}

The results of this formula are compared with Mr. Airy's experiments in the following Table, in which the less root is taken for $k$, and its sign is neglected.

The angles $\phi$, in the first column, are deduced from the observed inclinations of the rays in air to the axis of the crystal; and as $k$ was observed to be somewhat different for the ordinary and extraordinary rays, its mean values are given in the second column. The exact coincidence between these and the calculated values is, perhaps, in some degree accidental; but a less perfect agreement would be sufficient to confirm the theory. 
The magnitude of $k$ varies considerably with the colour of the light, increasing from the red to the violet, while the coeffieient of $\sin ^{2} \phi . k$, in formula (13) diminishes. If we take the violet ray $H$, for example, this coefficient will be 159 . But it would be useless to make any more ealculations, as we have no experiments with which they might be compared.

The figure of the wave surface yet remains to be examined.

Eliminating $k$ between formulæ (5) and (6), we obtain the equation

$$
\left(s^{2}-A\right)\left(s^{2}-B\right)=4 \pi^{2} \frac{C^{2}}{l^{2}},
$$

which expresses the nature of the surface, $s$ being a perpendicular from the origin on a tangent plane. From this equation it follows that the two values of 8 can never become equal in quartz, as they do in other crystals; for if we solve the equation for $s^{2}$, and put the radical equal to zero, we shall get the condition

$$
(A-B)^{2}+16 \pi^{2} \frac{C^{2}}{l^{2}}=0,
$$

which cannot be fulfilled, since the quantity which ought to vanish is the sum of two squares. The two sheets, or nappes, of the wave surface are therefore absolutely separated.

It is commonly assumed that one of the rays is refracted according to the ordinary law; but this is not the case, since neither of the values of $s$ is constant. However, the ray which has the greater velocity ( $a$ being greater than $b$ ) may still, for convenience, be called the ordinary ray. Of the two roots of equation (8), the one, $k_{0}$, whose numerical value (supposing $\phi$ not to vanish) is less than unity, corresponds to this ray. When $C$ is positive, $k_{\mathrm{o}}$ is negative ; and when $C$ is negative, $k_{\mathrm{o}}$ is positive : therefore in both kinds of quartz, by formulø (5) and (6), we have $s_{\mathrm{o}}{ }^{2}>A$, and $s_{\mathrm{e}}{ }^{2}<B$; denoting by $s_{\mathrm{o}}$ and $s_{\mathrm{e}}$ the respective velocities of propagation of the ordinary and extraordinary waves. Hence, if we conceive a sphere of the radius $a$, with its centre at the origin, and a concentrie prolate spheroid, whose semiaxis of 


\section{On the Laws of the Double Refraction of Quartz.}

revolution is also equal to $"$, and parallel to the axis of the crystal, while the radius of its equator is equal to $b$, the ordinary nappe of the wave surface will fall entirely without the sphere, and the extraordinary nappe entirely within the spheroid, whether the crystal be right-handed or left-handed. With respect to the little ellipse in which the vibrations are performed, and of which the semiaxes parallel to $x$ and $y$ are represented by $p$ and $q$ respectively, it is evident that $p>q$ for the ordinary wave, since $k_{0}<1$; and that $p<q$ for the extraordinary wave. When $C$ vanishes, the minor axis of each ellipse also vanishes, and the rays become plane-polarized, the ordinary vibrations being then parallel to the direction of $x$, and the extraordinary parallel to that of $y$. This is exactly what ought to happen on the supposition that the vibrations of a plane-polarized ray* are parallel to its plane of polarization-a supposition which was kept in view in framing the fundamental equations (1) and (2).

To show, with precision, how the two kinds of quartz are to be distinguished by the sign of $C$, we must give definite directions to the axes of co-ordinates. To this end, let us imagine the plane of $x y$ to be horizontal, and a circle to be described in it with the origin $O$ for its centre; and let the north, east, and south points of this circle be marked respectively with the letters $\mathrm{N}, \mathrm{E}, \mathrm{S}$. Let the direction of $+x$ be eastward, from $O$ to $E$; that of $+y$ northward, from $O$ to $N$; and that of $+z$ vertically downwards; the progress of the light through the crystal being

* On this point there are two very different opinions. Fresnel supposed, as is well known, that the vibrations of a plane-polarized ray are perpendicular to its plane of polarization; whereas, according to M. Cauchy, whom I have followed, they are parallel to that plane. I am induced to adopt the latter supposition, because I have succeeded, by means of hypotheses which are grounded on it, in discovering the laws of reflexion from crystallized surfaces; laws which include, as a particular case, those discovered by Fresnel for ordinary media. The hypotheses alluded to, along with-some of their results, are published in the London and Edinburgh Philosophical Magazine, Vol. vur., p. 103, in a letter to Sir David Brewster (suprre, pp. 75, et seq.) See also Vol. vil., p. 295, of the same Journal (supra, pp. 55, et seq.) I hope soon to offer the Academy a detailed account of my researches on this subject. 
also downwards, and the plane of the wave moving parallel, as before, to the plane of $x y$. 'Then the crystal will be right-handed or left-handed, according as $C$ is positive or negative. For, if $C$ be positive, $k_{\mathrm{o}}$ will be negative, and formulæ (4) will become, by exhibiting the sign of $k_{\mathrm{o}}$,

$$
\xi=p \cos \left\{\frac{2 \pi}{l}(s t-z)\right\}, \quad \eta=-k_{\circ} p \sin \left\{\frac{2 \pi}{l}(s t-z)\right\},
$$

for the ordinary vibration; and

$$
\xi=k_{0} q \cos \left\{\frac{2 \pi}{l}(s t-z)\right\}, \quad \eta=q \sin \left\{\frac{2 \pi}{l}(s t-z)\right\},
$$

for the extraordinary vibration. Now if we suppose the arc $\frac{2 \pi}{l}(s t-z)$ either to vanish, or to be a multiple of the circumference, the molecule will be at the east point of its vibration; and upon increasing the time a little, the value of $\eta$ will become negative in (15), and positive in (16), so that the movement will be towards the south in the first case, and towards the north in the second. Therefore, when $C$ is positive, the ordinary vibration takes place in the direction NES, or from left to right, and the extraordinary in the direction $S E N$, or from right to left, supposing a spectator to look in the direction of the progress of the light. It may be shown, in like manner, that when $C$ is negative, the ordinary and extraordinary vibrations are in the directions $S E N$ and $N E S$, or from right to left and from left to right respectively. Now if a plane-polarized ray be transmitted along the axis of the crystal, the plane of polarization will be turned in the direction of the ordinary vibration, because this vibration, being propagated more quickly, will be in advance of the other, upon emerging from the crystal. Hence, the rotation is from left to right when $C$ is positive, and from right to left when $C$ is negative; and the crystal is called right-handed in the first case, and left-handed in the second.

We have all along supposed that $C$ is a constant quantity, and the agreement of our results with experiment proves that 


\section{On the Lawes of the Double Refraction of Quartz.}

this supposition is at least very nearly true in the neighbourhood of the axis. It is probable, however, not only that $C$ varies with $\phi$, but that it becomes different in equations (1) and (2); that is to say, it is probable that the following equations

$$
\begin{aligned}
& \frac{d^{2} \xi}{d t^{2}}=A \frac{d^{2} \xi}{d z^{2}}+C \frac{d^{3} \eta}{d z^{3}}, \\
& \frac{d^{2} \eta}{d t^{2}}=B \frac{d^{2} \eta}{d z^{2}}-C^{\prime} \frac{d^{3} \eta}{d z^{2}},
\end{aligned}
$$

in which $C^{\prime}$ is a little different from $C$, would be more correct than those which we have assumed. Indeed Mr. Airy's experiments seem to indicate that $C^{\prime}$ is greater than $C$; for he found, as we have already said, that the ratio of the axes of the little ellipse described by a vibrating molecule is somewhat different for the two rays, being more nearly a ratio of equality for the ordinary than for the extraordinary ray. Now if we set out from equations (17), instead of (1) and (2), and proceed in all respects as before, we shall arrive at the formula

$$
k^{2}-\frac{l}{2 \pi C}\left(a^{2}-b^{2}\right) \sin \phi \cdot k=\frac{C^{\prime}-}{C}
$$

instead of formula (8). The quantity $\frac{C^{\prime}}{C}$ will be greater than unity, if $C^{\prime}$ be greater than $C$, and the value of $k_{0}$ will be greater than before. This seems to be the explanation of the difference between the ratios observed by Mr. Airy.

It may be proper to state briefly the considerations which led to the foregoing theory. Beginning with the simple case of a ray passing along the axis, the first thing to be explained was the law of M. Biot, that the angle of rotation varies inversely as the square of $l$ or of $\lambda$. Now it was remarked by Fresnel, who first resolved the phenomena of rotation into the interference of two circularly polarized waves, that the interval $\delta$ between these waves, at their emergence from the crystal, must be inversely as 1, if the angle of rotation be inversely as the square of $l$. This re- 
mark suggested* to me the idea of adding, to the equations of the common theory, terms containing the third differential coefficients of the displacements; for it was evident that such additional terms would give, in the value of $s^{2}$, a part inversely proportional to 7 . It was also evident that the third differential coefficient of $\xi$ should be combined with the second differential coefficients of $\eta$, and the third of $\eta$ with the second of $\xi$, in order that, after substitutions such as we have indicated in deducing formulæ (5) and (6), the sines or cosines might disappear by division, and that thus the value of $s^{2}$ might be independent of the time, as it ought to be. This kind of reasoning led me to assume the equations

$$
\begin{aligned}
& \frac{d^{2} \xi}{d t^{2}}=a^{2} \frac{d^{2} \xi}{d z^{2}}+C \frac{d^{3} \eta}{d z^{3}}, \\
& \frac{d^{2} \eta}{d t^{2}}=a^{2} \frac{d^{2} \eta}{d z^{2}}+D \frac{d^{3} \xi}{d z^{3}},
\end{aligned}
$$

for the case of a ray passing along the axis of quartz; and then, substituting in these equations the values of the differential coefficients obtained by differentiating the formulø

$$
\xi=p \cos \left\{\frac{2 \pi}{l}(s t-z)\right\}, \quad \eta= \pm p \sin \left\{\frac{2 \pi}{l}(s t-z)\right\},
$$

which express a circular vibration (from right to left, or from left to right, according to the sign of the second $p$ ), the result was

from $(19)$, and

$$
s^{2}=a^{2} \mp \frac{2 \pi}{l} C
$$

$$
s^{2}=a^{2} \pm \frac{2 \pi}{l} D
$$

* "The singular relation between the interval of retardation $[\delta]$ and the length of the wave $[l]$ seems to afford the only clue to the unravelling of this difficulty." "Report on Physical Optics," by Professor Lloyd ("Fourth Report of the British Association," p. 409). It was in reading this Report that Fresnel's remark, about the relation between $\delta$ and $l$, first came to my knowledge. 
74 On the Lazus of the Double Refraction of Quartz.

from (20); which showed that $D=-C$, since the values of $s$, corresponding to the same circular vibration, ought to be equal. The transition from this simple case to that of a ray inclined at a given angle $\phi$ to the axis was easily made, by taking into account the doubly refracting structure of the crystal. This was done by supposing $\xi$ and $\eta$ parallel to the principal directions in the plane of the wave, and by changing $a^{2}$, in equation (20), into $a^{2}-\left(a^{2}-b^{2}\right) \sin ^{2} \phi$; and thus the fundamental equations $(1)$ and (2) were obtained. 


\section{$(75)$}

VIII.-ON THE LAWS OF REFLEXION FROM CRYSTALLIZED SURFACES.

[From the Philosophical Magazine, VoL. vIII., 1835.]

\section{To Sir David Brewster.}

DE.AR Sir-I have great pleasure in sending you an account of the laws by which I conceive that the vibrations of light are regulated when a ray is reflected and refracted at the separating surface of two media; especially as the only guide which I had, in my inquiry after these laws, was your Paper on the action of crystallized surfaces upon light, published in the Philosophical Transactions for the year 1819. The observation which I found there, that the polarizing angle was the same for a given plane of incidence, "whether the obtuse angle of the rhomb [of Iceland spar] was nearest or furthest from the eye, or whether it was to the right or left hand of the observer," disappointed me at first, being contrary to what I had anticipated from principles analogous to those which had been employed by Fresnel in the problem of reflexion from ordinary media. I then sought other principles, and the observation is now a result of theory.

Assuming, as a basis for calculation, that Fresnel's law of double refraction is rigorously true, I have been obliged to make an essential change in his physical ideas. Conceive an ellipsoid whose semiaxes are parallel to the three principal directions of the crystal, and equal respectively to its three principal indices of refraction, and let a central section of the ellipsoid be made by a plane parallel to the plane of a wave passing 
through the crystal. The section will be an ellipse, and the wave will be polarized by the crystal in a plane parallel to either semiaxis of this ellipse, the index of refraction for the wave being equal to the other semiaxis. This is Fresnel's law of double refraction; and the theory which led him to it makes it necessary to admit that the vibrations of the wave are perpendicular to its plane of polarization; whereas, according to the views which $I$ have adopted, the vibrations of the wave are parallel to its plane of polarization, and to one semiaxis of the elliptic section, while its index of refraction is equal to the other semiaxis. These views nearly agree with the theory of M. Cauchy, according to whom the vibrations of polarized light are parallel to its plane of polarization, but inclined at small angles to the plane of the wave in crystallized media, instead of being exactly parallel to the latter plane, as I have supposed them to be. Besides, the theory of M. Cauchy, founded on the six equations of pressure in a crystallized medium, implies the existence of a third ray of feeble intensity, and for the other two rays gives a law somewhat different from that of Fresnel. Being obliged, in order to account for your experiments, to abandon the physical ideas of Fresnel, and to approximate towards those of M. Cauchy, I was embarrassed by this third ray ; and wishing to get rid of it, as well as of the slight deviations from the symmetrical law of Fresnel, I adopted the expedient of altering the equations of pressure, in such a way as to make them afford only two rays, and give a law of refraction exactly the same as Fresnel's. The equations which $\mathrm{I}$ found to answer this purpose are the following :-

$$
\begin{aligned}
& A=-2 \rho\left(c^{2} \frac{d \eta}{d y}+b^{2} \frac{d \xi}{d z}\right) V^{2} \rho, \\
& B=-2\left(a^{2} \frac{d \xi}{d z}+c^{2} \frac{d \xi}{d x}\right) V^{2} \rho, \\
& C=-2\left(b^{2} \frac{d \xi}{d x}+a^{2} \frac{d \eta}{d y}\right) V^{2} \rho
\end{aligned}
$$




$$
\begin{aligned}
& D=a^{2}\left(\frac{d \eta}{d z}+\frac{d \zeta}{d y}\right) V^{2} \rho, \\
& E=b^{2}\left(\frac{d \zeta}{d x}+\frac{d \xi}{d z}\right) V^{2} \rho, \\
& F=c^{2}\left(\frac{d \xi}{d y}+\frac{d \eta}{d x}\right) V^{2} \rho .
\end{aligned}
$$

In these equations, the axes of co-ordinates are perpendicular to each other, and parallel to the principal directions of the crystal ; $x, y, z$ are the co-ordinates of a vibrating molecule at the time $t ; \xi, \eta, \zeta$ are the components of the displacement of the same molecule at the same time; $a, b, c$ are the three principal indices of refraction out of the crystal into an ordinary medium in which the velocity of light is equal to $V$; and $\rho$ is the density of the ether, which density I suppose to be the same in all media. The quantities $A, F, E$ are the components, parallel to the axes of $x, y, z$, respectively, of the pressure upon a plane perpendicular to the axis of $x ; F, B, D$ are the components of the pressure upon a plane perpendicular to the axis of $y$; and $E, D, C$ the components of the pressure upon a plane perpendicular to the axis of $z$. The values of $D, E, F$ are the same as those given by M. Cauchy; but the values of $A, B, C$ are different from his, and much simpler. By introducing into the equations of M. Cauchy the condition that the vibrations shall be performed without any change of density, the resulting values of $A, B, C$ might be shown to agree nearly with those given above. The six pressures, $A, B, C, D, E, F$, being known, it is easy to find the pressure upon a plane making any given angles with the axes of co-ordinates.

These things being premised, it is time to mention the laws, or rather hypotheses, which I have imagined for discovering the relations that exist, as to direction and magnitude, among the vibrations in each ray, when reflexion and refraction take place at the separating surface of two media, whether crystallized or not. In stating the two very simple laws that have occurred to me 
for this purpose, it will be convenient, when the first medium is an ordinary one, to suppose that the incident light is polarized. Then, by the first law, the vibrations in one medium are equivalent to those in the other; that is to say, if the incident and reflected. vibrations be compounded, like forces acting at a point, their resultant will be the same, both in length and direction, as the resultant of the refracted vibrations similarly compounded. By the second law, the lateral pressure upon the separating surface is the same in both media; the lateral pressure being understood to mean the pressure in a direction perpendicular to the plane of incidence.

As it would engage us too long to follow these laws into detail, I shall merely state some of the results which I have obtained from them, for the case of a uniaxal crystal into which the light passes out of an ordinary medium.

Imagine the surface of the crystal to be horizontal, and call the point of incidence $I$. With the centre $I$ and any radius, conceive a sphere to be described, cutting in the point $Z$ a vertical line $I Z$ drawn through the centre, and let a radius $I P$, parallel to the axis of the crystal, meet the surface of the sphere in $P$. Let the great circle $Z O E$ be the plane of incidence, containing both the direction $I O$ of the ordinary refracted ray produced backwards, and the direction IE of a normal to the extraordinary wave;

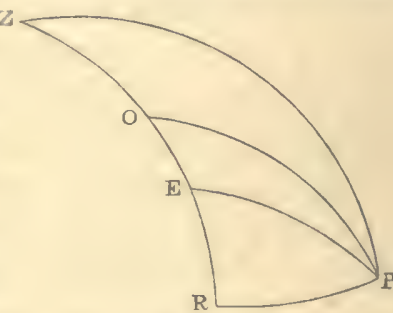

Fig. 16. and draw the great circles $P Z, P O, P E$. The angle $Z$ will be the azimuth of the plane of incidence. Let $Z O=\phi, Z E=\phi^{\prime}, P O=\psi$, $P E=\psi^{\prime}$, the angle $Z O P=\theta$, and the angle $Z E P=\theta^{\prime}$. Call the angle of incidence $i$, and suppose $b$ to be the reciprocal of the ordinary refractive index and $a$ the reciprocal of the extraordinary.

Each of the refracted rays, in turn, may be made to disappear, by polarizing the incident ray in a certain direction assigned by theory. When the extraordinary ray disappears, the reflected ray is polarized in a plane inclined to the plane of incidence at 


\section{from Crystallized Surfaces.}

an angle $\beta$ determined by the formula

$\tan \beta=\cos (i+\phi) \tan \theta+2\left(a^{2}-b^{2}\right) \sin \theta \sin \psi \cos \psi \frac{\sin ^{2} i}{\sin (i-\phi)}$.

When the ordinary ray disappears, the plane of polarization of the reflected ray is inclined to the plane of incidence at an angle $\beta$ determined by the formula

$$
\begin{aligned}
& -\tan \beta^{\prime}=\cos \left(i+\phi^{\prime}\right) \operatorname{cotan} \theta^{\prime} \\
& +\left(a^{2}-b^{2}\right) \frac{\cos 2 \theta^{\prime}}{\sin \theta^{\prime}} \sin \psi^{\prime} \cos \psi^{\prime} \frac{\sin ^{2} i}{\sin \left(i-\phi^{\prime}\right)} .
\end{aligned}
$$

And when the angles $\beta, \beta^{\prime}$, become equal, the plane of polarization of the reflected ray becomes independent of the plane of polarization of the incident ray; and the angle of incidence $i$, at which this equality takes place, is the polarizing angle of the crystal. Hence we have the equation of condition

$$
\left.\begin{array}{r}
\cos (i+\phi) \tan \theta+2\left(a^{2}-b^{2}\right) \sin \theta \sin \psi \cos \psi \frac{\sin ^{2} i}{\sin (i-\phi)} \\
+\cos \left(i+\phi^{\prime}\right) \operatorname{cotan} \theta^{\prime}+\left(a^{2}-b^{2}\right) \frac{\cos 2 \theta^{\prime}}{\sin \theta^{\prime}} \sin \psi^{\prime} \cos \psi^{\prime} \frac{\sin ^{2} i}{\sin \left(i-\phi^{\prime}\right)}
\end{array}\right\}=
$$

to be fulfilled at the polarizing angle.

Since $i+\phi$, in this equation, is nearly equal to a right angle, put $i+\phi=\frac{\pi}{2}+\delta$, and $\delta$ will be a small quantity. Draw $P R$ an are of a great circle perpendicular to $Z O E$, and let $Z R=p$, $P R=q$. Then we shall find from equation (4), after various substitutions and reductions,

$$
\delta=K \cos ^{2} q\left(\cos ^{2} \phi-\cos ^{2} p\right) \text {; where } K=\frac{\left(a^{2}-b^{2}\right)\left(1+b^{2}\right)}{2 b\left(1-b^{2}\right)} .
$$

In deducing this value of $\delta$, the approximations were made with a tacit reference to the case of reflexion in air from a common rhomb of Iceland spar. The coefficient $K$, in this case, is equal to about nine degrees, and the resulting numerical values of 
the polarizing angles in various azimuths agree very well with your experiments. You will perceive that the value of $\delta$ is the same in supplementary azimuths, which explains the observation, cited in the beginning of my letter, relative to the equality of the polarizing angles at opposite sides of the perpendicular $I Z$ in a given plane of incidence.

When the point $R$ falls upon $O$, we have $\delta=0$, and $i+\phi$ equal to a right angle. Hence, when the cotangent of $Z R$ is equal to the ordinary index, the tangent of the polarizing angle is equal to the same index. This theorem, though deduced from an approximate equation, might be shown to be exact.

When the axis of the crystal lies in the plane of incidence, we may obtain an exact expression for the polarizing angle. The condition of polarization then becomes

$$
\cos \left(i+\phi^{\prime}\right)-\left(a^{2}-b^{2}\right) \sin \psi^{\prime} \cos \psi^{\prime} \frac{\sin ^{2} i}{\sin \left(i-\phi^{\prime}\right)}=0 ;
$$

from which, by the proper substitutions, we obtain the following expression:-

$$
\sin ^{2} i=\frac{1-a^{2} \cos ^{2} \lambda-b^{2} \sin ^{2} \lambda}{1-a^{2} b^{2}} ;
$$

where $\lambda$ denotes the complement of $Z P$, or the inclination of the axis to the face of the crystal, and $i$ is the polarizing angle. This formula, in a shaye somewhat different, was communicated, above a year ago, to Professor Lloyd, who has noticed it, in connexion with your Tappr. in his "Report on Physical Optics." When $a$ and $b$ becongles nal, the formula gever your law of the tangent for ordinary inojia. $^{3}$

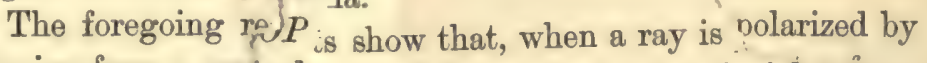
reflexion from a cry ing, the plane of polarization deviates from the plane of inciden $\mathrm{a}_{3}$, except when the axis lies in the latter plane; and that ther deviation may be made very great by placing the crys tal in contact with a medium whose refractive power is nearly eq ual to that of the crystal itself; for when $i$ is nearly equal to $\phi{ }^{0} \mathrm{r}$ to $\phi^{\prime}$, the divisor $\sin (i-\phi)$ or $\sin \left(i-\phi^{\prime}\right)$ is 


\section{Laws of Reflexion from Crystallized Surfaces. 8I}

very small, and therefore $\tan \beta$ or $\tan \beta^{\prime}$ is very great. But this remark is of no value whatever in explaining the very singular phenomena which you have observed in the extreme case just mentioned; nor can I imagine any reason why there should be a deviation, as there was in some of your experiments, when the axis lies in the plane of incidence, since everything is then alike on both sides of this plane. Indeed the whole of this subject, which occupies the latter part of your Paper of 1819 , is very extraordinary and interesting; and I was glad to hear that you had resumed the investigation of it, and made many experiments which have not been published.

I wish you would publish them. They seem to be of great importance in the present state of optical science.

I am, dear Sir, ever truly yours,

J. Mac Cullagh.

Trux. Coll., Dublin, Dec. 22, 1835. 
IX.-ON THE PROBABLE NATURE OF THE IIGHT TRANS. MITTED BY THE DIAMOND AND BY GOLD LEAF.

[Proceedings of the Royal Irish Academy, VoL. I. p. 27.-Read Jan. 9, 1837.]

Professor Mac Cullagh made a verbal communication on the probable nature of the light transmitted by the diamond and by gold leaf. He conceives that as there is a change of phase caused by reflexion from these bodies, so there is also a change of phase produced by refraction; the change being different according as the incident light is polarized in the plane of incidence, or in the perpendicular plane. Consequently, if the incident ray be polarized in any intermediate plane, the refracted ray should be elliptically polarized; and on examining the light transmitted by gold leaf, this was found to be the case. Of course the same thing is true of the light which enters the other metals, and which subsequently absorbed. The same remark explains the appearance of double refraction in specimens of the diamond which give only a single image; and it is likely that other precious stones will be found to possess similar properties. Mr. Mac Cullagh has obtained a general formula for the difference of phase between the two component portions of the refracted light-one polarized in the plane of incidence, and the other perpendicular to it. He finds from this formula, that the difference of phase, which is nothing at a perpendicular incidence, increases until it becomes equal to the characteristic at an incidence of $90^{\circ}$; and when the light emerges into air, the difference of phase is doubled. The formula has not yet been submitted to the test of experiment. 


\section{$(83)$}

\section{X.-ON THE LAWS OF CRYSTALLINE REFLEXION.}

[From the Philosophical Magazine, Vou. x., 1837.]

In a Number of Poggendorff's Annalen (No. 6, for 1836), which reached Dublin late in November, there are some remarks by M. Seebeck on a Paper of mine which appeared in the last February Number of this Journal (vol. viii. p. 103). That Paper contains a general theory of reflexion at the surfaces of crystallized media; and M. Seebeck, in comparing the results with his own experiments, has fully confirmed some of my formulæo, while he has shown that others are defective. I have therefore been obliged to revise my theory, and I have ascertained that it was vitiated by the introduction of a certain relation among the quantities denominated pressures, which, following the example of M. Cauchy, I had supposed to be concerned in the problem. This relation I had observed to hold in the case of singly refracting media, and I concluded, without any other reason, that it would hold good generally. But though it led to the correct formula for the polarizing angles in different azimuths, it was nevertheless arbitrary and unfounded; and therefore it is now banished entirely from the investigation, the place which it occupied being supplied by the natural and simple law of the preservation of vis viva, while everything else remains as before. I hope the imperfection of my first essay will be excused, when it is considered that the erroneous proposition bears but a small proportion to the whole theory; and, moreover, that the general problem, which I undertook to resolve, is one that has not been attempted by any other 
person, although the want of a solution has long been felt. The difficulties which we have to deal with, in entering upon this problem, are not mere mathematical difficulties, but difficulties arising from the want of first principles; and, in physical questions of this kind, where we must, at the outset, have recourse to conjecture, in order to supply the very principles of our reasoning, it can hardly be expected that the whole truth should be divined at once. I think, however, that I have now obtained a true mechanical theory; and if so, it will help to decide, not only the question immediately before us, but also the other much-disputed, though more elementary, questions concerning the density of the ether in different media, and the direction of the vibrations in polarized light. In fact, a particular supposition respecting each of the latter questions is included in my theory, the several principles of which, making the single alteration that has been mentioned, I shall here enumerate :-

1. The density of the ether is the same in all media.

2. The vibrations of plane-polarized light are parallel to the plane of polarization.

3. The vis viva is preserved.

4. The vibrations are equivalent at the common surface of two media.

To these may be added the definition of the polarizing angle of a crystal; namely, the angle of incidence at which the plane of polarization of the reflected ray becomes independent of the plane of polarization of the incident ray. At the polarizing angle, the former plane does not, in general, coincide with the plane of reflexion, but makes with it a small angle which may be called the deviation.

It is curious that, about a year and a half ago, I employed these four principles, precisely as I have now enumerated them, in deducing Fresnel's well-known laws of reflexion for ordinary media; but I did not then apply the law of vis vira to crystals, because my mind was preoccupied by the notion that there existed some relation among the pressures. This notion I had 
taken up from reading a little Paper, by M. Cauchy, in the Bulletin des Sciences Mathématiques for July 1830; and by combining such a relation with the three conditions afforded by my own law of equivalent vibrations, I had actually obtained, for the polarizing angles in different azimuths, a formula (that marked (5) in my former Paper), which I found to agree very well with Sir David Brewster's experiments, and which M. Seebeck has found to agree still better with his own.

The formula for the polarizing angle is obtained by equating two values of the deviation; and it is remarkable that the very same formula comes out in my present theory, although the values of the deviation are entirely different. Referring, for brevity, to the notation of my former Paper, I find, for the case of a uniaxal erystal,

$$
\begin{gathered}
\tan \beta=\cos (i+\phi) \tan \theta, \ldots \ldots \ldots \ldots \ldots \ldots \ldots \\
\quad-\tan \beta^{\prime}=\cos \left(i+\phi^{\prime}\right) \operatorname{cotan} \theta^{\prime}+\left(a^{2}-b^{2}\right) \frac{\sin \psi^{\prime} \cos \psi^{\prime} \sin ^{2} i}{\sin \theta^{\prime} \sin \left(i-\phi^{\prime}\right)} .
\end{gathered}
$$

These equations $(a)$ and $(b)$ are to be substituted for equations (2) and (3), ${ }^{*}$ which are the equations that M. Seebeck found to be at variance with his experiments.

By means of formula (5), equation $(a)$ becomes

$$
\beta=\frac{K}{2} \sin 2 q \sin (p-\phi),
$$

from which the deviation in any azimuth may be readily calculated. The azimuth (as M. Seebeck reckons it) begins when $\boldsymbol{\theta}$ $=0$, and $p$ is then positive. This formula $(c)$ perfectly represents the experiments of M. Seebeck on Iceland spar. The corresponding expressions for biaxal crystals may be easily deduced, and will be given in a Paper which I am preparing to lay before the Royal Irish Academy.

At the time of my last communication I was not aware that the case in which the plane of incidence is a principal section of the crystal (or the azimuth $=0$ ) had been solved by M. Seebeck, and that formula ( $(\tilde{\tau}), \dagger$ which I regarded as my own, had been obtained by him long before. 
It remains to say a word respecting the new principle of equivalent vibrations, the most important, perhaps, of all, as it is certainly the simplest that can be imagned. If we conceive an ethereal molecule situated at the common surface of two media, it would seem that its motion ought to be the same, whether we regard the molecule as belonging to the first medium or to the second. Now the incident and réflected vibrations are superposed in the first medium, and the refracted vibrations in the second; and therefore we may infer (when the phase is not changed by reflexion or refraction), that if the incident and reflected vibrations be compounded, like forces acting at a point, their resultant will be the same, both in length and direction, as the resultant of the refracted vibrations similarly compounded. This is the law of equivalent vibrations, and it gives, at once, three equations. A fourth equation is afforded by Fresnel's law of the vis viva; and thus we have the four conditions necessary for a general solution of the problem. From the principle of equivalent vibrations, as we have stated it, it follows that the vibrations resolved parallel to the separating surface are equivalent in the two media; and, in fact, this part of the general principle was assumed by Fresnel; but the other part, namely, that the vibrations perpendicular to the separating surface are equivalent, was not assumed by him, nor is it by any means true in his theory. It appears then that three conditions only are afforded by the hypotheses which Fresnel successfully employed in solving the problem of reflexion from ordinary media. These hypotheses, therefore, are not sufficient when applied to crystals; except, indeed, in the case before alluded to, where the azimuth $=0$, which has been solved by M. Seebeck. It should be observed, that though the reasons which I have assigned for the principle of equivalent vibrations are extremely simple, yet it was not by such simple reasoning that I was led to it originally.

Trinity College, Dublin, December 13, 1836. 


\section{( 87$)$}

XI.-ON THE LAWS OF CRYSTALLINE REFLEXION AND REFRACTION.

[Transactions of the Royal Irish Academy, VoL. xvirI.-Read Jan. 9, 1837.]

When a ray of light, which has been polarized in a given plane, suffers reflexion and refraction at the surface of a transparent medium, the rays into which it is divided are found to be polarized in certain other planes; and it becomes a question to determine the positions of these planes, as well as the relative intensities of the different rays; or, in theoretical language, to find the direction and magnitude of the reflected and refracted vibrations, supposing those of the incident vibration to be given. The transparent medium may be either a singly-refracting substance, such as glass, or a doubly-refracting crystal, like Iceland spar. When the medium is of the first kind, the problem is comparatively simple, being, in fact, nothing more than a particular case of the problem which we have to consider when the medium is supposed to be of the second kind. In the progress of knowledge it was natural that the simpler question should be first attended to ; and accordingly Fresnel, during his brief and brilliant career, found time to solve it. But the general problem, relative to doubly-refracting media, had not been attempted by anyone, when, in the year 1834, my thoughts were turned to the subject. I then recollected a conclusion to which I had been led some years before, and which, on this occasion, proved of essential service to me. Being fond of geometrical construc- 
tions, I amused myself, when I first became acquainted with Fresnel's theories, by throwing his algebraical expressions, whenever I could, into a geometrical form; and treating in this way the well-known formulæ in which he has embodied his solution of the problem just alluded to, I obtained a remarkable result, which gave me the first view of the principle that I have since employed under the name of the principle of the equivalence of vibrations. In order to state this result briefly, I will take leave to introduce a new term for expressing a right line drawn parallel to the plane of polarization of a ray, and perpendicular to the direction of the ray itself. Calling such a right line the transversal of the polarized ray, I found, from the formulæ of Fresnel, that when polarized light falls upon a singlyrefracting medium, the transversals of the incident, of the reflected, and of the refracted rays are all parallel to the same plane, which is the plane of polarization of the refracted ray; and that the magnitudes of the vibrations, or the greatest excursions of the ethereal molecules, in the incident and the reflected rays, are to each other inversely as the sines of the angles which the respective transversals of those rays make with the transversal of the refracted ray. I was struck by the strong analogy which these relations among the transversals bore to the composition of forces or of small vibrations in mechanics; but it happened unfortunately that, in the theory of Fresnel, the vibrations of light were supposed to take place, not in the direction of the transversals, but perpendicular to them, so that there was no physical circumstance to support the analogy, there being no motion in the direction of the transversals; while, on the other hand, no such analogy existed among the vibrations themselves in the directions which Fresnel had assigned to them. It was therefore with some interest that I afterwards learned, upon the publication of the tenth volume of the Memoirs of the Institute, that M. Cauchy* had actually inferred, from mechanical principles, that the vibrations of polarized light are in the 
direction of the transversals; but this inference was to be received with caution, as being contrary to the hypothesis of Fresnel; and besides, I had in the meantime contrived a way of adapting my analogy, in some degree, to that hypothesis, by supposing areas to be compounded instead of vibrations; so that I hesitated which of the two opinions to prefer. Taking, however, the opinion of $\mathbf{M}$. Cauchy as that which fell in more naturally with the aforesaid analogy, I was led to the conclusion, that the vibration in the refracted ray is probably the resultant of the incident and reflected vibrations; and I saw that if this principle were true for singly-refracting media, it should also, from its very nature, be true, when properly generalized, for doubly-refracting erystals; so that in such crystals the resultant of the two refracted vibrations would be the same, both in length and direction, as the resultant of the incident and reflected vibrations.

This was the principle of equivalent vibrations. But I had no sooner begun to regard it as probable, than an objection started up against it. In the case of a ray ordinarily refracted out of a rarer into a denser medium, the magnitude of the refracted vibration, as deduced from this principle, was greater than that which came out from the theory of Fresnel, in the proportion of the sine of the angle of incidence to the sine of the angle of refraction. Consequently, assuming with Fresnel that the ether is more dense in the denser medium, the law of the preservation of vis viva was violated.

There was another embarrassment which I felt in my early efforts to find out the laws of crystalline reflexion. Taking for granted the hypothesis of Fresnel, that the density of the ether in an ordinary medium is inversely as the square of its refractive index, I was at a loss what hypothesis to make, in this respect, for doubly-refracting crystals, wherein the refractive index changes with the direction of the ray. For the density, being independent of direction, could not be conceived to vary with the refractive index. About two years ago I got over this difficulty, by supposing the density of the 
ether to be the same in all media.* At the same time I was compelled to employ the principle of equivalent vibrations, in order to have a sufficient number of conditions, though for a while I overlooked the perfect agreement which now subsisted between this principle and the law of vis viva: it happened, in fact, that the new hypothesis of a constant density made the vis viva of the refracted ray exactly the same as in the theory of Fresnel.†

But to see why it was necessary to assume the principle of equivalent vibrations, we must observe, that when a polarized ray is incident on a crystal there are four things to be determined, namely, the direction and magnitude of the reflected vibration, and the magnitudes of the two refracted vibrations. Hence we must have four conditions, or we must have relations affording so many equations. But the hypotheses of Fresnel, by which he solved the problem of reflexion for ordinary media, afford only three conditions. We will state his hypotheses at length :-

1st. The vibrations of polarized light are in the plane of the wave, and perpendicular to the plane of polarization.

2nd. The density of the ether is inversely as the square of the refractive index of the medium.

3rd. The vis viva is preserved.

4th. The vibrations parallel to the separating surface of two media are equivalent; that is, the refracted vibration parallel to the surface is the resultant of the incident and reflected vibrations parallel to the same.

We see that the fourth hypothesis gives two conditions, and the law of vis viva gives a third.

Let us now take the more general principle of equivalent vibrations, in place of the fourth hypothesis of Fresnel, altering

* This bypothesis is maintained by Mr. Challis; and certainly it falls in extremely well with the astronomical phenomenon of the aberration of light. - See, on this subject, Professor Lloyd's Report on Physical Optics, "Fourth Report of the British Association for the Advancement of Science," pp. 311, 313.

† Supra, p. 100, note. 
the first hypothesis in the way that we have shown to be necessary in order to suit that principle, and making the ethereal density constant. Then, if we retain the law of vis viva, our new hypotheses will be these:-

1st. The vibrations of polarized light are in the plane of the wave, and parallel to the plane of polarization; which may be expressed in a word, by saying that the vibrations are transver$s a l$, according to the peculiar sense in which I use the term.

2nd. The density of the ether is the same in all bodies as in vacuo.

3rd. The vis viva is preserved.

4th. The vibrations in two contiguous media are equivalent; that is, the resultant of the incident and reflected vibrations is the same, both in length and direction, as the resultant of the refracted vibrations.

It is evident that the last hypothesis affords three equations, by resolving the vibrations parallel to three axes of co-ordinates; and the law of vis viva supplies a fourth equation. Thus we have the requisite number of conditions.

The hypotheses that we have last enumerated are those which will be employed in the present Paper. They have been made to include the law of vis viva, because I lately found that this law must necessarily accompany the rest; but at first I neglected it, and even made considerable progress without it; for, by the help of another hypothesis, I obtained formula which represented such experiments as I was aware of at the time. This other hypothesis I took up from reading an article by M. Cauchy in the Bulletin des Sciences Mathématiques, ${ }^{*}$ in which he arrives, by a peculiar process, at the formulø of Fresnel for the case of ordinary reflexion. The hypotheses

* "Sur la Réfraction et la Réflexion de la Lumière," Bulletin des Sci. Math., Juillet, 1830. 'In this Paper the vibrations of polarized light must be supposed perpendicular to the plane of polarization, though the Paper was published immediately after the author had promulged the contrary opinion. The latter opinion, which I adopted from him because it harmonized with my analogy before mentioned, he has formally renounced of late, and has returned to the 
which he chiefly employs are relations among certain quantities called pressures; and it was such a relation that $I$ adopted instead of the law of vis viva. I supposed that, at the confines of two media, the pressure on the separating surface, in a direction perpendicular to the plane of incidence, ought to be the same, whether it be considered as resulting from the vibrations in the first medium or in the second. This hypothesis I conceived to be true in general, because I found it to be true for ordinary media; but I could never assign any better reason for it. Combining it, however, with the principle of equivalent vibrations, I deduced several expressions for uniaxal crystals, and among others a formula for the polarizing angles in different azimuths of the plane of reflexion. When this formula was compared with the experiments of Sir David Brewster* on the polarizing angles of Iceland spar, the accordance was so satisfactory as to leave no doubt upon my mind that I had arrived at the true formula for these angles; and though the truth of the conclusion did not allow me to argue that the premises

hypothesis of Fresnel. M. Cauchy supposed too, in the above Paper, that the ethereal density is the same in different media; but he has found cause to abandon this hypothesis also. See his notes addressed to M. Libri, in the Comptes rendus des Séances de l'Académie des Sciences, Séance du 4 Avril, 1836, where he gives the reasons for his present opinions. He says, "Ainsi Fresnel a eu raison de dire, non-seulement que les vibrations des molécules éthérées sont généralement comprises dans les plans des ondes, mais encore que les plans de polarisation sont perpendiculaires aux directions des vitesses ou des déplacements moléculaires. J'arrive au reste à cette dernière conclusion d'une autre manière, en établissant les lois de la réflexion et de la réfraction à l'aide d'une nouvelle méthode qui sera développée dans mon mémorie ...... [cette méthode] ne m’oblige plus à supposer, comme je l'avais fait dans un article du Bulletin des Sciences, que la densité de l'éther est la même dans tous les milieux. Mes nouvelles recherches donnent lieu de croire que cette densité varie en général quand on passe d'un milieu à un autre." More lately, in his Nouveaux Exercices de Mathématiques, 7e Livraison, M. Cauchy states positively that his principles do not permit him to adopt the hypothesis that the density of the ether is the same in all media. $\mathrm{He}_{\boldsymbol{\theta}}$ also gives the differential equations which, as he has found by his new method, ought to subsist at the separating surface of two media, and from which he has obtained the formule of Fresnel for ordinary reflexion. But these equations do not include the laws of crystalline reflexion.

* Phil. Trans., 1819, p. 150. 
were true, yet the presumption in their favour was very strong, insomuch that, upon remarking, as I did soon after, that the law of vis viva harmonized with my other hypotheses, I did not think it worth while* to try what would be the consequence of using this law, instead of the relation which I had put in its place. In this state of my theory, I gave an account of it at the meeting of the British Associationt in Dublin, in August, 1835 ; and the leading steps and results were afterwards published in a letter to Sir David Brewster. $\neq$

Now we are to observe, that when common light is polarized by reflexion at the surface of a doubly-refracting crystal, the polarization does not, in general, coincide with the plane of reflexion, as in the case of ordinary media, but is inclined to it at a certain angle, which may be called the deviation; and it was by equating two values of the deviation that I obtained the formula above mentioned for the polarizing angle. This formula, as we have seen, was correct; but it happened, singularly enough, that the expressions for the deviation, which were

* I had, besides, an objection to the law of vis viva, on the ground that it would give an equation of the second degree; and I wished to have all my equations linear, lest, in the seemingly complicated question of crystalline reflexion, they should give two answers when the nature of the question required but one. This has actually happened, since the present Paper was read, in applying my hypotheses to the case of internal reflexion at the second surface of a uniaxal crystal. Supposing an ordinary ray to emerge after double reflexion, and putting $\theta$ for the angle which the emergent transversal makes with the plane of incidence, I found, for determining $\theta$, an equation of the form

$$
A+B \tan \theta+C \tan ^{2} \theta=0,
$$

wherein $A$ is very small, but does not vanish; so that the equation gives two roots, one very small, the other about the proper value. It is clear, therefore, that there is a want of adjustment somewhere: but $I$ am now inclined to think that the fault is not in the principle of vis viva. Possibly our laws of the propagation of light in doubly refracting media are not quite accurate. Whatever supplementary law shall be found to remedy this untorard result will probably, at the same time, account for the extraordinary phenomena observed by Brewster, in reflexion at the first surface when the crystal is in contact with a medium of nearly equal refractive power.

† London and Edinburgh Philosophical Magazine, Vol. vII. p. 295.

Ibid., Vol, vir. p. 103 ; February, 1836. (Supra, p. 75.) 
used in obtaining the formula, were erroneous. It is to M. Seebeck that I am obliged for pointing out this curious circumstance. In Poggendorff's Amnals, ${ }^{*}$ he gave an abstract of my letter to Sir David Brewster, and compared my results with his own numerous and accurate experiments, both on the polarizing angles of Iceland spar and on the angles of deviation. $\mathrm{He}$ found that my formula represented the former class of experiments as well as could be wished; but the theoretical values of the deviations did not at all agree with his experimental measures. These measures of the deviation he published on this occasion; and, with their assistance, I traced the error to its source, which was the relation among the pressures. The principle of vis vica was therefore introduced, instead of that relation, and the theory became much simpler by the change. I now obtained, for the deviation, a new expression, which agreed with the experiments of M. Seebeck; but the formula for the polarizing angle came out the very same as before. This correction was made on the 6th of December, and was published in the Philosophical Magazine $\dagger$ on the first of the present month.

In the interval I have arrived at very elegant geometrical laws, which can be easily remembered, and which embrace the whole theory of crystalline reflexion. In enunciating these, it will be convenient to draw our transversals always through the same origin $O$, which we shall suppose to be the point of incidence, as this point is common to all the rays, whether incident, reflected, or refracted; and we may imagine wave planes to be drawn through the origin, parallel to the plane of each wave, so that every transversal will lie in its own wave plane. The incident and reflected wave planes will be perpendicular to the incident and reflected rays, but the two refracted wave planes will in general be oblique to their respective rays. In the latter case, a right line drawn through the origin perpendicular to

* Annalen der Physik und Chemie, Vol. xxxvur. p. 276.

+ London and Edinburgh Philosophical Magazine, Vol. x. p. 43. (Supra, p. 84.) 
the wave plane is called the wave normal. It is scarcely necessary to remark, that all the four wave planes intersect the surface of the crystal in the same right line which is perpendicular to the plane of incidence; and that the angles of refraction are the angles which the refracted wave normals make with a perpendicular to that surface. The index of refraction is the ratio of the sine of the angle of incidence to the sine of the angle of refraction, just as in ordinary media; but here it is a variable ratio, and has different values for the same angle of incidence. I have elsewhere* shown how to find the refracted rays and waves when the incident ray is given.

As we suppose the ethereal molecules to vibrate parallel to the transversals, we may take the lengths of the transversals proportional to the magnitudes or amplitudes of the vibrations; these lengths being always measured from the common origin o. Then, in virtue of our fourth hypothesis, the transversals will be compounded and resolved exactly by the same rules as if they were forces acting at the point $O$.

We must now conceive a wave surface of the crystal, with its centre at $O$, the point of incidence. As the velocities of rays which traverse the crystal in directions parallel to the radii of its wave surface are represented by those radii, so let a concentric sphere be described with a radius $O S$, which shall represent, on the same scale, the constant velocity of light in the medium external to the crystal. At any point $T$ on the wave surface apply a tangent plane, on which let fall, from $O$, a perpendicular $O G$, meeting the plane in $G$. On this perpendicular take the length Fig. 17. $O P$ from towards $G$, so that $O P$ shall be a third proportional to $O G$ and the constant line $O S$. Then, while the point $T$ describes the wave surface, the point $P$ will describe another surface reciprocal $\dagger$ to the wave surface. This other surface may

- Irish Academy Transactions, Vol. xvII. p. 202.

$\dagger$ For the general theory of reciprocal surfaces, see Irish Academy Transactions, Vol. xvIr. p. 241. 
very properly be called the index surface, $O P$ is the refractive index of the ray whose velocity is $O T$, or rather of the wave $T G$, which belongs to that ray; for if we conceive an incident wave, touching the sphere, to be refracted into the wave $T G$, touching the wave surface in $T$, the sine of the angle of incidence will be to the sine of the angle of refraction as $O S$ to $O G$, or as $O P$ to $O S$; so that, taking the constant $O S$ for unity, the index of refraction will be represented by $O P$. The wave surface and the index surface will thus be reciprocal to each other, every point $T$ on the one having a point $P$ reciprocally corresponding to it on the other.

It is remarkable that the transversal of the ray $O T$ is perpendicular to the plane OPT; for in the theory of Fresnel, as I formerly proved, $\uparrow$ the direction of the vibrations is the right line $T G$; and as I suppose the transversal to be perpendicular to the vibrations of that theory, and to be, at the same time, in the wave plane, which is perpendicular to $O P$, it follows that the transversal must be perpendicular to both the right lines $T G$ and $O P$, and therefore perpendicular to their plane $O P T$. Therefore conceiving the transversal to be drawn through $O$ at right angles to the plane $O P T$, the plane of polarization of the ray $O T$ must needs pass through it. But there is nothing else to fix the position of this last plane. We may make it pass through the ray itself $O T$, as an ordinary media, or we may draw it through the wave normal $O P$ with Fresnel. Or, instead of drawing it through either of these two sides of the triangle OPT, we may make it parallel to the third side $P T$. The last is what I should prefer, because the plane so determined possesses important properties. I shall call it, however, the polar plane, because the name, plane of polarization, is a long one; and the signification of the latter may, if any one

* This is the surface which I formerly called (Trans., p. 252) the surface of refraction; a name not sufficiently descriptive. Sir W. Hamilton has called it the surface of wave slowness, and sometimes the surface of components. But the name index surface seems to recommend itself, as both short and expressive.

+ Ibid. Vol. Xvi. p. 76. (Supra, p. 12.) 
chooses, be kept distinct, though in an ordinary medium both terms must mean the same thing. The polar plane then of the ray $O T$ is a plane passing through its transversal and parallel to the right line $P T$; so that if $O K$ be drawn parallel to $P T$, the polar plane will pass through $O K$. In general, to find the transversals and the polar plane of any ray, we take the point where the ray meets its own nappe of the wave surface, and join it with the corresponding point on the index surface, drawing a plane through the origin and the joining line. Then a right line perpendicular to this plane at the origin will be the transversal, and a plane drawn through the transversal parallel to the joining line will be the polar plane.

Now let a polarized ray be incident at $O$ upon the crystal. It will in general be divided into two rays. But each of these rays in turn may be made to disappear by polarizing the incident ray in a certain plane. Let us suppose then that there is only one refracted ray OT. In what direction must the incident ray be polarised, or, in other words, what must be the position of its transversal, in order that this may be the case? and what will be the corresponding transversal of the reflected ray? The answer is simple-both transversals will lie in the polar plane of the refracted ray. Let us pursue this remark a little.

The refracted ray $O T$ being given, we can find its polar plane, and thence the intersections of this plane with the incident and reflected wave planes. These intersections will be the positions of the incident and reflected transversals when $O T$ is the sole refracted ray. The refracted transversal lies also in the polar plane; and this transversal is, by our fourth hypothesis, the diagonal of a parallelogram, whose sides are the other two transversals, which determines the relative lengths of the three transversals, or the relative amplitudes of the vibrations. The intensities of the reflected and incident rays are, of course, proportional to the squares of their transversals. When the ray $O T$ dissappears, we must take the polar plane of the other ray, and proceed as before.

Thus there are, in the incident wave plane, two transversal 
directions which give only a single refracted ray. These, as well as the corresponding ones in the reflected wave plane, may be called uniradial transversals. They are the intersections of the two refracted polar planes with the incident and reflected wave planes.

When the incident transversal does not coincide with either of the uniradial directions, it is to be resolved parallel to them, and then each component transversal will supply a refracted ray, according to the foregoing rules. The reflected transversals, arising from the component incident ones, are to be found separately by the same rules, and then to be compounded.

In ordinary reflexion, if the incident transversal be in the jlane of incidence, or perpendicular to it, the reflected transversal will be so likewise. But this does not hold in crystalline reflexion. The general method just given will, however, enable us to determine the positions and magnitudes of the reflected transversals in these two remarkable cases; and then, if we choose, we can reduce any other case to these two, by resolving the incident transversal in directions parallel and perpendicular to the plane of incidence.

If we conceive a pair of incident transversals, at right angles to each other, to revolve about the origin, it is evident that there will be a position in which the reflected transversals corresponding to them will also be at right angles to each other. There is no difficulty in finding this position, and there will be an advantage in using it when common unpolarized light is incident on the crystal. For, the incident transversals being rectangular, we may suppose the light to be equally divided between them, and then the intensities of the corresponding reflected portions can be found by the preceding rules. As the reflected transversals are also rectangular, the sum of these intensities will be the whole intensity of the reflected light, and their difference will be the intensity of the polarized part of it. This part will be polarized in a plane passing through the greater of the two reflected transversals.

Common light will be completely polarized by reflexion when 
the two uniradial directions in the reflected wave plane coincide with each other; that is, when this plane and the two refracted polar planes have a common intersection. For then, if the ineident light be polarized, it is manifest that the reflected transversal will lie in that intersection, whatever be the position of the incident transversal; and therefore if common light be incident, with its transversals in every possible direction, the reflected transversals will have but one direction. Thus the reflected light will be completely polarized in a plane passing through the above intersection.

Hence, as the reflected ray is perpendicular to its wave plane, it follows that, at the polarizing angle of a erystal, the reflected ray is perpendicular to the intersection of the polar planes of the two refracted rays. The reflected transversal, as we have seen, is this very intersection. This transversal is inclined, in general, to the plane of incidence, and we have had occassion to speak of its inclination under the name of the deviation. If we now suppose the double refraction to diminish until it disappears, the intersection of the polar planes will at last coincide* with the refracted ray. There will then be no deviation, and the reflected and refracted rays will be at right angles to each other, agreeably to the law of Brewster, which prevails at the polarizing angle of an ordinary medium.

There is a case in which the construction that we have given for determining the polar plane of a ray becomes useless. It is when the ray $O T$ is a normal to the wave surface; for then $O P$ coincides with $O T$, and we cannot fix the transversal by our construction. But it is precisely in such a case that the polar plane is most easily ascertained, for it is then nothing more than the plane of polarization of the common theory. For example, if we take the ordinary ray of a uniaxal erystal, its polar plane will pass through the ray itself. and the axis of the crystal. Of course in an ordinary medium the polar plane and the plane of polarization are synonymous.

* For the polar planes will become two planes of polarization at right angles to each other. 
It may not be amiss to apply our general rules to the case of ordinary reflexion and refraction. Suppose then a polarized ray to fall on the surface of an ordinary medium. Draw a plane through the incident transversal and the refracted ray; this will be the plane of polarization of the refracted ray, and it will intersect the reflected wave plane in the reflected transversal. The refracted transversal will be the diagonal of a parallelogram, whose sides are the other two transversals; hence we have the relative lengths of the transversals, and thus everything is determined.*

- This construction was mentioned at the meeting of the British Association in Dublin. - See the Reports of the Association, or London and Edinburgh Phil. Mag. vol, vii. p. 295. The following is an extract from the Paper which I read at that meeting :-

"The formulæ given by Fresnel for the same purpose will be found to agree exactly with this rule, in determining the positions of the planes of polarization; and his expression for the amplitude of the reflected vibration is also in accordance with our construction. But the coincidence does not hold with regard to the amplitude of the refracted vibration, though the vis viva of the refracted ray is the same in both theories.

"Now it is very remarkable that if we alter the hypotheses of Fresnel where they are at variance with the preceding principles, we shall, from his own equations of condition, deduce formulæ agreeing in every respect, even as to the amplitude of the refracted wave, with the construction which we have accounted for in a different way (i.e. by using the relation among the pressures instead of the law of vis viva). The requisite alterations are two in number. First, the vibrations are to be supposed parallel to the plane of polarization, and not perpendicular to it, as Fresnel conceived; and secondly, the density of the ether is to be considered the same in both media, from which it follows, that the corresponding ethereal masses, imagined by Fresnel, are to each other as the sine of twice the angle of incidence to the sine of twice the angle of refraction. Substituting in Fresnel's equations of condition this value of the ratio of the masses, we obtain the formulø which I am inclined to regard as correct."

The equations spoken of in this extract are those which arise from the principle of vis viva, and from the equivalence of vibrations parallel to the separating surface of the two media. But it is worth while to observe, that when the vibrations are all in the same direction, that is, when the light is polarized perpendicular to the plane of incidence, the very same formulæ will come out from Young's remarkable analogy of the two elastic balls, one of which impinges directly on the other, supposed previously at rest, the masses of the balls being to each other in the ratio of the ethereal masses mentioned above. And, perhaps, this consideration affords the simplest possible explanation of Brewster's law relative to the pola- 
The reason of this construction will be evident, if we consider that, in an ordinary medium, the polar plane is the same as the plane of polarization; and that, when there is only one refracted ray, the three transversals lie in the polar plane of that ray, aceording to the general remark with which we set out. We now proceed to show that the theorem asserted in this remark is a consequence of our hypotheses, and we shall afterwards deduce a few results which may be readily compared with experiments.

Let us suppose then that the direction of the incident trans-

rizing angle; for, as there is no reflected motion when the balls are equal, the whole velocity of impact being communicated to the ball that was at first quiescent, so there is no reflected vibration when the ethereal masses are equal; that is, when the sine of twice the angle of incidence is equal to the sine of twice the angle of refraction, or when the angles of incidence and refraction are together equal to a right angle. The whole of the incident vibration then passes into the refracted ray. In general, if $t_{1}, t_{2}$ denote the angles of incidence and refraction, the masses of the imaginary balls will be as $\sin 2 t_{1}$, to $\sin 2 \iota_{2}$; and, if the velocity of the original impact be taken for unity, the common theory of the collision of elastic bodies will give

$$
\frac{\sin 2 t_{1}-\sin 2 t_{2}}{\sin 2 t_{2}+\sin 2 t_{3}} \text { or } \frac{\tan \left(t_{1}-t_{2}\right)}{\tan \left(t_{1}+t_{3}\right)}
$$

for the velocity retained by the impinging ball after the impact; and

$$
\frac{2 \sin 2 t_{1}}{\sin 2 t_{1}+\sin 2 t_{2}^{i}} \text { or } \frac{\sin 2 t_{1}}{\sin \left(t_{1}+t_{2}\right) \cos \left(t_{1}-t_{2}\right)} \text {, }
$$

for the velocity communicated to the other ball. These expressions (I.) and (Ir.), are the same as the values of $\tau_{3}$ and $\tau_{2}$, which we should deduce from equations (1) and (2), on the next page, by supposing $\tau_{1}$ to be unity, and the angles $\theta_{1}, \theta_{2}, \theta_{3}$ to be right angles. The general construction given in the text will lead to the same results, if we find from it the limiting ratios of the transversals, on the supposition that their directions approach each other indefinitely, and ultimately coincide in a right line perpendicular to the plane of incidence.

When the transversals are all in the plane of incidence, or when the light is polarized in that plane, the incident, the reflected, and the refracted transversals are to each other as $\sin \left(t_{1}+t_{4}\right), \sin \left(t_{1}-t_{2}\right)$, and $\sin 2 t_{2}$ respectively; because each transversal is proportional to the sine of the angle between the other two; and, in the present case, the angle between any two transversals is equal to the angle between the corresponding rays. Hence, taking the incident transversal for unity, the reflected transversal is

$$
\frac{\sin \left(i_{1}^{\prime}-t_{2}\right)}{\sin \left(t_{1}+t_{2}\right)}
$$


versal is such that there is only one refracted ray. It is evident that, in this case, the three transversals must lie in the same plane, since, by the fourth hypothesis, the refracted vibration is the resultant of the other two vibrations; and, therefore, we have only to prove that the plane of the transversals is the same as the polar plane of the refracted ray. Let $\tau_{1}, \tau_{2}, \tau_{3}$ be the respective lengths of the incident, refracted, and reflected transversals; let $\theta_{1}, \theta_{2}, \theta_{3}$ be the angles which they make with the plane of incidence, the angle $\theta_{2}$ being known from the theory of Fresnel; put $\iota_{1}, \iota_{2}, \iota_{3}$ for the angles made by the respective wave planes with the surface of the crystal, and $m_{1}, m_{2}, m_{3}$ for the relative quantities of ether set in motion by each wave. Then our hypotheses will give us the four following equations:-

$$
\begin{gathered}
m_{1} \tau_{1}{ }^{2}=m_{2} \tau_{2}{ }^{2}+m_{3} \tau_{3}{ }^{2}, \\
\tau_{1} \sin \theta_{1}+\tau_{3} \sin \theta_{3}=\tau_{2} \sin \theta_{2}, \\
\tau_{1} \cos \theta_{1} \cos \iota_{1}+\tau_{3} \cos \theta_{3} \cos \iota_{3}=\tau_{2} \cos \theta_{2} \cos \iota_{2}, \\
\tau_{1} \cos \theta_{1} \sin \iota_{1}+\tau_{3} \cos \theta_{3} \sin \iota_{3}=\tau_{2} \cos \theta_{2} \sin \iota_{2} .
\end{gathered}
$$

The first equation is manifestly the translation of the law of

and the refracted transversal is

$$
\frac{\sin 2 t_{x}}{\sin \left(t_{1}+\imath\right)}
$$

It has been already observed that our theory differs from that of Fresnel with regard to the magnitude of the refracted transversals. The expressions (II.) and (Iv.) must, in fact, be multiplied each by $\frac{\sin t_{2}}{\sin t_{1}}$ in order to produce the corresponding expressions which result from Fresnel's hypotheses. But the two theories also differ as to the relative directions of the incident and reflected transversals. For, supposing the light to fall upon the denser medium, or $\iota_{1}$ to be greater than $\iota_{2}$, our construction indicates that these transversals, when the angle of incidence is small, point in the same direction; whereas Fresnel concludes the contrary to be the case. However, the disagreement in this respect ceases as we approach the limiting incidence of $90^{\circ}$; for then, according to both theories, the incident and reflected transversals point in opposite directions. This last conclusion is conformable to the - inference which Professor Lloyd has drawn from his experiments on the interference of direct light with light reflected at a very oblique incidence. - See Irish Acad. Transactions, voL. xvi1. p. 176. 
the preservation of vis viva ; the other three are obtained from the principle of equivalent vibrations, by resolving the vibrations, or transversals, in three rectangular directions. In the second equation, the transversals are resolved perpendicular to the plane of incidence; in the fourth, perpendicular to the surface of the crystal; and in the third equation they are resolved parallel to the intersection of these two planes. When the angles $\theta_{1}, \theta_{2}, \theta_{3}$ begin, the transversals are in the plane of incidence in such a relative position, that, if they were turned round together in that plane through a right angle, they would point each in the direction of its own wave's progress. These angles increase on the same side of the plane of incidence, and range through the whole circumference. The angles $t_{1}, t_{2}, t_{3}$ are those of incidence, refraction, and reflexion; but, for the sake of symmetry, they are taken to be the angles which the wave normals, drawn from the origin in the direction of each wave's motion, make with the perpendicular to the surface, this perpendicular being directed towards the interior of the erystal. Thus it happens that $\iota_{3}$ is the supplement of $\iota_{1}$. Attending to this circumstance, equations (3) and (4) give us

$$
\begin{aligned}
& \tau_{1} \cos \theta_{1}-\tau_{3} \cos \theta_{3}=\tau_{2} \cos \theta_{2} \frac{\cos \iota_{2}}{\cos \iota_{1}}, \\
& \tau_{1} \cos \theta_{1}+\tau_{3} \cos \theta_{3}=\tau_{2} \cos \frac{\sin \iota_{2}}{\sin \iota_{1}} ;
\end{aligned}
$$

and, by adding and subtracting these, we find

$$
\begin{aligned}
& \tau_{1}=\tau_{2} \frac{\cos \theta_{2}}{\cos \theta_{1}} \frac{\sin \left(\iota_{1}+\iota_{2}\right)}{\sin 2 \iota_{1}}, \\
& \tau_{3}=\tau_{2} \frac{\cos \theta_{2}}{\cos \theta_{3}} \frac{\sin \left(\iota_{2}-\iota_{1}\right)}{\sin 2 \iota_{1}} ;
\end{aligned}
$$

which values if we substitute in equations (1) and (2), observing that $m_{3}=m_{1}$, as is evident, we shall get

$$
\frac{\sin ^{2}\left(\iota_{1}+\iota_{2}\right)}{\cos ^{2} \theta_{1}}-\frac{\sin ^{2}\left(\iota_{1}-\iota_{2}\right)}{\cos ^{2} \theta_{3}}=\frac{m_{2} \sin ^{2} 2 \iota_{1}}{m_{1} \cos ^{2} \theta_{2}},
$$


IO4

$$
\sin \left(\iota_{1}+\iota_{2}\right) \tan \theta_{1}-\sin \left(\iota_{1}-\iota_{2}\right) \tan \theta_{3}=\sin 2 \iota_{1} \tan \theta_{2} .
$$

Subtracting from (7) the identity

$$
\sin ^{2}\left(\iota_{1}+\iota_{2}\right)-\sin ^{2}\left(\iota_{1}-\iota_{2}\right)=\sin 2 \iota_{1} \sin 2 \iota_{2},
$$

there remains

$$
\begin{aligned}
& \sin ^{2}\left(\iota_{1}+\iota_{2}\right) \tan ^{2} \theta_{1}-\sin ^{2}\left(\iota_{1}-\iota_{2}\right) \tan ^{2} \theta_{3} \\
&=\frac{\sin ^{2} 2 \iota_{1}}{\cos ^{2} \theta_{2}}\left(\frac{m_{2}}{m_{1}}-\frac{\sin 2 \iota_{2}}{\sin 2 \iota_{1}} \cos ^{2} \theta_{2}\right) ;
\end{aligned}
$$

and this, by making

becomes

$$
\frac{m_{2}}{m_{1}}=\frac{\sin 2 \iota_{2}+2 h \sin ^{2} \theta_{2}}{\sin 2 \iota_{1}}
$$

$\sin ^{2}\left(\iota_{1}+\iota_{2}\right) \tan ^{2} \theta_{1}-\sin ^{2}\left(\iota_{1}-\iota_{2}\right) \tan ^{2} \theta_{3}=\sin 2 \iota_{1}\left(\sin 2 \iota_{2}+2 h\right) \tan ^{2} \theta_{2}$,

which is divisible by equation (8), the quotient being

$$
\sin \left(\iota_{1}+\iota_{2}\right) \tan \theta_{1}+\sin \left(\iota_{1}-\iota_{2}\right) \tan \theta_{3}=\left(\sin 2 \iota_{2}+2 h\right) \tan \theta_{2} \text {. }
$$

Then, by adding and subtracting equations (8) and (12) we obtain

$$
\left.\begin{array}{c}
\tan \theta_{1}=\cos \left(\iota_{1}-\iota_{2}\right) \tan \theta_{2}+\frac{h \tan \theta_{2}}{\sin \left(\iota_{1}+\iota_{2}\right)}, \\
\tan \theta_{3}=-\cos \left(\iota_{1}+\iota_{2}\right) \tan \theta_{2}+\frac{h \tan \theta_{2}}{\sin \left(\iota_{1}-\iota_{2}\right)} .
\end{array}\right\}
$$

These equations give the positions of the incident and reflected transversals when $h$ is known.

Now let the directions in which the transversals have been resolved in equations (2), (3), (4), be taken for the axes of $z, x, y$ respectively; so that, the origin being at $O$, the plane of $x y$ may be the plane of incidence, and the axis of $x$ may lie in the surface of the crystal. And, the reflected ray being conceived to lie within the angle made by the positive directions of $x$ and $y$, let the initial condition that we have assumed for the angles $\boldsymbol{\theta}_{1}, \boldsymbol{\theta}_{2}$, $\theta_{3}$ be satisfied by supposing that, when these angles begin, the 
transversals $\tau_{1}, \tau_{2}$ lie between the negative directions of $x$ and $y$, and the transversal $\tau_{3}$ between the directions of $+x$ and $-y$. Then if $\theta_{1}, \theta_{2}, \theta_{3}$ be reckoned towards the positive axis of $z$, so that each angle may be $90^{\circ}$ when the corresponding transversal points in the direction of $z$ positive, the equations of the transversal $\tau_{1}$ will be

$$
\frac{z}{\tan \theta_{1}}=-\frac{x}{\cos \iota_{1}}=-\frac{y}{\sin \iota_{1}}
$$

and those of $\tau_{3}$ will be

Let

$$
\frac{z}{\tan \theta_{3}}=\frac{x}{\cos \iota_{1}}=-\frac{y}{\sin \iota_{1}} .
$$

$$
z+A x+B y=0
$$

be the equation of a plane passing through the directions of $\tau_{1}$, $\tau_{2}$ and $\tau_{3}$. To determine $A$ and $B$, let the variables be eliminated from this equation by means of (14) and (15) successively, and we shall get the two equations of condition,

$$
\begin{aligned}
& \tan \theta_{1}-A \cos \iota_{1}-B \sin \iota_{1}=0, \\
& \tan \theta_{3}+A \cos \iota_{1}-B \sin \iota_{1}=0 ;
\end{aligned}
$$

which, by addition and subtraction, give

$$
\begin{aligned}
& B=\frac{\tan \theta_{1}+\tan \theta_{3}}{2 \sin \iota_{1}}, \\
& A=\frac{\tan \theta_{1}-\tan \theta_{3}}{2 \cos \iota_{1}} ;
\end{aligned}
$$

substituting, in these values, the expressions (13) for $\tan \theta_{1}$, $\tan \theta_{3}$, we have

$$
\left.\begin{array}{l}
B=\tan \theta_{2}\left(\sin \iota_{2}+\frac{h \cos \iota_{2}}{\sin ^{2} \iota_{1}-\sin ^{2} \iota_{2}}\right), \\
A=\tan \theta_{2}\left(\cos \iota_{2}-\frac{h \sin \iota_{2}}{\sin ^{2} \iota_{1}-\sin ^{2} \iota_{2}}\right) ;
\end{array}\right\}
$$

whence, by making

$$
\tan \kappa=\frac{h}{\sin ^{2} \iota_{1}-\sin ^{2} \iota_{2}},
$$


we find

$$
\frac{B}{A}=\frac{\tan \iota_{2}+\tan \kappa}{1-\tan \iota_{2} \tan \kappa}=\tan \left(\iota_{2}+\kappa\right)
$$

But if $z=0$ in (16), we have

$$
A x+B y=0,
$$

for the equation of the right line in which the plane of the transversals intersects the plane of incidence. This right line, lying; like the refracted wave normal, between the directions of $+x$ and $-y$, makes with the direction of $-y$ an angle $v$ which obviously has $\frac{B}{A}$ for its tangent; and therefore, by (21),

$$
v=\iota_{2}+\kappa ;
$$

which shows that the intersection of the two planes is inclined to the refracted wave normal at an angle equal to $\mathrm{k}$.

We must now find the value of $h$, which depends on the relative ethereal.masses put in motion by the incident and refracted waves. Conceiving the incident and refracted rays to be cylindrical pencils, having of course a common section in the plane of $x z$, which is the surface of the crystal, let each pencil be cut by a pair of planes parallel to its wave plane, and distant a wave's length from each other; then the cylindrical volumes so cut out will represent the corresponding masses, since, by our second hypothesis, the densities are equal. These volumes are to each other in the compound ratio of their altitudes, whieh are the wave lengths, and of the areas of their bases. The altitudes are evidently as $\sin \iota_{1}$ to $\sin \iota_{2}$. The first base is a perpendicular section of the incident pencil ; the second base an oblique section of the refracted one, the obliquity being equal to the angle $\varepsilon$ at which the wave normal is inclined to the ray. The perpendicular sections are to each other as the cosines of the angles which they make with the common section of the cylinders, or as $\cos \iota_{1}$ to $\cos \iota_{(2)}$; putting $\iota_{(2)}$ for the angle which the refracted ray makes with the negative direction of $y$. The second base is greafer than the perpendicular section of the refracted pencil in the pro- 
portion of unity to $\cos \varepsilon$. Therefore, compounding all these ratios, we find

$$
\frac{m_{2}}{m_{1}}=\frac{\sin \iota_{2} \cos \iota_{(2)}}{\sin \iota_{1} \cos \iota_{1} \cos \dot{\varepsilon}} .
$$

The same result may be otherwise obtained by observing that, in a system of waves, the corresponding masses are proportional to the ordinates $y$ of the points where the rays meet their wave surfaces. By a system of waves, I mean an ineident wave with all that are derived from it by reflexion or refraction at the same surface of the crystal, or at parallel surfaces. If, at the point where the incident ray intersects its spherical wave surface, we apply a tangent plane intersecting the plane of $x \approx$ in a right line parallel to $z$, through which right line other planes are drawn touching the wave surface of the crystal in four points, these tangent planes will be the waves derived from the incident wave which touches the sphere; and the points of contact, including that on the sphere, will be the points where the rays meet the wave surfaces. Then the corresponding masses will be represented by prisms having a common rectangular base in the plane $x z$, one side of this rectangle being the distance, on the axis of $x$, between the origin and the common intersection of the tangent planes; and the triangular face of each prism having the same distance for one side, and a point of contact for the opposite angle. These prisms, as they have a common base, will be proportional to their altitudes, which are the ordinates $y$ of the points of contact, The expression (24) may be easily deduced from this relation.

Let $O T, O P$, and the negative direction of $y$ meet the surface of the wave sphere (described with the radius $O S$ ) in the points $T, P_{\theta}, Y_{\text {; }}$; and let the right line, in which the plane of the transversals intersects the plane of incidence, meet the sphere in $L_{i}$. Then the points $Y_{i}, P_{i}, L_{i}$,

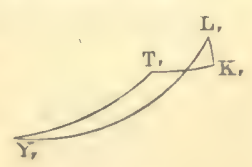

Fig. 18. being all in the plane of incidence, will be on the same great circle $Y, P, L$; and drawing the great circles $T, P, Y, T$, we 
shall have $Y, P_{1}=\iota_{2}, Y, T_{1}={\left(\iota_{2}\right)}_{T}, P,=\varepsilon, Y, L,=v=\iota_{2}+\kappa$, by (23) ; whence $P, L_{\mathrm{s}}=\kappa$.

As the transversal $\tau_{2}$ is perpendicular to the plane OTP, or to the plane of the great circle $T, P$, the cosine of the spherical angle $T, P, Y$, is the sine of $\theta_{2}$; and therefore, from the triangle $T, P, Y$, we have

$$
\cos \iota_{(2)}=\cos \iota_{2} \cos \varepsilon+\sin \iota_{2} \sin \varepsilon \sin \theta_{2},
$$

which being substituted in (24), gives

$$
\frac{m_{2}}{m_{1}}=\frac{\sin 2 \iota_{2}+2 \sin ^{2} \iota_{2} \sin \theta_{2} \tan \varepsilon}{\sin 2 \iota_{1}} ;
$$

and comparing this result with (10), we find

$$
h=\frac{\sin ^{2} \iota_{2} \tan \varepsilon}{\sin \theta_{2}}
$$

whence, and from (20), it follows that

$$
\tan \kappa=\frac{\sin ^{2} \iota_{2} \tan \varepsilon}{\left(\sin ^{2} \iota_{1}-\sin ^{2} \iota_{2}\right) \sin \theta_{2}} .
$$

Draw the great circle $L, K$, at right angles to $T, P$, and meeting it in $K_{j}$; then the plane of $L, K$, will be the plane of the transversals, since the latter plane passes through $L$, and is perpendicular to $T, P$, But the tangent of $P, K$, is equal to the tangent of $P, L$, multiplied by the cosine of the angle $P$, or by the sine of $\theta_{2}$; therefore, denoting $P, K$, by $\varepsilon_{1}$, and recollecting that $P L_{1}=\kappa$, we find, by (28),

$$
\frac{\tan \varepsilon_{1}}{\tan \varepsilon}=\frac{\sin ^{2} t_{2}}{\sin ^{2} \iota_{1}-\sin ^{2} \iota_{2}}
$$

Now we have seen that the ratio of $O P$ to $O S$, or $O S$ to $O G$ (Fig. 17), is the index of refraction; so that $\sin ^{2} \iota_{1}$ is to $\sin ^{2} \iota_{2}$ as $O P$ to $O G$. Therefore, by (29),

$$
\frac{\tan \varepsilon_{1}}{\tan \varepsilon}=\frac{O G}{O P-O G}=\frac{O G}{G P}
$$


but $O G$ is to $G P$ as the tangent of the angle $G P T$ is to the tangent of the angle GOT; and since $\varepsilon$ is the angle GOT, it follows that $\varepsilon_{1}$ is equal to the angle GPT or $K O P$. Consequently, $O K$ will meet the surface of the sphere in the point $K$, Thus we have proved our assertion, that, when there is only one refracted ray, the plane of the transversals is the polar plane of that ray.

The sign of the quantity $h$ is always the same as that of the cosine of the spherical angle $T, P, Y_{1}$. But to remove all ambiguity respecting signs, we must make a few additional conventions. Supposing, as we have hitherto done, that the refracted light moves from $O$ to $T$, and conceiving a right line to be drawn from the origin parallel to $G T$, and directed from $G$ towards $T$, let the angle $\sim_{2}$, which this right line makes with the plane of incidence, be reckoned, like $\theta_{1}, \theta_{2}$, from an initial position comprised between the negative directions of $x$ and $y$; and let $\vartheta_{2}$, like the angles $\theta_{1}, \theta_{2}, \theta_{3}$, increase on the side of $z$ positive, and range from $0^{\circ}$ to $360^{\circ}$. Then $\vartheta_{2}$ will always be equal either to the angle $P$, of the spherical triangle $T, P, Y$, or to the reentrant angle, which is the difference between $P$, and $360^{\circ}$. In either case, the cosine of $\vartheta_{2}$ will be the same, both in magnitude and sign, as the cosine of the angle $T, P, Y_{,}$. Consequently, if, instead of (25), we use the direct trigonometrical formula

$$
\cos \iota_{(2)}=\cos \iota_{2} \cos \varepsilon+\sin \iota_{2} \sin \varepsilon \cos \sim_{2},
$$

we shall find

$$
h=\frac{\sin ^{2} \iota_{2} \tan \varepsilon \cos \vartheta_{2}}{\sin ^{2} \theta_{2}} ;
$$

showing that the sign $h$ is always the same as the sign of $\cos \approx_{2}$. Now as $\theta_{2}$ differs from $\vartheta_{2}$ by a right angle, we will suppose

$$
\theta_{2}=\vartheta_{2}+90^{\circ} \text {, }
$$

and then we shall have $\sin \theta_{2}=\cos \vartheta_{2}$, algebraically as well as numerically. Thus we see that, by adopting these conventions, the value of $h$ in (27) will have the proper sign. Therefore, substituting this value of $h$ in formulæ (13), we obtain 


$$
\left.\begin{array}{l}
\tan \theta_{1}=\cos \left(\iota_{1}-\iota_{2}\right) \tan \theta_{2}+\frac{\sin ^{2} \iota_{2} \tan \varepsilon}{\cos \theta_{2} \sin \left(\iota_{1}+\iota_{2}\right)}, \\
\tan \theta_{3}=-\cos \left(\iota_{1}+\iota_{2}\right) \tan \theta_{2}+\frac{\sin ^{2} \iota_{1} \tan \varepsilon}{\cos \theta_{2} \sin \left(\iota_{1}-\iota_{2}\right)} \cdot
\end{array}\right\}
$$

These formulæ give the uniradial directions, or the positions of the incident and reflected transversals, when the sole refracted ray is that with which we have been occupied. The like directions, when the other ray exists alone, will be given by the formulæ

$$
\left.\begin{array}{l}
\tan \theta_{1}^{\prime}=\cos \left(\iota_{1}-\iota_{2}^{\prime}\right) \tan \theta_{2}^{\prime}+\frac{\sin ^{2} \iota_{2}^{\prime} \tan \varepsilon^{\prime}}{\cos \theta_{2}^{\prime} \sin \left(\iota_{1}+\iota_{2}^{\prime}\right)}, \\
\tan \theta^{\prime}=-\cos \left(\iota_{1}+\iota_{2}^{\prime}\right) \tan \theta_{2}^{\prime}+\frac{\sin ^{2} \iota_{2}^{\prime} \tan \varepsilon^{\prime}}{\cos \theta_{2}^{\prime} \sin \left(\iota_{1}-\iota_{2}^{1}\right)} ;
\end{array}\right\}
$$

where all the quantities, except $\iota_{1}$, which remains the same, are marked with accents, to show that they belong to the second refracted ray.

The uniradial directions having been found by these equations, the relative magnitudes of the uniradial transversals are determined by equations (6). When the incident transversal is not uniradial, it is evident, as we said before, that it may be resolved* in the two uniradial directions; that each component

* That, if an incident transversal be resolved in any two directions, the reflected and refracted transversals arising from it will be the resultants of those which would arise from each of its components separately, is a principle which appears very evident, insomuch that we can hardly suppose it to be untrue, without doing violence to our physical conceptions. Nevertheless, it is necessary to prove that this principle is not contrary to the law of vis viva; for though the vis viva may be preserved by each set of components (as it is when these are uniradial), yet we cannot therefore conclude that it will be preserved by their resultants. Here then is a test of the consistency of our theory; for we are bound to show that the law of vis viva is not infringed by the adoption of the principle in question. Now it is easy to see that, whatever be the two directions in which the incident transversal is resolved, the final results will always be the same; because, taking the component in each of these directions separately, the reflected and refracted transversals belonging to it must be obtained, in the first place, by the help of a resolution per- 
transversal, as if the other component did not exist, will furnish a refracted ray and a partial reflected transversal uniradial in its direction; and that the total (or actual) reflected transversal will be the resultant of the two partial ones.

When $\theta_{3}=\theta_{3}^{\prime}$, the partial reflected transversals will coincide, and their resultant will have a fixed direction, independent of the direction of the incident transversal. The angle of incidence at which this takes place is the polarizing angle, and the common value of $\theta_{3}$ and $\theta_{3}^{\prime}$ is the deviation. If, at the polarizing angle, the partial reflected transversals be equal in magnitude, and opposite in direction, their resultant will vanish, and the reflected ray will disappear. This will happen when the incident transversal is in the plane of the two refracted transversals, and therefore in the intersection of this plane with the incident wave plane; for, when there is no reflected ray, the incident transversal alone must be equivalent to the two refracted transversals.

Since the reflected transversal can be made to vanish at the polarizing angle, this angle might be found directly by putting the vis viva of the incident ray equal to the sum of the vires vivae of the two refracted rays, and by making the incident transversal the resultant of the two refracted transversals. Resolving the transversals parallel to the axes of co-ordinates, these conditions would give four equations, from which we could

formed in the uniradial directions. We need not, therefore, consider any case but that in which the resolution is uniradial throughout.

The incident transversal being denoted by $T_{1}$, let $T_{3}$ be the reflected transversal determined by the rules given in the text; and let the uniradial components of the former be $\tau_{1}, \tau_{1}^{\prime}$, while those of the latter are $\tau_{3}, \tau_{3}^{\prime}$. Then will

$$
\begin{aligned}
& T_{1}^{2}=\tau_{1}^{2}+\tau_{1}^{2}+2 \tau_{1} \tau_{1}^{\prime} \cos \left(\theta_{1}-\theta_{1}^{\prime}\right), \\
& T_{3}^{2}=\tau_{3}^{2}+\tau_{3}^{\prime}+2 \tau_{3} \tau_{3}^{\prime} \cos \left(\theta_{3}-\theta_{3}^{\prime}\right) ;
\end{aligned}
$$

where the signification of $\theta_{1}, \theta_{1}^{\prime}, \theta_{3}, \theta_{3}^{\prime}$ is the same as in the text. The vis viva of one refracted ray is $m_{1}\left(\tau_{1}^{2}-\tau_{3}^{2}\right)$, and that of the other is $m_{1}\left(\tau_{1}^{\prime}{ }^{2}-\tau_{3}^{\prime}{ }^{2}\right)$; therefore the vis viva of both refracted rays is

$$
m_{1}\left(\tau_{1}^{2}+\tau_{1}^{2}-\tau 3^{2}-\tau^{\prime} 3^{2}\right),
$$


eliminate the two ratios of the three transversals, together with the angle at which the incident transversal is inclined to the plane of incidence. In the equation produced by this elimination, the angle of incidence would be the polarizing angle, and the other quantities would be known functions of that angle; whence the angle itself would be known.

a quantity which ought to be equal to

$$
m_{1}\left(T_{1}^{2}-T_{3}^{2}\right)
$$

and consequently the equation

$$
\tau_{1} \tau_{1}^{\prime} \cos \left(\theta_{1}-\theta_{1}^{\prime}\right)=\tau_{3} \tau_{3}^{\prime} \cos \left(\theta_{3}-\theta_{3}^{\prime}\right)
$$

ought to be true, This equation, by help of the expressions (6) for $\tau_{1}, \tau_{3}$, and the like expressions for $\tau_{1}^{\prime}, \tau^{\prime}$, becomes

$$
\begin{aligned}
& \sin \left(t_{1}+t_{2}\right) \sin \left(t_{1}+t_{3}^{\prime}\right)\left(1+\tan \theta_{1}^{\circ} \tan \theta_{1}^{\prime}\right) \\
& =\sin \left(t_{1}-t_{3}\right) \sin \left(t_{1}-t_{9}^{\prime}\right)\left(1+\tan \theta_{3} \tan \theta_{3}^{\prime}\right) ;
\end{aligned}
$$

which again, by substituting the values (13) and the other similar values, is changed into

$$
\sin \left(t_{2}+i_{2}^{\prime}\right)\left\{\cos \left(t_{9}-t_{9}^{\prime}\right)+\operatorname{cotan} \theta_{9} \operatorname{cotan} \theta_{2}\right\}+h+h^{\prime}=0,
$$

where $h^{\prime}$ denotes for one refracted ray what $h$ denotes for the other, the value of $h$ being given by formula (27), and that of $h^{\prime}$ by the same formula with accented letters. The angle of incidence, we may observe, has disappeared from the equation.

If, therefore, the laws of reflexion, which we have endeavoured to establish, are consistent with cach other, this last equation must be satisfied by means of the relations which the laws of propagation afford; or rather, the equation must express a property of the wave surface of the crystal, however strange it may be thought that such a property should be derived from the laws of reflexion-laws which would seem, at first sight, to have no connexion at all with the form of the wave surface. Now I have found that the equation (vir.) really does express a rigorous property of the biaxal wave surface of Fresnel; a very curious fact, which not only shows that the laws of reflexion and the laws of propagation are perfectly adapted to each other, but also indicates that both sets of laws have a common source in other and more intimate laws not yet discovered. Indeed the laws of reflexion are not independent even among themselves; for the expressions (III.) and (IV.) in the note on ordinary reflexion (page 101) have been deduced solely from the principle of equivalent vibrations, and yet they satisfy the law of vis viva. Perhaps the next step in physical optics will lead us to those higher and more elementary principles by which the laws of reflexion and the laws of propagation are linked together as parts of the same system. 
It deserves to be remarked, that, at any angle of incidence, if the incident and reflected wave planes be intersected by a plane drawn through the two refracted transversals, the intersections will be corresponding transversal directions; that is to say, if the incident transversal coincide with one intersection, the reflected transversal will coincide with the other. For it is evident, from our fourth hypothesis, that if three of the transversals be in one plane, the fourth transversal must be in the same plane.

We come now to apply our theory to the case of uniaxal crystals; and, in doing so, we shall take the crystal to be of the negative kind, like Iceland spar, so that the ordinary refraction will be more powerful than the extraordinary. On the sphere described with the centre $O$ and rädius $O S$, let $X Y$ be a great circle in the plane of incidence, the radii $O X, O Y$ being the positive directions of the co-ordinate axes of $x$ and $y$. Suppose the right lines $i O$ and $O i^{\prime}$, intersecting the sphere in $i$ and $i$, to be the incident and reflected rays; let the ordinary refracted ray and the extraordinary wave normal be produced backwards from $O$ to meet the sphere, at the side of the incident light, in the points $o$ and $e$ respectively; let the right line $O A$, eutting the sphere in $A$, be the direction of the axis of the crystal; and draw the great circles $A o, A e, A Y$. The points $i, e, o, i^{\prime}$ are all on the cirole $X Y$. The point $E$, where the extraordinary ray $O E$ produced backwards meets the sphere, will be on the oircle $A e$; and if, as in the figure, the are $A e$ be less than a quadrant, the point $e$ will lie between $A$ and $E$. The polar plane of the ordinary ray is obviously the plane of the circle Ao ; but the polar plane of the other ray must be found by a construction. On the are $\boldsymbol{A e E}$

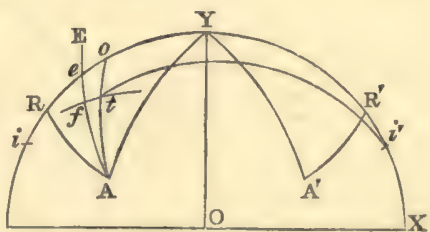

Fig. 19. take the portion $e f$, so that the point $e$ may lie between the points $E$ and $f$, and so that the tangent of $e f$ may be to the tangent of $E e$ as the square of the sine of the are $e Y$ is to the dif- 
ference between the squares of the sines of $i Y$ and $e Y$. Through $f$ draw the great circle $f t$ perpendicular to the circle $A c E$; and it is manifest from (29) that the plane of $f t$ is the polar plane of the extraordinary ray. On each circumference $A o$ and $f t$, the points which are distant $90^{\circ}$ from $i$ and $i$, the distances being measured by ares of great circles, are the points where the uniradial transversals, prolonged from the centre, intersect the sphere. Let $A 0$ and $f t$ intersect each other in $t$, and let $t i^{\prime}$ be an are of a great circle connecting the point $t$ with the point $i$. When the connecting are $t i$ is a quadrant, the two uniradial transversals, belonging to the reflected ray, coincide with each other and with the right line $O t$; the angle of incidence is then the polarizing angle; the plane of $t i^{\prime}$ is the plane of polarization of the reflected ray; and the angle $t i^{\prime} Y$ is the deviation.

To find the equations appropriate to uniaxal crystals, we may suppose formulø (34) to belong to the ordinary, and formulæ (35) to the extraordinary ray. Then will $\varepsilon=0$, and $\varepsilon^{\prime}=$ the are Ee. Putting $\theta$ and $\boldsymbol{\theta}^{\prime}$ for the spherical angles $A o i$ and $A e i$, we shall easily see that $\theta_{2}=\theta+180^{\circ}$, and $\theta_{2}^{\prime}=\theta^{\prime}+90^{\circ}$, if we conceive the point $A$ and the positive axis of $z$ to be both on the upper side of the plane $X O Y$. And if $\omega^{\prime}$ denote the are $A e$, while $b$ and $a$ respectively express the reciprocals of the principal indices, ordinary and extraordinary, the law of Huyghens, for the double refraction of uniaxal crystals, will give us

$$
\tan \varepsilon^{\prime}=\frac{a^{2}-b^{2}}{s^{2}} \sin \omega^{\prime} \cos \omega^{\prime},
$$

where

$$
\delta^{2}=\frac{\sin ^{2} \iota_{2}}{\sin ^{2} \iota_{1}}=b^{2}+\left(a^{2}-b^{2}\right) \sin ^{2} \omega^{\prime}
$$

Observing these relations, we have, from (34),

$$
\left.\begin{array}{l}
\tan \theta_{1}=\cos \left(\iota_{1}-\iota_{2}\right) \tan \theta, \\
\tan \theta_{3}=-\cos \left(\iota_{1}+\iota_{2}\right) \tan \theta,
\end{array}\right\}
$$

for the ordinary ray; and from (35) we get 
$\tan \theta_{1}=-\cos \left(\iota_{1}-\iota_{2}^{\prime}\right) \operatorname{cotan} \theta-\left(a^{2}-b^{2}\right) \frac{\sin \omega^{\prime} \cos \omega^{\prime} \sin ^{2} \iota_{1}}{\sin \theta \sin \left(\iota_{1}+\iota_{2}^{\prime}\right)}$,

$$
\left.\tan \theta_{3}^{\prime}=\cos \left(\iota_{1}+i_{2}^{\prime}\right) \operatorname{cotan} \theta^{\prime}-\left(a^{2}-b^{2}\right) \frac{\sin \omega^{\prime} \cos \omega^{\prime} \sin ^{2} \iota_{1}}{\sin \theta^{\prime} \sin \left(\iota_{1}-\iota_{2}^{\prime}\right)},\right\}
$$

for the extraordinary ray.

The four preceding equations determine the uniradial directions; and the following equation,

$\cos \left(\iota_{1}+\iota_{2}\right) \tan \theta+\cos \left(\iota_{1}+\iota_{2}^{\prime}\right) \operatorname{cotan} \theta-\left(a^{2}-b^{2}\right) \frac{\sin \omega^{\prime} \cos \omega^{\prime} \sin ^{2} \iota}{\sin \theta^{\prime} \sin \left(\iota_{1}-\iota_{2}^{\prime}\right)}=0$

obtained by putting $\tan \theta_{3}=\tan \theta_{3}^{\prime}$, is that which determines the polarizing angle.

In making use of this last equation to deduce the law of the polarizing angles in various positions of the axis of the crystal, we shall confine ourselves to the case in which the reflexion from the crystal takes place in air, because the angle $\iota_{1}-i_{2}$ will then be considerable, and the quantities $\cos \left(\iota_{1}+\iota_{2}\right)$ and $\cos \left(\iota_{1}+\imath_{2}^{\prime}\right)$ will be small, so that it will be easy to arrive at approximate results. For we shall have, in the first place,

$$
\cos \left(\iota_{1}+i_{2}^{\prime}\right)=\cos \left(\iota_{1}+\iota_{2}\right)-\left(\iota_{3}^{\prime}-\iota_{2}\right)
$$

nearly, since $\iota_{1}+\iota_{2}$ will not differ much from a right angle; and because

$$
\sin \iota_{2}=b \sin \iota_{1}, \sin \iota_{2}=s \sin \iota_{1},
$$

we shall also have, rigorously,

$$
\sin ^{2} \iota_{2}^{\prime}-\sin ^{2} \iota_{2}=\left(s^{2}-b^{2}\right) \sin ^{2} \iota_{1}=\left(a^{2}-b^{2}\right) \sin ^{2} \omega^{\prime} \sin ^{2} \iota_{1},
$$

or

$$
\sin \left(\iota_{2}^{\prime}-\iota_{2}\right)=\left(a^{2}-b^{2}\right) \frac{\sin ^{2} \omega^{\prime} \sin ^{2} \iota_{1}}{\sin \left(\iota_{2}+\iota_{2}\right)},
$$

which may be written

$$
\iota_{2}-\iota_{2}=\left(a^{2}-b^{2}\right) \frac{\sin ^{2} \omega^{\prime} \sin ^{2} \iota_{1}}{\sin 2 \iota_{2}},
$$

with sufficient accuracy. This value of $\iota_{2}-\iota_{2}$ having been substituted in (41), the resulting expression for $\cos \left(\iota_{1}+i_{2}^{\prime}\right)$ must be 
substituted in equation (40), which will then become

$$
\begin{gathered}
\cos \left(\iota_{1}+\iota_{2}\right)(\tan \theta+\operatorname{cotan} \theta)-\left(a^{2}-b^{2}\right) \sin ^{2} \iota_{1} \sin \omega\left(\frac{\sin \omega \operatorname{cotan} \theta}{\sin 2 \iota_{2}}\right. \\
\left.+\frac{\cos \omega}{\sin \theta \cos 2 \iota_{2}}\right)=0
\end{gathered}
$$

if, denoting the are $A_{0}$ by $\omega$, we confound $\omega^{\prime}$ with $\omega, \theta^{\prime}$ with $\theta$, and write $\cos 2 \iota_{2}$ instead of $\sin \left(\iota_{1}-i_{2}\right)$. Multiplying all the terms of (46) by $\sin \theta \cos \theta$, we find

$\cos \left(\iota_{1}+\iota_{2}\right)=\left(a^{2}-b^{2}\right) \sin ^{2} \iota_{1} \sin \omega \cos \theta\left(\frac{\sin \omega \cos \theta}{\sin 2 \iota_{2}}+\frac{\cos \omega}{\cos 2 \iota_{2}}\right)$.

From $A$ draw the are $A R$ meeting the are $i Y$ at right angles in the point $R$, and put $R Y=p, A R=q$. Then by means of the values

$$
\left.\begin{array}{l}
\cos \omega=\cos q \cos \left(p-\iota_{2}\right), \\
\sin \omega \cos \theta=\cos q \sin \left(p-\iota_{2}\right),
\end{array}\right\}
$$

afforded by the right-angled triangle $A R o$, the equation (47) will take the form

$$
\cos \left(\iota+\iota_{2}\right)=\frac{\left(a^{2}-b^{2}\right) \sin ^{2} \iota_{1}}{\sin 2 \iota_{2} \cos \iota_{\iota_{2}}} \cos ^{2} q \sin \left(p-\iota_{2}\right) \sin \left(p+\iota_{2}\right),
$$

or

where

$$
\cos \left(\iota+\iota_{2}\right)=K \cos ^{2} q\left(\sin ^{2} p-\sin ^{2} \iota_{2}\right),
$$

$$
K=\frac{\left(a^{2}-b^{2}\right)\left(1+b^{2}\right)}{2 b\left(1-b^{2}\right)} ;
$$

this value of $K$ being found by assuming $\tan \iota_{2}=\operatorname{cotan} \iota_{1}=b$, which is accurate enough for the purpose.

Thus we have obtained $\iota_{1}+\iota_{2}$, or the sum of the polarizing angle and the angle of ordinary refraction. The former angle itself may be inferred from formula (50) by help of the relation $\sin \iota_{2}=b \sin \iota_{1}$. In this way, if we use $\varpi_{1}$ instead of $\iota_{1}$ to distinguish the polarizing angle from other angles of incidence, and if we put

$$
k=\frac{K}{1+b^{2}}=\frac{a^{2}-b^{2}}{2 b\left(1-b^{2}\right)},
$$


we shall find

$$
\varpi_{1}=\varpi-k \cos ^{2} q\left(\sin ^{2} p-\sin ^{2} \iota_{2}\right),
$$

in which $w$ is the angle whose cotangent is equal to $b$; in other words, $\varpi$ is the polarizing angle of an ordinary medium whose refractive index is equal to the ordinary index of the crystal.

This result accounts for a remarkable fact observed by $\mathrm{Sir}$ David Brewster, who, in the year 1819, led the way in the experimental investigation of the laws of crystalline reflexion. $\mathrm{He}_{\mathrm{e}}$ found that the polarizing angle remains the same when the crystal is turned round through $180^{\circ}$, though one of the angles of refraction is changed, and though the situation of the refracted rays, with respect to the axis of the crystal, becomes quite different from what it was. This circumstance, which surprised me when I first met with it, is an immediate consequence of formula (53); for the effect of a semi-revolution of the erystal is to ehange the signs of $p$ and $q$; but the nature of the formula is such that these changes of sign do not alter the value of $\varpi_{1}$. Neither is that value altered by turning the crystal until the azimuth, as the spherical angle $A Y i$ is usually called, is ohanged into its supplement; for then the sign of $p$ alone is affected.

Another remark, made by the same distinguished observer, is also a consequence of formula (53). From his experiments it appears that, on a given surface of the cirystal, the polarizing angle differs from a constant angle by a quantity proportional to the square of the sine of the azimuth $A Y i$. Now, ealling this azimuth $a$, and putting $\lambda$ for the acute angle at which the axis of the crystal is inclined to its surface, so that $\lambda$ may be the complement of the are $A Y$, we have

$$
\sin q=\cos \lambda \sin a, \quad \tan p=\operatorname{cotan} \lambda \cos \alpha \text {; }
$$

and by making these substitutions in formula (53), after having changed $\sin \iota_{2}$ into $\cos w$, that formula becomes

$$
\varpi_{1}=\varpi-k\left(\sin ^{2} \varpi-\sin ^{2} \lambda\right)+k \sin ^{2} \varpi \cos ^{2} \lambda \sin ^{2} a,
$$

which agrees with the remark of Brewster. 
The deviation $\theta_{3}$ or $\theta_{3}^{\prime}$ is found from the second of equations (38), by putting $\frac{\tan q}{\sin \left(p-\iota_{2}\right)}$ for $\tan \theta$, and by substituting for $\cos \left(\iota_{1}+\iota_{2}\right)$ the value (49) or (50) which it has at the polarizing angle. The result is

$$
\theta_{3}=\boldsymbol{\theta}_{3}=-\frac{\boldsymbol{K}}{2} \sin 2 q \sin \left(p+\iota_{2}\right),
$$

since the small are $\theta_{3}$ may be taken for its tangent. This result is easily transformed into

$$
\theta_{3}=\theta_{3}^{\prime}=-\Pi \sin q \cos \phi,
$$

where $\phi$ denotes the are $A$, or the angle which the incident ray makes with the axis of the crystal; and this last expression is equivalent to the following,

$$
\theta_{3}=\theta_{3}^{\prime}=-K \cos \lambda \sin a(\sin \lambda \cos \varpi+\cos \lambda \sin \varpi \cos \alpha),
$$

which gives the deviation in terms of $\lambda$ and $\boldsymbol{a}$.

As an example of the application of our formulæ, we shall make some computations relative to Iceland spar. According to M. Rudberg, the ordinary index of that crystal, for a ray situated in the brightest part of the spectrum, at the boundary of the orange and yellow, is 1.66 ; and the least extraordinary index for the same ray is 1.487 . Dividing unity by each of these numbers, we get $a={ }^{\circ} 6725, b={ }^{\circ} 6024$; whence $\approx=58^{\circ} 56^{\prime}$; $k=\cdot 1164=6^{\circ} 40^{\prime} ; K=\cdot 1587=9^{\circ} 5^{\prime}$. Having thus determined the constants, we can readily calculate the polarizing angle and the deviation, for any given values of $\lambda$ and $\alpha$.

First, let us see how the polarizing angle varies on different faces of the crystal.

1. When $\lambda=90^{\circ}$, the face of the crystal is perpendicular to its axis, and $\varpi_{1}$ is independent of $a$. In this case the formula (55) gives

$$
\varpi_{1}=\varpi+k \cos ^{2} \varpi=60^{\circ} 42^{\prime},
$$

which is the maximun value of the polarizing angle. 
2. When $\lambda=0$, the axis lies in the face of the crystal, and formula ( $\tilde{5} 5)$ becomes

$$
\varpi_{1}=\varpi-k \sin ^{2} \approx \cos ^{2} \alpha,
$$

showing that $\varpi_{1}=\varpi$, when $a$ is either $90^{\circ}$ or $270^{\circ}$. But when $a$ is 0 or $180^{\circ}$, we have

$$
\varpi_{1}=\varpi-k \sin ^{2} \varpi=54^{\circ} 2^{\prime},
$$

which is the minimum value of the polarizing angle.

3. For the natural fracture-faces of the crystal the value of $\lambda$ is $45^{\circ} 23^{\prime}$. Hence, when $a=0$ or $180^{\circ}$,

$$
\varpi_{1}=\varpi-k\left(\sin ^{2} \pi-\sin ^{2} \lambda\right)=57^{\circ} 26^{\prime} ;
$$

and when $a=90^{\circ}$ or $270^{\circ}$,

$$
\varpi_{1}=\varpi+k \cos ^{2} \approx \sin ^{2} \lambda=59^{\circ} 50^{\prime} .
$$

These values of the polarizing angles agree very well with the experiments of Sir David Brewster, and still better with those of M. Seebeck.

If we wish to know in what azimuths $\varpi_{1}$ is equal to $\varpi$, on a given surface of the crystal, it is obvious from (55) that we must make

$$
\sin ^{2} \varpi-\sin ^{2} \lambda=\sin ^{2} \varpi \cos ^{2} \lambda \sin ^{2} a,
$$

whence we have, simply,

$$
\cos a= \pm \frac{\tan \lambda}{\tan \varpi}
$$

which shows that the thing is impossible when $\lambda$ is greater than $\varpi$; and that, when $\lambda$ is less than $\varpi$, there are four such azimuths; as indeed there are, generally speaking, four values of a corresponding to any other particular value of the polarizing, angle. If $a^{\prime}$ be the least of these azimuths, the others will be $180^{\circ}-a^{\prime}, 180^{\circ}+a^{\prime}$, and $360^{\circ}-a^{\prime}$. On a natural face of the crystal, the value of $a^{\prime}$, answering to the supposition $\varpi_{1}=\varpi$, is found to be $52^{\circ} 22^{\prime}$.

Next, let us trace the changes which the deviation undergoes in some remarkable cases. 
1. When the face of the orystal is perpendicular to its axis there is evidently no deviation.

2. When the axis lies in the face of the crystal the deviation vanishes in the azimuths $0,90^{\circ}, 180^{\circ}, 270^{\circ}$. In the intermediate azimuths, differing $45^{\circ}$ from each of these, the deviation is a maximum; for if we put $\lambda=0$ in formula (55) the result will be

$$
\theta_{3}=-\frac{K}{2} \sin \varpi \sin 2 a ;
$$

and this quantity (neglecting its sign) is a maximum when sin $2 a= \pm 1$. The coefficient of $\sin 2 a$ is equal to $3^{\circ} 54^{\prime}$, which is consequently the greatest value of the deviation. According to the experiments of M. Seebeck, the value is $3^{\circ} 57^{\prime}$.

3. On the fracture-faces of the crystal the deviation $v a-$ nishes in the azimuths 0 and $180^{\circ}$, as also in two other azimuths for which

$$
\cos a=-\frac{\tan \lambda}{\tan \varpi}
$$

and in which, therefore, $\varpi_{1}$ is equal to $\varpi$. In the azimuth $45^{\circ}$ the deviation is $-3^{\circ} 35^{\prime}$; in the azimuth $90^{\circ}$ it is $-2^{\circ} 32^{\prime}$; and in the azimuth $127^{\circ} 38^{\prime}$ it vanishes; after which it attains a small maximum with a positive sign, and vanishes again in azimuth $180^{\circ}$. The calculated values of the deviation agree pretty well with the values observed by M. Seebeck.

The sign of the deviation shows at what side of the plane of incidence the plane of polarization lies. But the position of the latter plane is best indicated by that of the transversal of the reflected ray. If this transversal and the axis of the crystal be produced from the origin, towards the same side of the plane of $x z$, until they intersect the sphere in the points $t$ and $A$ respectively, these points will be on the same side of the great circle $X Y$ when the deviation and the sine of the azimuth have unlike algebraic signs; and they will be on opposite sides of that circle when those quantities have like signs. Therefore if the crystal be supposed to revolve in its own plane, be- 
ginning at the azimuth 0 , the points $t$ and $A$ will lie on the same side of $X Y$ until $A$ reaches the position $A^{\prime}$, where the angle $A^{\prime} Y i$ is equal to $127^{\circ} 38^{\prime}$; the point $t$ will then pass over to the side opposite $A$, at which side it will remain until $A$ arrives at the azimuth $232^{\circ} 22^{\prime}$. Thenceforward, to the end of the revolution, both points will be found on the same side of the circle $X Y$.

We have seen that the deviation always vanishes when the axis of the crystal lies in the plane of incidence. The reason is, because the crystal is then symmetrical on opposite sides of that plane. In this case the problem of reflexion offers peculiar facilities for solution, since the uniradial directions are obviously parallel and perpendicular to the plane of incidence. Let us, therefore, consider the case at length.

1. In the first place, when the only refracted ray is the ordinary one, the three transversals are in the plane of incidence, and the transversal of each ray is proportional to the sine of the angle between the other two rays. Hence the proportions are

$$
\frac{\tau_{1}}{\sin \left(t_{1}+t_{2}\right)}=\frac{\tau_{2}}{\sin 2 t_{1}}=\frac{\tau_{3}}{\sin \left(t_{1}-t_{2}\right)},
$$

the same as in ordinary media.

2. In the second place, when the sole refracted ray is the extraordinary one, the three transversals are perpendicular to the plane of incidence; and, if we use accents to mark the quantities connected with this ray, we have the equations

$$
\begin{gathered}
\boldsymbol{\tau}_{1}+\boldsymbol{\tau}_{3}^{\prime}=\boldsymbol{\tau}_{2}{ }_{2}, \\
m_{1} \tau_{1}^{\prime}{ }_{1}^{2}=m_{2}^{\prime} \tau_{2}^{\prime}{ }^{2}+m_{1} \tau_{3}^{\prime}{ }_{3}^{2},
\end{gathered}
$$

which give the proportions

wherein

$$
\frac{\tau_{1}^{\prime}}{m_{1}+m_{2}^{\prime}}=\frac{\tau_{2}^{\prime}}{2 m_{1}}=\frac{\tau_{3}^{\prime}}{m_{1}-m_{2}^{\prime}},
$$

$$
\frac{m_{2}^{\prime}}{m_{1}}=\frac{\sin 2 \ell_{2}^{\prime} \pm 2}{\sin 2 \sin ^{2} \ell_{2}^{\prime} \tan \varepsilon^{\prime}}
$$

loy (26) ; the upper or lower sign being taken, in the numera- 
tor of (63), according as the refracted ray or its wave normal makes the smaller angle with a perpendioular to the face of the erystal.

To find the polarizing angle, we have only to make $m_{1}=m_{2}^{\prime}$, for then $\tau_{3}^{\prime}$ will vanish by (62); and therefore, if common light be incident, the whole reflected pencil will be polarized in the plane of incidence. Supposing the crystal to be a negative one, let us conceive the refracted ray to lie within the acute angle made by the axis of the crystal with a perpendicular to its surface. We shall then have to take the positive sign in the numerator of (63), and the polarizing angle will be given by the condition

$$
\sin 2 t_{1}=\sin 2 \ell_{2}^{\prime}+2 \sin ^{2} \iota_{2}^{\prime} \tan \varepsilon^{\prime} .
$$

But from (36) we have, in general,

$$
\sin ^{2} \iota_{2}^{\prime} \tan \varepsilon^{\prime}=\left(a^{2}-b^{2}\right) \sin \omega^{\prime} \cos \omega^{\prime} \sin ^{2} \iota_{1},
$$

and in the present instance it is evident that

$$
\omega^{\prime}=90^{\circ}-\lambda-i_{2}^{\prime},
$$

where $\lambda$ denotes, as before, the angle which the axis of the crystal makes with its surface. Substituting these values in (64), and multiplying all the terms by $\tan i_{2}$, we get

$\sin ^{2} \imath_{2}^{\prime}=\sin \iota_{1} \cos \iota_{1} \tan \imath_{2}-\left(a^{2}-b^{2}\right) \sin \left(\lambda+i_{2}\right) \cos \left(\lambda+i_{2}\right) \tan \imath_{2} \sin ^{2} \iota_{1}$.

Again, from (37) we have

$$
\sin ^{2} \iota_{2}^{\prime}=b^{2} \sin ^{2} \iota_{1}+\left(a^{2}-b^{2}\right) \cos ^{2}\left(\lambda+\iota_{2}^{\prime}\right) \sin ^{2} \iota_{1}^{\prime}
$$

and by equating these two expressions for $\sin ^{2} \ell_{2}$, we find

$$
\tan \iota_{2}^{\prime}=\frac{a^{2} \cos ^{2} \lambda+b^{2} \sin ^{2} \lambda}{\operatorname{cotan} \iota_{1}+\left(a^{2}-b^{2}\right) \sin \lambda \cos \lambda} .
$$

Then, if this value of $\tan i_{2}$ be substituted in equation (66), after all its terms have been divided by $\cos ^{2} i_{2}$, we shall obtain the simple and rigorous formula

$$
\sin ^{2} t_{1}=\frac{1-a^{2} \cos ^{2} \lambda-b^{2} \sin ^{2} \lambda}{1-a^{2} b^{2}}=\sin ^{2} \varpi_{1},
$$


for determining the polarizing angle $\varpi_{1}$, when the axis of the crystal lies in the plane of incidence. It is manifest, from the nature of the formula, that this angle is the same, whether the azimuth is 0 or $180^{\circ}$; that is, whether the light is incident at the right or left side of the perpendicular to the surface of the crystal.

This formula might be deduced more briefly by recollecting what we have already proved, that the corresponding masses $m_{1}$ and $m_{2}^{\prime}$ are proportional to the ordinates $y$ of the points where the incident ray and the extraordinary refracted ray meet their respective wave surfaces; whence it follows that these ordinates must be equal at the polarizing angle; and thus the question is reduced at once to a geometrical problem. For as both rays are in the plane of incidence, the axis of $x$ will be intersected in one and the same point by right lines touching the wave surfaces, or their sections, at the extremities of the ordinates. Now the sections in the plane of $x y$ are a circle and ellipse with their common centre at the origin, the radius of the circle being unity, and the semiaxes of the ellipse being $a$ and $b$, of which $b$ is inclined at the angle $\lambda$ to the axis of $x$; and therefore it is required to draw, parallel to the axis of $x$, a right line intersecting the circle and ellipse; so that if tangents be applied to them at two points of intersection which lie on the same side of the axis of $y$, these tangents, when produced, may cut each other on the axis of $x$. The angle which the tangent to the circle makes with the axis of $x$ is then the polarizing angle $\varpi_{1}$; and the solution of the problem just stated leads directly and easily to the formula (68). From this way of viewing the matter we see the reason why the polarizing angle is the same in the azimuths 0 and $180^{\circ}$; for if tangents be applied at the two remaining points where the parallel that we have spoken of intersects the circle and ellipse, it is evident that these tangents also will cut each other on the axis of $x$; since tangents drawn at the extremities of any chord, either of a circle or an ellipse, intersect the parallel diameter at equal distances from the centre.

Let the reflecting surface of the crystal be in contact with 
a fluid medium whose index of refraction out of vacuo is represented by $N$, and let $B$ and $A$ respectively denote the ordinary and the principal extraordinary indices of refraction out of vacuo into the crystal. Then putting $\frac{N}{A}$ for $a$, and $\frac{N}{B}$ for $b$, in the preceding formula, and making

$$
L^{2}=A^{2} \sin ^{2} \lambda+B^{2} \cos ^{2} \lambda,
$$

we readily deduce

$$
\tan ^{2} \varpi_{1}=\frac{A^{2} B^{2}-L^{2} N^{2}}{N^{2}\left(L^{2}-N^{2}\right)}
$$

Hence we perceive that if $L^{2}=A B$, that is, if

$$
\tan \lambda=\sqrt{ } \frac{B}{A}
$$

(in which case $\lambda$ will never be much above or below $45^{\circ}$ ), the value of $\varpi_{1}$ will be always possible; for then we shall have

$$
\tan ^{2} \varpi_{1}=\frac{A B}{N^{2}} .
$$

But if $\lambda$ be different from this, and of course $L^{2}$ not equal to $A B$, the value of $\varpi_{1}$ may become impossible for certain values of $N$. For it is clear that if $N$ lie between the limits $L$ and $\frac{A B}{I}$, the numerator and denominator of the fraction (69) will have unlike signs, and the tangent of $\varpi_{1}$ will be the square root of a negative quantity. In this case, therefore, if common light be incident, it will "refuse to be polarized," as Brewster expresses it; in other words, it will be impossible to find an angle of ineidence at which the reflected pencil will cease to contain light polarized perpendicularly to the plane of incidence, or at which the reflected transversal $\tau_{3}^{\prime}$ will vanish. With all values of $N$, except those which are included between the narrow limits $L$ and $\frac{A B}{L}$, the polarizing angle is possible. It is zero at the latter limit, and $90^{\circ}$ at the former. Outside these limits it 
changes rapidly at first, until $N$ has passed either of them by a quantity considerable in proportion to the interval between them.

From (68) we find $\varpi_{1}=\lambda$, when $a=1$, or $N=A$; and also $\varpi_{1}=90^{\circ}-\lambda$, when $b=1$, or $N=B$. In the latter case it is remarkable that no light is reflected when common light is incident at the angle $90^{\circ}-\lambda$. For then we have $\tau_{3}^{\prime}=0$; and because $\iota_{1}=\iota_{2}$, we have likewise $\tau_{3}=0$. Therefore no light can enter the reflected pencil. But this case deserves that we should consider it more at large, without restricting ourselves to the supposition that the axis of the crystal lies in the plane of incidence.

Assuming then that $N=B$, or that the refractive index of the fluid, which covers the reflecting surface, is equal to the ordinary index of the crystal itself, we may observe that, in this case, every angle of incidence, in every azimuth, has a right to be regarded as a polarizing angle. In fact, common light cannot suffer reflexion at the separating surface of the crystal and the fluid, without becoming completely polarized. For if polarized light be incident, and if $\tau_{3}$ and $\tau_{3}^{\prime}$ be the uniradial reflected transversals, respectively belonging to the ordinary and to the extraordinary ray, the former transversal must necessarily vanish, for the same reason that no reflexion can take place at the separating surface of two ordinary media whose refractive indices are equal; and thus the actual reflected transversal will always coincide in direction with $\tau_{3}^{\prime}$, whatever be the direction of the incident transversal. Consequently, if common light be incident, the whole reflected pencil will be polarized in a plane passing through $\tau_{3}^{\prime}$, and making with the plane of incidence an angle $\theta_{3}^{\prime}$ determined by the second of formulæ (39). By putting $\iota_{2}=t_{1}$ in that formula, and employing the expression (44), we first obtain

$$
\begin{aligned}
\tan \theta_{3}^{\prime}=\frac{\cos \left(\iota_{1}+i_{2}^{\prime}\right) \cos \theta^{\prime} \tan \omega^{\prime}+\sin \left(\iota_{1}+i_{2}\right)}{\sin \theta^{\prime} \tan \omega^{\prime}} \\
=\frac{\cos \left(\iota_{1}+i_{2}\right) \tan \left(p-i_{2}^{\prime}\right)+\sin \left(\iota_{1}+i_{2}^{\prime}\right)}{\sin \theta^{\prime} \tan \omega^{\prime}} ;
\end{aligned}
$$


and thence

$$
\tan \theta_{3}^{\prime}=\frac{\sin \left(p+\iota_{1}\right)}{\sin \theta^{\prime} \tan \omega^{\prime} \cos \left(p-\iota_{2}^{\prime}\right)}=\frac{\sin \left(p+\iota_{1}\right) \cos \omega^{\prime}}{\sin q \cos \left(p-\iota_{2}^{\prime}\right)} ;
$$

and finally,

$$
\tan \theta^{\prime}=\sin \left(p+\iota_{1}\right) \operatorname{cotan} q ;
$$

a result which shows that the plane of polarization of the reflected ray is perpendicular to a plane drawn through the ray itself and the axis of the crystal.

Moreover, we find, from the first of formulæ (39), by proceeding as above,

$$
\tan \theta_{1}^{\prime}=-\sin \left(p-\iota_{1}\right) \operatorname{cotan} q=-\operatorname{cotan} \theta ;
$$

and from (38) it is evident that $\boldsymbol{\theta}_{1}=\boldsymbol{\theta}$. Therefore all that relates to the case under our consideration may be summed up in the following statement:

When $N=B$, and the incident light is polarized in a plane passing through the axis, the course of the light is unaltered, and there is neither reflexion nor refraction. When it is polarized in the perpendicular plane, all the light which enters the crystal undergoes extraordinary refraction. Whatever light is reflected is always polarized in a plane at right angles to that which passes through the reflected ray and the axis of the crystal; and this is true, whether the incident light is polarized or not.

Here, for the present, we must terminate our deductions from the general theory propounded in this Paper. Several other questions remain to be discussed, such as the reflexion of common light* at the first surface, and the internal $\dagger$ reflexion

* The mode of treating the case in which common light is incident has been pointed out at the bottom of p. 100 .

$+I$ have since found that the problem of reflexion at the second surface may be reduced to that of reflexion at the first surface by means of a very simple rule. Let us suppose the two surfaces of the crystal to be parallel; and let a ray $R_{1}$, uniradially polarized, and incident on the first surface, give the ray $R_{3}$ by reflexion, and the single ray $R_{2}$ by refraction. Let $R_{2}$ be the ray which suffers internal reflexion at the second surface, thereby giving the two reflected rays $R_{\text {, }}, R^{\prime}$," and the 
at the second surface of a crystal; but these must be reserved for a future communication. It would be easy, indeed, to write down the algebraical solutions resulting from our theory; but this we are not content to do, because the expressions are rather complicated, and, when rightly treated, will probably contract themselves into a simpler form. It is the character of all true

single refracted ray $R_{(1)}$ emerging from the crystal in a direction parallel to $R_{1}$. Put $\tau_{1}, \tau_{3}, \tau_{2}$, and $\tau_{\text {, }} \tau^{\prime}$, , $\tau_{(1)}$ for the transversals of the rays in the order in which they have been named. As the transversal $\tau_{2}$ is supposed to be given in magnitude, the lengths as well as the directions of $\tau_{1}$ and $\tau_{3}$ can be found by the construction in page 97.

Now, the direction of $\tau_{3}$ being changed, and its magnitude retained, let the ray $R_{3}$ be turned directly back, so as to be incident again on the crystal, and to suffer reflexion and refraction at the first surface. Then the two refracted rays which it gives will be parallel to $R_{,}, R^{\prime}$, , and their transversals will be equal and parallel to $\tau_{\text {, }}, \tau^{\prime}$, . The reflected ray which it gives will coincide with $R_{1}$; and the reflected transversal, when compounded with $\tau_{1}$, will furnish a resultant equal and parallel to the emergent transversal $\tau(1)$.

Thus the constructions, which have been given for the first surface, may be made available for the second surface, and every question relative to crystalline reflexion may be solved geometrically by means of the polar planes.

The foregoing rule was not, properly speaking, deduced from theory. I first formed a clear conception of what the rule ought to be, and then verified it for the simple case of singly-refracting media, and finally proved it for doubly-refracting crystals. The truth of the rule, in crystals, depends upon the truth of the three following equations:-

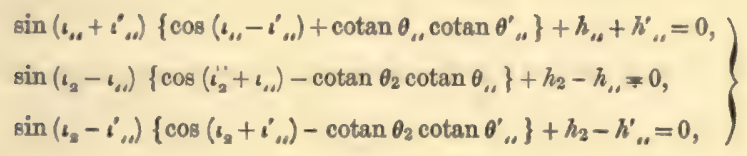

in which the notation is intelligible without any explanation. The first equation is the same as equation (vII.) already noticed; and the other two differ from it only in appearance, the change in the signs being occasioned by a change in the relative position of the rays.

When the reflexion is total, I suppose we may follow the example which Fresnel has set us in the case of ordinary media. The general algebraic expression for each reflected transversal will then become imaginary ; and by putting it under the form

$$
T(\cos \phi+\sqrt{ }-1 \sin \Phi),
$$

we shall hare $T$ for the reflected transversal, and $\Phi$ for the change of phase. 
theories that the more they are studied, the more simple they appear to be. And we may add, that a close examination of such theories always meets with its reward, in the unexpected* consequences which present themselves to view. Nothing can be simpler than the laws of double refraction, as they were delivered by Fresnel; yet the properties of his wave surface still continue to furnish the geometer with beautiful and curious relations. So we may hope that a little more time, devoted to the laws of reflexion, will not be spent in vain. They promise to supply many other theorems, not undeserving of attention, though perhaps not as simple and comprehensive as those that have already been made known.

From the nature of the rules which we have given for treating the question of reflexion at either surface of the crystal, it follows that the final equation, for determining the position of a transversal, is always linear, though the equation of vis viva is of the second degree. This result very strongly confirms the theory; but it shows, at the same time, that the law of the preservation of vis viva is not to be regarded as an ultimate principle, but rather as a consequence of some elementary law not yet discovered.

It now appears that the conjectures put forward in the note, p. 93, were hasty, and that there was some mistake in the calculations which gave rise to them. It $i^{8}$ scarcely necessary to mention, that the sheet in which that note is found was printed off before I had obtained the result announced in the subsequent note, p. 111. Various delays occurred while my Paper was going through the press; and $I$ took advantage of them to increase its value, by appending notes on some of the questions which I had overlooked or omitted in the first consideration of the subject.

* As an instance of this, it may be mentioned, that the conclusion arrived at in the note, p. 111, was wholly unexpected. And in verifying the equation (vir.), an unexpected and useful theorem was obtained; for it became necessary to find a manageable expression for the tangent of the angle $\epsilon$ which the wave normal makes with the ray. This expression is wanted in applying the formulæ (34) and (35) to biaxal erystals, and therefore I shall make no apology for introducing it here.

Having described a sphere concentric with the wave surface, let the wave normal $O P$ and the two optic axes (which are the nodal diameters of the index surface) be produced from the centre $O$ to meet the sphere in the points $P, A, A_{\text {, }}$, respectively, thus marking out the angles of a spherical triangle $P, A A_{i}$. The same wave normal may belong to two different rays; and if we select one of these rays, its transversal must lie in a plane drawn through the wave normal, and bisecting either the internal angle $A P, A$, of the spherical triangle, or the ex- 
If we are asked. what reasons can be assigned for the hypotheses on which the preceding theory is founded, we are far from being able to give a satisfactory answer. We are obliged to confess that, with the exception of the law of vis viva, the hypotheses are nothing more than fortunate conjectures. These conjectures are very probably right, since they have led to elegant laws which are fully borne out by experiments; but this is all that we can assert respecting them. We cannot attempt to deduce them from first principles; because, in the theory of light, such principles are still to be sought for. It

ternal supplementary angle. By producing the optic axes in the proper directions, we may always make the above plane (which Fresnel calls the plane of polarization) bisect the internal angle. Supposing this to have been done for the ray which was selected, put $\omega$ and $\omega$, for the sides $P, A$ and $P, A$, of the spherical triangle, and $\psi$ for the contained angle $A P, A$. Let $s$ be the length of the wave normal from the centre $O$ to the point where it intersects the tangent plane applied at the extremity of the ray, that is, applied at the point where the ray meets its own nappe of the wave surface; and let $a$ and $c$ be the greatest and least semiaxes of the ellipsoid which generates the wave surface. Then we shall have

$$
\tan \epsilon=\frac{a^{2}-c^{2}}{2} \sin \left(\omega-\omega_{0}\right) \sin \frac{1}{2} \psi \text {. }
$$

And it is now manifest that if $\epsilon$, be the angle which the other ray mukes with the same wave normal, and 8 , the length of the wave normal intercepted between the centre and the tangent plane at the extremity of this ray, we shall also have

$$
\tan \epsilon_{\mathrm{s}}=\frac{a^{2}-c^{2}}{2 s_{\mathrm{s}}^{2}} \sin \left(\omega+\omega_{s}\right) \cos \frac{1}{2} \psi .
$$

If a ray is given in direction it will have two wave normals; and then the angles $\epsilon$, $\epsilon$, which it makes with each normal, may be found from the formulx

$$
\left.\begin{array}{l}
\tan \epsilon=\frac{r^{2}}{2}\left(\frac{1}{c^{2}}-\frac{1}{a^{2}}\right) \sin \left(\omega-\omega_{0}\right) \sin \frac{1}{2} \psi, \\
\tan \epsilon=\frac{r_{1}^{2}}{2}\left(\begin{array}{c}
1 \\
c^{2}
\end{array}-\frac{1}{a^{2}}\right) \sin \left(\omega+\omega_{1}\right) \cos \frac{1}{2} \psi,
\end{array}\right\}
$$

where $r$ and $r$, are the two radii of the wave surface which are in the direction of the ray ; the spherical triangle $P, A A_{\text {, }}$ of which the sides and contained angle are expressed by the same letters as before, being now formed by producing the ray and the two nodal diameters of the wave surface, until they intersect the sphere in the points $P_{s}, \mathcal{A}, \mathcal{A}_{\text {, }}$ 
is certain, indeed, that light is produced by undulations, propagated, with transversal vibrations, through a highly elastic ether; but the constitution of this ether, and the laws of its connexion (if it has any connexion) with the particles of bodies, are utterly unknown. The peculiar mechanism of light is a secret that we have not yet been able to penetrate. As a proof of this, we might observe, that some of the simplest and most familar phenomena have never been explained. Not to mention dispersion, about which so much has been fruitlessly written, we may remark, that the very cause of ordinary refraction, or of the retardation which light undergoes upon entering a transparent medium, is not at all understood. Much less can it be said that double refraction has been rigorously explained; its laws alone have been clearly developed by Fresnel. In short, the whole amount of our knowledge, with regard to the propagation of light, is confined to the laws of phenomena: scarcely any approach has been made to a mechanical theory of those laws. And if the case of uninterrupted propagation through a continuous medium presents such difficulties, it would be useless to think of accounting for the laws which subsist at the confines of two media, where the continuity is broken.

But perhaps something might be done by pùrsuing a contrary course; by taking those laws for granted, and endeavouring to proceed upwards from them to higher principles. In this point of view, our second law, or hypothesis, is extremely remarkable; for it seems to be opposed, in some degree, to the notion that the ethereal molecules are strongly attracted or repelled by the particles of bodies. However that may be, it would appear that a true theory must be in accordance with this hypothesis; and that any mechanical ideas, which would make the mean density of the ether vary from one medium to another, ${ }^{*}$ cannot be admitted to represent the real state of

* Those who maintain that the density of the ether is different in different media, ought to consider the following question:-What function of the three principal indices of a doubly-refracting crystal represents the density of the ether within the crystal ? 
things in nature. It is no objection to the hypothesis in question, to say that it increases the difficulty of accounting for refraction; for, as there is positive evidence in favour of the hypothesis, we ought rather to conclude that the common opinion, which attributes refraction to a change of density in the ether, is altogether erroneous.

In the next place we may remark, that our first hypothesis, ${ }^{*}$ concerning the direction of vibrations in polarized light, will be useful in testing any proposed theory; for as it now seems to be certain that the vibrations are parallel to the plane of polarization, and not perpendicular to it, as Fresnel supposed, such a direction of the vibrations ought to be a consequence of the theory which we adopt.

The third hypothesis, or the principle of the preservation of vis viva, is the most natural that can be imagined, inasmuch as it implies only this, that the incident light is equal to the sum of the reflected and refracted lights. Yet it is probable that even this principle, like the law of vis viva in ordinary mechanics, is a result of simpler laws, and will be shown to be so as soon as the true mechanism of light shall be discovered.

The fourth hypothesis is a very important one, because the whole theory turns upon it; and therefore, in the beginning of this Paper, a particular account has been given of the manner in which it was originally suggested. If we wish to give a reason for this hypothesis, we might say that the motion of a particle of ether, at the common surface of two media, ought to be the same, to whichsoever medium the particle is conceived to belong; and as the incident and reflected vibrations are superposed in one medium, and the refracted vibrations in the other, we might infer that the resultant of the former vibrations ought to be the same, both in length and direction, as the resultant of the latter. At first sight this reasoning appears sufficiently plausible; but it will not bear a close examination. For as the argument is general, it would prove that the principle of

* This hypothesis properly belongs to the laws of propagation, as it relates only to what passes within a given medium. 
the equivalence of vibrations is true for metals,* as well as for crystals, which it certainly is not. - It is not easy to see why the principle should hold in the one case and not in the other; but it is probably prevented from holding, in the case of metals,

- A few days after this Paper was read, I found reason to persuade myself that in metals the vibrations parallel to the surface are equivalent, but not those perpendicular to it; and that in metals, as well as in crystals, the vis viva is preserved. This persuasion was founded on a system of formulæ which I had invented for expressing the laws of metallic reflexion and refraction; and which seem to represent very satisfactorily the experiments of Brewster, Phil. Trans., 1830. As metallic and crystalline reflexion are kindred subjects, and will one day be brought under the same theory, however distinct they may now appear, it will not be out of place to insert the formulæ for metals here. These formulæ are not proposed as true, but as likely to be true; and they will be found to express, at least with general correctness, all the circumstances that have hitherto been regarded as anomalies in the action of metals upon light.

I suppose that for every metal there are two constants, $\boldsymbol{M}$ and $\boldsymbol{\chi}$, of which the first is a number greater than unity, and the second is an angle included between 0 and $90^{\circ}$. The number $M$ I call the modulus, and the angle $\chi$ the characteristic of the metal. Both $M$ and $\chi$ vary with the colour of the light, and the ratio $\frac{M}{\cos \chi}$ is probably the index of refraction. From Brewster's experiments it appears that $\boldsymbol{M}$ diminishes from the red to the violet; and therefore I should suppose that $\cos \chi$ diminishes in a greater ratio, in order that the index of refraction may increase as in transparent substances.

Put $t_{1}$ for the angle of incidence, and $t_{2}$ for the angle of refraction, so that

$$
\frac{\sin t_{1}}{\sin t_{2}}=\frac{M}{\cos \chi}
$$

and let $\mu$ be a variable determined by the condition

$$
\mu=\frac{\cos \iota_{1}}{\cos t_{3}}
$$

These two relations combined will give

$$
\frac{1}{\mu^{3}}=1+\left(1-\frac{\cos ^{2} \chi}{M^{2}}\right) \tan ^{2} t_{1},
$$

Twhich shows that $\mu$ is equal to unity at a perpendicular incidence, and that it ranishes at an incidence $90^{\circ}$, decreasing always during the interval.

Now if plane polarized light be incident on the metal, we must distinguish two principal cases, according as the light is polarized in the plane of incidence, or in the perpendicular plane. In the first case, denoting the reflected and refracted transversals by $\tau_{3}$ and $\tau_{2}$ respectively, let us put $\Delta_{3}$ for the change of phase in 
by the same cause, whatever it is, which produces a change of phase in metallio reflexion.

It will be proper to conclude this Essay with a brief sketch of the researches of Sir David Brewster and M. Seebeck, the

the reflected ray, and $\Delta_{2}$ for the change of phase in the refracted ray. Let the same symbols, marked with accents, be used in the second case with similar significations. Then if the incident transversal be taken for unity, we shall have the following formulø:

1. When the incident transversal is in the plane of incidence,

$$
\left.\begin{array}{c}
\tau_{9}^{2}=\frac{M \Gamma^{2}+\mu^{2}-2 M \mu \cos \chi}{M^{2}+\mu^{2}+2 M \mu \cos \chi}, \\
\tau_{2}^{2}=\frac{4 M^{2} \mu^{2}}{M^{2}+\mu^{2}+2 M \mu \cos \chi}, \\
\tan \Delta_{3}=\frac{2 M \mu \sin \chi}{M^{2}-\mu^{2}}, \quad \tan \Delta_{2}=\frac{\mu \sin \chi}{M+\mu \cos \chi},
\end{array}\right\}
$$

2. When the incident transversal is perpendicular to the plane of incidence,

$$
\left.\begin{array}{c}
\tau_{3}^{\prime}=\frac{1+M^{2} \mu^{2}-2 M \mu \cos \chi}{1+M^{2} \mu^{2}+2 M \mu \cos \chi}, \\
\tau_{2}^{\prime 2}=\frac{4 M^{2} \mu^{2}}{1+M^{2} \mu^{2}+2 M \mu \cos }, \\
\tan \Delta_{3}^{\prime}=\frac{2 M \mu \sin \chi}{M^{2} \mu^{2}-1^{\prime}} . \quad \tan \Delta_{2}^{\prime}=\frac{\sin \chi}{M \mu+\cos \chi} .
\end{array}\right\}
$$

When $\chi=0$, there is no change of phase, and the formulæ become identical with those given in the note, p. 101. When $\chi=90^{\circ}$, there is total reflexion at all incidences. The case of pure silver approximates to this. For good speculum metal, $\chi$ is about $70^{\circ}$. The value of $M$ ranges from $2 \frac{1}{2}$ to 5 in different metals.

When the incident transversal is inclined to the plane of incidence, its components, parallel and perpendicular to that plane, will give two reflected transversals with a difference of phase equal to $\Delta_{3}^{\prime}-\Delta_{3}$. The reflected vibration will then be performed in an ellipse; and the position and magnitude of the axes of the ellipse may be deduced from the preceding formulæ. The consequences of these formulæ are very simple and elegant, but I cannot dwell upon them here. Suffice it to observe, that every angle of incidence has another angle corresponding to it, which I call its conjugate angle of incidence; and that the value of $\Delta_{3}^{\prime}-\Delta_{3}$ at one of these angles is the supplement of its value at the other, while the ratio $\frac{\tau^{\prime}{ }_{3}}{\tau_{3}}$ is the same at both angles; whence it follows that, ceteris paribus, the elliptic vibrations, reflected at conjugate angles, are similar to each other, and have their homologous 
only other writers who have treated of the subject of erystalline reflexion.

So early as the year 1819, Sir David Brewster published, in the Philosophical Transactions, a Paper "On the Action of Crystallized Surfaces upon Light." * In this Paper the Author details a great variety of experiments on the polarizing effects of Iceland spar. He gives the measures of the polarizing angles in different azimuths, when the reflexion takes place in air; but he does not notice the accompanying deviations, which were probably too small to attract his attention. In another instance, however, he obtained very large deviations. He conceived the idea of pushing his experiments into an extreme case, by masking, as it were, the ordinary reflecting action of the crystal, and leaving the extraordinary energy at full liberty to display itself. This was done by dropping on the reflecting surface a little oil of cassia, a fluid whose refractive index is nearly equal to the ordinary index of Iceland spar. When common light, incident at $45^{\circ}$, was reflected at the separating surface of the oil and the spar, the reflected pencil was found to be partially, and sometimes completely, polarized in planes variously inclined to the plane of incidence, the inclination going through all magnitudes from 0 to $180^{\circ}$, as the crystal was turned round in azimuth. This general result is no more than what theory would lead us to expect, when the angle of incidence is nearly equal to one of the angles of refraction; but to institute a minute comparison of theory with experiment would require troublesome calcula-

axes equally inclined to the plane of incidence, but on opposite sides of it. When $\Delta_{3}^{\prime}-\Delta_{3}=90^{\circ}$, the conjugate incidences are equal, the ratio $\frac{\tau_{3}^{\prime}}{\tau_{3}}$ is a minimum, and the axes of the elliptic vibration are parallel and perpendicular to the plane of incidence. When $\Delta_{3}^{\prime}=90^{\circ}$, or $M \mu=1$, the value of $\tau_{3}^{\prime}$ is a minimum, and equal to $\tan \frac{1}{2} x$.

The foregoing formulæ differ slightly from those which I have given in No. I. of the Proceedings of the Royal Irish Academy. The small quantity $\chi$, which occurs in the latter, has been purposely neglected, as its presence interferes with the simplicity of the expressions.

* Phil. Trans. 1819, p. 145. 
tions, which I have not had time to make. With the view, however, of showing clearly, from theory, that the range of the deviation is unlimited, I have considered the simple case in which $N=B$, or in which the refractive index of the fluid is exactly equal to the ordinary index of the crystal. This case, moreover, is remarkable on its own account; and it might be worth while to try whether it could not be verified by direct experiment. If a fluid could be procured whose refractive index, for some definite ray of the spectrum, should be equal to the ordinary index of the erystal for the same ray, and if common light, incident at any angle and in any azimuth, were reflected at the confines of the fluid and the crystal, then, supposing the theory to be exact, the definite ray aforesaid would, as we have seen, be completely polarized by reflexion, and the plane of polarization would always be perpendicular to a plane drawn through the direction of the reflected ray and the axis of the erystal. This experiment would be an elegant test of the theory in its applieation to these extreme and trying cases; and if it were successful, no doubt could be entertained* as to the rigorous accuracy of the geometrical laws of reflexion.

* I was at this time in doubt whether the phenomena observed with oil of cassia could be reconciled to theory; and when the note in page 93 was written, I was almost certain that they could not. But I have since, I think, found out the cause of this perplexity. Some of Brewster's experiments were made with natural surfaces of Iceland spar; others with surfaces arificially polished. I believe (though I have made very few calculations relative to the point) that the former class of experiments will be perfectly explained by the theory; the latter I am certain cannot be so explained, nor ought we to expect that they should. For the process of artificial polishing must necessarily occasion small inequalities, by exposing little elementary rhombs with their faces inclined to the general surface; and the action of these faces may produce the unsymmetrical effects which Brewster notices as so extraordinary (Sixth Report of the British Association, Transactions of the Sections, p. 16). If this will not account for such effects, I do not know what will. From an old observation of Brewster (Phil. Trans., 1819), it would appear that imperfect polish does actually produce a want of symmetry in the phenomena; for when common light was reflected between oil of cassia and a badly polished surface perpendicular to the axis, he found that the reflected ray was polarized neither in the plane of incidence, nor perpendicular 
The experiments with oil of eassia must be very difficult on account of the great feebleness of the reflected light. Sir David Brewster, however, resumed them at different times; and he laid an extensive series of his results before the Physieal Section of the British Association at its late meeting in Bristol.

It was not until the latter end of November, 1836, that I became acquainted with the investigation of M. Seebeck, who has contributed greatly to the advancement of the subject. He made very acurate experiments on the light reflected in air from Iceland spar. He detected the deviation, notwithstanding its smallness, and measured it with great care. He also made the first step in the theory of crystalline reflexion; and the remarkable formula (68), which gives the polarizing angle when the axis lies in the plane of incidence, is due to him. The hypotheses which he employed were similar to those of Fresnel, and they enabled him to solve the problem of reflexion in the case just mentioned, but not to attempt it generally. The date of his first Papers* is the year 1831; but he did not publish his experiments on the deviation until a recent occasion, when he was led to compare them $\uparrow$ with the theory which I had originally given in my letter to Sir David Brewster. I have already stated the correction which the theory underwent in conse-

to it, but $75^{\circ}$ out of it. The same surface, when the light was reflected in air, gave the polarizing angle more than two degrees below its proper value.

To show that, in other respects, the general character of the phenomena is in accordance with theory, we may observe that when $N=B$, and $\lambda=0$ or $90^{\circ}$, if common light be incident at $45^{\circ}$ in the plane of the principal section of the crystal, the whole of the reflected light will be polarized perpendicularly to that plane; and therefore if $N$ be nearly equal to $B$, while every thing else remains the same, the reflected pencil will contain some vnpolarized light, and will be only partially polarized in a plane perpendicular tu the plane of incidence; so that (as Brewster has found by experiment) the erystal will then produce by reflexion the same effect which is produced by ordinary riraction. This (as he also found) will not happen when $\lambda$ and the angle of incidence are each equal to $45^{\circ}$, because the light is then incident at the polarizing angio.

* Poggendorff's Annals, Vol xxi. p. 290 ; Vol. xxir. p. 126.

+ Ibid., Vol. xxxvir. p. 280.1 
quenoe of those experiments, and by which it was brought to its present simple form.*

* Two or three months after this correction had been published in the Philosophical Magazine, a notice of it was inserted in Poggendorff's Annals, vol. xl. p. 462. Up to that time, I believe, nothing had been published in Germany on the general theory of crystalline reflexion; at least the writer of the notice (whom I take to be M. Seebeck) does not seem to have heard of any other theory, or any other principles than mine. But in the next number of Poggendorff, vol. xl. p. 497 , there appeared a letter from M. Neumann, in which the writer speaks of a theory of his own, founded on principles exactly the same as those which I had already announced, and refers to a Paper which he had communicated on the subject to the Academy of Berlin. The Paper has been printed in the Transactions of that Academy for the year 1835; and through the kindness of the author I have received a copy of it, just in time to acknowledge it here. On casting my eye over it, I recognize several equations which are familiar to me-in particular, the equations (VIr.), (vIII.), (IX.), (x.), which I discovered independently in November last. M. Neumann's Paper is very elaborate, and supersedes, in a great measure, the design which I had formed of treating the subject more fully at my leisure; nor can I do better than recommend it to those who wish to pursue the investigations through all their details.

Thintty College, Dublin, March, 1838. 
XII. - ON A NEW OPTICAL INSTRUMENT, INTENDED CHIEFLY FOR THE PURPOSE OF MAKING EXPERIMENTS ON THE LIGHT REFLECTED FROM METALS.

[Proceedings of the Royal Irish Academy, April 9, 1838.]

The instrument consists of two hollow arms or tubes, moveable about the centre, and in the plane, of a large divided circle, each arm being provided with a Nicol's eye-piece, or some equivalent contrivance for polarizing light in a single plane; while in one arm, which is of course crooked, a Fresnel's rhomb is interposed between the eye-piece and the centre of the circle. At this centre is placed a stage for carrying the reflector, with its plane perpendicular to the plane of the circle, and having a motion to and fro for adjustment. Each eye-piece, as well as the Fresnel's rhomb, turns freely about the axis of the arm to which it belongs, and is provided with a small circle for measuring its angle of rotation. When the two arms are set at equal angles with the reflector, and the observer looks through the crooked arm, he will see a light admitted through the straight one; and then, by turning the Fresnel's rhomb, and the eye-piece next his eye, he will be able, by means of their combined movements, to find a position in which the light will entirely disappear. An observation will then have been made; for the light, before its incidence on the metal, is polarized in a given plane by the first eye-piece; but after reflexion from the metal (as we know from Sir David Brewster's experiments) it is elliptically polarized ; and our object is to determine the position and species of the 
little ellipse in which the reflected vibration is supposed to be performed. Now, the axes of this ellipse are parallel and perpendicular to the principal plane of the rhomb, when it is in the situation above deseribed, where the light completely disappears; and the ratio of the axes is the tangent of the angle which that plane makes with the principal section of the eyepiece next the eye. The angles are read off from the divided circles; and thus, for any angle of incidence, and any plane of primitive polarization, we can at once ascertain the nature of the reflected elliptic vibration. Professor Mac Cullagh mentioned that the instrument was made last year with the view of testing certain formulæe which he has proposed for the case of metallio reflexion, and which have been printed in Vol. xvirI. pp. 70, 71, of the Transactions of the Academy*; but that he had not yet found leisure to make the various adjustments which are necessary in order to obtain satisfactory results with it. The instrument is beautifully executed by Mr. Grubb, who himself contrived the subordinate mechanism, by which the requisite movements are effected with perfect ease to the observer.

* Supra, pp. 132, 133. 


\section{XIII.-LAWS OF CRYSTALLINE REFLEXION.-QUESTION OF PRIORITY.}

[Procedings of the Royal Irish Academy, Nov. 30, 1838.]

The President, Sir William R. Hamilton, read the following letter which had been addressed to him by M. Neumann of Königsberg, on some points connected with the history of the Laws of Crystalline Reflexion:-

\section{Monsirug,}

Le haut prix que j'attache à votre suffrage et à celui de l'illustre Académie, à laquelle vous présidez, et l'honorable mention, que vous avez voulu faire de mon mémoire sur la théorie de la lumière dans la séance de cette Académie du 25 Juin, m'engagent à vous dresser la lettre suivante. Vous avez donné dans cette séance un jugement dans la question de priorité, qui pouvait s'élever entre Mr. Mac Cullagh et moi par rapport à la découverte des lois suivant lesquelles la lumière est reflechie et refractée par des milieux crystallins-j'ai l'honneur de vous communiquer dans ce qui suit quelques faits et quelques reflexions fondées sur ces faits, et qui auraient été peut-être de quelque influence sur ce jugement.

Au commencement de l'année 1833 j'ai communiqué à $\mathbf{M}$. Seebeck de Berlin non seulement l'ensemble des principes de ma théorie tels qu'ils se trouvent imprimés dans le $\$ 2$ de mon mémoire, mais j'avais illustré encore ces principes par leur application aux milieux non crystallins. En même tems j'ai annoncé à M. Seebeck, que les résultats tirés de ces principes par rapport aux milieux erystallins étaient parfaitement d'accord avec ses observations sur l'angle de polarisation du kalkspath, et je lui fis part de la formule même, qui exprime l'inclinaison du plan do polarisation du rayon polarisé par réflexion vers le plan de reflexion. Sous la date du 11 Mai, 1833, M. Seebeck m'écrivit, que cette formule aussi s'accordait parfaitement avec ses observations, qu' il n'avait pas encore publiées et qu' il avait la complaisance de me communiquer en manuscrit. Dans le printems de 1834 le manuscrit de mon mémoire tel qu' il a paru depuis allait être achevé; mais un voyage que je fis dans ce tems et qui m'éloigna 


\section{Question of Priority.}

assez long-tems de Königsberg, m'empêcha de la publier incessamment. Cependant $\mathrm{j}^{\prime}$ avais pris soin d'en faire un abrégé dans lequel je développai complètement les principes de ma théorie et les résultats auxquels elle m'avait conduit par rapport aux crystaux ì un axe.

J'envoyai cet extrait en Mai ou Juin, 1834, par la librairie de M. Schropp de Berlin à M. Arago, en le priant do le faire imprimer dans les Annales de Chimie et de Physique, ce savant ayant dans une note publiée dans ce tems marqué un grand intérêt pour l'investigation des lois des intensités du rayon ordinaire et extraordinaire, lois qui se trouvaient parmi les résultats mentionnés. Il n'y a pas de doute que cet extrait ne soit parvenu dans les mains de M. Arago, entre lesquelles il doit se trouver encore à présent. Du reste, M. Jacobi en avait pris une connaissance détaillée, et à Berlin il a été entre les mains de MM. Weiss et Poggendorf.

En passant par Vienne dans l'été de 1834, j'avais lo plaisir d'entretenir de mes résultats et de ma méthode $\mathrm{M}$. Ettinghausen, savant très distingué et très versé dans les parties les plus épineuses de l'optique. Antérieurement j'avais enseigné mes doctrines à $\mathbf{M}$. Senff, maintenant professeur à l'Université de Dorpat, pendant le séjour que fit à Koenigsberg ce jeune et habile physicien, qui vient de publier un excellent travail sur les propriétés optiques et crystallographiques du fer sulfaté.

Il suit de tout ce qui précède, que déjà en 1834, mes resultats trouvés par rapport aux lois de reflexion et de refraction des crystaux n'étaient guère inconnus aux physiciens de l'Allemagne, qui s'occupent de l'optique, et si dès lors ils n'ont pas reçu une plus grande publicité, vous voyez, Monsieur, cela tenait aux Annales de Chimie. La publication de mon mémoire a été rétardée par l'espoir que j'avais conçu de pouvoir lui ajouter une partie expérimentale. Mais l'exécution des appareils me faisant attendre trop long tems, j'ai présenté vers la fin de 1835 à l'Académie de Berlin mon ouvrage tel qu'il a été imprimé depuis parmi les mémoires de cette Académie. La partie expérimentale a été publiée en 1837 dans le volume 42 des Annales de M. Poggendorf.

Je vois du discours que vous avez tenu, Monsieur, dans la Séance de votre Académie du 25 Juin passé, et qui vient de m'être communiqué, que c'est déjà en Août, 1835, que Mr. Mac Cullagh a fait à l'Association Britannique une communication sur les lois de reflexion et refraction par les crystaux, et qui a été imprimée dans le Lond. and Edinb. Phil. Mag., Février, 1836. Je crois très volontiers, que Mr. Mac Cullagh est parvenu aux résultats qui se trouvent dans cette publication, par ses propres efforts et sans avoir eu connaisance de mes travaux sur ce même sujet. Toutefois ce ne sont pas ces résultats qui, pourraient être l'objet d'une question de priorité. En effet dans une note publiée dans les Annales de M. Poggendorf (vol. xxxviii. 1836), M. Seebeck a montré que les formules auxquelles est parvenu Mr. Mac Cullagh ne sunt pas justes, et qu'elles ne représentent pas les lois de reflexion et de refraction par les crystaux. Dans la même note M. Seebeck a exposé, comment les lois de reflexion et de refraction des milieux non crystallins conformes à cette definition du plan de polarisation, à laquelle on est conduit dans la théorie de la double refraction, peuvent être déduites des suppositions faites par Fresnel, avec la seule modification de l'homogénéité 
de l'éther dans tous les milieux. Mais les suppositions de Fresnel ainsi modifiées forment la base principale de ma méthode, dont j'avais déjà fait part à M. Seebeck depuis plusieurs années. Il est vrai, que dans les deux milieux Fresnel ne suppose que l'égalité de deux composantes parallèles au plan de séparation, mais l'égalité de la troisième n'est qu'une simple conséquence de celle des deux autres et des autres suppositions. Ce sont les suppositions de Fresnel modifiées de la dite manière, qu'a adoptées Mr. Mac Cullagh, après s'être convaincu par la note de M. Seebeck de la faussete des résultats qu'il avait jusque-là obtenus, conviction qui l'engagea à rejeter tout ce qui n'était pas conforme à ces suppositions, et dès lors seulement on 1837, dans le Lond. and Edinb. Phil. Mag., Mr. Mac Cullagh est parvenu aux mêmes lois de reflexion et de refraction que j'avais eues l'honneur de présenter à l'Academie des Sciences de Berlin en 1835.

Vous voyez par tout ceci, Monsieur, que dès 1833 j'ai été en pleine possession de la méthode, et que dès le commencement de 1834 j'ai été en pleine possession des résultats qu'elle fournit, que dans ce même tems j'ai envoyé un abrégé contenant ces résultats et lu en manuscrit par plusieurs savans bien connus à $\mathbf{M}$. le redacteur des Annales de Physique et Chimie pour le publier dans ce recueil, et qu'à la fin de 1835, j'ai présenté l'ouvrage complet à present imprimé à l'Académie de Berlin; vous voyez en même tems, que Mr. Mac Cullagh ayant communiqué à l'Association Britanique en $\mathbf{1 8 3 5}$ des lois de réflexion et de refraction crystallin, ces lois ont été demontrées être fautives par M. Seebeck in 1836, et que Mr. Mac Cullagh n'est parvenu en 1837 aux vraies lois qu' après avoir pris connaissance du fondement de ma méthode, et s'en être servi.

De tout cela résulte, Monsieur, que la priorité de la découverte des lois de réflexion et réfraction par des crystaux n'est pas douteuse, et, qu'il n'y a pas de simultanéité entre mes travaux et ceux de Mr. Mac Cullagh, dont du reste personne ne peut estimer plus que moi le talent distingué.

Daignez, Monsieur, agréer les assurances de la plus haute considération arec laquelle je suis, \&c.,

Köniabbera, 5 Octobre, 1838.

F. E. NEUMANN.

When this letter was read, Professor Mac Cullagh requested permission to make a few remarks. After expressing much regret that his researches in the theory of light should have clashed with those of any other person (though in the present state of science such collisions were perhaps inevitable), he proceeded to say, that he did not think it necessary to detain the Academy with a formal reply to the communication which had just been read; it would be sufficient for him to observe, in general, that the facts brought forward by the writer, with reference to the history of his own investigations, were all, without exception, of a private nature, not one of them being 
taken from any published document; that the first document of the kind, which professed to give any account of M. Neumann's "method," or any statement of the principles employed in it, appeared in the Annals of Poggendorf (Vol. xL. p. 497), some months after Mr. Mac Cullagh had published his last Paper on the subject in the Philosophical Magasine (Vol. x. p. 43), and even after that Paper had been noticed in the aforesaid Annals (Vol. xL. p. 462); that M. Neumann's Memoir in the Berlin Transactions was not published until a later period; that, therefore, there could be no question about priority of publication; and that, consequently, if it were to be imagined for a moment that either author had borrowed from the other, the presumption must necessarily be against $\mathbf{M}$. Neumann. With respect to M. Seebeck's note, it would be enough to state, that M. Neumann is not mentioned there at all; that the principles there given by M. Seebeck are not adequate to the general solution of the problem; and that such of them as differ from those of Fresnel had been previously published by Mr. Mac Cullagh. It was clear, therefore, that Mr. Mac Cullagh owed nothing on the score of theory to anyone but Fresnel. He had, indeed, made one alteration in his theory as it originally stood; for he had at first rejected Fresnel's law of the vis vira, and had been obliged to restore it afterwards, in order to account for certain experiments of M. Seebeck, which M. Seebeck himself, from want of sufficient principles, had not attempted to account for; but the real service which M. Seebeek had rendered him, and for which he had frequently acknowledged his obligations, was the communication of these experiments, and not any suggestion of the law of vis vixa, which he knew well enough before. In all this, however, it was plain that M. Neumann had no concern, unless he chose to say that he had appropriated to himself Fresnel's law of the vis riva, that he had determined to regard it as the foundation of his method (le fondement de sa méthode), and that thenceforward no one else (however ignorant of such appropriation) could have any right to use it. 
Having thus endeavoured to prove his olaim to priority of publication, and to establish the independence of his own researches, which was all that was necessary for self defence, Mr. Mas Cullagh concluded by saying, that he would there drop the argument, without discussing his claim to priority in the abstract, as he had an objection to disputes of such a kind, and did not wish to pursue them any farther than he was compelled to do. But if anyone thought it worth while to examine the merits of this second question, he would find the circumstances relating to it very fully and clearly stated in the last number of the Proceedings of the Academy, ${ }^{*}$ and would thence be enabled to form a judgment for himself.

* Vol. I. p. 217. 


\section{( 145$)$}

\section{XIV.-AN ESSAY TOWARDS A DYNAMICAL THEORY OF CRYSTALLINE REFLEXION AND REFRACTION.}

[Transactions of the Royal Irish Academy, VoL. xxI,-Read December 9, 1839.]

Sect. I.-Introductory Observations.-Equation of Motion.

Nearly three years ago I communicated to this Academy* the laws by which the vibrations of light appear to be governed in their reflexion and refraction at the surfaces of crystals. These laws-remarkable for their simplicity and elegance, as well as for their agreement with exact experiments-I obtained from a system of hypotheses which were opposed, in some respects, to notions previously received, and were not bound together by any known principles of mechanics, the only evidence of their truth being the truth of the results to which they led. On that occasion, however, I observed that the hypotheses were not independent of each other ; and soon afterwards I proved that the laws of reflexion at the surface of a crystal are connected, in a very singular way, with the laws of double refraction, or of propagation in its interior ; from which I was led to infer that "all these laws and hypotheses have a common source in other and more intimate laws which remain to be discovered ;" and that "the next step in physical opties would probably lead to those higher and more elementary principles by which the laws of reflexion

"In a Paper "On the Laws of Crystalline Reflexion and Refraction." - Transactions of the Royal-Irish Academy, Vou. xvir. p. 31. (Supra, p. 87.) 
and the laws of propagation are linked together as parts of the same system." This step has since been made, and these anticipations have been realised. In the present Paper I propose to supply the link between the two sets of laws by means of a very simple theory, depending on certain special assumptions, and employing the usual methods of analytical dynamics.

In this theory, the two kinds of laws, being traced from a common origin, are at once connected with each other and severally explained; and it may be observed, that the explanation of each, as well as the source of their connexion, is now made known for the first time. For though the laws of crystalline propagation have attracted much attention during the period which has elapsed since they were discovered by Fresnel, $\uparrow$ they have hitherto resisted every attempt that has been made to account for them by dynamical reasonings; and the laws of reflexion, when recently discovered, were apparently still more difficult to reach by such considerations. Nothing can be easier, however, than the process by which both systems of laws are now deduced from the same principles.

The assumptions on which the theory rests are these:-First, that the density of the luminiferous ether is a constant quantity; in which it is implied that this density is unchanged either by the motions which produce light or by the presence of material particles, so that it is the same within all bodies as in free space, and remains the same during the most intense vibrations. Second, that the vibrations in a plane-wave are rectilinear, and that, while the plane of the wave moves parallel to itself, the vibrations continue parallel to a fixed right line, the direction of this right line and the direction of a normal to the wave being functions of each other. This supposition holds in all known crystals, except quartz, in which the vibrations are elliptical.

Concerning the peculiar constitution of the ether we know

- Ibid, p. 63, note. (Supra, p. 112.) The note here referred to was added some time after the Paper itself was read.

† These laws were published in his Memoir on Double Refraction-Mémoires de l'Institut, tom, vii. p. 45. 
nothing, and shall suppose nothing, except what is involved in the foregoing assumptions. But with respect to its physical condition generally, we shall admit, as is most natural, that a vast number of ethereal particles are contained in the differential element of volume; and, for the present, we shall consider the mutual action of these particles to be sensible only at distances which are insensible when compared with the length of a wave.

By putting together the assumptions we have made, it will appear that when a system of plane waves disturbs the ether, the vibrations are transversal, or parallel to the plane of the waves. For all the particles situated in a plane parallel to the waves are displaced, from their positions of rest, through equal spaces in parallel directions; and therefore if we conceive a closed surface of any form, including any volume great or small, to be described in the quiescent ether, and then all its points to partake of the motion imparted by the waves, any slice cut out of that volume, by a pair of planes parallel to the wave-plane and indefinitely near each other, can have nothing but its thickness altered by the displacements; and since the assumed preservation of density requires that the volume of the slice should not be altered, nor conseguently its thickness, it follows that the displacements must be in the plane of the slice, that is to say, they must be parallel to the wave-plane. And conversely, when this condition is fulfilled, it is obvious that the entire volume, bounded by the arbitrary surface above described, will remain constant during the motion, while the surface itself will always contain within it the very same ethereal particles which it enclosed in the state of rest; and all this will be accurately true, no matter how great may be the magnitude of the displacements.

Let $x, y, z$ be the rectangular co-ordinates of a particle before it is disturbed, and $x+\xi, y+\eta, z+\zeta$ its co-ordinates at the time $t$, the displacements $\xi, \eta, \zeta$ being functions of $x, y, z$ and $t$. Let the ethereal density, which is the same in all media, be regarded as unity, so that $d x d y d z$ may, at any instant, represent indif- 
ferently either the element of volume or of mass. Then the equation of motion will be of the form

$$
\iiint d x d y d z\left(\frac{d^{2} \xi}{d t^{2}} \delta \xi+\frac{d^{2} \eta}{d t^{2}} \delta \eta+\frac{d^{2} \zeta}{d t^{2}} d \zeta\right)=\iiint d x d y d z \delta \nabla,
$$

where $\mathrm{V}$ is some function depending on the mutual actions of the particles. The integrals are to be extended over the whole volume of the vibrating medium, or over all the media, if there be more than one.

Setting out from this equation, which is the general formula of dynamies applied to the ease that we are considering, we perceive that our chief difficulty will consist in the right determination of the function $\mathrm{V}$; for if that function were known, little more would be necessary, in order to arrive at all the laws which we are in search of, than to follow the rules of analytical mechanics, as they have been given by Lagrange. The determination of $V$ will, of course, depend on the assumptions above stated respecting the nature of the ethereal vibrations; but, before we proceed further, it seems advisable to introduce certain lemmas, for the purpose of abridging this and the subsequent investigations.

\section{Sect. II.-Lemmas.}

Lemma I.-Let a right line making with three rectangular axes the angles $a, \beta, \gamma$, be perpendicular to two other right lines which make with the same axes the angles $a^{\prime}, \beta^{\prime}, \gamma^{\prime}$ and $a^{\prime \prime}, \beta^{\prime \prime}, \gamma^{\prime \prime}$ respectively, and which are inclined to each other at an angle denoted by $\theta$; then it is easy to prove that

$$
\begin{aligned}
& \sin \theta \cos \alpha=\cos \beta^{\prime} \cos \gamma^{\prime \prime}-\cos \beta^{\prime \prime} \cos \gamma^{\prime}, \\
& \sin \theta \cos \beta=\cos \gamma^{\prime} \cos \alpha^{\prime \prime}-\cos \gamma^{\prime \prime} \cos \alpha^{\prime}, \\
& \sin \theta \cos \gamma=\cos \alpha^{\prime} \cos \beta^{\prime \prime}-\cos \alpha^{\prime \prime} \cos \beta^{\prime} ;
\end{aligned}
$$

supposing the first right line to be prolonged in the proper direction from the origin, in order that the opposite members of any 
one of these equations may have the same sign, as well as the same magnitude.

If the last two right lines be perpendicular to each other, we have $\sin \theta=1$, and the formulæ become

$$
\begin{aligned}
& \cos \alpha=\cos \beta^{\prime} \cos \gamma^{\prime \prime}-\cos \beta^{\prime \prime} \cos \gamma^{\prime}, \\
& \cos \beta=\cos \gamma^{\prime} \cos \alpha^{\prime \prime}-\cos \gamma^{\prime \prime} \cos \alpha^{\prime}, \\
& \cos \gamma=\cos \alpha^{\prime} \cos \beta^{\prime \prime}-\cos \alpha^{\prime \prime} \cos \beta^{\prime} ;
\end{aligned}
$$

but in this case the three right lines are perpendicular to each other, and therefore we have, in like manner,

and also

$$
\begin{aligned}
& \cos a^{\prime}=\cos \beta^{\prime \prime} \cos \gamma-\cos \beta \cos \gamma^{\prime \prime}, \\
& \cos \beta^{\prime}=\cos \gamma^{\prime \prime} \cos \alpha-\cos \gamma \cos \boldsymbol{a}^{\prime \prime}, \\
& \cos \gamma^{\prime}=\cos a^{\prime \prime} \cos \beta-\cos a \cos \beta^{\prime \prime} ;
\end{aligned}
$$

$$
\begin{aligned}
& \cos a^{\prime \prime}=\cos \beta \cos \gamma^{\prime}-\cos \beta^{\prime} \cos \gamma, \\
& \cos \beta^{\prime \prime}=\cos \gamma \cos a^{\prime}-\cos \gamma^{\prime} \cos a, \\
& \cos \gamma^{\prime \prime}=\cos a \cos \beta^{\prime}-\cos a^{\prime} \cos \beta .
\end{aligned}
$$

The last three groups of formulæ will still be true, if we suppose the first right line to make with the axes the angles $a, a^{\prime}, a^{\prime \prime}$, the second the angles $\beta, \beta^{\prime}, \beta^{\prime \prime}$, and the third the angles $\gamma, \gamma^{\prime}, \gamma^{\prime \prime}$

Lemma II.-Let $\xi, \eta, \zeta$ denote, as before, the displacements of a particle whose initial co-ordinates are $x, y, z$; and after putting

$$
X=\frac{d \eta}{d z}-\frac{d \zeta}{d y}, \quad X=\frac{d \zeta}{d x}-\frac{d \xi}{d z}, \quad Z=\frac{d \xi}{d y}-\frac{d \eta}{d x},
$$

suppose the axes of co-ordinates, still remaining rectangular, to have their directions changed in space, whereby the quantities $X, Y, Z$ will be changed into $X^{\prime}, Y^{\prime}, Z^{\prime}$, answering to the new co-ordinates $x^{\prime}, y^{\prime}, z^{\prime}$, and to the new displacements $\xi^{\prime}, \eta^{\prime}, \zeta^{\prime}$; then will the quantities $X^{\prime}, Y^{\prime}, Z^{\prime}$ be connected with $X, Y, Z$ by 
the very same relations which connect the co-ordinates $x^{\prime}, y^{\prime}, z^{\prime}$ with $x, y, z$, or the displacements $\xi^{\prime}, \eta^{\prime}, \zeta^{\prime}$ with $\xi, \eta, \zeta$.

That is to say, if the axes of $x, y, z$ make with the axis of $x^{\prime}$ the angles $a, \beta, \gamma$, with the axis of $y^{\prime}$ the angles $a^{\prime}, \beta^{\prime}, \gamma^{\prime}$, and with the axis of $z^{\prime}$ the angles $a^{\prime \prime}, \beta^{\prime \prime}, \gamma^{\prime \prime}$ respectively, we shall have

and

$$
\begin{aligned}
& X=X^{\prime} \cos a+Y^{\prime} \cos a^{\prime}+Z^{\prime} \cos a^{\prime \prime}, \\
& Y=X^{\prime} \cos \beta+Y^{\prime} \cos \beta^{\prime}+Z^{\prime} \cos \beta^{\prime \prime}, \\
& Z=X^{\prime} \cos \gamma+Y^{\prime} \cos \gamma^{\prime}+Z^{\prime} \cos \gamma^{\prime \prime}
\end{aligned}
$$

$$
Z^{\prime}=X \cos \boldsymbol{\alpha}^{\prime \prime}+Y \cos \beta^{\prime \prime}+Z \cos \gamma^{\prime \prime} ;
$$

just as we have, for example,

$$
\begin{aligned}
& \xi=\xi^{\prime} \cos \alpha+\eta^{\prime} \cos \boldsymbol{a}^{\prime}+\zeta^{\prime} \cos \boldsymbol{a}^{\prime \prime}, \\
& \eta=\xi^{\prime} \cos \beta+\eta^{\prime} \cos \beta^{\prime}+\zeta^{\prime} \cos \beta^{\prime \prime}, \\
& \zeta=\xi^{\prime} \cos \gamma+\eta^{\prime} \cos \gamma^{\prime}+\zeta^{\prime} \cos \gamma^{\prime \prime},
\end{aligned}
$$

and

$$
\begin{aligned}
& x^{\prime}=x \cos a+y \cos \beta+z \cos \gamma, \\
& y^{\prime}=x \cos a^{\prime}+y \cos \beta^{\prime}+z \cos \gamma^{\prime}, \\
& z^{\prime}=x \cos a^{\prime \prime}+y \cos \beta^{\prime \prime}+z \cos \gamma^{\prime \prime} .
\end{aligned}
$$

For, the change of the independent variables $x, y, z$ into $x^{\prime}, y^{\prime}, z^{\prime}$ gives us the equations

$$
\begin{aligned}
& \frac{d \eta}{d z}=\frac{d \eta}{d x^{\prime}} \frac{d x^{\prime}}{d z}+\frac{d \eta}{d y^{\prime}} \frac{d y^{\prime}}{d z}+\frac{d \eta}{d z^{\prime}} \frac{d z^{\prime}}{d z}, \\
& \frac{d \zeta}{d y}=\frac{d \zeta}{d x^{\prime}} \frac{d x^{\prime}}{d y}+\frac{d \zeta}{d y^{\prime}} \frac{d y}{d y}+\frac{d \zeta}{d z^{\prime}} \frac{d z^{\prime}}{d y},
\end{aligned}
$$

in the right-hand members of which we have to substitute the 
values of the differential coefficients obtained from $(d)$ and $\left(d^{\prime}\right)$. Thus we get

$$
\begin{aligned}
\frac{d \eta}{d z} & =\left(\frac{d \xi^{\prime}}{d x^{\prime}} \cos \beta+\frac{d \eta^{\prime}}{d x^{\prime}} \cos \beta^{\prime}+\frac{d \zeta^{\prime}}{d x^{\prime}} \cos \beta^{\prime \prime}\right) \cos \gamma \\
& +\left(\frac{d \xi^{\prime}}{d y^{\prime}} \cos \beta+\frac{d \eta^{\prime}}{d y^{\prime}} \cos \beta^{\prime}+\frac{d \zeta^{\prime}}{d y^{\prime}} \cos \beta^{\prime \prime}\right) \cos \gamma^{\prime} \\
& +\left(\frac{d \xi^{\prime}}{d z^{\prime}} \cos \beta+\frac{d \eta^{\prime}}{d z^{\prime}} \cos \beta^{\prime}+\frac{d \xi^{\prime}}{d z^{\prime}} \cos \beta^{\prime \prime}\right) \cos \gamma^{\prime \prime}, \\
\frac{d \zeta}{d y} & =\left(\frac{d \xi^{\prime}}{d x^{\prime}} \cos \gamma+\frac{d \eta^{\prime}}{d x^{\prime}} \cos \gamma^{\prime}+\frac{d \zeta^{\prime}}{d x^{\prime}} \cos \gamma^{\prime \prime}\right) \cos \beta \\
& +\left(\frac{d \xi^{\prime}}{d y^{\prime}} \cos \gamma+\frac{d \eta^{\prime}}{d y^{\prime}} \cos \gamma^{\prime}+\frac{d \xi^{\prime}}{d y^{\prime}} \cos \gamma^{\prime \prime}\right) \cos \beta^{\prime} \\
& +\left(\frac{d \xi^{\prime}}{d z^{\prime}} \cos \gamma+\frac{d \eta^{\prime}}{d z^{\prime}} \cos \gamma^{\prime}+\frac{d \zeta^{\prime}}{d z^{\prime}} \cos \gamma^{\prime \prime}\right) \cos \beta^{\prime \prime} ;
\end{aligned}
$$

and when we subtract these equations, attending to the formulæ in Lemma I., we find

or simply,

$$
\begin{aligned}
\frac{d \eta}{d z}-\frac{d \zeta}{d y}=\left(\frac{d \eta^{\prime}}{d z^{\prime}}-\frac{d \zeta^{\prime}}{d y^{\prime}}\right) \cos a+ & \left(\frac{d \zeta^{\prime}}{d x^{\prime}}-\frac{d \xi^{\prime}}{d z^{\prime}}\right) \cos \boldsymbol{a}^{\prime} \\
& +\left(\frac{d \xi^{\prime}}{d x^{\prime}}-\frac{d \eta^{\prime}}{d x^{\prime}}\right) \cos \boldsymbol{a}^{\prime \prime},
\end{aligned}
$$

$$
X=X^{\prime} \cos a+Y^{\prime} \cos a^{\prime}+Z^{\prime} \cos a^{\prime \prime},
$$

which is the first of formulø (D). And in like manner the others may be proved.

The same things will obviously hold with respect to quantities derived from $X, Y, Z$ in the same way that these are derived from $\xi, \eta, \zeta$. That is, if we put

$$
X_{1}=\frac{d Y}{d z}-\frac{d Z}{d y}, \quad Y_{1}=\frac{d Z}{d x}-\frac{d X}{d z}, \quad Z_{s}=\frac{d X}{d y}-\frac{d Y}{d x} ;
$$

and then suppose the axes of co-ordinates to be changed, the 
formulø for the transformation of the quantities $X, Y, Z$, will be similar to those for the transformation of the co-ordinates themselves. The like will be true of the quantities $X_{\text {, }} Y_{\text {", }} Z_{\text {", }}$ if we put

$$
X_{1}=\frac{d Y_{1}}{d z}-\frac{d Z_{1}}{d y}, \quad Y_{1}=\frac{d Z_{i}}{d x}-\frac{d X_{i}}{d z}, \quad Z_{1}=\frac{d X_{\prime}}{d y}-\frac{d Y_{1}}{d x}
$$

and so on successively.

It is to be observed that, in this Lemma, the displacement is not limited by any restriction whatever. Each of its components may be any function of the co-ordinates. But the displacements produced by a system of plane waves are restricted by our definition of such waves; they must be the same for all particles situated in the same wave plane. If the waves be parallel, for instance, to the plane of $x^{\prime}, y^{\prime}$, the quantities $\xi^{\prime}, \eta^{\prime}, \zeta^{\prime}$ will be independent of the co-ordinates $x^{\prime}, y^{\prime}$, and will be functions of $z^{\prime}$ only. This consideration reduces formulæ (D) to the following :

$$
\begin{aligned}
& X=\frac{d \eta^{\prime}}{d z^{\prime}} \cos a-\frac{d \xi^{\prime}}{d z^{\prime}} \cos a^{\prime}, \\
& Y=\frac{d \eta^{\prime}}{d z^{\prime}} \cos \beta-\frac{d \xi^{\prime}}{d z^{\prime}} \cos \beta^{\prime}, \\
& Z=\frac{d \eta^{\prime}}{d z^{\prime}} \cos \gamma-\frac{d \xi^{\prime}}{d z^{\prime}} \cos \gamma^{\prime},
\end{aligned}
$$

in which it is remarkable that the normal displacement $\zeta^{\prime}$ does not appear. If $\xi^{\prime}=0$, these formulæ become

$$
X=\frac{d \eta^{\prime}}{d z^{\prime}} \cos \alpha, \quad Y=\frac{d \eta^{\prime}}{d z^{\prime}} \cos \beta, \quad Z=\frac{d \eta^{\prime}}{d z^{\prime}} \cos \gamma ;
$$

or if $\eta^{\prime}=0$, then we have

$$
X=-\frac{d \xi^{\prime}}{d z^{\prime}} \cos \alpha^{\prime}, \quad Y=-\frac{d \xi^{\prime}}{d z^{\prime}} \cos \beta^{\prime}, \quad Z=-\frac{d \xi^{\prime}}{d z^{\prime}} \cos \gamma^{\prime} .
$$

Lemma III.-If, in an ellipsoid whose semiaxes are equal to $a, b, c$, there be two rectangular diameters, one making with 
the semiaxes the angles $\alpha, \beta, \gamma$, and the other the angles $a^{\prime}, \beta^{\prime}, \gamma^{\prime}$, such as to satisfy the condition

$$
\frac{\cos a \cos a^{\prime}}{a^{2}}+\frac{\cos \beta \cos \beta^{\prime}}{b^{2}}+\frac{\cos \gamma \cos \gamma^{\prime}}{c^{2}}=0,
$$

these diameters will be the axes of the ellipse in which their plane intersects the ellipsoid.

For, the above condition expresses that either diameter is parallel to the tangent plane at the extremity of the other; they are therefore conjugate diameters of the elliptic section; and hence, as they are at right angles to each other, they must be its axes.

If the semiaxes of the ellipsoid be represented by $\frac{1}{a}, \frac{1}{b}, \frac{1}{c}$, the equation of condition will become

$$
a^{2} \cos \boldsymbol{a} \cos \boldsymbol{a}^{\prime}+b^{2} \cos \beta \cos \beta^{\prime}+c^{2} \cos \gamma \cos \gamma^{\prime}=0 .
$$

Lemma IV.-Let $s, s^{\prime}$ be the lengths of perpendiculars let fall from the centre of an ellipsoid upon any two tangent planes, and $r, r^{\prime}$ the lengths of radii drawn to the respective points of contact. Then putting $\omega$ for the angle between the directions of $r$ and $s^{\prime}$, and $\omega^{\prime}$ for the angle between the directions of $r^{r}$ and $s$, we shall have

$$
r 8 \cos \omega=r^{\prime} s^{\prime} \cos \omega^{\prime} .
$$

For if the semiaxes of the ellipsoid, having their lengths denoted by $a, b, c$, make with the direction of $s$ the angles $a, \beta, \gamma$, and with that of $s^{\prime}$ the angles $\alpha^{\prime}, \beta^{\prime}, \gamma^{\prime}$; with the direction of $r$ the angles $\boldsymbol{\alpha}_{0}, \boldsymbol{\beta}_{0}, \gamma_{0}$, and with that of $r^{\prime}$ the angles $\alpha_{1}, \beta_{1}, \gamma_{1}$, there will exist the relations.

$$
\begin{aligned}
& a^{2} \cos \alpha=r s \cos \alpha_{0}, \quad b^{2} \cos \beta=r s \cos \beta_{0}, \quad c^{2} \cos \gamma=r s \cos \gamma_{0}, \\
& a^{2} \cos a^{\prime}=r^{\prime} s^{\prime} \cos a_{1}, \quad b^{2} \cos \beta^{\prime}=r^{\prime} s^{\prime} \cos \beta_{1}, \quad c^{2} \cos \gamma^{\prime}=r^{\prime} s^{\prime} \cos \gamma_{1},
\end{aligned}
$$

by one set of which the quantity

$$
a^{2} \cos , \alpha \cos \alpha^{\prime}+b^{2} \cos \beta \cos \beta^{\prime}+c^{2} \cos \gamma \cos \gamma^{\prime}
$$


will be converted into

$r s\left(\cos \alpha_{0} \cos \alpha^{\prime}+\cos \beta_{0} \cos \beta^{\prime}+\cos \gamma_{0} \cos \gamma^{\prime}\right)=r s \cos \omega$, and by the other set into

$$
r^{\prime} s^{\prime}\left(\cos a_{1} \cos a+\cos \beta_{1} \cos \beta+\cos \gamma_{1} \cos \gamma\right)=r^{\prime} s^{\prime} \cos \omega^{\prime} ;
$$

so that we shall get

$r s \cos \omega=r^{\prime} s^{\prime} \cos \omega^{\prime}=a^{2} \cos \alpha \cos a^{\prime}+b^{2} \cos \beta \cos \beta^{\prime}$

Corollary. - When the condition

$$
+c^{2} \cos \gamma \cos \gamma^{\prime}
$$

$$
a^{2} \cos \alpha \cos \alpha^{\prime}+b^{2} \cos \beta \cos \beta^{\prime}+c^{2} \cos \gamma \cos \gamma^{\prime}=0
$$

is satisfied, each of the angles $\omega, \omega^{\prime}$ is a right angle. Let us suppose, at the same time, that the direction of $s$ is perpendicular to that of $s^{\prime}$. Then will the directions of $s$ and $r^{\prime}$ coincide with the axes of the ellipse in which their plane intersects the ellipsoid; for $s$ is perpendicular to $r^{\prime}$ and parallel to the tangent plane at its extremity. The directions of $s^{\prime}$ and $r$, in the same manner, will coincide with the axes of another elliptic section.

\section{Sect. III.-Determination of the Function on which the Motion depends. Principal Axes of a Crystal.}

We come now to investigate the particular form which must be assigned to the function $\mathrm{V}$, in order that the formula (1) may represent the motions of the ethereal medium. For this purpose conceive the plane of $x^{\prime} y^{\prime}$ to be parallel to a system of plane waves whose vibrations are entirely transversal and parallel to the axis of $y^{\prime}$, so that $\xi^{\prime}=0, \zeta^{\prime}=0$. Imagine an elementary parallelepiped $d x^{\prime} d y^{\prime} d z^{\prime}$, having its edges parallel to the axes of $x^{\prime}, y^{\prime}, z^{\prime}$, to be described in the ether when at rest, and then all its points to move according to the same law as the ethereal particles which compose it. The faces of the parallelepiped which are perpendicular to the edge $d z^{\prime}$ will be shifted, each in its own plane, in a direction parallel to the axis of $y^{\prime}$; but their displacements will be unequal, and will differ by $d \eta^{\prime}$, 
so that the edges connecting their corresponding angles will no longer be parallel to the axis of $z^{\prime}$, but will be inclined to it at an angle $\kappa$ whose tangent is $\frac{d \eta^{\prime}}{d z^{\prime}}$.

Now the function $\nabla$ can only depend upon the directions of the axes of $x^{\prime}, y^{\prime}$, $z^{\prime}$ with respect to fixed lines in the crystal, and upon the angle $\kappa$, which measures the change of form produced in the parallelepiped by vibration. This is the most general supposition which can be made concerning it. Since, however, by our second assumption, any one of these directions, suppose that of $x^{\prime}$, determines the other two, we may regard $\mathrm{V}$ as depending on the angle $\kappa$ and on the direction of the axis of $x^{\prime}$ alone. But from the equations (F) it is manifest that the angle $\mathrm{k}$ and the angles which the axis of $x^{\prime}$ makes with the fixed axes of $x, y, z$ are all known when the quantities $X, Y, Z$ are known. Consequently $\mathrm{V}$ is a function of $X, Y, Z$.

Supposing the angle $\kappa$ to be very small, the quantities $X, Y, Z$ will also be very small; and if $V$ be expanded according to the powers of these quantities, we shall have

$$
\begin{aligned}
\mathrm{V}=K+A X+B Y+C Z+D X^{2}+E Y^{2}+F Z^{2} \\
+G Y Z+H X Z+I X Y+\& c .,
\end{aligned}
$$

the quantities $K, A, B, C, D$, \&c., being constant. But in the state of equilibrium the value of $\delta \mathrm{V}$ ought to be nothing, in whatever way the position of the system be varied; that is to say, when the displacements $\xi, \eta, \zeta$, and consequently the quantities $X, Y, Z$, are supposed to vanish, the quantity

$$
\delta \mathrm{V}=A \delta X+B \delta Y+C \delta Z+2 D X \delta X+\& c .
$$

ought also to vanish independently of the variations $\delta \xi, \delta \eta, \delta \zeta$, or, which comes to the same thing, independently of $\delta X, \delta Y$, $\delta Z$. Hence* we must have $A=0, B=0, C=0$; and therefore, if we neglect terms of the third and higher dimensions, we may conclude that the variable part of $\mathrm{V}$ is a homogeneous function

* See the reasoning of Lagrange in an analogous case, Mécanique Analytique, tom. 1. p. 68 . 
of the second degree, containing, in its general form, the squares and products of $X, Y, Z$, with six constant coefficients.

Of these coefficients, the three which multiply the products of the variables may always be made to vanish by changing the directions of the axes of $x, y, z$. For this is a known property of functions of the second degree, when the co-ordinates are the variables; and we have shown, in Lemma II., that the quantities $X, Y, Z$ are transformed by the very same relations as the co-ordinates themselves. Therefore, in every crystal there exist three rectangular axes, with respect to which the function $\nabla$ contains only the squares of $X, Y, Z$; and as it will presently appear that the coefficients of the squares must all be negative, in order that the velocity of propagation may never become imaginary, we may consequently write, with reference to these axes,

$$
\mathrm{V}=-\frac{1}{2}\left(a^{2} X^{2}+b^{2} Y^{2}+c^{2} Z^{2}\right)
$$

omitting the constant $K$ as having no effect upon the motion.

The axes of co-ordinates, in this position, are the principal axes of the crystal, and are commonly known by the name of axes of elasticity. Thus the existence of these axes is proved without any hypothesis respecting the arrangement of the particles of the medium. The constants $a, b, c$ are the three principal velocities of propagation, as we shall see in the next section.

Having arrived at the value of $V$, we may now take it for the starting point of our theory, and dismiss the assumptions by which we were conducted to it. Supposing, therefore, in the first place, that a plane wave passes through a crystal, we shall seek the laws of its motion from equations (1) and (2), which contain everything that is necessary for the solution of the problem. The laws of propagation, as they are called, will in this way be deduced, and they will be found to agree exactly, so far as magnitudes are concerned, with those discovered by Fresnel; but the direction of the vibrations in a polarized ray will be different from that assigned by him. In the second place, we shall investigate the conditions which are fulfilled when light passes out of one medium into another, and we shall thus obtain the laws of reflexion and refraction at the surface of a crystal. 
Sect. IV.-Propagation of Light in a Crystalimeed Medium.-Laws of Fresnel.-Alteration required to be MADE IN THEM. - WAVE-SURFACE, INDEX-SURFACE, AND their Properties.

The principal axes of the crystal being the axes of $x, y, z$, we have, by equation (2),

$$
-\delta V=a^{2} X \delta X+b^{2} Y \delta Y+c^{2} Z \delta Z
$$

or, by taking the variations from formulæ (c), and interchanging the characteristies $d$ and $\delta$,

$$
-\delta \nabla=a^{2} \boldsymbol{X}\left(\frac{d \delta \eta}{d z}-\frac{d \delta \zeta}{d y}\right)+b^{2} Y\left(\frac{d \delta \zeta}{d x}-\frac{d \delta \xi}{d z}\right)+c^{2} Z\left(\frac{d \delta \xi}{d y}-\frac{d \delta \eta}{d x}\right) ;
$$

and if we substitute this value in equation (1), and then integrate by parts the right-hand member, in order to get rid of the differential coefficients of the variations, we shall obtain

$$
\begin{gathered}
\iiint d x d y d z\left(\frac{d^{2} \xi}{d t^{2}} \delta \xi+\frac{d^{2} \eta}{d t^{2}} \delta \eta+\frac{d^{2} \zeta}{d t^{2}} \delta \zeta\right) \\
=\iint d y d z\left(c^{2} Z \delta \eta-b^{2} Y \delta \xi\right)+\iint d x d z\left(a^{2} X \delta \zeta-c^{2} Z \delta \xi\right) \\
+\iint d x d y\left(b^{2} Y \delta \xi-a^{2} X \delta \eta\right) \\
+\iiint d x d y d z\left\{\left(c^{2} \frac{d Z}{d y}-b^{2} \frac{d Y}{d z}\right) d \xi+\left(a^{2} \frac{d X}{d z}-c^{2} \frac{d Z}{d x}\right) \delta \eta\right. \\
\left.+\left(b^{2} \frac{d Y}{d x}-a^{2} \frac{d X}{d y}\right) \delta \xi\right\} .
\end{gathered}
$$

But as the variations $\delta \xi, \delta \eta, \delta \zeta$ are arbitrary and independent, this equation cannot hold unless the double integrals, which relate to the limits of the system, reduce themselves to zero, leaving the equality to subsist, independently of the variations, between the triple integrals alone. Equating, therefore, the 
coefficients of the corresponding variations in the triple integrals, we get

$$
\begin{aligned}
& \frac{d^{2} \xi}{d t^{2}}=c^{2} \frac{d Z}{d y}-b^{2} \frac{d Y}{d z}, \\
& \frac{d^{2} \eta}{d t^{2}}=a^{2} \frac{d X}{d z}-c^{2} \frac{d Z}{d x}, \\
& \frac{d^{2} \zeta}{d t^{2}}=b^{2} \frac{d Y}{d x}-a^{2} \frac{d X}{d y},
\end{aligned}
$$

which are the equations of propagation, giving the expression for the accelerating force parallel to each axis of co-ordinates.

When there is a single medium extending indefinitely on all sides, the conditions relative to the limits are of no importance, and we have only to consider the equations (5), from which we shall now deduce the laws by which a system of plane waves is propagated.

Supposing the waves to be parallel to the plane of $x^{\prime} y^{\prime}$, the displacements will be functions of $z^{\prime}$ only; and if $\psi$ be any function of the displacements, we shall have, by formulæ $\left(d^{\prime}\right)$,

$$
\frac{d \psi}{d x}=\frac{d \psi}{d z^{\prime}} \frac{d z^{\prime}}{d x}=\frac{d \psi}{d z^{\prime}} \cos a^{\prime \prime}, \frac{d \psi}{d y}=\frac{d \psi}{d z^{\prime}} \cos \beta^{\prime \prime}, \frac{d \psi}{d z}=\frac{d \psi}{d z^{\prime}} \cos \gamma^{\prime \prime},
$$

so that the equations (5) may be written

$$
\begin{aligned}
& \frac{d^{2} \xi}{d t^{2}}=c^{2} \frac{d \dot{Z}}{d z^{\prime}} \cos \beta^{\prime \prime}-b^{2} \frac{d Y}{d z^{\prime}} \cos \gamma^{\prime \prime}, \\
& \frac{d^{2} \eta}{d t^{2}}=a^{2} \frac{d X}{d z^{\prime}} \cos \gamma^{\prime \prime}-c^{2} \frac{d Z}{d z^{\prime}} \cos a^{\prime \prime}, \\
& \frac{a^{2} \zeta}{d t^{2}}=b^{2} \frac{d Y}{d z^{\prime}} \cos a^{\prime \prime}-a^{2} \frac{d X}{d z^{\prime}} \cos \beta^{\prime \prime} ;
\end{aligned}
$$

and when we combine these with the following,

$$
\frac{d^{2} \xi^{\prime}}{d t^{2}}=\frac{d^{2} \xi}{d t^{2}} \cos a+\frac{d^{2} \eta}{d t^{2}} \cos \beta+\frac{d^{2} \zeta}{d t^{2}} \cos \gamma,
$$




$$
\begin{aligned}
& \frac{d^{2} \eta^{\prime}}{d t^{2}}=\frac{d^{2} \xi}{d t^{2}} \cos a^{\prime}+\frac{d^{2} \eta}{d t^{2}} \cos \beta^{\prime}+\frac{d^{2} \zeta}{d t^{2}} \cos \gamma^{\prime}, \\
& \frac{d^{2} \eta^{\prime}}{d t^{2}}=\frac{d^{2} \xi}{d t^{2}} \cos \alpha^{\prime \prime}+\frac{d^{2} \eta}{d t^{2}} \cos \beta^{\prime \prime}+\frac{d^{2} \zeta}{d t^{2}} \cos \gamma^{\prime \prime},
\end{aligned}
$$

attending to the relations $(\mathrm{B}),\left(\mathrm{B}^{\prime}\right)$, we find

$$
\begin{aligned}
& \frac{d^{2} \xi^{\prime}}{d t^{2}}=-\left(a^{2} \frac{d X}{d z^{\prime}} \cos a^{\prime}+b^{2} \frac{d Y}{d z^{\prime}} \cos \beta^{\prime}+c^{2} \frac{d Z}{d z^{\prime}} \cos \gamma^{\prime}\right), \\
& \frac{d^{2} \eta^{\prime}}{d t^{2}}=a^{2} \frac{d X}{d z^{\prime}} \cos a+b^{2} \frac{d Y}{d z^{\prime}} \cos \beta+c^{2} \frac{d Z}{d z^{\prime}} \cos \gamma, \\
& \frac{d^{2} \zeta^{\prime}}{d t^{2}}=0
\end{aligned}
$$

from which it appears that there is no accelerating force in the direction of a normal* to the wave, and consequently no vibration in that direction. Introducing now the values of $X, Y, Z$ from formulæ ( $\mathrm{E})$, the first two of these equations become

$$
\begin{aligned}
\frac{d^{2} \xi^{\prime}}{d t^{2}}= & \left(a^{2} \cos ^{2} a^{\prime}+b^{2} \cos ^{2} \beta^{\prime}+c^{2} \cos ^{2} \gamma^{\prime}\right) \frac{d^{2} \xi^{\prime}}{d z^{\prime 2}} \\
& -\left(a^{2} \cos a \cos a^{\prime}+b^{2} \cos \beta \cos \beta^{\prime}+c^{2} \cos \gamma \cos \gamma^{\prime}\right) \frac{d^{2} \eta^{\prime}}{d z^{\prime 2}}, \\
\frac{d^{2} \eta^{\prime}}{d t^{2}}= & \left(a^{2} \cos ^{2} a+b^{2} \cos ^{2} \beta+c^{2} \cos ^{2} \gamma\right) \frac{d^{2} \eta^{\prime}}{d z^{\prime 2}} \\
& -\left(a^{2} \cos a \cos \alpha^{\prime}+b^{2} \cos \beta \cos \beta^{\prime}+c^{2} \cos \gamma \cos \gamma^{\prime}\right) \frac{d^{2} \xi^{\prime}}{d z^{\prime 2}} .
\end{aligned}
$$

But as the axes of $x^{\prime}, y^{\prime}$ are arbitrarily taken in the plane of $x^{\prime} y^{\prime}$ we may subject their directions to the condition

$$
a^{2} \cos a \cos a^{\prime}+b^{2} \cos \beta \cos \beta^{\prime}+c^{2} \cos \gamma \cos \gamma^{\prime}=0 ;
$$

* In the ingenious, but altogether unsatisfactory theory, by which Fresnel has endeavoured to account for his beautiful laws, the direction of the elastic force brought ir ruplay by the displacement of the ethereal molecules is, in general, inclined to jective of the wave. He supposes, however, that the force normal to that $\mathrm{pl}$. - n w produce any appreciable effect, by reason of the great resistance whic plane Wffers to compression.-Mémoires de l'Institut, tom. vii. p. 78. 
and then, if we put

$$
\begin{aligned}
& s^{2}=a^{2} \cos ^{2} \alpha+b^{2} \cos ^{2} \beta+c^{2} \cos ^{2} \gamma, \\
& s^{\prime 2}=a^{2} \cos ^{2} a^{\prime}+b^{2} \cos ^{2} \beta^{\prime \prime}+c^{2} \cos ^{2} \gamma^{\prime},
\end{aligned}
$$

the equations (6) will be reduced to the well-known form

$$
\frac{d^{2} \xi^{\prime}}{d t^{2}}=s^{2} \frac{d^{2} \xi^{\prime}}{d z^{\prime 2}}, \quad \frac{d^{2} \eta^{\prime}}{d t^{2}}=s^{2} \frac{d^{2} \eta^{\prime}}{d z^{2}} .
$$

This result shows that, when the directions of $x^{\prime}$ and $y^{\prime}$ fulfil the condition (7), the vibrations ' $\xi^{\prime}$ and $\eta^{\prime}$ are propagated independently of each other, the former with the velocity of $s^{\prime}$, the latter with the velocity $s$. The vibrations must therefore be parallel exclusively to one or other of these directions, else the system of waves will split into two systems, one vibrating parallel to $x^{\prime}$, the other parallel to $y$.

When the plane of the wave is parallel to one of the principal axes, it is easy to infer that the vibrations must be either parallel or perpendicular to that axis; and that, in the latter case, the velocity of propagation is constant, being equal to $a, b$, or $c$, according as the wave is parallel to the axis of $x, y$, or z. These constants are therefore called the principal velocities of propagation; and we now perceive the reason of the negative sign in equation (2); for if any of the terms in the right-hand member of that equation were positive, the corresponding velocity would be imaginary.

According to Fresnel, the wave which is propagated with the velocity $a$ has its vibrations not perpendicular to the axis of $x$, but parallel to it; and it is to be observed that a difference of the same character distinguishes his views, throughout, from the results of the present theory. It will appear in fact, by what immediately follows, that the equations (7), (8), (9), express exactly the laws of Fresnel, provided the quantities $\xi^{\prime}$ and $\eta^{\prime}$, in the equations (9), be interchanged. To make these laws agree with our theory, it is therefore necessary tc . them in one particular, and in one only ; it is necessary ti, se that 
the direction of the ribrations is always perpendicular to that assigned by Fresnel. And Fresnel was obliged to say that, in agree with the phenomena, an ordinary medium, the vibrations of a ray plarized in a certain plane are perpendicular to that plane, it is clear that, on the present principles, we must come to a different co say that the vibrations of a polarized ray are parallel to its plane of polarization.

Conceive an ellips $y, z, x^{\prime}, y^{\prime}, z^{\prime}$; and let its semiaxes be parallel to $x, y, z$, their 11 respectively. From the idento $\frac{1}{a}, \frac{1}{b}, \frac{1}{c}$ respectively. tity of the condition (III., it is evimarked $\left(G^{\prime}\right)$ in Lems of $x^{\prime}$ and $y^{\prime}$, when dent that the directions of vibration, they are the two directions the ellipse in coincide with the axes of the el which the plane of $x y^{\prime}$ interse $O R$, meet-

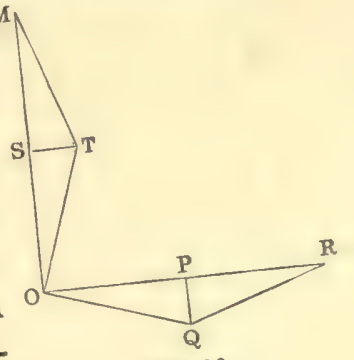

Fig. 20. lipsoid; and if the right line direction of $x^{\prime}$, we have ing the ellipsoid in $R$, $1=a^{2} \cos ^{2} \alpha+b^{2} \cos ^{2} \beta+c^{2} \cos ^{2} \gamma$,

$$
\text { or, by }(8), \quad \frac{1}{O R}=s \text {, }
$$

so that $O R$ is the reciprocal of the velocity with which the vibrations parallel to $y^{\prime}$ are propagated. vibrations parallel to either semiaxis measured by the reciprocal propagated with a velocity whis. Again, conceive an ellipsoid with its before, but equal to $a, b, c$ resemiaxes parallel to $x, y, z$, as be touched in the point $Q$ by a spectively. Let this ellipsoidicularly in $P$, and draw the right plane which cuts $O R$ perpendicon $(7)$ is identical with that lines $O P, P Q$. Then as $\quad$ M 
marked (I) in the corollary to Lemma IV., it follows that $O y^{\prime}$ (if we so call the direction of $y^{\prime}$ ) is perpendicular to $O Q$, and also that $O y^{\prime}$ and $O Q$ coincide with the axes of the elliptic section made in this ellipsoid by the plane $Q O y^{\prime}$, just as $O y^{\prime}$ and $O R$ coincide with the axes of the section $R O y^{\prime}$ in the first ellipsoid. The plane $Q O R$ is therefore perpendicular to $O y^{\prime}$ and to the plane of the wave. Moreover, we have

$$
(O P)^{2}=a^{2} \cos ^{2} \alpha+b^{2} \cos ^{2} \beta+c^{2} \cos ^{2} \gamma=s^{2},
$$

so that $O P$ is the reciprocal of $O R$, and is equal to the velocity $s$ with which the wave is propagated when its vibrations are parallel to $O y^{\prime}$.

Now let the figure $T O S M$ be equal in all respects to $Q O P R$, but in a position perpendicular to it, so that if $Q O P R$ were turned round in its own plane through a right angle, the point $O$ being fixed, the points $Q, P, R$ would fall upon $T, S, M$ respectively; and supposing the wave-plane $R O y^{\prime}$ to take various positions passing through $O$, imagine a construction similar to the preceding one to be always made by means of the two ellipsoids. Then while the points $R$ and $Q$ describe the ellipsoids, the points $M$ and $T$ describe two biaxal* surfaces reciprocal to each other, the latter surface being touched $\dagger$ in the point $T$ by a plane which cuts $O M$ perpendicularly in $S$. But this plane is parallel to the central wave-plane $R O y^{\prime}$, and distant from it by an interval $O S(=O P)$ which represents the velocity of the wave; and as the surface whose tangent planes possess this property is, by definition, the vave-surface of the crystal, it is obvious that the point $T$ describes the wave-surface. The radius $O T$, drawn to the point of contact, is then, by the theory of waves, the direction of the ray which belongs to the wave $R O y^{\prime}$, and the length $O T$ represents the velocity of light along the ray. As to the surface described by the point $M$, it is that

* See Transactions of the Royal Irish Academy, Vou. xvir. p. 244 (supra, p. 24).

† Ibid. Vou. xvI. p. 6 (supra, p. 4). 
which I have called the surface of indices, or the index-surface, because its radius $O M$, being the reciprocal of $O S$, represents the index of refraction, or the ratio of the sine of the angle of incidence to the sine of the angle of refraction, when the wave $R O y^{\prime}$, to which $O M$ is perpendicular, is supposed to have passed into the crystal out of an ordinary medium in which the velocity of propagation is unity. The angles of incidence and refraction are understood to be the angles which the incident and refracted waves respectively make with the refracting surface of the crystal.

The wave-surface and the index-surface have the same geometrical properties since they are both biaxal surfaces. Let us consider the former, which is generated by the ellipsoid whose semiaxes are $a, b, c$; and let us conceive this ellipsoid to be intersected by a concentric sphere of which the radius is $r$. Then the equations of the ellipsoid and the sphere being respectively

$$
\frac{x^{2}}{a^{2}}+\frac{y^{2}}{b^{2}}+\frac{z^{2}}{c^{2}}=1, \quad \frac{x^{2}+y^{2}+z^{2}}{r^{2}}=1,
$$

we get, by subtracting the one from the other,

$$
x^{2}\left(\frac{1}{a^{2}}-\frac{1}{r^{2}}\right)+y^{2}\left(\frac{1}{b^{2}}-\frac{1}{r^{2}}\right)+z^{2}\left(\frac{1}{c^{2}}-\frac{1}{r^{2}}\right)=0,
$$

for the equation of the cone $A$ which has its vertex at $O$, and passes through the curve of intersection. If $O Q$ be equal to $r$, it will be a side of this cone; and a plane touching the cone along $O Q$ will make in the ellipsoid a section of which $O Q$ will be a semiaxis; so that $O T$ will be perpendicular to that plane, and equal in length to $r$. Therefore, as $O Q$ describes the con $\theta$ $A$, the right line $O T$ describes another cone $B$ reciprocal to $A$, and the point $T$ describes the curve in which the wave-surface is intersected by the sphere above mentioned; this curve being a spherical ellipse, reciprocal to that which the point $Q$ describes on

- See Transactions of the Royal Irish Academy, Vox. xvirr. p. 38 (supra, p. 96). I had previously called it the surface of refraction, Vox. xviI. p. 252 (supra, p. 36). 
the surface of the ellipsoid. The equation of the cone $B$ is found from that of $A$, by changing the coefficients of the squares of the variables into their reciprocals, and is therefore

$$
\frac{a^{2} x^{2}}{r^{2}-a^{2}}+\frac{b^{2} y^{2}}{r^{2}-b^{2}}+\frac{c^{2} z^{2}}{r^{2}-c^{2}}=0 \text {, }
$$

which, of course, is also the equation of the wave-surface, if $r$ be supposed to be the radius drawn from $O$ to the point whose co-ordinates are $x, y, z$. Combining this equation with that of the sphere, we have

$$
\frac{x^{2}}{r^{2}-a^{2}}+\frac{y^{2}}{r^{2}-b^{2}}+\frac{z^{2}}{r^{2}-c^{2}}=1,
$$

which represents a hyperboloid passing through the common intersection of the sphere, the cone $B$, and the wave-surface.

Since the differences between the coefficients of the squares of the variables in the equation (10) are the same as the corresponding differences in the equation of the ellipsoid, the cone $A$ has its planes of circular section coincident with those of the ellipsoid. The cone $B$, being reciprocal to $A$, has therefore its focal lines perpendicular to the circular sections of the ellipsoid. These focal lines are consequently the nodal diameters* of the wave-surface, that is, the diameters which pass through the points where the two sheets of that surface intersect each other.

If the direction of $O T$ eut the other sheet of the wavesurface in $T^{\prime}$, and if two radii of constant lengths, equal to $O T$ and $O T^{\prime}$ respectively, revolve within the surface, the cones $B$ and $B^{\prime}$ described by these radii will intersect each other at right angles, since they have the same focal lines. And supposing the axis of $y$ to be the mean axis of the ellipsoid, so that the nodal diameters lie in the plane of $x z$, the axis of $x$ will lie within one of the cones, as $B$, and the axis of $z$ within the other cone $B^{\prime}$. Now the angle contained by the two sides of either

* See Transactions of the Royal Irish Academy, Vol. xvir. p. 247 (supra, p. 29). 
cone, which lie in the plane of $x$, is given by the angles $\theta$ and $\theta^{\prime}$ which the direction of the right line $O T T^{\prime}$ makes with the nodal diameters; because the angles which any side of a cone makes with its focal lines have a constant sum, or a constant difference, according to the way in which they are reckoned. But if the angles $\theta$ and $\theta^{\prime}$ be reckoned (as they may be) so that their sum shall be equal to the angle contained by the two sides of the cone $B$ which are in the plane of $x z$, their difference will be equal to the angle contained by the two sides of the cone $B^{\prime}$ which are in the same plane; the contained angle, in each case, being that which is bisected by the axis of $x$. Therefore, the lengths $O T$ and $O T^{\prime}$, which we denote by $r$ and $r^{\prime}$, are equal to two radii of the ellipse whose equation is

$$
\frac{x^{2}}{a^{2}}+\frac{z^{2}}{c^{2}}=1,
$$

these radii making with the axis of $z$ the angles $\frac{1}{2}\left(\theta+\theta^{\prime}\right)$ and $\frac{1}{2}\left(\theta-\theta^{\prime}\right)$ respectively. Hence

$$
\begin{aligned}
\frac{1}{r^{2}}=\frac{\sin ^{2} \frac{1}{2}\left(\theta+\theta^{\prime}\right)}{a^{2}}+\frac{\cos ^{2} \frac{1}{2}\left(\theta+\theta^{\prime}\right)}{c^{2}} & =\frac{1}{2}\left(\frac{1}{a^{2}}+\frac{1}{c^{2}}\right) \\
& -\frac{1}{2}\left(\frac{1}{a^{2}}-\frac{1}{c^{2}}\right) \cos \left(\theta+\theta^{\prime}\right), \\
\frac{1}{r^{\prime 2}}=\frac{\sin ^{2} \frac{1}{2}\left(\theta-\theta^{\prime}\right)}{a^{2}}+\frac{\cos ^{2} \frac{1}{2}\left(\theta-\theta^{\prime}\right)}{c^{2}} & =\frac{1}{2}\left(\frac{1}{a^{2}}+\frac{1}{c^{2}}\right) . \\
& -\frac{1}{2}\left(\frac{1}{a^{2}}-\frac{1}{c^{2}}\right) \cos \left(\theta-\theta^{\prime}\right) .
\end{aligned}
$$

These formulæ give the two velocities of propagation along a ray which makes the angles $\theta, \theta^{\prime}$ with the nodal diameters. Subtracting them, we have

$$
\frac{1}{r^{2}}-\frac{1}{r^{\prime 2}}=\left(\frac{1}{a^{2}}-\frac{1}{c^{2}}\right) \sin \theta \sin \theta .
$$

All the preceding equations, relative to the wave-surface, 
may be transferred to the index-surface, by changing the quantities $a, b, c$ into their reciprocals. For example, if the normal to a wave make the angles $\boldsymbol{\theta}_{0}, \boldsymbol{\theta}_{1}$ with the nodal diameters of the index-surface, the formulæ (13) give

$$
\begin{aligned}
& s^{2}=\frac{1}{2}\left(a^{2}+c^{2}\right)-\frac{1}{2}\left(a^{2}-c^{2}\right) \cos \left(\theta_{0}+\theta_{1}\right), \\
& s^{2}=\frac{1}{2}\left(a^{2}+c^{2}\right)-\frac{1}{2}\left(a^{2}-c^{2}\right) \cos \left(\theta_{0}-\theta_{1}\right) ;
\end{aligned}
$$

observing that $s$ and $s^{\prime}$, the two normal velocities of propagation, are the reciprocals of the radii of this surface which coincide with the wave-normal. Subtracting these expressions, we get

$$
s^{2}-s^{\prime 2}=\left(a^{2}-c^{2}\right) \sin \theta_{0} \sin \theta_{1} .
$$

As the position of the tangent plane, at any point $T$ of a biaxal surface, depends on the position of the axes of the section QOy made in the generating ellipsoid by a plane perpendioular to $O T$, it is obvious that when this section is a circle, that is, when the point $T$ is a node of the surface, the position of the tangent plane is indeterminate, like that of the axes of the section; and it is easy to show that the cone which that plane touches in all its positions is of the second order. Again, when the section $R O y^{\prime}$ of the reciprocal ellipsoid is a circle, the right line $O S$ is given both in position and length; and the tangent plane, which cuts $O S$ in $S$, is fixed; but the point of contact $T$ is not fixed, since the semiaxis $O R$, to which the right line $S T$ is parallel, may be any radius of the circle $R O y^{\prime}$. In this case, the point $T$ describes a curve in the tangent plane, and this curve is found to be a circle. But both these cases have been fully discussed elsewhere.*

* See Transactions of the Royal Irish Academy, Vor. xvir.pp. 245, 260 (supra, pp. 25-7, 49-51). 
Sect. V.-Conditions to be satisfied when Light passes out of one Medium into another.-Remarkable CircumSTANCES CONNECTED With THEM. -Relations aMoNG THE Transversals of the Incident, Reflected, and ReFRACTED RAYS.

Now let light pass out of one medium into another-suppose out of an ordinary into a doubly-refracting medium; and taking the origin of rectangular co-ordinates $x_{\theta}, y_{\mathrm{n}}, z_{0}$ at a point $O$ on the surface which separates the two media, let this surface be the plane of $x_{0} y_{0}$. Then if the components of the displacement of a particle whose initial co-ordinates are $x_{0}, y_{0}, z_{0}$ be denoted by $\xi_{0}^{\prime}, \eta_{0}^{\prime}, \zeta_{0}^{\prime}$ when the particle is in the first medium, and by $\xi_{0}^{\prime \prime}, \eta_{0}^{\prime \prime}, \zeta_{0}^{\prime \prime}$ when it is in the second, the equation (1), adapted to the present case, will be

$$
\begin{gathered}
\iiint d x_{0} d y_{0} d z_{0}\left(\frac{d^{2} \xi_{0}^{\prime}}{d t^{2}} \delta \xi_{0}^{\prime}+\frac{d^{2} \eta_{0}^{\prime}}{d t^{2}} \delta \eta_{0}^{\prime}+\frac{d^{2} \zeta_{0}}{d t^{2}} \delta \xi_{0}^{\prime}\right) \\
+\iiint d x_{0} d y_{0} d z_{0}\left(\frac{d^{2} \xi_{0}^{\prime \prime}}{d t^{2}} \delta \xi^{\prime \prime}{ }_{0}+\frac{d^{2} \eta_{0}^{\prime \prime}}{d t^{2}} \delta \eta_{0}^{\prime \prime}+\frac{d^{2} \xi_{0}^{\prime \prime}}{d t^{2}} \delta \xi_{0}^{\prime \prime}\right) \\
=\iiint d x_{0} d y_{0} d z_{0} \delta \mathrm{V}^{\prime}+\iiint d x_{0} d y_{0} d z_{0} \delta \mathrm{V}^{\prime \prime} ;
\end{gathered}
$$

wherein $\delta \mathrm{V}^{\prime}$ and $\delta \mathrm{V}^{\prime \prime}$ are the respective values of $\delta \mathrm{V}$ for the two media, which are conceived to extend indefinitely on each side of the plane of $x_{0} y_{0}$; that plane being an upper limit of the integrations relative to one medium, and a lower limit of the integrations relative to the other. Each medium is conceived to be occupied by systems of plane waves-the first by incident and reflected waves, the second by refracted waves; and, except where they are bounded by the plane of $x_{0} y_{0}$, these waves are regarded as unlimited in extent.

For the ordinary medium, if we put

$$
X_{0}^{\prime}=\frac{\prime d \eta_{0}^{\prime}}{d z_{0}}-\frac{d \xi_{0}^{\prime}}{d y_{0}}, \quad Y_{0}^{\prime}=\frac{d \xi_{0}^{\prime}}{d x_{0}}-\frac{d \xi_{0}^{\prime}}{d z_{0}}, \quad Z_{0}=\frac{d \xi_{0}^{\prime}}{d y_{0}}-\frac{d \eta_{0}^{\prime}}{d x_{0}} ;
$$


and suppose the velocity of propagation to be unity, we have*

$$
-\delta V^{\prime}=\bar{X}_{0}^{\prime}\left(\frac{d \delta \eta_{0}^{\prime}}{d z_{0}}-\frac{d \delta \zeta_{0}^{\prime}}{d y_{0}}\right)+Y_{0}^{\prime}\left(\frac{d \delta \zeta_{0}^{\prime}}{d x_{0}}-\frac{d \delta \xi_{0}^{\prime}}{d z_{0}}\right)+Z_{0}^{\prime}\left(\frac{d \delta \xi_{0}^{\prime}}{d y_{0}}-\frac{d \delta \eta_{0}^{\prime}}{d x_{0}}\right)
$$

For the crystallized medium, if its principal axes be those of $x, y, z$, the value of $\delta V^{\prime \prime}$ will be the same as that of $\delta V$ in formula (3) ; but instead of the variations of $\xi, \eta$, $\zeta$, we must use those of $\xi^{\prime \prime}, \eta^{\prime \prime}, \zeta^{\prime \prime}{ }_{0}$. Denoting the cosines of the angles which the principal axes respectively make with the axis of $x_{0}$ by $l, m, n$; with the axis of $y_{0}$ by $l^{\prime}, m^{\prime}, n^{\prime}$; with the axis of $z_{0}$ by $l^{\prime \prime}, m^{\prime \prime}, n^{\prime \prime}$; and putting

$$
\begin{gathered}
X_{0}^{\prime \prime}=\frac{d \eta_{0}^{\prime \prime}}{d z_{0}}-\frac{d \zeta_{0}^{\prime \prime}}{d y_{0}}, \quad Y_{0}^{\prime \prime}=\frac{d \zeta_{0}^{\prime \prime}}{d x_{0}}-\frac{d \xi_{0}^{\prime \prime}}{d z_{0}}, \quad Z_{0}^{\prime \prime}=\frac{d \xi_{0}^{\prime \prime}}{d y}-\frac{d \eta_{0}^{\prime \prime}}{d x_{0}}, \\
\delta X^{\prime \prime}=\frac{d \delta \eta_{0}^{\prime \prime}}{d z_{0}}-\frac{d \delta \xi_{0}^{\prime \prime}}{d y_{0}}, \quad \delta Y_{0}^{\prime \prime}=\frac{d \delta \zeta_{0}^{\prime \prime}}{d x_{0}}-\frac{d \delta \xi_{0}^{\prime \prime}}{d z_{0}}, \quad \delta Z_{0}^{\prime \prime}=\frac{d \delta \xi^{\prime \prime}}{d y_{0}}-\frac{d \delta \eta_{0}^{\prime \prime}}{d x_{0}} ;
\end{gathered}
$$

we have

$$
\begin{aligned}
& \delta X=l \delta X_{0}^{\prime \prime}+l \delta Y_{0}^{\prime \prime}+l^{\prime \prime} \delta Z^{\prime \prime}{ }_{0} \\
& \delta Y=m \delta X_{0}^{\prime \prime}+m^{\prime} \delta Y_{0}^{\prime \prime}+m^{\prime \prime} \delta Z_{0}^{\prime \prime} \\
& \delta Z=n \delta X_{0}^{\prime \prime}+n^{\prime} \delta Y^{\prime \prime}{ }_{0}+n^{\prime \prime} \delta Z^{\prime \prime}{ }_{0} .
\end{aligned}
$$

These expressions for $\delta X, \delta Y, \delta Z$ having been written in formula (3), the resulting value of $\delta V^{\prime \prime}$, as well as the above value of $\delta V^{\prime}$, is to be substituted in the equation (17), and then the right-hand member of that equation is to be integrated by parts, in order to get rid of the differential coefficients of the variations. When this operation is performed, the triple integrals on one side of the equation will be equal to those on the other; and by equating the coefficients of the corresponding variations

* It is assumed here, and in what follows, that when there are two or more coexisting waves in a given medium, the form of the function $V$ is the same as for a single wave, provided the displacements which enter into the function be the resultants of the displacements due to each wave separately. This, however, ought evidently to be the case, in order that the principle of the superposition of vibrations may hold good. 
in each medium, we should get the laws of propagation in each. But we are not now considering these laws, and we need only attend to the double integrals produced by the operation aforesaid. The double integrals are together equal to zero; but we are concerned only with that part of them which relates to the common limit of the media, the plane of $x_{0} y_{0}$; and this part must be separately equal to zero, since the conditions to be fulfilled at the plane of $x_{0} y_{0}$ are independent of anything that might take place at other limiting surfaces, if such were supposed to exist. Collecting therefore the terms produced by integrating with respect to $z_{0}$, and observing that a negative sign must be interposed between those which belong to different media, we get

$\iint d x_{0} d y_{0}\left(\boldsymbol{Y}_{0}^{\prime} \delta \xi_{0}^{\prime}-X_{0}^{\prime} \delta \eta_{0}^{\prime}\right)-\iint d x_{0} d y_{0}\left(Q \delta \xi^{\prime \prime}{ }_{0}-P \delta \eta^{\prime \prime}{ }_{0}\right)=0$,

where

$$
\boldsymbol{P}=a^{2} l \boldsymbol{X}+b^{2} m \boldsymbol{Y}+c^{2} n \boldsymbol{Z}, \quad \boldsymbol{Q}=a^{2} l^{\prime} \boldsymbol{X}+b^{2} m^{\prime} \boldsymbol{Y}+c^{2} n^{\prime} \boldsymbol{Z} .
$$

In each of these equations it is understood that $z_{0}=0$. But when $z_{0}=0$, we have obviously

and therefore

$$
\xi_{0}^{\prime}=\xi^{\prime \prime}, \quad \eta_{0}=\eta_{0}^{\prime \prime},
$$

$$
\delta \xi_{0}^{\prime}=\delta \xi^{\prime \prime}{ }_{0}, \quad \delta \eta_{0}^{\prime}=\delta \eta_{0}^{\prime \prime} ;
$$

so that the equation (18) becomes

$$
\iint d x_{0} d y_{0}\left\{\left(\boldsymbol{Y}_{0}^{\prime}-Q\right) \delta \xi_{0}^{\prime}-\left(\boldsymbol{X}_{0}^{\prime}-P\right) \delta \eta_{0}^{\prime}\right\}=0,
$$

which, as the variations $d \xi^{\prime}$ and $d \eta^{\prime}$ are arbitrary and independent, is equivalent to the two equations

$$
X_{0}^{\prime}=P, \quad Y_{0}^{\prime}=Q .
$$

Thus, to find the relations which subsist among the vibrations incident, reflected, and refracted, at the common surface of two media, we have four conditions, expressed by the equations (20) and (21); and these conditions are sufficient to determine the reflected and refracted vibrations, when the incident 
vibration is given. But though, by the nature of the question, four conditions only are required for its solution, there remains another condition which ought to be satisfied; for we ought evidently to have

$$
\zeta_{0}^{\prime}=\zeta^{\prime \prime} \text {, when } z_{0}=0 \text {. }
$$

This condition is apparently independent of the rest; but it cannot really be so, if the preceding theory is consistent with itself. We shall accordingly see, in what follows, that the last condition is included in the other four; which is a remarkable circumstance, and a singular confirmation of the theory.*

As the incident and reflected waves coexist in the first medium, and two sets of refracted waves in the second, the resolved displacements, and all the quantities which depend upon them, are composed of two parts, due to the coexisting waves. Let the point $O$ be, for each set of waves, the origin of a system of rectangular co-ordinates, which we shall call $x_{1}, y_{1}, z_{1}$ for the incident, and $x_{1}^{\prime}, y_{1}^{\prime}, z_{1}^{\prime}$ for the reflected wave, the axes of $z_{1}$ and $z_{1}^{\prime}$ being perpendicular to the respective waves, and their positive directions being those of propagation. Let the displacements in these wares be parallel to $y_{1}, y_{1}^{\prime}$, and be denoted by $\eta_{1}, \eta_{1}^{\prime}$, respectively. Then if the axes of $x_{0}, y_{0}, z_{0}$ make with the axis of $x_{1}$ the angles $a_{1}, \beta_{1}, \gamma_{1}$, and with the axis of $x_{1}^{\prime}$ the angles $a_{1}^{\prime}, \beta_{1}^{\prime}, \gamma_{1}^{\prime}$, we have, by the formulø $(F)$,

$X_{0}^{\prime}=\frac{d \eta_{1}}{d z_{1}} \cos \alpha_{1}+\frac{d \eta_{1}^{\prime}}{d z_{1}^{\prime}} \cos \alpha_{1}^{\prime}, \quad Y_{0}^{\prime}=\frac{d \eta_{1}}{d z_{1}} \cos \beta_{1}+\frac{d \eta_{1}^{\prime}}{d z_{1}^{\prime}} \cos \beta_{1}^{\prime}$.

Again, let the co-ordinates $x_{2}, y_{2}, z_{2}$ have reference to one set of refracted waves, and $x_{2}^{\prime}, y_{2}^{\prime}, z_{2}^{\prime}$ to the other, the axes of $z_{2}$ and $\Sigma_{2}^{\prime}$ being perpendicular to the respective waves, and their positive directions being those in which the waves are propa-

* In considering the question of reflexion at the common surface of two ordinary media (Mémoires de l'Institut, tom. ix. p. 396), Fresnel assumes the conditions (20) ; but his other suppositions violate the condition (22). In fact, this last condition is inconsistent with the supposition that, in a polarized ray, the direction of the vibrations is perpendicular to the plane of polarization. See the Transactions of the Royal Irish Academy, Vou. xviri. p. 32 (supra, p. 88). 
gated. Suppose the displacements to be parallel to $y_{2}, y^{\prime}$, and to be denoted by $\eta_{2}, \eta_{2}^{\prime}$ respectively. Then if the axes of $x, y, z$ make with the axis of $x_{2}$ the angles $\boldsymbol{a}_{(2)}, \boldsymbol{\beta}_{(2)}, \gamma_{(2)}$, and with the axis of $x_{2}^{\prime}$ the angles $\boldsymbol{a}_{(2)}^{\prime}, \beta_{(2)}^{\prime}, \gamma_{(2)}$, we have, by the formulæ $(F)$,

$$
\begin{aligned}
& X=\frac{d \eta_{2}}{d z_{2}} \cos a_{(2)}+\frac{d \eta_{2}^{\prime}}{d z_{2}^{\prime}} \cos a_{(2)}^{\prime}, \\
& Y=\frac{d \eta_{2}}{d z_{2}} \cos \beta_{(2)}+\frac{d \eta_{2}^{\prime}}{d z_{2}^{\prime}} \cos \beta_{(2)}^{\prime}, \\
& Z=\frac{d \eta_{2}}{d \Sigma_{2}} \cos \gamma(2)+\frac{d \eta_{2}^{\prime}}{d z_{2}^{\prime}} \cos \gamma_{(2)}^{\prime} ;
\end{aligned}
$$

and thence, by the relations (19),

$$
\begin{aligned}
P & =\frac{d \eta_{1}}{d z_{2}}\left(a^{2} l \cos \boldsymbol{a}_{(2)}+b^{2} m \cos \beta_{(2)}+c^{2} n \cos \gamma_{(2)}\right) \\
& +\frac{d \eta_{2}^{\prime}}{d z_{2}^{\prime}}\left(a l^{2} \cos \boldsymbol{a}_{(2)}^{\prime}+b^{2} m \cos \beta_{(2)}+c^{2} n \cos \gamma_{(2)}^{\prime}\right), \\
Q & =\frac{d \eta_{2}}{d z_{2}}\left(a^{2} l^{\prime} \cos \boldsymbol{a}_{(2)}+b^{2} m^{\prime} \cos \beta_{(2)}+c^{2} n^{\prime} \cos \gamma_{(2)}\right) \\
& +\frac{d \eta_{2}^{\prime}}{d z_{2}^{\prime}} l\left(a^{2} l^{\prime} \cos \boldsymbol{a}_{(2)}^{\prime}+b^{2} m^{\prime} \cos \beta_{(2)}^{\prime}+c^{2} n^{\prime} \cos \gamma_{(2)}^{\prime}\right) .
\end{aligned}
$$

Suppose the ellipsoid which generates the wave-surface of the second medium to have its centre at $O$, and to be touched in the points $Q$ and $Q^{\prime}$ by two planes which cut the axes of $x_{2}$ and $x_{2}^{\prime}$ perpendicularly in the points $P$ and $P^{\prime}$; the lengths $O P$ and $O P^{\prime}$ being expressed by $s, s^{\prime}$, and the lengths $O Q$ and $O Q^{\prime}$ by $r, r^{\prime}$. Let the axes of $x_{0}, y_{0}, z_{0}$ make with the direction of $O Q$ the angles $\alpha_{2}, \beta_{2}, \gamma_{2}$, and with the direction of $O Q$ the angles $a_{2}^{\prime}, \beta_{2}^{\prime}, \gamma_{2}^{\prime}$. Then, from the equation (н) in ${ }^{\prime} L e m m a$ IV., it is manifest that

$$
\begin{aligned}
& a^{2} l \cos \boldsymbol{a}_{(2)}+b^{2} m \cos \beta_{(2)}+c^{2} n \cos \gamma_{(2)}=r s \cos \boldsymbol{\alpha}_{2,} \\
& a^{2} l \cos \boldsymbol{a}_{(2)}^{\prime}+b^{2} m \cos \beta_{(2)}^{\prime}+c^{2} n \cos \gamma_{(2)}^{\prime}=r^{\prime} s^{\prime} \cos \boldsymbol{a}_{2,}^{\prime}, \\
& a^{2} l^{\prime} \cos \boldsymbol{\alpha}_{(2)}+b^{2} m^{\prime} \cos \beta_{(2)}+c^{2} n^{\prime} \cos \gamma_{(2)}=r s \cos \beta_{2}, \\
& a^{2} l^{\prime} \cos \boldsymbol{a}_{(2)}^{\prime}+b^{2} m^{\prime} \cos \beta_{(2)}^{\prime}+c^{2} n^{\prime} \cos \gamma_{(2)}^{\prime}=r^{\prime} s^{\prime} \cos \beta_{2}^{\prime} .
\end{aligned}
$$


Hence,

$$
\begin{aligned}
& P=\frac{d \eta_{2}}{d z_{2}} r s \cos a_{2}+\frac{d \eta_{2}^{\prime}}{d z_{2}^{\prime}} r^{\prime} s^{\prime} \cos \alpha_{2}^{\prime}, \\
& Q=\frac{d \eta_{2}}{d z_{2}} r s \cos \beta_{2}+\frac{d \eta_{2}^{\prime}}{d z_{2}^{\prime}} r^{\prime} s^{\prime} \cos \beta_{2}^{\prime} .
\end{aligned}
$$

The quantities $s, s^{\prime}$ are, as appears by the last section, the normal velocities with which the two sets of refracted waves are propagated. The velocity with which the incident and reflected waves are propagated is taken as unity. Therefore, if $\tau_{1}, \tau_{1}^{\prime}$ be the transversals, or amplitudes of vibration in the incident and reflected waves, and $\tau_{2}, \tau^{\prime}{ }_{2}$ the transversals of the refracted waves, the lengths of the latter waves being denoted by $\lambda_{2}, \lambda_{2}^{\prime}$, and the length of an incident or reflected wave by $\lambda_{1}$, and if we put

$$
\begin{array}{ll}
\phi_{1}=\frac{2 \pi}{\lambda_{1}}\left(t-z_{1}+v_{1}\right), & \phi_{1}^{\prime}=\frac{2 \pi}{\lambda_{1}}\left(t-z_{1}^{\prime}+\nu_{1}^{\prime}\right), \\
\phi_{2}=\frac{2 \pi}{\lambda_{2}}\left(s t-z_{2}+\nu_{2}\right), & \phi_{2}^{\prime}=\frac{2 \pi}{\lambda_{2}^{\prime}}\left(s^{\prime} t-z_{2}^{\prime}+v_{2}^{\prime}\right),
\end{array}
$$

where $v_{1}, v_{1}^{\prime}, v_{2}, v_{2}^{\prime}$ are constants, and $\pi$ is the ratio of the circumference to the diameter of a circle, we may write $\eta_{1}=\tau_{1} \cos \phi_{1}, \quad \eta_{1}^{\prime}=\tau_{1}^{\prime} \cos \phi_{1}^{\prime}, \quad \eta_{2}=\tau_{2} \cos \phi_{2}, \quad \eta_{2}^{\prime}=\tau_{2}^{\prime} \cos \phi_{2}^{\prime}$.

By means of these values the formula (23) and (24) become

$$
\begin{aligned}
& X_{0}^{\prime}=\frac{2 \pi}{\lambda_{1}}\left(\tau_{1} \cos \alpha_{1} \sin \phi_{1}+\tau_{1}^{\prime} \cos a_{1}^{\prime} \sin \phi_{1}^{\prime}\right), \\
& Y_{0}^{\prime}=\frac{2 \pi}{\lambda_{1}}\left(\tau_{1} \cos \beta_{1} \sin \phi_{1}+\tau_{1}^{\prime} \cos \beta_{1}^{\prime} \sin \phi_{1}^{\prime}\right), \\
& P=2 \pi\left(\frac{r s}{\lambda_{2}} \tau_{2} \cos \boldsymbol{a}_{2} \sin \phi_{2}+\frac{r^{\prime} s^{\prime}}{\lambda_{2}^{\prime}} \tau_{2}^{\prime} \cos a_{2}^{\prime} \sin \phi_{2}^{\prime}\right), \\
& Q=2 \pi\left(\frac{r s}{\lambda_{2}} \tau_{2} \cos \beta_{3} \sin \phi_{2}+\frac{r^{\prime} s^{\prime}}{\lambda_{2}^{\prime}} \tau_{2}^{\prime} \cos \beta_{2}^{\prime} \sin \phi_{2}^{\prime}\right) .
\end{aligned}
$$

The angles $\phi_{1}, \phi_{1}^{\prime}, \phi_{2}, \phi_{2}^{\prime}$ are the phases of vibration in the 
different waves at the time $t$. To see how they depend on the co-ordinates $x_{0}, y_{0}, z_{0}$, conceive the axis of $z_{0}$ to be directed from $O$ towards the interior of the second medium, and the axis of $x_{0}$ to lie in the plane of incidence, so that the positive directions of $z_{1}, z_{2}, z_{2}^{\prime}$ may lie within the angle made by the positive directions of $x_{0}$ and $z_{0}$, while the positive direction of $z_{1}^{\prime}$ lies within the angle made by the positive direction of $x_{0}$ and the negative direction of $z_{0}$. Let $i_{1}$ be the angle of incidence, and $i_{2}$, $i_{2}^{\prime}$ the angles of refraction; then

$$
\begin{array}{ll}
z_{1}=x_{0} \sin i_{1}+z_{0} \cos i_{1}, & z_{1}^{\prime}=x_{0} \sin i_{1}=z_{0} \cos i_{1}, \\
z_{2}=x_{0} \sin i_{2}+z_{0} \cos i_{2}, & z_{2}^{\prime}=x_{0} \sin i_{2}^{\prime}+z_{0} \cos i_{2}^{\prime} .
\end{array}
$$

These values are to be written in the expressions (25). They show that the phases, and therefore the displacements, are independent of $y_{0}$.

Since the conditions relative to the plane of $x_{0} y_{0}$ must hold at every instant of time, and for every point of that plane, the co-efficients of $t$, as well as those of $x_{0}$, in the values of the different phases, must be identical; so that we must have

$$
\frac{1}{\lambda_{1}}=\frac{8}{\lambda_{2}}=\frac{8^{\prime}}{\lambda_{2}^{\prime}}, \quad \frac{\sin i_{1}}{\lambda_{1}}=\frac{\sin i_{2}}{\lambda_{2}}=\frac{\sin i_{2}^{\prime}}{\lambda_{3}^{\prime}}
$$

Therefore, when $z_{0}=0$, the supposition

$$
\nu_{1}=\nu_{1}^{\prime}=\nu_{2}=v_{2}^{\prime}
$$

renders the phases identical, independently of $t$ and $x_{0}$. And, from the form of the equations of condition, it is easy to see that this supposition is necessary; because the equations (20), when the values (26) are substituted in them, contain only the cosines of the phases; and the equations (21), when the values (27) are substituted in them, contain only the sines of the 
phases. Making the latter substitution, and attending to the relations just mentioned, we find

$$
\begin{aligned}
& \tau_{1} \cos a_{1}+\tau_{1}^{\prime} \cos a_{1}^{\prime}=r \tau_{2} \cos a_{2}+r^{\prime} \tau_{2}^{\prime} \cos \alpha_{2}^{\prime}, \\
& \tau_{1} \cos \beta_{1}+\tau_{1}^{\prime} \cos \beta_{1}^{\prime}=r \tau_{2} \cos \beta_{2}+r^{\prime} \tau_{2}^{\prime} \cos \beta_{2}^{\prime} .
\end{aligned}
$$

In these equations, the angles by whose cosines each transversal is multiplied are the angles which a plane, passing through the directions of that transversal and of the corresponding ray, makes with the planes of $y_{0} z_{0}$ and $x_{0} z_{0}$. This is evident with regard to the incident and reflected rays. And if we refer to the diagram in the preceding section, it will also be evident with regard to the refracted rays; for $O Q$ is perpendicular to the transversal $\tau_{2}$, and to the right line $O T$, which is the direction of the corresponding ray.

Taking $O$ for the point of incidence, let right lines proceeding from it represent the different rays; and let the length of each ray, measured from $O$ in the direction of propagation, be assumed proportional to the velocity with which the light is propagated along it. Through the extremity of each ray conceive its transversal to be drawn, and let the transversals so drawn have their moments taken, with respect to the point $O$, as if they represented forces applied to a rigid body. The length of the incident or reflected ray being considered as unity, the lengths of the refracted rays (as appears by the last Section) are $r$ and $r^{\prime}$ respectively. Hence, as each transversal is perpendicular to its ray, the moments of the incident and reflected transversals are proportional to $\tau_{1}, \tau^{\prime}{ }_{1}$, and the moments of the refracted transversals to $r \tau_{2}, r^{\prime} \tau_{2}^{\prime}$ respectively. The equations (31) therefore signify that when the moments are projected, either upon the plane of $y_{0} z_{0}$, or upon the plane of $x_{0} z_{0}$, the total projected moments are the same for the two media; or that, if the transversals themselves be projected on either of these planes, the moments of the projections of the incident and reflected transversals are together equal to the moments of the projections of the refracted transversals. 
But the second of the equations (31) has another signification. For if the transversals applied at the extremities of the refracted rays be projected on the plane of $x_{0} z_{0}$, which is the plane of incidence, and contains the axes of $z_{2}$ and $z_{2}^{\prime}$, the projections will be perpendicular to these axes, since the transversals themselves are perpendicular to them; and the distances of the projections from the point $O$ will be proportional to $s$ and $s^{\prime}$, or, by the relations (29), to $\sin i_{2}$ and $\sin i_{2}^{\prime}$; so that if $\theta_{2}$ and $\theta_{2}^{\prime}$ be the angles which the transversals make with the plane of incidence, the moments of the projections will be represented by $\tau_{2} \cos \theta_{2} \sin i_{2}$ and $\tau_{2}^{\prime} \cos \theta_{2}^{\prime} \sin i_{2}^{\prime}$. At the same time, if $\theta_{1}$ and $\theta_{1}^{\prime}$ be the angles which the incident and reflected transversals make with the plane of incidence, the moments of the corresponding projections of these transversals will evidently be represented by $\tau_{1} \cos \theta_{1} \sin i_{1}$, and $-\tau_{1}^{\prime} \cos \theta_{1}^{\prime} \sin i_{1}$; the latter quantity being taken with a negative sign, because the extremity of the reflected ray, where the transversal $\tau_{1}^{\prime}$ is applied, lies in the first medium, while the extremities of the incident and refracted rays lie in the second, and it is supposed that when any of the angles $\theta_{1}, \theta_{1}^{\prime}, \theta_{2}, \theta_{2}^{\prime}$ is zero, the direction of the corresponding transversal makes an acute angle with the axis of $x_{0}$. Hence we have

$$
\tau_{1} \cos \theta_{1} \sin i_{1}-\tau_{1}^{\prime} \cos \theta_{1}^{\prime} \sin i_{1}=\tau_{2} \cos \theta_{2} \sin i_{2}+\tau_{2}^{\prime} \cos \theta_{2}^{\prime} \sin i_{2}^{\prime} ;
$$

an equation which expresses that if each transversal be projected upon the axis of $z_{0}$, the sum of the projections of the ineident and reflected transversals will be equal to the sum of the projections of the refracted transversals. Therefore, since the phases of the different vibrations are identical when $z_{0}=0$, the condition (22) is fulfilled, as it ought to be.

On account of this identity of phases, it follows from the conditions (20) and (22), that if the transversals be drawn through the point $O$, and those which belong to each medium be compounded like forees acting at a point, their resultants will be the same; that is, the resultant of the incident and 
reflected transversals will be the same as the resultant of the refracted transversals.

Hence, recollecting what has been proved respecting the moments of the transversals applied at the extremities of the rays, we have the following theorem :

Supposing the length of each ray, measured from the point of incidence and in the direction of propagation, to be taken proportional to the velocity with which the light is propagated along it, and its transversal to be drawn through the extremity of this length, the incident and reflected transversals having their proper directions, but the refracted transversals having their directions reversed; if all the transversals so drawn be compounded like forces applied to a rigid body, their resultant will be a couple, lying in a plane parallel to the plane which separates the two media.

This theorem affords a complete solution of the question of reflexion and refraction.* Expressed analytically it gives five equations, of which four are independent.

To apply the preceding results to a simple case, suppose the second medium, as well as the first, to be an ordinary one. We have then only one refracted ray, and one refracted transversal $\tau_{\mathrm{a}}$.

$1^{\circ}$. When the incident ray is polarized in the plane of incidence, the transversals are all in that plane; and as they are perpendicular to the rays, and the refracted transversal is the resultant of the other two, we have evidently

$$
\tau_{1}^{\prime}=\tau_{1} \frac{\sin \left(i_{1}-i_{2}\right)}{\sin \left(i+i_{2}\right)}, \quad \tau_{2}=\tau_{1} \frac{\sin 2 i_{1}}{\sin \left(i_{1}+i_{2}\right)} .
$$

$2^{\circ}$. When the incident ray is polarized perpendicularly to

- The same theorem applies to the other case of reflexion and refraction, when a ray which has entered the crystal emerges from it into an ordinary medium, undergoing double reflexion at the surface where it emerges. In fact, the conditions (20) and (21) hold good whether the ordinary medium is the first or the second; and in the latter case, as well as in the former, it may be shown that the condition (22) is fulfilled, and that the theorem above mentioned is true. 
have, by Lemacidence, the transversals are all perpendicular 9. Taking $2 \sin i_{1}$ to represent the length of the $\cos a_{2}=$ reflected ray, the proportional length of the resy is $2 \sin i_{2}$, and the projections of these lengths on T. slane of $y_{0} z_{0}$ are $2 \sin i_{1} \cos i_{1}$ and $2 \sin i_{2} \cos i_{2}$, or $\sin 2 i_{1}$ and $\sin 2 i_{2}$. The transversals applied at the extremities of the rays are not altered by being projected on the plane of $y_{0} z_{0}$; therefore the moments of the incident, reflected, and refracted transversals, projected on this plane, are represented by the quantities $\tau_{1}$ sin $2 i_{1}-\tau_{1}^{\prime}$ sin $2 i_{1}$, and $\tau_{2}$ sin $2 i_{2}$ respectively. Equating the last moment to the sum of the other two, and the refracted transversal to the sum of the other two transversals, we get

$$
\left(\tau_{1}-\tau_{1}^{\prime}\right) \sin 2 i_{1}=\tau_{2} \sin 2 i_{2}, \quad \tau_{1}+\tau_{1}^{\prime}=\tau_{2} ;
$$

and thence

$$
\tau_{1}^{\prime \prime}=\tau_{1} \frac{\tan \left(i_{1}-i_{2}\right)}{\tan \left(i_{1}+i_{2}\right)}, \quad \tau_{2}=\tau_{1} \frac{\sin 2 i_{1}}{\sin \left(i_{1}+i_{2}\right) \cos \left(i_{1}-i_{2}\right)} .
$$

This case has been considered by Fresnel. The relative magnitudes of the incident and reflected transversals, as given by him, are in accordance* with the formulæ (32) and (33); but with respect to the refracted transversals, his results do not agree with the formulø.

Sect. VI.-Presertation of Vis $V_{I V A}$-Theorem of the Polar Plane-Conclusiox.

Returning to the general question, if we resolve the transversals parallel to the axes of $x_{0}, y_{0}, z_{0}$, and equate the sums of the parallel components in one medium to the corresponding

- There is, however, a difference as to the relative dinections of the incident and reflected transversals. When the second medium is the denser, and the incidence is perpendicular, these transversals, according to the preseat theory, have the sume direction, but according to Fresnel they have opposite directions. 
sums in the other, we get the three conditions lant of the

$$
\begin{aligned}
& \left(\tau_{1} \cos \theta_{1}+\tau_{1}^{\prime} \cos \theta_{1}^{\prime}\right) \cos i_{1}=\tau_{2} \cos \theta_{2} \cos i_{2}+\tau_{2}^{\prime} \cos \theta_{\text {ing the }} \\
& \tau_{1} \sin \theta_{1}+\tau_{1}^{\prime} \sin \theta_{1}=\tau_{2} \sin \theta_{2}+\tau_{2}^{\prime} \sin \theta_{2}^{\prime} \text {, the }
\end{aligned}
$$

$\left(\tau_{1} \cos \theta_{1}-\tau_{1}^{\prime} \cos \theta_{1}^{\prime}\right) \sin i_{1}=\tau_{2} \cos \theta_{2} \sin i_{2}+\tau_{2}^{\prime} \cos \theta_{2}^{\prime} \sin i_{2}^{\prime \prime}$.

A fourth condition is supplied by the first of the equations (31), in which equation we have to write

$$
\cos a_{1}=\sin \theta_{1} \cos i_{1}, \quad \cos \boldsymbol{a}_{1}^{\prime}=-\sin \theta_{1}^{\prime} \cos i_{1},
$$

and to substitute similar expressions for $\cos \boldsymbol{a}_{2}, \cos \boldsymbol{a}_{2}^{\prime}$.

The right line $O Q$ is perpendicular to the transversal $\tau_{2}$ and to the ray OT. The cosines of the angles $a_{2}, \beta_{2}, \gamma_{2}$ may therefore be found by means of the cosines of the angles which the transversal and the ray make with the axes of $x_{0}, y_{0}, z_{0}$.

The cosines of the angles which the transversal $\tau_{2}$ makes with these axes are respectively

$$
\cos \theta_{2} \cos i_{2}, \quad \sin \theta_{2}, \quad-\cos \theta_{2} \sin i_{2} .
$$

As the plane which passes through the ray and the wavenormal $O S$ is perpendicular to the transversal $\tau_{2}$, this plane makes with the plane of incidence an angle equal to $90^{\circ}+\theta_{2}$ or $90^{\circ}-\theta_{2}$. Let a sphere, having its centre at $O$, be intersected in the points $S_{0}, T_{0}$ by the right lines $O S, O T$, and in the points $\boldsymbol{X}_{0}, \boldsymbol{Y}_{0}, Z_{0}$ by the axes of $x_{0}, y_{0}, z_{0}$; and conceive the points $T_{0}$ and $Y_{0}$ to be at the same side of the plane $x_{0}, z_{0}$, the spherical angle $T_{0} S_{0} X_{0}$ being $90^{\circ}+\theta_{2}$, and the spherical angle $T_{0} S_{0} Z_{0}$ being $90^{\circ}-\theta_{2}$. Let $\varepsilon$ be the angle which the ray makes with the wave-normal. Then, the angles which the ray makes with the axes of co-ordinates being measured by the arcs $T_{0} X_{0}, T_{0} Y_{0}$, $T_{0} Z_{0}$, the cosines of these angles respectively are

$$
\begin{gathered}
\sin i_{2} \cos \varepsilon-\sin \theta_{2} \cos i_{2} \sin \varepsilon, \quad \cos \theta_{2} \sin \varepsilon, \\
\cos i_{2} \cos \varepsilon+\sin \theta_{2} \sin i_{2} \sin \varepsilon .
\end{gathered}
$$

Hence, as the transversal is at right angles to the ray, we 
have, by Lemma I.,

$\cos \alpha_{2}=\sin i_{2} \sin \varepsilon+\sin \theta_{2} \cos i_{2} \cos \varepsilon, \quad \cos \beta_{2}=-\cos \theta_{2} \cos \varepsilon$,

$$
\cos \gamma_{2}=\cos i_{2} \sin \varepsilon-\sin \theta_{2} \sin i_{2} \cos \varepsilon .
$$

In like manner, putting $\varepsilon^{\prime}$ for the angle which the other refracted ray makes with its wave-normal, we have

$$
\begin{gathered}
\cos {\boldsymbol{a}_{2}^{\prime}}_{2}=\sin i_{2}^{\prime} \sin \varepsilon^{\prime}+\sin \theta_{2} \cos i_{2}^{\prime} \cos \varepsilon^{\prime}, \quad \cos \beta_{2}^{\prime}=-\cos \theta_{2}^{\prime} \cos \varepsilon^{\prime}, \\
\cos \gamma_{2}^{\prime}=\cos i_{2}^{\prime} \sin \varepsilon^{\prime}-\sin \theta_{2}^{\prime} \sin i_{2}^{\prime} \cos \varepsilon^{\prime} .
\end{gathered}
$$

If we substitute, in the first of the equations (31), the values just given for $\cos \boldsymbol{a}_{2}, \cos \boldsymbol{a}_{2}^{\prime}$, along with the above values of $\cos \alpha_{1}, \cos \alpha^{\prime}$, and attend to the relations

$$
\begin{array}{ll}
r \cos \varepsilon=s . & r^{\prime} \cos \varepsilon^{\prime}=s^{\prime}, \\
\sin i_{2}=s \sin i_{1}, & \sin i_{2}^{\prime}=s^{\prime} \sin i_{1,}
\end{array}
$$

we find, after multiplying all the terms of the equation by $\sin i_{1}$,

$$
\begin{aligned}
& \left(\tau_{1} \sin \theta_{1}-\tau_{1}^{\prime} \sin \theta_{1}\right) \sin i_{1} \cos i_{1}=\tau_{2}\left(\sin \theta_{2} \sin i_{2} \cos i_{2}\right. \\
& \left.\quad+\sin ^{2} i_{2} \tan \varepsilon\right)+\tau_{2}^{\prime}\left(\sin \theta_{2}^{\prime} \sin i_{2}^{\prime} \cos i_{2}^{\prime}+\sin ^{2} i_{2}^{\prime} \tan \varepsilon\right) .
\end{aligned}
$$

Joining this equation to the equations (34) we have all the oonditions that are necessary for the solution of the question.

Multiplying the first of the equations (34) by the third, also the second of these equations by the equation (38), adding the products together, and then dividing by $\sin i_{18}$ we obtain

$$
\mu_{1}\left(\tau_{1}^{2}-\tau_{1}^{\prime 2}\right)=\mu_{2} \tau_{2}^{2}+\mu_{2}^{\prime} \tau_{2}^{\prime 2}+M \tau_{2} \tau_{2}^{\prime},
$$

where we have put

$$
\begin{gathered}
\mu_{1}=\cos i_{1}, \quad \mu_{2}=s\left(\cos i_{2}+\sin \theta_{2} \sin i_{2} \tan \varepsilon\right), \\
\mu_{2}^{\prime}=8^{\prime}\left(\cos i_{2}^{\prime}+\sin \theta_{2} \sin i_{2}^{\prime} \tan \varepsilon^{\prime}\right),
\end{gathered}
$$

$\boldsymbol{M} \sin i_{1}=\sin \left(i_{2}+i_{2}^{\prime}\right)\left\{\cos \theta_{2} \cos \theta_{2}^{\prime}+\sin \theta_{2} \sin \theta_{2}^{\prime} \cos \left(i_{2}-i_{2}^{\prime}\right)\right\}$

$+\sin \theta_{3} \sin ^{2} i_{2} \tan \varepsilon+\sin \theta_{2} \sin ^{2} i_{2} \tan \varepsilon^{\prime}$. 
The last expression may be put under the form

$$
\begin{gathered}
M \sin i_{1} \sin \left(i_{2}-i_{2}^{\prime}\right) \\
=\sin ^{2} i_{2}\left\{\cos \theta_{2} \cos \theta_{2}^{\prime}+\sin \theta_{2} \sin \theta_{2}^{\prime} \cos \left(i_{2}-i_{2}^{\prime}\right)\right. \\
\left.+\sin \theta_{2} \sin \left(i_{2}-i_{2}^{\prime}\right) \tan \varepsilon\right\} \\
-\sin ^{2} i_{2}^{\prime}\left\{\cos \theta_{2} \cos \theta_{2}^{\prime}+\sin \theta_{2} \sin \theta_{2}^{\prime} \cos \left(i_{2}-i_{2}^{\prime}\right)\right. \\
\left.-\sin \theta_{2} \sin \left(i_{2}-i_{2}^{\prime}\right) \tan \varepsilon^{\prime}\right\} .
\end{gathered}
$$

Let the axes of $x_{0}, y_{0}, z_{0}$ make with the direction of $O P$ the angles $\alpha_{\|}, \beta_{\|,} \gamma_{,}$, and with the direction of $O P^{\prime}$ the angles $a^{\prime}, \beta^{\prime}, \gamma^{\prime}$. The cosines of these angles may be found from the expressions (35) and (36) by supposing $\varepsilon$ and $\varepsilon^{\prime}$ to vanish. Therefore

$\cos \alpha_{\text {" }}=\sin \theta_{2} \cos i_{2}, \quad \cos \beta_{\text {" }}=-\cos \theta_{2}, \quad \cos \gamma_{\text {" }}=-\sin \theta_{2} \sin i_{2}$, $\cos \boldsymbol{a}^{\prime}{ }_{1 \prime}=\sin \theta_{2}^{\prime} \cos i_{2}^{\prime}, \cos \beta^{\prime}{ }_{1 \prime}=-\cos \theta_{2}^{\prime}, \quad \cos \gamma^{\prime}{ }_{1}=-\sin \theta_{2} \sin i_{{ }_{2}}$.

If $\omega$ be the angle which $O Q$ makes with $O P^{\prime}$, and $\omega^{\prime}$ the angle which $O Q^{\prime}$ makes with $O P$, so that

$$
\begin{aligned}
& \cos \omega=\cos \alpha_{2} \cos \alpha^{\prime}{ }_{1 /}+\cos \beta_{2} \cos \beta^{\prime}{ }_{1,}+\cos \gamma_{2} \cos \gamma_{\text {, , }}^{\prime} \\
& \cos \omega^{\prime}=\cos a_{2}^{\prime} \cos a_{\|}+\cos \beta_{2}^{\prime} \cos \beta_{\mu}+\cos \gamma_{2}^{\prime} \cos \gamma_{\text {, }}
\end{aligned}
$$

we find, by the formulæ (35), (36), (41),

$$
\begin{aligned}
\cos \omega=\cos \varepsilon\left\{\cos \theta_{2} \cos \theta_{2}^{\prime}+\sin \theta_{2} \sin \theta_{2}^{\prime} \cos \left(i_{2}-i_{2}^{\prime}\right)\right. \\
\left.+\sin \theta_{2}^{\prime} \sin \left(i_{2}-i_{2}^{\prime}\right) \tan \varepsilon\right\}, \\
\cos \omega^{\prime}=\cos \varepsilon^{\prime}\left\{\cos \theta_{2} \cos \theta_{2}^{\prime}+\sin \theta_{2} \sin \theta_{2} \cos \left(i_{2}-i_{2}^{\prime}\right)\right. \\
\left.-\sin \theta_{2} \sin \left(i_{2}-i_{2}^{\prime}\right) \tan \varepsilon^{\prime}\right\} .
\end{aligned}
$$

Hence, observing the relations (37), we see that the right-hand member of the equation (40) is equal to the quantity

$$
\sin ^{2} i_{1}\left(r s \cos \omega-r^{\prime} 8^{\prime} \cos \omega^{\prime}\right) \text {. }
$$


But, by the property of the ellipsoid (Lemma IV.), this quantity is zero; ; therefore $\boldsymbol{M}=0$, and the equation (39) becomes

$$
\mu_{1} \tau_{1}^{2}=\mu_{1} \tau_{1}^{\prime 2}+\mu_{2} \tau_{2}^{2}+\mu_{2}^{\prime} \tau_{2}^{\prime 2}
$$

On each ray let a length, representing the velocity with which the light is propagated along it, be measured, as before, from the point $O$. The distances of the plane of $x_{0} y_{0}$ from the extremities of these lengths will be proportional to the coefficients of the squares of the transversals in the preceding equation. For if we take, on the incident or reflected ray, a length equal to unity, its projection on the axis of $z_{0}$ will be $\cos i_{1}$ or $\mu_{1}$; and if, on the refracted ray $O T$, we take a length equal to $r$, its projection on the same axis will be

$$
r\left(\cos i_{2} \cos \varepsilon+\sin \theta_{2} \sin i_{2} \sin \varepsilon\right),
$$

which is equal to $\mu_{2}$. Similarly, the length $r^{\prime}$, assumed on the other refracted ray, will have its projection equal to $\mu_{2}^{\prime}$. The quantities by which the squares of the transversals are multiplied, in the equation (42), are therefore the corresponding ethereal volumest which we may conceive to be put in motion by the different waves; and as we suppose the density of the ether to be the same in both media, the equation expresses a principle analogous to that of the preservation of vis viva.‡

By giving a certain direction to the incident transversal, that is, by polarizing the incident ray in a certain plane, we may make one of the refracted rays disappear. If $O T$ be the ray which remains, we have $\tau^{\prime}{ }_{2}=0$, and the equations (34) and (38) become

* The equation $M=0$ is the same as the equation (vir.) in my former Paper.Transactions, R.I.A., VoL. xvII. p. 52 (supra, p. 112).

+ Ibid., p. 48 (oupra, p. 106).

\# A similar equation of vis viva holds when the light passes out of a crystal into an ordinary medium. 


$$
\begin{aligned}
& \left(\tau_{1} \cos \theta_{1}+\tau_{1}^{\prime} \cos \theta_{1}\right) \cos i_{1}=\tau_{2} \cos \theta_{2} \cos i_{2}, \\
& \tau_{1} \sin \theta_{1}+\tau_{1}^{\prime} \sin \theta_{1}^{\prime}=\tau_{2} \sin \theta_{2}, \\
& \left(\tau_{1} \cos \theta_{1}-\tau_{1}^{\prime} \cos \theta_{1}\right) \sin i_{1}=\tau_{2} \cos \theta_{2} \sin i_{2}, \\
& \left(\tau_{1} \sin \theta_{1}-\tau_{1}^{\prime} \sin \theta_{1}\right) \sin i_{1} \cos i_{1} \\
& \quad=\tau_{2}\left(\sin \theta_{2} \sin i_{2} \cos i_{2}+\sin ^{2} i_{2} \tan \varepsilon\right) .
\end{aligned}
$$

In this case, the three transversals are in the same plane, the refracted transversal being the resultant of the other two. Therefore if we find this plane, everything will be determined.

The axes of $x_{0}, y_{0}, z_{0}$ make, with the incident transversal, angles whose cosines are

$$
\cos \theta_{1} \cos i_{1}, \quad \sin \theta_{1}, \quad-\cos \theta_{1} \sin i_{1},
$$

and, with the reflected transversal, angles whose cosines are

$$
\cos \theta_{1}^{\prime} \cos i_{1}, \quad \sin \theta_{1}^{\prime}, \quad \cos \theta_{1}^{\prime} \sin i_{1} ;
$$

therefore, by Lemma I., the cosines of the angles which these axes make with a right line perpendicular to the plane of the transversals are proportional to the quantities

$$
\sin \left(\theta_{1}+\theta_{1}^{\prime}\right) \sin i_{1}, \quad-\cos \theta_{1} \cos \theta_{1}^{\prime} \sin 2 i_{1}, \quad \sin \left(\theta_{1}^{\prime}-\theta_{1}\right) \cos i_{1} .
$$

Now from the product of the first and second of the equations (43), combined with the product of the third and fourth, we find, by the help of the relations (37),

$$
\begin{aligned}
2 \tau_{1} \tau_{1}^{\prime} \sin \left(\theta_{1}+\theta_{1}\right) \sin i_{1} & =\tau_{2}{ }^{2} \tan i_{1} \cos \theta_{2}\left\{\sin \theta_{2} \cos i_{2}\right. \\
& \left.-s^{2}\left(\sin \theta_{2} \cos i_{2}+\sin i_{2} \tan \varepsilon\right)\right\} .
\end{aligned}
$$

From the squares of the first and third of those equations we find

$$
-2 \tau_{1} \tau_{1}^{\prime} \cos \theta_{1} \cos \theta_{1} \sin 2 i_{1}=\tau_{2}^{2} \tan i_{1} \cos ^{2} \theta_{2}\left(s^{2}-1\right),
$$

and from the product of the first and fourth, combined with the product of the second and third,

$$
\begin{aligned}
2 \tau_{1} \tau_{1}^{\prime} \sin \left(\theta_{1}^{\prime}-\theta_{1}\right) \cos i_{1}=r_{2}{ }^{2} & \tan i_{1} \cos \theta_{2}\left\{-\sin \theta_{2} \sin i_{2}\right. \\
& \left.+8^{2}\left(\sin \theta_{2} \sin i_{2}-\cos i_{2} \tan \varepsilon\right)\right\} .
\end{aligned}
$$


In the three equations just found, the left-hand members are proportional, as we have seen, to the cosines of the angles which a right line perpendicular to the plane of the transversals makes with the axes of co-ordinates; and the right-hand members, as appears by the formulæ (35) and (41), are proportional to the quantities

$$
\frac{\cos \alpha_{u \prime}}{8}-r \cos a_{2}, \quad \frac{\cos \beta_{u \prime}}{8}-r \cos \beta_{2}, \quad \frac{\cos \gamma_{11}}{8}-r \cos \gamma_{2},
$$

which are obriously the differences between the corresponding co-ordinates of the points $R$ and $Q$. The plane of the transversals is therefore perpendioular to the right line $Q R$, which joins those points.

A plane parallel to the right line $T M$, and passing through the transversal of the ray $O T$, is that which $I$ have called the polar plane of the ray ; ${ }^{*}$ and this plane is perpendicular to $Q R$. Therefore, when there is only one refracted ray, the incident and reflected transversals lie in the polar plane of that ray; and their directions being thus determined, the relative magnitudes of the three transversals are known. In this case the incident and reflected transversals are called uniradial; and as each refracted ray in turn may be 'made to disappear, there are two uniradial directions in the plane of the incident wave, and two in that of the reflected wave.

When the incident transversal is not uniradial, it may be considered as the resultant of two uniradial transversals, each of whioh will supply a refracted ray, and will produce a uniradial component of the reflected transversal.

It is needless to extend these deductions further. They have been carried far enough to show that the results of the foregoing theory are in perfect accordance with the laws established in my former Paper on the subject of erystalline reflexion. The theory itself suggests much matter for consideration; but at present we shall eonfine ourselves to one remark, which may

* Transactions, Royal Irish Academy, VoL. xviIr. p. 39 (supra, p. 96). 
be necessary to prevent any misconception as to the nature of the foundation on which it stands. In this theory, everything depends on the form of the function $V$; and we have seen that, when that form is properly assigned, the laws by which crystals act upon light are included in the general equation of dynamics. This fact is fully proved by the preceding investigations. But the reasoning which has been used to account for the form of the function* is indirect, and cannot be regarded as sufficient, in a mechanical point of view. It is, however, the only kind of reasoning which we are able to employ, as the constitution of the luminiferous medium is entirely unknown.

* Since this Paper was read to the Academy, I have found that the form of the function $\mathrm{V}$ is more general than it would seem to be from the mode in which it is here deduced; and I have obtained from it a theory of the Total Reflexion of Light. For a sketch of this theory, see the Proceedings of the Royal Irish Academy, VoL. II. p. 96 (supra, p. 187). 


\section{$\left(\begin{array}{l}185\end{array}\right)$}

XV.-ON THE OPTICAL LAWS OF ROCK-CRYSTALS.

[Proceedings of the Royal Irish Academy, VoL. I. p. 385.--Read Jan. 13, 1840.]

Professor Mac Cullagh made a communication respecting the optical Laws of Rock-crystal (Quartz).

In a Paper read to the Academy in February 1836, and published in the Transactions (VoL. xvII. p. 461), ${ }^{*}$ he had shown how the peculiar properties of that crystal might be explained, by adding to the usual equations of vibratory motion eertain terms depending on differential co-efficients of the third order, and containing only one new constant $C$. This hypothesis, which was very simple in itself, not only involved as consequences all the laws that were previously known, but led to the discovery of a new one-the law, namely, by which the ellipticity of the vibrations depends on the direction of the ray within the crystal. He was not able, however, to account for his hypothesis, nor has it since been accounted for by anyone.

But the theory developed in the Paper which he read at the last meeting of the Academy now enables him to assign, with a high degree of probability, the origin of the additional terms above mentioned, and, if not to account for them mechanically, at least to advance a step higher in the inquiry. In that theory it was supposed (and the supposition holds good in all known crystals, except quartz), that the molecules of the ether vibrate in right lines, the displacements remaining always parallel to each other as the wave is propagated; and it was shown that the function $\mathrm{V}$, by which the motion is determined,

\footnotetext{
* Supra, p. 63.
} 
then depends only on the relative displacements of the molecules. But when this is not the case-when, as in quartz, each molecule is supposed to vibrate in a curve-then it is natural to conceive that the function $\mathrm{V}$ may depend, not only on the relative displacements, but also on the relative areas which each molecule describes about every other more or less advanced in its vibration. This idea, analytically expressed, introduces a new term $v$ into the value of the function $2 \mathrm{~V}$; and, if the plane of the ware be taken for the plane of $x y$, it is easy to show that

$$
v=C\left(\frac{d \eta}{d s} \frac{d^{2} \xi}{d s^{2}}-\frac{d \xi}{d s} \frac{d^{2} \eta}{d z^{2}}\right)
$$

Now if we integrate by parts the expression

$$
\iiint d x d y d z \delta v,
$$

so as to get rid of the variations of differential co-efficients, the reduced form of the triple integral will be

$$
2 C \iiint d x d y d z\left(\frac{d^{3} \eta}{d z^{3}} \delta \xi-\frac{d^{3} \xi}{d z^{3}} \delta \eta\right)
$$

from which it appears that the quantities

$$
C \frac{d^{3} \eta}{d z^{3}}, \quad-C \frac{d^{3} \xi}{d z^{3}},
$$

are to be added to the usual expressions for the force in the directions of $x$ and $y$ respectively. These are the very terms in the addition of which the hypothesis before alluded to consists. 


\section{$(187)$}

\section{XVI.-ON A DYNAMICAL THEORY OF CRYSTALLINE REFLEXION AND REFRACTION.}

[Proceedings of the Royal Irish Academy, Vor. II. p. 96.-Read May 24, 1841.]

Professor Mac Cullagh read a supplement to his Paper "On a Dynamical Theory of Crystalline Reflexion and Refraction."

In his former Paper on that subject," the author had given the general principles for solving all questions relative to the propagation of light in a given medium, or its reflexion and refraction at the separating surface of two media; but he had applied them only to the common case of waves, which suffer no diminution of intensity in their progress, and in which the vibration may be represented by the sine or cosine of an are multiplied. by a constant quantity. Some months after that Paper was read, it occurred to him that he might obtain new and important results by substituting in his differential equations of motion a more general expression for the integral, that is (as usual in such problems), by making the displacements proportional to the sine or cosine of an are, multiplied by a negative exponential, of which the exponent should be a linear function of the co-ordinates. Such vibrations would become very rapidly insensible, and would, therefore, be fitted to represent the disturbance which, in the case of total reflexion, takes place immediately bebind the reflecting surface; and, the laws of this disturbance being thus discovered, the laws of polarization in the totally

* See Proceedings, 9th December, 1839 (supra, p. 146). 
reflected light would also become known, by means of the general formulre which the author had established for all cases of reflexion at the common surface of two media.

The present supplement is the fruit of these considerations. It contains the complete theory of the new kind of vibrations, not only in ordinary media, but in doubly-refracting crystals ; and also the complete discussion of the laws of total reflexion at the first or second surface of a crystal, including, as a particular case, the well-known empirical formulæo of Fresnel for total reflexion at the surface of an ordinary medium.

The existence of vibrations represented by an expression containing a negative exponential as a factor had been recognised by other writers, and was indeed sufficiently indicated by the phenomenon of total reflexion; but it was impossible to obtain the laws of such vibrations, so long as the general equations for the propagation of light were unknown.

The method of deducing these equations was given in the abstract of the author's former Paper ;* but as they were not there stated, it may be well to transcribe them here. If then we put

$$
X=\frac{d \eta}{d z}-\frac{d \xi}{d y}, \quad Y=\frac{d \zeta}{d x}-\frac{d \xi}{d z}, \quad Z=\frac{d \xi}{d y}-\frac{d \eta}{d x},
$$

and suppose the axes of co-ordinates to be the principal axes of the crystal, the equations in question may be thus written:

$$
\begin{aligned}
& \frac{d^{2} \xi}{d t^{2}}=c^{2} \frac{d Z}{d y}-b^{2} \frac{d Y}{d z}, \\
& \frac{d^{2} \eta}{d t^{2}}=a^{2} \frac{d X}{d z}-c^{2} \frac{d Z}{d x}, \\
& \frac{d^{2} \zeta}{d t^{2}}=b^{2} \frac{d Y}{d x}-a^{2} \frac{d X}{d y} ;
\end{aligned}
$$

and if we further put

$$
\xi=\frac{d \eta_{1}}{d z}-\frac{d \zeta_{1}}{d y}, \quad \eta=\frac{d \zeta_{1}}{d x}-\frac{d \xi_{1}}{d z}, \quad \zeta=\frac{d \xi_{1}}{d y}-\frac{d \eta_{1}}{d x},
$$

* See Proceedings, 9th December, 1839 (supra, p. 157). 
they will take the following simple form :

$$
\frac{d^{2} \xi_{1}}{d t^{2}}=-a^{2} \boldsymbol{X}, \quad \frac{d^{2} \eta_{1}}{d t^{2}}=-b^{2} \boldsymbol{Y}, \quad \frac{d^{2} \boldsymbol{\zeta}_{1}}{d t^{2}}=-c^{2} \boldsymbol{Z},
$$

in which it is remarkable that the auxiliary quantities $\xi_{1}, \eta_{1}, \zeta_{1}$, are exactly, for an ordinary medium, the components of the displacement in the theory of Fresnel. In a doubly-refracting crystal, the resultant of $\xi_{1}, \eta_{1}, \zeta_{1}$ is perpendicular to the ray, and comprised in a plane passing through the ray and the wave-normal. Its amplitude, or greatest magnitude, is proportional to the amplitude of the vibration itself, multiplied by the velocity of the ray.

The conditions to be fulfilled at the separating surface of two media were given in the abstract already referred to. From these it follows, that the resultant of the quantities $\xi_{1}, \eta_{1}, \zeta_{1}$, projected on that surface, is the same in both media; but the part perpendicular to the surface is not the same; whereas the quantities $\xi, \eta, \zeta$, are identical in both. These assertions, analytically expressed, would give five equations, though four are sufficient; but it can be shown that any one of the equations is implied in the other four, not only in the case of common, but of total reflexion; which is a very remarkable circumstance, and a very strong confirmation of the theory.

The laws of double refraction, diseovered by Fresnel, but not legitimately deduced from a consistent hypothesis, either by himself or any intermediate writer, may be very easily obtained, as the author has already shown, from equations (2), by assuming

$$
\xi=p \cos a \sin \phi, \quad \eta=p \cos \beta \sin \phi, \quad \zeta=p \cos \gamma \sin \phi,
$$

where

$$
\phi=\frac{2 \pi}{\lambda}(l x+m y+n z-s t) ;
$$

but the new laws, which are the object of the present supplement, are to be obtained from the same equations by making

$$
\begin{aligned}
& \xi=\varepsilon\left(p \cos a \sin \phi+q \cos a^{\prime} \cos \phi\right), \\
& \eta=\varepsilon\left(p \cos \beta \sin \phi+q \cos \beta^{\prime} \cos \phi\right), \\
& \zeta=\varepsilon\left(p \cos \gamma \sin \phi+q \cos \lambda^{\prime} \cos \phi\right),
\end{aligned}
$$


where $\phi$ has the same signification as before, and

$$
\varepsilon=e^{-\frac{2 \pi r}{\lambda} Y(f x+c y+i h z)} ;
$$

the vibrations being now elliptical, whereas in the former case they were rectilinear. In these elliptic vibrations the motion depends not only on the distance of the vibrating particle from the plane whose equation is

$$
l x+m y+n z=0,
$$

but also on its distance from the plane expressed by the equation

$$
f x+g y+h z=0 ;
$$

and if the constants in the equation of each plane denote the cosines of the angles which it makes with the co-ordinate planes, we shall have $\lambda$ for the length of the wave, and $s$ for the velocity of propagation; while the rapidity with which the motion is extinguished, in receding from the second plane, will depend upon the constant $r$. The constants $p$ and $q$ may be any two conjugate semi-diameters of the ellipse in which the vibration is performed; the former making, with the axes of co-ordinates, the angles $\alpha, \beta, \gamma$, the latter the angles $\alpha^{\prime}, \beta^{\prime}, \gamma^{\prime}$.

As vibrations of this kind cannot exist in any medium, unless they are maintained by total reflexion at its surface, we shall suppose, in order to contemplate their laws in their utmost generality, that a crystal is in contact with a fluid of greater refractive power than itself, and that a ray is incident at their common surface, at such an angle as to produce total reflexion. The question then is, the angle of incidence being given, to determine the laws of the disturbance within the crystal.

The author finds that the refraction is still double, and that two distinct and separable systems of vibration are transmitted into the crystal. He shows that the surface of the crystal itself (the origin of co-ordinates being upon it at the point of incidence) must coincide with the plane expressed by equation (8), a circumstance which determines the three constants $f, g, h$. The plane expressed by (7) is parallel to the plane of the re- 
fracted wave; and a normal, drawn to it through the origin, lies in the plane of incidence, making with a perpendicular to the face of the crystal an angle $\omega$, which may be called the angle of refraction; so that, if $i$ be the angle of incidence, we have

$$
\sin \omega=8 \sin i,
$$

the velocity of propagation in the fluid being regarded as unity.

To each refracted wave, or system of vibration, corresponds a particular system of values for $r, s, \omega$. These the author shows how to determine by means of the index-surface (the reciprocal of Fresnel's wave-surface), which he has employed on other occasions," and the rule which he gives for this purpose affords a remarkable example of the use of the imaginary roots of equations, without the theory of which, indeed, it would have been difficult to prove, in the present instance, that there are two, and only two, refracted waves. Taking a new system of co-ordinates $x^{\prime}, y^{\prime}, z^{\prime}$, of which $z^{\prime}$ is perpendicular to the surface of the crystal, and $y^{\prime}$ to the plane of incidence, while $x^{\prime}$ lies in the intersection of these two planes; put $y^{\prime}=0$ in the equation of the index-surface referred to those co-ordinates, the origin being at its centre; we shall then have an equation of the fourth degree between $x^{\prime}$ and $z^{\prime}$, which will be the equation of the section made in the index-surface by the plane of incidence. In this equation put $x^{\prime}=\sin i$, and then solve it for $z^{\prime}$. When $i$ exceeds a certain angle $i$, the four values of $z^{\prime}$ will be imaginary; and if they be denoted by

$$
u \pm v \sqrt{-1}, \quad u^{\prime} \pm v^{\prime} \sqrt{-1},
$$

each pair will correspond to a refracted system, and we shall have, for the first,

$$
\tan \omega=\frac{\sin i}{u}, \quad s=\frac{\sin \omega}{\sin i}, \quad r=s v ;
$$

and for the second,

$$
\tan \omega^{\prime}=\frac{\sin i}{u^{\prime}}, \quad s^{\prime}=\frac{\sin \omega^{\prime}}{\sin i}, \quad r^{\prime}=8^{\prime} v^{\prime} .
$$

* Transactions of the Academy, VoLs. xvir. and xvir. (supra, pp. 36, 96). 
When $i$ lies between $i^{\prime}$ and a certain smaller angle $i^{\prime \prime}$, two of the roots will be real, and two imaginary. The real roots correspond to waves which follow the law of Fresnel; the imaginary roots give a single wave, following the other laws just mentioned.

Lastly, when $i$ is less than $i^{\prime \prime}$, all the roots are real, the refraction is entirely regulated by Fresnel's law, and the reflexion by the laws already discovered and published by the author.

If the crystal be uniaxal, and all the values of $z^{\prime}$ imaginary, the ordinary wave-normal will coincide with the axis of $x^{\prime}$; whilst the extraordinary wave-normal and the axis of $z^{\prime}$ will be conjugate diameters of the ellipse in which the index surface is cut by the plane of incidence.

When $a=b=c$, the crystal becomes an ordinary medium; there is then only single refraction, and the refracted wave is always perpendicular to the axis of $x^{\prime}$.

With regard to the ellipse in which the vibrations are performed, it may be worth while to observe, that if it be projected perpendicularly on the plane of incidence, the projected diameters which are parallel to the surface of the crystal and to the waveplane will, in all cases, be conjugate to each other, and their respective lengths will be in the proportion of $r$ to unity. The vibrations, it is obvious, are not performed in the plane of the wave, though they take place without changing the density of the ether.

The new laws here announced are, properly speaking, laws of double refraction, and are necessary to complete our knowledge of that subject. Between them and the laws of Fresnel a curious analogy exists, founded on the change of real into imaginary constants.

The laws of the total reflexion, which accompanies the new kind of refraction, need not be dwelt upon in this abstract, as nothing is now more easy than to form the equations which contain them. In fact, the difficulties which formerly surrounded the problem of reflexion, even in the simplest cases, have completely disappeared, since the author made known the conditions which must be fulfilled at the separating surface of two media. 
In what precedes, it has been supposed that the reflexion and refraction take place at the first surface of the crystal, because this is the more difficult and complicated of the two cases into which the question resolves itself. But it will usually happen in practice that a ray which has entered the crystal will suffer total reflexion at the second surface, while the new kind of vibration is propagated into the air without. The refracted wave will then be always perpendicular to the axis of $x^{\prime}$; the two reflected rays, within the crystal, will be plane-polarized, according to the common law, but they will each undergo a change of phase; and the vis viva of the two rays together will be equal to that of the incident ray, the vis viva being measured by the square of the amplitude multiplied by the proportional mass.

In conclusion, the author states a mathematical hypothesis by which both the laws of dispersion, and those of the elliptic polarization of rock crystal, may be connected with the laws already developed. 


\section{XVII.-NOTES ON SOME POINTS IN THE THEORY OF LIGHT.}

[Proceedings of the Royal Irish Academy, Vor. II. p. 139.-Read Nov. 8, 1841.]

I.

On a Mechanical Theory which has been proposed for the Explanation of the Phenomena of Circular Polariation in Liquids, and of Circular and Elliptic Polarization in Quartz or Rock-crystal; with Remarks on the corresponding Theory of Rectilinear Polarisation.

THE theory of elliptic polarization, which I feel myself called upon to notice, was first stated by M. Cauchy, and has been made the subject of elaborate investigation by other writers. That celebrated analyst, conceiving (though without sufficient reason, as will presently appear) that he had fully explained the known laws of the propagation of rectilinear vibrations by the hypothesis that the luminiferous ether, in media transmitting such vibrations, consists of separate molecules symmetrically arranged with respect to each of three rectangular planes, and acting on each other by forces which are some function of the distance, was led very naturally to imagine that he would find the laws of circular and elliptic vibrations; in other media, to be included in the more general hypothesis of an unsymmetrical arrangement. Accordingly, in a letter read to the French Academy on the 22nd of February, 1836-a letter to which he attached so much importance that he desired it might not only be published in 
the Proceedings, but also "deposited in the Archives" of that body*-he gave a precise statement of his more extended views, informing the Academy that he had submitted his new theory to calculation, and that, among other remarkable results, he had obtained (with a slight variation or correction) the laws of eircular polarization, discovered by Arago, Biot, and Fresnel. Referring to his Memoir on Dispersion, published at Prague, under the title of Nouveaux Exercices de Mathématiques, he observes, that the results therein contained may be generalized, by "ceasing to neglect" in the equations of motion [the equations marked (24) in $\S 2$ of that memoir] certain terms which vanish in the case of a symmetrical distribution of the ether. He then goes on to say-

"Nos formules ainsi généralisées représentent les phénomènes de l'absorption de la lumière ou de certains rayons, produite par les verres colorés, la tourmaline, \&e., le phenomène de la polarisation circulaire produite par le cristal de roche, l'huile de térébenthine, \&c. (Voir les expériences de MM. Arago, Biot, Fresnel). Elles servent même à déterminer les conditions et les lois de ces phénomènes; elles montrent que généralement, dans un rayon de lumière polariseé, une molecule d'éther décrit une ellipse. Mais dans certains cas particuliers, cette ellipse se change en une droite, et alors on obtient la polarisation rectiligne." "Enfin le calcul proure que, dans le cristal de roche, l'huile de térébenthine, \&e., la polarisation des rayons transmis parallèlement à l'axe (s'il s'agit du cristal de roche) n'est pas rigoureusement cireulaire, mais qu'alors l'ellipse diffère très peu du cercle."

Thus, to say nothing for the present of the questions of dispersion and absorption, it appears that M. Cauchy conceived he had completely accounted for the facts of circular and elliptic polarization, and that he had deduced the formulas "which serve to determine the conditions and laws of these phenomena." But neither in this letter, nor in any subse-

See the Comptes Rendus des Séances de l'Académie des Sciences, tom. ii. p. 182. 


\section{yo Notes on some Points in the Theory of Light.}

quent version* of his theory, has he given the formulas themselves. Nor has he told us the nature of the calculations by which he was enabled to correct the received opinion, and to prove that the vibrations in a ray transmitted along the axis of quartz, or through oil of turpentine, are not rigorously circular, as Fresnel and others have supposed, but slightly elliptical. Now-to take the case of quartz-if we consider that the vibrations of a ray passing along the axis are in a plane perpendicular to it, and if we admit, as M. Cauchy always does in the case of other uniaxal crystals, that there is a perfect optical symmetry all round the axis, we shall find it hard to conceive on what grounds he could have come to the conclusion that the vibrations of such a ray are performed in an ellipse. For if all planes passing through the axis of the crystal be alike in their optical properties, there will be absolutely nothing to determine the position and ratio of the axes of the ellipse; there will be no reason why its major axis, for example, should lie in one of these planes, rather than in any other. But, whatever may be thought of this case independently of observation, it is manifestly absurd to suppose that the vibrations are elliptical in the case of a ray passing through oil of turpentine, or any other liquid possessing the property of rotatory polarization; for, in a liquid, all planes drawn through the ray itself are circumstanced alike. From these simple considerations it is evident that the theory of M. Cauchy is unsound; but a closer examination will show that it is entirely without foundation, and that it is directly opposed to the very phenomena which it professes to explain. To make this appear, however, in the easiest way that the abstruseness of the subject will allow, it will be necessary to

* From some statements that have been made within the last few days by Professor Powell (Phil. Mag. Vor. xIx. p. 374), at the request of M. Cauchy himself, it appears that the latter republished his views about circular and elliptic polarization, in a lithographed memoir of the date of August, 1836; but I do not find that he published, either then or since, the detailed calculations which he seems to have made. 


\section{Notes on some Points in the Theory of Light. ?OI}

advert to some former researches of my own, which have a direct bearing on the question.

The same day on which M. Cauchy's letter was read to the French Academy, I had the honour of reading to the Royal Irish Academy a Paper "On the Laws of Double Refraction in Quartz" wherein I showed that everything which we know respecting the action of that crystal upon light is comprised mathematically in the following equations:-

$$
\begin{aligned}
& \frac{d^{2} \xi}{d t^{2}}=A \frac{d^{2} \xi}{d z^{2}}+C \frac{d^{3} \eta}{d z^{3}}, \\
& \frac{d^{2} \eta}{d t^{2}}=B \frac{d^{2} \eta}{d z^{2}}-C \frac{d^{3} \xi}{d z^{3}},
\end{aligned}
$$

which differ from the common equations of vibratory motion by the two additional terms containing third differential coefficients multiplied by the same constant $C$, this constant having opposite signs in the two equations. The quantities $\xi$ and $\eta$ are, at any time $t$, the displacements parallel to the axes of $x$ and $y$, which are supposed to be the principal directions in the plane of the wave, one of them being therefore perpendicular to the axis of the crystal. The constants $A$ and $B$ are given by the expressions

$$
A=a^{2}, \quad B=a^{2}-\left(a^{2}-b^{2}\right) \sin ^{2} \psi,
$$

where $a$ and $b$ are the principal velocities of propagation, ordinary and extraordinary, and $\psi$ is the angle made by the wave-normal (or the direction of $z$ ) with the axis of the crystal. The only new eonstant introduced is $C$, which, though the peculiar phenomena of quartz depend entirely on its existence, is almost inconceivably small: its value is determined in the Paper just referred to. The equations are there proved to afford a strict geometrical representation of the facts; not only connecting together all the laws disoovered by the distinguished observers to whom M. Cauchy refers, and includ-

* See Transactions, R. I. A., VoL. Xvir. p. 461 (supra, p. 63). 


\section{y Notes on some Points in the Theory of Light.}

ing the subsequent additions for which we are indebted to Mr. Airy, but leading to new results, one of which establishes a relation between two different classes of phenomena, and is verified by the experiments of $\mathrm{M}$. Biot and Mr. Airy. Having, therefore, such conclusive proofs of the truth of these equations, we are entitled to assume them as a standard whereby to judge of any theory; so that any mechanical hypothesis which leads to results inconsistent with them may be at once rejected.

Now I assert that the mechanical hypothesis of M. Cauchy contradicts these equations, and therefore contradicts all the phenomena and experiments which he supposed it to represent. But before we proceed to the proof of this assertion, it may perhaps be proper to remark, that previously to the date of M. Cauchy's communication, and of my own Paper, I had actually tried and rejected this identical hypothesis, and had even gone so far as to reject along with it the whole of M. Cauchy's views about the mechanism of light. For though, in my Paper, I have said nothing of any mechanical investigations, yet, as a matter of course, before it was read to the Academy, I made every effort to connect my equations in some way with mechanical principles; and it was because I had failed in doing so to my own satisfaction, that $I$ chose to publish the equations without comment," as bare geometrical assumptions, and contented myself with stating orally to the Academy, as I did some months after to the Physical Section of the British Association in Bristol t that a mechanical account of the phenomena still remained a desideratum which no attempts of mine had been able to supply. I am not sure that on the first occasion I stated the precise nature of these attempts, though I

* The circumstances here related will account for what Mr. Whewell (History of the Inductive Sciences, Vor. Ir. p. 449) calls the "obscure and oracular form " in which those equations were published. Having, at that time, no good explanation of them to give, I thought it better to attempt none. But in the general view which I have since taken (see p. 224 of this volume), they do not offer any peculiar diffculty.

+ See "Transactions of the Sections," p. 18. 
incline to think I did; but I have a distinct recollection of havinge done so on the second occasion, in reply to questions that were asked me by some Members of the Association.* Now, my first attempt to explain those equations, which was made almost as soon as I disoovered them, actually turned upon the very idea which about the same time found entrance into the mind of M. CauchyI mean the idea of an unsymmetrical arrangement of the ether. For as it was generally believed, at that period, that the hypothesis of ethereal molecules symmetrically distributed had led, in the hands of M. Cauchy, to a complete theory of rectilinear polarization in crystals, $\uparrow$ the notion of endeavouring to account for the phenomena of elliptio polarization, by freeing the hypothesis from any restriction as to the distribution of the ether, would naturally occur to anyone who was thinking on the subject, no less than to M. Cauchy himself. And though, for my own part, I never was satisfied with that theory, which seemed to me to possess no other merit than that of following out in detail the extremely curious, but (as I thought) very imperfect, analogy which had been perceived to exist between the vibrations of the luminiferous medium and those of a common elastio $\ddagger$ solid (for

* At the period of this meeting, M. Cauchy's letter on Elliptic Polarization had been published for some months; but I was not then aware of its existence. Indeed the letter appears not to have attracted any general notice; for the theory which it contains was afterwards advanced in England as a new one, and M. Cauchy has been lately obliged to assert his prior claim to it, through the medium of Professor Powell.-See notes, pp. 196, 202-3.

† See his Exercices de Mathématiques, Cinquième Année, Paris, 1830, and the Mémoires de l'Institut, tom. x. p. 293.

‡ The analogy was suggested by the hypothesis of transversal vibrations, which, when viewed in its physical bearing, was considered by Dr. Young to be "perfectly appalling in its consequences," as it was only to solids that a "lateral resistance" tending to produce such vibrations had ever been attributed. (Supplement to the Encyclopadia Britannica, VoL. vi. p. 862, Edinburgh, 1824.) He admits, however, that the question, whether fluids may not "transmit impressions by lateral adhesion, remains completely open for discussion, notwithstanding the apparent difficulties attending it." As far as I am aware, Fresnel always regarded the ether as a fluid. M. Poisson affirms that it must be so regarded, and attributes its apparent peculiarities to the immense rapidity of its vibrations, which does not 


\section{, \\ Votes on some Points in the Theory of Light.}

$\therefore$ is usual to regard such a solid as a rigid system of attracting or repelling molecules, and $\mathrm{M}$. Cauchy has really done nothing more than transfer to the luminiferous ether both the constitution of the solid and differential formulas of its vibration), still I should have been glad, in the absence of anything better, to find my equations supported by a similar theory, and their form at least countenanced by the like mechanical analogy. Besides, I recollected that Fresnel himself, in his Memoir on Double Refraction, had indicated a "helicoidal arrangement," or something of that sort, as a probable cause of circular polarization* and as this was an hypothesis of the same kind as the other, only not so general, I was prepared to find that the supposition of an arbitrary arrangement, whatever might be thought of its physical reality, would lead to equations of the same form as those which I had assumed. Upon trial, however, the very contrary proved to be the case; for though it was possible to obtain additional terms, containing differential co-efficients of the third order, multiplied by the same constant $C$, yet this constant always came out with the same sign in both equations, whereas a difference of sign was essential for the expression of the phenomena. I had no sooner arrived at this result than $I$ perceived it to be fatal to the theory of M. Cauchy, and to afford a demonstration of its insufficiency, not only in the particular application which I had made of it, but in all its applications. For the hypothesis

allow the law of equal pressure to hold good in the state of motion (Annates de Chimie, tom. xliv. p. 432). M. Cauchy calls the ether a fluid, though he treats it as a solid. My own impression is, that the ether is a medium of a peculiar kind, differing from all ponderable bodies, whether solid or fluid, in this respect, that it absolutely refuses, in any case, to change its density, and therefore propagates to a distance transversal vibrations only; while ordinary elastic fluids transmit only normal vibrations, and ordinary solids admit vibrations of both kinds. This hypothesis also includes the supposition that the density of the ether is unchanged by the presence of ponderable matter. As to M. Cauchy's third ray, with vibrations nearly normal to the wave, there is no reason to believe that it has even the faintest existence; but it is necessarily introduced by his identification of the vibrations of light with those of an indefinitely extended elastic solid.

* Mémoires de I Institut, tom. vii. p. 73. 


\section{Notes on some Points in the Theory of Light. 20 I}

which I used was, in fact, identical with that theory, in the most general form of which it is susceptible, when unrestricted by any particular supposition as to the arrangement of the ethereal molecules; and therefore the fundamental conception of the theory could not be true, as it not merely failed to explain a large and most remarkable class of phenomena-those of circular and elliptical polarization-but absolutely excluded them, and left no room for their existence. It followed from this, that the mechanical explanation, which the same theory was supposed to have given, of the phenomena of rectilinear polarization and double refraction in crystals, could not be well founded: indeed, as I havio said, I had always distrusted it, and that for various reasons, of which one has been already mentioned, and another was suggested by the forced relations which M. Cauchy had found it necessary to establish among the constants of his theory, and by which he had compelled, as it were, his complicated formulas to assume the appearance of an agreement (though, after all, a very imperfect one) with the simple laws of Fresnel.

Such were the conclusions at which I arrived, and the reflections which they forced upon me, nearly six years ago. They have been frequently mentioned in conversation to those who took an interest in such matters, and their general tenor may be gathered from what I have elsewhere written; ${ }^{*}$ but I did not think it worth while to publish them in detail, because it seemed probable that juster notions would prevail in the course of a few years, and that the ingenious speculations to which I have alluded would gradually come to be estimated at their proper value. But from whatever cause it has arisenwhether from the real difficulties of the subject, or the extreme vagueness of the ideas that most persons are content to form of it, or from deference to the authority of a distinguished mathematician-certain it is that the doctrines in question have not only been received without any expression of dissent, but have been eagerly adopted, both in this country and abroad, by

*Transactions, R. I. A., Vor. xvrr. p. 68 (supra, p. 129). 
a host of followers; and even the extraordinary error, which it is my more immediate object to expose, has been continually gaining ground up to the very moment at which I write, and has at last begun to be ranked among the elementary truths of the undulatory theory of light. Notwithstanding my unwillingness, therefore, to be at all concerned in such discussions, I do not think myself at liberty to remain silent any longer. There are occasions on which every consideration of this kind must give way to a regard for the interests of science.

To show that the principles of $M$. Cauchy contradict, instead of explaining, the phenomenon of elliptio polarization, let us take the axes of co-ordinates as before; and let us suppose, for the sake of simplicity, and to avoid his third ray, that the normal displacements vanish. Then his fundamental equations take the form

$$
\begin{aligned}
& \frac{d^{2} \xi}{d t^{2}}=\Sigma f \Delta \xi+\Sigma h \Delta \eta, \\
& \frac{d^{2} \eta}{d t^{2}}=\Sigma g \Delta \eta+\Sigma h \Delta \xi,
\end{aligned}
$$

where $f, g, h$ are quantities depending on the law of force and the mutual distances of the molecules.* If, therefore,

* I have not thought it necessary to transcribe the original equations of M. Cauchy, which are rather long. He has presented them in different forms; but the system marked (16) at the end of $\$ 1$ of his Memoir on Dispersion, already quoted, is the most convenient, and it is the one which I have here used. The directions of the co-ordinates being arbitrary, I have supposed the axis of $z$ to be perpendicular to the wave-plane. Then, on putting $\zeta=0, \Delta \zeta=0$, in order to get rid of the normal vibration, the last equation of the system becomes useless, and the other two are reduced to the equations (2), given above; the letters $f, g, h$, being written in place of certain functions depending on the mutual actions of the molecules. It will be proved, further on, that this simplification does not at all affect the argument. As the directions of $x$ and $y$ still remain arbitrary, I have made them parallel to the axes of the supposed elliptic vibration.

It may be right to observe, for the sake of clearness, that, when the medium is arranged symmetrically, it is always possible to take the directions of $x$ and $y$ such that the two sums depending on the quantity $h$ may disappear from the equations (2), and then the vibrations are rectilinear. But when the arrangement is unsymmetrical, this is no longer possible. 
we assume that each molecule describes an ellipse, the axes of which are parallel to those of $x$ and $y$; that is to say, if we make

$$
\begin{gathered}
\xi=p \cos \phi, \quad \eta=q \sin \phi, \\
\phi=\frac{2 \pi}{\lambda}(s t-z),
\end{gathered}
$$

and consequently,

$$
\begin{aligned}
& \Delta \xi=p\left(\sin 2 \theta \sin \phi-2 \sin ^{2} \theta \cos \phi\right), \\
& \Delta \eta=-q\left(\sin 2 \theta \cos \phi+2 \sin ^{2} \theta \sin \phi\right),
\end{aligned}
$$

where $\theta=\frac{\pi \Delta z}{\lambda}$, we shall find, by substituting these values in the equations (2), which must hold good independently of $\phi$,

$$
\begin{aligned}
& 8^{2}=A^{\prime}+C^{\prime} k, \quad 8^{2}=B^{\prime}-\frac{C^{\prime}}{k}, \\
& \Sigma f \sin 2 \theta-2 k \Sigma h \sin ^{2} \theta=0, \\
& \Sigma g \sin 2 \theta+\frac{2}{k} \Sigma h \sin ^{2} \theta=0,
\end{aligned}
$$

The equations (2) are precisely the same as those which have been employed by Mr. Tovey and by Professor Powell, the latter of whom, in his lately published work, entitled, "A General and Elementary View of the Undulatory Theory, as applied to the Dispersion of Light, and other Subjects," has dwelt at great length on the theory of elliptic polarization which they have been supposed to afford, and which he regards as a most important accession to the Science of Light. Professor Powell has also made some communications on the subject to the British Association, and has written two Papers about it in the Philosophical Transactions (1838, p. 253 : and 1840, p. 157), besides several others in the Philosophical Magazine. He, however, always attributed this theory of elliptic polarization to Mr. Tovey, until his attention was directed, by a letter from M. Cauchy, to some investigations of the latter which he had not previously seen (Phil. Mag. Vor. XIX. p. 374). Mr. Tovey set out with the principles of M. Cauchy, and therefore naturally struck into the same track, in pursuit of the same object, apparently quite unconscious that anyone had preceded him. It was, indeed, an obvious reflection, that these principles, when generalized to the utmost, ought to include, not only the laws of elliptic polarization, but (as realiy has been thought by $\mathbf{M}$. Cauchy and his followers) of dispersion and absorption, and, in short, of all the phenomena of optics. 
204 Notes on some Points in the Theory of Light.

wherein $k=\frac{q}{p}$ expresses the ratio of the semiaxes of the elliptio vibration, and

$$
\begin{gathered}
A^{\prime}=\frac{\lambda^{2}}{2 \pi^{2}} \Sigma f \sin ^{2} \theta, \quad B^{\prime}=\frac{\lambda^{2}}{2 \pi^{2}} \Sigma g \sin ^{2} \theta, \\
C^{\prime}=\frac{\lambda^{2}}{4 \pi^{2}} \Sigma h \sin 2 \theta .
\end{gathered}
$$

Equating the two values of $s^{2}$, we get, for the determination of $k$, the following quadratio:-

$$
k^{2}+\frac{A^{\prime}-B^{\prime}}{C^{\prime}} k+1=0 .
$$

Now making the substitutions (3) in equations (1), page 197, we have

and thence

$$
8^{2}=A-\frac{2 \pi}{\lambda} C k, \quad 8^{2}=B-\frac{2 \pi}{\lambda} \frac{C}{k},
$$

$$
k^{2}-\frac{\lambda}{2 \pi C}(A-B) k-1=0,
$$

a result which is perfectly inconsistent with the former, since the two roots of (5) have the same sign, if they are not imaginary, while those of (7) have opposite signs, and cannot be imaginary. If, therefore, one equation agrees with the phenomena, the other must contradict them. The last equation indicates that, in the double refraction of quartz, the two elliptio vibrations are always possible, and performed in opposite directions, which is in accordance with the facts; whereas the equation (5), deduced from M. Cauchy's theory, would inform us that the vibrations of the two rays are either impossible or in the same direction."

To apply the results to a particular instance, let us conceive a circularly polarized ray passing along the axis of quartz, or through one of the rotatory liquids, such as oil of

* This conclusion, which shows that M. Cauchy's Theory is in direct opposition to the phenomena, might have been obtained without any reference to the equations (1). But these equations are necessary in what follows. 
turpentine; the position of the co-ordinates $x$ and $y$, in the plane of the wave, being now, of course, arbitrary. In each of these cases we have $k= \pm 1$, and $A=B=a^{2}$, so that the value of $s^{2}$ in equation (6) is expressed by the constant $a^{2}$, plus or minus a term which is inversely proportional to the wave-length $\lambda$; the sign of this term depending on the direction of the circular vibration. Now it will not be possible to obtain a similar value of $s^{2}$ from the formulas (4), unless we suppose $A^{\prime}=B^{\prime}=a^{2}$, since it is only in the expansion. of $C^{\prime}$ that a term inversely proportional to $\lambda$ can be found; but on this supposition the formulas are inconsistent with each other, nor can they be reconciled by any value of $k$. Indeed, when $A^{\prime}=B^{\prime}$, the equation (5) give $k= \pm \sqrt{-1}$. Thus it appears that circular vibrations, such as are known to be propagated along the axis of quartz, and through certain fluids, cannot possibly exist on the hypothesis of $\mathbf{M}$. Cauchy. It was probably some partial perception of this fact that caused M. Cauchy to assert that the vibrations, in these cases, are not exactly circular, but in some degree elliptical-a supposition which, if it were at all conceivable, which we have seen it is not (p. 196), would be at once set aside by what has just been proved; for no assumed value of $k$, whether small or great, will in any way help to remove the difficulty.

But this is not all. Rectilinear vibrations are excluded as well as circular; for we cannot suppose $k=0$ in the equations (4), so long as the quantity $C^{\prime}$, resulting from the hypothesis of unsymmetrical arrangement, has any existence. Thus the inconsistency of that hypothesis is complete, and the equations to which it leads are utterly devoid of meaning.

The foregoing investigation does not differ materially from that which I had recourse to in the beginning of the year 1836. To render the proof more easily intelligible, and to get rid of M. Cauchy's "third ray," which has no existence in the nature of things, I have suppressed the normal vibrations; a procedure which is not, in general, allowable on the principles of M. Cauchy. It will readily appear, however, that this simplifica- 
tion still leaves the demonstration perfectly rigorous in the case of circular vibrations, and does not affect its force when the vibrations are elliptical. For in the rotatory fluids it is obvious that the normal vibrations, supposing such to exist, must, by reason of the symmetry which the fluid eonstitution requires, be independent of the transversal vibrations, and separable from them, so that the one kind of vibrations may be supposed to vanish when we wish merely to determine the laws of the other. The equations (2) are, therefore, quite exact in this case; and they are also exact in the case of a ray passing along the axis of quartz, since such a ray is not experimentally distinguishable from one transmitted by a rotatory fluid, and its vibrations must consequently be subject to the same kind of symmetry. In these two cases, therefore, it is rigorously proved that the values of $k$, which ought to be equal to phus and minus unity, are imaginary, and equal to $\pm \sqrt{-1}$. And if we now take the most general case with regard to quartz, and suppose that the ray, which was at first coincident with the axis of the crystal, becomes gradually inclined to it, the values of $k$ must evidently continue to be imaginary, until such an inclination has been attained that the two roots of equation (5) become possible and equal, in consequence of the increased magnitude of the co-efficient of the second term. Supposing the last term of that equation to remain unchanged, this would take place when the co-efficient of $k$ (without regarding its sign) became equal to the number 2, and the values of $k$ each equal to unity, both values being positive or both negative. The vibrations which before were impossible would, at this inclination, suddenly become possible; they would be circular, which is the exclusive property of vibrations transmitted along the axis; and they would have the same direction in both rays, which is not a property of any vibrations that are known to exist. At greater inclinations the vibrations would be elliptical, but they would still have the same direction in the two rays. These results would not be sensibly altered by regarding the equation (5) as only approximate in the case of rays inclined to the axis; for the last term of that equation, if it 
does not remain the same, can never differ much from unity; since it must become exactly equal to unity, whatever be the direction of the ray, when the crystalline structure is supposed to disappear, and the medium to become a rotatory fluid.

That a theory involving so many inconsistencies should have been advanced by a person of M. Cauchy's reputation would, perhaps, appear very extraordinary, if we did not recollect that it was unavoidably suggested by the general principles which he had previously adopted, and which were supposed, not merely by himself, but by the scientific world generally, to have already afforded the only satisfactory explanation of the laws of double refraction in the common and well-known case where the vibrations are rectilinear. This supposed explanation was obtained, as has been said, by restricting the application of M. Cauchy's principles to the hypothesis of a vibrating medium arranged symmetrically, in which case it was shown that the vibrations were necessarily rectilinear; and of course the removal of this restriction was the only way in which it was possible, on those principles, to account for the existence of circular and elliptical vibrations. Accordingly, when M. Cauchy perceived that, on the hypothesis of unsymmetrical arrangement, the existence of rectilinear vibrations became impossible, and that of elliptic vibrations, generally speaking, possible, he found it very easy to persuade himself that he had obtained a new proof of the correctness of his views, and a new and most important application of the fundamental equations by which his general principles were analytically expressed. To have supposed otherwise would have been to admit that his general principles were false. If the elliptical or quasi-circular vibrations which he was now contemplating were not capable of being identified with those which had been recognized in the phenomena presented by quartz and the rotatory fluids-if their laws were essentially or very considerably different-his theory would be inconsistent with a wide range of well-known 


\section{Notes on some Points in the Theory of Light.}

facts, and, notwithstanding its so-called explanations of other laws, should be finally abandoned. Under these circumstances, therefore, he very naturally supposed that his new results must be in complete harmony with the phenomena discovered by M. Arago, and analyzed so successfully by M.M. Biot and Fresnel; although, had he taken the precaution of acquiring such a clear notion of the phenomena as would have enabled him to translate them into analytical language, he must have perceived that they were entirely opposite to his results, and that this opposition furnished an argument which swept away the very foundations of his theory. For, if the constitution of the luminiferous medium were such as M. Cauchy supposes, the well-known phenomena of circular and elliptio polarization would, as we have seen, be absolutely impossible.

Thus the argument which overturns the particular theory of elliptical polarization destroys at the same time all the other optical theories of M. Cauchy, because they are all built on the principles which we have now demonstrated to be false. But though the principles of M. Cauchy are now, for the first time, formally refuted, they were objected to, on general grounds, so long ago as the year 1830 , by a person whose opinion, on a question of mechanics, ought to have had considerable weight. This was M. Poisson, who, having deduced from the equations of motion of an elastic solid the consequence that such a body admitted vibrations perpendicular to the direction of their propagation, thought it right to remark that this conclusion could not be supposed to account for transversal vibrations in the theory of light, because (as he expressed himself) "the same equations of motion could not possibly apply to two systems [of molecules] so essentially different from each other" as the ethereal fluid and an elastio solid."-(See the Annales de Chimie, tom. xliv.

* As the theory of M. Cauchy (Mem. de l'Institut, tom. x.) had been communicated to the Academy of Sciences some months before the period (October, 1830) at which M. Poisson wrote, there can be no doubt that M. Poisson's remark was directed against that theory, though he did not expressly mention it. 
p. 432). The remark, however, did not meet with much attention from mathematicians, who were, perhaps, not disposed to scrutinize too elosely any hypothesis which gave transversal vibrations as a result. Besides, the hypothesis appeared to go much further, as it offered prima facie explanations of a great variety of phenomena; it was one to which calculation could be readily applied, and therefore it naturally found favour with the calculator ; and as to M. Poisson's objection, it was easily removed by a change of terms, for when the elastic solid was called an "elastic system," there was no longer anything startling in the announcement that the motions of the ether are those of such a system. The hypothesis was therefore embraced by a great number of writers in every part of Europe, who reproduced, each in his own way, the results of M. Cauchy, though sometimes with considerable modifications. Every day saw some new investigation purely analytical-some new mathematical research uncontrolled by a single physical conception-put forward as a "mechanical theory" of double refraction, of circular polarization, of dispersion, of absorption; until at length the Journals of Science and Transactions of Societies were filled with a great mass of unmeaning formulas. This state of things was partly occasioned by the great number of "disposable" constants entering into the differential equations of M. Cauchy and their integrals; for it was easy to introduce, among the constants, such relations as would lead to any desired conclusion; and this method was frequently adopted by $\mathbf{M}$. Cauchy himself. Thus, in his theory of double (or rather triple) refraction, given in the works already cited (p. 145), he supposes three out of his nine constants to vanish, and assumes, among the other six, three very strange and improbable relations, by means of which each of the principal sections of his wave-surface (considering only two out of its three sheets) is reduced to the circle and ellipse of Fresnel's law; and the tiree principal sections being thus forced to coincide, it would not be very surprising if the two sheets were found to coincide in every part with the wave-surface of Fres- 


\section{Notes on some Points in the Theory of Light.}

nel. The coincidence, however, is only approximate; but M. Cauchy is so far from being embarrassed by this ciroumstance, that he does not hesitate to regard his own theory as rigorously true, and that of Fresnel as bearing to it, in point of accuracy, the same relation which the elliptical theory of the planets, in the system of the world, bears to that of gravitation.* Nor is he at all embarrassed by the supernumerary ray belonging to the third sheet of his wave-surface; he assumes at once that such a ray exists, though it was never seen, and promises, for the satisfaction of philosophers, to make known the means of ascertaining its existence. $\dagger$ But he afterwards contented himself with observing that as its vibrations are in the direction of propagation they probably make no impression on the eye, and he then gave it the name of the "invisible ray." ¥

In these investigations, the suppositions which M. Cauchy had made respecting the constants led to the result that the vibrations of a polarized ray are parallel to its plane of polarization; but in the year 1836 he changed his opinion on this point, and then, by reinstating the constants that he had before supposed to vanish, and establishing proper relations amongst them and the rest, he arrived at the conclusion that the vibrations are perpendicular to the plane of polarization.\$ All his other results, of course, underwent some corresponding change; and it is this new theory which must now be regarded as rigorous, while that of Fresnel is to be looked on as approximate. But it is needless to say, that if the accuracy of Fresnel's law of double refraction is to be disputed, it must be on much better grounds than these; and the results of M. Cauchy are certainly too far removed from that law to have any chance of being consonant with truth. Although, for example, his new views respecting the direction of the vibra-

\footnotetext{
* Mémoires de l'Institut, tom, x. p. 313.

† Ibid. p. 305.

† Nouveaux Exercices, p. 40.

\&omptes Rendus, tom. ii. p. 342.
} 
tions agree, in a general way, with those of Fresnel, there is yet, in one particular, an important difference between them; for, according to Fresnel, the vibrations are always exactly in the surface of the wave, while, according to M. Cauchy (in his old theory as well as the new), they are only so in ordinary media. In a biaxal crystal he finds-and this is one of the ways in which the "invisible ray" manifests its influence-that the direction of vibration, in each of the two rays that are visible, is inclined at a certain angle to the wave-plane; but this angle, though small, is by no means inconsiderable, as M. Cauchy seems to intimate, overlooking the fact, which appears from his own equations, that it is of the same order of magnitude as the quantities on which the double refraction depends. It is true, the deviation measured by this angle cannot, if it exists, be directly observed in the refracted light; but its indirect effects on reflected light ought to be very great, since the action of the crystal on a ray reflected at its surface differs from that of an ordinary medium by a quantity of the same order merely as the aforesaid angle; and as the problem of crystalline reflexion has been already solved* on the supposition (which is an essential one in the solution) that the vibrations are exactly in the plane of the wave, it is highly improbable, considering the complex nature of the question, that it will be solved, in any satisfactory way, on a supposition so different as that which is required by the theory of $\mathbf{M}$. Cauchy. However, as the laws of such reflexion are now well known, by means of the solution alluded to, it is possible that M. Cauchy may, as in the case of double refraction, succeed in deducing the same laws, or, if not the same, what may seem to be more exact laws, from certain principles $\uparrow$ of his own, helped out, if need be,

* Transactions, Royal Irish Academy, Vor. xvir., p. 31 (supra, p. 87).

+ In applying these principles to the question of reflexion and refraction at the surface of an ordinary medium (Comptes Rendus, tom. ii. p. 348), M. Cauchy has arrived at the singular conclusion, that light may be greatly increased by refraction through a prism, at the same time that it is almost totally reflected within it. Supposing the refracting angle of the prism to be very little less than the angle of 


\section{I 2 Notes on some Points in the Theory of Light.}

by proper relations among his constants ; especially if, to allow greater scope for such relations, the number of constants be increased by the hypothesis of two coexisting systems of molecules, an hypothesis which M. Cauchy has already considered with his usual generality, but without making any precise application of it.*

Perhaps one cause why M. Cauchy's views on the subject of double refraction have met with such general acceptance may be found in the fact, that a theory setting out from the

total reflexion for the substance of which it is composed, a ray incident perpendicularly on one of the faces will emerge, making a very small angle with the other face; and as the reflexion at the latter face is nearly total, it is self-evident that the intensity of the emergent light, as compared with that of the incident, must be very small. M. Cauchy, however, finds by an elaborate analysis that a prodigious multiplication of light ["une prodigieuse multiplication de a lumiere" ] takes place, the emergent ray being nearly six times more intense than the incident when the prism is made of glass, and nearly nine times when the prism is of diamond. This result was, in a general way, actually verified experimentally by himself and another person; so easy it is, in some cases, to see anything that we expect to see. Had the result been true, it would have been a very brilliant discovery indeed; for then we should have been able, by a simple series of refractions, to convert the feeblest light into one of any intensity we pleased; but the very absurdity of such a supposition should have taught M. Cauchy to distrust both his theory and his experiment. Far from doing so, however, he considers the fact to be perfectly established, and to afford a new argument against the system of emission. "Ici," says he, "un rayon, réfléchi en totalité, est de plus transmis avec accroissement de lumière; ce qui est un nouvel argument contre le système d'émission." The system of emission has at least this advantage, that by no possible error could such a conclusion be deduced from it. For if all the particles of light be reflected, certainly none of them can be refracted.

The truth is, that M. Cauchy mistook the measure of intensity in the hypothesis of undulations, supposing it to be proportional simply to the square of the amplitude of vibration; whereas it is really measured by the vis viva, or by that square multiplied by the quantity of ether put in motion, a quantity which in the present case is evanescent, since the corresponding volumes of ether, moved by the ray within in the prism and by the emergent ray, are to each other as the sine of twice the angle of the prism to the sine of twice the very small angle which the emergent ray makes with the second face of the prism. The intensity of the emergent light is therefore very small, as it ought to be, though the amplitude of its vibrations is considerable.

* Exercices d'Analyse et de Physique Mathématique, tom. i. p. 33. 
same principles, and leading, by the same relations among constants, to formulas identical in every respeot with his earlier results, was advanced independently, and nearly at the same time, by M. Neumann of Königsberg.** A coincidence so remarkable would be looked upon, not unreasonably, as a strong argument in favour of the theory; though it must be allowed that, in the effort to extend the knowledge of any subject, there is a tendeney in different minds to adopt the same errors respecting it, as well as the same truths; a fact of which we have seen other examples in the course of the present article.

According to M. Neumann, $\uparrow$ the "third ray," not being perceived as light, must manifest its existence as radiant heat, or as a chemical power, or as some other agent ["als strahlende Wärme, oder chemisch wirkend, oder als irgend ein anderes Agens"] and he thinks that the nature of this ray will be more easily investigated, if the laws of reflexion shall be deduced from the aforesaid theory. But we have seen that the laws of reflexion are, to all appearance, at variance with the theory, and they take no account whatever of the third ray. Besides, the discoveries which have been made of late years respecting the polarization of radiant heat, and the strong analogies that have been traced between it and light, amount to a demonstration that its vibrations are transversal, and of course essentially different from those of the supposed third ray, which are normal, or nearly so. There is every reason to believe that the vibrations of the chemical rays are also transversal; and we may confidently assert, that the three species of rays-those of light and heat, and the chemical rays-are produced not only by vibrations of the same medium, but by the same kind of vibrations, propagated with nearly the same velocities. If, therefore, the third ray of MM. Cauchy and Neumann has any existence, it must be referred to "some other agent," the nature of which it is impossible to conjecture.

Enough has now been said to show that the optical theory

- Poggendorff's Annals, Vol. xxv. p. 418.

† Ibid. p. 454. 
which we have examined, and which has passed eurrent in the scientifie world for a considerable period, is quite inadequate to explain the leading phenomena of light, and that it is based upon principles which are altogether inapplicable to the subject. M. Cauchy states, in the memoir so often quoted," that the first application which he had made of his principles was to the theory of sound, and that the formulas which he had deduced from them agreed remarkably well with the experiments of Savart and others on the vibrations of elastio solids. As I have already intimated, it is in the solution of such questions (which, however, have long been familiar to mathematicians) that the fundamental equations of $\mathbf{M}$. Cauchy may be most advantageously employed; and had he pursued, his researches in this direction, his labours would doubtless have been attended with more success, and with greater benefit to science.

\section{II.-On Fresnel's Formula for the Intensity of Reflected Light, with Remarks on Metallic Reflexion.}

When Mr. Potter discovered, by experiment, that more light is reflected by a metal at a perpendicular ineidence than at any oblique incidence (at least as far as $70^{\circ}$ ), the fact was looked upon, by himself and others, as contrary to all received theories; and certainly the universal opinion, ûp to that time, was, that the intensity of reflexion always increases with the incidence. It may therefore be worth while to remark, that the formula given by Fresnel for reflexion at the surface of a transparent body, though not of course applicable, except in a very rude way, to the case of metals, would yet lead us to expect, for highly refracting bodies, as the metals are supposed to be, precisely such a result as that obtained by Mr. Potter. For when the index of refraction exceeds the number $2+\sqrt{ } \overline{3}$, or the tangent of $75^{\circ}$; the expression for the intensity of reflected light will be found to have a minimum value at a certain angle of in- 
Notes on some Points in the Theory of Light. 215

cidence; while for all less values of the refractive index the intensity will be least at the perpendicular incidence.

Let $i$ and $i$ be the angles of incidense and refraction, and put;

$$
M=\frac{\sin i}{\sin i^{\prime \prime}}, \quad \mu=\frac{\cos i}{\cos i^{\prime \prime}}
$$

then if $I$ be the intensity of the reflected light, when common light is incident, Fresnel's expression

$$
1=\frac{1}{2}\left\{\frac{\sin ^{2}\left(i-i^{\prime}\right)}{\sin ^{2}\left(i+i^{\prime}\right)}+\frac{\tan ^{2}\left(i-i^{\prime}\right)}{\tan ^{2}\left(i+i^{\prime}\right)}\right\},
$$

in which the intensity of the incident light is taken for unity, may be put under the form

$$
I=\frac{\left(\frac{1}{\mu}-\mu\right)^{2}+\left(\frac{1}{M}-M\right)^{2}}{\left(\frac{1}{\mu}+\mu+\frac{1}{M}+M\right)^{2}},
$$

which has a minimum value when

$$
\mu+\frac{1}{\mu}=M+\frac{1}{M}-\frac{8}{M+\frac{1}{M}} ;
$$

the value of $I$ being in that case

$$
I=\frac{\left(M-\frac{1}{M}\right)^{2}-4}{2\left(M-\frac{1}{M}\right)^{2}}=\frac{\left(M+\frac{1}{M}\right)^{2}-8}{2\left(M+\frac{1}{M}\right)^{2}-8}
$$

and the corresponding angle of inoidence being given by the formula

$$
\sin i=\frac{\sqrt{M} \sqrt[V]{\varepsilon^{2}-1}}{\varepsilon+\sqrt{\varepsilon^{2}-1}}, \text { where } \varepsilon=\frac{1}{4}\left(M+\frac{1}{M}\right) .
$$

Since $\mu+\frac{1}{\mu}$ cannot be less than 2 , it is easy to see that, when 
there is a minimum, $M+\frac{1}{M}$ cannot be less than 4 , and therefore $M$ cannot be less than $2+\sqrt{3}$, or $3 \cdot 732$.

As an example, let $M+\frac{1}{M}=6$. Then, at a perpendicular incidence, one-half the incident light will be reflected. The minimum will be when $i=65^{\circ} 36^{\prime}$, and at this angle only $\frac{7}{16}$ of the incident light will be reflected. The value here assumed for the refractive index is that which Sir J. Hersehel* assigns to mercury ; but if my ideas be correct, it is far too low for that metal.

The only person who supposes that the refractive index of a metal is not a large number is M. Cauchy, It has always been held as a maxim in optics, that the higher the reflective power of any substance, the higher also is its refractive index. But M. Cauchy completely reverses this maxim; for, as I have elsewhere shown, $\uparrow$ it follows from his theory that the most reflective metals are the least refractive, and even that the index of refraction, which for transparent bodies is always greater than unity, may for metals descend far below unity. Thus, according to his formula, the index of refraction for pure silver is the fraction $\frac{1}{4}$, so that the dense body of the silver actually plays the part of a very rare medium with respect to a vacuum. It appears to me that such a result as this is quite sufficient to overturn the theory from which it is derived. The formulas, however, which he gives for the intensity of the reflected light, are identical with the empirical expressions which I had given long before, and are at least approximately true.

In framing my own empirical theory, lative to the value of the refractive index presented themselves. Putting $\boldsymbol{M}$ for the modulus, and $\chi$ for the characteristic, I had to choose between the values $M \cos \chi$ and $\frac{M}{\cos \chi}$. The latter value is that which I adopted; the former, which is M. Cauchy's, was

* Treatise on Light, Art. 594.

+ Comptes Rendus, tom. viii. p. 964 ; vid. note at the end of this volume.

‡ See Proceedings, Vor. I. p. 2 (supra, p. 58). 
rejested, because $I$ saw that it would lead to the result above mentioned.

Another result of M. Cauchy's, which he has given twice in the Comptes Rendus," requires to be noticed. When a polarized ray is reflected by a metal, the phase of its vibration is altered; and if the incidence be oblique, the change of phase is different, according as the light is polarized in the plane of incidence, or in the perpendicular plane. But when the ray is reflected at a perpendicular incidence, it is manifest that the change is a constant yuantity, whatever be the plane of polarization. In fact, the distinction between the plane of incidence and the perpendicular plane no longer exists, and the phenomena must be the same in all planes passing through the ray. Yet M. Cauchy, in the two places above quoted, asserts it to be a consequence of his theory, that in this case the alterations of phase are different for two planes of polarization at right angles to each other, and that the difference of the alterations amounts to half an undulation. The same singular hypothesis had been previously made by M. Neumann, $\uparrow$ whom M. Cauchy appears to have followed; but M. Neumann has since admitted it to be erroneous. +

* Tom. ii. p. 428, and tom. viii. p. 965.

+ Poggendorff's Annals, Vol. xxvr. p. 90.

$\ddagger$ Ibid. VoL. xI. p. 513. 
XVIII.-ON THE PROBLEM OF TOTAI REFLEXICN.

[Proceedings of the Royal Irish Academy, Vor. II.-Read November 30, 1841.]

Professor Mac Cullagh communicated to the Academy a very simple geometrical rule, which gives the solution of the problem of total reflexion, for ordinary media, or for uniaxal crystals.

First, let the total reflexion take place at the common surface of two ordinary media, as between glass and air, and let it be proposed to determine the incident and reflected vibrations, when the refracted vibration is known. It is to be observed, that the refracted vibration (which is in general elliptical) cannot be arbitrarily assumed; for, as may be inferred from what has been already stated," it must be always similar to the section of a certain cylinder, the sides of which are perpendicular to the plane of incidence, and the base of which is an ellipse lying in that plane, and having its major axis perpendicular to the reflecting surface, the ratio of the major to the minor axis being that of unity to the constant $r$. The value of $r$, as determined by the general rule in p. 191, is

$$
r=\sqrt{1-\frac{1}{n^{2} \sin ^{2} i}}
$$

where $i$ is the angle of incidence, and $n$ the index of refraction out of the rarer into the denser medium. The ellipse is greatest

* Proceedings of the Academy, Vor. Ir. p. 102 (supra, p. 192). 
for a particle at the common surface of the media; and for a particle situated in the rarer medium, at the distance $\approx$ from that surface, its linear dimensions are proportional to the quantity $e^{-\frac{2 \pi r z}{\lambda}}$; so that for a very small value of $z$ the refracted vibration becomes insensible.

Now, taking any plane section of the aforesaid cylinder to represent the refracted vibration for a particle situated at the common surface of the two media, let $O P$ and $O Q$ be the semiaxes of the section, and let them be drawn, with their proper lengths and directions, from the point of incidence $O$; through which point also let two planes be drawn to represent the incident and reflected waves. Then conceive a plane passing through the semiaxis $O P$, and intersecting the two waveplanes, to revolve until it comes into the position where the semiaxis makes equal angles with the two intersections; and in this position let the intersections be made the sides of a parallelogram, of which the semiaxis $O P$ is the diagonal. Let $O A$ and $O A^{\prime}$, which are of course equal in length, denote these two sides. Make a similar construction for the other semiaxis $O Q$, and let $O B, O B^{\prime}$, which are also equal, denote the two sides of the corresponding parallelogram. Then will the incident vibration be represented by the ellipse of which $O A$ and $O B$ are conjugate semidiameters, and the reflected vibration by the ellipse of which $O A^{\prime}$ and $O B^{\prime}$ are conjugate semidiameters. And the correspondence of phase in describing the three ellipses will be such that the points $A, A^{\prime}, P$ will be simultaneous positions, as also the points $B, B^{\prime}, Q$.

The same construction precisely will answer for the case of total reflexion at the surface of a uniaxal crystal, which is covered with a fluid of greater refractive power than itself. It is to be applied successively to the ordinary and extraordinary refracted vibrations, and we thus get the uniradial incident and reflected vibrations, or rather the ellipses which are similar to them. And as any incident vibration may be resolved into two which shall be similar to the uniradial ones, we can find the re- 
flected vibration which corresponds to it, by compounding the uniradial reflected vibrations.

It may be well to mention that, in a uniaxal crystal, the plane of the extraordinary refracted vibration is always perpendicular to the axis, and therefore the ellipse in which the vibration is performed may be easily determined by the remark in p. 192. The plane of the ordinary vibration has no fixed position in the crystal; but if we conceive the auxiliary quantities $\boldsymbol{\xi}_{1}, \eta_{1}, \boldsymbol{\zeta}_{1}$ (p. 188), to be compounded into an ellipse (as if they were displacements), the plane of this auxiliary ellipse will be perpendicular to the axis of the crystal.

Whether the preceding very simple construction, for finding the incident and reflected vibrations by means of the refracted vibration, extends also to the case of biaxal crystals, is a point which has not yet been determined, on account of the complicated operations to which the investigation leads, at least when attempted in any way that obviously suggests itself. 


\section{$(221)$}

XIX.-ON THE DISPERSION OF THE OPTIC AXES, AND OF THE AXES OF ELASTICITY, IN BIAXAL CRYSTALS.

[From the Philosophical Magazine, Vox. xxI., October, 1842.]

Is the last number of the Philosophical Magazine (p. 228), there appeared an extract from the Proceedings of the Royal Irish Academy, containing a notice of a memoir which I had the honour of reading to that body on the 24th of May, 1841; and in the concluding paragraph of the notice a brief allusion is made to a "mathematical hypothesis" by which I had connected the laws of dispersion and those of the elliptio polarization of rock-crystal with the other laws that were there announced. My present object is to indicate the development of that hypothesis, with reference more particularly to the subject of dispersion in erystals, and to communicate a very simple result which I have lately had occasion to obtain from it. The result is remarkable as embracing and explaining a class of intricate phenomena which hitherto have not been connected with any theory, or rather have stood in opposition to all theories; I mean the phenomena of the dispersion of the optic axes, and of the axes of elasticity (as they are called) in biaxal crystals.

The name of axes of elasticity was given by Fresnel to three rectangular directions, which, according to his theory, exist in every crystallized medium, and which are distinguished by the property, that if a partiele of the medium be slightly displaced in the direction of any one of them, the elastio foree thereby called into play will act precisely in the line of the dis- 
placement. These directions coincide with the axes of the ellipsoid by which he constructs his wave-surface; and the position of the axes being thus fixed, it is only their lengths that can be supposed to vary for the differently coloured rays. Such is the view taken by Fresnel with regard to crystalline dispersion, and it is obviously the only view that his theory admits. Succeeding theorists, in their numerous attempts to deduce Fresnel's beautiful laws from dynamical principles, have always been obliged to assume that the medium is symmetrically arranged with respect to three rectangular planes; and as, in this hypothesis, the axes of elasticity, or of optical symmetry, necessarily coincide with those of symmetrical arrangement, their directions are fixed, as before, independently of colour.

From these principles it follows that the optic axes for different colours all lie in the same plane, namely, the plane of the greatest and least axes of the ellipsoid, and that they are equally inclined to each of the latter axes, so that the angle made by any pair, to whatever colour they belong, is always bisected by the same right line. This was accordingly, for a long time, believed to be the case; and the earlier experiments of Sir J. Herschel,* which are appealed to by Fresnel, as well as the observations of Sir David Brewster, seemed to establish it as a general law. But it was afterwards discovered by Sir J. Herschel that, in borax, the optic axes for different colours lie in different planes inclined at very sensible angles to each other; and the same discovery was made about the same time (1832) by M. Nörrenberg. The latter observer further ascertained, that even when the optic axes all lie in the same plane there are cases, as in sulphate of lime, wherein their angles are not bisected by the same right line. These facts, and others of a like nature that have been since observed, show the falsehood of the supposition that the lines called the axes of elasticity have always the same directions whatever be the colour of the 
light; they are inconsistent with all received notions, and contradict every theory that has been hitherto proposed. No person, as far as I am aware, has even attempted to explain them.

But in the theory which I have constructed to represent the laws of the action of crystallized bodies upon light, and which has already brought so much within its grasp, the phenomena in question do not offer any difficulty whatever: on the contrary, they are of a kind that would naturally be looked for, antecedently to experiment. For, in this theory, I make no hypothesis as to the constitution of the ether, or the arrangement of its molecules; nor any hypothesis, like that of Fresnel, respecting the mechanical signification of the axes of elasticity. The existence of three rectangular axes possessing peculiar properties is not a principle, but a result, of theory : their directions are determined by conditions perfectly analogous to those which determine the principal axes of an ellipsoid from its general equation; and these directions are functions of certain quantities which are constant when differentials of the second and subsequent orders are neglected, but which vary when these are taken into account. The differentials of higher orders introduce terms depending on the wave-length; and thus the directions, as well as the lengths, of the principal lines depend on the colour of the light, or, to speak more accurately, on the length of the wave.

All this will be easily understood if we recur to the first principles of the theory. According to these, everything depends on the form assigned to the function $V$ in the general dynamical equation

$$
\iiint d x d y d z\left(\frac{d^{2} \xi}{d t^{2}} \delta \xi+\frac{d^{2} \eta}{d t^{2}} \delta \eta+\frac{d^{2} \zeta}{d t^{2}} d \zeta\right)=\iiint d x d y d z \delta \mathrm{V},
$$

from which the motion of the ether is deduced. In my first memoir on the subject (read to the Academy on the 9th of December, 1839), I showed that when differentials of the first order only are preserved, the function $\mathrm{V}$-which may perhaps 
with propriety be called the potential, since the motion of the system is potentially, or virtually, included in it-is a function of the second degree, composed of the three quantities $X, Y, Z$, which are connected with the displacements $\xi, \eta, \zeta$, by the following relations :-

$$
X=\frac{d \eta}{d z}-\frac{d \zeta}{d y}, \quad Y=\frac{d \zeta}{d x}-\frac{d \xi}{d s}, \quad Z=\frac{d \xi}{d y}-\frac{d \eta}{d x} .
$$

To show this, I make use simply of the consideration that the motion must be such as to satisfy the condition

$$
\frac{d \xi}{d x}+\frac{d \eta}{d y}+\frac{d \zeta}{d z}=0
$$

which seems to be characteristic of the vibrations of light. But the same condition allows us to suppose that the potential contains not only the quantities $X, Y, Z$, but their differential ooefficients of any order with respect to the co-ordinates. This supposition, however, is too general, and requires to be limited by other considerations. Now the most natural restriction which can be imposed consists in the assumption that the quantities of all orders are formed on the same type, those of any order being derived from the preceding in the same way that the quantities $X, Y, Z$ are derived from $\xi, \eta, \zeta:$ there are particular reasons also which go to strengthen this hypothesis, and have led me to adopt it. Putting therefore

$$
\begin{array}{lll}
X_{1}=\frac{d Y}{d z}-\frac{d Z}{d y}, & Y_{1}=\frac{d Z}{d x}-\frac{d X}{d z}, & Z_{1}=\frac{d X}{d y}-\frac{d Y}{d x} ; \\
X_{2}=\frac{d Y_{1}}{d z}-\frac{d Z_{1}}{d y}, & Y_{2}=\frac{d Z_{1}}{d x}-\frac{d X_{1}}{d z}, & Z_{2}=\frac{d X_{1}}{d y}-\frac{d Y_{1}}{d x},
\end{array}
$$

and so on, I suppose the potential to be a function of the second degree, composed of all the quantities $X, Y, Z, X_{1}, Y_{1}, Z_{1}, X_{2}$, $Y_{2}, Z_{2}$, \&c. ; and this is the "mathematical hypothesis" alluded to in the beginning of this article. The hypothesis occourred to me more than three years ago (June, 1839), but I did not ven- 
ture to communicate. it to the Academy until the date of my second memoir (May, 1841); and even then I had not studied it with the attention which I now conceive it merits. It was only very lately, in fact, in some conversations which I had with M. Babinet during a short visit to Paris, that my attention was strongly drawn to the subject of dispersion in crystals, particularly the dispersion of the axes of elasticity. My thoughts then naturally reverted to the hypothesis which I have mentioned, and since my return $I$ have found that it affords a complete explanation of all the phenomena.*

I have also found that it gives the general law, extended to biaxal crystals, of that elliptic and circular polarization which has hitherto been detected only in quartz and in certain fluids; while for the case of rectilinear polarization it gives a law (very possibly a true one) more general than that of Fresnel, but quite as elegant, and differing very slightly from it. The hypothesis, therefore, is still too general for our present purpose. To make it include only those crystals to which the law of Fresnel is rigorously applicable, the alternate derivatives $X_{1}$, $\bar{Y}_{1}, Z_{1}, X_{3}, Y_{3}, Z_{3}$, \&e., must be supposed to vanish in the function which represents the potential. Then, the axes of coordinates having any fixed directions within the crystal, the axes of elasticity will be the principal axes of an ellipsoid represented by an equation of the form

$$
A x^{2}+B y^{2}+C z^{2}+2 D y z+2 E x z+2 F x y=1,
$$

in which each of the six coefficients-the first, for example-expresses a series of the form

$$
A_{0}+\frac{A_{1}}{\lambda^{2}}+\frac{A_{2}}{\lambda^{4}}+\frac{A_{3}}{\lambda^{6}}+\& c
$$

where $\lambda$ denotes the wave-length, and all the other quantities

* I am indebted, for my information on the subject, to a short article, drawn up by MM. Quetelet and Babinet, in the Bulletin of the Royal Academy of Brussels, Vou. II. p. 100; as also to Poggendorff's Annals, Vou. xxvi. p. 309 ; VoL. XXxv. p. 81. 
are constant. The ellipsoid itself is the reciprocal of that ellipsoid by which the wave-surface is constructed, and its semiaxes are the three principal indices of refraction. As $\lambda$ is supposed to vary, not only the lengths but the directions of the principal axes vary, and thus we have a different wave-surface for every different wave-length within the crystal.

The optic axes are perpendicular to the circular sections of the above ellipsoid, and describe, in general, two fragments of a cone, the equation of which may be found by supposing $\lambda$ to be variable in the equation of the ellipsoid. But only very particular cases have been hitherto observed, and I shall not stop to discuss them.

J. Mac Cullagh.

Trin. Corl., Dublas, September, 1842. 
XX. - ON THE LAW OF DOUBLE REFRACTION.

[From the Philosophical Magazine, Vou. xxi., 1842].

Having mentioned, in an article* which I sent a few days ago for insertion in the Philosophical Magazine, that I had been led, in following out an hypothesis, to a law of double refraction more general than that of Fresnel, I think it may be well to state very briefly the nature of that law, and to point out the difference between it and the law of Fresnel, especially as I have since observed that the difference is one of a very extraordinary kind, and one which, if it has a real existence (a question which experiment only can decide), may serve to account for phenomena that have seemed hitherto inexplicable.

I have said, in the article referred to, that when the potential $\nabla$, which is a function of the second degree, is supposed to contain only the squares and products of the derivatives $X, Y, Z, X_{2}, Y_{2}, Z_{2}, X_{4}$, \&c., we get the law of Fresnel, as well as the law of crystalline dispersion; but if we make the more general, and apparently the more natural supposition, that it contains also the squares and products of the alternate derivatives $X_{1}, Y_{1}, Z_{1}, X_{3}, Y_{3}, Z_{3}$, \&c., then we get, of course, a different law. Now I find that there will still be two optic axes for each colour, and that the two directions of vibration in a given wave-plane will have the same relation to them as be-

* " On the Dispersion of the Optic Axes, and of the Axes of Elasticity, in Biaxal Crystals" (supra, p. 221): 
fore; while the difference of the squares of the two velocities of propagation will continue proportional to the product of the sines of the angles which the wave normal makes with the optic axes; but the sum of the squares of these velocities will be increased or diminished by a quantity proportional to the square of a perpendicular let fall from the centre on the tangent plane of a certain very small ellipsoid, this tangent plane being supposed parallel to the wave. Such is the general result for biaxal crystals; but its bearing will be best perceived by taking the case of a uniaxal crystal, wherein the law of Fresnel reduces itself to that of Huyghens.

In this case the wave-surface will, instead of the sphere and spheroid of Huyghens, consist of two ellipsoids touching each other at the extremities of a common diameter, which coincides with the axis of the erystal; one ellipsoid differing slightly from a sphere, the other slightly from a spheroid. Neither of the rays will be refracted according to the ordinary law, nor will the wave-surface be symmetrical round the axis. As the law of refraction is unsymmetrical, that of reflexion will be so likewise, and thus we may perhaps obtain an explanation of the extraordinary phenomena observed by Sir David Brewster in reflexion at the common surface of oil of cassia and Iceland spar.

It will no doubt appear strange to eall in question the accuracy of the Huyghenian law, which is generally considered to be established beyond dispute by the experiments of Wollaston and Malus. But the fact is, that no exact experiments have ever been made on the refraction of the ordinary ray. Neither of those philosophers seems to have entertained any suspicion that the ordinary law might be inapplicable to it; they both took for granted that it followed the law of Snellius. But their results seem to be quite consistent with the supposition that the ordinary index, for rays passing in different directions through Iceland spar, may vary in the third place of decimals, perhaps even in the second. The experiments of Rudberg throw no light upon the question, for it happens, 
oddly enough, that though he had two prisms in every other case, he used only one of Iceland spar : he could not therefore compare the velocities of rays passing in different directions. On comparing his numbers, however, with those of Wollaston and Malus, there is, as Sir David Brewster has observed," a "surprising discrepancy," so great indeed as to be quite "alarming." After remarking the difficulty of finding any explanation of it, Sir David concludes that it must arise from the different refractive powers possessed by different specimens. But though this cause must operate in some degree, we cannot tell to what extent it is effeetive, and the discrepancy may notwithstanding be occasioned, in a great measure, by a deviation from the Huyghenian law. The whole question must therefore be reopened, and the ordinary indices for the fixed lines of the spectrum must be determined by means of different prisms cut out of the same piece of Iceland spar.

Whatever the result may be-whether it shall confirm the law of Huyghens, or show that another must be substituted for it-it will at least be useful for science, by removing the uncertainty in which the subject is at present involved.

* Phil. Mag., S. 3, VoL. I. p. 8.

Tris. ColL., Dublin, Sept. 24, 1842. 
XXI.-ON THE LAWS OF METALLIC REFLEXION, AND ON THE MODE OF MAKING EXPERIMENTS UPON ELLIPTIC POLARIZATION.

[Proceedings of the Royal Irish Academy, Vou. II. p. 376.-Read, May 8, 1843.]

Several years ago, as the Academy are aware, I made an attempt to investigate the laws according to which light is reflected at the surface of metals, and I then proposed certain formulø which represented, with sufficient accuracy, all the facts and experiments which I was able to collect upon the subject.* But in order to test these formula satisfactorily, it was nécessary to obtain measurements far more exact than any that had previously been made; and for this end I devised an instrument, which was constructed for me by Mr. Grubb, and of which a brief description has been given in the Proceedings. $\dagger$ I regret to say, however, that nothing of much consequence has yet been done with the instrument. Some preliminary trials of its performance were indeed made in the summer of 1837 , and the results of one of these shall presently be given; but an accidental strain which it suffered, while I.was preparing to undertake a series of experiments, caused me to discontinue the observations at the time; and being then obliged to superintend the printing of my essay on the "Laws of Crystalline Reflexion and Refraction," $\neq$ my attention was drawn afresh to this latter

* See the Proceedings of the Academy, Vou. I. p. 2, October, 1836 (supra, p. 58); Transactions, VoL. xvur. p. 71, note (supra, p. 133).

+ Voz. I. p. 159 (supra, p. 138).

* Transactions R. I. A. (supra, p. 87). 
subject, respecting which some new questions suggested themselves, which I thought it right to discuss in notes appended to the essay. I was not afterwards at leisure to take up the experimental inquiry, until the beginning of the year 1839, when I began to think of putting the instrument in order for that purpose. The strain which it had suffered rendered some slight alterations necessary; and I now resolved to make additions to it also, with the view of operating upon the fixed lines of the spectrum, as a few trials had convinced me that measures sufficiently precise could not be obtained without employing light of definite refrangibility. I wished, moreover, to take the opportunity which the nature of the proposed experiments presented, of verifying the theory of Fresnel's rhomb, or rather of verifying, by means of the rhomb, the formulæ which Fresnel has given for computing the effects of total reflexion, when it takes place at the common surface of two ordinary media. I wrote therefore to Munich for several articles which I wanted; among others, for a set of rhombs cut at different angles, out of different kinds of glass. But while I was waiting for these some months elapsed, and in the meantime $I$ got sight of a new theory, which, from its connexion with my former researches, possessed more immediate interest, and the pursuit of which, in conjunction with other studies and various engagements, caused me again to suspend the inquiry respecting the laws of metallic reflexion. I allude to the "Dynamical Theory of Crystalline Reflexion and Refraction," communicated to the Academy in December, 1839.* This was followed soon after by a general "Theory of Total Reflexion," $\uparrow$ founded on the same principles. The latter theory, forming a new department of physical optics, and involving the solution of questions not previously attempted, was analytically complete when it was communicated to the Academy in May, 1841; but its geometrical development has since required my attention from time to time, and has not yet been brought to that degree of simplicity of which it appears to

- Proceedings, Vol。 I. p. 374, (supra, p. 145).

† Ibid. Vou. II. p. 96 (supra, p. 187). 
be susceptible.* Indeed I have found that, in this instance, the geometrical laws of the phenomena are by no means obvious interpretations of the equations resulting from the analytical solution of the problem; and in endeavouring to verify such supposed laws I have often been led to algebraical calculations of so complicated a nature that it has been impracticable to bring them to any conclusion, and I have been obliged, from mere weariness, to abandon them altogether. On returning, however, to the investigation, after perhaps a long interval of time, I have usually perceived some mode of eluding the calculations, or of directly deducing the geometrical law; and, when the theory comes to be published in its final form, no trace of these difficulties will probably appear.

From the causes above mentioned, combined with frequent absence from Dublin, the researches which I had entered upon, respecting the action of metals upon light, have been hitherto interrupted; and as it may still be some time before they are resumed, I venture, in the meanwhile, to submit to the Academy the results already spoken of, which were obtained on the first trial of the instrument, and which afford the best data that can yet be had for comparison with theory.

The results, it must be confessed, are those of very rough experiments, made one evening (about the month of July, 1837) in company with $\mathrm{Mr}$. Grubb, before I had received the instrument from his hands, and merely with the view of showing him, when it was finished, the kind of phenomena that I proposed to observe with it, and the mode of observing them. But the instrument was so far superior (in workmanship at least) to any apparatus previously employed for this sort of experiments, that it was impossible, without great negligence in using it, not to obtain measures of considerable accuracy. I did not, however, at the time, set much value on these measures, because I expected shortly to possess a series of observations made with every possible precaution; but having chanced to preserve the paper on which they were noted down, I was tempted, a few.

*Proceedings, VoI. II, p. 174 (supra, p. 218). 
lays ago, to try how far they agreed with my formulæ; and the agreement turns out to be so close, that I think myself justified in publishing them. Besides, it will be curious hereafter to compare them with more careful measurements.

Before we proceed, however, to the details of the experiments, it may be well to give the formulæ in a state fitted for immediate application. The light incident on the metal being polarized in a certain plane, let $\boldsymbol{a}$ denote the azimuth of this plane, or the angle which it makes with the plane of incidence ; and as the reflected light will be elliptically polarized, or, in other words, will perform its vibrations in ellipses all similar and equal to each other, as well as similarly placed, put $\boldsymbol{\theta}$ for the angle which either axis of any one of these ellipses makes with the plane of incidence, and let $\beta$ be another angle, such that its tangent may represent the ratio of one axis of the ellipse to the other. Then when the optical constants $M$ and $\chi$ (of which I suppose the first to be a number greater than unity, and the second an angle less than $90^{\circ}$ ) are known for the particular metal, the angles $\theta$ and $\beta$ may be computed for any value of $a$, at any given angle of incidence, by the following formulæ:

$$
\begin{aligned}
& \tan 2 \theta=\frac{\left(v^{\prime}-\nu\right) \sin 2 a}{2 f+\left(\nu^{\prime}+v\right) \cos 2 a}, \\
& \sin 2 \beta=\frac{2 g \sin 2 a}{\nu^{\prime}+\nu+2 f \cos 2 a^{\prime}},
\end{aligned}
$$

in which $f$ and $g$ are constant quantities given by the expressions

$$
f=\left(M-\frac{1}{M}\right) \cos \chi, \quad g=\left(M+\frac{1}{M}\right) \sin \chi,
$$

and $v, v^{\prime}$ are quantities depending on the angle of incidence $i$, in the following way. Let $i^{\prime}$ be an angle such that

$$
\frac{\sin i}{\sin i^{\prime}}=\frac{M}{\cos \chi},
$$


and put

then will

$$
\frac{\cos i}{\cos i}=\mu,
$$

$$
v=\frac{1}{\mu}-\mu, \quad \nu^{\prime}=\frac{f^{2}+g^{2}}{\nu} .
$$

The angles $\theta$ and $\beta$ are given by immediate observation with the instrument; and from their values at any incidence, and for any azimuth $a$ of the plane of primitive polarization, we can find the constants $M$ and $\chi$, which we may afterwards use to calculate the values of $\theta$ and $\beta$ for all other incidences and azimuths, in order to compare them with the observed values. It is indifferent, in the formulø, whether $\theta$ be referred to the major or the minor axis of the elliptic vibration, as also whether $\tan \beta$ be the ratio of the minor to the major axis, or the reciprocal of that ratio ; but in what follows we shall suppose $\theta$ to be the inclination of the plane of incidence to that axis, which, when $a$ is $45^{\circ}$ or less, is always the major axis ; and $\beta$ shall be supposed less than $45^{\circ}$, in order that its tangent may represent the ratio of the minor axis to the major.

When the azimuth $a$ is equal to $45^{\circ}$, the formulø (A) become

$$
\tan 2 \theta=\frac{v^{\prime}-v}{2 f}, \quad \sin 2 \beta=\frac{2 g}{v^{\prime}+v} ;
$$

from which we may deduce the remarkable relation

$$
\frac{\tan 2 \beta}{\cos 2 \theta}=\frac{g}{f}
$$

showing that, in the case supposed, the ratio of $\tan 2 \beta$ to $\cos 2 \theta$ is independent of the angle of incidence. In the experiments which I made with $\mathrm{Mr}$. Grubb this azimuth was always $45^{\circ}$; and the following Table contains the results of observation compared with those obtained by calculation from formulæ $(\mathrm{F})$. The experiments were made upon a small disk of speculum metal; and in the calculations I have taken

$$
M=2 \cdot 94, \chi=64^{\circ} 25^{\prime} .
$$




\begin{tabular}{|c|c|c|c|c|}
\hline \multirow{2}{*}{$\begin{array}{l}\text { Angle of } \\
\text { Incidence. }\end{array}$} & \multicolumn{2}{|c|}{ Value of $\theta$} & \multicolumn{2}{|c|}{ Value of $\beta$. } \\
\hline & Observed. & Calculated. & Observed. & Calculated. \\
\hline $\begin{array}{l}65^{\circ} \\
70 \\
75 \\
80 \\
84\end{array}$ & $\begin{array}{r}27^{\circ} 55^{\prime} \\
1541 \\
-\quad 846 \\
-\quad 3015 \\
-3722\end{array}$ & $\begin{array}{r}27^{\circ} 53^{\prime} \\
1544 \\
-\quad 916 \\
-2925 \\
-3725\end{array}$ & $\begin{array}{lr}28^{\circ} & 0^{\prime} \\
33 & 7 \\
34 & 10 \\
27 & 0 \\
16 & 47\end{array}$ & $\begin{array}{lr}28^{\circ} & 0^{\prime} \\
33 & 1 \\
34 & 6 \\
26 & 53 \\
17 & 17\end{array}$ \\
\hline
\end{tabular}

The light used in these observations was that of a candle placed at a short distance, and was admitted through small apertures at the ends of the tubes.* The Nicol's prism in the first tube having been secured in a position in which its principal plane was inclined $45^{\circ}$ to the plane of incidence, and the two arms having been set at the proper angle with the surface of the metal, the Fresnel's rhomb and the Nicol's prism in the second tube were moved simultaneously, until the image of the candle became as faint as possible. Had light perfectly homogeneous been employed, the image could have been made to vanish altogether; but instead of vanishing, it became highly coloured; and our rule in observing was to make the blue at one side of it, and the red at the other, equally vivid, so as to get results which should belong, as nearly as possible, to the mean ray of the spectrum. When this was done, the angles $\theta$ and $\beta$ (subject, however, to certain corrections which will be hereafter explained) were respectively read off from the divided circles belonging to the rhomb and the prism. The observations were made at large incidences, because it is within the last thirty degrees of incidence that the phenomena go through their most rapid changes.

If we now cast our eyes on the above Table, making due allowance for the uncertainty arising from the dispersion of the metal, we shall be struck with the agreement between the calculated and observed numbers. The differences are greatest in

* Sce the description of the instrument in the Procecdings, VoL. 1. p. 159 (sup $m$, p. 138). 


\section{Laws of Metallic Reflexion, and Mode of}

the last two observations, which, however, were really the first; for the observations were made in the inverse order of the incidences, and their accuracy may have improved as they went on. However that may be, the differences are quite within the limits of the errors of observation; and they are actually less than those which Fresnel found to exist between calculation and experiment in the much simpler case of reflexion at the surface of a transparent ordinary medium, when he proceeded to verify the formula which he had discovered for computing the effect of such reflexion.*

It may seem extraordinary that these experiments should have been in my possession for nearly six years before $\mathrm{I}$ became aware of their close agreement with my formulæ; but the fact is, that I did not regard them with much interest, because, from the circumstances in which they were made, I did not expect more than a general accordance with theory. And even now I am in no haste to infer the absolute exactitude of the formulæ, though they are found to represent the phenomena so well. It was far more allowable to infer that the formula of Fresnel was exact in the case just mentioned, though it appeared to represent the phenomena less perfectly. For, to say nothing of the small number of our experiments, the present is a much more complicated case, and the phenomena depend on two constants instead of one, so that the formulæ might be slightly altered, and yet perhaps continue to agree very well with rough experiments. Where there is only one constant this is not so probable. Again, there is one of the quantities in the preceding formulæ which may be greatly altered without producing more than a slight effect on the values of $\theta$ and $\beta$. This quantity is the ratio of $\sin i$ to $\sin i$, which, according to the value in formula (c), is a number so large as to make the angle $i$ always small, so that its cosine never differs much from unity; and therefore if the above ratio were taken equal to any other large number, the value of $\mu$ in formula (D) would remain nearly the

* See the Table which he has given in the Annales de Chimie, tom. xviii. p. 314. 
same, and consequently the values of $\theta$ and $\beta$ would be but slightly changed.

It is with regard to the value of $\mu$ as a function of the incidence that I entertain the greatest doubts, and if any defect shall be found in the formulø I think it will be here. The relations (c) and (D), from which $\mu$ may be deduced in terms of $i$, were not indeed adopted without strong reasons; but $I$ am not entirely satisfied with them; because, when we reverse the problem, and seek to determine the constants $\boldsymbol{M}$ and $\chi$ from the observed values of $\theta$ and $\beta$ at a given incidence, the results are rather complicated and involved, though the approximate determination is easy enough. As the formulre are in a great measure built upon conjecture, we must not be disposed to receive them without the strongest experimental proofs; and it will certainly require experiments of no ordinary accuracy to decide some of the questions which may be raised respecting them.

When plane-polarized light is incident on a metal, if its vibrations be resolved in directions parallel and perpendicular to the plane of incidence, the effect of the reflexion is to change unequally the phases of the resolved vibrations ; and it may be useful to have the formulø which express the difference of phase after reflexion, and the ratio of the amplitudes of vibration. Put $\phi$ for the difference of phase; and supposing, for simplicity, the incident light to be polarized in an azimuth of $45^{\circ}$, let $\sigma$ be an angle less than $45^{\circ}$, such that $\tan \sigma$ may represent the ratio of the reflected amplitudes respectively perpendicular and parallel to the plane of incidence; then we shall have

$$
\operatorname{tn} \phi=\frac{2 g}{\nu^{\prime}-\nu}, \quad \cos 2 \sigma=\frac{2 f}{\nu^{\prime}+\nu} ;
$$

from which we may infer that

$$
\sin \phi \tan 2 \sigma=\frac{g}{f}
$$

or that the product on the left side of the last equation is independent of the angle of incidence. It is to be observed that the 
relations (G) and (I) are independent of the value of $\mu$, and may hold good though that value should require to be changed.

All the preceding formulæe are merely mathematical consequences of those which I published long ago in the Transactions* of the Academy. The formule which I had previously given in the Proceedings, $\dagger$ are slightly different, and, I think, less likely to be exact, because they are less simple, and do not lead to any of the remarkable relations which may be deduced from the others.

Having had occasion, in the course of the few experiments which I made with the instrument before mentioned, to study the nature of Fresnel's rhomb, which constitutes an important part of it, I shall here describe the method which must be followed in order to obtain true results, when the rhomb is employed in observations on light elliptically polarized. A ray in which the vibrations are supposed to be elliptical is given, and what we want is to determine the ratio of the axes of the elliptic vibration, and their directions with respect to a fixed. plane passing through the ray; in other words, to determine the angles which we have denoted by $\beta$ and $\theta$ in the case of a ray reflected from a metal. For this purpose the ray is admitted perpendicularly to the surface at one end of the rhomb, and after having suffered two total reflexions within, passes out perpendicularly to the surface at the other end. Then causing the rhomb to revolve about the ray, we shall find two positions of it in which the emergent light will be the plane-polarized, these positions being readily indicated by a Nicol's prism interposed between the rhomb and the eye; for such a prism, by being turned round the ray, can make the light totally disappear when it is plane-polarized, but not otherwise. These two positions of the rhomb will be exactly $90^{\circ}$ from each other; in one of them the principal plane of the rhomb (the plane of reflexion within it) will be parallel to the major axis of the elliptic vibration, and the angle which it makes with the plane of inci- 
dence on the metal will be equal to $\theta$ : while in the same position the angle which the principal plane makes with the plane of polarization of the emergent ray (as given by the Nicol's prism) will be equal to $\beta$. In the other position, the principal plane will be parallel to the minor axis of the elliptic vibration, and the corresponding angles will be equal to $90^{\circ}-\theta$ and $90^{\circ}-\beta$ respectively. This, however, proceeds on the supposition that the rhomb is exact. When it is not so, which is of course the proper supposition, and a very necessary one in the experiments with which we are concerned, there will still be, generally speaking, two positions of it in which the emergent ray will be plane-polarized, or in which a disappearance of the light may be produced by the Nicol's prism; but these positions will no longer be $90^{\circ}$ from each other, nor will the principal plane, in either of them, coincide with an axis of the elliptic vibration. If we now measure the angles between the different planes as before, and denote them by $\theta, \beta^{\prime}$ in the first position, and by $90^{\circ}-\theta^{\prime \prime}, 90^{\circ}-\beta^{\prime \prime}$ in the second, we shall find that $\theta^{\circ}$ and $\theta^{\prime \prime}$ are unequal, but we shall have $\beta^{\prime}$ equal to $\beta^{\prime \prime}$. The values of $\theta$ and $\beta$ will then be given by the formulæ

$$
\frac{\theta^{\prime}+\theta^{\prime \prime}}{2}, \quad \cos 2 \beta=\frac{\cos 2 \beta^{\prime}}{\cos \left(\theta^{\prime}-\theta^{\prime \prime}\right)} .
$$

The error of the rhomb may easily be found. Supposing the vibrations to be resolved in directions parallel and perpendicular to its principal plane, the rhomb is intended to produce a difference of $90^{\circ}$ between the phases of the resolved vibrations, or to alter by that amount the difference of phase which may already exist; but the effect really produced is usually different from $90^{\circ}$, and this difference, which I call $\varepsilon$, is the error of the rhomb. The value of $\varepsilon$ is given by the formula

$$
\tan \varepsilon=\frac{\sin \left(\theta^{\prime}-\theta^{\prime \prime}\right)}{\tan 2 \beta} ;
$$

and as the error of the rhomb is a constant quantity, we have 
thus an equation of condition which must always subsist between the angles $\boldsymbol{\theta}^{\prime}-\boldsymbol{\theta}^{\prime \prime}$ and $\beta$. For any given rhomb the sine of the first of these angles is proportional to the tangent of twice the second, and therefore $\theta^{\prime}-\theta^{\prime \prime}$ constantly increases as $\beta$ increases towards $45^{\circ}$, that is, as the axes of the elliptic vibration approach to equality. When $\beta$ is equal to $45^{\circ}-\frac{1}{2} \varepsilon$, we have $\theta^{\prime}-\theta^{\prime \prime}=90^{\circ}$; and for values of $\beta$ still nearer to $45^{\circ}$, the value of $\sin \left(\theta^{\prime}-\theta^{\prime \prime}\right)$ becomes greater than unity, that is to say, it becomes impossible, by means of the rhomb, to reduce the light to the state of plane-polarization. This is a case that may easily happen with an ordinary rhomb in making experiments on the light reflected from metals; because at a certain incidence, and for a certain azimuth of the plane of primitive polarization, the reflected light will be circularly polarized.

The rhomb which I used in the experiments tabulated above was made by Mr. Dollond, and was perhaps as accurate as rhombs usually are; it was cut at an angle of $54 \frac{1}{2}^{\circ}$, as prescribed by Fresnel. Its error was about $3^{\circ}$, and the value of $\theta^{\prime}-\theta^{\prime \prime}$, at the incidence of $75^{\circ}$, was about $7^{\circ}$. But in another rhomb, also procured from Mr. Dollond, and cut at the same angle, the value of $\theta^{\prime}-\theta^{\prime \prime}$, under the same circumstances, was about $20^{\circ}$, and the value of $\varepsilon$ was therefore about $8^{\circ}$. The angle given by Fresnel was calculated for glass of which the refractive index is 1.51 ; and the errors of the rhombs are to be attributed to differences in the refractive powers of the glass. I was not at all prepared to expect errors so large as these when I began to work with the rhomb, and they perplexed me a good deal at first, until I found the means of taking them into account, and of making the rhomb itself serve to measure and to eliminate them. The value of the rhomb as an instrument of research is much increased by the circumstance that it can thus determine its own effect, and that it is not at all necessary to adapt its angle exactly to the refractive index of the glass. It may also be remarked, that this circumstance affords a method of directly and accurately testing the truth of the formulø which Fresnel has given for the case of total reflexion at the sc- 
parating surface of two ordinary media; for we have only to measure the angle of the rhomb and the refractive index of the glass, and to compute, by Fresnel's formula, the alteration which the rhomb ought to produce in the difference between the phases of the resolved vibrations; which alteration of phase we may then compare with that deduced, by means of the formulæ (K) and (L), from direct experiment.

If, in each position of the rhomb, we measure the angle which the plane of polarization of the emergent ray makes with the plane of incidence on the metal, and call the two angles respectively $\gamma^{\prime}, \gamma^{\prime \prime}$, we shall have

$$
\gamma^{\prime}=\theta-\beta^{\prime}, \quad \gamma^{\prime \prime}=\theta^{\prime \prime}+\beta^{\prime},
$$

and therefore

$$
\gamma^{\prime}+\gamma^{\prime \prime}=\theta^{\prime}+\theta^{\prime \prime}=2 \theta, \quad 2 \beta^{\prime}=\gamma^{\prime \prime}-\gamma^{\prime}+\theta^{\prime}-\theta^{\prime \prime} ;
$$

from which it appears that if the rhomb were perfectly exact, that is, if $\theta^{\prime}$ and $\theta^{\prime \prime}$ were equal to each other, the angle $\theta$ would be half the sum of $\gamma^{\prime}, \gamma^{\prime \prime}$, and the angle $\beta$ half their difference. It would then be sufficient to measure the angles $\gamma^{\prime}$ and $\gamma^{\prime \prime}$, in order to get $\theta$ and $\beta$ accurately. And if the rhopb were erroneous, the true value of $\theta$ would still be half the sum of $\gamma^{\prime}$, $\gamma^{\prime \prime}$; but the true value of $\beta$ would not be discoverable without measuring the angles $\theta^{\prime}, \theta^{\prime \prime}$, by the help of which it can be deduced from the second of formulø (N), combined with the second of formulø (K). Nor can we discover whether the rhomb is erroneous or not, without measuring the angles $\theta^{\prime}$, $\theta^{\prime \prime}$; and therefore as these angles must be measured in any oase, the former method of determining $\theta$ and $\beta$ is to be preferred.

In making experiments on elliptically polarized light, a plate of mica, or any other doubly-refracting crystal, placed perpendicular to the ray, may be used instead of Fresnel's rhomb. If the thickness of the crystalline plate be such that the interval between the two rays which emerge from it is equal to the fourth part of the length of a wave, for light of a given refrangibility, the plate will, for such light, perform all the functions 
of the rhomb; the principal plane of the rhomb being represented by the plane of polarization of one of the emergent rays. But unless the light be perfectly homogeneous, this method is liable to great inaccuracy in practice, since the effect of the plate in producing or altering the difference of phase between the two rays which interfere on their emergence from it is inversely proportional to the length of a wave, and will therefore be extremely different for light of different colours, and will change sery perceptibly even within the limits of the same colour. It is true, the effect of the rhomb always varies with the colour of the light: but this variation is trifling compared with that which exists in the other case. It was for this reason that I employed. the rhomb in my experiments, instead of a crystalline plate. The apparatus, however, is much simplified by using such a plate; and if any one chooses to do so, and to work with homogeneous light, he must take care to follow, in every respect, the directions which I have given for conducting experiments with the rhomb. The two cases are precisely similar; and if it be necessary not to neglect the errors of the rhomb, it is certainly not less necessary to take into account those which may arise from a want of accuracy in the thickness of the plate, considering how difficult it is to make the thickness correspond exactly to the particular ray which we wish to observe.

I have been induced to enter into these particulars, respecting the mode of making experiments on elliptic polarization, because the subject is one which has not hitherto been studied; nor does it seem to have occurred to anyone that any precaution was requisite beyond that of getting the rhomb cut as nearly as possible at the proper angle, or the crystalline plate made as nearly as possible of the proper thickness. This, indeed, was quite sufficient for ordinary purposes. For example, light polarized in a plane inclined $45^{\circ}$ to the principal plane of the rhomb or of the plate, would, as far as the eye could judge, be circularly polarized after passing through either of them. Notwithstanding a certain error in the angle of the one, or in the thickness of the other, such light would, when analyzed by a rhomboid of Iceland- 
spar, give two images always sensibly equal in intensity. But an error whioh could not be at all detected in this way might produce a very great effect in such experiments as those upon the metals, and, for the purpose of eomparison with theory, might render them entirely useless, if in the first method of observing we relied upon one set of observations, taking (suppose) the values of $\theta^{\prime}$ and $\beta^{\prime}$ for the true values of $\theta$ and $\beta$; or if, in the second method, we contented ourselves with merely measuring the angles $\gamma^{\prime}$ and $\gamma^{\prime \prime}$.

The necessity of attending to the foregoing rules and remarks will appear from an examination of the experiments of M. de Senarmont, published in the Annales de Chimie.* In these very elaborate experiments, which were made upon light reflected at various incidences from steel and speculum metal, the author followed a plan similar to that which I have adopted, and which, in a general way, I had previously sketched in the Proceedings $\dagger$ of the Academy. There was this differenoe, however, that he used a plate of mica instead of Fresnel's rhomb. Now as he worked with common white light, the use of the mica plate must have rendered two kinds of errors unavoidable. In the first place, it would be impossible always to take the observations for the same ray of the spectrum; and next, as a consequence of this, the thickness of the plate would be generally inexact for the particular ray to which the observations happened to correspond. If the thickness of the plate were exact for a certain ray, it would be very sensibly inexact even for the neighbouring parts of the spectrum; and as the part of the spectrum to which the observations belonged was continually changing, the results obtained for different incidences and azimuths would not be comparable with each other, even though, in each separate case, the error of the plate were allowed for and eliminated. The values of $\theta$, however, as determined by M. de Senarmont, would be correct, so far as this error is concerned; those of $\beta$ alone would be erroneous. For the values of $\theta$ were determined in two ways: by measuring the angles

- Tom. 1xxiii. pp. 351-358. 
$\theta^{\prime}, \theta^{\prime}$, and taking their sum for $2 \theta$; also by measuring the angles $\gamma^{\prime}, \gamma^{\prime \prime}$, and taking their sum for the same quantity. Now each of these methods gives a true value of $\theta$, because by the preceding formulø we have $2 \theta=\theta^{\prime}+\theta^{\prime \prime}=\gamma^{\prime}+\gamma^{\prime \prime}$; and this accounts for the agreement, shown by the tables of M. de Senarmont, between the values* of $2 \theta$ obtained by these different methods. But the values of $\beta$ were deduced from the angles $\gamma^{\prime}, \gamma^{\prime \prime}$, by simply making their difference equal to $2 \beta$; and we see by the second of formulø (N) that, when the plate is not of the proper thickness, this value of $2 \beta$ is erroneous by the whole amount of the angle $\theta^{\prime}-\theta^{\prime \prime}$, the difference between $\beta^{\prime}$ and $\beta$ being supposed so small that it may be neglected. As M. de Senarmont proceeded on the common assumption that when the thickness of the plate has been adjusted to that part of the spectrum to which the observations are intended to refer, it may afterwards, through the whole series of experiments, be regarded as exact, he necessarily conceived $\theta^{\prime}$ and $\theta^{\prime \prime}$ to be the same angle; and it was on the principle of taking an average between two measures of the same quantity that he made the supposition $2 \theta=\theta^{\prime}+\theta^{\prime \prime}$, which happened to be correct. When therefore he found $\theta^{\prime}$ and $\theta^{\prime \prime}$ to be different, he of course looked upon the difference as merely an error of observation, which it would be superfluous to tabulate. Not having the values of this difference, therefore, we have not the means of immediately correcting the values of $2 \beta$. But as observations were made for several azimuths at each angle of incidence, we may use the values of $\theta$ to determine those of $\beta$; for when at any incidence (except that of maximum polarization, where $\theta=0$ for all azimuths) the values of $\theta$ are known for two given values of $\boldsymbol{a}$, we can deduce the corresponding values of $\beta$, without any other theory than that of the composition of vibrations. The values

* Or rather the values of $180^{\circ}+2 \theta$; because the angle $\omega$, the double of which appears in the tables of $\mathbf{M}$. de Senarmont, is equal to $90^{\circ}+\theta$. The angles which he calls $\gamma_{1}$ and $\gamma_{2}$ are equal to $90^{\circ}+\gamma^{\prime \prime}$ and $90^{\circ}+\gamma^{\prime}$ respectively. It therefore comes to the same thing, whether the one set of angles or the other is supposed to be measured. The letter $\beta$ has the same signification in both notations. 
of $\beta$ so deduced must indeed be expected to be very inaccurate, partly because of errors in the observed values of $\theta$, partly because the observations in different aximuths do not answer to the same ray of the spectrum ; but they will be accurate enough to show the great amount of the error committed by neglecting the difference $\theta^{\prime}-\theta^{\prime \prime}$. For example, putting $\theta_{0}$ and $\beta_{0}$ for the values of $\theta$ and $\beta$ when $a=45^{\circ}, \mathbf{M}$. de Senarmont gives, at the incidence of $60^{\circ}$ upon steel, $2 \theta_{0}=64^{\circ} 15^{\prime}$ (taking the mean of his two determinations), and for the aximuths $55^{\circ}, 30^{\circ}, 25^{\circ}$, he gives $2 \theta$ equal to $88^{\circ} 5^{\prime}, 37^{\circ} 2^{\prime}$, and $29^{\circ} 36^{\prime}$ respectively. Combining these values of $2 \theta$ in succession with that of $2 \theta_{0}$, we get for $2 \beta_{0}$ the series of values $32^{\circ} 38^{\prime}, 33^{\circ} 28^{\prime}, 34^{\circ} 30^{\prime}$; the differences between which are to be attributed to the causes above stated. The mean value of $2 \boldsymbol{\beta}_{0}$ thus found is $33^{\circ} 32^{\prime}$; while its value, as given by $\mathbf{M}$. de Senarmont, is only $28^{\circ} 41^{\prime}$. The difference $4^{\circ} 51^{\prime}$ is the value of $\theta^{\prime}-\theta^{\prime \prime}$, which, divided by the tangent of $2 \beta_{0}$, gives $7^{\circ} 19^{\prime}$ for the mean value of $\varepsilon$, the error of the mica plate corresponding to that part of the spectrum which was observed at the incidence of $60^{\circ}$.

At incidences nearer the angle of maximum polarization the errors are probably much greater. Beyond that angle they again diminish, and in some cases they almost vanish. Thus, at the incidence of $85^{\circ}$ upon steel, with the value of $2 \theta_{0}$ and the value of $2 \theta$ corresponding to $a=20^{\circ}$, we get, by com putation, a value of $2 \beta_{0}$, which differs only by a few minutes from that given by M. de Senarmont. Nearly the same thing happens at the same incidence when we take $a=25^{\circ}$. In these eases, therefore, the results belong to that particular ray for which the thickness of the plate was exact.

The observations of M. de Senarmont on speculum metal were not carried beyond the incidence of $60^{\circ}$. He $_{\theta}$ states that he was unable to observe at higher incidences, on account of the uncertainty arising from the dispersion of the metal; but though this cause operated in some degree, his embarrassment must have been really occasioned by the increasing magnitude of the difference $\theta^{\prime}-\theta^{\prime \prime}$, as he approached the angle of maximum po- 
larization; that difference being perhaps twice as great as in the case of steel. My own experiments on speculum metal were all made, as has been seen, at incidences greater than $60^{\circ}$.

The experiments of M. de Senarmont do not at all agree with the formulø; and therefore I have been obliged to analyze his mothod of observation, and to show that it could not lead to correct results. It is to be regretted that his method was defective, as the zeal and assiduity which he has displayed in the inquiry would otherwise have put us in possession of a large collection of valuable data.

I shall conclude by saying a few words respecting the intensity of the light reflected by metals. The formulø for computing this intensity have been given in the Transactions of the Academy, in the place already referred to; but they may be here stated in a form better suited for calculation. If we suppose $\psi$ and $\psi^{\prime}$ to be two angles, such that

$$
\operatorname{cotan} \psi=\frac{M}{\mu}, \quad \operatorname{cotan} \psi^{\prime}=M \mu
$$

and then take two other angles $\omega, \omega^{\prime}$, such that

$$
\cos \omega=\sin 2 \psi \cos \chi, \quad \cos \omega^{\prime}=\sin 2 \psi^{\prime} \cos \chi,
$$

we shall have

$$
\tau=\tan \frac{1}{2} \omega, \quad \tau^{\prime}=\tan \frac{1}{2} \omega^{\prime},
$$

where $r$ is the amplitude of the reflected rectilinear vibration, when the incident light is polarized in the plane of incidence, and $\tau^{\prime}$ is the amplitude of the reflected vibration when the incident light is polarized perpendicularly to that plane; the amplitude of the incident vibration being in each case supposed to be unity. Hence when common light is incident, if its intensity be taken for unity, the intensity $I$ of the reflected light will be given by the formula

$$
I=\frac{1}{2}\left(\tan ^{2} \frac{1}{2} \omega+\tan ^{2} \frac{1}{2} \omega^{\prime}\right) .
$$

If with the values of $\boldsymbol{M}$ and $\chi$ determined by my experi- 
ments we compute, by the last formula, the intensity of reflexion for speculum metal at a perpendicular incidence, in which case $\mu=1$, we shall find $I=\cdot 583$. This is considerably lower than the estimate of Sir William Herschel, who, in the Philosophical Transactions for 1800 (p. 65), gives 673 as the measure of the reflective power of his specula. The same number, very nearly, results from taking the mean of Mr. Potter's observations.* It might seem therefore that the formula is in fault; but I am inclined to think that the metal which I employed had really a low reflective power. Its angle of maximum polarization was certainly much less than that of the speoulum metal used by Sir David Brewster, $\uparrow$ who states the angle to be $76^{\circ}$, whereas in my experiments it was only about $73 \frac{1}{2}^{\circ}$; and 'any increase in this angle, by increasing the value of $M$, raises the reflective power. On the other hand, the maximum value of $\beta$ (when $a=45^{\circ}$ ) was greater than that given by Sir David Brewster, namely, $32^{\circ}$; and any increase in $\beta$ tends also to increase the reflective power. Now it is not unreasonable to suppose that the highest values of both angles may be most nearly those which belong to the best specula; and accordingly, if we take $76^{\circ}$ for the incidence of maximum polarization, and retain the maximum value of $\beta$, namely, $34^{\circ} 37^{\prime}$, which results from my experiments, we shall get $M=3 \cdot 68, \chi=66^{\circ} 16^{\prime}$, and the value of $I$ at the perpendicular incidence will come out equal to .662, which soarcely differs from the number given by Herschel.

It is clear from what precedes that the optical constants are different for different specimens of speculum metal, and this is no more than we should expect, from the circumstance that the metal is a compound, and therefore liable to vary in its eptical properties from variations in the proportion of its constituents; but I am disposed to believe that the same thing is generally true, though of course in a less degree, of the simple metals; so that in order to render the comparison satisfactory, the measures of intensity should always be made on the same spe-

* Edinburgh Journal of Science, New Series, Vor. IIr. p. 280.

† Philosophical Transactions, 1830, p. 324. 
cimen which has furnished the values of $M$ and $\chi$. There is one metal, however, with respect to which there can be no doubt that the experiments of different observers are strictly comparable, when it is pure, and at ordinary temperatures-I mean mercury. For this metal Sir David Brewster states the angle of maximum polarization to be $78^{\circ} 27^{\prime}$, and the maximum value of $\beta$, when $\alpha=45^{\circ}$, to be $35^{\circ}$; from which $I$ find $M=4 \cdot 616$, $\chi=68^{\circ} 13^{\prime}$, and, at the perpendioular incidence, $I=\cdot 734$. Now Bouguer observed the quantity of light reflected by mercury, but not at a perpendicular incidence. His measures were taken at the incidences of $69^{\circ}$ and $78 \frac{1}{2}^{\circ}$, for the first of which he gives, by two different observations, $\cdot 637$ and $\cdot 666$; for the second, by two observations, $\cdot 754$ and $\cdot 703$, as the intensity of reflexion." If we make the computation from the formula, with the above values of $M$ and $\chi$, we find the quantities of light reflected at these two incidences to be, as nearly as possible, equal to each other, and to seven-tenths of the incident light, the intensity of reflexion being a minimum at an intermediate incidence; and if we suppose these quantities to be really equal at the incidences observed by Bouguer, we must take the mean of all his numbers, which is 69 , as the most probable result of observation. This result differs but little from one of the two numbers given by him at each incidence, and scarcely at all from the result of calculation.

The angle at which the intensity of reflexion is a minimum, when common light is incident, may be found from the formula

$$
\left(M+\frac{1}{M}\right)\left(\mu+\frac{1}{\mu}\right)=\left(M-\frac{1}{M}\right) \sqrt{\left(f^{2}+g^{2}\right)}-4 \cos \chi,
$$

which gives the values of $\mu$, and thence that of $i$. This incidence for mercury is, by calculation, $75^{\circ} 15^{\prime}$, and the minimum value of $I$ is $\cdot 693$, which is less than its value at a perpendicular incidence by about one-eighteenth of the latter. According to the formulio, the reflexion is always total at an incidence of $90^{\circ}$.

* See his Traité d' Optique sur la Gradation de la Lumière: Paris, 1760; pp. 124, 126. 
XXII. ON THE ATTEMPT LATELY MADE BY M. LAURENT TO EXPLAIN, ON MECHANICAL PRINCIPLES, THE PHENOMENON OF CIRCULAR POLARIZATION IN LIQUIDS.

[Abstract of a Communication addressed to the British Assocration, in the year 1843. Report, p. 7.]

THE Author showed that this attempt had not succeeded. M. Laurent supposes the particles of the luminiferous ether not to be simply material points, but to have dimensions which are not insensible when compared with their distances; and on this hypothesis he deduces a system of differential equations, the integrals of which he conceives to represent the phenomenon in question. The integrals given by M. Laurent are, however, altogether erroneous, though this circumstance was not noticed by M. Cauchy in the remarks and comments which he made on M. Laurent's Memoir. The true integrals of these equations (supposing the equations to be correctly deduced) were shown by Professor MacCullagh to indicate motions of the ether which do not correspond to the observed phenomenon. The account of M. Laurent's theory, with M. Cauchy's remarks upon it, will be found in the eighteenth volume of the Comptes Rendus of the Academy of Sciences of Paris. 
XXIII.ON TOTAL REFLEXION.

Proceedings of the Royal Irish Academy, Vou. IIr. p. 49.-Read, January 13, 1845.

Professor Mac Collagh made a communication on the subject of Total Reflexion.

In the case of total reflexion the vibrations which take place in the rarer medium are in general elliptical, and when this medium is a crystal, the equations by which the ellipse of vibration is determined are very complicated. The projection of this ellipse upon the plane of incidence may, however, be easily found by the remark in p. 12 of the present volume; the projecting cylinder is therefore known, and as the ellipse of vibration is a section of this cylinder, the question of determining the ellipse is reduced to that of determining its plane. For this purpose Mr. Mac Cullagh gave the following rule. Having constructed the ellipsoid of indices (that whose axes are parallel to the axes of elasticity, and inversely proportional to the three principal velocities of propagation in the crystal), let its two planes of circular section intersect the aforesaid cylinder. The curves of intersection will be ellipses, which shall be supposed to have a common centre $O$ in the axis of the cylinder. Let $O P, O P^{\prime}$ be the greater semiaxes of these ellipses, and $O Q, O Q^{\prime}$ the less semiaxes; the lengths of the two former being denoted by $p, p^{\prime}$, and the lengths of the two latter by $q, q^{\prime}$. Join the extremities $P, P^{\prime}$ of the greater semiaxes, and the extremities $Q, Q^{\prime}$ of the less semiaxes; and 
divide each of the right lines $P P^{\prime}, Q Q^{\prime}$, in the ratio of $\sqrt{p^{2}-q^{2}}$ to $\sqrt{p^{\prime 2}-q^{\prime 2}}$. Then a plane drawn through the centre $O$ and the two points of division will be the plane of vibration. In the application of this rule some precautions are to be observed, but they need not here be insisted on.

The foregoing rule was deduced (in the year 1843) from the general equations by a peculiar use of imaginary quantities, after the author had several times tried in vain to obtain a geometrical interpretation of those equations by considerations of a more obvious and ordinary kind. This use of imaginaries is founded on a remarkable theorem relative to the ellipse, by which it appears, that the plane of an ellipse and its species (that is, the directions and the ratio of its axes) may be expressed by two imaginary constants, just as the direction of a right line in space is expressed by two real constants. By means of this theorem-which it is unnecessary to repeat, as it has been published in the University Calendar*-we may find such properties of elliptical vibrations as are analogous to those of rectilinear vibrations; and it was in this way that the above rule was discovered. It is analogous (though it searcely appears so at first sight) to the rule by which, in the theory of Fresnel, the direction of rectilinear vibrations is determined, when the plane of the wave is given. follows:-

" "Examination Papers" of the year 1842, p. Ixxxiv. [The theorem is as

Given an ellipse in space, the origin of co-ordinates being taken at the centre. Let the points $x, y, z, x^{\prime}, y^{\prime}, z^{\prime}$, be the extremities of conjugate diameter. Then the imaginary quantities

$$
\frac{y+y^{\prime} \sqrt{-1}}{x+x^{\prime} \sqrt{-1}}, \quad \frac{z+z^{\prime} \sqrt{-1}}{x+x^{\prime} \sqrt{-1}}
$$

are constant for every system of conjugate diameters.] 



\section{PART II.}

G E O M E T Y. 



\section{I.-GEOMETRICAL THEOREMS ON THE RECTIFICATION OF THE CONIC SECTIONS.}

[Travisactions of the Royal Irish Academy, VoL. XVI. p. 79.-Read, June 21, 1830.]

Lemma 1. Let $T$ and $t$ be two points indefinitely near each other on any given curve $A T$, and let tangents at $T$ and $t$ meet in the points $P$ and $p$ any other given line $M N$, straight or curved, and. draw $P_{q}$ perpendicular to $t p$; then the difference between the aro $A T$ and the tangent $T P$ will exceed or fall short of the

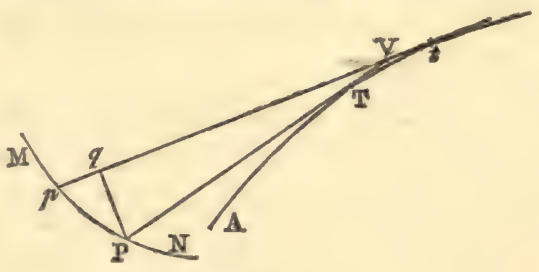

Fig. 1.

difference between the are $A t$ and the tangent $t p$ by a quantity which is ultimately to $p q$ in a ratio of equality.

For the increment of $T P$, or the difference of $T P$ and $t p$, is ultimately equal to the sum of $p q, V T$, and $V t$ ( $V$ being the intersection of $p t$ and $P T$ produced); and $T t$, or the increment of the arc $A T$, is ultimately equal to the sum of $V T$ and $V t$; therefore the difference of the increments is ultimately equal to $p q$. Whence the proposition is manifest.

Problem.-To find the Length of the Are of a Parabola.

Let $F$ be the focus and $A$ the vertex of a parabola $A T$ : draw $A K$ perpendicular to $A F$, and let a tangent at the point $T$ meet it in $P$; take $p$ indefinitely near to $P$, and let $F p$ 
intersect $P T$ in $q$ : then since $F P$ and $F p$ are perpendicular to the tangents $P T$ and $p t$, it follows (by the preceding lemma) that $P q$ is ultimately equal to the increment of the difference between the are $A T$ and the tangent TP. With the centre $E$ and semiaxis $F A$ describe the equilateral hyperbola $G A H$, and bisect the angles $A F P, A F p$ by the straight lines $F L, F l$. Then (since the square of the radius vector of an equilateral hyperbola is inversely as the cosine of twice the angle which it makes with the axis) the square of $L F$ will be equal to $A F \times P F$; and because the angle $L F l$ is half the angle $P F q$, there-

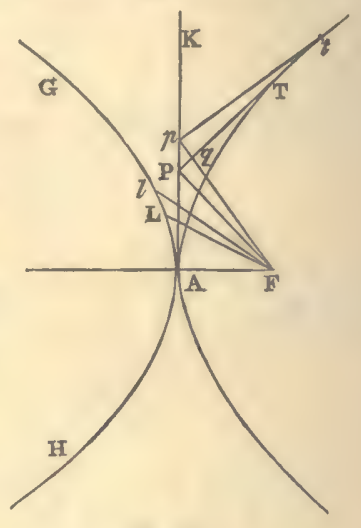

Fig. 2. fore the area $L F l$ will be equal to one-fourth of the rectangle under $A F$ and $P q$.

Hence the hyperbolic sector $A F L$ is equal to one-fourth of the rectangle under $A F$ and the difference between the parabolio are $A T$ and the tangent $T P$.

Lemma 2. If on either axis of an ellipse a semicircle be described, of which $C D$ and $C H$ are two radii at right angles to each other, and if $D N$ and $H M$ be drawn perpendicular to the axis $a A$, and meeting the ellipse in $E$ and $L$; then $C E$ and $C L$ will be conjugate semidiameters.

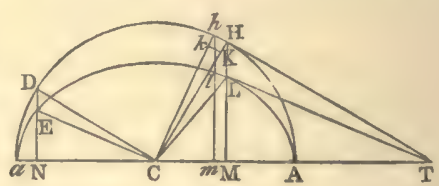

Fig. 3.

For tangents at $H$ and $L$ will meet in a point $T$ in $A a$ produced; the triangles $H M T$ and $D N C$ will be similar, and $D N$ and $H M$ will be similarly divided in $E$ and $L$; therefore $C E$ and $T L$ will be parallel, and consequently $C E$ and $C L$ will be conjugate semidiameters.

Lemma 3. Take in the ellipse a point $l$ indefinitely near to $L$, and draw through it $m h$ parallel to $M H$; join $C h$, and with the centre $C$ and a radius equal to $C E$ describe the are $K k$ 
meeting $C H$ and $C h$ in $K$ and $k$ : then $K k$ will be ultimately equal to $\mathrm{Ll}$.

For $L l: H h:: L T: H T:: C E: C D:: C K: C H:: K k: H h$. Therefore $L l=K k$.

Lemma 4. If the semiaxes $A C$ and $B C$ of an ellipse be equal to the sum and difference of the sides $P Q, Q R$, of a triangle $P Q R$, and if the angle $B C D$ be equal to half the contained angle $P Q R(A D a$ being the semicircle on $A a)$; then, $D E N$ being drawn perpendicular to $A a, C E$ will be

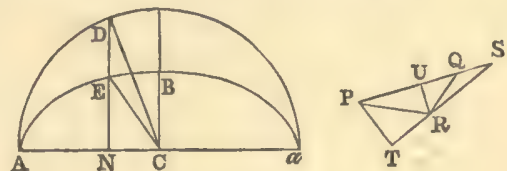

Fig. 4. equal to the base $P R$. Take $Q U$ and $Q S$ equal to $Q R$; then $P S$ and $P U$ will be equal to $A C$ and $C B$, and the angle $C D N$ to $T S P$; therefore drawing $P T$ perpendicular to $T S, D N$ and $N C$ will be equal to $T S$ and $T P$. But $U R S$ being a right angle, $U R$ is parallel to $P T$, and therefore $T S: T R:: P S: P U:: A C$ $: B C:: D N: E N$; but $T S=D N$, therefore $T R=E N$; and since $P T=C N$, it follows that $P R=C E$.

\section{Theorem.}

Let $A T$ and $A^{\prime} T^{\prime}$ be an ellipse and hyperbola, the semiaxis ( $C A$ or $C^{\prime} A^{\prime}$ ) of either being equal to $\left(C^{\prime} F^{\prime}\right.$ or $\left.C F\right)$ the distance between the focus and centre of the other; and let tangents at the points $T$ and $T^{\prime \prime}$ meet in $P$ and $P^{\prime}$ the circles desoribed on the axes, so that $F P=F^{\prime} P^{\prime}$ : let

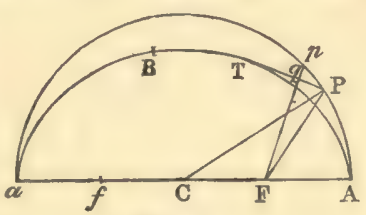

Fig. 5 . also $a^{\prime \prime} B^{\prime \prime} A^{\prime \prime}$ be another ellipse whose semiaxes $\left(A^{\prime \prime} C^{\prime \prime}\right.$ and $B^{\prime \prime} C^{\prime \prime}$ ) are equal to $a F$ and $F A$, and take in its circumference a point $L$ so that the semidiameter conjugate to that passing through $L$ may be equal to $F P$ or $F^{\prime} P^{\prime}$; then will the excess of the ellip-

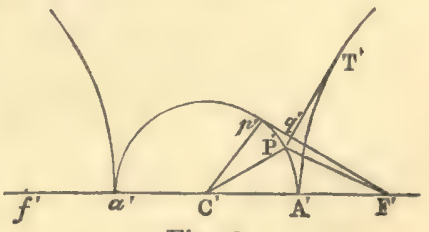

Fig. 6 . tic are $A T$ above its tangent $T P$ be greater than the excess of 
the hyperbolic are $A^{\prime} T^{\prime}$ above its tangent $T^{\prime} P^{\prime}$, by twice the elliptio are $A^{\prime \prime} L$.

Take the point $L$ so that when the ordinate $M L H$ is drawn to meet in $H$ the semicircle described on the axis, the angle $H C^{\prime \prime} M$ may be equal to half the angle $P C F$ (or $P^{\prime} C^{\prime} F^{\prime}$, for the triangles $P C F$ and $P^{\prime} C^{\prime} F^{\prime}$ have all their sides and angles equal); draw $C^{\prime \prime} D$ perpendicular to $C^{\prime \prime} H$, and $D E$ to $A^{\prime \prime} d^{\prime \prime}$, meeting the

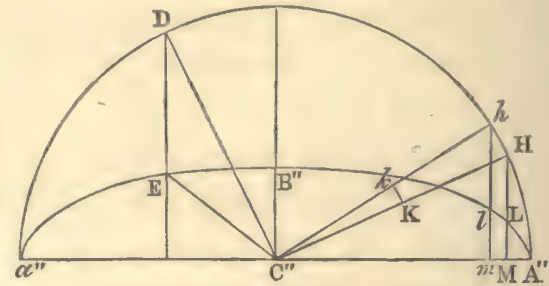

Fig. 7. ellipse in $E$; then, by lem. 2, $C^{\prime \prime} E$ will be conjugate to $C^{\prime \prime} L$, and by lem. 4 it will be equal to $F P$, since the angle $B^{\prime \prime} C^{\prime \prime} D$ is equal to $H C^{\prime \prime} M$, and is therefore half of $P C F$, whilst the semiaxes $C^{\prime \prime} A^{\prime \prime}, C^{\prime \prime} B^{\prime \prime}$, are the sum and difference of $P C$ and $C F$. Hence the point $L$ thus found is that required by the enunciation. Take $p, p^{\prime}, h$, indefinitely near to $P, P^{\prime}, H$, and similarly related to each other; let $F p$ and $F p^{\prime}$ intersect $T P$ and $T^{\prime} P^{\prime}$ in $q$ and $q^{\prime}$, and with the centre $C^{\prime \prime}$ and a radius equal to $C^{\prime \prime} E$ describe the evanescent are $K k$. Then $F P, F^{\prime} P^{\prime}$ are always perpendicular to $T P, T^{\prime} P$, and therefore $P q$ is ultimately the increment of the difference between the aro $A T$ and the tangent $T P$ (lem. 1.) and $P^{\prime} q^{\prime}$ the increment of the difference between the are $A^{\prime} T^{\prime}$ and the tangent $T^{\prime} P^{\prime}$; also by lem. $3 . K k$ is ultimately equal to $L l$, the increment of the are $A^{\prime \prime} L$. Now the angles $C F P$ and $C F p$ are equal to $C^{\prime} P^{\prime} F^{\prime}$ and $C^{\prime} p^{\prime} F^{\prime}$; therefore $P F p$ is equal to the sum of $P^{\prime} F^{\prime} p^{\prime}$ and $P^{\prime} C^{\prime} p$, or to $P^{\prime} F^{\prime} p^{\prime}$ with twice $H C^{\prime \prime} h$ : but $F P, F^{\prime} P^{\prime}$, and $C^{\prime \prime} K$ are all equal, and therefore $P q$ is equal to $P^{\prime} q^{\prime}$ and twice $K k$, or to $P^{\prime} q^{\prime}$ and twioe $L l$. Whence the proposition is manifest.

Schol. The angle $B^{\prime \prime} C^{\prime \prime} H$ is half the angle $f C P$ or $f^{\prime} C^{\prime} P^{\prime}$, and therefore by lem. 4 , the semidiameter $C^{\prime \prime} L$ is equal to the straight line $f P$ or $f^{\prime} P^{\prime}$. This gives another and easier method of finding the point $L$.

Hence every are of a hyperbola may be found by means of 


\section{Rectification of the Conic Sections.}

two elliptio arcs. This beautiful theorem was discovered by Landen, and is now for the first time demonstrated geometrically; but the manner in which it is stated by him and by succoeding writers differs from the above, and is much more complicated. The different forms under which they have presented it may be easily deduced from the preceding, by means of the theorem of Fagnani, of which a geometrical demonstration was first given in vol. ix. of the Tiransactions of the Royal Irish Academy, by the Lord Bishop of Cloyne.

December 23; 1829. 
II.-ON THE SURFACES OF THE SECOND ORDER.

[Proceedings of the Royal Irish Academy, VoL. II. p. 446.-Read Nov. 30, 1843.]

THere is hardly any geometrical theory which more requires to be studied, or which promises to reward better whatever thought may be bestowed upon it, than that of the surfaces of the second order. My attention was drawn to it, many years ago, by the consideration of mechanical and physical questions. In the dynamical problem of the Rotation of a Solid body, and in the investigation of the properties of the Wave-Surface of Fresnel, I found, so long since as the year 1829, that the ellipsoid could be employed with very great advantage; while the discussion of these questions, but especially of the former," suggested properties of the ellipsoid and its kindred surfaces which I might not otherwise have perceived. In this manner I was led to consider systems of confocal surfaces, and thence to notice the focal curves, which I discovered to be analogous, in the theory of the surfaces of the second order, to the foci in that of the plane conic sections. That theory now began to interest me on its own account, and, guided by analogy, I struck out the leading properties possessed by the surfaces in relation to their focal curves; but the interference of other matters prevented $m e$ from continuing the inquiry. I had done enough, however, in this

* The Theory of Rotation, here spoken of, was completed in the year 1831; but, from causes which need not be mentioned at present, it was not published. The investigations relative to Fresnel's Wave-Surface will be found in the Transactions of the Royal Irish Academy, Vou. xvi. p. 65; Vor. xvir. p. 241. See also Vor. xxi. p. 32, of the same Transactions. 
and other parts of the theory, to open new views respecting it; and the results at which $I$ had arrived seemed so fitted for instruction, that, when I was appointed Professor of Mathematics in the University, I made them the subject of the first lectures which I gave in that capacity, in the beginning of the year 1836. Next year the heads of these lectures were communicated to this Academy, in a Paper of which a very short abstract appeared in the Proceedings." The subject soon became a favourite one among the more advanced students in the University, who are, for the most part, excellent geometers, and in the present Article very little will be found which is not well known amongst them; very little, indeed, which was not communicated to the Academy on the occasion just mentioned, or which may not be gathered, in the shape of detached questions, out of the Examination Papers published in the University Calendar. But as nothing has yet been published on the subject in a connected form, except the brief notice in the Proceedings of the Academy, and as mathematicians in other countries attach some importance to researches of this kind, and appear to be in quest of certain principles which are familiar to us here, it seems proper to collect together the chief results that have already been obtained, in order that persons wishing to pursue these speculations may be better able to judge where their inquiries should begin, and in what direction further progress is most likely to be made.

\section{Part I.-Gleneration of Surfaces of the Second Order.}

$\S 1$. The different species of surfaces of the second order are obtained, as is usually shown in elementary treatises, by the discussion of the general equation of the second degree among three co-ordinates; but it is necessary that we should also be able to derive these surfaces from a common geometrical origin, if we would bring them completely within the grasp of geometry. Now as the different conic sections may (with the

* Proceedings of the Royal Irish Academy, Vor. I. p. 89. 
exception of the circle), be described in plano by the motion of a point whose distance from a given point bears a constant ratio to its distance from a given right line, ${ }^{*}$ it is natural to suppose that there must be some analogous method by which the surfaces of the second order may be generated in space. Accordingly I have sought for such a method, and I have found that (with certain analogous exceptions) every surface of the second order may be regarded as the locus of a point whose distance from a given point bears a constant ratio to its distance from a given right line, provided the latter distance be measured parallel to a given plane; this plane being, in general, oblique to the right line. The given point I call, from analogy, a focus, and the given right line a directrix; the given plane may be called a directive plane, and the constant ratio may be termed the modulus.

To find the equation of the surface so defined, let the axis of $z$ be parallel to the directrix; let the plane of $x y$ pass through the focus, and cut the directrix perpendicularly in $\Delta$, the coordinates being rectangular, and their origin arbitrarily assumed in that plane; and let the axis of $y$ be parallel to the intersection of the plane $x y$ with the directive plane, the angle between the two planes being denoted by $\phi$. Then if we put $x_{1}, y_{1}$ for the co-ordinates of the focus, and $x_{2}, y_{2}$ for those of the point $\Delta$, while the co-ordinates of a point $S$ upon the surface are denoted by $x, y, z$, the distance of this last point from the focus will be the square root of the quantity

$$
\left(x-x_{1}\right)^{2}+\left(y-y_{1}\right)^{2}+z^{2} ;
$$

and if a plane drawn through $S$, parallel to the directive plane, be conceived to cut the directrix in $D$, the distance $S D$ will be the square root of the quantity

$$
\left(x-x_{2}\right)^{2} \sec ^{2} \phi+\left(y-y_{2}\right)^{2} ;
$$

* This method of describing the conic sections is due to the Greek geometers. It is given by Pappus at the end of the Seventh Book of his Mathematical Collections. 
so that, $m$ being the modulus, the locus of the point $S$ will be a surface of the second order, represented by the equation

$$
\left.\left(x-x_{1}\right)^{2}+\left(y-y_{1}\right)^{2}+z^{2}=m^{2}\left\{x-x_{2}\right)^{2} \sec ^{2} \phi+\left(y-y_{2}\right)^{2}\right\},
$$

which, by making

$$
\begin{array}{ll}
A=1-m^{2} \sec ^{2} \phi, & B=1-m^{2}, \\
G=m^{2} x_{2} \sec ^{2} \phi-x_{1}, & \boldsymbol{H}=m^{2} y_{2}-y_{1}, \\
\boldsymbol{K}=m^{2}\left(x_{3}{ }^{2} \sec ^{2} \phi+y_{2}{ }^{2}\right)-x_{1}{ }^{2}-y_{1}{ }^{2},
\end{array}
$$

may be put under the form

$$
A x^{2}+B y^{2}+z^{2}+2 G x+2 H y=K,
$$

showing that the plane of $x y$ is one of the principal planes of the surface, and that the planes of $x z$ and $y z$ are parallel to principal planes.

Before we proceed to disouss this equation it may be well to observe, that as it remains the same when $\phi$ is changed into $-\phi$, or into $180^{\circ}-\phi$, the directive plane may have two positions equally inclined to the plane of $x y$, and therefore equally inclined to the directrix. Indeed it is obvious that, if through the point $S$ we draw two planes making equal angles with the directrix, and cutting it in the points $D$ and $D^{\prime}$ respectively, the distances $S D$ and $S D^{\prime}$ will be equal. Every surface described in this way has consequently two directive planes; and as each of these planes is parallel to the axis of $y$, their intersection is always parallel to one of the axes of the surface. This axis may therefore be called the directive axis. The directive planes have a remarkable relation to the surface, as may be shown in the following manner:-

Suppose a section of the surface to be made by a plane which is parallel to one of the directive planes, and which cuts the directrix in $D$; then the distance of any point $S$ of the section from the focus $F$ will have a constant ratio to its distance $S D$ 
from the point $D$; and, as the locus of a point whose distances from the two points $F$ and $D$ are in a constant ratio to each other is a plane or a sphere, according as the ratio is one of equality or not, it follows that the section aforesaid will be a right line in the one case, and a circle in the other. Hence it appears that all directive sections, that is, all sections made in the surface by planes parallel to either of the directive planes, are right lines when the modulus is unity, and circles when the modulus is different from unity.

Since the equation (3) is not altered by changing the sign of $\phi$, or by changing $\phi$ into its supplement, we may suppose this angle (when it is not zero) to be always positive and less than $90^{\circ}$; for the supposition $\phi=90^{\circ}$ is to be excluded, as it would make the secant of $\phi$ infinite, and the directive planes parallel to the directrix. In the discussion of the equation there are two leading cases to be considered, answering to two classes of surfaces. The first case, when neither $A$ nor $B$ vanishes, gives the ellipsoid, the two hyperboloids, and the $\operatorname{con} \theta$; the second, when either or each of these quantities is zero, includes the two paraboloids and the different kinds of cylinders.

$\S 2$. First Class of Surfaces. - When neither $A$ nor $B$ vanishes, we may make both $G$ and $H$ vanish, by properly assuming the origin of co-ordinates. Supposing this done, we have

$$
x_{1}=m^{2} x_{2} \sec ^{2} \phi, \quad y_{1}=m^{2} y_{2},
$$

the equation of the surface being then

$$
A x^{2}+B y^{2}+z^{2}=K,
$$

in which the axes of co-ordinates are of course the axes of the surface. When $K$ is not zero, the surface is an ellipsoid or hyperboloid, having its centre at the origin of co-ordinates; when $K=0$, the surface is a cone having its vertex at the origin.

Eliminating $x_{2}, y_{2}$ from the value of $K$, by means of the relations (4), we get

$$
K=\frac{A}{1-A} x_{1}^{2}+\frac{B}{1-B} y_{1}^{2}
$$


and eliminating $x_{1}, y_{1}$ in like manner, we get

$$
K=A(1-A) x_{2}{ }^{2}+B(1-B) y_{2}{ }^{2} ;
$$

from which expressions it appears that, everything else remaining, the focus and directrix may be changed without changing the surface described. For in order that the surface may remain unchanged, it is only necessary that $K$ should remain constant, since $A$ and $B$ are supposed constant. This condition being fulfilled, the focus may be any point $\mathrm{F}$ whose co-ordinates $x_{1}, y_{1}$ satisfy the equation (6), and $\Delta$ (the foot of the directrix) may be any point whose co-ordinates $x_{2}, y_{2}$ satisfy the equation (7); it being understood, however, that when one of these points is chosen, the other is determined. The locus of $\mathrm{F}$ (supposing $K$ not to vanish) is therefore an ellipse or a hyperbola, ${ }^{*}$ which may be called the focal curve, or the focal line; and the locus of $\Delta$ is another ellipse or hyperbola, which may be called the dirigent eurve or line: the centre of each curve is the centre of the surface, and its axes coincide with the axes of the surface which lie in the plane of $x y$. Moreover, as the quantities $1-A$ and $1-B$ are essentially positive, the two curves are always of the same kind, that is, both ellipses, or both hyperbolas ; and when they are hyperbolas, their real axes have the same direction. The directrix, remaining always parallel to the axis of $z$, describes a cylinder which may be called the dirigent cylinder.

Since, by the relations (4), the corresponding co-ordinates of $F$ and $\Delta$ have always the same sign, these points either lie within the same right angle made by the axes of $x$ and $y$, or lie on the same axis, at the same side of the centre. And as these relations give

$$
x_{2}-x_{1}=\frac{A}{1-A} x_{1}, \quad y_{2}-y_{1}=\frac{B}{1-B} y_{1},
$$

it is easy to see that the right line $\Delta \mathrm{F}$ is a normal to the focal

* In the Proceedings of the Academy, Vou. I. p. 90, it was stated inadvertently that "if we confine ourselves to the central surfaces, the locus of the foci will be an ellipse." 
curve; for the quantities $x_{2}-x_{1}$ and $y_{2}-y_{1}$ are proportional to the cosines of the angles which that right line makes with the. axes of $x$ and $y$ respectively, while the values just given for these quantities are, in virtue of the equation (6), proportional to the cosines of the angles which the normal to the focal curve at the point $\mathrm{F}$ makes with the same axes.

It may also be shown, that if the directrix prolonged through $\Delta$ intersects a directive plane in a certain point, and if a right line drawn through $\mathrm{F}$, parallel to the directrix, intersect the same plane in another point, the right line joining those points will be a normal to the curve described in that plane by the first point.

§ 3. To find in what way the focal and dirigent curves are connected with the surface, let the equations (5), (6), (7) (when $K$ does not vanish), be put under the forms

$$
\begin{gathered}
\frac{x^{2}}{\bar{P}}+\frac{y^{2}}{Q}+\frac{z^{2}}{\bar{R}}=1, \\
\frac{x_{1}{ }^{2}}{P_{1}}+\frac{y_{1}{ }^{2}}{Q_{1}}=1, \quad \frac{x_{2}{ }^{2}}{P_{2}}+\frac{y_{2}{ }^{2}}{Q_{z}}=1,
\end{gathered}
$$

so that the quantities $P, Q, R$ may represent the squares of the semiaxes of the surface, and $P_{1}, Q_{1}, P_{2}, Q_{2}$ the squares of the semiaxes of the curres, these quantities being positive or negative, according as the corresponding semiaxes are real or imaginary. Then we have

$$
\begin{array}{ll}
P=\frac{K}{A}, & Q=\frac{K}{\bar{B}}, \quad R=K, \\
P_{1}=P(1-A), & Q_{1}=Q(1-B), \\
P_{2}=\frac{P}{1-A}, & Q_{2}=\frac{Q}{1-B} ;
\end{array}
$$

whence it follows that

and also that

$$
P_{1} P_{2}=P^{2}, \quad Q_{1} Q_{2}=Q^{2},
$$

$$
P_{1}=P-R, \quad Q_{1}=Q-R \text {. }
$$


From equations (12) we see that $P_{1}$ and $P_{2}$ have always the same sign, as also $Q_{1}$ and $Q_{2}$; and that, neglecting signs, the semiaxes of the surface are mean proportionals between the corresponding semiaxes of the focal and dirigent curves. These curves are therefore reciprocal polars with respect to the section made in the surface by the plane of $x y$; and it would be easy to show that the points $F$ and $\Delta$ are reciprocal points, or that a tangent applied at one of them to the curve which is its locus has the other for its pole.

The focal curve, when we know in which of the principal planes it lies, is determined by the conditions (13); and as it depends on the relative magnitudes of the quantities $P, Q, R$, it will be convenient to distinguish the axes of the surface, with relation to these magnitudes. Supposing, therefore, the quantities $P, Q, R$ to be taken with their proper signs, as they are in the equation (9), that axis to which the greatest of them (which is always positive) refers shall be called the primary axis ; and that to which the quantity algebraically least has reference shall be termed the secondary axis; while the quantity which has an intermediate algebraic value shall mark the middle or mean axis. Then, since $P_{1}$ and $Q_{1}$ will be negative, if $R$ be the greatest of the quantities aforesaid, the focal curve cannot lie in the plane of the mean and secondary axes. Its plane must therefore pass through the primary axis: it will be the plane of the primary and mean axes, if $R$ be the least of the three quantities; but the plane of the primary and secondary axes, if $R$ be the intermediate quantity. In the former case the curve will be an ellipse, in the latter a hyperbola ; and we shall extend the name of focal curves to both the curves so determined, though it may happen that only one of them ean be used in the generation of the surface by the moctular method, as the method of which we are treating may be called, from its employment of the modulus. A focal curve which can be so used shall be distinguished as a modular focal; but each focal, whether modular or not, shall be supposed to have a dirigent curve and a dirigent eylinder connected with it by the relations already laid down. 
Since $P_{1}-Q_{1}=P-Q$, the foci of a focal ourve are the same as those of the principal section in the plane of which it lies, and they are therefore on the primary axis of the surface. It will sometimes contribute to brevity of expression, if we also give the name of primary to the major axis of an ellipse and to the real axis of a hyperbola. We may then say that the primary axes of the surface and of its two focal curves are coincident in direction; and that (as is evident) the foci of either curve are the extremities of the primary axis of the other.

If $K$ be supposed to approach gradually to zero, while $A$ and $B$ remain constant, the focal and dirigent ellipses will gradually contract, and the focal and dirigent hyperbolas will approach to their asymptotes, which remain fixed. When $K$ actually vanishes, the surface becomes a cone; the two ellipses are each reduced to a point coinciding with the vertex of the cone, and each hyperbola is reduced to the pair of right lines which were previously the asymptotes. The dirigent cylinder, in the one case, is narrowed into a right line; in the other case it is converted into a pair of planes, which we may call the dirigent planes of the curve.

$\S 4$. We have now to show how the different kinds of surfaces belonging to the first class are produced, according to the different values of the modulus and other constants concerned in their generation.

I. When $m$ is less than $\cos \phi$, the quantities $A, B, K, P, Q, R$ are all positive, and $Q$ is intermediate in value between $P$ and $R$. The surface is therefore an ellipsoid, and its mean axis is the directive. As the quantities $1-A$ and $1-B$ are always positive, the focal and dirigent curves are ellipses.

Here we cannot suppose $K$ to vanish, as the surface would then be reduced to a point.

When $\phi=0$, that is, when the directive planes coincide with each other, and therefore with a plane perpendicular to the directrix, so that SD is the shortest distance of the point $S$ from the directrix, the surface is a spheroid produced by the revo- 
lution of an ellipse round its minor axis, and the focal and dirigent curves are circles.

II. When $m$ is greater than unity; $A$ and $B$ are negative; and if $K$ be finite, it is also negative; whence $P$ and $Q$ are positive, and $K$ is negative. Also, supposing $\phi$ not. to vanish, $Q$ is greater than $P$. The surface is therefore a hyperboloid of one sheet, with its real axes in the plane of $x y$; and the directive axis is the primary. The focal and dirigent curves are ellipses. But when $\phi=0$, the surface is that produced by the revolution of a hyperbola round its imaginary axis, and the focal and dirigent* are circles.

If $K=0$, which implies, since $A$ and $B$ have the same sign, that $x_{1}, y_{1}, x_{2}, y_{2}$ are each zero, the surface is a cone having the axis of $z$ for its internal axis; and the focal and dirigent are each reduced to a point. The focus and directrix are consequently unique; the focus can only be the vertex of the cone; the directrix can only be the internal axis; and the directrix therefore passes through the focus. The directive axis, which coincides with the axis of $y$, is one of the external axes; that one, namely, which is parallel to the greater axes of the elliptie sections made in the cone by planes perpendicular to its internal axis. This is on the supposition that $\phi$ is finite; for, when $\phi=0$, the cone becomes one of revolution round the axis of $z$.

III. When $m$ is greater than $\cos \phi$, but less than unity, we have $A$ positive and $B$ negative, and the species of the surface depends on $K$. It is inconsistent with these conditions to suppose $\phi=0$, and therefore the surface cannot, in this case, be one of revolution. The value of $K$ may be supposed to be given by the formula

$$
K=\frac{1-A}{A}\left(x_{2}-x_{1}\right)^{2}+\frac{1-B}{B}\left(y_{2}-y_{1}\right)^{2},
$$

which contains only the relative co-ordinates of the focus and the foot of the directrix, and is a consequence of the equations (6) and (7).

* When the term dirigent stands alone, it is understood to mean a dirigent line. 
$1^{\circ}$. If $K$ is a positive quantity, the surface is a hyperboloid. of one sheet, with its secondary axis in the direction of $x$; the primary axis, as before, in the directive, but the focal and dirigent are now hyperbolas.

$2^{\circ}$. If $K$ is a negative quantity, the surface is a hyperboloid of two sheets, having its primary axis coincident with that of $x$. The secondary axis is the directive; the focal and dirigent are hyperbolas.

$3^{\circ}$. If $K=0$, the surface is a cone, having the axis of $x$ for its internal axis ; the directive axis being, as before, that external axis to which the greater axes of the elliptic sections, made by planes perpendicular to the internal axis, are parallel. The axis of $\approx$ is the other external axis, which may be called the mean axis of the cone, because it coincides with the mean axis of any hyperboloid to which the cone is asymptotic. As $A$ and $B$ have different signs, it is evident, from the equations (6) and (7), that the focal and dirigent are each a pair of right lines passing through the vertex, each pair making equal angles with the internal axis. Two planes, each of which is drawn through the mean axis and a dirigent line, are the dirigent planes of the cone.

The corresponding focal and dirigent lines are those which lie within the same right angle made by the internal and directive axes ; and since by the equations (6) and (8) the value of $K$ may be written

$$
\Pi=x_{1}\left(x_{2}-x_{1}\right)+y_{1}\left(y_{2}-y_{1}\right),
$$

we see that, as $K$ now vanishes, the right line joining corresponding points $F$ and $\Delta$ upon these lines is perpendicular to the focal line. Of the two sides of the cone which are in the plane $x y$, one lies between each focal and its dirigent; and it may be inferred from the equations, that the tangents of the angles which the internal axis makes with a focal line, with one of these sides of the cone, and with a dirigent line, are in continued proportion, the proportion being that of the cosine of $\phi$ to unity. And hence it follows, that these two sides of the cone, 
with a focal line and its dirigent, cut harmonically any right line which orosses them.

§. From this discussion it appears, that the ellipsoid and the hyperboloid of two sheets can be generated modularly, each in one way only, the modular focal being the ellipse for the former, and the hyperbola for the latter; but that the hyperboloid of one sheet can be generated in two ways, each of its focals being modular, and each focal having its proper modulus. The cone also admits two modes of generation," in one of which, however, the focus is limited to the vertex of the cone, and the directrix to its internal axis. But when the hyperboloid of one sheet, or the cone, is a surface of revolution, it has only one mode of modular generation. In cases of double generation, the directive planes of course remain the same, as they have a fixed relation to the surface. A modular focal, it may be observed (and the remark applies equally to surfaces of the second class), is distinguished by the circumstance that it does not intersect the surface. The only exception to this rule are the focal lines of the cone, which pass through its vertex. A focal which is not modular may be called umbilicar, because it intersects the surface in the umbilios; an umbilic being a point on the surface where the tangent plane is parallel to a directive plane. Thus the focal hyperbola of the ellipsoid, and the focal ellipse of the hyperboloid of two sheets, are umbilicar focals, and pass through the umbilios of these surfaces; but the hyper-

* The double generation of the cone, when its vertex is the focus, may be proved synthetically by the method indicated in the Examination Papers of the year 1838, p. xlvi. (published in the University Catendar for 1839). Supposing the cone to stand on a circular base (one of its directive sections), and to be circumscribed by a sphere, the right lines joining its vertex with the two points where a diameter perpendicular to the plane of the base intersects the sphere, will be its internal and mean axes. Then if $P$ be either of these points, $V$ the vertex, $C$ the point where the axis $P V$ cuts the plane of the base, and $B$ any point in the circumference of the base, the triangles $P V B$ and $P B C$ will be similar, since the angles at $V$ and $B$ are equal, and the angle at $P$ is common to both triangles; therefore $B V$ will be to $B C$ as $P V$ to $P B$, that is, in a constant ratio. It is not difficult to complete the demonstration, when the focus is supposed to be any point on one of the focal lines. 
boloid of one sheet has no umbilics, and accordingly both its focals are modular, and neither of them intersects the surface. The umbilicar focals and dirigents have properties which shall be mentioned hereafter.

An umbilicar focal and the principal section whose plane coincides with that of the focal are curves of different kinds, the one being an ellipse when the other is a hyperbola; but a modular focal is always of the same kind with the coincident section of the surface, being an ellipse, a hyperbola, or a pair of right lines, according as the section is an ellipse, a hyperbola, or a pair of right lines; and when the section is reduced to a point, so likewise is the modular focal.

The plane of a modular focal always passes through the directive axis. When the directive axis is the primary, as in the hyperboloid of one sheet, both focals are modular. But in the ellipsoid and the hyperboloid of two sheets, where the primary axis is not directive, only one of the focals can be modular. The plane of an umbilicar focal is always perpendicular to the directive axis; and therefore, when that axis is the primary, there is no umbilicar focal.*

When the surface is doubly modular, the two moduli $m, m^{\prime}$ are connected by the relation

$$
\frac{\cos ^{2} \phi}{m^{2}}+\frac{\sin ^{2} \phi}{m^{\prime 2}}=1
$$

* If the first of the equations (10), when $P_{1}$ and $Q_{1}$ are both negative, be supposed to express an imaginary focal, there will, in a central surface, be three focals, two modular and one umbilicar; the two modular focals being in the principal planes which pass through the directive axis, and the umbilicar focal in the remaining principal plane. Then, when we know which of the axes is the divective axis, we know which of the three focals is imaginary, because the plane of the imaginary focal is perpendicular to the primary axis. A modular focal may be imaginary, and yet have a real modulus; this occurs in the hyperboloid of two sheets. In the ellipsoid, the imaginary focal has an imaginary modulus. In all cases the two moduli are connected by the relation (16).

It will appear hereafter that the vertex of the cone is an umbilicar focus. The cone has therefore three focals, none of which is imaginary; but two of them are single points coinciding with the vertex. 
where $\phi$ is the angle made by a directive plane with the plane of the focal to which the modulus $m$ belongs. One modulus is greater than unity; the other is less than unity, but greater than the cosine of the angle which the plane of the corresponding focal makes with a directive plane. In the hyperboloid of one sheet, the less modulus is that which belongs to the focal hyperbola. In the cone, the less modulus belongs to the focal lines. Of the two moduli of a cone, that which belongs to the focal lines may be termed the linear modulus; and the other, to which only a single focus corresponds, may be called the singular modulus.

\$6. Second Class of Surfaces.-In this class of surfaces, one of the quantities $A, B$ vanishes, or both of them vanish.

I. When $m=\cos \phi$, and $\phi$ is not zero, $A$ vanishes, but $B$ does not; and the surface is either a paraboloid or a cylinder.

$1^{\circ}$. If the surface is a paraboloid, we may suppose the origin of co-ordinates to be at its vertex, in which case both $H$ and $K$ vanish, and we have the relations

$$
\begin{gathered}
G=x_{2}-x_{1}, \quad y_{1}=y_{2} \cos ^{2} \phi, \\
x_{2}{ }^{2}+y_{2}{ }^{2} \cos ^{2} \phi-x_{1}{ }^{2}-y_{1}{ }^{2}=0 ;
\end{gathered}
$$

the equation of the surface being

$$
y^{2} \sin ^{2} \phi+z^{2}+2 G x=0,
$$

which shows that the paraboloid is elliptic, having its axis in the direction of $x$, and the plane of $x y$ for that of its greater principal section. From the relations (17) we obtain the following:

$$
\begin{gathered}
y_{1}^{2} \tan ^{2} \phi+2 G x_{1}+G^{2}=0, \\
y_{2}^{2} \sin ^{2} \phi \cos ^{2} \phi+2 G x_{2}-G^{2}=0 ;
\end{gathered}
$$

from which we see that the focal and dirigent curves are parabolas, having their axes the same as that of the surface; and their vertices equidistant from the vertex of the surface, but at opposite sides of it. The cavity of each ourve is turned in the 
same direction as that of the section $x y$. The focus of the focal parabola is the focus of the section $x y$, and its vertex is the focus of the section $x z$ of the surface; its parameter being the difference of the parameters of these two sections. The parameter of the section $x y$ is a mean proportional between the parameters of the focal and dirigent parabolas.

$2^{\circ}$. If the surface is a cylinder, we may make $G$ and $H$ vanish, by taking the origin on its axis. We then have

$$
\begin{gathered}
x_{2}=x_{1}, \quad y_{1}=y_{2} \cos ^{2} \phi, \\
K=y_{1}{ }^{2} \tan ^{2} \phi=y_{2}^{2} \sin ^{2} \phi \cos ^{2} \phi ;
\end{gathered}
$$

the equation of the cylinder, which is elliptic, being

$$
y^{2} \sin ^{2} \phi+z^{2}=K \text {. }
$$

Here the focal and dirigent are each a pair of right lines parallel to the axis of the cylinder, and passing through the foci and directrices of a section perpendicular to the axis. The corresponding focal and dirigent lines lie at the same sides of the axis.

II. When $m=1$, and $\phi$ is not zero, $B$ vanishes, but $A$ does not.

$1^{\circ}$. If the surface is a paraboloid, and the origin of co-ordinates at its vertex, the quantities $G$ and $K$ vanish; and the equation of the surface becomes

$$
x^{2} \tan ^{2} \phi-z^{2}=2 H y,
$$

and we have the relations

$$
\begin{gathered}
H=y_{2}-y_{1}, \quad x_{1}=x_{2} \sec ^{2} \phi, \\
x_{2}{ }^{2} \sec ^{2} \phi+y_{2}{ }^{2}-x_{1}{ }^{2}-y_{1}{ }^{2}=0 .
\end{gathered}
$$

The paraboloid is therefore hyperbolic, its axis being that of $y$, which is also the directive axis; and as the tangent of $\phi$ may have any finite value, the plane of $x y$, which is that of the focal 
curve, may be either of the principal planes passing through the axis of the surface. The relations (23) give

$$
\begin{gathered}
x_{1}^{2} \sin ^{2} \phi-2 H y_{1}-H^{2}=0, \\
x_{2}^{2} \tan ^{2} \phi \sec ^{2} \phi-2 H y_{2}+H^{2}=0,
\end{gathered}
$$

for the equations of the focal and dirigent, which are therefore parabolas, having their axes the same as those of the surface, and their concavities turned in the same direction as that of the section $x y$; their vertices being equidistant from the vertex of the surface, and at opposite sides of it. The focus of the focal parabola is the focus of the section $x y$, and its vertex is the focus of the section $y z$, its parameter being the sum of the parameters of these two sections. The parameter of the section $x y$ is a mean proportional between the parameters of the focal and dirigent parabolas.

$2^{\circ}$. If the surface is a cylinder, and the origin on its axis, $G$ and $H$ vanish, and we have

$$
\begin{gathered}
x_{1}=x_{2} \sec ^{2} \phi, \quad y_{1}=y_{2}, \\
-K=x_{1}^{2} \sin ^{2} \phi=x_{2}^{2} \tan ^{2} \phi \sec ^{2} \phi ;
\end{gathered}
$$

the equation of the cylinder, which is hyperbolic, being

$$
x^{2} \tan ^{2} \phi-z^{2}=-K .
$$

The focal and dirigent are each a pair of right lines parallel to the axis of the oylinder; the corresponding lines passing through a focus and the adjacent directrix of any section perpendicular to the axis. The directive planes are parallel to the asymptotio planes of the oylinder.

In this case, if $K=0$, the surface is reduced to two directive planes, and the focal and dirigent to the intersection of these planes.

III. When $m=1$, and $\phi=0$, both $A$ and $B$ vanish, and the surface is the parabolio cylinder. If, as is allowable, we sup- 
pose $G$ and $K$ to vanish, the equation of the cylinder becomes

and we have

$$
z^{2}+2 H y=0
$$

whence

$$
\begin{aligned}
& H=y_{2}-y_{1}, \quad x_{1}=x_{2}, \\
& x_{2}^{2}+y_{2}^{2}-x_{1}^{2}-y_{1}^{2}=0 ;
\end{aligned}
$$

$$
y_{1}=-\frac{1}{2} H, \quad y_{2}=\frac{1}{2} H \text {. }
$$

The focal and dirigent are each a right line parallel to the axis of $x$, the former passing through the focus, the latter meeting the directrix of the parabolic section made by the plane of $y \approx$. The plane of $x y$ is the directive plane.

§ 7. We learn from this discussion that, among the surfaces of the second class, the hyperbolic paraboloid is the only one which admits a twofold modular generation; the modulus, however, being the same for both its focals. In the elliptic paraboloid the modular focal is restricted to the plane of that principal section which has the greater parameter; we shall therefore suppose a parabola to be described in the plane of the other principal section, according to the law of the modular focals; the law being, that the focus of the parabola shall be the focus of the principal section in the plane of which the parabola lies, and its vertex the focus of the principal section in the perpendicular plane. The parabola so described will have its concavity opposed to that of the surface; it will cut the surface in the umbilics, and will be its umbilicar focal, the only such focal to be found among the surfaces of the second class. We shall of course suppose further, that this focal has a dirigent parabola connected with it by the same law as in the other cases, the vertices of the focal and dirigent being equidistant from that of the surface and at opposite sides of it, while the parameter of the dirigent is a third proportional to the parameters of the focal and of the principal section in the plane of which the curve lies. The two focals of a paraboloid are so related, that the focus of the one is the vertex of the other. The cylinders have no other focals than those which occur above. 
§ 8. In this, as in the first class of surfaces, the right line $\mathrm{F} \Delta$, joining a focus $\mathrm{F}$ with the foot of its corresponding directrix, is perpendicular to the focal line; and the focal and dirigent are reciprocal polars with respect to the section $x y$ of the surface. These properties are easily inferred from the preceding results; but, as they are general, it may be well to prove them generally for both classes of surfaces. Supposing, therefore, the origin of co-ordinates to be anywhere in the plane of $x y$, and writing the equation of the surface in the form

$$
\left(x-x_{1}\right)^{2}+\left(y-y_{1}\right)^{2}+z^{2}=L\left(x-x_{2}\right)^{2}+M\left(y-y_{2}\right)^{2},
$$

which, when identified with (3), gives the relations

$$
\begin{aligned}
& A=1-L, \quad B=1-M, \\
& G=L x_{2}-x_{1}, \quad H=M y_{2}-y_{1}, \\
& K=L x_{2}{ }^{2}+M y_{2}{ }^{2}-x_{1}{ }^{2}-y_{1}{ }^{2},
\end{aligned}
$$

we find, by differentiating the values of the constants $G, H$, and $K$,

$$
\begin{gathered}
L d x_{2}=d x_{1}, \quad \boldsymbol{M} d y_{2}=d y_{1}, \\
L x_{2} d x_{2}+\mathbb{M} y_{2} d y_{2}-x_{1} d x_{1}-y_{1} d y_{1}=0 .
\end{gathered}
$$

Hence we obtain

$$
\left(x_{2}-x_{1}\right) d x_{1}+\left(y_{2}-y_{1}\right) d y_{1}=0 ;
$$

an equation which expresses that the right line joining the points $\mathrm{F}$ and $\Delta$ is perpendicular to the line whioh is the locus of the point $\mathrm{F}$.

Again, the equation of the section $x y$ of the surface being

$$
A x^{2}+B y^{2}+2 G x+2 H y=K,
$$

the equation of the right line which is, with respect to this section, the polar of a point $\Delta$ whose co-ordinates are $x_{2}, y_{2}$, is

$$
\left(A x_{2}+G\right) x+\left(B y_{2}+H\right) y=\boldsymbol{K}-G x_{2}-H y_{2} ;
$$

but the relations (31) give

$$
\begin{aligned}
& A x_{2}+G=x_{2}-x_{1}, \quad B y_{2}+H=y_{2}-y_{1}, \\
& K-G x_{2}-H y_{2}=x_{1}\left(x_{2}-x_{1}\right)+y_{1}\left(y_{2}-y_{1}\right) ;
\end{aligned}
$$


and hence the equation (35) becomes

$$
\left(x_{2}-x_{1}\right)\left(x-x_{1}\right)+\left(y_{2}-y_{1}\right)\left(y-y_{1}\right)=0,
$$

which, as is evident from (33), is the equation of a tangent applied to the focal at the point $\mathbf{F}$ corresponding to $\Delta$. This shows that the focal and dirigent are reciprocal polars with respect to the section $x y$, and that in this relation, as well as in the other, the points $F$ and $\Delta$ are corresponding points.

Supposing $F^{\prime}$ and $\Delta^{\prime}$ to be two other corresponding points on the focal and dirigent, if tangents applied to the focal at F and $\mathrm{F}^{\prime}$ intersect each other in $\mathrm{T}$, the point $\mathrm{T}$ will be the pole of the right line $\Delta \Delta^{\prime}$ with respect to the section $x y$, as well as the pole of the right line $\mathrm{FF}^{\prime}$ with respect to the focal; and hence if any right line be drawn through $\mathrm{T}$, and if $\mathrm{P}$ be the pole of this right line with respect to the section, and $\mathrm{N}$ its pole with respect to the focal, the points $\mathrm{P}$ and $\mathrm{N}$ will be on the right lines $\Delta \Delta^{\prime}$ and $\mathrm{FF}^{\prime}$ respectively. Now it is useful to observe that the distances $\Delta \Delta^{\prime}$ and $\mathrm{FF}^{\prime}$ are always similarly divided (both of them internally or both of them externally) by the points $\mathrm{P}$ and $\mathrm{N}$, so that we have $\Delta \mathrm{P}$ to $\Delta^{\prime} \mathrm{P}$ as $\mathrm{FN}$ to $\mathrm{F}^{\prime} \mathrm{N}$. This property may be proved directly by means of the foregoing equations; or it may be regarded as a consequence of the following theorem:-If through a fixed point in the plane of two given conics having the same centre, or of two given parabolas having their axes parallel, any pair of right lines be drawn, and their poles be taken with respect to each ourve, the distance between the poles relative to one curve will be in a constant ratio to the distance between the poles relative to the other curve.* In fact, the poles of the right lines TF, TF', with respect to the focal, are $\mathrm{F}, \mathrm{F}^{\prime}$; and their poles with respect to the section $x y$ are $\Delta, \Delta^{\prime}$; therefore, since the focal and the section $x y$ may be taken for the given curves, and the point $T$

* There is an analogous theorem for two surfaces of the second order which have the same centre, or two paraboloids which have their axes parallel. If through a fixed right line any two planes be drawn, and their poles be taken with respect to each surface, the distance between the poles relative to the one surface will be in a constant ratio to the distance between the poles relative to the other. 
for the fixed point, the ratio of $\mathrm{FF}^{\prime}$ to $\Delta \Delta^{\prime}$ is the same as the ratio of $\mathrm{FN}$ to $\Delta \mathrm{P}$, or of $\mathrm{F}^{\prime} \mathrm{N}$ to $\Delta^{\prime} \mathrm{P}$; and consequently the distanoes $\mathrm{FF}^{\prime}$ and $\Delta \Delta^{\prime}$ are similarly divided in the points $\mathrm{N}$ and $P$.

§9. In the equation (30), considered as equivalent to the equation (1), the constants $L$ and $\boldsymbol{M}$ are both positive; but the properties which have been deduced from the former equation are independent of this ciroumstance, and equally subsist when one of these constants is supposed to be negative (for they cannot both be negative). This leads us to inquire what surfaces the equation (30) is capable of representing when the constants $L$ and $M$ have different signs; as also, for a given surface, what lines are traced in the plane of $x y$ by points $F$ and $\Delta$, of which $x_{1}, y_{1}$, and $x_{2}, y_{2}$ are the respective co-ordinates. After the examples already given, this question is easily disenssed, and the result is, that the only surfaces which ean be so represented are the ellipsoid, the hyperboloid of two sheets, the cone, and the elliptio paraboloid-that is to say, the umbilicar surfaces together with the oone; and that, for an umbilicar surface, the locus of $\mathrm{F}$ is the umbilicar focal, and therefore the locus of $\Delta$ is the corresponding dirigent; while for the cone the points $\mathrm{F}$ and $\Delta$ are unique, coinciding with each other and with the vertex of the cone. A geometrical interpretation of this case is readily found; for as $L$ and $\boldsymbol{M}$ have different signs, the right-hand member of the equation (30), if $\boldsymbol{M}$ be the negative quantity, is the product of two factors of the form

$$
f\left(x-x_{2}\right)+g\left(y-y_{2}\right), \quad f\left(x-x_{2}\right)-g\left(y-y_{2}\right),
$$

in which $f$ and $g$ are constant; and these factors are evidently proportional to the distances of a point whose co-ordinates are $x, y, z$, from two planes whose equations are

$$
f\left(x-x_{2}\right)+g\left(y-y_{2}\right)=0, \quad f\left(x-x_{2}\right)-g\left(y-y_{2}\right)=0,
$$

which planes always pass through a directrix, and are inclined at equal and constant angles to the axis of $x$ or of $y$. Therefore, if $\mathrm{F}$ be the focus which belongs to this directrix, the square 
of the distance of $\mathrm{F}$ from any point of this surface is in a constant ratio to the rectangle under the distances of the latter point from the two planes. And these planes are directive planes; because, if a section parallel to one of them be made in the surface, the distance of any point of the section from the other plane will be proportional to the square of the distance of the same point from the focus; and, as the locus of a point, whose distance from a given plane is proportional to the square of its distance from a given point, is obviously a sphere, it follows that the section aforesaid is the section of a sphere, and consequently a circle; which shows that the plane to which the section is parallel is a directive plane. Thus, ${ }^{*}$ the square of the

: In attempting to find a geometrical generation for the surfaces of the second order, one of the first things which I thought of, before I fell upon the modular method, was to try the locus of a point such that the square of its distance from a given point should be in a constant ratio to the rectangle under its distances from two given planes; but when I saw that this locus would not represent all the species of surfaces, I laid aside the discussion of it. Some time since, however, Mr. Salmon, Fellow of Trinity College, was led independently, in studying the modular method, to consider the same locus; and he remarked to me, what I had not previously observed, that it offers a property supplementary, in a certain sense, to the modular property; that when the surface is an ellipsoid, for example, the given point or focus is on the focal hyperbola, which the modular property leaves empty. This remark of Mr. Salmon served to complete the theory of the focals, by indicating a simple geometrical relation between a non-modular focal and any point on the surface to which it belongs.

In a memoir "On a new method of Generation and Discussion of the Surfaces of the second Order," presented by M. Amyot to the Academy of Sciences of Paris, on the 26th December, 1842, the author investigates this same locus, conceiving it to involve that property in surfaces which is analogous to the property of the focus and directrix in the conic sections; and the importance attached to the discovery of such analogous properties induced $\mathbf{M}$. Cauchy to write a very detailed report on M. Amyot's memoir, accompanied with notes and additions of his own (Comptes rendus des Séances de l'Académie des Sciences, tom. xvi. pp. 783-828, 885-890; April, 1843); and also occasioned several discussions, principally between $\mathbf{M}$. Poncelet and M. Chasles, relative to that Memoir (Comptes rendus, tom. xvi. pp. $829,938,947,1105,1110)$. But the property involved in this locus cannot be said to afford a method of generation of the surfaces of the second order, since it applies only to some of the surfaces, and gives an ambiguous result even where it does apply. It is therefore not at all analogous to the aforesaid general property of the conic sections, and moreover it was not new when M. Amyot brought it 
distance of any point of the surface from an umbilicar focus bears a constant ratio to the rectangle under the perpendicular distances of the same point from two directive planes drawn through the directrix corresponding to that focus; and it is easy to see that this ratio, the square root of which we shall denote by $\mu$, is equal to $L-M$, or, neglecting signs, to the sum of the numerical values of $L$ and $M$. Of course, if the distances from the directive planes, instead of being perpendicular, be measured parallel to any fixed right line, the ratio will still be constant, though different. For example, if the fixed right line for each plane be that which joins the corresponding umbilic with either focus of the section $x y$, the ratio

forward. Mr. Salmon had in fact proposed it for investigation to the students of the University of Dublin, at the ordinary Examinations in October, 1842 ; and it was published, towards the end of that year, in the University Calendar for 1843, some months before the date of M. Cauchy's report, by which the contents of M. Amyot's memoir were first made known. The parallelism of the two given planes to the circular sections of the surface is also stated in the Calendar; but this remarkable relation is not noticed by $\mathbf{M}$. Amyot, nor by M. Cauchy (see the "Examination Papers" of the year 1842, p. xlv., quest. 17, 18; in the Calendar for 1843). It is scarcely necessary to add, that the analogue which M. Amyot and other mathematicians have been seeking for, and which was long felt to be wanting in the theory of surfaces of the second order, is no other than the modular property of these surfaces, which appears to be not yet known abroad. M. Poncelet insists much on the importance of extending the signification of the terms focus and directrix, so as to make them applicable to surfaces; and he supposes this to have been effected, for the first time, by M. Amyot. These terms, however, applied in their true general sense to surfaces, had been in use, several years before, among the mathematical students of Dublin, as may be seen by referring to the Calendar ("Examination Papers" of the year 1838, p. c. 1839, p. xxxi.).

The locus above mentioned, being co-extensive with the umbilicar property, does not represent any surface which ean be generated by the right line, except the cone. To remedy this want of generality, M. Cauchy proposes to consider a surface of the second order as described by a point, the square of whose distance from a given point bears a constant ratio either to the rectangle under its distances from two given planes, or to the sum of the squares of these distances. This enunciation, no doubt, takes in both kinds of focals, and all the species of surfaces; but the additional conception is not of the kind required by the analogy in question, nor has it any of the characters of an elementary principle. For the given planes, according to M. Cauchy's idea, do not stand in any simple or natural relation to the surface; and besides there is no reason why, instead of the sum of the squares 
of the square to the rectangle will be the square of the number $m$ sec $\phi$, where $m$ is the modulus, and $\phi$ the angle which the primary axis makes with a directive plane.

When the umbilicar property is applied to the cone, the vertex of which is, as we have seen, to be regarded as an umbilicar focus, having the directive axis for its directrix, it indicates that the product of the sines of the angles which any side of the cone makes with its two directive planes is a constant quantity.

It is remarkable that the vertex of the cone affords the only instance of a focal point which is at once modular and umbilicar, as well as the only instance of a focal point which is doubly modular. This union of properties it may be conceived to owe to the circumstance that the cone is the asymptotic limit of the two kinds of hyperboloids. For if a series of hyperboloids have the same asymptotic cone, and their primary axes be indefinitely diminished, they will approach indefinitely to the cone; and, in the limit, the focal ellipse and hyperbola of the hyperboloid of one sheet will pass into the vertex and the focal lines of the cone, thus making the vertex doubly modular; while the focal ellipse of the hyperboloid of two sheets will also be contracted into the vertex, and will make that point umbilicar.

of the distances from the given planes, we should not take the sum after multiplying the one square by any given positive number, and the other square by another given positive number; nor is there any reason why we should not take other homogeneous functions of these distances. This conception would therefore be found of little use in geometrical applications; while the modular principle, on the contrary, by employing a simple ratio between two right lines, both of which have a natural connexion with the surface, lends itself with the greatest ease to the reasonings of geometry. Indeed the whole difficulty, in extending the property of the directrix to surfaces of the second order, consisted in the discovery of such a ratio inherent in all of them-a ratio having nothing arbitrary in its nature, and for which no other of equal simplicity can be substituted.

It may be proper to mention that the term modulus, which I have used for the first time in the present Paper, with reference to surfaces of the second order, has been borrowed from M. Cauchy, by whom it is employed, however, in a signification entirely different. Several other new terms are also now introduced, from the necessity of the case. 
When the two directive planes coincide, and become one directive plane, the umbilicar property is reduced to this, that the distances of any point in the surface from the point $\mathrm{F}$ and from the directive plane are in a constant ratio to each other; and therefore the surface becomes one of revolution round an axis passing through $\mathrm{F}$ at right angles to that plane; the point $F$ being a focus of the meridional section, or the vertex if the surface be a cone. When the directive planes are supposed to be parallel, but separated by a finite interval, we get the same class of surfaces of revolution, with the addition of the surface produced by the revolution of an ellipse round its minor axis; the point $\mathrm{F}$ being still on the axis of revolution, but not having any fixed relation to the surface.

$\S 10$. If in the equation (30) we supposed the right-hand member to have an additional term containing the product of the quantities $x-x_{2}$ and $y-y_{2}$, with a constant coefficient, all the foregoing conclusions regarding the geometrical meaning of that equation would remain unchanged, because the additional term could always be taken away by assigning proper directions to the axes of $x$ and $y$. If, after the removal of this term, the coefficients of the squares of the aforesaid quantities were both positive, the locus of $\mathrm{F}$ would be a modular focal of the surface expressed by the equation; but if one coefficient were positive and the other negative, the locus of $\mathrm{F}$ would be an umbilicar focal. The equation in its more general form is evidently that which we should obtain for the locus of a point $\mathrm{S}$, such that the square of its distance SF from a given point $\mathrm{F}$ should be a given homogeneous function of the second degree of its distances from two given planes; the plane of $x y$ being drawn through $F$ perpendicular to the intersection of these planes, and $x_{2}, y_{2}$ being the co-ordinates of any point on this intersection, while $x_{1}, y_{1}$ are the co-ordinates of $\mathrm{F}$. The point F might be any point on one of the focals of the surface described by $\mathrm{S}$; the intersection of the two planes (supposing them always parallel to fixed planes) being the corresponding directrix. 
These considerations may be further generalized, if we remark that the equation of any given surface of the second order may be put under the form

$$
\begin{gathered}
\left(x-x_{1}\right)^{2}+\left(y-y_{1}\right)^{2}+\left(z-z_{1}\right)^{2}=L\left(x-x_{2}\right)^{2}+M\left(y-y_{2}\right)^{2}+N\left(z-z_{2}\right)^{2} \\
+L^{\prime}\left(y-y_{2}\right)\left(z-z_{2}\right)+M^{\prime}\left(x-x_{2}\right)\left(z-z_{2}\right)+N^{\prime}\left(x-x_{2}\right)\left(y-y_{2}\right),
\end{gathered}
$$

where $L, M, N, L^{\prime}, M^{\prime}, N^{\prime}$ are constants, and $x_{1}, y_{1}, z_{1}$ are conceived to be the co-ordinates of a certain point F, and $x_{2}, y_{2}, z_{2}$ the co-ordinates of another point $\Delta$. The constants $L^{\prime}, M^{\prime}, N^{\prime}$ may, if we please, be made to vanish by changing the directions of the axes of co-ordinates; and when this is done, the new coordinate planes will be parallel to the principal planes of the surface. Then, by proceeding as before, it may be shown that, without changing the surface, we are at liberty, under certain conditions, to make the points $F$ and $\Delta$ move in space. The conditions are expressed geometrically by saying that the two surfaces, upon which these points must be always found, are reciprocal polars with respect to the given surface, the points $F$ and $\Delta$ being, in this polar relation, corresponding points; and that the surface which is the locus of $F$ is a surface of the second order, confocal with the given one, it being understood that confocal surfaces are those which have the same focal lines. The surface on which $\Delta$ lies is therefore also of the second order, and the right line $\Delta \mathrm{F}$ is a normal at $\mathrm{F}$ to the surface which is the loous of this point. Moreover, if through the point $\Delta$ three or more planes be drawn parallel to fixed planes, and perpendiculars be dropped upon them from any point $\mathrm{S}$ whose co-ordinates are $x, y, z$, the right-hand member of the equation (38) may be conceived to represent a given homogeneous function of the second degree of these perpendiculars; and the given surface may therefore be regarded as the locus of a point $\mathrm{S}$, such that the square of the distance SF is always equal to that function.

$\S 11$. In the enumeration of the surfaces capable of being generated by the modular method, we miss the five following 
varieties, which are contained in the general equation of the second degree, but are excluded from that method of generation by reason of the simplicity of their forms-namely, the sphere, the right cylinder on a circular base, and the three surfaces which may be produced by the revolution of a conic section (not a circle) round its primary axis.* These three surfaces are the prolate spheroid, the hyperboloid of two sheets, and the paraboloid of revolution; and the circumstance, that the foci of the generating curves are also foci of the surfaces, renders it easy to investigate their focal properties. $\dagger$ In point of simplicity, the excepted surfaces are to the other surfaces of the second order what the eircle is to the other conic sections, the eircle being, in like manner, excepted from the curves which can be generated by the analogous method in plano; and the geometry of the five excepted surfaces may therefore be regarded as comparatively elementary. These five surfaces were, in fact, studied by the Greek geometers, $\ddagger$ and, along with the oblate spheroid and the cone, they make up all the surfaces of the second order with which the ancients were acquainted. Except the cone, the surfaces considered by them are all of revolution; and there is only one surface of revolution, the hyperboloid of one sheet, which was not noticed until modern times. This surface is mentioned (under the name of the hyperbolic cylindroid) by Wren, $\S$ who remarks that it can be generated by the revolution of a right line round another right line not in the same plane. As to the general conception of surfaces of the second order, the suggestion of it was reserved for the algebraio geometry of Descartes. In that geometry the

* The case of two parallel planes is also excluded, but it is not here taken into account. The case of two parallel right lines is in like manner excluded from the corresponding generation of lines of the second order.

+ A Paper by M. Chasles, on these surfaces of revolution, will be found in the "Memoirs," of the Academy of Brussels, tom. v. (An. 1829).

₹ The hyperboloid of two sheets, and the paraboloid of revolution, were known by the name of conoids. Archimedes has left a treatise on Conoids and Spheroids, as well as a treatise on the Sphere and Cylinder.

\$ In the Philosophical Transactions for the year 1669, p. 961. 
curves previously known as sections of the cone are all expressed by the general equation of the second degree between two coordinates; and hence it occurred to Euler* about a century ago, to examine and classify the different kinds of surfaces comprised in the general equation of the second degree among three coordinates. The new and more general forms thus brought to light have since engaged a large share of the attention of geometers; but the want of some other than an algebraic principle of connexion has prevented any great progress from being made in the investigation of such of their properties as do not immediately depend on transformations of co-ordinates. This want the modular method of generation perfectly supplies, by ovolving the different forms from a simple geometrical conception, at the same time that it brings them within the range of ideas familiar to the ancient geometry, and places their relation to the conic sections in a striking point of view.

It may be well to remark that the excepted. surfaces are limits of surfaces which can be generated modularly, as the circle is the limit of the ellipse in the analogous generation of the conic sections. Thus the sphere is the limit of an oblate spheroid, one of whose axes remains constant, while its focal circle is indefinitely diminished; and the right circular cylinder is the limit of an elliptic cylinder, whose focal lines are conceived to approach indefinitely to coincidence with each other and with the axis of the cylinder, while one of the axes of the principal elliptic section remains constant. In these cases the dirigent lines, along with the directrices, move off to infinity. The other three excepted surfaces correspond to the supposition $\phi=90^{\circ}$, which was excluded in the discussion of the general equation (1). For if we make $m$ sec $\phi=n$, the quantity which constitutes the right-hand member of that equation may be written

$$
n^{2}\left(x-x_{2}\right)^{2}+n^{2}\left(y-y_{2}\right)^{2} \cos ^{2} \phi
$$

and if we suppose $n$ to remain finite and constant, while $\phi$

* See his Introductio in Analysin Infinitorum, p. 373. Lausanne, 1748. 
approaches to $90^{\circ}$, and $m$ indefinitely diminishes, this quantity will approach indefinitely to $n^{2}\left(x-x_{2}\right)^{2}$, which will be its limiting value when $\phi=90^{\circ}$. But $x-x_{2}$ is the distance of the point $\mathrm{S}$ from a fixed plane intersecting the axis of $x$ perpendicularly at the distance $x_{2}$ from the origin of co-ordinates; and therefore, in the limit, the equation expresses that the distances of any point $\mathrm{S}$ of the surface, from the focus $\mathrm{F}$ and from this fixed plane, are to each other as $n$ to unity, that is, in a constant ratio, which is a common property of the three surfaces in question. This property also belongs to the right cone, but the right cone does not rank among the excepted surfaces.

\$12. We have seen that, when the modulus is unity, any plane parallel to either of the directive planes intersects the surface in a right line; whence it follows, that through any point on the surface of a hyperbolic paraboloid two right lines may be drawn which shall lie entirely in the surface. The plane of these right lines is of course the tangent plane at that point, and therefore every tangent plane intersects the surface in two right lines. This is otherwise evident from considering that the sections parallel to a given tangent plane are similar hyperbolas, whose centres are ranged on a diameter passing through the point of contact, and whose asymptotes, having always the same directions, are parallel to two fixed right lines which we may suppose to be drawn through that point. For as the distance between the plane of section and the tangent plane diminishes, the axes of the hyperbola diminish; and they vanish when that distance vanishes, the hyperbola being then reduced to its asymptotes. The tangent plane therefore intersects the surface in the two fixed right lines aforesaid. The same reasoning, it is manifest, will apply to any other surface of the second order which has hyperbolic seotions parallel to its tangent planes; and therefore the hyperboloid of one sheet, which is the only other such surface, ${ }^{*}$ is also intersected in two

* The double generation of these two surfaces by the motion of a right line has been long known. It appears to have been discovered and fully discussed by some of the first pupils of the Polytechnic School of Paris. This mode of generation had, 
right lines by any of its tangent planes. These right lines are usually called the generatrices of the surface.

From what has been said, it appears that the generatrices of the hyperbolic paraboloid, and the asymptotes of its sections (all its sections, except those made by planes parallel to the axis, being hyperbolas), are parallel to the directive planes. The generatrices of the hyperboloid of one sheet, and the asymptotes of its hyperbolic sections, are parallel to the sides of the asymptotic cone; because any section of the hyperboloid is similar to a parallel section of the asymptotic cone; and when the latter section is a hyperbola its asymptotes are parallel to two sides of the cone.

\section{Part II.-Properties of Surfaces of the Second Order.}

$\S 1$. In the preceding part of this Paper it has been necessary to enter into details for the purpose of communicating fundamental notions clearly. In the following part, which will contain certain properties of surfaces of the second order, we shall be as brief as possible; giving demonstrations of the more elementary theorems, but confining ourselves to a short statement of the rest.

Many consequences follow from the principles already laid down.

Through any directrix of a surface of the second order let a fixed plane be drawn cutting the surface, and let $S$ be any point of the section. If the directrix and its focus $\mathrm{F}$ be modular, and if a plane always parallel to the same directive plane be conceived to pass through $\mathrm{S}$ and to cut the directrix in $\mathrm{D}$, the directive distance SD will be always parallel to a given right line, and will therefore be in a constant ratio to the perpendicular distance of $\mathrm{S}$ from the directrix. This perpendicular distance will

however, been remarked by Wren, with regard to the hyperboloid of revolution. It does not seem to have been observed, that the existence of rectilinear generatrices is included in the idea of hyperbolic sections parallel to a tangent plane. 
consequently bear a given ratio to SF, the distance of the point $\mathrm{S}$ from the focus. And the same thing will be true when the directrix and focus are umbilicar, because the perpendicular distance of the point $\mathbb{S}$ from the directrix will be in a constant ratio to its distance from each directive plane drawn through the directrix.

The fixed plane of section will in general contain another directrix parallel to the former, and belonging to the same focal ; and it is evident that the perpendicular distance of $S$ from this other directrix will be in a given ratio to its distance $\mathrm{SF}^{\prime}$ from the corresponding focus $\mathrm{F}^{\text {}}$, the ratio being the same as in the former case. Hence, according as the point $\mathrm{S}$ lies between the two directrices, or at the same side of both, the sum or difference of the distances $\mathrm{SF}$ and $\mathrm{SF}^{\prime}$ will be constant.

If the plane of section pass through either of the foci, as $\mathrm{F}$, this focus and its directrix will manifestly be the focus and directrix of the section. In this case the plane of section will be perpendicular to the focal at $F$. And if the surface be a cone, the point $\mathrm{F}$ being anywhere on one of its focal lines, the distance of the point $\mathrm{S}$ from the direetrix will be in a constant ratio to its perpendicular distance from the dirigent plane which contains the directrix, and therefore this perpendicular distance will be in a given ratio to the distance SF. Now, calling $\mathrm{V}$ the vertex of the cone, and taking SV for radius, the perpendicular distance aforesaid is the sine of the angle which the side SV of the cone makes with the dirigent plane; and SF, which is perpendicular to VF, is the sine of the angle SVF. Consequently the sines of the angles which any side of a cone makes with a dirigent plane and the corresponding focal line are in a given ratio to each other.

$\S 2$. Conceive a surface of the second order to be intersected in two points $\mathrm{S}, \mathrm{S}^{\prime}$ by a right line which cuts two parallel directrices in the points $\mathrm{E}, \mathrm{E}^{\prime}$, and let $\mathrm{F}, \mathrm{F}^{\prime}$ be the foci corresponding respectively to these directrices. The perpendicular distances of the points $S, S^{\prime}$ from the first directrix and from the second are to each other as the lengths $\mathrm{SE}, \mathrm{S}^{\prime} \mathrm{E}, \mathrm{SE}, \mathrm{S}^{\prime} \mathrm{E}^{\prime}$ 
respectively, and therefore the ratios of $\mathrm{FS}$ to $\mathrm{SE}$, of $\mathrm{FS}^{\prime}$ to $\mathrm{S}^{\prime} \mathrm{E}$, of $\mathrm{F}^{\prime} \mathrm{S}$ to $\mathrm{SE}^{\prime}$, and of $\mathrm{F}^{\prime} \mathrm{S}^{\prime}$ to $\mathrm{S}^{\prime} \mathrm{E}^{\prime}$, are all equal.

Hence, the right line FE bisects one of the angles made by the right lines FS and FS'; and the right line $\mathrm{F}^{\prime} \mathrm{E}^{\prime}$ bisects one of the angles made by $F^{\prime} S$ and $F^{\prime} S^{\prime}$.

When the points $\mathrm{S}, \mathrm{S}^{\prime}$ are at the same side of $\mathrm{E}$, the angle supplemental to SFS' is that which is bisected by the right line FE. Now if the point $S$ be fixed, and $S^{\prime}$ approach to it indefinitely, the angle SFE will approach indefinitely to a right angle. Therefore if a right line touching the surface meet a directrix in a certain point, the distance between this point and the point of contact will subtend a right angle at the focus which corresponds to the directrix. And if a cone circumscribing the surface have its vertex in a directrix, the curve of contact will be in a plane drawn through the corresponding focus at right angles to the right line which joins that focus with the vertex.

When the surface intersected by the right line $\mathrm{SS}^{\prime}$ is a cone, suppose this line to lie in the plane of the focus $\mathrm{F}$ and its directrix, that is, in the plane which is perpendicular at $\mathrm{F}$ to the focal line VF (the vertex of the cone being denoted, as before, by V); the angles made by the right lines FE, FS, FS', are then the same as the angles made by planes drawn through VF and each of the right lines VE, VS, VS'; and the last three right lines are the intersections of a plane VSS' with the dirigent plane on which the point $\mathrm{E}$ lies, and with the surface of the cone. Therefore if a plane passing through the vertex of a cone intersect its surface in two right lines, and one of its dirigent planes in another right line, and if a plane be drawn through each of these right lines respectively and the focal line which belongs to the dirigent plane, the last of the three planes so drawn will bisect one of the angles made by the other two. And hence, if a plane touching a cone along one of its sides intersect a dirigent plane in a certain right line, and if through this right line and the side of contact, respectively, two planes be drawn intersecting each other in the focal line which corresponds to the dirigent plane, the two planes so drawn will be at right angles to each other. 
Let a right line touching a surface of the second order in $\mathrm{S}$ meet two parallel directrices in the points $\mathrm{E}, \mathrm{E}^{\prime}$, and let $\mathrm{F}, \mathrm{F}^{\prime}$ be the corresponding foci. Then the triangles FSE and $\mathrm{F}^{\prime} \mathrm{SE}^{\prime}$ are similar, because the angles at $\mathrm{F}$ and $\mathrm{F}^{\prime}$ are right angles, and the ratio of FS to $\mathrm{SE}$ is the same as the ratio of $\mathrm{F}^{\prime} \mathrm{S}$ to $\mathrm{SE}^{\prime}$. Therefore the tangent EE' makes equal angles with the right lines drawn from the point of contact $S$ to the foci $F, F^{\prime}$. When the surface is a cone, let the tangent be perpendicular to the side VS which passes through the point of contact; the angles FSE and $\mathrm{F}^{\prime} \mathrm{SE}^{\prime}$ are then the angles which the tangent plane $\mathrm{VEE}^{\prime}$ makes with the planes VSF and $\mathrm{VSF}^{\prime}$, because the right line FE is perpendicular to the plane VSF, and the right line $\mathrm{F}^{\prime} \mathrm{E}^{\prime}$ is perpendicular to the plane VSF'. Therefore the tangent plane of a cone makes equal angles with the planes drawn through the side of contact and each of the focal lines.

Supposing a section to be made in a surface of the second order by a plane which cuts any directrix in the point $\mathrm{E}$, if the focus $\mathrm{F}$ belonging to this directrix be the vertex of a cone having the section for its base, the right line FE will be an axis of the cone. For if through FE any plane be drawn cutting the base of the cone in the points $\mathrm{S}, \mathrm{S}^{\prime}$, one of the angles made by the sides $\mathrm{FS}, \mathrm{FS}^{\prime}$ which pass through these points will always be bisected by the right line $\mathrm{FE}$; and this is the characteristio property of an axis.

\& 3. Two surfaces of the second order being supposed to have the same focus, direetrix, and directive planes, so that they differ only in the value of the modulus $m$, or of the umbilicar ratio $\mu$ (see Part I. § 9) : let a right line passing through any point $\mathrm{E}$ of the directrix cut one surface in the points $\mathrm{S}, \mathrm{S}^{\prime}$, and the other in the points $S_{0}, S_{1}$, and conceive right lines to be drawn from all these points to the common focus F. Since, if ratios be expressed by numbers, the ratio of FS to SE (or of $\mathrm{FS}^{\prime}$ to $\mathrm{S}^{\prime} \mathrm{E}$ ) is to the ratio of $\mathrm{FS}_{0}$ to $\mathrm{S}_{0} \mathrm{E}$ (or of $\mathrm{FS}_{1}$ to $\mathrm{S}_{1} \mathrm{E}$ ) as the value of $m$ for the one surface is to its value for the other, when the focus is modular, or as the value of $\mu$ for the one surface is to its value for the other when the focus is umbilicar, the 
sines of the angles EFS 0 and EFS (or of the angles EFS End $_{1}$ EFS') are in a constant proportion to each other, because these sines are proportional to those ratios. And since the right line FE bisects the angles SFS' and $\mathrm{S}_{0} \mathrm{FS}_{1}$, both internally or both externally, in which case the angles $\mathrm{SFS}_{0}$ and $\mathrm{S}^{\prime} \mathrm{FS}_{1}$ are equal, or else one internally and the other externally, in which case the angles $\mathbf{S F S}_{0}$ and $\mathbf{S}^{\prime} \mathrm{FS}_{1}$ are supplemental, it is easy to infer, from the constant ratio of the aforesaid sines, that in the first case the product, in the second case the ratio of the tangents of the halves of the angles SFS $_{0}$ and S $^{\prime} \mathrm{FS}_{0}$ (or of the halves of the angles $\mathrm{SFS}_{1}$ and $\mathrm{S}^{\prime} \mathrm{FS}_{1}$ ) is a consequent quantity.

If the point $S^{\prime}$ approximate indefinitely to $S$, the right line passing through these points will approach indefinitely to a tangent. Therefore when two surfaces are related as above, if a right line passing through any point $\mathbf{E}$ of their common directrix intersect one surface in the points $S_{0}, \mathbb{S}_{1}$, and touch the other in the point $S$, the chord $S_{0} S_{1}$ will subtend a constant angle at the common focus F, and this angle will be bisected, either internally or externally, by the right line FS drawn from the focus to the point of contact. And the angle EFS being then a right angle, the cosine of the angle $\mathrm{SFS}_{0}$ or $\mathrm{SFS}_{1}$ will be equal to the ratio of the less value of $m$ or $\mu$ to the greater.*

$\S 4$. Among the surfaces of the second order, the only one which has a point upon itself for a modular focus is the cone, the vertex of which is such a focus, related either to the internal or to the mean axis as directrix. In the latter relation the vertex belongs to the series of foci which are ranged on the focal lines. To see the consequence of this, let $\mathrm{V}$ be the vertex of the cone, and VW its mean axis perpendicular to the plane of the focal lines. On one of the focal lines and its dirigent assume any corresponding points $F$ and $\Delta$, and let $\Delta D$ be the directrix passing through $\Delta$. Then if a directive plane, drawn through any point $S$ of the surface, cut this directrix in $\mathrm{D}$ and the mean

* See Exam. Papers, An. 1839, p. xxxi., questions 9, 10. These and some of the preceding theorems were originally stated with reference to modular foci only. They are now extended to umbilicar foci. 
axis in W, the ratio of SF to SD will be expressed by the linear modulus, as will also the ratio of $\mathrm{VF}$ to $\mathrm{WD}$, since $\mathrm{V}$ is a point of the surface, and WD is equal to the directive distance of $\mathrm{V}$ from $\Delta \mathrm{D}$. But since $\nabla$ is a focus to which the mean axis is directrix, the ratio of $\mathrm{SV}$ to $\mathrm{SW}$ is expressed by the same modulus. Thus the triangles SVF and SWD are similar, the sides of the one being proportional to those of the other. Therefore the angle SVF is equal to the angle SWD ; that is to say, the angle which the side VS of the cone makes with the focal line $\mathrm{VF}$ is equal to the angle contained by two right lines WD and WS, of which one is the intersection of the directive plane with the dirigent plane VWD corresponding to VF, and the other is the intersection of the directive plane with the plane VWS passing through the mean axis and the side VS of the cone.

Hence it appears that the sum of the angles (properly reckoned) which any side of the cone makes with its two focal lines is constant. For if $\mathrm{F}^{\prime}$ be a point on the other focal line, and $\mathrm{D}^{\prime}$ the point where the directrix corresponding to $\mathrm{F}^{\prime}$ is intersected by the same directive plane SWD, it may be shown, as above, that the angle $\mathrm{SVF}^{\prime}$ is equal to the angle $\mathrm{SWD}^{\prime}$, that is, to the angle made by the right line WS with the right line WD', in which the directive plane intersects the dirigent plane corresponding to $\mathrm{VF}^{\prime}$. Conceiving therefore the points $\mathrm{F}, \mathrm{F}^{\prime}, \mathrm{S}$, and with them the points $D, D^{\prime}$, to lie all on the same side of the principal plane which is perpendicular to the internal axis, the right line WS will lie between the right lines WD and WD', and the sum of the angles SVF and $\mathrm{SVF}^{\nu}$ will be equal to the angle DWD', which is a constant angle, being contained by the right lines in which a directive plane intersects the two dirigent planes of the cone. This constant angle will be found to be equal, as it ought to be, to one of the angles made by the two sides of the cone which are in the plane of the focal lines, namely, to the angle within which the internal axis lies.

If we conceive the cone to have its vertex at the centre of a sphere, and the points $\mathrm{F}, \mathrm{F}^{\prime}, \mathrm{S}$ to be on the surface of this sphere, the ares of great circles connecting the point $\mathrm{S}$ with each of the 
fixed points $\mathrm{F}, \mathrm{F}^{\prime}$ will have a constant sum. The curve formed by the intersection of the sphere and the cone may therefore, from analogy, be called a spherical ellipse, or, more generally, a spherical conic, because, by removing one of its foci $F, F^{\prime}$ to the opposite extremity of the diameter of the sphere, the difference of the ares $\mathrm{SF}$ and $\mathrm{SF}^{*}$ will be constant, which shows that the spherical curve is analogous to the hyperbola as well as to the ellipse. Either of these plane curves may, in fact, be obtained as a limit of the spherical curve when the sphere is indefinitely enlarged, according as the diameter along which the enlargement takes place, and of which one extremity may be conceived to be fixed while the other recedes indefinitely, coincides with the internal or with the directive axis of the cone. The fixed extremity becomes the centre of the limiting curve, which is an ellipse in the first case, and a hyperbola in the second.

The great circle touching a spherical conic at any point makes equal angles with the two ares of great circles which join that point with the foci, because the sum of these arcs is constant. This is identical with a property already demonstrated relative to the tangent planes of the cone. Indeed it is obvious that the properties of the cone may also be stated as properties of the spherical conic, and this is frequently the more convenient way of stating them.

$\S 5$. If the sides of one cone be perpendicular to the tangent planes of another, the tangent planes of the former will be perpendicular to the sides of the latter. For the plane of two sides of the first cone is perpendicular to the intersection of the two corresponding tangent planes of the second cone; and as these two sides approach indefinitely to each other, their plane approaches to a tangent plane, while the intersection of the two corresponding tangent planes of the second cone approaches indefinitely to a side of the cone. Thus any given side of the one cone corresponds to a certain side of the other; and any side of either cone is perpendicular to the plane which touches the other along the corresponding side. This reasoning applies to cones of any kind. 
Two cones so related may be called reciprocal cones. When one is of the second order, it will be found that the other is also of the second order, and that, in their equations relative to their axes, which are obviously parallel or coincident, the coefficients of the squares of the corresponding variables are reciprooally proportional, so that the equations

$$
\boldsymbol{P} x^{2}+Q y^{2}+R z^{2}=0, \quad \frac{x^{2}}{\boldsymbol{P}}+\frac{y^{2}}{\mathbf{Q}}+\frac{\boldsymbol{z}^{2}}{R}=0,
$$

express two suoh cones which have a common vertex. These cones have the same internal axis, but the directive axis of the one coincides with the mean axis of the other, and it may be shown from the equations that the directive planes of the one are perpendieular to the focal lines of the other. The two curves in which these cones are intersected by a sphere, having its centre at their common vertex, are reciprocal spherical conics. In general, two curves traced on the surface of a sphere may be said to be reciprocal to each other, when the cones passing through them, and having a common vertex at the centre of the sphere, are reciprocal cones. Any given point of the one curve corresponds to a certain point of the other, and the great circle which touches either eurve at any point is distant by a quadrant from the corresponding point of the other curve.

By means of these relations any property of a cone of the second order, or of a spherical conic, may be made to produce a reciprocal property. Thus, we have seen that the tangent plane of a cone makes equal angles with two planes passing through the side of contact and through each of the focal lines; therefore, drawing right lines perpendicular to the planes, and planes perpendicular to the right lines here mentioned, we have, in the reciprocal cone, a side making equal angles with the right lines in which the directive planes of this cone are intersected by a plane touching it along that side. It is therefore a property of the cone, that the intersections of a tangent plane with the two directive planes make equal angles with the side of contact; a 
property which it is easy to prove without the aid of the reciprocal cone.

The two directive sections drawn throngh any point $\mathbf{S}$ of a given surface of the second order may, when they are circles, be made the directive sections of a cone, and this may obviously be done in two ways. Each of the two cones so determined will be touched by the plane which touches the given surface at the point $S$, because the right lines which are tangents to the two circular sections at that point are tangents to each cone as well as to the given surface; therefore the side of contact of each cone bisects one of the angles made by these two tangents; and hence the two sides of contact are the principal directions in the tangent plane at the point $\mathrm{S}$, that is, they are the directions of the greatest and least curvature of the given surface at that point; for these directions are parallel to the axes of a section made in the surface by a plane parallel to the tangent plane, and the axes of any section bisect the angles contained by the right lines in which the plane of section cuts the two directive planes.

$\S 6$. It has been shown that the sum of the angles which any side of a cone makes with its focal lines is constant. Hence we obtain the reciprocal property, ${ }^{*}$ that the sum of the angles (properly reckoned) which any tangent plane of a cone makes with its two directive planes is constant. This property may be otherwise proved as follows :-

Through a point assumed anywhere in the side of contact

* This property, and that to which it is reciprocal, as well as some other properties of the cone, were, together with the idea of reciprocal cones and of spherical conics, suggested by my earliest researches connected with the mechanical theory of rotation and the laws of double refraction. I was not then aware that the focal lines of the cone had been previously discovered, nor that the spherical conic had been introduced into geometry. Indeed all the properties of the cone which are given in this Paper were first presented to me in my own investigations. Its double modular property, related to the vertex as focus, was one of the propositions in the theory of the rotation of a solid body, and was used in finding the position of the axis of rotation within the body at a given time. But the modular property common to all the surfaces of the second order was not discovered until some years later. 
let two directive planes be drawn. As the circles in which the cone is cut by these planes have a common chord, they are circles of the same sphere; and a tangent plane applied to this sphere, at the aforesaid point, coincides with the tangent plane of the cone, because each tangent plane contains the tangents drawn to the two circles at that point. The common chord of the circles is bisected at right angles by the principal plane which is perpendicular to the directive axis, and therefore that principal plane contains the centres of the two eircles and the centre of the sphere. Now the acute angle made by a tangent plane of a sphere with the plane of any small eircle passing through the point of contact is evidently half the angle subtended at the centre of the sphere by a diameter of that circle; therefore the acute angles, which the common tangent plane of the cone and of the sphere above mentioned makes with the planes of the directive sections, are the halves of the angles subtended at the centre of the sphere by the diameters of the sections. But the diameters which lie in the principal plane already spoken of, and are terminated by two sides of the cone, are chords of the great circle in which that plane intersects the sphere; and the halves of the angles which they subtend at its centre are equal to the angles in the greater segments of which they are the chords, and consequently equal to the two adjacent acute angles of the quadrilateral which has these chords for its diagonals. Hence, as two opposite angles of the quadrilateral are together equal to two right angles, it follows that the four angles of the quadrilateral represent the four angles, the obtuise as well as the acute angles, which the tangent plane of the cone makes with the planes of the directive sections; the two angles of the quadrilateral which lie opposite to the same diagonal being equal to the acute and obtuse angles made by the tangent plane with the plane of the section of which that diagonal is the diameter.

Thus any two adjacent angles of the quadrilateral may be taken for the angles which the tangent plane of the cone makes with the directive planes. If we take the two adjacent angles which lie in the same triangle with the angle $\kappa$ contained by the 
two sides of the cone that help to form the quadrilateral, the sum of these two angles will be equal to two right angles diminished by $\kappa$; and if we take the two remaining angles of the quadrilateral, their sum will be equal to two right angles increased by $\kappa$; both which sums are constant. But if we take either of the other pairs of adjacent angles, the difference of the pair will be constant, and equal to $\kappa$.

The same conclusion may be deduced as a property of the spherical conio. Let a great circle touching this curve be intersected in two points, one on each side of the point of contact, by the two directive circles, that is, by two great circles whose planes are directive planes of the cone which passes through the conic and has its vertex at the centre of the sphere. Since the right lines in which the tangent plane of a cone intersects the directive planes are equally inclined to the side of contact, the aro intercepted between the points where the tangent circle of the conic intersects the directive circles is bisected in the point of contact; therefore, either of the spherical triangles whose base is the tangent are so intercepted, and whose other two sides are the directive circles, has a constant area; because, if we suppose the tangent arc to change its position through an indefinitely small angle, and to be always terminated by the directive circles, the two little triangles bounded by its two positions and by the two indefinitely small directive ares which lie between these positions, will have their nascent ratio one of equality, so that the area of either of the spherical triangles mentioned above will not be changed by the change in the position of its base. But in each of these triangles the angle opposite the base is constant; therefore the sum of the angles at the base is constant.

From this reasoning it appears that if a spherical triangle have a given area, and two of its sides be fixed, the third side will always touch a spherical conic having the fixed sides for its directive arcs, and will be always bisected in the point of contact.

$\S 7$. The intersection of any given central surface of the second order with a concentric sphere is a spherical conic, since the cone which passes through the curve of intersection, and has 
its vertex at the common centre, is of the second order. The cylinder also, which passes through the same curve and has its side parallel to any of the axes of the given surface, is of the second order; and the cone, the cylinder, and the given surface are condirective, that is, the directive planes of one of them are also the directive planes of each of the other two. This may be seen from the equations of the different surfaces; for, in general, two surfaces whose principal planes are parallel will be condirective, if, when their equations are expressed by co-ordinates perpendicular to these planes, the differences of the coefficients of the squares of the variables in the equation of the one be proportional to the corresponding differences in the equation of the other.

If any given surface of the second order be intersected by a sphere whose centre is any point in one of the principal planes, the cylinder passing through the curve of intersection, and having its side perpendicular to that principal plane, will be of the second order, and will be condirective with the given surface. This cylinder, when its side is parallel to the directive axis, is hyperbolio; otherwise it is elliptic. If a paraboloid be cut by any plane, the eylinder which passes through the curve of section, and has its side parallel to the axis of the paraboloid, will be condirective with that surface; and it will be elliptic or hyperbolic, according as the paraboloid is elliptic or hyperbolic.*

If two concentric surfaces of the second order be reciprocal polars with respect to a concentric sphere, the directive axis of the one surface will coincide with the mean axis of the other, and the directive planes of the one will be perpendicular to the asymptotes of the focal hyperbola of the other. When one of the surfaces is a hyperboloid, the other is a hyperboloid of the same kind; the asymptotes of the focal hyperbola of each sur-

* I have introduced the terms directive and condirective, as more general than the terms cyclic and biconcyclic employed by M. Chasles. The latter terms suggest the idea of circular sections, and therefore could not properly be used with reference to the hyperbolic paraboloid, or to the hyperbolic or parabolic cylinder, in each of which surfaces a directive section is a right line. 
face are the focal lines of its asymptotio cone; and the two asymptotio cones are reciprocal.

When any number of central surfaces of the second order are confocal, or, more generally, when their focal hyperbolas have the same asymptotes, it is obvious that their reciprocal surfaces, taken with respect to any sphere concentric with them, are all condirective.

$\S 8$. If a diameter of constant length, revolving within a given central surface, describe a cone having its vertex at the centre, the extremities of the diameter will lie in a spherical conic. And if the cone be touched by any plane, the side of contact will evidently be normal to the section which that plane makes in the given surface, and will therefore be an axis of the section. As the axes of a section always bisect the angles made by the two right lines in which its plane intersects the directive planes of the surface, and as the cone aforesaid has the same directive planes with the given surface, it follows that the right lines in which a tangent plane of a cone cuts its directive planes are equally inclined to the side of contact-a theorem which has been already obtained in another way.

If a section be made in a given central surface by any plane passing through the centre, the cone described by a constant semidiameter equal to either semiaxis of the section will touch the plane of section; for if it could cut that plane, a semiaxis would be equal to another radius of the section. Denoting by $r, r^{\prime}$ the semiaxes of the section, conceive two cones to be described by the revolution of two constant semidiameters equal to $r$ and $r^{\prime}$ respectively. These cones are condirective with the given surface, and have the plane of section for their common tangent plane. Supposing that surface to be expressed by the equation

$$
\frac{x^{2}}{\bar{P}}+\frac{y^{2}}{Q}+\frac{z^{2}}{\bar{R}}=1
$$

and the directive axis to be that of $y$, the axis of $x$ will be the internal axis of one cone, say of that described by $r$, and the axis 
of $z$ will be the internal axis of the other cone. Let $\kappa$ be the angle made by the two sides of the first cone which lie in the plane $x \approx$, and $\kappa^{\prime}$ the angle made by the two sides of the second cone which lie in the same plane; the former angle being taken so as to contain the axis of $x$ within it, and the latter so as to contain within it the axis of $z$. Then, considering $r, r^{\prime}$ as radii of the section $x z$ of the surface, we have obviously

$$
\begin{aligned}
& \frac{1}{r^{2}}=\frac{\cos ^{2} \frac{1}{2} \kappa}{P}+\frac{\sin ^{2} \frac{1}{2} \kappa}{R}=\frac{1}{2}\left(\frac{1}{P}+\frac{1}{R}\right)+\frac{1}{2}\left(\frac{1}{P}-\frac{1}{R}\right) \cos \kappa, \\
& \frac{1}{r^{\prime 2}}=\frac{\cos ^{2} \frac{1}{2} \kappa^{\prime}}{R}+\frac{\sin ^{2} \frac{1}{2} \kappa^{\prime}}{P}=\frac{1}{2}\left(\frac{1}{P}+\frac{1}{R}\right)-\frac{1}{2}\left(\frac{1}{P}-\frac{1}{R}\right) \cos \kappa^{\prime} ;
\end{aligned}
$$

observing that when these formulø give a negative value for $r^{2}$ or $r^{\prime 2}$, in which case the surface expressed by the equation (2) must be a hyperboloid, the direction of $r$ or $r^{\prime}$ meets, not that surface, but the surface of the conjugate hyperboloid expressed. by the equation

$$
\frac{x^{2}}{P}+\frac{y^{2}}{Q}+\frac{z^{2}}{R}=-1
$$

Now calling $\theta$ and $\theta^{\prime}$ the angles made by the tangent plane of the cones with the directive planes of the given surface, which are also the directive planes of each cone, the angles $\kappa, \kappa^{\prime}$ depend on the sum or difference of $\theta$ and $\theta$. If the latter angles be taken so that their sum may be equal to the supplement of $\kappa$, their difference will be equal to $\kappa^{\prime}$, and the formulø (3) will become

$$
\begin{aligned}
& \frac{1}{r^{2}}=\frac{1}{2}\left(\frac{1}{P}+\frac{1}{R}\right)-\frac{1}{2}\left(\frac{1}{P}-\frac{1}{R}\right) \cos \left(\theta+\theta^{\prime}\right) \\
& \frac{1}{r^{\prime 2}}=\frac{1}{2}\left(\frac{1}{P}+\frac{1}{R}\right)-\frac{1}{2}\left(\frac{1}{P}-\frac{1}{R}\right) \cos \left(\theta-\theta^{\prime}\right),
\end{aligned}
$$

by which the semiaxes of any central section are expressed in terms of the non-directive semiaxes of the surface, and of the 
angles which the plane of section makes with the directive planes.*

$\S 9$. From the centre $O$ of the surface expressed by equation (2) let a right line $\mathrm{O} \Sigma$ be drawn cutting perpendicularly in $\Sigma$ the plane which touches the surface at $\mathbf{S}$. Let $\sigma$ denote the length of the perpendicular $0 \Sigma$, and $a, \beta, \gamma$ the angles which it makes with $x, y, z$. Then

$$
\sigma^{2}=P \cos ^{2} a+Q \cos ^{2} \beta+R \cos ^{2} \gamma .
$$

From this formula it is manifest that, if three planes touching the surface be at right angles to each other, the sum of the squares of their perpendicular distances from the centre will be equal to the constant quantity $P+Q+R$, and therefore the point of intersection of the planes will lie in the surface of a given sphere. If another surface represented by the equation

$$
\frac{x^{2}}{P_{0}}+\frac{y^{2}}{Q_{0}}+\frac{z^{2}}{R_{0}}=1
$$

be touched by a plane cutting $\mathrm{O} \Sigma$ perpendicularly in $\boldsymbol{\Sigma}_{0}$, and if $\sigma_{0}$ be the length of $\mathrm{O} \Sigma_{0}$, then

$$
\sigma_{0}^{2}=P_{0} \cos ^{2} a+Q_{0} \cos ^{2} \beta+R_{0} \cos ^{2} \gamma ;
$$

and therefore when the two surfaces are confocal, that is, when

$$
\boldsymbol{P}-\boldsymbol{P}_{0}=\boldsymbol{Q}-\boldsymbol{Q}_{0}=\boldsymbol{R}-\boldsymbol{R}_{0}=k,
$$

we have $\sigma^{2}-\sigma_{0}^{2}=k$, which is a constant quantity. Hence, if three confocal surfaces be touched by three rectangular planes, the sum of the squares of the perpendiculars dropped on these planes from the centre will be constant, and the locus of the intersection of the planes will be a sphere.

The focal curves of a given surface are the limits of surfaces confocal with it, $\uparrow$ when these surfaces are conceived, by the pro-

* See Transactions of the Royal Irish Academy, Vor. xxI., as before cited. The formulx (5) were first given, for the case of the ellipsoid, by Fresnel, in his Theory of Double Refraction, Mémoires de l'Institut, tom. vii., p. 155.

$\uparrow$ It was by this consideration, arising out of the theorems given in this and the next section about confocal surfaces, that I was led to perceive the nature of the 
gressive diminution of their mean or secondary axes, to become flattened, and to approach more and more nearly to a plane passing through the primary axis. And it will appear hereafter, that if a bifocal right line, that is, a right line passing through both focal curves, be the intersection of two planes touching these curves, those two planes will be at right angles to each other. Therefore the locus of the point where a tangent plane of a given central surface is intersected perpendicularly by a bifocal right line is a sphere. The primary axis of the surface is evidently the diameter of this sphere.

Hence we conclude that the locus of the point where a tangent plane of a paraboloid is intersected perpendicularly by a bifocal right line is a plane touching the paraboloid at its vertex. For a paraboloid is the limit of a central surface whose primary axis is prolonged indefinitely in one direction, and a plane is the corresponding limit of the sphere described on that axis as diameter. As this consideration is frequently of use in deducing properties of paraboloids from those of central surfaces, it may be well to state it more particularly. It is to be observed, then, that the indefinite extension of the primary axis at one extremity may take place according to any law which leaves the other extremity always at a finite distance from a given point, and. gives a finite limiting parameter to each of the principal sections of the surface which pass through that axis. The simplest supposition is, that one extremity of the axis and the adjacent foci of those two principal sections remain fixed, while the other extremity and the other foci move off, with the centre, to distances which are conceived to increase without limit. Then, at any finite distances from the fixed points, the focal curves approach indefinitely to parabolas, as do also all sections of the surface which pass through the primary axis, while the surface itself approaches indefinitely to a paraboloid; so that the limit

focal curves, and the analogy between their points and the foci of conics. And I regarded that analogy as fully established when I found (in March or April, 1832) that the normal at any point of a surface of the second order is an axis of the cone which has that point for its vertex and a focal for its base. 
of the central surface is a paraboloid having parabolas for its focal ourves. The limit of an ellipsoid, or of a hyperboloid of two sheets, is an elliptic paraboloid, having one of its focals modular and the other umbilicar, like each of the central surfaces from which it may be derived; and the limit of a hyperboloid of one sheet is a hyperbolic paraboloid, having, like that hyperboloid, both its focals modular.

$\S 10$. Let the plane touching at $\mathbf{S}$ the surface expressed by equation (2) intersect the axis of $x$ in the point $\mathbf{X}$, and let the normal applied at $\mathrm{S}$ intersect the planes $y \approx, x z, x y$, in the points $\mathrm{L}, \mathbf{M}, \mathbf{N}$ respectively. Since the section made in the surface by a plane passing through $O X$ and the point $S$ has one of its axes in the direction of $\mathrm{OX}$, it appears, by an elementary property of conies, that the rectangle under $\mathrm{OX}$ and the co-ordinate $x$ of the point $\mathrm{S}$ is equal to the quantity $P$; but that co-ordinate is to LS as $\mathrm{O \Sigma}$ or $\sigma$ is to $\mathrm{OX}$, and therefore the rectangle under $\sigma$ and LS is equal to $P$. Similarly the rectangle under $\sigma$ and MS is equal to $Q$, and the rectangle under $\sigma$ and NS is equal to $R$. Thus the parts of the normal intercepted between the point $S$ and each of the principal planes are to each other as the squares of the semiaxes respectively perpendicular to these planes; the square of an imaginary semiaxis being regarded as negative, and the corresponding intercept being measured from $S$ in a direction opposite to that which corresponds to a real semiaxis.

The rectangle under $\sigma$ and the part of the normal intercepted between two principal planes is equal to the difference of the squares of the semiaxes which are perpendicular to these planes. This rectangle is therefore constant, not only for a given surface, but for all surfaces which are confocal with it.

Hence the part of the normal intercepted between two principal planes bears a given ratio to the part of it intercepted between one of these and the third principal plane, whether the normal be applied at any point of a given surface, or at any point of a surface confocal with it.

If therefore normals to a series of confocal surfaces be all parallel to a given right line, they must all lie in the same plane 
passing through the common centre of the surfaces, because otherwise the parts of any such normal, which are intercepted between each pair of principal planes, would not be in a constant ratio to each other.

The point $S$ being the point at which any of these parallel normals is applied, the plane touching the surface at $S$ is parallel to a given plane; the perpendicular $0 \Sigma$ dropped upon it from the centre has a given direction, the plane OS $\Sigma$ is fixed, and the directions of the lines OL, OM, ON, in which the plane intersects the principal planes, are also fixed. And as the angle $0 \Sigma \mathrm{S}$ is always a right angle, and the normal at $\mathbf{S}$ is always parallel to $G \Sigma$, the distance $\mathbf{S} \boldsymbol{\Sigma}$ bears a given ratio to each of the distances OL, OM, ON, and therefore also to each of the intercepts MN. Hence, since the rectangle under $0 \Sigma$ and any one of these intercepts is constant, the rectangle under $0 \mathbf{\Sigma}$ and $\mathbf{S} \boldsymbol{\Sigma}$ is constant.

Therefore if a series of confocal central surfaces be touched by parallel planes, the points of contact will all lie in one plane, and their locus, in that plane, will be an equilateral hyperbola, having its centre at the centre of the surfaces, and having one of its asymptotes perpendicular to the tangent planes. This hyperbola evidently passes through two points on each of the focal curres, namely the points where the tangent to each curve is parallel to the tangent planes.

If a series of confocal paraboloids be touched by parallel planes, it will be found that the points of contact all lie in a bifocal right line, and that the normals at these points lie in a plane parallel to the axis of the surfaces; so that the part of any normal which is intercepted by the two principal planes is constant. This theorem may be proved from the two following properties of the paraboloid:-1. A normal being applied to the surface at the point $\mathbb{S}$, the segments of the normal, measured from $S$ to the points where it intersects the planes of the two principal sections, are to each other inversely as the parameters of these sections. 2. Supposing the axis $x$ to be that of the surface, the difference between the co-ordinates $x$ of the point $\mathbf{S}$ and of the point where the normal meets the plane of the prineipal 
sections, is equal to the semiparameter of the other principal section.

$\S$ 11. Let a tangent plane, applied at any point $\mathrm{S}$ of a surface of the second order, intersect the plane of one of its focals in the right line $\theta$, and let $\mathrm{P}$ be the foot of the perpendicular dropped from $S$ upon the latter plane. The pole of the right line $\theta$, with respect to the principal section lying in this plane, is the point $P$. Let $\mathrm{N}$ be its pole with respect to the focal. Then if $\mathrm{T}$ be any point of the right line $\Theta$, the polar of this point with respect to the section will pass through $P$, and its polar with respect to the focal will pass through $\mathrm{N}$; and if the former polar intersect the dirigent curve in $\Delta, \Delta^{\prime}$, and the latter intersect the focal in $\mathrm{F}, \mathrm{F}^{\prime}$, the points $\mathrm{F}, \mathrm{F}^{\prime}$ will correspond respectively to the points $\Delta, \Delta^{\prime}$, and the distances $\Delta \Delta^{\prime}$ and $\mathrm{FF}^{\prime}$ will be similarly divided by the points $\mathrm{P}$ and $\mathrm{N}$ (see Part I. $\S 8$ ). But since the point $\mathrm{S}$ is in the plane of the two directrices which pass through $\Delta$ and $\Delta^{\prime}$, the lengths $\Delta \mathrm{P}$ and $\Delta^{\prime} \mathrm{P}$, which are the perpendicular distances of $\mathrm{S}$ from the directrices, are proportional to the lengths FS and F'S. Therefore FN is to $F^{\prime} N$ as FS is to $F^{\prime} S$, and the right line NS bisects one of the angles made by the right lines FS and F'S. And as this holds wherever the point $T$ is taken on the right line $\theta$, that is, in whatever direction the right line $\mathrm{FF}^{\prime}$ passes through the point $\mathrm{N}$, it follows that the right line NS is an axis of the cone which has the point $\mathrm{S}$ for its vertex and the focal for its base. Further, if $\mathrm{FF}^{\prime}$ intersect $\theta$ in the point $Q$, we have $F N$ to $F^{\prime} N$ as $F Q$ is to $F^{\prime} Q$, because $N$ is the pole of $\Theta$ with respect to the focal; therefore $F Q$ is to $F^{\prime} Q$ as FS is to F'S, and hence the right line QS also bisects one of the angles made by FS and F'S. The right lines NS and QS are therefore at right angles to each other; and as the latter always lies in the tangent plane, the former must be perpendicular to that plane.

Consequently the normal at any point of a surface of the second order is an axis of the cone which has that point for its vertex and either of the focals for its base.

It is known that when two confocal surfaces intersect each 
other, they intersect everywhere at right angles; and that through any given point three surfaces may in general be described, which shall have the same focal ourves. If three confocal surfaces pass through the point $S$, the normal to each of them at $\mathbf{S}$ is an axis of each of the cones which stand on the focals and have $\mathrm{S}$ for their common vertex. The normals to the three surfaces are therefore the three axes of each cone.

If the points at which a series of confocal surfaces are touched by parallel planes be the vertices of cones having one of the focals for their common base, each of these cones will have ane of its axes perpendicular to the tangent planes. Therefore when an axis of a cone which stands on a given base is always parallel to a given right line, the locus of the vertex is an equilateral hyperbola or a right line, according as the base is a central conic or a parabola.

$\S 12$. A system of three confocal surfaces intersecting each other consists of an ellipsoid, a hyperboloid of one sheet, and a hyperboloid of two sheets, if the focals be central conics; but it consists of two elliptio paraboloids and a hyperbolic paraboloid, if the focals be parabolas. In the central system, the ellipsoid has the greatest primary axis, and the hyperboloid of two sheets the least; and the focal which is modular in one of these surfaces is umbilicar in the other. The asymptotic cones of the hyperboloids are confocal, the focal lines of each cone being the asymptotes of the focal hyperbola. In the system of paraboloids, the two elliptic paraboloids are distinguished by the circumstance that the modular focal of the one is the umbilicar focal of the other.

The curve in which two confocal surfaces intersect each other is a line of curvature of each, as is well known;" and a series of lines of curvature on a given surface are found by making a series of confocal surfaces intersect it.

Now if a series of the lines of curvature of a given surface be projected on one of its directive planes by right lines parallel

- See Dupin's Développements de Géométrie. 
to either of its non-directive axes, the projections will be a series of confocal conics; and when the surface is umbilicar, the faci of all these conics will be the corresponding projections of the umbilics." When the surface is not umbilicar, its directive axis will be parallel to the primary axis of the projections.

The same line of ourvature has two projections, according as it is projected by right lines parallel to the one or to the other non-directive axis. In the ellipsoid these projections are always curves of different kinds, the one being an ellipse when the other is a hyperbola; but in a hyperboloid the projections are either both ellipses or both hyperbolas. In the hyperbolic paraboloid the projections are parabolas. In the elliptic paraboloid one of the projections is always a parabola, and the other is either an ellipse or a hyperbola.

The corresponding projections of two lines of curvature which pass through a given point of the surface are confocal conics intersecting each other in the projection of that point, and of course intersecting at right angles.

$\S 13$. A bifocal chord is a bifocal right line terminated both ways by the surface.t In a central surface, the length of a bifocal chord is proportional to the square of the diameter which is parallel to it; the square of the diameter being equal to the rectangle under the chord and the primary axis.

More generally, if a chord of a given central surface touch two other given surfaces confocal with it, the length of the chord will be proportional to the square of the parallel diameter of the first surface, the square of the diameter being equal to the rectangle under the chord and a certain right line $2 l$, determined by the formula

$$
l^{2}=\frac{P Q R}{\left(P-P^{\prime}\right)\left(P-P^{\prime \prime}\right)}
$$

wherein it is supposed that the equation (2) represents the first

* "Exam. Papers," An. 1838, p. xlvi., question 4; p. xcix., question 70.

+ The theorems in $\$ 13$ are now stated for the first time. 
surface, and that $P^{\prime}, P^{\prime \prime}$ are the quantities corresponding to $P$ in the equations of the other two surfaces.

In any surface of the second order, the lengths of two bifocal chords are proportional to the rectangles under the segments of any two intersecting chords to which they are parallel.

In the paraboloid expressed by the equation

$$
\frac{y^{2}}{p}+\frac{z^{2}}{q}=x,
$$

if $\chi$ be the length of a bifocal chord making the angles $\beta$ and $\gamma$ with the axes of $y$ and $z$ respectively, we have

$$
\frac{1}{\chi}=\frac{\cos ^{2} \beta}{p}+\frac{\cos ^{2} \gamma}{q} \text {. }
$$

$\S 14$. At the point $\mathrm{S}$ on a given central surface expressed by the equation (2), let a tangent plane be applied, and let $k, k^{\prime}$ be the squares of the semiaxes of a central section made in the surface by a plane parallel to the tangent plane; each of the quantities $k, k^{\prime}$ being positive or negative, according as the corresponding semiaxis of the section is real or imaginary, that is, according as it meets the given surface or not. Then the equations* of two other surfaces confocal with the given one, and passing through the point $\mathbf{S}$, are

$\frac{x^{2}}{P-k}+\frac{y^{2}}{Q-k}+\frac{z^{2}}{R-k}=1, \quad \frac{x^{2}}{P-k^{\prime}}+\frac{y^{2}}{Q-k^{\prime}}+\frac{z^{2}}{R-k^{\prime}}=1$.

The given surface is intersected by these two surfaces respectively in the two lines of curvature which pass through the point $\mathbf{S}$; the tangent drawn to the first line of curvature at $\mathbf{S}$ is parallel to the second semiaxis of the section, and the tangent drawn to the second line of curvature at $\mathbf{S}$ is parallel to the first semiaxis of the section.

When two confocal surfaces intersect, the normal applied to * "Exam. Papers," An. 1837, p. c., questions 4, 5, 6; An.1838, p. c., questions 71, 72. 
one of them at any point $S$ of the line of curvature formed by their intersection lies in the tangent plane of the other, and is parallel to an axis of any section made in the latter by a plane parallel to the tangent plane. Supposing the surfaces to be central, if two normals be applied at the point $\mathrm{S}$, and a diameter of each 'surface be drawn parallel to the normal of the other, the two diameters so drawn will be equal and of a constant length, wherever the point $S$ is taken on the line of curvature; the square of that length being equal to the difference of the squares of the primary axes of the surfaces, and the diameter of the surface which has the greater primary axis being real, while that of the other surface is imaginary. As the point $\mathrm{S}$ moves along the line of curvature, each constant diameter describes a cone condirective with the surface to which it belongs; the two cones so described are reciprocal, and the focal lines of the cone whioh belongs to one surface are perpendicular to the directive planes of the other surface.

When two confocal paraboloids intersect, if normals be applied to them at any point $\mathbf{S}$ of their intersection, and a bifocal chord of each surface be drawn parallel to the normal of the other, the two chords so drawn will be equal and of a constant length, wherever the point $S$ is taken in the line of intersection of the surfaces; that constant length being equal to the difference between the parameters of either pair of coincident prinoipal sections.

$\S 15$. The point $\mathrm{S}$ being the common intersection of a given system of confocal surfaces, of which the equations are

$$
\begin{gathered}
\frac{x^{2}}{\bar{P}}+\frac{y^{2}}{\bar{Q}}+\frac{z^{2}}{R}=1, \quad \frac{x^{2}}{\boldsymbol{P}^{\prime}}+\frac{y^{2}}{\mathbf{Q}^{\prime}}+\frac{z^{2}}{\bar{R}^{\prime}}=1, \\
\frac{x^{2}}{\bar{P}^{\prime \prime}}+\frac{y^{2}}{Q^{\prime \prime}}+\frac{z^{2}}{\bar{R}^{\prime \prime}}=1,
\end{gathered}
$$

suppose that another surface $A$ confocal with these, and expressed by the equation

$$
\frac{x^{2}}{P_{0}}+\frac{y^{2}}{Q_{0}}+\frac{z^{2}}{R_{0}}=1,
$$


is circumscribed by a cone having its vertex at $\mathrm{S}$. If the normals applied at $\mathbf{S}$ to the given surfaces, taken in the order of the equation (10), be the axes of new rectangular co-ordinates $\xi, \eta, \xi$, the equation of the cone, referred to these co-ordinates, will be*

$$
\frac{\xi^{2}}{P-P_{0}}+\frac{\eta^{2}}{P^{\prime}-P_{0}}+\frac{\xi^{2}}{P^{\prime \prime}-P_{0}}=0
$$

The surfaces of the given system, in the order of their equations, may be supposed to be an ellipsoid, a hyperboloid of one sheet, and a hyperboloid of two sheets; the axes of $x, y, z$ being respectively the primary, the mean, and the secondary axes of each surface. Then $\mathrm{P}$ is greater than $\mathrm{P}^{\prime}$, and $\mathrm{P}^{\prime}$ greater than $\mathrm{P}^{\prime \prime}$.

The normals to the given surfaces are the axes of the cone -expressed by the equation (12); and if the surface $\mathrm{A}$ be ohanged, but still remain confocal with the given system, it is obvious from that equation that the focal lines of the eiroumscribing cone will remain unchanged, since the differences of the quantities by which the squares of $\xi, \eta, \zeta$ are divided are independent of the surface $\mathrm{A} . \mathrm{As}_{\mathrm{s}} \mathrm{P}^{\prime}$ is intermediate in value between $\mathrm{P}$ and $\mathrm{P}^{\prime \prime}$, the normal to the hyperboloid of one sheet is always the mean axis of the cone; the focal lines lie in the plane $\xi \xi$, and their equation is

$$
\frac{\xi^{2}}{P^{\prime}-P}+\frac{\zeta^{2}}{P^{\prime}-P^{\prime \prime}}=0
$$

* The equation (12) was obtained in the year 1832, and was given at my Lectures in Hilary Term, 1836. The most remarkable properties of cones circumscribing confocal surfaces are immediate consequences of this equation. That such cones, when they have a common vertex, are confocal, their focal lines being the generatrices of the hyperboloid of one sheet passing through the vertex, was first stated by Professor C. G. J. Jacobi, of Königsberg, in 1834 (see Crelle's Journal, Vou. xIr. p. 137). See also the excellent work of M. Chasles, published in 1837, and entitled Aperçu historique sur l'Origine et le Développement des Méthodes en Gémétrie, p. 387. The analogy which exists between the focals of surfaces and the foci of curves of the second order was supposed by M. Chasles to have been pointed out in that work for the first time (Comptes Rendus, tom. xvi., pp. 833, 1106); but that analogy had been previously taught and developed in the Lectures just alluded to. 
which shows that they are parallel to the asymptotes of a central section made in the hyperboloid of one sheet by a plane parallel to the plane $\xi \zeta$, since the quantities $P^{\prime}-P$ and $P^{\prime}-P^{\prime \prime}$ are (including the proper signs) the squares of the semiaxes of the section which are parallel to $\xi$ and $\xi$ respectively. The focal lines are therefore the generatrices of that hyperboloid at the point $S$.

When $R_{0}=0$, the equation (12) becomes

$$
\frac{\xi^{2}}{R}+\frac{\eta^{2}}{R^{\prime}}+\frac{\xi^{2}}{R^{\prime \prime}}=0
$$

which is that of the cone standing on the focal ellipse and having its vertex at $S$. When $Q_{0}=0$, the same equation becomes

$$
\frac{\xi^{2}}{Q}+\frac{\eta^{2}}{Q^{\prime}}+\frac{\zeta^{2}}{Q^{\prime \prime}}=0
$$

which is that of the cone standing on the focal hyperbola, and having its vertex at $\mathrm{S}$. The normal to the hyperboloid of one sheet at the point $S$ is the mean axis of both cones; the normal to the ellipsoid is the internal axis of the first cone and the directive axis of the second, while the normal to the hyperboloid of two sheets is the directive axis of the first and the internal axis of the second.

The three surfaces expressed by the equations

$$
\begin{gathered}
\frac{\xi^{2}}{\bar{P}}+\frac{\eta^{2}}{P^{\prime}}+\frac{\zeta^{2}}{\bar{P}^{\prime \prime}}=1, \quad \frac{\xi^{2}}{\bar{Q}}+\frac{\eta^{2}}{Q^{\prime}}+\frac{\zeta^{2}}{\bar{Q}^{\prime \prime}}=1, \\
\frac{\xi^{2}}{R}+\frac{\eta^{2}}{R^{\prime}}+\frac{\xi^{2}}{R^{\prime \prime}}=1,
\end{gathered}
$$

are a confocal system, having their centres at $S$, and being respectively an ellipsoid, a hyperboloid of one sheet, and a hyperboloid of two sheets. They intersect each other in the centre of the system expressed by the equations (10), and their normals at that point are the axes of $x, y, z$ respectively. The relations 
between the two systems of surfaces are therefore perfectly reoiprocal. From the equations (14) and (15) it is manifest that the asymptotio cones of the hyperboloids of one system pass through the focals of the other.

$\$ 16$. The point $\mathrm{S}$ being the intersection of a given system of confocal paraboloids whose equations are

$$
\begin{gathered}
\frac{y^{2}}{p}+\frac{z^{2}}{q}=x+h, \quad \frac{y^{2}}{p^{\prime}}+\frac{z^{2}}{q^{\prime}}=x+h^{\prime}, \\
\frac{y^{2}}{p^{\prime \prime}}+\frac{z^{2}}{q^{\prime \prime}}=x+h^{\prime \prime},
\end{gathered}
$$

where $p-p^{\prime}=q-q^{\prime}=4\left(h-h^{\prime}\right)$, and $p-p^{\prime \prime}=q-q^{\prime \prime}=4\left(h-h^{\prime \prime}\right)$ : suppose that another paraboloid $\mathrm{A}$ confocal with these, and expressed by the equation

$$
\frac{y^{2}}{p_{0}}+\frac{z^{2}}{q_{0}}=x+h_{0},
$$

is circumscribed by a cone having its vertex at $\mathbf{S}$. Then if the normals applied at $\mathrm{S}$ to the given system of surfaces, taken in the order of their equations, be the axes of the co-ordinates $\xi, \eta, \zeta$ respectively, the equation of the circumscribing cone will be

$$
\frac{\xi^{2}}{p-p_{0}}+\frac{\eta^{2}}{p^{\prime}-p_{0}}+\frac{\zeta^{2}}{p^{\prime \prime}-p_{0}}=0 ；
$$

showing that those normals are the axes of the cone, and that the focal lines of the cone are independent of the surface $\mathbf{A}$, provided it be confocal with the given surfaces. If the hyperbolic paraboloid be the second surface of the given system, the parameter $p^{\prime}$ will be intermediate in value between $p$ and $p^{\prime \prime}$, and the equation of the fooal lines of the cone will be

$$
\frac{\xi^{2}}{p^{\prime}-p}+\frac{\xi^{2}}{p^{\prime}-p^{\prime \prime}}=0
$$

which is the equation of a pair of right lines parallel to the asymptotes of a section made in the hyperbolic paraboloid by 
a plane parallel to the plane $\xi \zeta$, since the quantities $p^{\prime}-p$ and $p^{\prime}-p^{\prime \prime}$ are proportional to the squares of the semiaxes of the section which are parallel to $\xi$ and $\zeta$ respectively. The focal lines are therefore the generatrices of the hyperbolio paraboloid at the point $\mathrm{S}$.

Putting $p_{0}$ and $q_{0}$ alternately equal to zero in the equation (19), we get

$$
\frac{\xi^{2}}{p}+\frac{\eta^{2}}{p^{\prime}}+\frac{\xi^{2}}{p^{\prime \prime}}=0, \quad \frac{\xi^{2}}{q}+\frac{\eta^{2}}{q^{\prime}}+\frac{\xi^{2}}{q^{\prime \prime}}=0,
$$

the equations of two cones which have a common vertex at $\mathrm{S}$, the first of them standing on the focal which lies in the plane $x z$, the second on the focal which lies in the plane $x y$. The mean axis of each of these cones is the normal at $S$ to the hyperbolic paraboloid; the internal axis of either cone is the normal to the elliptic paraboloid which has the base of that cone for its modular focal.

As the cones which have a common vertex, and stand on the focals of any surface of the second order, are confocal, they intersect at right angles. Therefore when two planes passing through a bifocal right line touch the focals, these planes are at right angles to each other. And as cones which have a common vertex, and circumscribe confocal surfaces, are confocal, two such cones, when they intersect each other, intersect at right angles. Therefore when a right line touches two confocal surfaces, the tangent planes passing through this right line are at right angles to each other.

$\S 17$. When two surfaces are reciprocal polars* with respect to any sphere, and one of them is of the second order, the other is also of the second order. Let the surface $\mathrm{B}$ be reciprocal to the surface A before mentioned, with respect to a sphere of which the centre is $\mathrm{S}$; and suppose $\mathrm{R}^{\prime}$ and $\mathrm{R}$ to be any corresponding points on these surfaces. Then the plane which

* Transactions of the Royal Irish Academy, Vor. xvir. p. 241; "Examination Papers," An. 1841, p. exxvi., question 4. 
touches the surface $A$ at the point $\mathrm{R}$ intersects the right line $\mathrm{SR}^{\prime}$ perpendieularly in a point $\mathrm{K}$, such that the rectangle under $\mathrm{SR}^{\prime}$ and $\mathrm{SK}$ is constant, being equal to the square of the radius of the sphere. Now if the point $\mathrm{K}$ approach indefinitely to $\mathrm{S}$, the distance $\mathrm{SR}^{\prime}$ will increase without limit, the surface $\mathrm{B}$ being of course a hyperboloid; and if through $\mathrm{S}$ any plane be drawn touching the surface $\mathrm{A}$, a right line perpendicular to this plane will evidently be parallel to a side of the asymptotic cone of the hyperboloid. The asymptotic cone of B is therefore reciprocal to the cone which, having its vertex at $\mathrm{S}$, ciroumscribes the surface A. Hence, as the directive planes of a hyperboloid are the same as those of its asymptotic cone, it follows that the directive planes of the surface B are perpendicular to the generatrices of the hyperboloid of one sheet, or the hyperbolic paraboloid, which passes through $\mathrm{S}$, and is confocal with the surface A. And this relation between two reciprocal surfaces ought to be general, whatever be the position of the point $S$ with respect to them; * for though it has been deduced by the aid of the circumscribing cone aforesaid, it does not, in its enunciation, imply the existence of such a cone. This conclusion may be verified by investigating the equation of the surface $\mathrm{B}$ in terms of the co-ordinates $\xi, \eta, \xi$. Suppose $\rho$ to be the radius of the sphere with respect to which the surfaces $\mathrm{A}$ and $\mathrm{B}$ are reciprocal. Then if $\mathrm{A}$ be a central surface expressed by the equation (11), and having $\xi_{0}, \eta_{0}, \xi_{0}$ for the co-ordinates of its centre, the surface $B$ will be represented by the equation

$$
\begin{gathered}
\left(P-P_{0}\right) \xi^{2}+\left(P^{\prime}-P_{0}\right) \eta^{2}+\left(P^{\prime \prime}-P_{0}\right) \xi^{2} \\
=2 \rho^{2}\left(\xi_{0} \xi+\eta_{0} \eta+\zeta_{0} \zeta\right)-\rho^{4} ;
\end{gathered}
$$

but if $\Delta$ be a paraboloid expressed by the equation (18), the equation of $\mathrm{B}$ will be

$$
\begin{gathered}
\left(p-p_{0}\right) \xi^{2}+\left(p^{\prime}-p_{0}\right) \eta^{2}+\left(p^{\prime \prime}-p_{0}\right) \zeta^{2} \\
=\boldsymbol{\rho} 4^{2}(\boldsymbol{\xi} \cos \boldsymbol{a}+\boldsymbol{\eta} \cos \boldsymbol{\beta}+\boldsymbol{\zeta} \cos \gamma),
\end{gathered}
$$

* This relation was first noticed by Mr. Salmon. 
where $a, \beta, \gamma$ are the angles which the axis of $x$ makes with the axes of $\xi, \eta, \zeta$ respectively. In the first case, the equation (22) shows that the directive planes of B are perpendicular to the right lines expressed by the equation (13); in the second case, the equation (23) shows that the directive planes of B are perpendicular to the right lines expressed by the equation (20).

When the surface $A$ is a paraboloid, and the distance of the point $\mathrm{R}$ from its vertex is indefinitely increased, the plane touching the surface at $\mathrm{R}$ approaches indefinitely to parallelism with its axis, and the right line SK, perpendicular to that plane, increases without limit. Therefore the surface B passes through the point $\mathrm{S}$, and is touched in that point by a plane perpendicular to the axis of $\mathrm{A}$.

When the point $S$ lies upon the surface $A$, the co-efficient of the square of one of the variables, in the equation (22) or (23), is reduced to zero, and the surface B is a paraboloid having its axis parallel to the normal applied at $\mathrm{S}$ to the surface $\mathbf{A}$. This also appears from considering that when $\mathrm{S}$ is a point of the surface $\mathbf{A}$, the normal at that point is the only right line passing through $\mathrm{S}$, which meets the surface $\mathrm{B}$ at an infinite distance.

If a series of surfaces be confocal, their reciprocal surfaces, taken with respect to any given sphere, will be condirective.

When the equations of any two condirective surfaces are expressed by co-ordinates perpendicular to their principal planes, the constants in the equations may be always so taken that the differences of the co-efficients of the squares of the variables in one equation shall be equal to the corresponding differences in the other. Then, by subtracting the one equation from the other, we get the equation of a sphere. Therefore when two condirective surfaces intersect each other, their intersection is, in general, a spherical curve. But when the surfaces are two paraboloids of the same species, their intersection is a plane curve.

\$18. Through any point $\mathrm{S}$ of a given surface four bifocal right lines may in general be drawn. Supposing the surface 
to be central, let a plane drawn through the centre, parallel to the plane which touches the surface at $\mathrm{S}$, intersect any one of these right lines. Then the distance of the point of intersection from the point $\mathrm{S}$ will always be equal to the primary semiaxis of the surface.*

If through any point $\mathrm{S}$ of a given central surface a right line be drawn touching two other given surfaces confocal with it, and if this right line be intersected by a plane drawn through the centre parallel to the plane which touches the first surface at $\mathrm{S}$, the distance of the point of intersection from the point $\mathbf{S}$ will be constant, wherever the point $\mathbf{S}$ is taken on the first surface. If this constant distance be called $l$, and the other denominations be the same as in the formula (7), the value of $l$ will be given by that formula. $\uparrow$

* "Examination Papers," An. 1838, p. xlvii., question 9.

+ In the notes to the last-mentioned work of M. Chasles, on the History of Methods in Geometry, will be found many theorems relative to surfaces of the second order. Among them are some of the theorems which are given in the present Paper; but it is needless to specify these, as M. Chasles's work is so well known. 


\section{$(318)$}

III.-NOTE RELATIVE TO THE COMPARISON OF ARCS OF CURVES, PARTICULARLY OF PLANE AND SPHERICAL CONICS.

[Proceedings of the Royal Irish Academy, Vor. II. p. 446.-Read Nor. 30, 1843.]

The first Lemma given in my Paper on the rectification of the conic sections* is obviously true for curves described on any given surface, provided the tangents drawn to these curves be shortest lines on the surface. The demonstration remains exactly the same; and the Lemma, in this general form, may be stated as follows :-

Understanding a tangent to be a shortest line, and supposing two given curves $\mathrm{E}$ and $\mathrm{F}$ to be described on a given surface, let tangents drawn to the first curve at two points $\mathrm{T}, t$, indefinitely near each other, meet the second curve in the points $\mathbf{P}, p$. Then taking a fixed point $\mathbf{A}$ on the curve $\mathbf{E}$, if we put $s$ to denote (according to the position of this point with respect to T) the sum (or difference) of the are AT and the tangent $\mathrm{TP}$, and $s+d s$ to denote the sum (or difference) of the are At and the tangent $t p$, we shall have $d s$ equal to the projection of the infinitesimal are $\mathrm{P} q$ upon the tangent; that is, if $a$ be the angle which the tangent TP makes with the curve $\mathrm{F}$ at the point $\mathrm{P}$, we shall have ds equal to $\mathrm{P} p$ multiplied by the cosine of $\alpha$.

Now thrqugh the points $\mathrm{P}, p$ conceive other tangents $\mathrm{T}^{\prime} \mathrm{P}$,

* Transactions of the Royal Irish Academy, Vou. xvi. p. 79 (supra, p. 20). 


\section{Note relative to the comparison of Arcs of Curves. 3 I9}

$t^{\prime} p$ to be drawn, touching the curve $\mathrm{E}$ in the points $\mathrm{T}^{\prime}, t^{\prime}$; and let $s^{\prime}$ and $d s^{\prime}$ have for these tangents the same signification which $s$ and $d s$ have for the former tangents. Supposing the nature of the curve $\mathrm{F}$ to be such that it always bisects, either internally or externally, the angle made at the point $\mathrm{P}$ by the tangents TP and $\mathrm{T}^{\prime} \mathrm{P}$, it is evident that $d s= \pm d s^{\prime}$, and therefore either $s+s^{\prime}$ or $s-s^{\prime}$ is a constant quantity.

A simple example of this theorem is afforded by the plane and spherical conics. If the curves $\mathrm{E}$ and $\mathrm{F}$ be two confocal conics, either plane or spherical, and tangents TP, T'P be drawn to $\mathrm{F}$ from any point $\mathrm{P}$ of $\mathrm{E}$ (the tangents being of course right lines when the curves are plane, and ares of great circles when they are spherical; in both cases shortest lines), it is well known that the angle TPT' made by the tangents is always bisected by the conic $\mathrm{E}$. The angle is bisected internally or externally according as the conics intersect or not. Hence we have the two following properties* of confocal conios:-

1. When two confocal conics do not intersect, if one of them be touched in the points $T, T^{\prime}$ by tangents drawn from any point $\mathrm{P}$ of the other, the sum of the tangents $T P, T^{2} \mathrm{P}$ will exceed the convex arc 'TT' lying between the points of contact, by a constant quantity.

2. When two focal conics intersect in the point $\mathbf{A}$, if one of them be touched in the points $T, T^{\prime}$ by tangents drawn from any point $P$ of the other, the differenoe between the tangents

* The first of these properties was originally given for spherical conics by the Rev. Charles Graves, Fellow of Trinity College, in the "notes and additions" to his translation of M. Chasles's Memoirs on Cones and Spherical Conics, p. 77 (Dublin, 1841). Mr. Graves obtained it as the reciprocal of the proposition, that when two spherical conics have the same directive circles, any tangent are of the inner conic divides the outer one into two segments, each of which has a constant area. Both properties, with the general theorem relative to curves described on any surface and touched by shortest lines, were afterwards given in the University Calendar. See "Examination Papers," An. 1841, p. xli., questions 3-6; An. 1842, p. Ixxxiii., questions 30-34. These two properties of conics were communicated, in October, 1843, to the Academy of Sciences of Paris, by M. Chasles, who supposed them to be new. See the Comptes Rendus, tom. xvii. p. 838 . 
320 Note relative to the comparison of Arcs of Curves.

$\mathrm{TP}, \mathrm{T}^{\prime} \mathrm{P}$ will be equal to the difference between the ares $\mathrm{AT}$, $\mathrm{AT}^{*}$.

These properties give the readiest and most elegant solution of problems concerning the comparison of different ares of a plane or spherical conio. Any are being given on a conic, we may find another aro beginning from a given point, which shall differ from the given are by a right line if the conic be plane, or by a circular are if the conic be spherical. 
IV.-NOTE ON SURFACES OF THE SECOND ORDER.

[Proceedings of the Royal Irish Academy, Vou. IIr. p. 429.-Read Apri] 12, 1847.]

LET a surface $A$ of the second order be represented by the equation

$$
\frac{x^{2}}{P_{0}}+\frac{y^{2}}{Q_{0}}+\frac{z^{2}}{R_{0}}=1
$$

its primary axis being that of $x$. Through a given point $S$ whose co-ordinates are $x^{\prime}, y^{\prime}, z^{\prime}$, conceive three surfaces confocal with $A$ to be described, and let $P, P^{\prime}, P^{\prime \prime}$, be the squares of their primary semiaxes. Then if normals drawn to these surfaces respectively at the point $S$ be the axes of a new system of co-ordinates $\xi, \eta, \zeta$, and if we put

$$
P-P_{0}=k, \quad P^{\prime}-P_{0}=k^{\prime}, \quad P^{\prime \prime}-P_{0}=k^{\prime \prime}, \quad \frac{x^{2}}{P_{0}}+\frac{y^{2}}{Q_{0}}+\frac{z^{\prime 2}}{R_{0}} f,
$$

the equation of the surface $A$, referred to the new co-ordinates, will be

$$
\frac{\xi^{2}}{k}+\frac{\eta^{2}}{k^{\prime}}+\frac{\xi^{2}}{k^{\prime \prime}}=(f-1)\left(\frac{\xi_{0} \xi}{k}+\frac{\eta_{0} \eta}{k^{\prime}}+\frac{\xi_{0} \xi}{k^{\prime \prime}}-1\right)^{2},
$$

where $\xi_{0}, \eta_{0}, \zeta_{0}$ are the co-ordinates of its centre.

From the form of this equation it is evident that, if the surface be intersected by the plane whose equation is

$$
\frac{\xi_{0} \xi}{k}+\frac{\eta_{0} \eta}{k^{\prime}}+\frac{\xi_{0} \zeta}{k^{\prime \prime}}=1,
$$


it will be touched along the curve of intersection by the cone whose equation is

$$
\frac{\xi^{2}}{k}+\frac{\eta^{2}}{k^{\prime}}+\frac{\zeta^{2}}{k^{\prime \prime}}=0
$$

This mode of deducing, in its simplest form, the equation of a cone circumscribing a surface of the second order, is much easier than the direct investigation by which the equation $(c)$ was originally obtained.

Let a right line passing through $S$ intersect the plane expressed by the equation $(b)$, in a point whose distance from $S$ is equal to $\approx$, while it intersects the surface $A$ in two points, $P$ and $P^{\prime}$, the distance of either of which from $S$ is denoted by $\rho$. Let the surface $B$, represented by the equation

$$
\frac{\xi^{2}}{k}+\frac{\eta^{2}}{k^{\prime}}+\frac{\xi^{2}}{k^{\prime \prime}}=f-1
$$

be intersected by the same right line in a point whose distance from $S$ is equal to $r$, the distance $r$ being, of course, a semidiameter of this surface. Then it is obvious that the equation $(a)$ may be written

$$
\frac{1}{r^{2}}=\left(\frac{1}{\varpi}-\frac{1}{\rho}\right)^{2},
$$

so that, if $\rho$ and $\rho^{\prime}$ represent the distances $S P$ and $S P^{\prime}$ respectively, we have

and therefore

$$
\frac{1}{\rho}=\frac{1}{\varpi}+\frac{1}{r}, \quad \frac{1}{\rho^{\prime}}=\frac{1}{\varpi}-\frac{1}{r}
$$

$$
\frac{1}{\rho}-\frac{1}{\rho^{\prime}}=\frac{2}{r} .
$$

This result is useful in questions relating to attraction. For if $A$ be an ellipsoid, every point of which attracts an external point $S$ with a force varying inversely as the fourth power of the distance, and if the point $S$ be the vertex of a pyramid, one 
of whose sides is the right line $S P P^{\prime}$, and whose transverse section, at the distance unity from its vertex, is the indefinitely small area $\omega$, the portion $P P^{\prime}$ of the pyramid will attract the point $S$, in the direction of its length, with a force expressed by the quantity

$$
\left(\frac{1}{\rho}-\frac{1}{\rho^{\prime}}\right) \omega, \text { or } \frac{2 \omega}{r} ;
$$

and putting $\theta$ for the angle which the right line $S P$ makes with the axis of $\xi$, the attraction in the direction of $\xi$ will be

$$
\frac{2 \omega \cos \theta}{r} \text {. }
$$

Now, supposing the axis of $\xi$ to be normal to the confocal ellipsoid described through $S$, it will be the primary axis of the surface $B$, which will be a hyperboloid of two sheets; and the surface being symmetrical round this axis, it is easy to see, from the expression for the elementary attraction, that the whole attraction of the ellipsoid will be in the direction of $\xi$. Therefore when the force is inversely as the fourth power of the distance, the attraction of an ellipsoid on an external point is normal to the confocal ellipsoid passing through that point.

Hence we infer, that if $U$ be the sum of the quotients found by dividing every element of the volume of an ellipsoid by the cube of its distance from an external point, the value of $U$ will remain the same, wherever that point is taken on the surface of an ellipsoid confocal with the given one.

The question of the attraction of an ellipsoid, when the law of force is that of the inverse square of the distance, has been treated by Poisson, in an elegant but very elaborate memoir, presented to the Academy of Sciences in 1833.* In the preceding year I had obtained the theorems just mentioned, by considering the law of the inverse fourth power; and, as well as I remember, they were deduced exactly as above, by setting out from the equation (a). But I did not then succeed in applying

- Mémoires de l'Institut, tom. xiii. 
the same method to the case where the law of force is that of nature, probably from not perceiving that, in this case, the ellipsoid ought to be divided (as Poisson has divided it) into concentric and similar shells. This application requires the following theorem, which is easily proved:-

Supposing $A^{\prime}$ to be another ellipsoid, concentric, similar, and similarly placed with $A$; let the right line $S P P^{\prime}$ intersect it in the points $p$ and $p^{\prime}$, respectively, adjacent to $P$ and $P^{\prime}$; then if the direction of that right line be conceived to vary, the rectangle under $P p$ and $P^{\prime} p$ (or under $P p^{\prime}$ and $P^{\prime} p^{\prime}$ ) will be to the rectangle under $S P$ and $S P^{\prime}$ in a constant ratio.

Denoting the constant ratio by $m$, and combining this theorem with the formula $(f)$, we have

$$
\frac{P p \times P^{\prime} p}{P P^{\prime}}=\frac{m r}{2}
$$

Now let the two surfaces $A$ and $A^{\prime}$ be supposed to approach indefinitely near each other, so as to form a very thin shell, then ultimately $P^{\prime} p$ will be equal to $P P^{\prime}$, and we shall have

$$
P p=P^{\prime} p^{\prime}=\frac{n v}{2}
$$

where $m$ is indefinitely small. Therefore if the point $S$, external to the shell, be the vertex of a pyramid whose side is the right line $S P$, and whose section, at the unit of distance from the vertex, is $\omega$, the attraction of the two portions $P p$ and $P^{\prime} p^{\prime}$ of this pyramid, which form part of the shell, will be equal to $m r \omega$. Hence it appears, as before, on account of the symmetry of the surface $B$ round the axis of $\xi$, that the whole attraction of the shell on the point $S$ is in the direction of that axis, and consequently (as was found by Poisson) in the direction of the internal axis of the cone whose vertex is $S$, and which circumscribes the shell.

To find the whole attraction of the shell, the expression

$m r \omega \cos \theta$ 
must be integrated. Let $\phi$ be the angle which a plane, passing through $S P$ and the axis of $\xi$, makes with the plane $\xi \eta$, then

$$
\begin{aligned}
& \omega=\sin \theta d \theta d \phi
\end{aligned}
$$

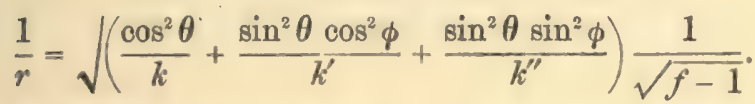

When these values are substituted in (i), that expression may be readily integrated, first with respect to $\theta$, and then with respect to $\phi$.

It is evident that, by the same substitutions, the expression (g) may be twice integrated.

An investigation similar to the preceding has been given by M. Chasles, for the case in which the force varies inversely as the square of the distance.* He uses a theorem equivalent to the formula $(f)$, but deduces it in a different way.

From what has been proved it follows that, if $V$ be the sum of the quotients found by dividing every element of the shell by its distance from an external point $S$, the value of $V$ will be the same wherever that point is taken on the surface $\Sigma$ of an ellipsoid confocal with the surface $A$ of the shell.

Let $\boldsymbol{\Sigma}^{\prime}$ be another ellipsoid confocal with $A$, and indefinitely near the surface $\Sigma$. The normal interval between the two surfaces $\boldsymbol{\Sigma}$ and $\boldsymbol{\Sigma}^{\prime}$, at any point $S$ on the former, will be inversely as the perpendicular dropped from the common centre of the ellipsoids on the plane which touches $\boldsymbol{\Sigma}$ at $\boldsymbol{S}$. Hence, supposing the point $S$ to move over the surfaee $\Sigma$, that perpendicular will vary as the attraction exerted by the shell on the point $S$, when the force is inversely as the square of the distanoe, or as the attraction exerted by the whole ellipsoid $A$ on the point $S$, when the force is inversely as the fourth power of the distance.

When the point $S$ is on the focal hyperbola, the integrations, by which the actual attraction is found in either case, are simplified, for the surface $B$ is then one of revolution round the axis of $\xi$, and its semidiameter $r$ is independent of the angle $\phi$.

* Mémoires des Sacants Etrangers, tom. ix. 
From the expression for the attraction of a shell we can find, by another integration, the attraction of the entire ellipsoid when the law of force is that of nature. And thus the well-known problem of the integral calculus, in which it is proposed to determine directly the attraction of an ellipsoid on an external point, without employing the theorem of Ivory to evade the difficulty, is solved in what appears to be the simplest manner. 
PART III.

R O T A T I N . 

I.-ON THE ROTATION OF A SOLID BODY ROUND A FIXED POINT; BEING AN ACCOUNT OF THE LATE PROFESSOR MACCULLAGH'S LECTURES ON THAT SUBJECT.* COMPILED BY THE REV. SAMUEL HAUGHTON, FELLOW OF TRINITY COLLEGE, DUBLIN.

[Tiunsactions of the Royal Irish Academy, VoL. xxir.p. 139.-Read April 23, 1849.]

\section{I.-Composition of Rotations.}

LET $O$ be the intersection of two axes of rotation, OR, $\mathrm{OR}^{\prime}$; and let the magnitudes of the rotations be represented by $\omega, \omega^{\prime}$; then the motion impressed upon the body by these two rotations will be the same as the motion produced by a single rotation round an axis, which is represented in magnitude and position by the diagonal of the parallelogram formed by $\omega, \omega^{\prime}$. For, draw through any point $I$ of the body a plane perpendicular to the line OI, and project upon this plane the parallelogram formed by $\omega, \omega^{\prime}$; the sides of this parallelogram will be $\omega$ sin ROI and $\omega^{\prime} \sin \mathrm{R}^{\prime} \mathrm{OI}$. Now the velocities impressed upon the

* This Essay - "On the Rotation of a Solid Body round a Fixed Point," has been compiled from my notes of Professor Mac Cullagh's Lectures, delivered in the Hilary Term of the year 1844, in Trinity College. A short account of some of the results contained in it was published by Professor Mac Cullagh himself, in the Proceedings of the Royal Irish Academy. $t$ As it has appeared to many of $\mathbf{M r}$. Mac Cullagh's friends desirable that a somewhat more detailed account of his researches in this subject should be published, I have, in accordance with this desire, drawn up and presented to the Academy an account of his Lectures on Rotation. I have endeavoured to arrange the subject in a systematic order, and to give the results proved by him during the course of the Lectures, carefully excluding all theorems and proofs of theorems, which were not originally given by him, as here stated. - Samuel HaUghton. 
point I by the rotations $\omega$ and $\omega^{\prime}$ are OI. $\omega$ sin ROI and OI. $\omega^{\prime} \sin \mathrm{R}^{\prime} \mathrm{OI}$; and the directions of these velocities are perpendicular to the sides of the projected parallelogram. Hence, if this parallelogram be turned in its plane through $90^{\circ}$, its sides will represent in magnitude and direction the actual velocities: the resultant of these velocities is perpendicular to the projection of the diagonal of the parallelogram $\left(\omega, \omega^{\prime}\right)$ : this projection, turned round through $90^{\circ}$, will represent the actual velocity, which is therefore the same in magnitude and direction as would be produced by a single rotation represented by the diagonal of $\left(\omega, \omega^{\prime}\right)$. Hence rotations may be resolved along three rectangular axes by the same laws as couples, and they must be counted positive when the motion produced is from $z$ to $x, x$ to $y, y$ to $z$, and vice versa.

\section{II.-Linear Velocities produced by a given Rotation.}

Let the origin of co-ordinates be assumed on the axis of rotation, and let the magnitude of the rotation and of its components be represented by $(\omega, p, q, r)$ : the velocity of any point $(x, y, z)$ is in a direction perpendicular to the plane containing the axis of rotation and the point $(x, y, z)$; and its magnitude is represented by the area of the triangle whose angles are situated at the origin, the point $(x, y, z)$, and the point $(p, q, r)$. Hence, the components of the linear velocity are represented by the projections of this triangle on the coordinate planes. These projections are

$$
\begin{aligned}
& u=q z-r y ; \\
& v=r x-p z ; \\
& w=p y-q x .
\end{aligned}
$$




\section{III.-To Represent geometrically the Moments of INertia} of a Body with respect to Axes drawn throvgh a Fixed Point.

The moment of inertia of a body with respect to any axis $(a, \beta, \gamma)$ is

$$
\begin{aligned}
M=A^{\prime} \cos ^{2} \alpha+B^{\prime} \cos ^{2} \beta & +C^{\prime \prime} \cos ^{2} \gamma-2 L^{\prime} \cos \beta \cos \gamma \\
& -2 M^{\prime} \cos \boldsymbol{\alpha} \cos \gamma-2 N^{\prime} \cos \boldsymbol{\alpha} \cos \beta ;
\end{aligned}
$$

where

$$
\begin{array}{ll}
A^{\prime}=\int\left(y^{2}+z^{2}\right) d m, & L^{\prime}=\int y z d m ; \\
B^{\prime}=\int\left(x^{2}+z^{2}\right) d m, & M^{\prime}=\int x z d m ; \\
C^{\prime}=\int\left(x^{2}+y^{2}\right) d m, & N^{\prime}=\int x y d m .
\end{array}
$$

Assume $M=\frac{\mu}{r^{2}} ; \mu$ being the mass of the body, and $r$ a distance measured on the line $(a, \beta, \gamma)$, and construct the ellipsoid whose equation is

$$
A^{\prime} x^{2}+B^{\prime} y^{2}+C^{\prime} z^{2}-2 L^{\prime} y z-2 M^{\prime} x z-2 N^{\prime} x y=\mu ;
$$

then it is evident that the moments of inertia of the body with respect to axes passing through the fixed point are represented by the squares of the reciprocals of the radii vectores of this ellipsoid. Assume $A=\mu a^{2}, B=\mu b^{2}, C=\mu c^{2}$, and let the axes of co-ordinates be the axes of the ellipsoid; its equation will thus become

$$
a^{2} x^{2}+b^{2} y^{2}+c^{2} z^{2}=1 ;
$$

and the equation of the reciprocal ellipsoid will be

$$
\frac{x^{2}}{a^{2}}+\frac{y^{2}}{b^{2}}+\frac{z^{2}}{c^{2}}=1 .
$$

This latter ellipsoid may be called the ellipsoid of gJration, as the perpendiculars on its tangent planes represent the radii of 


\section{Rotation of a Solid Body round a Fixed Point.}

gyration; this is evident from the consideration, that these perpendiculars are reciprocal to the radii vectores of the ellipsoid (3). In fact, the moment of inertia with respect to any axis will be represented by the formula

$$
M=\left(a^{2} \cos ^{2} a+b^{2} \cos ^{2} \beta+c^{2} \cos ^{2} \gamma\right) \mu=\mu P^{2}=\frac{\mu}{R^{\prime 2}}
$$

$(R, P)$ denoting the radius vector and perpendicular on tangent plane of the ellipsoid (4); and $\left(R^{\prime}, P^{\prime}\right)$ the corresponding lines in the ellipsoid (3).

IV.-To find the Magnitude, Position, and Direction of the Statical Couple produced by the Centrifugal Forces.

If from any point $(x, y, z)$ of the body, a perpendicular be let fall on the axis of rotation $(a, \beta, \gamma)$, the centrifugal force will be represented by the product of the square of the angular velocity and this perpendicular : the corresponding elementary statical couple will be found by multiplying the centrifugal force by the distance from the foot of the perpendicular to the origin, which is represented by the quantity $(x \cos \alpha+y \cos \beta$ $+z \cos \gamma$ ). The components of the elementary couple will be proportional to the projections of the triangle formed by the lines before mentioned. The components of the elementary couples must be integrated for the entire extent of the body, and the integrals thus found will be the components of the couple produced by centrifugal force: the expressions are as follows:

$$
\begin{aligned}
& \omega^{2}(x \cos \alpha+y \cos \beta+z \cos \gamma)(z \cos \beta-y \cos \gamma) d m \\
& \omega^{2}(x \cos \alpha+y \cos \beta+z \cos \gamma)(x \cos \gamma-z \cos \alpha) d m \\
& \omega^{2}(x \cos \alpha+y \cos \beta+z \cos \gamma)(y \cos \alpha-x \cos \beta) d m
\end{aligned}
$$

If the axes of co-ordinates be principal axes, these expressions, when integrated, will become

$$
\begin{aligned}
& \omega^{2} \cos \beta \cos \gamma(B-C)=q r(B-C) ; \\
& \omega^{2} \cos a \cos \gamma(C-A)=p r(C-A) ; \\
& \omega^{2} \cos a \cos \beta(A-B)=p q(A-B):
\end{aligned}
$$


$p, q, r$ being the components of the angular velocity $\omega$. The position of the resultant couple may be expressed by means of the ellipsoid (4). If a tangent plane be drawn to this ellipsoid at the point $(x, y, z)$, and perpendieular to the line $(a, \beta, \gamma)$, it may be easily shown that the projections of the triangle formed by the radius vector and perpendicular are represented by the quantities

$$
\cos \beta \cos \gamma\left(b^{2}-c^{2}\right), \quad \cos \boldsymbol{a} \cos \gamma\left(c^{2}-a^{2}\right), \quad \cos \boldsymbol{a} \cos \beta\left(a^{2}-b^{2}\right):
$$

these three expressions multiplied by $\mu \omega^{2}$ will produce the quantities used in (6). Hence it appears that the couple produced by the centrifugal forces lies in the plane of the radius vector and perpendicular to a tangent plane of the ellipsoid (4); the tangent plane being perpendicular to the axis of rotation. Also, the magnitude of the resultant couple is proportional to the triangle formed by the radius vector and perpendicular.

The differential equations of motion commonly used in the solution of this problem may be deduced immediately from equations (6). In fact, as the axes of co-ordinates are axes of permanent rotation, the increment of angular velocity round each axis will be equal to the statical couple of the applied forces (including centrifugal forces), divided by the moment of inertia round that axis. The statement of this fact, in analytical language, will give the equations of motion :

$$
\begin{aligned}
& A \frac{d p}{d t}=(B-C) q r+L ; \\
& B \frac{d q}{d t}=(C-A) p r+M ; \\
& C \frac{d r}{d t}=(A-B) p q+N
\end{aligned}
$$

$(L, M, N)$ being the components of the applied statical couple.

The position and magnitude of the couple produced by the centrifugal forees are easily found by the method which has been just given; but the direction will be found more readily by 


\section{Rotation of a Solid Body round a Fixed Point.}

taking more particular axes of co-ordinates. Let the axis of rotation be the axis $\mathrm{OZ}$, and the plane of radius vector and perpendicular be the co-ordinate plane $\mathrm{XOZ}$. In the accompanying figure $\mathrm{OR}^{\prime}$ and $\mathrm{OP}^{\prime}$ are the radius vector and perpendicular of the ellipsoid (2), and OR, $\mathrm{OP}$ the radius vector and perpendicular of the ellipsoid (4), which is reciprocal to the former; the rotation is positive, in the direction indieated by the arrow. As the rotation is round the axis of $z$, it is

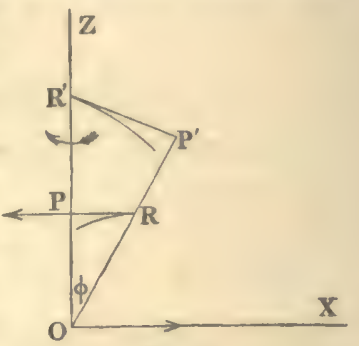

Fig. 1. easy to see that the statical couple produced by centrifugal force will have for components, round the axes of $x$ and $y$ respectively, the quantities $\omega^{2} \int y z d m, \omega^{2} \int x z d m$ taken with their proper sign; i.e. the components are $\pm \omega^{2} L^{\prime}, \pm \omega^{2} M^{\prime} ; L^{\prime}, M I^{\prime}$ being coefficients in the equation of the ellipsoid.

$$
A^{\prime} x^{2}+B^{\prime} y^{2}+C^{\prime} z^{2}-2 L^{\prime} y z-2 M^{\prime} x z-2 N^{\prime} x y=\mu .
$$

The tangent plane to this ellipsoid, applied at the point $(x, y, z)$, will be

$$
\left(A^{\prime} x-M \boldsymbol{I}^{\prime} z-N^{\prime} y\right) x^{\prime}+\left(B^{\prime} y-N^{\prime} x-L^{\prime} z\right) y^{\prime}+\left(C^{\prime} z-L^{\prime} y-M^{\prime} x\right) z^{\prime}=\mu .
$$

At the point $\mathrm{R}^{\prime}$ the tangent plane will be perpendicular to the plane $\mathrm{XOZ}$, and will be found by making $x=0, y=0$, and destroying the coefficient of $y^{\prime}$ in the preceding equation. These conditions give us $L^{\prime}=0$, which proves that the statical couple produced by centrifugal force lies altogether in the plane XOZ. The equation of the tangent plane is the same as the equation of the line $\mathrm{R}^{\prime} \mathrm{P}^{\prime}$, and is

Hence we obtain

$$
C^{\prime} z^{\prime}-M^{\prime} x^{\prime}=-\frac{\mu}{z}
$$

$$
\tan \phi=-\frac{M^{\prime}}{C^{\prime}} .
$$

The value of the centrifugal couple is $\omega^{2} \boldsymbol{M} \boldsymbol{I}^{\prime}$, which is found 
from the preceding equation by replacing $C^{\prime}$ and $\tan \phi$ by their values $\mu P^{2}$, and $\frac{Q}{P} ; Q$ being the line $R P$.

We thus obtain finally the centrifugal couple lying in the plane $\mathrm{XOZ}$, and expressed by the equation

$$
\omega^{2} \int x \approx d m=-\mu \omega^{2} P Q .
$$

It thus appears that the centrifugal couple lies in the plane of radius vector and perpendicular, is proportional to the area of the triangle ROP, and has a direction opposite to the direction of rotation.

\section{V.-To find the Relation between the Plane of Principal Moments and the Axis of Rotation at any Instant.}

The motion of the body at any instant consists of a rotation of a certain magnitude round a certain axis; this rotation might be produced by an impulsive couple of a determinate magnitude and direction. The statical impulsive couple thus conceived is the couple of principal moments. Let this couple be represented by $G$, and act round the axis OR (fig. 1, p. 334); then the corresponding axis of rotation will be the perpendicular $\mathrm{OP}$, and the relation between $G$ and $\omega$ may be thus found :-Let the axes of co-ordinates be the axes of the ellipsoid (4), the radius vector being determined by the angles $(\lambda, \mu, v)$, and the axes of rotation by the angles $(a, \beta, \gamma)$. From mechanical considerations we obtain the equations

$$
\begin{aligned}
& G \cos \lambda=A p=\mu \omega a^{2} \cos a ; \\
& G \cos \mu=B q=\mu \omega b^{2} \cos \beta ; \\
& G \cos \nu=C r=\mu \omega c^{2} \cos \gamma .
\end{aligned}
$$

Hence we obtain

$$
\begin{gathered}
\frac{\cos \lambda}{\cos \nu}=\frac{a^{2} \cos a}{c^{2} \cos \gamma}, \quad \frac{\cos \mu}{\cos \nu}=\frac{b^{2} \cos \beta}{c^{2} \cos \gamma}, \\
\omega=\frac{G}{\mu P R}=\frac{G \cos \phi}{\mu P^{2}} .
\end{gathered}
$$




\section{Rotation of a Solid Body round a Fixed Point.}

The first two of these equations prove that the axis of rotation is the perpendicular on tangent plane of the ellipsoid, and the last equation gives the magnitude of the rotation in terms of the impressed couple and quantities determined by the nature of the body itself. Equations (9) are true, whatever be the forces acting on the body; if no forces act, $G$ will be fixed in magnitude and position in space, by the principle of conservation of areas, but will change its position in the body, the axis of rotation accompanying it, and changing its position both in the body and in space.

VI.-Rotation produced by Centrifugal Force; Particular Properties of the Motion when no Forces act.

The axis of rotation produced by the centrifugal couple always lies in the plane of principal moments. This theorem may be thus proved: Let the radius vector and perpendicular be drawn, which coincide with the axis of principal moment and axis of rotation at any instant; a line perpendicular to the plane of radius vector and perpendicular is the axis of centrifugal couple; this line and the original radius vector are axes of the section of the ellipsoid made by their plane : at the point where the axis of the centrifugal couple pierces the ellipsoid let a tangent plane be applied; the perpendicular let fall on this tangent plane is the axis of rotation produced by centrifugal forces. From the construction it is evident that the plane of the second radius vector and perpendicular is perpendicular to the axis of $G$; hence the axis of the centrifugal couple and the axis of rotation produced by it always lie in the plane of principal moment. Two important corollaries follow from the theorem just demonstrated, in the case where no forces act:-First, the component of angular velocity round the axis of primitive impulse is constant during the motion. Secondly, the radius vector which coincides with the axis of $G$ is of constant length during the motion. The first theorem is obvious; for as the axis of rotation produced by centrifugal force is always perpendicular to the axis of $G$, it 
cannot alter the rotation round that axis. The second theorem follows from equation (9), from which we deduce

$$
\omega \cos \phi=\frac{G}{\mu R^{2}} \text {. }
$$

The left-hand member of this equation is constant by the preceding theorem; and $G$ is constant, since there is no external force; therefore $R$ is constant.

As the axis of $G$ is fixed in space, and the line $R$ is constant, it is evident that the axis of $G$ will describe in the body the cone of the second degree, determined by the intersection of the ellipsoid (4) with the sphere whose radius is $R$. The equation of this cone is

$$
\frac{R^{2}-a^{2}}{a^{2}} x^{2}+\frac{R^{2}-b^{2}}{b^{2}} y^{2}+\frac{R^{2}-c^{2}}{c^{2}} z^{2}=0 .
$$

As the axis of principal moments describes this cone in the body, it is accompanied by the axis of rotation, which is always the corresponding perpendicular on tangent plane of the ellipsoid. The cone described by the axis of rotation might be found thus. Let tangent planes be applied to the ellipsoid along the spherical conic in which the cone (11) cuts the ellipsoid. From the centre let fall perpendiculars on these tangent planes; the locus of these perpendiculars is the required cone.

\section{VII.-The Axis of principal Moments is fixed in SPACE.}

This is evident from D'Alembert's prinoiple, but may be shown by geometrical considerations in the particular case under consideration. The axis varies in position in the body, in consequence of the centrifugal couple, which must be compounded with the impressed couple at each instant. Referring to equation (8), the value of the centrifugal couple is $-\mu \omega^{2} P Q d t$, the principal moment being $G=\mu \omega P R$ (vid. (9)). Hence the angle 


\section{Rotation of a Solid Body roind a Fixed Point.}

through which the axis of principal moments shifts in an element of time is $-\frac{\omega Q d t}{R}$ : this angle, multiplied by the constant radius vector, will give the elementary motion on the spherical oonic traced by the axis of principal moment on the surface of the ellipsoid : this motion is therefore - $\omega Q d t$; but in the same time the point of the body which coincides with the point where the axis of moments pierces the spherical conic will describe the angle $+\omega Q d t$ in consequence of the angular rotation. Hence the axis of moments will remain fixed in space, and will move in the body with a velocity proportional to the tangent of the angle between the radius vector and perpendicular, the motion being in a direction opposite to the direction of the rotation. This is evident from the consideration that $Q \omega=P \omega \tan \phi, P \omega$ being constant and equal to $\frac{G}{\mu R}(v i d .(9))$.

VIII.-To find the Motion of the principal Axis in the BoDy.

\section{First Method.}

The point of the principal axis of moments, which is situated at the distance $R$ from the centre, moves on the spherical conic which has been determined. Let this point be projected on the three co-ordinate planes; then, since the spherical conic is projected into a conio section, the movement of the axis of moments is reduced to the movement of a point on a conic section, according to a law which must be determined. The radius vector describes an elementary triangle in the surface of the cone (11): let the projections of this triangle on the co-ordinate planes be $\left(d A_{1}, d A_{2}, d A_{3}\right)$; we obtain easily

$$
\frac{d A_{1}}{d t}=y \frac{d z}{d t}-z \frac{d y}{d t}, \quad \frac{d A_{2}}{d t}=z \frac{d x}{d t}-x \frac{d z}{d t}, \frac{d A_{3}}{d t}=x \frac{d y}{d t}-y \frac{d x}{d t} .
$$

Substituting in these equations the values of the velocities given by (1), we obtain 


$$
\begin{aligned}
& \frac{d A_{1}}{d t}=P \omega \frac{R^{2}-a^{2}}{a^{2}} x=\left(R^{2}-a^{2}\right) p ; \\
& \frac{d A_{2}}{d t}=P \omega \frac{R^{2}-b^{2}}{b^{2}} y=\left(R^{2}-b^{2}\right) q ; \\
& \frac{d A_{3}}{d t}=P \omega \frac{Q^{2}-c^{3}}{c^{2}} z=\left(R^{2}-c^{2}\right) r .
\end{aligned}
$$

These equations prove that the areolar velocity of the projection on a co-ordinate plane varies as the ordinate to that plane. By means of the method of quadratures, we may determine from equations (12) the position of the projections of the principal axis at any instant, and hence deduce the position of the axis itself.

\section{Second Method.}

If the spherical conic be projected on a cyclio plane of the ellipsoid of gyration, by lines parallel to $x$ and $z$, the projections will be two concentric circles, and the corresponding projections will lie on the same ordinate $\mathrm{SII}^{\prime}$ (fig. 2). The inner circle will belong to the projection parallel to $x$, if $R$ be greater than $b$, and will belong to the projection parallel to $\approx$ if $R$ be less than $b$; and if $R$ be equal to $b$, the two circles will coincide with each other and with the

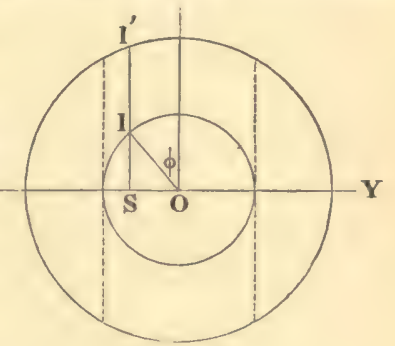

Fig. 2. spherical conic, which in this case becomes the circular section of the ellipsoid. The projected point will revolve round the circumference of the inner circle, and will vibrate on the circumference of the outer circle between the dotted lines. It is evident that the mean axis of the ellipsoid OY lies in the plane of the figure. Let SI and SI' be equal to $\rho, \rho^{\prime}$, and let $C, C^{\prime}$ denote the radii of the two circles: the velocities of the projections in the circles will evidently be

$$
V=\frac{C}{\rho} \frac{d y}{d t}, \quad V_{2}^{\prime}=\frac{C^{\prime}}{\rho^{\prime}} \frac{d y}{d t} ;
$$




\section{Rotation of a Solid Body round a Fixed Point.}

$C$ and $C^{\prime}$ having the values

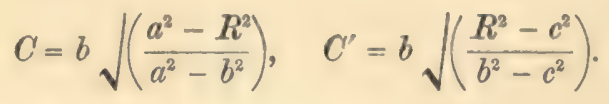

The value of $\frac{d y}{d t}$ deduced from (1) is

$$
\frac{d y}{d t}=P \omega\left(\frac{1}{c^{2}}-\frac{1}{a^{2}}\right) z x=P \omega\left(\frac{1}{c^{2}}-\frac{1}{a^{2}}\right) \rho \rho^{\prime} \sin \theta \cos \theta .
$$

$\theta$ being the angle made by the plane of the circular section with the plane $(x, y)$,

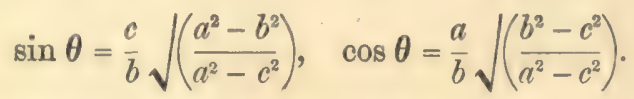

Introducing these values of $\frac{d y}{d t}, \sin \theta$ and $\cos \theta$, and for $P_{\omega}$ its value $\frac{G}{\mu R}$, we obtain finally for the velocities

$$
\begin{aligned}
& V=\frac{G}{\mu} \sqrt{\left\{\left(\frac{1}{R^{2}}-\frac{1}{a^{2}}\right)\left(\frac{1}{c^{2}}-\frac{1}{b^{2}}\right)\right\} \rho^{\prime}=K \rho^{\prime} ;} \\
& V^{\prime}=\frac{G}{\mu} \sqrt{\left\{\left(\frac{1}{R^{2}}-\frac{1}{c^{2}}\right)\left(\frac{1}{a^{2}}-\frac{1}{b^{2}}\right)\right\} \rho=K^{\prime} \rho .}
\end{aligned}
$$

The velocity of each projection, therefore, varies as the ordinate of the other. This theorem enables us to find a simple expression for the time. Using the angle $(\phi)$ marked in fig. 2, we obtain

$$
\frac{C d \phi}{d t}=K \sqrt{ }\left(C^{\prime 2}-C^{2} \sin ^{2} \phi\right) ;
$$

$(\phi, C, K)$ belonging to the projection parallel to axis of $x$. If $\left(\psi, C^{\prime}, K^{\prime}\right)$ be the corresponding quantities for the other projection, we obtain also

$$
\frac{C^{\prime} d \psi}{d t}=\pi^{\prime} \sqrt{ }\left(C^{2}-C^{\prime 2} \sin ^{2} \psi\right) ;
$$


or, since it is easily seen that $\frac{K}{K^{\prime}}=\frac{C}{C^{\prime}}$, we obtain finally

$$
\begin{aligned}
& K^{\prime} d t=\frac{d \phi}{\sqrt{\left(1-\frac{C^{2}}{C^{\prime 2}} \sin ^{2} \phi\right)}} \\
& K d t=\frac{d \psi}{\sqrt{\left(1-\frac{C^{\prime 2}}{C^{2}} \sin ^{2} \psi\right)}}
\end{aligned}
$$

The motion of the principal axis of moments is, therefore, expressed by an elliptic function of the first kind.

The motion of the axis of moments is determined by the magnitude of the radius vector of the ellipsoid, which is the axis of the original couple impressed upon the body; if this radius vector be greater than the mean axis of the ellipsoid, the corresponding spherical conio will have the axis of $x$ for its internal axis; and if the radius be less than the mean axis, the axis of z will be the internal axis of the conic; in no case will the mean axis be the internal axis of the spherical conic. If the radius $R$ be nearly equal to either the greatest or least semi-axis, the expression (14) for the time may be integrated. Let $R$ be nearly equal to the greatest semi-axis. The first of the equations (14) belongs to the interior circle, which is of small dimensions in the case supposed; the second equation expresses the vibratory motion of the projection, through a small are of the outer circle, which will have a radius much greater than the inner circle; we may, therefore, suppose the angle $\psi$ to be equal to its sine. Multiplying both sides of the equation by $\frac{C^{\prime}}{C}$ we obtain.

Hence

$$
\frac{C^{\prime} K}{C} d t=K d t \frac{\frac{C^{\prime}}{C} d \psi}{\sqrt{\left(1-\frac{C^{2 / 2}}{C^{2}} \psi^{2}\right)}} .
$$

$$
\frac{C^{\prime} \psi}{C}=\sin \left(\Pi^{\prime} t+A\right)
$$

If $T_{0}$ denote the time of a complete oscillation or revolution of 


\section{Rotation of a Solid Body round a Fixed Point.}

the axis of moments about the axis of $x$, and $T_{r}$ the time of a revolution of the body round the axis of $x$, the following relation between these two periods may be readily deduced from (15):

$$
T_{0}=T_{r} \frac{b c}{\sqrt{ }\left(\left(a^{2}-b^{2}\right)\right)\left(a^{2}-c^{2}\right)^{\circ}} .
$$

If the axis of moments, and consequently the axis of revolution, be situated near the axis of greatest or least inertia, it will always continue near this axis ; if, however, it be situated near the mean axis, the movement of the body will be determined by the following construction:-Let the two cyclic planes of the ellipsoid be drawn through the mean axis; they will divide the ellipsoid into two regions, in one of which is situated the axis of maximum inertia, and in the other the axis of minimum inertia. The spherical eonic described by the axis of principal moments will have the first or second of these axes for its internal axis, according as $R$ is greater or less than the mean axis. If the axis of principal moments lie in one of the eyclic planes, the spherical conic becomes a circle, and its two projections become identical with itself (fig. 2, p. 339); the expressions (14) are reduced to the form

which when integrated gives

$$
K d t=\frac{d \phi}{\cos \phi} ;
$$

$$
K t+A=\log \cot \left(\frac{\pi}{4}-\frac{\phi}{2}\right) ;
$$

or,

$$
\cot \left(\frac{\pi}{4}-\frac{\phi}{2}\right)=\cot \left(\frac{\pi}{4}-\frac{\phi_{0}}{2}\right) \varepsilon^{K t} ;
$$

$\phi_{0}$ being the value of $\phi$ corresponding to $t=0$, and $K$ being expressed by the following quantity :

$$
K=\frac{G}{\mu} \frac{\sqrt{ }\left\{\left(a^{2}-b^{2}\right)\left(b^{2}-c^{2}\right)\right\}}{b^{2} a c} .
$$

It is evident from the equation (17) that the axis of moments will coincide with the mean axis of inertia at the end of an infinite time. 
IX. - To find the Position of the Body in Space at the End of Any given Time.

\section{First Method.}

The radius vector of the ellipsoid of gyration, which is perpendicular to the plane containing the axes of principal moment and of rotation, always lies in the plane of principal moment, and describes in that plane areas proportional to the time.

Let $O G, O \Omega$ be the axes of principal moment and of rotation ; $\mathrm{OR}^{\prime}, \mathrm{O} \Omega^{\prime}$, the axes of centrifugal couple and of corresponding rotation; the plane $\Omega O \Omega^{\prime}$ will contain the two successive positions of the axis of rotation. Let OI be the position of the axis of rotation at the end of the time $\delta t$; then $\delta u$ will be equal to the angle deseribed in the fixed plane by the line OR'. Let $R^{\prime}$ and $P^{\prime}$ be the radius vector and perpendicular correspond- $\mathrm{R}^{\prime}$ ing to the centrifugal couple and its

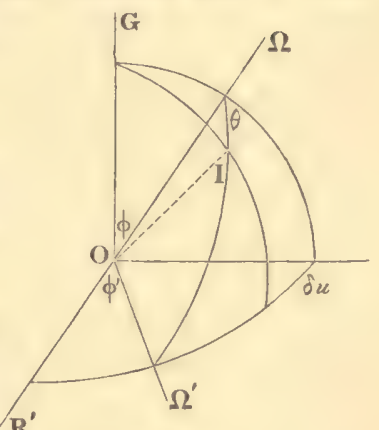

Fig. 3. axis of rotation. The following relations are evident from the figure :

because

$$
\frac{\omega}{\omega^{\prime}}=-\frac{\sin \Omega^{\prime} \mathrm{OI}}{\sin \Omega \mathrm{OI}}=\frac{\cos \phi^{\prime}}{\sin \phi \delta u} ;
$$

$$
\sin \Omega^{\prime} \mathrm{OI}=\frac{\cos \phi^{\prime}}{\sin \theta}, \quad \sin \Omega \mathrm{OI}=\frac{\sin \phi \delta u}{\sin \theta} ;
$$

but, from mechanical considerations,

$$
\frac{\omega}{\omega^{\prime}}=-\frac{P^{\prime} R^{\prime}}{P R \omega \sin \phi \delta t} ; \text { because } \omega=\frac{G}{\mu P R}, \omega^{\prime}=-\frac{G \omega \sin \phi \delta t}{\mu P^{\prime} R^{\prime}} .
$$

Hence, by equating the geometrical and mechanical expression, we obtain

$$
-R^{\prime 2} \delta u=\omega P R \delta t=\frac{G}{\mu} \delta t
$$




\section{Rotation of a Solid Body round a Fixed Point.}

The position of the body in space is thus reduced to quadratures; but the problem may be solved more readily in the following manner.

\section{Second Method.}

The axis of principal moments, appearing to move in a direction opposite to the rotation, describes in the body the cone whose equation has been given (11). If the cone reciprocal to this cone be described, one of its sides will lie in the fixed plane, and the whole motion of the body in space will be the same as the motion of this cone, which partly slides and partly rolls on the fixed plane, the sliding motion being uniform. This theorem is evident by resolving the angular velocity $\omega$ into two components, one round the axis of principal moments, and the other in a direction perpendicular to this, round the side of the reciprocal cone, which is in contact with the fixed plane. These components are $\omega \cos \phi$ and $\omega \sin \phi ; \omega \cos \phi$ being constant and producing the sliding motion, while $\omega \sin \phi$ represents the angular velocity round the side of the cone in contact with the fixed plane. The angle described by the side of the reciprocal cone in the fixed plane at the end of a given time is, therefore, the algebraic sum of two angles, one of which is proportional to the time, and the other is the angle described in the cone in consequence of the rotation $\omega \sin \phi$, and is, therefore, measured by the are of a spherical conic. The position of the body at the end of the time $t$ is thus found:-determine by equation (14) the position of the axis of principal moments in the cone (11); the corresponding position of the component axis of rotation in the reciprocal cone is therefore known. Hence the angle described in the time $t$ in the fixed plane is

$$
\Theta=\int \omega \cos \phi d t \pm \int \frac{d s}{R}=\omega \cos \phi \cdot t \pm \frac{s}{R} .
$$

The equation of the reciprocal cone is

$$
\frac{a^{2} x^{2}}{R^{2}-a^{2}}+\frac{b^{2} y^{2}}{R^{2}-b^{2}}+\frac{c^{2} z^{2}}{R^{2}-c^{2}}=0 .
$$


In (19) the positive or negative sign must be used according as $R$ is less or greater than the mean axis of the ellipsoid; this is evident from the composition of rotations, and from the consideration that in the former case the axis of rotation falls inside the cone (11), while in the latter case it falls outside.

\section{X.-To find a Point, if any, in A Given Axis of Rotation,}

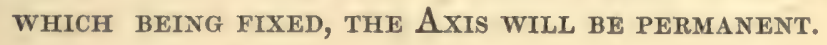

Let $\mathrm{R}^{\prime} \mathrm{R}^{\prime \prime}$ (fig. 4) be the given axis, round which the body revolves with a rotation expressed by $\omega$; describe the ellipsoid of gyration round the centre of gravity $\mathrm{O}$, and draw $O \mathrm{Y}^{\prime}$ parallel to $\mathrm{R}^{\prime} \mathrm{R}^{\prime \prime}$. The centrifugal force $\omega^{2} r d m$ at any point $(x, y, z)$ may be resolved into two components, $\omega^{2} \rho d m$ and $\omega^{2} \cdot \mathrm{R}^{\prime} \mathrm{P}^{\prime} . d m$; $r$ and $\rho$ denoting the distances of the point from the axes $\mathrm{R}^{\prime} \mathrm{R}^{\prime \prime}$ and $\mathrm{OP}^{\prime} \mathrm{re-}$ spectively; the effect of the rotation round $R^{\prime} R^{\prime \prime}$ is therefore the same as an equal rotation round $O \mathrm{P}^{\prime}$, together with

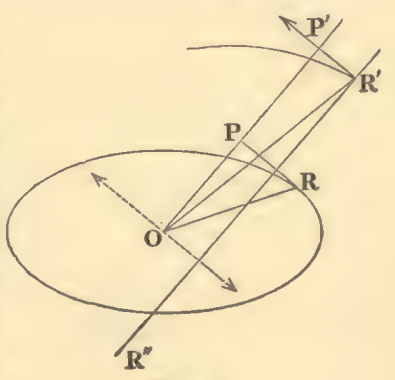

Fig. 4. a number of parallel and equal forees applied to each point of the body. The rotation round $\mathrm{OP}^{\prime}$ produces a centrifugal couple represented by $-\mu \omega^{2}$. OP. PR (vid. (8)); or, determining the point $\mathrm{R}^{\prime}$ by the condition $\mathrm{OP} . \mathrm{PR}=O \mathrm{P}^{\prime}$. $\mathrm{P}^{\prime} \mathrm{R}^{\prime}$, the centrifugal couple is $-\mu \omega^{2} . O P^{\prime} . P^{\prime} R^{\prime}$. The resultant of the parallel forces is a force applied at the centre of gravity, acting in the direction parallel to $\mathrm{R}^{\prime} \mathrm{P}^{\prime}$, and equal to $\mu \omega^{2}$. $\mathrm{R}^{\prime} \mathrm{P}^{\prime}$. Comparing this with the centrifugal couple, it is evident that the forces at $O$ destroy each other, and, therefore, the total result of the rotation round $\mathrm{R}^{\prime} \mathrm{R}^{\prime \prime}$ is to produce a force acting at the point $\mathrm{R}^{\prime}$, which has been just determined. If this point be fixed, the axis $\mathrm{R}^{\prime} \mathrm{R}^{\prime \prime}$ will be a permanent axis of rotation. The condition by which the point $R^{\prime}$ is found is, that the triangle $O R^{\prime} \mathrm{P}^{\prime}$ is equal to and in the same plane with the triangle $\mathrm{ORP}$ : hence, if an ellipsoid confocal to 
346 Rotation of a Solid Body round a Fixed Point.

the ellipsoid of gyration be described through the point $R$ ', it will be perpendicular to the line $\mathrm{R}^{\prime} \mathrm{R}^{\prime \prime}$. The general construction for permanent axes is, therefore, the following:-Let the ellipsoid of gyration be described, and confocal ellipsoids : any line which pierces one of these ellipsoids at right angles is a permanent axis of rotation for the point of intersection. 


\section{PART IV.}

ATTRACTION. 



\section{( 349$)$}

\section{I.-ON A DIFFICULTY IN THE THEORY OF THE ATTRAC- TION OF SPHEROIDS.}

[Transactions of the Royal Irish Academy, VoL. xvI. p. 237.-Read May 28, 1832.]

Av approximate theorem, discovered by Laplace, and relating to the attraction of a solid slightly differing from a sphere, on a point placed at its surface, has given rise to many disputes among mathematicians.* I hope the question will be set in a clear light by the following remarks.

Let us consider the function which expresses the sum of every element of a solid divided by its distance from a fixed point, and let us denote it, as Laplace has done, by the letter $V$. It is necessary to find the value of $V$ for a pyramid of indefinitely small angle, the fixed point being at its vertex. Calling $\phi$ the small solid angle of the pyramid (or the area which it intercepts on the surface of a sphere whose radius is unity and centre at the vertex), it is manifest that the element of the pyramid at the distance $r$ from the vertex is $\phi r^{2} d r$; dividing therefore by $r$, and integrating, we have $\frac{1}{2} \phi r^{2}$, or $\phi$ multiplied into half the square of the length, for the value of $\mathrm{V}$.

Again, supposing the force to vary inversely as the square of the distance-the only hypothesis that can be of use in the present inquiry - the attraction of the same pyramid on a point at its vertex, and in the direction of its length, is manifestly equal to $\phi r$.

Let us now consider a solid of any shape, regular or irregular, terminated at one end by a plane to which the straight line $P Q$ is perpendicular at the point $P$; and let there be a sphere of any magnitude, whose diameter $P^{\prime} Q^{\prime}$ is parallel to $P Q$. Let $P^{\prime \prime}$ be a fixed point, and from the points $P, P^{\prime}, P^{\prime \prime}$, draw three parallel straight lines $P p, P^{\prime} p^{\prime}, P^{\prime \prime} p^{\prime \prime}$, the first two

* See Pontécoulant, Théorie analytique du système du monde, tome ii. p. 380 ; with the references there given. 


\section{Difficulty in Theory of Attraction of Spheroids.}

terminated by the surfaces of the solid and of the sphere, the third, $P^{\prime \prime} p^{\prime \prime}$, in the same direction with them and equal to their difference, without regarding which of them is the greater, and
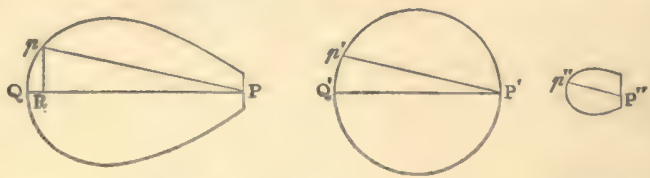

suppose all the points $p^{\prime \prime}$, taken according to the same law, to trace the surface of a third solid. Let $P p, P^{\prime} p^{\prime}, P^{\prime \prime} p^{\prime \prime}$, be edges of three small pyramids with their other edges proceeding from $P, P^{\prime}, P^{\prime \prime}$, parallel, and having of course the same solid angle, which we shall call $\phi$, and denote by $r, r^{\prime}, r^{\prime \prime}$, their respective lengths, and by $V, V^{\prime}, V^{\prime \prime}$, the values of the function $\mathrm{V}$ for each of them. Drawing $p R$ perpendicular to $P Q$, the attraction of the pyramid $P p$ in the direction of $P Q$ will be equal to $\phi \times P R$. Call this attraction $A$, and let $a$ be the radius of the sphere.

Since $r^{\prime \prime}$ is the difference of $r$ and $r^{\prime}$, we have $r^{2}+r^{\prime 2}-r^{\prime \prime 2}$ $=2 r r^{\prime}=2 P R \times P^{\prime} Q^{\prime}$, and multiplying by $\frac{1}{2} \phi$ we find $\frac{1}{2} \phi r^{\dot{2}}$ $+\frac{1}{2} \phi r^{\prime 2}-\frac{1}{2} \phi r^{\prime \prime 2}=2 a \phi \times P R$, that is $V+V^{\prime}-V^{\prime \prime}=2 a A$. The same thing is true for any other three pyramids similarly related to each other, throughout the whole extent of the three solids which are exhausted by them at the same time; and hence, if we now denote by $V, V^{\prime}, V^{\prime \prime}$, the whole values of the function $\mathrm{V}$ for the three solids, and by $A$ the whole attraction of the first of them parallel to $P Q$ on a point at $P$, we shall still have $V+V^{\prime}$ $-V^{\prime \prime}=2 a A$.

To express this general theorem in the notation of Laplace, we have merely to observe that the attraction $A$ is synonymous with $-\left(\frac{d V}{d r}\right)$, and that the quantity $V^{\prime}$ for the sphere is equal to $\frac{4}{3} \pi a^{2}$. Substituting these values, we find

$$
V+2 a\left(\frac{d V}{d r}\right)=-\frac{4}{3} \pi a^{2}+V^{\prime \prime} ;
$$

an exact equation, differing from the approximate one of Laplace only in containing the quantity $V^{\prime \prime}$, and totally independent of 
the nature of the surface or of the magnitude of the sphere; the only things supposed being that all the lines drawn from $\boldsymbol{P}$ meet the surface again but once, and that no part of it passes beyond a plane through $P$ at right angles to $P Q$.

With respect to the limit of the quantity $V^{\prime \prime}$, it is obvious that if a hemisphere be described from $P^{\prime \prime}$ as a centre, with a radius equal to the greatest difference $\delta$ between the lines $P p$, $P^{\prime} p^{\prime}$, the solid $P^{\prime \prime} p^{\prime \prime}$ will lie wholly within this hemisphere, and consequently $V^{\prime \prime}$ will be less than the value of $V$ for the hemisphere, that is, less than $\pi \delta^{2}$; for here all the little pyramids from the centre have the same length $\delta$, and their bases are spread over the hemispherical surface; wherefore $V^{\prime \prime}=2 \pi$ $\times \frac{1}{2} \delta^{2}=\pi \delta^{2}$. All this is independent of anything but the supposition just mentioned.

If now $P Q$ be supposed to be a spheroid of any sort, slightly differing from the sphere $P^{\prime} Q^{\prime}$, and such that the line $P Q$, perpendicular to the surface at $P$, passes nearly through the centre, then all the differences, of which $\delta$ is the greatest, being of the first order, the quantity $V^{\prime \prime}$, which is less than $\pi \delta^{2}$, will be of the second order; and therefore neglecting, as Laplace has done, the quantities of that order, we get the theorem in question.

It may be well to apply the general theorem to the simple case in which the first solid is a sphere of the radius $a^{\prime}$, because both Lagrange and Ivory have used this case to show that the reasonings of Laplace are incorrect. In this instance, then, the surface described by the point $p^{\prime \prime}$ is that of a sphere whose radius is the difference between $a$ and $a^{\prime}$; and the values of $V, V^{\prime}, V^{\prime \prime}$, and $A$, are $\frac{4}{3} \pi a^{\prime 2}, \frac{4}{3} \pi a^{2}, \frac{4}{3} \pi\left(a^{\prime}-a\right)^{2}$ and $\frac{4}{3} \pi a^{\prime}$ respectively.

Substituting these values in the equation $V+V^{\prime}-V^{\prime \prime}=2 a A$, and omitting the common factor $\frac{4}{3} \pi$, the resulting equation

$$
a^{\prime 2}+a^{2}-\left(a^{\prime}-a\right)^{2}=2 a a^{\prime}
$$

ought to be identical; and so it manifestly is.

November, 1831. 
II.-ON THE ATTRACTION OF ELLIPSOIDS, WITH A NEW DEMONSTRATION OF CLAIRAUT'S THEOREM, BEING AN ACCOUNT OF THE LATE PROFESSOR MAC CULLAGH'S LECTURES ON THOSE SUBJECTS. COMPILED BY GEORGE JOHNSTON ALLMAN, LL.D., OF TRINITY COLLEGE, DUBLIN.

[Transactions of the Royal Irish Academy, VoL. xxrr. p. 379.-Read June 13, 1853.]

\section{Proposition I.}

If $\mathrm{P}$ be any point on the surface of an ellipsoid, and $\mathrm{PC}_{1}$ be drawn perpendicular to an axis $\mathrm{OC}$, and an ellipsoid be described through $\mathrm{C}_{1}$ concentric, similar, and similarly placed to the given ellipsoid; then the component of the attraction of the given ellipsoid on $\mathrm{P}$ in a direction parallel to $\mathrm{OC}$ is equal to the attraction of the inner ellipsoid on the point $\mathrm{C}_{1}$.

This theorem is an extension of that given by MacLaurin* relating to the attraction of a spheroid on a point placed on its surface. It may, moreover, be established by means of the same geometrical proposition from which MacLaurin deduced his theorem.

Through the point $\mathrm{P}$ let a chord $\mathrm{PP}^{\prime}$ of the given ellipsoid be drawn parallel to the axis OC. Now, suppose both ellipsoids to be divided into wedges by planes parallel to each other, and passing respectively through this chord and the parallel axis of the inner; and suppose the wedges to be divided into pyramids, the common vertex of one set being at $P$, and of the other at $C_{1}$.

- De caus. Phys. Flux. et Ref. Maris, sect. 3; or see Airy's Tract on the Figure of the Earth, Prop. 8. 
Observing that any two of these parallel planes cut the two surfaces in similar ellipses, such that the semi-axis of one is equal to the parallel ordinate of the other, it is easy to see that

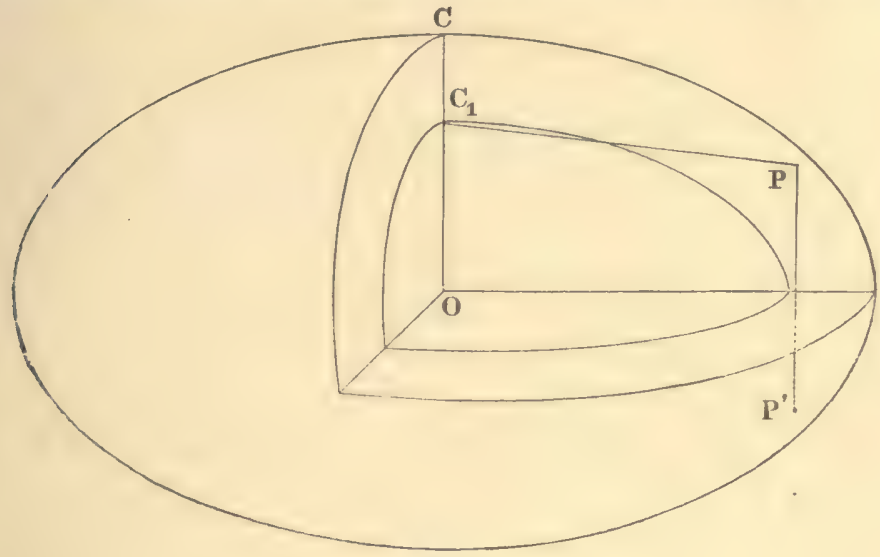

Fig. 1.

the reasoning employed by Mac Laurin may be used to establish the truth of the theorem stated above.

\section{Proposition II.}

To calculate the Attraction of an Ellipsoid on a Point placed at the extremity of an Axis.*

Let the semi-axes of the ellipsoid be $a, b$, $c$, where $a>b>c$, and let the point on which it is required to find the attraction be $\mathrm{C}$ (Fig. 1), the extremity of the least axis.

Suppose the ellipsoid to be divided by a series of cones of revolution which have a common vertex $\mathrm{C}$ and a common axis $\mathrm{CC}^{\prime}, \mathrm{C}^{\prime}$ being the vertex of the ellipsoid opposite to $C$; it will be sufficient to find an expression for the attraction of the part of the ellipsoid contained between two consecutive conical surfaces, whose semiangles are $\theta$ and $\theta+d \theta$ respectively. Suppose now the part of the ellipsoid between two consecutive cones to be divided into

- Proceedings of the Royal Irish Academy, Vor. Irr. p. 367. 
eleznentary pyramids with a common vertex C. Let CP be one of these elementary pyramids, whose solid angle is $\omega$; let $\mathrm{PQ}$ be drawn perpendicular to $\mathrm{CC}^{\prime}$; from the centre $\mathrm{O}$ draw a radius

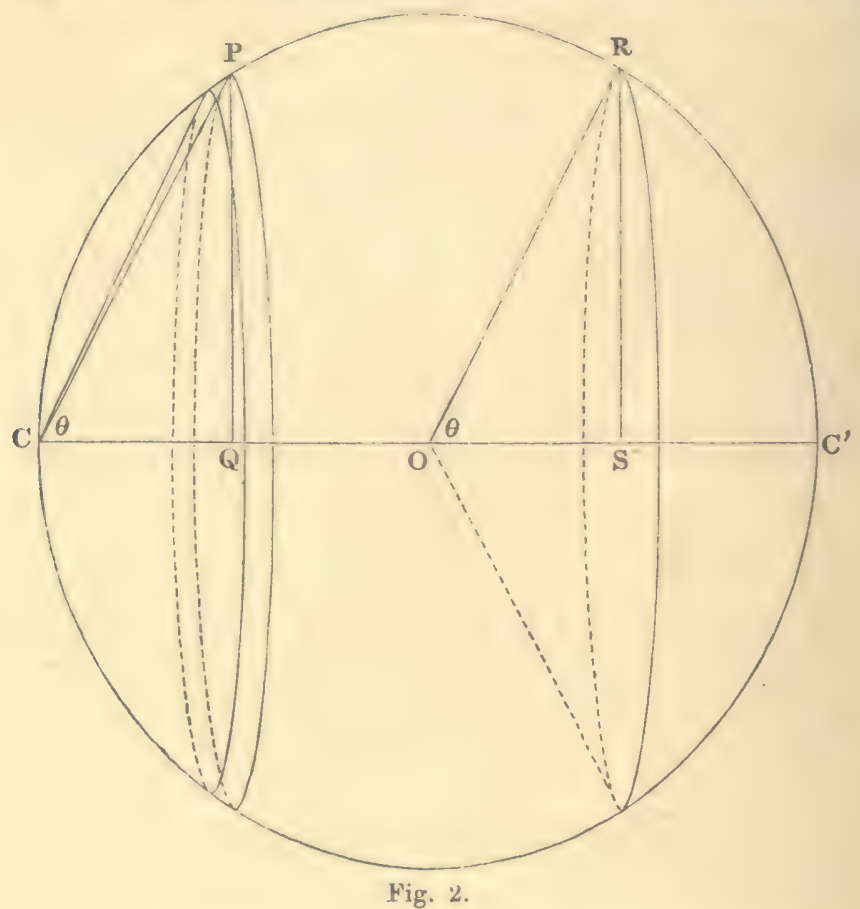

vector $\mathrm{OR}$ parallel to $\mathrm{CP}$, and from the extremity $\mathrm{R}$ let fall a perpendicular RS on the axis $\mathrm{CC}^{\prime}$.

Now the attraction of the elementary pyramid CP on the material point $\mu$, placed at its vertex $=\mu f \rho \omega . \mathrm{CP}$; and the component of this attraction in the direction of the axis is

$$
\mu f \rho \omega . \mathrm{CQ}=2 \mu f \rho \omega \cdot \frac{\overline{\mathrm{OR}}^{2} \cos ^{2} \theta}{c} .
$$

Now suppose the radius vector $O R$ to revolve around the axis $\mathrm{OC}^{\prime}$, then the attraction on the point $\mathrm{C}$ of the portion of the ellipsoid bounded by the two cones of revolution, whose semi- 
angles are $\theta$ and $\theta+d \theta$ respectively, since it is made up of the components in the direction $\mathrm{CC}^{\prime}$ of the attractions of all the elementary pyramids $\mathrm{CP}$, is

$$
\frac{2 \mu f \rho}{c} \cos ^{2} \theta \cdot \Sigma\left(\overline{\mathrm{OR}}^{2} \omega\right)=\frac{2 \mu f \rho}{c} \cos ^{2} \theta d \theta \cdot \Sigma\left(\overline{\mathrm{OR}}^{2} d \phi\right),
$$

$d \phi$ being the angle between two consecutive sides of the cone generated by the revolution of $\mathrm{OR}$.

But $\mathbf{\Sigma}\left(\overline{\mathrm{OR}}^{2} d \phi\right)$ is equal to twice the superficial area of the part of this cone which is enclosed within the ellipsoid. Moreover, the projection on the plane $a b$ of this portion of the surface of the cone is an ellipse, whose semi-axes are $r_{1} \sin \theta, r_{2} \sin \theta$, and whose area is $\pi r_{1} r_{2} \sin ^{2} \theta, r_{1}$ and $r_{2}$ being the maximum and minimum values of $\mathrm{OR}$ : the superficial area of the portion of the cone within the ellipsoid is therefore $\pi r_{1} r_{2} \sin \theta$.

Hence it follows that

$$
\Sigma\left(\overline{\mathrm{OR}}^{2} d \phi\right)=2 \pi r_{1} r_{2} \sin \theta .
$$

The attraction on the point $\mathrm{C}$ of the part of the ellipsoid contained between the two cones of revolution, whose common vertex is at $\mathrm{C}$, and whose semi-angles are $\theta$ and $\theta+d \theta$ respecspeotively, is therefore

where

$$
\frac{4 \pi \mu f \rho}{c} \cos ^{2} \theta d \theta \cdot r_{1} r_{2} \sin \theta,
$$

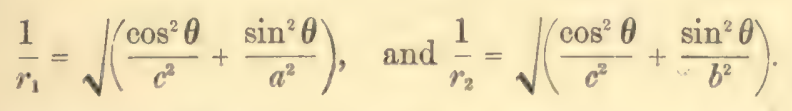

On substituting these values, the expression given above becomes

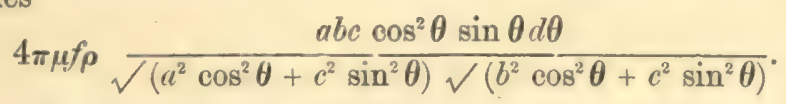

Hence the attraction of the solid ellipsoid on the point $\mathrm{C}$ at the extremity of the least axis is

$$
4 \pi \mu f_{\rho} \int_{0}^{\frac{\pi}{2}} \frac{a b c \cos ^{2} \theta \sin \theta d \theta}{\left.2 \operatorname{A~} 2^{2} a^{2} \cos ^{2} \theta+c^{2} \sin ^{2} \theta\right) \sqrt{\left(b^{2} \cos ^{2} \theta+c^{2} \sin ^{2} \theta\right)}} .
$$


Let $\cos \theta=u$, this expression becomes

$$
4 \pi \mu f \rho \int_{0}^{1} \frac{a b c u^{2} d u}{\sqrt{ }\left\{c^{2}+u^{2}\left(a^{2}-c^{2}\right)\right\} \sqrt{ }\left\{c^{2}+u^{2}\left(b^{2}-c^{2}\right)\right\}} .
$$

In the same way it may be shown that the attraction of the ellipsoid on a point $\mu$ placed at the extremity of the mean axis is

$$
4 \pi \mu f \rho \int_{0}^{1} \frac{a b c u^{2} d u}{\sqrt{ }\left\{b^{2}+u^{2}\left(c^{2}-b^{2}\right)\right\} \sqrt{ }\left\{b^{2}+u^{2}\left(a^{2}-b^{2}\right)\right\}} ;
$$

and on a point at the extremity of the greatest axis,

$$
4 \pi \mu f_{\rho} \int_{0}^{1} \frac{a b c u^{2} d u}{\sqrt{ }\left\{a^{2}+u^{2}\right.} \frac{\left.\left(b^{2}-a^{2}\right)\right\} \sqrt{ }\left\{a^{2}+u^{2}\left(c^{2}-a^{2}\right)\right\}}{} .
$$

It will be seen in a subsequent proposition, that these three expressions are not independent of each other, the values of the three attractions in question being connected by an equation.

\section{Proposition III.}

To give Geometrical Representations of the Attraction of an Ellipsoid on Points placed at the extremities of its least and mean Axes."

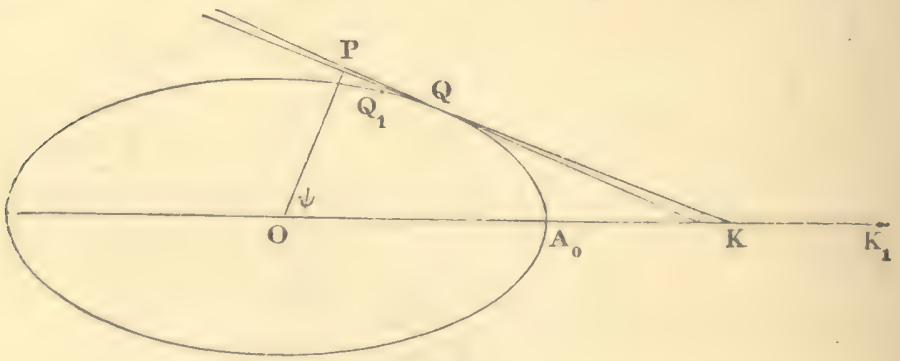

Fig. 3.

On the greater axis $\mathrm{OA}_{0}$ of the focal ellipse assume a point $\mathrm{K}_{1}$ such that $\mathrm{OK}_{1}=\frac{b}{c} \mathrm{OA}_{0}$; from the point $\mathrm{K}_{1}$ draw a tangent

* Proceedings of the Royal Irish Academy, Voz. In. p. 367. 
$\mathrm{K}_{1} \mathrm{Q}_{1}$ to the focal ellipse, and let $T=\tan \mathrm{K}_{1} \mathrm{Q}_{1}-\operatorname{arc} \mathrm{A}_{0} \mathrm{Q}_{1}$; then the attraction of the ellipsoid on the particle $\mu$ placed at the extremity $\mathrm{C}$ of the least axis is

$$
\frac{4 \pi \mu f \rho a b c^{2}}{\left(a^{2}-c^{2}\right)\left(b^{2}-c^{2}\right)} T \text {. }
$$

For let a point $\mathrm{K}$ (see fig. 3 ) be assumed on the greater axis $\mathrm{OA}_{0}$ of the focal ellipse, such that

$$
\mathrm{OK}=\frac{\mathrm{OA}_{0}}{c} \sqrt{ }\left\{c^{2}+u^{2}\left(b^{2}-c^{2}\right)\right\} ;
$$

from $K$ let a tangent $K Q$ be drawn to the focal ellipse, and let $\mathrm{OP}$ be the perpendicular let fall from $\mathrm{O}$ and $\mathrm{KQ}$; then $\psi$ denoting the angle $\mathrm{A}_{0} \mathrm{OP}$,

$$
\overline{\mathrm{OK}^{2}} \cdot \cos ^{2} \psi=\frac{a^{2}-c^{2}}{c^{2}}\left\{c^{2}+u^{2}\left(b^{2}-c^{2}\right)\right\} \cdot \cos ^{2} \psi .
$$

Moreover,

$$
\overline{\mathrm{OK}^{2}} \cdot \cos ^{2} \psi=\overline{\mathrm{OP}^{2}}=\left(a^{2}-c^{2}\right) \cos ^{2} \psi+\left(b^{2}-c^{2}\right) \sin ^{2} \psi .
$$

Equating these values, and solving for $\sin ^{2} \psi$, we get

Now

$$
\sin ^{2} \psi=\frac{\left(a^{2}-c^{2}\right) u^{2}}{c^{2}+u^{2}\left(a^{2}-c^{2}\right)} .
$$

$$
\begin{gathered}
d \cdot\left(\tan \mathrm{KQ}-\operatorname{arc} \mathrm{A}_{0} \mathrm{Q}\right)=\sin \psi d . \mathrm{OK}^{*} \\
=\frac{\left(a^{2}-c^{2}\right)\left(b^{2}-c^{2}\right)}{c} \frac{u^{2} d u}{\sqrt{ }\left\{c^{2}+u^{2}\left(a^{2}-c^{2}\right)\right\} \sqrt{ }} \frac{c^{2}+u^{2}\left(b^{2}-c^{2}\right)}{}
\end{gathered}
$$

By comparing this expression with (1), given in the last proposition, it appears that the attraction on the point $\mathrm{C}$ of the portion of the ellipsoid contained between the two conical surfaces whose semi-angles are $\theta$ and $\theta+d \theta$, respectively, is

$$
\frac{4 \pi \mu f \rho a b c^{2}}{\left(a^{2}-c^{2}\right)\left(b^{2}-c^{2}\right)} d \cdot\left(\tan \mathrm{KQ}-\operatorname{arc} \mathrm{A}_{0} \mathrm{Q}\right) .
$$

* Transactions of the Royal Irish Academy, VoL. xvr. p. 79. Proceedings of the Royal Irish Academy, VoL. II. p. 607 (supra, p. 255). 
Now, in order to obtain the attraction of the whole ellipsoid on the point $\mathrm{C}$, we have to integrate the expression given above between the limits $u=0$ and $u=1$, or $\mathrm{OK}=\mathrm{OA}_{0}$ and $\mathrm{OK}=\mathrm{OK}_{1}$; from which it appears that its value is

$$
\frac{4 \pi \mu f \rho a b c^{2}}{\left(a^{2}-c^{2}\right)\left(b^{2}-c^{2}\right)} T \text {. }
$$

It is easy to see that the attraction of the part of the ellipsoid contained within the conical surface, whose semi-angle $\theta$ is equal to the angle $\cos ^{-1} u$, is

$$
4 \pi \mu f \rho \frac{a b c^{2}}{\left(a^{2}-c^{2}\right)\left(b^{2}-c^{2}\right)}(T-t),
$$

where $t=\tan \mathrm{KQ}-\operatorname{are} \mathrm{A}_{0} \mathrm{Q}$.

To represent the attraction on a point $\mu$ placed at the extremity of the mean axis, assume on the transverse $\mathrm{OA}_{0}$ of the focal hyperbola a point $\mathrm{K}_{1}$ such that $\mathrm{OK}_{1}=\mathrm{OA}_{0} \frac{c}{b}$, and from $\mathrm{K}_{1}$ draw a tangent $K_{1} Q_{1}$ to the hyperbola, and let $T=\tan K_{1} Q_{1}$ $-\operatorname{arc} A_{0} Q_{1}$; then the attraction of the ellipsoid on the point $\mu$ is

$$
-4 \pi \mu f \rho \frac{a b^{2} c}{\left(a^{2}-b^{2}\right)\left(c^{2}-b^{2}\right)} T .
$$

To prove this, assume a point $\mathrm{K}$ such that

$$
\mathrm{OK}=\frac{\mathrm{OA}_{0}}{b} \sqrt{ }\left\{b^{2}+u^{2}\left(c^{2}-b^{2}\right)\right\} ;
$$

from $\mathrm{K}$ draw a tangent $\mathrm{KQ}$ to the hyperbola, and from $\mathrm{O}$ let fall a perpendicular $O P$ on this tangent; then if $\psi=$ angle $\mathrm{A}_{0} \mathrm{OP}$,

$$
\sin ^{2} \psi=\frac{\left(a^{2}-b^{2}\right) u^{2}}{b^{2}+u^{2}\left(a^{2}-b^{2}\right)} .
$$

Hence, by following a method similar to that used in finding the representation of the attraction on a point at the extremity of the least axis, the expression given above may be easily obtained.

The attractions $C, B$ of the ellipsoid on points placed at the extremity of the least and mean axes are thus represented by 
means of ares of the focal ellipse and hyperbola respectively. In consequence of the third focal conic of the ellipsoid being imaginary, no direct geometrical representation can be given for the attraction $\boldsymbol{A}$ on a point placed at the extremity of its greatest axis. It will, however, be found, as was intimated above, that a simple relation exists between the three attractions, which enables us to represent this last by means of arcs of both focal conios.

The relation alluded to is

$$
\frac{A}{a}+\frac{B}{b}+\frac{C}{c}=4 \pi \mu f_{\rho} .^{*}
$$

This can be easily proved by the help of the following geometrical theorem :-

If from the extremities A, B, C of the three axes of an ellipsoid three parallel chords $\mathrm{A} p, \mathrm{~B} q, \mathrm{C} r$, be drawn, and if these chords be projected each on the axis from whose extremity it is drawn, then the sum of these three projections, $\mathrm{A} a, \mathrm{~B} \beta, \mathrm{C} \gamma$, divided respectively by the lengths of the axes $\mathrm{AA}^{\prime}, \mathrm{BB}^{\prime}, \mathrm{CC}^{\prime}$, on which they are measured, will be equal to unity.

Now conceive three chords $\mathrm{A} p, \mathbf{A} p^{\prime}, \mathbf{A} p^{\prime \prime}$, to be drawn from A. making each with the other two very small angles, and so forming a pyramid with a very small vertical solid angle $\omega$; and from $\mathrm{B}$ and $\mathrm{C}$ let two systems of chords $\mathrm{B} q, \mathrm{~B} q^{\prime}, \mathrm{B} q^{\prime \prime}$, and $\mathrm{C} r, \mathrm{C} r^{\prime}, \mathrm{C}^{\prime \prime}$, be drawn, each system forming a very small pyramid whose three edges are parallel to the three edges $\mathrm{A} p, \mathrm{~A}^{\prime}$, $\mathrm{A} p p^{\prime \prime}$, of the pyramid which has its vertex at $\mathrm{A}$.

The attractions of the three pyramids, reduced each to the direction of the axis passing through its vertex, will be equal to $\mu f \rho \omega . \mathrm{A} a, \mu f \rho \omega . \mathrm{B} \beta, \mu f \rho \omega . \mathrm{C} \gamma$ respectively; and, therefore, the sum of those attractions divided respectively by the lengths of the axes will be

$$
\mu f \rho \omega)\left(\frac{\mathrm{A} a}{\mathrm{AA}^{\prime}}+\frac{\mathrm{B} \beta}{\mathrm{BB}^{\prime}}+\frac{\mathrm{C} \gamma}{\mathrm{C} \mathrm{C}^{\prime}}\right)=\mu f \rho \omega .
$$


Let pyramids thus related be indefinitely multiplied, and the ellipsoid will be simultaneously exhausted from the three points A, B, C.

Hence the sum of the whole attractions at A, B, C, divided respectively by the lengths of the corresponding axes, will be $2 \pi \mu f \rho$, or,

$$
\frac{A}{a}+\frac{B}{b}+\frac{C}{c}=4 \pi \mu f \rho \text {. }
$$

\section{Proposition IV.}

To find an expression for the potential $V$ of a system of particles at a point $\mathrm{M}$, whose distance from the centre of gravity of the system is very great compared with the mutual distances of the particles.

It is proved by Poisson, ${ }^{*}$ that if the origin of co-ordinates be at the centre of gravity of the system

$$
V=\frac{M}{r^{\prime}}+\frac{3}{2 r^{\prime 3}} \mathbf{\Sigma}\left(x x^{\prime}+y y^{\prime}+z z^{\prime}\right)^{2} d m-\frac{1}{2 r^{\prime 3}} \mathbf{\Sigma}\left(x^{2}+y^{2}+z^{2}\right) d m,
$$

$x^{\prime}, y^{\prime}, z^{\prime}$ being the co-ordinates of the distant point, and $r^{\prime}$ its distance from the origin. Let now the principal axes at that centre be taken as axes of co-ordinates; then, since

$$
\begin{gathered}
\Sigma x y d m=0, \quad \Sigma x z d m=0, \quad \Sigma y z d m=0 ; \\
V=\frac{M}{r^{\prime}}+\frac{3}{2 r^{\prime 5}} \mathbf{\Sigma}\left(x^{2} x^{\prime 2}+y^{2} y^{\prime 2}+z^{2} z^{\prime 2}\right) d m-\frac{1}{2 r^{\prime 3}} \mathbf{\Sigma}\left(x^{2}+y^{2}+z^{2}\right) d m .
\end{gathered}
$$

Hence, if $A, B, C$ be the three principal moments of inertia, and $I$ the moment of inertia of the system round OM,

$$
V=\frac{M}{r^{\prime}}+\frac{1}{2 \gamma^{\prime 3}}(A+B+C-3 I) .
$$




\section{Proposition V.}

A system of material particles attracts a point $\mathrm{M}$, whose distance from the centre of gravity $\mathrm{O}$ of the attracting system is very great compared with the mutual distances of the particles; then if a tangent plane be drawn to the "ellipsoid of gyration," perpendicular to $\mathrm{OM}$, the whole attraction lies in the plane $\mathrm{OST}$, where $\mathrm{S}$ is the point in which this tangent plane intersects $\mathrm{OM}$, and $\mathrm{T}$ its point of contact with the ellipsoid.

Let $a, \beta, \gamma$ be the direction angles of $\mathrm{OT} ; \boldsymbol{a}^{\prime}, \beta^{\prime}, \gamma^{\prime}$ of OM; and $a_{1}, \beta_{1}, \gamma_{1}$ of TS ; and $a_{0}, \beta_{0}, \gamma_{0}$ of the normal to the

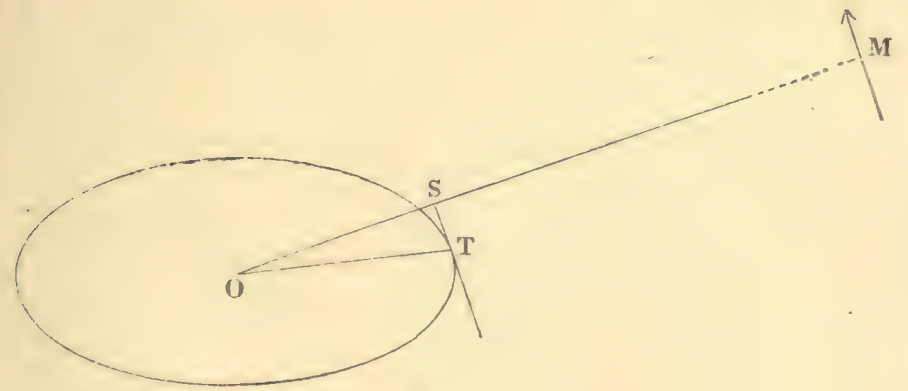

Fig. 4.

plane OST; and let OS, OT and the angle SOT, be denoted by $p, r$, and $\phi$ respectively. It will be sufficient to prove that the component $Q$ of the attraction in the direction of the normal to the plane OST is zero.

We shall first find the components $X, Y, Z$, of the attraction in the directions of the axes, and thence deduce the value of $Q$.

* The centre of this ellipsoid is at the centre of gravity; its axes are in the directions of the principal axes, and their lengths are determined by the equations

$$
M a^{2}=A, \quad M b^{2}=B, \quad M \sigma^{2}=C .
$$

This ellipsoid is used by Professor Mac Cullagh in his "Theory of Rotation": see Rev. S. Haughton's Account of P'rofessor Mac Cullagh's Lectures on that subject, Transactions R. I. A., VoL. xxir. p. 139 (supra, p. 329). 
Now,

$$
\begin{aligned}
& X=-\frac{d V}{d x^{\prime}}=\frac{M}{r^{\prime 2}} \cos a^{\prime}+\frac{3}{2 r^{\prime 4}}(A+B+C-3 I) \cos a^{\prime}+\frac{3}{2 r^{\prime 3}} \frac{d I}{d x^{\prime}} \\
& Y=-\frac{d V}{d y^{\prime}}=\frac{M}{r^{\prime 2}} \cos \beta^{\prime}+\frac{3}{2 r^{\prime 4}}(A+B+C-3 I) \cos \beta^{\prime}+\frac{3}{2 r^{\prime 3}} \frac{d I}{d y^{\prime \prime}} \\
& Z=-\frac{d V}{d z^{\prime}}=\frac{M}{r^{2}} \cos \gamma^{\prime}+\frac{3}{2 r^{\prime 4}}(A+B+C-3 I) \cos \gamma^{\prime}+\frac{3}{2 r^{\prime 3}} \frac{d I}{d z^{\prime}}
\end{aligned}
$$

but,

$$
\frac{d I}{d x^{\prime}}=\frac{2(A-I) \cos a^{\prime}}{r^{\prime}} ; \frac{d I}{d y^{\prime}}=\frac{2(B-I) \cos \beta^{\prime}}{r^{\prime}} ; \frac{d I}{d z^{\prime}}=\frac{2(C-I) \cos \gamma^{\prime}}{r^{\prime}}
$$

Hence we have

$$
\begin{aligned}
& X=\frac{M}{r^{\prime 2}} \cos a^{\prime}+\frac{3}{2 r^{\prime 4}}(A+B+C-5 I) \cos a^{\prime}+\frac{3 A \cos a^{\prime}}{r^{\prime 4}}, \\
& Y=\frac{M}{r^{\prime 2}} \cos \beta^{\prime}+\frac{3}{2 r^{\prime 4}}(A+B+C-5 I) \cos \beta^{\prime}+\frac{3 B \cos \beta^{\prime}}{r^{\prime 4}}, \\
& Z=\frac{M}{r^{\prime 2}} \cos \gamma^{\prime}+\frac{3}{2 r^{\prime 4}}(A+B+C-5 I) \cos \gamma^{\prime}+\frac{3 C \cos \gamma^{\prime}}{r^{\prime 4}} .
\end{aligned}
$$

Now,

but,

$$
Q=X \cos a_{0}+Y \cos \beta_{0}+Z \cos \gamma_{0} ;
$$

$$
\begin{aligned}
& \sin \phi \cos \alpha_{0}=\cos \beta \cos \gamma^{\prime}-\cos \gamma \cos \beta, \\
& \sin \phi \cos \beta_{0}=\cos \gamma \cos \alpha^{\prime}-\cos a \cos \gamma^{\prime}, \\
& \sin \phi \cos \gamma_{0}=\cos \alpha \cos \beta^{\prime}-\cos \beta \cos \alpha^{\prime} ;
\end{aligned}
$$

The following relations, moreover, exist:

$$
a^{2} \cos \boldsymbol{a}^{\prime}=r p \cos \boldsymbol{a}, \quad b^{2} \cos \beta^{\prime}=r p \cos \beta, \quad c^{2} \cos \gamma^{\prime}=r p \cos \gamma:
$$

hence, by substitution, we have

$$
\begin{gathered}
\cos \alpha_{0}=\frac{b^{2}-c^{2}}{p r \sin \phi} \cos \beta^{\prime} \cos \gamma^{\prime}, \quad \cos \beta_{0}=\frac{c^{2}-a^{2}}{p r \sin \phi} \cos \gamma^{\prime} \cos a^{\prime}, \\
\cos \gamma_{0}=\frac{a^{2}-b^{2}}{p r^{\prime} \sin \phi} \cos a^{\prime} \cos \beta^{\prime} .
\end{gathered}
$$


Substituting these values for $\cos \boldsymbol{\alpha}_{0}, \cos \beta_{0}, \cos \gamma_{0}$, in the expression for $Q$, and observing that

we get

$$
\cos a^{\prime} \cos \alpha_{0}+\cos \beta^{\prime} \cos \beta_{0}+\cos \gamma^{\prime} \cos \gamma_{0}=0,
$$

$$
Q=\frac{3 M}{r^{\prime 4}} \frac{a^{2}\left(b^{2}-c^{2}\right)+b^{2}\left(c^{2}-a^{2}\right)+c^{2}\left(a^{2}-b^{2}\right)}{p r \sin \phi} \cos a^{\prime} \cos \beta^{\prime} \cos \gamma^{\prime}=0 .
$$

\section{Proposition VI.}

The same things being supposed, to find the other Components of the Attraction, namely $R$ in the direction of the centre of gravity $\mathrm{MO}$, and $P$ in the transverse direction TS.

To find $R$;

$$
\begin{aligned}
R & =X \cos a^{\prime}+Y \cos \beta^{\prime}+Z \cos \gamma^{\prime} ; \\
\therefore R & =\frac{M I}{r^{\prime 2}}+\frac{3}{2 r^{\prime 4}}(A+B+C-5 I)+\frac{3 I}{r^{\prime 4}}, \\
R & =\frac{M}{r^{\prime 2}}+\frac{3}{2 r^{\prime 4}}(A+B+C-3 I) .
\end{aligned}
$$

To find $P$;

but,

$$
P=X \cos \alpha_{1}+Y \cos \beta_{1}+Z \cos \gamma_{1} ;
$$

$$
\begin{aligned}
& \sin \phi \cos a_{1}=\cos a^{\prime} \cos \phi-\cos a, \\
& \sin \phi \cos \beta_{\mathrm{t}}=\cos \beta^{\prime} \cos \phi-\cos \beta, \\
& \sin \phi \cos \gamma_{1}=\cos \gamma^{\prime} \cos \phi-\cos \gamma .
\end{aligned}
$$

Substituting for $\cos a, \cos \beta, \cos \gamma$ their values from (8), we get

$$
\begin{gathered}
\cos \alpha_{1}=-\frac{a^{2}-p^{2}}{p r \sin \phi} \cos a^{\prime}, \quad \cos \beta_{1}=-\frac{b^{2}-p^{2}}{p r \sin \phi} \cos \beta^{\prime}, \\
\cos \gamma_{1}=-\frac{c^{2}-p^{2}}{p r \sin \phi} \cos \gamma^{\prime} .
\end{gathered}
$$

Substituting these values of $\cos \alpha_{1}, \cos \beta_{1}, \cos \gamma_{1}$, and observing that

$$
\cos \boldsymbol{a}^{\prime} \cos \boldsymbol{\alpha}_{1}+\cos \beta^{\prime} \cos \beta_{1}+\cos \gamma^{\prime} \cos \gamma_{1}=0,
$$


we have

$$
P=-\frac{3 M}{r^{\prime 4}} \frac{p^{2}\left(r^{2}-p^{2}\right)}{p r \sin \phi}=-\frac{3 M I}{r^{4}} p r \sin \phi ;
$$

or,

$$
P=-\frac{3 M}{r^{\prime 4}} \mathrm{OS} \times \mathrm{ST} .
$$

The negative sign indicates* that the force $P$ acts in the direction TS, i.e. from the radius vector towards the perpendicular of the ellipsoid of gyration. If the force $P$ be resolved into three others in the direction of the axes, it is evident from the values given in Proposition V. for $X, Y, Z$, that these components are

$$
\frac{3(A-I)}{r^{\prime 4}} \cos \alpha^{\prime}, \quad \frac{3(B-I)}{r^{\prime 4}} \cos \beta^{\prime}, \quad \frac{3(C-I)}{r^{\prime 4}} \cos \gamma^{\prime} \cdot \dagger
$$

* The direction of the force $P$, which Professor Mac Cullagh determines by the interpretation of the negative sign, may be very clearly seen from the following considerations. This force exists in every case where the three principal moments of inertia of the system at 0 are not all equal, that is, when the ellipsoid of gyration is not a sphere. The greatest axis of that ellipsoid is manifestly towards that part of the body in which there is a deficiency of attracting matter. If we now consider the position of a perpendicular on a tangent plane of an ellipsoid with relation to the corresponding radius vector, we shall find that it always lies away from the greatest axis. But the transverse force has been shown to be in the plane of radius vector and perpendicular. Therefore, the direction of the transverse force, being towards the preponderating matter, must be from $\mathrm{T}$ to $\mathrm{S}$.

+ The results given by Professor Mac Cullagh in Propositions V. and VI. may be otherwise obtained, and, perhaps, with greater facility, by introducing the consideration of the statical moment of the attracting force."

If the three principal moments of inertia were equal to each other, then the whole attraction would be in the direction of the centre of gravity, and its magnitude would be

$$
\frac{M}{r^{\prime 2}}
$$

In general, however, the attracting mass will be of an irregular shape; there will exist then, in addition to the principal part of the attraction, which will be central, a transverse force which will tend to cause a motion of rotation about the centre of gravity.

The components of the moment of this transverse force in the three principal planes are

$$
x^{\prime} Y-y^{\prime} X, \quad y^{\prime} Z-z^{\prime} Y, \quad z^{\prime} X-x^{\prime} Z
$$

\footnotetext{
"See Rev. R. Townsend, in the "Dublin University Examination Papers," 1849, p. 51.
} 


\section{Proposition VII.}

An Ellipsoid is composed of ellipsoidal strata of different densities and of variable but small ellipticities; to find the Components, central and transverse, of its Attraction on an external point.

The values found in the last Proposition for the components of the attraction of any mass on a very distant point will be found to hold in the present case, whatever be the position of the attracted point. In order to show this, we shall first prove it for a homogeneous ellipsoid of small ellipticities. Such an ellipsoid being given, another confocal with it ean be constructed so small, that the distance to the attracted point may be

but from (7),

$$
\begin{aligned}
& x^{\prime} Y-y^{\prime} X=-\frac{3(A-B)}{r^{\prime 3}} \cos \alpha^{\prime} \cos \beta^{\prime}=-\frac{3 M}{r^{\prime 3}}\left(a^{2}-b^{2}\right) \cos \alpha^{\prime} \cos \beta^{\prime}, \\
& y^{\prime} Z-z^{\prime} Y=-\frac{3(B-C)}{r^{\prime 3}} \cos \beta^{\prime} \cos \gamma^{\prime}=-\frac{3 M}{r^{\prime 3}}\left(b^{2}-c^{2}\right) \cos \beta^{\prime} \cos \gamma^{\prime}, \\
& z^{\prime} X-x^{\prime} Z=-\frac{3(C-A)}{r^{\prime 3}} \cos \gamma^{\prime} \cos \alpha^{\prime}=-\frac{3 M}{r^{\prime 3}}\left(c^{2}-a^{2}\right) \cos \gamma^{\prime} \cos \alpha^{\prime} .
\end{aligned}
$$

Now it is well known, that

$$
\frac{1}{2}\left(a^{2}-b^{2}\right) \cos \alpha^{\prime} \cos \beta^{\prime}, \frac{1}{2}\left(b^{2}-c^{2}\right) \cos \beta^{\prime} \cos \gamma^{\prime}, \frac{1}{2}\left(c^{2}-a^{2}\right) \cos \gamma^{\prime} \cos \alpha^{\prime},
$$

are the areas of the projections of the triangle OST on the principal planes. Hence it follows that the resultant moment lies in the plane of the radius vector OT and the perpendicular $\mathrm{OS}$ to the corresopnding tangent plane of the ellipsoid of gyration; the tangent plane being perpendicular to OM. It appears, also, that the magnitude of the resultant moment is

$$
-\frac{3 M}{r^{3}} \mathrm{OS} \times \mathrm{ST}
$$

and therefore that the transverse component of the attraction

$$
P=-\frac{3 M}{r^{2}} \mathrm{OS} \times \mathrm{ST} \text {. }
$$

Or, the values of the central force and the moment of the transverse force may

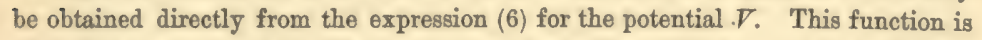
of such a nature, that its differential coefficient with relation to any line (the sign being changed) is equal to the resolved part of the attraction in that direction; and the differential coefficient with relation to any angle (the sign being changed as 
regarded as very great, compared with the axes of this ellipsoid. The components of the attraction of this small ellipsoid on the distant point are given by the expressions (10) and (11): now as the attractions of two confocal ellipsoids on an external point are in the same direction, and proportional to their masses: the components of the attraction of the proposed ellipsoid will, therefore, be

$$
\begin{aligned}
& R=\frac{M}{r^{\prime 2}}+\frac{3}{2 r^{\prime 4}} \frac{M}{M_{1}}\left(A_{1}+B_{1}+C_{1}-3 I_{1}\right), \\
& P=-\frac{3 M}{r^{\prime 4}} \mathrm{~S}_{1} \times \mathrm{OS}_{1} \mathrm{~T}_{1}
\end{aligned}
$$

the letters with suffixes referring to the small ellipsoid.

The attracting ellipsoids being confocal, their ellipsoids of gyration are confocal also; hence it follows that

and

$$
\frac{M}{M_{1}}\left(A_{1}+B_{1}+C_{1}-3 I_{1}\right)=A+B+C-3 I,
$$

$$
\mathrm{OS}_{1} \times \mathrm{S}_{1} \mathrm{~T}_{1}=\mathrm{OS} \times \mathrm{ST}
$$

before) gives the component in the plane of that angle of the moment of the attractive force.

Hence,

since

$$
R=-\frac{d \Gamma}{d r^{\prime}}=\frac{M}{r^{\prime 2}}+\frac{3}{2 r^{\prime 4}}(A+B+C-3 I),
$$

$$
\frac{d I}{d r^{\prime}}=0
$$

Again, if $N$ be the component of the moment of the attractive force round $0 Z$,

but

$$
N=-\left(x^{\prime} \frac{d}{d y^{\prime}}-y^{\prime} \frac{d}{d x^{\prime}}\right) V^{\prime}
$$

$$
\begin{gathered}
\left(x^{\prime} \frac{d}{d y^{\prime}}-y^{\prime} \frac{d}{d x^{\prime}}\right) F\left(x^{\prime 2}+y^{\prime 2}\right)=0 \text { where } F \text { is any function ; } \\
\therefore N^{-}=\frac{3}{2 r^{\prime 3}}\left(x^{\prime} \frac{d}{d y^{\prime}}-y^{\prime} \frac{d}{d x^{\prime}}\right)=\frac{3}{2 r^{\prime 3}}\left(x^{\prime} \frac{d}{d y^{\prime}}-y^{\prime} \frac{d}{d x^{\prime}}\right)\left(\frac{A x^{\prime 2}+B y^{\prime 2}+C z^{\prime 2}}{r^{\prime 2}}\right) ; \\
\therefore N=-\frac{3(A-B)}{r^{\prime 3}}\left(\cos \alpha^{\prime} \cos \beta^{\prime}\right) .
\end{gathered}
$$

The two other components of the moment may be similarly obtained. The remainder of the proof is the same as in the former part of this note. 
It appears, from this, that the central and transverse components of the attraction of a solid ellipsoid of uniform density, and whose ellipticities are small, on any external point whatever, are given by the same formulæ as the corresponding components of the action of any mass on a distant point.

Now it is a property of moments of inertia, that they are subtractive, that is, the difference of the moments of inertia of two masses with relation to any axis is equal to the moment of inertia of the difference of those masses with relation to the same axis. And the values at which we have arrived for the central force, and for the three components of the transverse force, contain in each term either a mass or a moment of inertia in the first power, and therefore, these values also are subtractive. Hence the two components of the attraction of a homogeneous mass contained between two concentric and coaxal ellipsoids of small ellipticities, are given by formulæ (10) and (11). Now suppose an ellipsoidal mass to be oomposed of strata bounded by ellipsoids of different but small ellipticities, each stratum being homogeneous throughout its extent, while the density varies from one stratum to another according to any law; then, since those formulæ hold for the action of each stratum separately, and since the terms of which they are made up are in their nature additive, they hold for the entire mass.*

\section{Proposition VIII.}

An oblate Spheroid is composed of spheroidal strata of different densities and of variable but small ellipticities; to find the Components of its Attraction on any external point.

The expressions given in the last Proposition for $R$ and $P$ become simplified in this case. Let $\mathrm{OZ}$ be the axis of revolution, and let $\lambda$ denote the angle which OM makes with the plane

"See Professor Mac Cullagh, in the "Dublin University Examination Papers," 1833 , p. 268. 
$\mathrm{XY}$; then since $A$ and $B$ are equal, we have

and therefore

$$
I=A \cos ^{2} \lambda+C \sin ^{2} \lambda
$$

also

$$
A+B+C-3 I=(C-A)\left(1-3 \sin ^{2} \lambda\right) ;
$$

$$
M \mathrm{OS} \times \mathrm{OT}=(A-C) \sin \lambda \cos \lambda .
$$

Substituting these values in the expressions for $R$ and $P$, we have

$$
\begin{aligned}
& R=\frac{M}{r^{\prime 2}}+\frac{3}{2} \frac{C-A}{r^{\prime 4}}\left(1-3 \sin ^{2} \lambda\right), \\
& P=3 \frac{C-A}{r^{\prime 4}} \cos \lambda \sin \lambda .
\end{aligned}
$$

The direction of the force $P$ is towards the plane of the equator; this appears from the shape of the " ellipsoid of gyration," which in this case is a prolate surface of revolution.

\section{Proposition IX.-Clairaut's Theorem.}

Whatever be the law of variation of the Earth's density at different distances from the centre, if the ellipticity of the surface be added to the ratio which the excess of the polar above the equatorial gravity bears to the equatorial gravity, their sum will be $\frac{5}{2} q$, where $q$ is the ratio of the centrifugal force at Equator to equatorial gravity.

For suppose the attracted point $\mathbf{M}$ to be on the surface of the earth, which is known to be an oblate spheroid of small ellipticity. Then, from the principles of Hydrostatics, since the tangential force is zero, we have

$$
R \cos \theta-P \sin \theta-\omega^{2} r \cos \lambda \cos (\theta-\lambda)=0,
$$

where $\omega$ denotes the angular velocity, and $\theta$ the angle which the tangent to the meridian through the attracted point makes with the radius vector. Developing $\cos (\theta-\lambda)$ and arranging, we obtain

$$
\left(R-\omega^{2} r \cos ^{2} \lambda\right) \cos \theta=\left(P+\omega^{2} r \cos \lambda \sin \lambda\right) \sin \theta .
$$


But, from the property of the elliptio section made by the plane of the meridian, we have

$$
\cot \theta=\frac{e^{2} \sin \lambda \cos \lambda}{1-e^{2} \cos ^{2} \lambda}=2 \varepsilon \sin \lambda \cos \lambda, \quad q \cdot p \cdot,
$$

where $e$ is the excentricity and $\varepsilon$ the ellipticity of this ellipse.

Substituting in (16) this value of $\cot \theta$, and the values of $R$ and $P$ from (13) and (14), the equation of equilibrium becomes

$$
\begin{gathered}
\left\{\frac{M}{r^{2}}+\frac{3}{2} \frac{C-A}{r^{4}}\left(1-3 \sin ^{2} \lambda\right)-\omega^{2} r \cos ^{2} \lambda\right\} 2 \varepsilon \sin \lambda \cos \lambda \\
=\left(3 \frac{C-A}{r^{4}}+\omega^{2} r\right) \sin \lambda \cos \lambda,
\end{gathered}
$$

or, approximately,

$$
\left\{\frac{M}{a^{2}}+\frac{3}{2} \frac{C-A}{a^{4}}\left(1-3 \sin ^{2} \lambda\right)-\omega^{2} a \cos ^{2} \lambda\right\} 2 \varepsilon=3 \frac{C-A}{a^{4}}+\omega^{2} a .
$$

If we neglect quantities of the second order, this equation becomes

$$
\frac{2 \varepsilon M}{a^{3}}=3 \frac{C-A}{a^{4}}+\omega^{2} a .
$$

We have thus arrived at a relation which enables us to express the unknown quantity $C-A$, in terms of quantities which are all known, and, therefore, to eliminate the former from any other equation in which it may occur.

Now let $R_{e}$ and $R_{p}$ denote the equatorial and polar attractions respectively; we have from the general value of $R(13)$,

but

$$
\begin{aligned}
& R_{e}=\frac{M}{a^{2}}+\frac{3}{2} \frac{C-A}{a^{4}}, \\
& R_{p}=\frac{M}{c^{2}}-3 \frac{C-A}{c^{4}} ;
\end{aligned}
$$

$$
\begin{gathered}
c=a(1-\varepsilon) \therefore \frac{1}{c^{2}}=\frac{1}{a^{2}}(1+2 \varepsilon) \text { and } \frac{1}{c^{4}}=\frac{1}{a^{4}}(1+4 \varepsilon) \\
\therefore R_{p}=\frac{M I}{a^{2}}+\frac{2 M \varepsilon}{a^{2}}-3 \frac{C-A}{a^{4}} . \\
2 \text { в }
\end{gathered}
$$


But

$$
\begin{gathered}
G_{p}=R_{p} \text { and } G_{\theta}=R_{e}-\omega^{2} a ; \\
\therefore G_{p}-G_{e}=\frac{2 \varepsilon M}{a^{2}}-\frac{9}{2} \frac{C-A}{a^{4}}+\omega^{2} a .
\end{gathered}
$$

Eliminating $\frac{C-A}{a^{4}}$ by means of equation (17), we get

$$
\frac{G_{p}-G_{e}}{G_{e}}=-\varepsilon+\frac{5}{2} \frac{\omega^{2} a}{G_{e}}
$$

or

$$
\frac{G_{p}-G_{e}}{G_{e}}+\varepsilon=\frac{5}{2} q
$$




\section{SUPPLEMEN T.}

\section{EGYPTIAN CHRONOLOGY.}





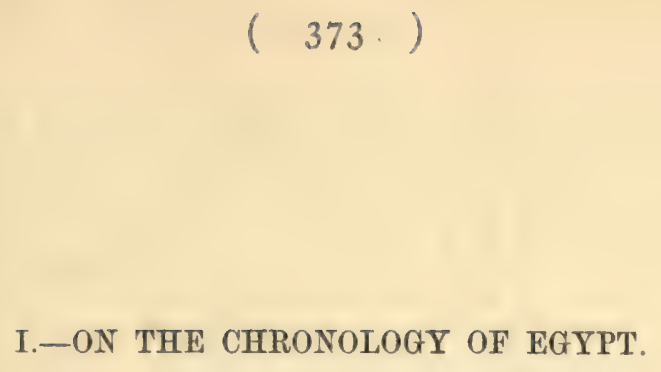

I.-ON THE CHRONOLOGY OF EGYPT.

[Proceedings of the Royal Irish Academy, Voc. I., p. 66.-Read April 24, 1837.]

IN this Paper the author endeavours to ascertain the names of the Egyptian sovereigns who were contemporary with Moses. For this purpose he finds it necessary to determine the interval between two celebrated epochs-the reign of Menes and the Exodus of the Israelites. He conceives that the former epoch is fixed by the "old chronicle" at the distance of 443 years from the beginning of a cynic (or canicular) cycle; and he thinks it strange that this simple meaning should not have occurred to chronologists, who have universally supposed the "cynic cycle" of the old chronicle to be a series of demi-god kings who derived that appellation from the dog-headed Anubis. The canicular cycle is a well-known period of 1460 years, which the Egyptians seemed to have used for computing time, as we sometimes use the Julian period. One of these cycles commenced in the year 2782 before the Christian era; and if we reckon 443 years in advance, we shall have the year B. c. 2339 for the commencement of the reign of Menes. This date agrees well with the computation of Josephus, who says that the interval from Menes to Solomon was upwards of 1300 years. Again, we are told by Clemens of Alexandria, that the Exodus of the Israelites took place 345 years before the beginning of a canicular cycle. This is evidently the cycle which commenced в. с. 1322 ; and hence we have в. c. 1667 for the date of the Exodus. The interval between Menes and the Exodus was, therefore, about 670 years. 
If, now, we take the catalogue of Eratosthenes, which commences with Menes, we shall find, at the distance of 670 years from Menes, a king named Achescus Ocaras, who reigned only one year; preceded by a king named Apappus, who reigned a hundred years, and succeeded by queen Nitocris, who reigned. six jears. Mr. Mao Cullagh thinks that Apappus is the king in whose reign Moses was born; that Ocaras is he who pursued the Israelites to the Red Sea; and that Nitocris is the famous queen mentioned by Herodotus. It may be objected that Eratosthenes gives us the succession of Theban kings, whereas the Pharaohs of the Mosaic history reigned in Lower Egypt; but it is remarkable that the three sovereigns mentioned above are found in Manetho's dynasties among those who reigned at Memphis; and it is singular that these are the only sovereigns (except Menes and his immediate successor) in which the dynas. ties of Manetho and the catalogue of Eratosthenes agree. All the other names are different. Of course the predecessors of Apappus, at Thebes and at Memphis, were different; and thus we can easily understand how there arose up at Memphis "a new king who knew not Joseph." It would appear, in fact, that A pappus was of a Theban family, and that he succeded, for some reason or another, to the throne of Lower Egypt. He was only six years old (as we learn from Manetho) when he came to the throne; and it is natural to suppose that his chief advisers, as he grew up, were the courtiers who accompanied the young king from his own country to Memphis, and who knew nothing of Joseph, and cared nothing for his people. Accordingly, when Apappus arrived at manhood he issued an order that erery male child of the Hebrews should be destrojed, lest they should grow too numerous for the Egyptians; and, under these circumstances, Moses was born in the twenty-first year of his reign, and was saved by the king's young daughter, a girl about ten jears old. About the sixtieth year of Apappus, Moses was obliged to fly to the land of Midian, for having killed an Egsptian; and when at length the king of Egypt died"after many days," as it is in the original-Moses returned in 
the beginning of the reign of Ocaras, before whom were performed those signs and wonders which prepared the way for the departure of the Israelites. On the night of the Passover, the king lost his first-born, perhaps his only son; and this may be the reason that he was succeeded by his sister Nitocris. The short reign of Ocaras (a single year) might be explained by supposing he was drowned in the Red Sea; but as there is nothing in the sacred narrative which obliges us to admit that the king perished in this manner, we may adopt the account of Herodotus, that he was murdered by his subjects. We may imagine that some of his nobles remained with Pharaoh on the shore; and that when they saw the sea return and swallow up all that had gone in after the Israelites, they murdered the king, whose obstinacy had brought such calamities on his people, and then placed his sister Nitocris on the throne. As Nitocris was the daughter of Apappus, there is nothing to prevent us from supposing that the queen, now ninety years old, was the princess who had saved the infant Moses. Weary of her life, she lived only to arenge her brother. For this purpose, says Herodotus, she constructed a large subterranean chamber, to which, when it was finished, she invited the principal agents in her brother's death; and there, by the waters of the Nile admitted through a secret canal, they were drowned in the midst of the banquet. The queen then threw herself into a room filled with ashes, where she perished. 


\section{$(376)$}

II.-ON THE CATALOGUE OF EGYPTIAN KINGS, WHICH IS USUALLY KNOWN BY THE NAME OF THE LATERCULUM OF ERATOSTHENES.

[Proceedings of the Royal Irish Academy, Vor. II. p. 366.-Read January 9, 1843.]

THIs Catalogue, which the distinguished mathematician and philosopher whose name it bears drew up by command of Ptolemy Euergetes, contains a long series of kings who reigned at Thebes in Upper Egypt, and has been preserved to us in the Chronographia of Georgius Syncellus, a Greek monk of the eighth century. It is a document which has been made much use of by ehronologers; by some of whom (as by Sir John Marsham for example, who calls it "venerandissimum antiquitatis monumentum"), it has been reckoned of the very highest authority; but it is extremely corrupt in the latter part, owing to the carelessness with which it was transcribed either by Syncellus himself or his immediate copyists. The writers on Egyptian antiquities have in consequence been much perplexed in settling the chronology of the reigns in which the errors exist, and the attempts that have been made to remove the confusion have only served to increase it. It was the object of the author to restore the document to its original state, and he showed that this might be effected, with complete certainty, by a proper attention to the manuscripts of Syncellus. Of these only two are 
known : one has been used by Father Goar, the first editor of the Chronographia (Paris, 1652); the other, which is a much better one, has been collated by Dindorf, the second and latest editor. Dindorf's edition was published at Bonn, in the year 1829, as part of the Corpus Scriptorum Historice Byzantince, and on its first appearance Mr. Mac Cullagh had satisfied himself as to the original readings of the Catalogue, and had seen how to account for the errors which, probably from Syncellus's own negligence, had crept into it; but he did not publish his conclusions at the time, thinking that similar considerations could not fail to occur to some of the numerous writers who were then giving their especial attention to such subjects. This, however, has not been the case. Chronologers have continued to follow in the footsteps of Goar, a man of little learning, and of no critical sagacity, who corrected the Catalogue most injudiciously, and whose corrections, strange to say, are left without any remark by Dindorf. Thus Mr. Cory, in his Ancient Fragments, a work much referred to, merely transcribes Goar's list ; and Mr. Cullimore, in attempting to reconcile ancient authors with each other and with the monuments, has adopted an hypothesis respecting the identity of two sovereigns which is not tenable when the true version of the Catalogue is known. Even in Goar's edition, however, there was quite enough to have led a person of ordinary judgment to the correct readings of the Catalogue, though perhaps they could not be said to be absolutely certain without the additional light obtained from that of Dindorf.

The Catalogue in question professes to entain the names of thirty-eight sovereigns, with the years of their reigns; the whole succession occupying, as is stated, a period of 1076 years ; but it is only in the last eight reigns that the errors and inconsistencies occur. The thirty-second prince is called Stamenemes $\beta$, that is, Stamenemes the Second, though there is, at present, no other of that name in the list ; and the beginning of his reignas appears from the years of the world, which Syncellus has annexed according to the Constantinopolitan reckoning-follows 
the termination of the preceding one by an interval of twentysix years. Jackson, in his Chronological Antiquities, is positive that this prince is called the Second by a mistake, and adds the years that are wanting to the reign of his predecessor, as Goar had previously done. In the first part of this view all authors, without exception, are agreed, though they do not explain how a mistake, so very odd, could have originated; but the learned Marsham-who, having adopted the short chronology of the Hebrew Bible, is so hard pressed to find room for the Egyptian dynasties that he is obliged to begin the reign of Menes the very year after the Deluge-is glad to omit the twenty-six years altogether, thus reducing the sum of all the reigns to 1050 years, contrary to what is expressly stated by Syncellus. The natural inference from the state of the MSS. is, however, simply this: that the thirty-second king was Stamenemes I., that he reigned twenty-six years, and was succeeded by Stamenemes II. We may easily conceive that the eye of the transcriber, deceived by the identity of names, passed over the first, and rested on the second, thus occasioning the error. Indeed there can now be no doubt that this was the fact; because, in the MS. marked (B) by Dindorf, the next king is numbered as the thirty-fourth, the next but one as the thirty-fifth, and so on; which shows that a name had dropped out, and this name could be no other than that of Stamenemes I., who must have filled the vacant interval, and must consequently have reigned the number of years that has been assigned to him.

As neither Groar nor any other writer perceived this omission, the successor of Stamenemes II. has always been reckoned as the thirty-third in the list, and the next following as the thirtyfourth, \&c. But as one error begets another, the omission was compensated by the insertion of an anonymous king, who is placed thirty-sixth in the list, with a reign of fourteen years; the insertion being necessary to complete the number (thirtyeight) which the Catalogue ought to contain. And, by a further error, these fourteen years are taken out of the reign of the 
thirty-seventh sovereign, who ought to have nineteen years instead of the five that have been hitherto assigned to him. This last error was occasioned by an ignorant correction of a mistake which is found in both the MSS., and which therefore probably arose from the carelessness of Syncellus himself. The thirtyseventh king and his predecessor are stated to have begun to reign in the same year of the world, and to have reigned the same number of years (five). Now from what goes before it is plain that both these numbers belong to the thirty-sixth king; and from the year of the world in which the thirty-eighth and last king began to reign, it is elear that the thirty-seventh reigned nineteen years. The mistake in the MSS. is one which might easily be made by a thoughtless writer; for the Catalogue is given in detached portions-a few reigns at a time-separated by a great quantity of other matter, and the name of the thirtysixth king ends one of these portions, while that of the thirtyseventh begins another; so that, not having both before his eyes at the same moment, a person so careless as Syncellus might, without being conscious of it, attach the same reign and date to the two names, by transcribing twice over the same line of numbers in the Catalogue which he was copying; the whole of which Catalogue, in all likelihood, he had previously drawn up in a tabular form, with the years of the world annexed according to his own chronology, that it might be ready, as any portion of it was wanted, for immediate transference to his pages. Such seems to be the natural account of the matter; but, as usual, it does not occur to Goar, who takes the opportunity, which the confusion affords him, of foisting in his supplementary king between the two last mentioned, giving each of these five years, as in the MS., by which means he obtains room for him; while on the other hand he alters the year of the world attached to the thirty-seventh king, so as to make it suit his hypothesis.

The following is a view of the last eight reigns, as they appear to have stood in the original document, compared with the erroneous list of Goar. The years of the world are omitted, as 
being of no importance, except so far as they are useful in the preceding argument.

I. GodR's List.

31. Peteathyres reigned

32. Stamenemes "

33. Sistosichermes ",

34. Maris

35. Siphoas

36. Anonymous

37. Phruoro

38. Amuthartous ,"
II. Corrected List.

Years.

42

23

55

43

5

14

5

63
31. Peteathyres reigned 16

32. Stamenemes I. , 26

33. Stamenemes II. , 23

34. Sistosichermes, , 55

35. Maris , , 43

36. Siphoas ", 5

37. Phruoro $\quad 19$

38. Amuthartaus , 63

The interval of time which has been shown to belong to the first Stamenenes, and which was added by Goar to the reign of Peteathyres, is differently disposed of by $\mathrm{Mr}$. Cullimore, in a chronological table which he has given in the second volume of the Transactions of the Royal Society of Literature. His object being to compare the lists of Eratosthenes, Manetho, \&c., with the supposed hieroglyphical series, he makes Saophis, the fifteenth in Eratosthenes' Catalogue, the same as a king whose name is read Phrathek Osirtesen; but the forty-third year of the latter is mentioned on the monuments, whereas Saophis has only twenty-nine years in the Catalogue. To escape from this difficulty, therefore, Mr. Cullimore adds the unappropriated interval to the reign of Saophis, thus giving him fifty-five years instead of twenty-nine. But it now appears that such a supposition is altogether inadmissible, and consequently the two personages in question cannot be identified-a circumstance which proves that there is some fault in Mr. Cullimore's assumptions, and that his other conclusions, at least in this part of his Table, cannot be relied on.

The corrections here given do not interfere with the inferences drawn by Professor Mac Cullagh from the Catalogue of Eratosthenes in a former Paper on Egyptian Chronology (Proceedings of the Royal Irish Academy, vol. i., p. 66), ${ }^{*}$ because the 
portion of the Catalogue with which he was there concerned terminates with the reign of Queen Nitooris, the twenty-second in the list. The corrections, indeed, though not hitherto published, were made long before the date (April, 1837) of that Paper, but not before he had adopted the hypothesis therein proposed, as an answer to the old and ever-recurring question-Who were the Egyptian sovereigns that were contemporary with Moses? For it was in consequence of this hypothesis, which had suggested itself to him at a very early period, that he was led to examine the Catalogue minutely, in order to diseover whether his chronology was affected by its errors.

Having been led to refer to his hypothesis, Mr. Mac Cullagh took occasion to observe that, in the interval which had elapsed since it was published, he had not met with any facts that were opposed to it: on the contrary, the more he considered it, the more he was inclined to believe in its reality; though it was entirely different from every other that had been proposed, either by modern chronologers or by the early Fathers of the Church, in their manifold attempts to connect the narrative of Moses with the remaining fragments of Egyptian history. The hypothesis, indeed, is the only one which, while it gives a probable date for the Exodus, also satisfies what Mr. Mac Cullagh conceives to be the necessary conditions of the question; namely, a very long reign - of at least eighty years-during which the Israelites were persecuted, succeeded by a very short oneapparently not more than a year-during which their deliverance was wrought ; and it is interesting in itself, on account of the remarkable connexion which it establishes between sacred and profane history, and the highly dramatic character of the events which are thus, for the first time, brought into view. 



\section{DUBLIN UNIVERSITY PRESS SERIES.}

Tнв Provost and Senior Fellows of Trinity College have undertaken the publication of a Series of Works, chiefly Educational, to be entitled the DUBLIN UNIVERsity Press Series.

The following volumes of the Series are now ready, viz. :-

Six Lectures on Physical Geography. By the Rev. S. HAUghton, M.D., Dubl., D.C.L., Oxon., F.R.S., Fellow of Trinity College, and Professor of Geology in the University of Dublin.

An Introduction to the Systematic Zoology and Morphology of Vertebrate Animals. By Alexander Macalister, M.D., Dubl., Professor of Comparative Anatomy in the University of Dublin.

The Codex Rescriptus Dublinensis of St. Matthew's Gospel (Z). First Published by Dr. Barrett in 180r. A New Edition, Revised and Augmented. Also, Fragments of the Book of Isaiah, in the LXX. Version, from an Ancient Palimpsest, now first Published. Together with a newly discovered Fragment of the Codex Palatinus. By T. K. Aввотт, B.D., Fellow of Trinity College, and Professor of Biblical Greek in the University of Dublin. With two Plates of Facsimiles.

The Parabola, Ellipse, and Hyperbola, treated Geometrically. By Robert William Griffin, A.M., LL.D., Ex-Scholar, Trinity College, Dublin.

An Introduction to Logic. By William Henry Stanley MoncK, M.A. Professor of Moral Philosophy in the University of Dublin.

Essays in Political and Moral Philosophy. By T. E. CLIfFe LESLIE, Hon. LL.D., Dubl., of Lincoln's Inn, Barrister-at-Law, Late Examiner in Poli: tical Economy in the University of London, Professor of Furisprudence and Political Economy in the Queen's University.

The Correspondence of Cicero : a revised Text, with Notes and Prolegomena.-Vol. I., The Letters to the end of Cicero's Exile. By RoBerT Y TyRreLL, M. A., Fellow of Trinity College, and Professor of Latin in the University of Dublin.

Faust from the German of Goethe. By Thomas E. WEBB, LL.D., Q.C., Regius Professor of Lazos, and Public Orator in the University of Dublin.

The Mathematical and other Tracts of the late James M'Cullagh, F.T.C.D., Professor of Natural Philosophy in the University of Dublin. Now first collected, and edited by Rev. J. H. Jellett, B.D., and Rev. Samuel Haughton, M.D., Fellows of Trinity College, Dublin.

The following will soon appear:-

Evangelia Antehieronymiana ex Codice vetusto Dublinensi. Ed. T. K. Aввот, B.D.

The Vell of Isis ; or, Idealism. By Thomas E. WEBB, LL.D., Q.C., Regius Professor of Laws, and Public Orator in the University of Dublin.

DUBLIN : HODGES, FIGGIS, AND CO.

LONDON: LONGMANS, GREEN, AND CO. 



\section{GENERAL LISTS OF WORKS}

PUBLISHED BY

Messrs. Longmans, Green \& Co.

\section{$-\infty 0 \% 000$ \\ HISTORY, POLITICS, HISTORICAL MEMOIRS, \&c.}

Russia Before and After the War. By the Author of 'Society in St. Petersburg' \&c. Translated from the German (with later Additions by the Author) by EDWARD FAIRFAX TAYLOR. Second Edition. 8vo, I4s,

Russia and England from I876 to I880; a Protest and an Appeal. By O. K. Author of 'Is Russia Wrong ?' With a Preface by J. A. Froude, M.A. Portrait and Maps. 8vo. I4s.

History of England from the Conclusion of the Great War in 1815. By SPeNCER WAlpole. 8vo. Vols. I. \& II. 1815-I832 (Second Edition, revised) price 36 s. VOL. III. $1832-1841$, price $18 s$.

History of England in the I8th Century. By W. E. H. LEcky, M.A. VOLS. I. \& II. I700-1760. Second Edition. 2 vols. 8vo. $36 s$.

\section{The History of England} from the Accession of James II. By the Right Hon. Lord Macaulay.

STUDENT's EDITION, 2 vols. cr. 8vo. I2s. PeOPle's Edition, 4 vols. cr. 8vo. I6s. Cabinet Edition, 8 vols. post 8 vo, 48 s. Library EDItion, 5 vols, 8vo. $f_{4}$.

\section{Lord Macaulay's Works.}

Complete and uniform Library Edition. Edited by his Sister, Lady TrkvELYAN. 8 vols. 8 vo. with Portrait, 25.55 .
Critical and Historical Essays contributed to the Edinburgh Review. By the Right Hon. Lord Macaulay.

Cheap Edition, crown 8vo. 3s. $6 d$. STUdent's Edition, crown 8 vo. $6 s$. PEOPLE'S EDition, 2 vols, crown 8vo, 8s. CABINET Edition, 4 vols. 24s. Library EDItion, 3 vols. 8vo. $36 s$.

\section{The History of England} from the Fall of Wolsey to the Defeat of the Spanish Armada. By J. A. Froude, M.A.

Cabinet Edition, 12 vols. crown, $\mathscr{L}_{3}$. $12 s$. Library Edition, 12 vols. demy, £8. 18 s.

The English in Ireland in the Eighteenth Century. By J.A. Froude, M.A. 3 vols. 8 vo. $\lfloor 2.8 s$.

Journal of the Reigns of King George IV, and King William IV. By the late C. C. F. Greville, Esq. Edited by H. ReEve, Esq. Fifth Edition. 3 vols. 8 vo, price $36 s$.

\section{The Life of Napoleon III.} derived from State Records, Unpul)lished Family Correspondence, and Personal Testimony. By BLANCHARD JERROLD. In Four Volumes, 8vo, with numerous Portraits and Facsimiles. VOLS. I. to III, price I8s, each. 
The Constitutional History of England since the Accession of George III. I760-1870. By Sir 'Thomas ERSKINe MAY, K.C.B. D.C.L. Sixth Edition. 3 vols, crown 8vo. 18s.

Democracy in Europe; a History. By Sir Thomas ERskine MAY, K.C.B. D.C.L. 2 vols, 8vo. $32 s$.

Introductory Lectures on Modern History delivered in I84I and 1842. By the late Thomas ARNOLD, D.D. 8vo. 7s. 6d.

On Parliamentary Government in England; its Origin, Development, and Practical Operation. By Alpheus Tond, 2 vols, 8vo. 37s.

History of Civilisation in England and France, Spain and Scotland. By Henry Thomas BUCKLE, 3 vols. crown 8 vo. 24 s.

Lectures on the History of England from the Earliest Times to the Death of King Edward II. By W. Longman, F.S.A. Maps and Illustrations. 8vo, 15 s.

History of the Life \& Times of Edward III. By W. LoNGMAN, F.S.A. With 9 Maps, 8 Plates, and 16 Woodcuts. 2 vols. 8vo. $28 s$.

History of the Life and Reign of Richard III. Including the Story of PerkiN IVARBeck. By JAMres GaIrdner. Second Edition, Portrait and Map. Crown 8vo. Ios. 6d.

Memoirs of the Civil War in Wales and the Marches, I642-I649. By JOHN ROLAND Phillips, of Lincoln's Inn, Barristerat-Law. 8vo, 16s.

History of England under the Duke of Buckingham and Charles I. I624-I628. By S. R. Gardiner, 2 vols, 8vo. Maps, 24.

\section{The Personal Govern-} ment of Charles I. from the Death of Buckingham to the Declaration in favour of Ship Money, 1628-1637. By S. R. GARDINER, 2 vols. 8vo. 24 s.
Memorials of the Civil

War between King Charles I. and the Parliament of England as it affected Herefordshire and the Adjacent Counties. By the Rev. J. WebB, M.A. Edited and completed by the Rev. T. W. WEBв, M.A. 2 vols. 8vo. Illustrations, $42 s$.

\section{Popular History of} France, from the Earliest Times to the Death of Louis XIV. By Miss Sewell. Crown 8vo. Maps, 7s, 6d.

A Student's Manual of the History of India from the Earliest Period to the Present By Col. Mendows TaYLor, M.R.A.S. Third Thousand, Crown 8vo. Maps, 7s. 6d.

Lord Minto in India; Correspondence of the First Earl of Minto, while Governor-General of India, from 1807 to 1814 , Edited by his Great-Niece, the Countess of Minto. Completing Lord Minto's Life and Letters published in 1874 by the Countess of Minto, in Three Volumes. Post 8vo. Maps, I2s.

Indian Polity; a View of the System of Administration in India. By Lieut.-Col, G. Chesney, 8vo. 21s,

Waterloo Lectures; a Study of the Campaign of 1815 . By Col. C. C. Chesney, R. E. 8vo. 1os. 6 \%.

The Oxford ReformersJohn Colet, Erasmus, and Thomas More; a History of their Fellow-Work. Ву F. Sеzвонм. Svo. I4s.

History of the Romans under the Empire. By Dean MErI. VALE, D.D. 8 vols. post 8 vo. 48 s.

\section{General History of Rome} from B.C. 753 to A.D. 476 . By Dean Merivale, D.D. Crown 8vo. Maps, price $7 s .6 d$.

\section{The Fall of the Roman}

Republic ; a Short History of the Last Century of the Commonwealth. By Dean Merivale, D.D. $12 \mathrm{mo} .7 \mathrm{~s} .6 \mathrm{~d}$. 
The History of Rome. By WILHelm IHNE. Vols. I. to III. $8 \mathrm{vo}$, price 45 s.

\section{Carthage and the Cartha-} ginians. By R. Bosworth Sмгтн, M.A. Second Edition. Maps, Plans, \&c. Crown 8vo. 10s, $6 d$.

\section{The Sixth Oriental Mo-} narchy; or, the Geography, History, and Antiquities of Parthia. Py G. Rawlinson, M.A. With Maps and Illustrations. 8vo. $16 s$.

\section{The Seventh Great Ori-} ental Monarchy ; or, a History of the Sassanians. By G. RAwlinson, M.A. With Map and 95 Illustrations. 8vo. 28s.

\section{The History of European} Morals from Augustus to Charlemagne. By W. E. H. Lecky, M.A. 2 vols. crown 8vo. I6s.

History of the Rise and Influence of the Spirit of Rationalism in Europe. By W. E. H. Lecky, II. 1. 2 vols. crown 8vo. $16 s$.

The History of Philosophy, from Thales to Comte. By George Henry Lewes. Fifth Edition. 2 vols. 8vo. 32s.

\section{A History of Classical} Greek Literature. By the Rev. J. P. MahaffY, M.A. Trin. Coll. Dublin. 2 vols. crown Svo, price $7 s, 6 d$. each.

\section{Zeller's Stoics, Epicu-} reans, and Sceptics. Translated by the Rev. O. J. Reichel, M.A. New Edition revised, Crown 8vo. I5s.

\section{Zeller's Socrates \& the} Socratic Schools. Translated by the Rev. O. J. Rrichel, M.A. Second Edition. Crown 8vo. Ios. 6 d.

\section{Zeller's Plato \& the Older}

Academy. Translated by S. Frances AlLEYNE and AlfReD GoODWIN, B.A. Crown 8vo. $18 s$.

'Aristotle and the Elder Peripatetics' and 'The Pro-Socratic Schools," completing the English Edition of Zeller's Work on Ancient Greek Philosophy, are preparing for publication.
Epochs of Modern History. Edited by C. Colbeck, M.A.

Church's Beginning of the Middle Ages, 2s. $6 d$.

Cox's Crusades, 2s. 6d.

Creighton's Age of Elizabeth, 2s. $6 d$.

Gairdner's Houses of Lancaster and York, 2s. 6d.

Gardiner's Puritan Revolution, 2s. $6 d$. Thirty Years' War, 2s. $6 d$.

Hale's Fall of the Stuarts, $2 s$. $6 d$.

Johnson's Normans in Europe, 2s. 6d.

Ludlow's War of American Independence, $2 s .6 d$.

Morris's Age of Anne, 2s. 6d.

Seebohm's Protestant Revolution, 2/6.

Stubbs's Early Plantagenets, 2s. $6 d$.

Warburton's Edward III. 2s. $6 d$.

\section{Epochs of Ancient His-} tory. Edited by the Rev. Sir G. W. Cox, Bart. M.A. \& C. Sankey, M.A.

Beesly's Gracchi, Marius \& Sulla, 2s. $6 d$. Capes's Age of the Antonines, 2s. $6 d$.

Early Roman Empire, 2s. 6d.

Cox's Athenian Empire, 2s. $6 d$.

Greeks \& Persians, 2s. $6 d$.

Curteis's Macedonian Empire, 2s. $6 d$.

Ihne's Rome to its Capture by the Gauls, 2s. 6d.

Merivale's Roman Triumvirates, 2s. $6 d$. Sankey's Spartan \& Theban Supremacies, $2 s, 6 d$.

Creighton's Shilling History of England, introductory to 'Epochs of English History.' Fcp. 8vo. Is.

Epochs of English History. Edited by the Rev. MANDELt. Creighton, M.A. Fcp. 8vo. 5s.

Browning's Modern England, 1820$1874,9 d$.

Cordery's Struggle against Absolute Monarchy, I603-I688, $9 d$.

Creighton's (Mrs.) England a Continental Power, 1066-1216, 9d.

Creighton's (Rev. M.) Tudors and the Reformation, I485-I603, 9d.

Rowley's Rise of the People, I215-1485, price $9 d$.

Rowley's Settlement of the Constitution, I688-1778, $9 d$.

Tancock's England during the American \& EuropeanWars, $1778-1820,9 d^{\prime}$

York-Powell's Early England to the Conquest, I $s$. 
The Student's Manual of Ancient History; the Political History, Cieography and Social State of the P'rincipal Nations of Antiquity. By IV. COOKE TAYLOR, LL. D. Cr. 8vo. 7s.6d.
The Student's Manual of Modern History; the Rise and Pro. gress of the Principal European Nations. Iry IV. Cooke Taylor, LL.D. Crown 8vo. 7 s. $6 d$.

\section{BIOGRAPHICAL WORKS.}

The Life of Henry Venn,

B.D. l'rebendary of St. Paul's, and IIon. Sec. of the Church Missionary Society ; with Extracts from his Letters and Papers. By the Rev. W. KNIGHT, II.A. With an Introduction by the Rev. J. VENI, M.A. [Fust ready'.

Memoirs of the Life of Anna Jameson, Author of 'Sacred and I.egendary Art' \&c. By her Niece, Glirardine Macpherson. 8vo, with Portrait, 12s. $6 d$.

Isaac Casaubon, I559I6r4. By MARK PATtison, Rector of Lincoln College, Oxford. 8vo. 18s.

The Life and Letters of Lord Macaulay. By his Nephew, G. Otto Trevelyan, M.P.

CABinet EDi'iton, 2 vols. crotrn 8vo. i2s. J.tBRARY EidTION, 2 vols. 8vo. $36 s$.

The Life of Sir Martin Frobisher, Knt. containing a Narrative of the Spanish Armada. By the Rev. Fraxk Jones, B.A. Portrait, Maps, and Facsimile. Clown 8vo. 6s.

The Life, Works, and Opinions of Heinrich Heine. By William Stigand. 2 vols 8vo. l'ortrait, $28 \mathrm{~s}$.

The Life of Mozart. Translated from the German Work of 1)r. LUDWIG Norrl by Lady WALlace. 2 vols, crown 8 vo. Portraits, 21 s.

The Life of Simon de Montfort, Earl of Leicester, with special reference to the Parliamentary Ilistory of his time. By G. W. Prothero, Crown 8vo. Maps, 9s.
Felix Mendelssohn's Letters, translated by Lady WALIACE. 2 vols, crown 8 vo. 5 s, each.

Autobiography. By JонN Stuart Mill, 8vo. $7 s, 6 d$.

Apologia pro Vitâ Suâ; Being a History of his Religious Opinions by JOHN HENRY NEWMAN, D.D. Crown 8 vo. $6 s$.

Leaders of Public Opinion in Ireland; Swift, Flood, Grattan, O'Connell. By W. E. H, LECKy, M.A. Crown 8vo. 7s, 6d.

Essays in Ecclesiastical Biography. By the Right Hon. Sir J. STEPHEN, LL.D. Crown 8vo. 7s. $6 d$.

Cæsar; a Sketch. By James ANTHONy FROUDE, M.A. formerly Fellow of Exeter College, Oxford. With Portrait and Map. 8vo. 16s.

\section{Life of the Duke of Wel-} lington. By the Rev, G. R. GLEIG, M.A. Crown 8vo. Portrait, $6 s$.

\section{Memoirs of Sir Henry} Havelock, K.C.B. By JoHN ClARK Marshman. Crown 8 vo. $3^{\text {s. }} 6 d$.

\section{Vicissitudes of Families.}

By Sir Bernard Burke, C.B. Two vols. crown 8 vo. $21 s$.

\section{Maunder's Treasury of} Biography, reconstructed and in great part re-written, with above 1,600 ad ditional Memoirs by W. L. R. CATEs. Fcp. 8vo. 6s. 
MENTAL and POLITICAL PHILOSOPHY.

Comte's System of Positive Polity, or Treatise upon Sociology :-

VoL. I. General View of Positivism and Introductory Principles. Translated by J. H. BRIDGes, M.B. 8vo. 21 s.

Vor. II. The Social Statics, or the Abstract Laws of Human Order. Translated by F. HARrison, M.A. 8vo. i4s.

Vol. III. The Social Dynamics, or the General Laws of Human Progress (the Philosophy of History). Translated by E. S. BEESLY, M.A. 8vo. 21 s 。

Vou. IV. The Theory of the Future of Man; with CoMTE's Early Essays on Social Philosophy. Translated by R. CoNGREVE, M.D. and H. D. HuTron, B.A. 8vo. 24 s.

\section{De Tocqueville's Demo-} cracy in America, translated by $\mathrm{H}$. REEVE. 2 vols, crown 8vo. I6s.

Analysis of the Phenomena of the Human Mind. By JAMes Milu. With Notes, Illustrative and Critical, 2 vols. 8 vo, 28 s.

On Representative Government. By John Stuart Mill. Crown 8 vo. 25 .

\section{On Liberty. By John} Stuart Mill. Post 8vo, 7s. 6d. crown 8 vo. I $s, 4 d$.

\section{Principles of Political} Economy. By John Stuart Mill. 2 vols. 8 vo. 30 s, or I vol, crown 8 vo. 5 s.

Essays on some Unsettled Questions of Political Economy. By John Stuart Mill, 8vo,6s.6d.

Utilitarianism. By JoHN Stuart Mili. 8vo. 5 s.

\section{The Subjection of Wo-} men. By John Stuart Mill. Fourth Edition, Crown 8vo. $6 s$.

\section{Examination of Sir Wil-} liam Hamilton's Philosophy. By John Stuart MilL. 8vo. 16s.
A System of Logic, Ratiocinative and. Inductive. By JoHN StuART MILl. 2 vols. 8vo. 25s.

\section{Dissertations and Dis-} cussions. By John Stuart Mul. 4 vols. 8vo. $£ 2.75$.

\section{The A B C of Philosophy;}

a Text-Book for Students. By the Rev. T. Griffith, M.A. Prebendary of St. Paul's. Crown 8vo. 5s.

\section{Philosophical Fragments} written during intervals of Business. By J. D. Morell, LL.D. Crown 8vo. 5 s.

\section{Path and Goal; a Discus-} sion on the Elements of Civilisation and the Conditions of Happiness. By M. M. Kalisch, Ph.D. M.A. 8vo. price 12s, $6 d$.

\section{The Law of Nations con-} sidered as Independent Political Communities. By Sir Travers Twiss, D.C.L. 2 vols. 8vo. £I. 13s.

A Systematic View of the Science of Jurisprudence. By SHILLDon Amos, M.A. 8vo. I8s.

A Primer of the English Constitution and Government. By S. Amos, M.A. Crown 8vo. $6 s$.

Fifty Years of the English Constitution, 1830-I880. By SHEI. Don Amos, M.A. Crown 8vo, Ios. 6d.

Principles of Economical Philosophy. By H. D. MAcleod, M.A. Second Edition in 2 vols. Vol. I. 8vo, I5s. VOL. II. PART I. 12s.

Lord Bacon's Works, collected \& edited by R. L. Eliss, M.A. J. Spedding, M. A, and D. D. Heath. 7 vols. 8 vo. 63.13 s. $6 d$.

\section{Letters and Life of Fran-} cis Bacon, including all his Occasional Works. Collected and edited, with a Commentary, by J. Speding. 7 rols. 8vo. f4. 4s. 
The Institutes of Justinian; with English Introduction, Translation, and Notes. By T. C. Sandars, M.A. 8vo. I8s.

The Nicomachean Ethics of Aristotle, translated into English by R. Williams, B.A. Crown 8vo, price $7 s, 6 \dot{d}$.

Aristotle's Politics, Books I. III. IV. (VII.) Greek Text, with an English Translation by W. E. BoLLAND, M.A. and Short Essays by $\mathbf{A}$. LANG, M.A. Crown 8vo. 7s. 6d.

The Politics of Aristotle; Greek Text, with English Notes. By Richard Congreve, M.A. 8vo, iss.

The Ethics of Aristotle; with Essays and Notes. By Sir A. Grant, Bart. LL.D. 2 vols. 8vo. 32 s.

Bacon's Essays, with Annotations. By R. Whately, D.D. 8vo. Ios. $6 d$.

Picture Logic ; an Attempt to Popularise the Science of Reasoning. By A. Swinbourne, B.A. Post 8vo. 5 s.

Elements of Logic. By R. Whately, D.D. 8vo. ros. $6 d$. Crown 8vo. 4s. $6 d$.

Elements of Rhetoric. By R. Whately, D.D. 8vo, Ios. $6 d$. Crown 8vo. 4s. $6 d$.
On the Influence of Authority in Matters of Opinion. By the lateSir. G. C. LEwIS, Bart. 8vo. I4s.

The Senses and the Intellect. By A. BAIn, LL.D. 8vo. 15 s:

The Emotions and the Will. By A. BAIN, LL.D. 8vo. 15s,

Mental and Moral Science; a Compendium of Psychology and Ethics, By A. BAIN, LL.D. Crown 8vo. 1os. $6 d$.

An Outline of the Necessary Laws of Thought; a Treatise on Pure and Applied Logic. By W. Thomson, D.D. Crown 8vo. $6 s$.

Essays in Political and Moral Philosophy. By T. E. Cliffe LEsLie, Hon. LL. D. Dubl. of Lincoln's Inn, Barrister-at-Law. 8vo. Ios. $6 d$.

Hume's Philosophical Works. Edited, with Notes, \&cc. by T. H. Green, M.A. and the Rev. T. H. Grose, M.A. 4 vols. 8vo. 56 s. Or separately, Essays, 2 vols. $28 s$. Treatise on Human Nature, 2 vols. 28 s.

Lectures on German Thought. Six Lectures on the History and Prominent Features of German Thought during the last Two Hundred Years, delivered at the Royal Institution of Great Britain. By KARL HILLEBRAND, Rewritten and enlarged. Crown 8vo. 7s. $6 d$.

\section{MISCELLANEOUS \& CRITICAL WORKS.}

Selected Essays, chiefly from Contributions to the Edinburgh and Quarterly Reviews. By A. HAYWARD, Q.C. 2 vols, crown Svo. $12 s$.

Miscellaneous Writings of J. Conington, M.A. Edited by J. A. Symonds, M.A. 2 vols, 8vo. $28 s$.

\section{Short Studies on Great} Subjects. By J. A. Froude, M. A. 3 vols. crown 8 vo, I8s.
Literary Studies. By the late Walter Bagehot, M.A. Fellow of University College, London. Edited, with a Prefatory Memoir, by R. II. HutTon. Second Edition. 2 vols. $8 v o$, with Portrait, $28 s$.

Manual of English Literature, Historical and Critical. By T. ARnold, M.A. Crown 8vo. 7 s. $6 d$.

The Wit and Wisdom of the Rev. Sydney Smith. Crown 8vo. 3s. 6d. 
Lord Macaulay's Miscellaneous Writings :-

LIBRARY EDITION, 2 vols. 8vo, 2 Is.

PeOPle's EDition, I vol. cr. 8 vo. $4 s, 6 d$.

Lord Macaulay's Miscellaneous Writings and Speeches. Student's Edition. Crown 8vo. $6 s$.

Speeches of the Right Hon. Lord Macaulay, corrected by Himself. Crown 8vo. $3^{s}, 6 d$ 。

Selections from the Writings of Lord Macaulay. Edited, with Notes, by G. O. Trevelyan, M.P. Crown. 8vo. $6 s$.

\section{Miscellaneous and Post-} humous Works of the late Henry Thomas Buckle. Edited by HeleN TAYLOR. 3 vols. 8 vo. 52 s. $6 d$.

\section{Miscellaneous Works of} Thomas Arnold, D.D. late Head Master of Rugby School. 8vo. 7s, 6d.

\section{The Pastor's Narrative;}

or, before and after the Battle of Wörth, 1870. By Pastor KI.EIN. Translated by Mrs. F. E. Marshall. Crown 8vo. Map, 6s.

German Home Life; a Series of Essays on the Domestic Life of Germany. Crown 8vo. $6 s$.

\section{Realities of Irish Life.}

By W. Steuart Trench. Crown 8vo. 2s. $6 d$. boards, or $3 s .6 d$. cloth.

\section{Two Lectures on South} Africa delivered before the Philoso. phical Institute, Edinburgh, Jan, 6\& 9, 1880. By James Axtmuny Frovde, M.A. 8vo. 5 s.

\section{Cetshwayo's Dutchman;}

the Private Journal of a White Trader in Zululand during the British Invasion. By Cornelius Vijn. Translated and edited with Preface and Notes by the Right Rev. J. W. Colenso, D.D. Bishop of Natal, Crown 8vo, Portrait, 5s.
Apparitions; a Narrative of Facts. By the Rev, B. W. Savile, M.A. Second Edition. Crown 8vo. price 5 s.

Max Müller and the Philosophy of Language. By LUDIWIG NOIRE, 8vo, $6 s$,

\section{Lectures on the Science} of Language. By F. MAX MüLLER, M.A. 2 vols, crown 8 vo. I6s,

Chips from a German Workshop ; Essays on the Science of Religion, and on Mythology, Traditions \& Customs. By F. MAX MüLler, M.A. 4 vols. 8vo. $f 2.18 s$.

\section{Language \& Languages.}

A Revised Edition of Chapters on Language and Families of Speech. By F. W. FARRAR, D.D. F.R.S. Crown 8 vo. 6s.

\section{The Essays and Contri-} butions of A. K. H. B. Uniform Cabinet Editions in crown 8vo.

Recreations of a Country Parson, Three Series, 3s. 6d. each.

Landscapes, Churches, and Moralities, price $3^{\text {s. }} 6 d^{\circ}$.

Seaside Musings, 3 s. $6 d$.

Changed Aspects of Unchanged Truths, $3 s_{0} 6 d$.

Counsel and Comfort from a City Pulpit, 3s. $6 d$.

Lessons of Middle Age, 3 s. $6 \%$.

Leisure Hours in Town, $35.6 d$.

Autumn Holidays of a Country Parson, price 3 s. $6 d$.

Sunday Afternoons at the Parish Church of a University City, 3s. 6d.

The Commonplace Philosopher in Town and Country, 3. 6d.

Present-Day Thoughts, 3s. $6 d$.

Critical Essays of a Country Parson, price 3 s. $6 d$.

The Graver Thoughts of a Country Parson, Three Series, 3s. 6d. each. 


\section{DICTIONARIES and OTHER BOOKS of REFERENCE.}

One-Volume Dictionary of the English Language. By $\mathrm{R}$. G. Latham, M.A. M.D. Medium 8vo. 24s.

Larger Dictionary of the English Language. By R, G. Latham, M.A. M.D. Founded on Johnson's English Dictionary as edited by the Rev. H. J. ToDD. 4 vols. 4 to. $£ 7$.

Roget's Thesaurus of English Words and Phrases, classified and arranged so as to facilitate the expression of Ideas, and assist in Literary Composition. Revised and enlarged by the Author's Son, J. L. ROGET: Crown 8vo, Ias. 6\%.

English Synonymes. By E. J. Whately. Edited by R. Whately, D.D. Fcp. 8vo. 3s.

Handbook of the English Language. By R. G. LAthaM, M.A. M.D. Crown 8vo. 6s.

Contanseau's Practical Dictionary of the French and English Languages. Post 8 vo. price $7 s .6 d$.

Contanseau's Pocket Dictionary, French and English, abridged from the Practical Dictionary by the Author. Square 18 mo. 3s. $6 d$.

\section{A Practical Dictionary} of the German and English Languages. By Rev. W. L. BlackleY, M.A. \& Dr. C. M. FRIEDLÄNDER. Post 8vo. 7s, 6d.

A New Pocket Dictionary of the German and English Languages. By F. W. LONGMAN, Ball. Coll. Oxford. Square $18 \mathrm{mo}$. $5^{5}$.

\section{Becker's Gallus ; Roman} Scenes of the Time of Augustus. Translated by the Rev. F. METCALFE, M.A. Post 8vo, 7s, 6d.

\section{Becker's Charicles;} Illustrations of the Private Life of the Ancient Greeks. Translated by the Rev. F. Metcalfe, M.A. Post 8vo. 7s. $6 d$.
A Dictionary of Roman and Greek Antiquities. With 2,000 Woodcuts illustrative of the Arts and Life of the Greeks and Romans. By A. Rich, B.A. Cron 8vo. 7s. 6d。

A Greek-English Lexicon. By H. G. LidDeli, D.D. Dean of Christchurch, and R. SCOTT, D.D. Dean of Rochester. Crown 4to. $36 s$.

Liddell \& Scott's Lexicon, Greek and English, abridged for Schools, Square 12mo. 7s. $6 d$.

\section{An English-Greek Lexi-} con, containing all the Greek Words used by Writers of good authority. By C. D. Yonge, M.A. 4to. 21s. School Abridgment, square $12 \mathrm{mo} .8 s .6 \mathrm{~d}$.

A Latin-English Dictionary. By John T'. White, D.D. Oxon. and J. E. RidDle, M.A. Oxon. Sixth Edition, revised. Quarto 2 Is.

White's College LatinEnglish Dictionary, for the use of University Students. 'Royal 8vo. I2s.

M'Culloch's Dictionary of Commerce and Commercial Navigation. Re-edited, with a Supplement shewing the Progress of British Commercial Legislation to the Year $\mathbf{1 8 8 0}$, by HugH G. REID. With II Maps and 30 Charts. 8vo. 63s. The Su PPLEMENT separately, price $5 s$.

\section{Keith Johnston's General} Dictionary of Geography, Descriptive, Physical, Statistical, and Historical ; a complete Gazetteer of the World. Medium 8vo. 42s.

\section{The Public Schools Atlas} of Ancient Geography, in 28 entirely new Coloured Maps. Edited by the Rev. G. Butler, M.A. Imperial 8vo. or imperial 4 to. 7 s. $6 d$.

\section{The Public Schools Atlas} of Modern Geography, in 3 I entirely new Coloured Maps. Edited by the Rev. G. Butler, M.A. Uniform, 5s. 


\section{ASTRONOMY and}

\section{Outlines of Astronomy.}

By Sir J. F. W. Herscher, Bart. M.A. Latest Edition, with Plates and Diagrams. Square crown 8vo. I2s.

\section{Essays on Astronomy.}

A Series of Papers on Planets and Meteors, the Sun and Sun-surrounding Space, Stars and Star Cloudlets. By R. A. Proctor, B.A. With ro Plates and 24 Woodcuts. 8vo. I2s.

\section{The Moon; her Motions,} Aspects, Scenery, and Physical Condition. By R. A. Proctor, B.A. With Plates, Charts, Woodcuts, and Lunar Photographs. Crown 8vo, Ios.6 $d$.

The Sun; Ruler, Light, Fire, and Life of the Planetary System. By R. A. Proctor, B.A. With Plates \& Woodcuts, Crown 8vo. I4s.

\section{The Orbs Around Us ;}

a Series of Essays on the Moon \& Planets, Meteors \& Comets, the Sun \& Coloured Pairs of Suns. By R. A. Procior, B.A. With Chart and Diagrams. Crown 8vo. 7s. 6d.

\section{Other Worlds than Ours;}

The Plurality of Worids Studied under the Light of Recent Scientific Researches. By R. A. Proctor, B.A. With I4 Illustrations. Cr. 8vo. ros. $6 d$.

\section{The Universe of Stars;}

Presenting Researches into and New Views respecting the Constitution of the Heavens. By R. A. Proctor, B.A. Second Edition, with 22 Charts (4 Coloured) and 22 Diagrams. 8vo. price ros. $6 d$.

\section{The Transits of Venus;}

A Popular Account of Past and Coming Transits. By R. A. Proctor, B.A. 20 Plates (I2 Coloured) and 27 Woodcuts, Crown 8vo, 8s. $6 \%$.
METEOROLOGY.

Saturn and its System. By R. A. Proctor, B.A. 8vo, with I4 Plates, I4s.

The Moon, and the Condition and Configurations of its Surface. By E. NeIson, F.R.A.S. With 26 Maps \& 5 Plates. Medium 8vo. 3 Is. $6 d$.

A New Star Atlas, for the Library, the School, and the Obser. vatory, in 12 Circular Maps (with 2 Index Plates). By R. A. Proctor, B.A. Crown 8vo. 5 s.

Larger Star Atlas, for the Library, in Twelve Circular Maps, with Introduction and 2 Index Plates. By R. A. Proctor, B.A. Folio, I5s. or Maps only, I2s. $6 d$.

A Treatise on the $\mathrm{Cy}$ cloid, and on all forms of Cycloidal Curves, and on the use of Cycloidal Curves in dealing with the Motions of Planets, Comets, \&cc. and of Matter projected from the Sun. By R. A. Proctor; B.A. With I6I Diagrams. Crown 8vo, ros, $6 d$.

\section{Dove's Law of Storms,}

considered in connexion with the Ordinary Movements of the Atmosphere. Translated by R. H. ScotT, M.A. 8vo. ros. $6 d$.

Air and Rain ; the Beginnings of a Chemical Climatology. By R. A. Sмiтн, F.R.S. 8vo. 24 s.

\section{Schellen's Spectrum} Analysis, in its Application to Terrestrial Substances and the Physical Constitution of the Heavenly Bodies. Translated by JANE and C. LASSEI.I., with Notes by W. HuGgins, LL.D. F.R.S. 8vo. Plates and Woodcuts, $28 s$. 


\section{NATURAL HISTORY and PHYSICAL SCIENCE.}

\section{Professor Helmholtz'}

Popular Lectures on Scientific Subjects. Translated by E. AtKinson, F.C.S. With numerous Wood Engravings. 8vo. 12s. 6d.

\section{Professor Helmholtz on} the Sensations of Tone, as a Physiological Basis for the Theory of Music. Translated by A. J. ElLIS, F.R.S. 8vo. $36 s$.

\section{Ganot's Natural Philo-} sophy for General Readers and Young Persons; a Course of Physics divested of Mathematical Formulæ and expressed in the language of daily life. Translated by E. ATkinson, F.C.S. Third Edition. Plates and Woodcuts. Crown 8vo. 7s. 6d.

Ganot's Elementary Treatise on Physics, Experimental and Applied, for the use of Colleges and Schools. Translated by E. ATKINson, F.C.S. Ninth Edition. Plates and Woodcuts, Large crown 8 vo. 15 s.

\section{Arnott's Elements of Phy-} sics or Natural Philosophy. Seventh Edition, edited by A. BAIN, LL.D. and A. S. TAYLOR, M.D. F.R.S. Crown 8vo. Woodcuts, 12s. $6 d$.

\section{The Correlation of Phy-} sical Forces. By the Hon. Sir W. R. Grove, F.R.S. \&c. Sixth Edition; revised and augmented. 8vo. 15s.

\section{Weinhold's Introduction} to Experimental Physics ; including Directions for Constructing Physical Apparatus and for Making Experiments. Translated by B. LoEwy, F.R.A.S. 8vo. Plates \& Woodcuts 3Is. 6d.

\section{A Treatise on Magnet-} ism, General and Terrestrial. By $\mathrm{H}$. ILOYD, D.D. D.C.L. 8vo. Ios. $6 d$.

\section{Elementary Treatise on} the Wave-Theory of Light. By I. Lioyo, D. D. D. C. L. 8vo. Ios, 6d.
Fragments of Science. By John TyNdall, F.R.S. Sixth Edition, revised and augmented. 2 vols. crown 8vo. $16 s$.

\section{Heat a Mode of Motion.} By John Tyndall, F.R.S. Fifth Edition in preparation.

Sound. By John Tyndall, F,R.S. Third Edition, including Recent Researches on Fog-Signalling. Crown 8vo, price Ios. $6 d$.

Contributions to Molecular Physics in the domain of Radiant Heat. By JoHN TYNDALL, F.R.S. Plates and Woodcuts. 8vo. I6s.

Professor Tyndall's Researches on Diamagnetism and Magne-Crystallic Action; including Diamagnetic Polarity. New Edition in preparation.

\section{Professor Tyndall's Lec-} tures on Light, delivered in America in 1872 and 1873 . With Portrait, Plate \& Diagrams. Crown 8vo. 7s, 6d.

\section{Professor Tyndall's Les-} sons in Electricity at the Royal Institution, 1875-6. With 58 Woodcuts. Crown 8vo, $2 s .6 d$.

\section{ProfessorTyndall's Notes} of a Course of Seven Lectures on Electrical Phenomena and Theories, delivered at the Royal Institution. Crown 8vo. Is. sewed, is. 6d. cloth.

ProfessorTyndall's Notes of a Course of Nine Lectures on Light, delivered at the Royal Institution. Crown 8vo, Is. swd,, Is, 6d. cloth.

\section{Principles of Animal Me-} chanics. By the Rev. S. HAughton, F.R.S. Second Edition. 8ro. 2 I $s$. 
Text-Books of Science, Mechanical and Physical, adapted for the use of Artisans and of Students in Public and Science Schools. Small 8vo. with Woodcuts, \&c.

Abney's Photography, 3s. 6d.

Anderson's (Sir John) Strength of Materials, $3 s .6 d$.

Armstrong's Organic Chemistry, 3s. 6d. Barry's Railway Appliances, 3s. $6 d$.

Bloxam's Metals, 3s. $6 d$.

Goodeve's Mechanics, 3s. $6 d$.

Mechanism, 3s. $6 d$.

Gore's Electro-Metallurgy, 6s.

Griffin's Algebra \& Trigonometry, $3 / 6$. Jenkin's Electricity \& Magnetism, 3/6. Maxwell's Theory of Heat, 3s. $6 d$.

Merrifield's Technical Arithmetic, $3 s .6 d$.

Miller's Inorganic Chemistry, $3^{s}, 6 d$.

Preece \& Sivewright's Telegraphy, $3 ; 6$.

Rutley's Study of Rocks, 4s. $6 d$.

Shelley's Workshop Appliances, 3s. 6d.

Thomés Structural and Physiological Botany, 6s.

Thorpe's Quantitative Analysis, 4s. 6d. Thorpe \& Muir's Qualitative Analysis, price $3 s .6 d$.

Tilden's Chemical Philosophy, 3s. $6 d$.

Unwin's Machine Design, 3s. $6 d$.

Watson's Plane \& Solid Geometry, 3/6.

\section{Light Science for Leisure}

Hours; Familiar Essays on Scientific Subjects, Natural Phenomena, \&c. By R. A. Proctor, B.A. 2 vols. crown 8vo. 7s. 6d. each.

\section{An Introduction to the} Systematic Zoology and Morphology of Vertebrate Animals. By A. Macalister, M.D. With 28 Diagrams. 8vo. Ios. $6 d$.

\section{The Comparative Ana-} tomy and Physiology of the Vertebrate Animals. By RICHARD OWEN, F.R.S. With 1,472 Woodclits. 3 vols. 8vo. 63.13 s. $6 d$.

\section{Homes without Hands;}

a Description of the Habitations of Animals, classed according to their Principle of Construction. By the Rev. J. G. WooD, M.A. With about I4O Vignettes on Wood. Svo. I4s.

\section{Wood's Strange Dwell-} ings; a Description of the Habitations of Animals, abridged from 'Homes without Hands.' With Frontispiece and 60 Woodcuts. Crown 8vo. $75.6 d$.

\section{Wood's Insects at Home;}

a Popular Account of British Insects, their Structure, Habits, and Transformations. 8vo. Woodcuts, I4s.

\section{Wood's Insects Abroad;}

a Popular Account of Foreign Insects, their Structure, Habits, and Transformations. 8vo. Woodcuts, I4s.

\section{Wood's Out of Doors; a}

Selection of Original Articles on Practical Natural History. With 6 Illustrations. Crown 8vo.7s. 6d.

\section{Wood's Bible Animals; a}

description of every Living Creature mentioned in the Scriptures, from the Ape to the Coral. With II 2 Vignettes. 8 vo. I4s.

\section{The Sea and its Living} Wonders, By Dr. G. HARTwig. 8 vo, with many Illustrations, 10s. $6 d$.

\section{Hartwig's Tropical} World. With about 200 Illustrations. 8vo. Ios. $6 d$.

\section{Hartwig's Polar World;}

a Description of Man and Nature in the Arctic and Antarctic Regions of the Globe. Maps, Plates \& Woodcuts. 8 vo. IOS, $6 d$.

\section{Hartwig's Subterranean} World. With Maps and Woodcuts. 8vo. ros. $6 d$.

\section{Hartwig's Aerial World;} a Popular Account of the Phenomena and Life of the Atmosphere. Map, Plates, Woodcuts, 8vo. Ias. 6u. 
Kirby and Spence's Introduction to Entomology, or Elements of the Natural History of Insects. Crown 8vo, 5 s.

A Familiar History of Birds. By E. Stanley, D.D. Fcp. 8vo. with Woodcuts, 3 s. $6 d$.

Rural Bird Life ; Essays on Ornithology, with Instructions for Preserving Objects relating to that Science. By Charles Dixon. With Coloured Frontispiece and 44 Woodcuts by G. Pearson, Crown 8vo. 7s, 6d. cloth extra, gilt edges.

\section{Rocks Classified and De-}

scribed. By BernHARd Von CotTA. An English Translation, by P. H. LAWRENCE, with English, German, and French Synonymes. Post 8vo, I4s.

\section{The Geology of England} and Wales; a Concise Account of the Lithological Characters, Leading Fossils, and Economic Products of the Rocks. By H. B. Woodward, F.G.S. Crown 8vo. Map \& Woodcuts, 14s.

\section{Keller's Lake Dwellings} of Switzerland, and other Parts of Europe. Translated by JoHN E. LEE, F.S.A. F.G.S. With 206 Illustrations. 2 vols. royal 8 vo. $42 s$.

\section{Heer's Primæval World} of Switzerland. Edited by JAMES Heywood, M.A. F.R.S. With Map, 19 Plates, \& 372 Woodcuts, 2 vols. 8vo. $16 s$.

\section{The Puzzle of Life and} How it Has Been Put Together; a Short History of Praehistoric Vegetable and Animal Life on the Earth. By A. Nicols, F.R.G.S. With 12 Illustrations. Crown 8 vo. $3^{s}$. $6 d$ 。
The Origin of Civilisation, and the Primitive Condition of Man; Mental and Social Condition of Savages, By Sir J. Luввоск, Bart. M.P. F.R.S. 8vo. Woodcuts, I8s.

A Dictionary of Science, Literature, and Art. Re-edited by the late W. T. BRANDE (the Author) and the Rev. Sir G. W. Cox, Bart. M.A. 3 vols, medium 8 vo. 63 s.

Hullah's Course of Lectures on the History of Modern Music. 8vo. 8s, $6 d$.

\section{Hullah's Second Course} of Lectures on the Transition Period. of Musical History. 8vo. ros. $6 d$.

\section{Loudon's Encyclopædia} of Plants; comprising the Specific Character, Description, Culture, History, \&ce. of all the Plants found in Great Britain. With upwards of I2,000 Woodcuts. 8vo. 42s.

\section{De Caisne \& Le Maout's} Descriptive and Analytical Botany. Translated by Mrs. HoOKER; edited and arranged by J. D. HookeR, M.D. With 5,500 Woodcuts. Imperial 8vo. price $3 \mathbf{I} s .6 d$.

\section{Rivers's Orchard-House ;} or, the Cultivation of Fruit Trees under Glass. Sixteenth Edition, re-edited by T. F. Rivers. Crown 8vo, with 25 Woodcuts, 5 s.

\section{The Rose Amateur's}

Guide. By Thomas Rrvers, Latest Edition. Fcp. 8vo. 4s. 6d.

\section{Town and Window Gar-} dening, including the Structure, Habits and Uses of Plants. By Mrs. BuCKTON With 127 Woodcuts. Crown 8vo, 2s. 


\section{CHEMISTRY and PHYSIOLOGY.}

Practical Chemistry; the Principles of Qualitative Analysis. By W. A. TILDEN, D.Sc. Lond. F.C.S. Professor of Chemistry in Mason's College, Birmingham: . Fcp. 8vo, Is. $6 d$.

\section{Miller's Elements of Che-} mistry, Theoretical and Practical. Re-edited, with Additions, by $\mathrm{H}$. Macleod, F.C.S. 3 vols. 8vo.

Part I. Chemical Physics. i6s.

PART II. INorganic Chemistry, 245.

Part III. Organic Chemistrey, 3 Is. $6 d$.

Annals of Chemical $\mathrm{Me}$ dicine; including the Application of Chemistry to Physiology, Pathology, Therapeutics, Pharmacy, Toxicology, and Hygiene. Edited by J. I. W. Thudichum, M.D. Yol. I. 8vo. I4s.

\section{Health in the House:} Twenty-five Lectures on Elementary Physiology in its Application to the Daily Wants of Man and Animals. By Mrs. Buckton. Crown 8vo. Woodcuts, $2 s$.
A Dictionary of Chemistry and the Allied Branches of other Scieaces. By Henry WatTs, F.C.S. assisted by eminent Scientific and Practical Chemists. 7 vols. medium 8vo. $£ 10.16 s, 6 d$.

Third Supplement, completing the Record of Chemical Discovery to the year I877. PART I. 8vo. 36s. PART II. completion, in the press.

Select Iethods in Chemical Analysis, chiefly Inorganic, By WM. CROOKES, F.R.S. With 22 Woodcuts. Crown 8vo. I2s. $6 d$.

The History, Products, and Processes of the Alkali Trade, including the most recent Improvements. By Charles T. KingzetT, F.C.S. With 32 Woodcuts. 8vo. 12s.

Animal Chemistry, or the Relations of Chemistry to Physiology and Pathology: a Manual for Medical Men and Scientific Chemists. By Charles T. Kingzetr, F.C.S. 8vo. price $18 s$.

\section{The FINE ARTS and ILLUSTRATED} EDITIONS.

In Fairyland; Pictures from the Elf-World. By RICHARD Doyle. With 16 coloured Plates, containing 36 Designs. Folio, 15 s.

\section{Lord Macaulay's Lays of} Ancient Rome. With Ninety Illustrations on Wood from Drawings by $G$. SCHARF. Fcp. 4 to. $2 \mathrm{I} s$.

\section{Miniature Edition}

of Macaulay's Lays of Ancient Rome, with Scharf's 90 Illustrations reduced in Lithography. Imp. 16 mo. ros. $6 d$.

Moore's Lalla Rookh. TENNIEL's Edition, with 68 Woodcut Illustrations. Crown 8vo. IOS. 6d.

Moore's Irish Melodies, MACLISE's Edition, with 161 Steel Plates. Super-royal 8vo. 21s.

\section{Lectures on Harmony,}

delivered at the Royal Institution. By G. A, MACFARREN. 8vo, I2s.
Sacred and Legendary Art. By Mrs. JAMESON. 6 vols, square crown 8 vo. $£ 5$. 15 s. $6 d$.

Jameson's Legends of the Saints and Martyrs. With 19 Etch. ings and 187 Woodcuts, 2 vols, $31 s .6 d$.

Jameson's Legends of the Monastic Orders. With II Etchings and 88 Woodcuts. I vol. $21 s$.

Jameson's Legends of the Madonna. With 27 Etchings and 165 Woodcuts. I vol. 2 Is.

Jameson's History of the Saviour, His Types and Precursors, Completed by Lady EAsTLAKE. With 13 Etchings and 28x Woodcuts. 2 vols. $42 s$.

The Three Cathedrals dedicated to St. Paul in London. By W. Longman, F.S.A. With numerous Illustrations. Square crown 8vo. $21 s$. 
The USEFUL ARTS,

The Art of Scientific Discovery. By G. Gore, LL.D. F.R.S. Crown 8vo. 15s.

The Amateur Mechanics' Practical Handbook; describing the different Tools required in the Workshop. By A. H. G. Hobson. With 33 Woodcuts. Crown 8vo. 2s. 6d.

The Engineer's Valuing Assistant. By H. D. IJoskOLD, Civil and Mining Engineer. 8vo. price $3 \mathrm{I} s, 6 d$.

\section{Industrial Chemistry; a}

Manual for Manufacturers and for Colleges or Technical Schools ; a Transla. tion (by Dr. T. H. BARRY) of Stoh. mann and Engler's German Edition of PAYen's 'Précis de Chimie Industrielle;' with Chapters on the Chemistry of the Metals, \&c. by B. H. PAUL, Ph.D. With 698 Woodcuts. Medium 8vo. 42 s.

\section{Gwilt's Encyclopædia of} Architecture, with above I, 600 Woodcuts. Revised and extended by W. PAPWORTH. 8vo. 52s. 6 d.

\section{Lathes and Turning, Sim-} ple, Mechanical, and Ornamental. By W. H. NorthCoTt. Second Edition, with 338 Illustrations. 8 vo. 18s.

\section{The Theory of Strains in}

Girders and similar Structures, with Observations on the application of Theory to Practice, and Tables of the Strength and other Properties of $\mathrm{Ma}$ terials. By B. B. Stoney, M.A. M. Inst. C.E. Royal 8vo. with 5 Plates and 123 Woodcuts, 36 s.

\section{A Treatise on Mills and} Millwork. By the late Sir W. FAIRBAIRN, Bart. C.E. Fourth Edition, with 18 Plates and 333 Woodcuts. I vol. 8vo. 25s.

\section{Useful Information for} Engineers. By the late Sir W. FAIRbairn, Bart. C.E. With many Plates and Woodcuts. 3 vols. crown 8vo. 3Is. 6d.
MANUFACTURES, \&o.

The Application of Cast and Wrought Iron to Building Purposes. By the late Sir W. FAIrBAIRN, Bart. C.E. With 6 Plates and I1 8 Woodcuts. 8vo, $16 s$.

\section{Hints on Household}

Taste in Furniture, Upholstery, and other Details. By C. L. EAs' LAKE. Fourth Edition, with 100 Illus. trations. Square crown 8vo, I4s.

\section{Handbook of Practical} Telegraphy. By R. S. Culley, Memb. Inst. C.E. Seventh Edition. Plates \& Woodcuts. 8vo. 16s.

\section{A Treatise on the Steam}

Engine, in its various applications to Mines, Mills, Steam Navigation, Railways and Agriculture. By J. BOURNE, C.E. With Portrait, 37 Plates, and 546 Woodcuts, 4 to. $42 s$.

Recent Improvements in the Steam Engine. By J. BOURNF, C.E. Fcp. 8vo. Woodcuts, 6 s.

\section{Catechism of the Steam}

Engine, in its various Applications. By John Bourne, C.E. Fcp. 8vo. Woodcuts, 6s.

\section{Handbook of the Steam} Engine, a Key to the Author's Catechism of the Steam Engine. By J. Bourne, C.E. Fcp. 8vo. Woodcuts, $9 s$.

Examples of Steam and Gas Engines of the most recent Approved Types as employed in Mines, Factories, Steam Navigation, Railways and Agriculture, practicaily described. By JOHN Bourne, C.E. With 54 Plates and 356 Woodcuts. 4to. 7os.

Cresy's Encyclopædia of Civil Engineering, Historical, Theoretical, and Practical. With above 3,000 Woodcuts. 8vo. $42 s$.

\section{Ure's Dictionary of Arts,} Manufactures, and Mines. Seventh Edition, re-written and enlarged by $\mathbf{R}$. HUNT, F.R.S. assisted by numerous contributors. With 2,604 Woodcuts. 4 vols. medium 8 vo. $£ 7.75$. 


\section{Practical Treatise on $\mathrm{Me}$ -} tallurgy. Adapted from the last German Edition of Professor KerL's Metallurgy by W. Crookes, F.R.S. \&c. and E. RöHRIG, Ph.D. 3 vols. 8vo. with 625 Woodcuts. $£ 4$. Igs.

\section{Anthracen; its Constitution,} Properties, Manufacture, and Derivatives, including Artificial Alizarin, Anthrapurpurin, \&c. with their Applications in Dyeing and Printing. By G. AUerbach. Translated by W. Crookes, F.R.S. 8vo. I2s.

\section{On Artificial Manures,}

their Chemical Selection and Scientific Application to Agriculture ; a Series of Lectures given at the Experimental Farm at Vincennes in 1867 and 1874 75. By M. Georges Ville. Translated and edited by W. Crookes, F.R.S. With 31 Plates, 8vo, 215.

\section{Practical Handbook of Dyeing and Calico-Printing. By W. CROOKES, F.R.S. \&c. With numerous Illustrations and specimens of Dyed Textile Fabrics. 8 vo. 42 s.}

The Art of Perfumery, and the Methods of Obtaining the Odours of Plants; the Growth and general Flower Farm System of Raising Fragrant Herbs; with Instructions for the Manufacture of Perfumes for the Handkerchief, Scented Powders, Odorous Vinegars and Salts, Snuff, Dentifrices, Cosmetics, Perfumed Soap, \&c. By G. W. S. PIEsse, Ph.D. F.C.S. Fourth Edition, with 96 Wood. cuts. Square crown 8vo, 21 s.

\section{Mitchell's Manual of} Practical Assaying. Fourth Edition, revised, with the Recent Discoveries incorporated, by W. CROOKES, F.R.S. Crown 8vo. Woodcuts, 3is. 6d.

\section{Loudon's Encyclopædia} of Gardening ; the Theory and Practice of Horticulture, Floriculture, Arboriculture \& Landscape Gardening. With I,000 Woodcuts, 8vo, $21 s$.

\section{Loudon's Encyclopædia} of Agriculture; the Laying-out, Improvement, and Management of Landed Property; the Cultivation and Economy of the Productions of Agriculture. With I, roo Woodcuts. 8vo, 21 s.

\section{RELIGIOUS and}

\section{A Handbook to the Bible,}

or, Guide to the Study of the Holy Scriptures derived from Ancient Monuments and Modern Exploration. By F. R. Conder, and Lieut. C. R. CONDER, R.E. late Commanding the Survey of Palestine. Second Edition; Maps, Plates of Cuins, \&c. Post 8vo. price $75.6 d$.

\section{Four Lectures on some} Epochs of Early Church History. By the .Very Rev. C. Merivale, D.D. Dean of Ely. Crown 8vo. 5s.

\section{A History of the Church} of England; Pre-Reformation Period. By the Rev. T. P. Boultbee, LL.D. 8vo. 15 s.

\section{Sketch of the History of} the Church of England to the Revolution of 1688. By T. V. SHORT, D.D. Crown 8vo, 7s, $6 d$.

\section{MORAL WORKS.}

\section{The English Church in} the Eighteenth Century. By CharLes $\mathrm{J}$. ABBEY, late Fellow of University College, Oxford; and JoHN H. OverTON, late Scholar of Lincoln College, Oxford. 2 vols. 8vo. 36 s.

\section{An Exposition of the 39}

Articles, Historical and Doctrinal. By E. H. Browne, D.D. Bishop of Winchester. Eleventh Edition. 8vo. I6s.

\section{A Commentary on the} 39 Articles, forming an Introduction to the Theology of the Church of England. By the Rev. T. P. Boultbee, LL.D. New Edition. Crown 8vo. 6s.

\section{Sermons preached most-} $1 y$ in the Chapel of Rugby School by the late T. ARNOLD, D.D. Collective Edition, revised by the Author's Daughter, Mrs. W. E. Forster. 6 vols. crown 8vo. 30s. or separately, 5 s. each. 
Historical Lectures on the Life of Our Lord Jesus Christ. By C. J. Elicoti, D.D. 8vo, $12 s$.

The Eclipse of Faith ; or a Visit to a Religious Sceptic. By Henry Rogers. Fcp. 8vo. 5 s.

Defence of the Eclipse of Faith. By H. Rogers. Fcp. 8vo.3s. $6 d$.

Nature, the Utility of Religion and Theism. Three Essays by JOHN STUART MILI. 8vo. IOs. $6 d$.

\section{A Critical and Gram-} matical Commentary on St. Paul's Epistles. By C. J. Ellicotr, D.D. 8vo. Galatians, 8s. 6d. Ephesians, 8s. 6d. Pastoral Epistles, 10s. 6d. Philippians, Colossians, \& Philemon, 10s. $6 d$. Thessalonians, $7 s .6 d$.

Conybeare \& Howson's Life and Epistles of St. Paul. Three Editions, copiously illustrated.

Library Edition, with all the Original Illustrations, Maps, Landscapes on Steel, Woodcuts, \&c. 2 vols. 4 to. $42 s$.

Intermediate Edition, with a Selection of Maps, Plates, and Woodcuts. 2 vols. square crown 8 vo. 21 s.

Student's Edition, revised and condensed, with 46 Illustrations and Maps. 1 vol. crown 8 vo. 9 s.

\section{The Jewish Messiah;}

Critical History of the Messianic Idea among the Jews, from the Rise of the Maccabees to the Closing of the Talmud. By J. DRUMmond, B.A. 8vo. 15s.

\section{Bible Studies. By M. M.} Kalisch, Ph.D. PaRT I. The Prophecies of Balaam. 8vo. I0s. 6d. PART II. The Book of Fonah. 8vo. price 10 . $6 d$.

\section{Historical and Critical}

Commentary on the Qld Testament; with a New Translation. By M. M. Kalisch, Ph.D. Vol, I. Genesis, 8vo. 18s. or adapted for the General Reader, 12s. Vol. II. Exodus, 15s. or adapted for the General Reader, I2s. Vol. III. Leviticus, Part I. 15s, or adapted for the General Reader, $8 s$. Vol. IV. Leviticus, Part II. I5s. or adapted for the General Reader, \&s.
Ewald's History of Israel.

Translated from the German by J. E. Carpenter, M.A. with Preface by R. Martineau, M.A. 5 vols, 8vo. 63s.

Ewald's Antiquities of Israel. Translated from the German by H. S. Solly, M. A. 8vo. 12s. $6 d$.

The Types of Genesis, briefly considered as revealing the Development of Human Nature. By A. JUkes, Crown 8vo. 7s, $6 d$.

The Second Death and the Restitution of all Things; with some Preliminary Remarks on the Nature and Inspiration of Holy Scripture. By A. Jukes. Crown 8vo. 3s. $6 d$.

The Gospel for the Nineteenth Century. Third Edition. 8vo, price ros. $6 d$.

\section{Supernatural Religion;} an Inquiry into the Reality of Divine Revelation. Complete Edition, thoroughly revised. 3 vols. 8 vo. 36 s.

Lectures on the Origin and Growth of Religion, as illus. trated by the Religions of India ; being the Hibbert Lectures, delivered at the Chapter House, Westminster Abbey, in 1878 , by F. MAx Müller, M.A. 8vo. Ios. $6 d$.

Introduction to the Science of Religion, Four Lectures delivered at the Royal Institution; with Essays on False Analogies and the Philosophy of Mythology. By F. MAx MÜller, M.A, Crown 8vo. 1os. $6 d$.

\section{The Four Gospels in} Greek, with Greek-English Lexicon. By John T. White; D.D. Oxon. Square $32 \mathrm{mo}$. 5 s.

Passing Thoughts on Religion. By Miss Sewelt. Fcp. 8vo. price $3 s .6 d$.

Thoughts for the Age. By Miss Sewell. Fep. 8vo. 3s. 6 .

\section{Preparation for the Holy} Communion; the Devotions chiefly from the works of Jeremy Taylor, $13 y$ y Miss SEIVEJL. - 32mo. $3^{5 .}$ 
Bishop Jeremy Taylor's Entire Works; with Life by Bishop Heber. Revised and corrected by the Rev. C. P. EDEN. Io vols. $\mathscr{E 5}$. 5 s.

Hymns of Praise and Prayer. Corrected and edited by Rev, John Martineau, LL.D. Crown 8vo. 4s. 6d. 32mo. 1s. $6 d$.

Spiritual Songs for the Sundays and Holidays throughout the Year. By J. S. B. Monsell, LL.D. Fcp. 8vo. 5s. 18 mo. 2s.

Christ the Consoler; a Book of Comfort for the Sick. By Ellice Hopkins. Second Edition. Fcp. 8vo. 2s. 6d.

Lyra Germanica; Hymns translated from the German by Miss C. WiNKWORTH, Fcp. 8vo. 5s.
The Temporal Mission of the Holy Ghost; or, Reason and Revelation. By HENRY EDWARD Manning, D.D. Crown 8vo. $8 s .6 d$.

\section{Hours of Thought on} Sacred Things ; Two Volumes of Sermons. By James Martineau, D.D. LL.D. 2 vols, crown 8vo. 7s. $6 d$. each.

Endeavours after the Christian Life ; Discourses. By James Martineau, D.D. LL.D. Fifth Edition. Crown 8vo. 7s, 6d.

\section{The Pentateuch \& Book} of Joshua Critically Examined. By J. W. Colenso, D.D. Bishop of Natal. Crown 8 vo. 6 s.

Lectures on the Pentateuch and the Moabite Stone; with Appendices. By J. W. Colenso, D.D. Bishop of Natal, 8vo. I2s.

\section{TRAVELS, VOYAGES, \&c.}

\section{Sunshine and Storm in} the East, or Cruises to Cyprus and Con. stantinople. By Mrs. Brassey. With 2 Maps and II 4 Illustrations engraved on Wood by G. Pearson, chiefly from Drawings by the Hon. A. Y. Bingham; the Cover from an Original Design by Gustave Doré. 8vo. 21 s.

\section{A Voyage in the 'Sun- beam, our Home on the Ocean for Eleven Months. By Mrs. Brassey. Cheaper Edition, with Map and 65 Wood Engravings. Crown 8vo. 7s. 6d.}

\section{One Thousand Miles up}

the Nile; a Journey through Egypt and Nubia to the Second Cataract. By Miss Amelia B. Edwards, Author of 'Untrodden Peaks and Unfrequented Valleys," 'Barbara's History,' \&c. With Facsimiles of Inscriptions, Ground Plans, Two Coloured Maps of the Nile from Alexandria to Dongola, and 8o Illustrations engraved on Wood from Drawings by the Author; bound in ornamental covers designed also by the Author. Imperial 8vo. $42 s$.
Wintering in the $\mathrm{Ri}$ viera; with Notes of Travel in Italy and France, and Practical Hints to Travellers. By William Miller, S.S.C. Edinburgh. With 12 Illus: trations. Post 8vo. 12s. 6d.

\section{San Remo and the Wes-}

tern Riviera ; comprising Bordighera, Mentone, Monaco, Beaulieu, Villefranche, Nice, Cannes, Porto Maurizio, Marina, Alassio, Verezzi, Noli, Monte Grosso, Pegli, Cornigliano, Genoa, and other Towns-climatically and medi cally considered, By A. Hill Hassall, M.D. Map and Woodcuts. Crown 8vo. Ios. $6 a$.

\section{Eight Years in Ceylon.} By Sir SAMuel W. BAKER, M.A. Crown 8vo. Woodcuts, 7s. $6 d$.

\section{The Rifle and the Hound} in Ceylon. By Sir Samuel W. Baker, M.A. Crown 8vo. Woodcuts, 7s, 6d. 
Himalayan and SubHimalayan Districts of British India, their Climate, Medical Topography, and Disease Distribution; with reasons for assigning a Malarious Origin to Goître and some other Diseases. By F. N. Macnamara, M.D. F.R.G.S. Surgeon-Major (retired) Indian Medical Service, late Professor of Chemistry, Calcutta Medical College, and Medical Inspector of Inland Labour Transport, Calcutta. 8vo.

[In the press.

\section{The Alpine Club Map of} Switzerland, with parts of the Neighbouring Countries, on the scale of Four Miles to an Inch. Edited by R. C. Nichols, F.R.G.S. 4 Sheets in Portfolio, 42s. coloured, or 34s. uncoloured.
The Alpine Guide. By John BalL, M.R.I.A. Post 8 vo, with Maps and other Illustrations :-

The Eastern Alps, ros. $6 d$.

Central Alps, including all the Oberland District, 7 s. $6 d$.

Western Alps, including Mont Blanc, Monte Rosa, Zermatt, \&c. Price $6 s .6 d$.

\section{On Alpine Travelling and} the Geology of the Alps. Price Is. Either of the Three Volumes or Parts of the 'Alpine Guide' may be had with this Introduction prefixed, Is, extra.

\section{WORKS of FICTION.}

Novels and Tales. By the Right Hon, the EARL of BEACONSFIELD, K.G. Cabinet Editions, complete in Ten Volumes, crown 8vo. 6s, each.

Lothair, 6s.

Venetia, $6 s$.

Coningsby, 6s.

Sybil, 6s.

Tancred, 6s.

Alroy, Ixion, \&c. 6s. Young Duke \&c. $6 s$.

Vivian Grey, $6 s$.

Henrietta Temple, $6 s$.

Contarini Fleming, \&c. $6 s$.

\section{Tales from Euripides;} Iphigenia, Alcestis, Hecuba, Helen, Medea. By Vincent K. Cooper, M.A. late Scholar of Brasenose College, Oxford. Fcp. 8vo. 3s. 6d.

Whispers from Fairyland. By the Right Hon. E. H. KNATChBUll-HugeSSEN, M.P. With 9 Illustrations. Crown 8vo. 3s. 6d.

Higgledy - Piggledy ; or, Stories for Everybody and Everybody's Children. By the Right Hon. E. H. Knatchbull-Hugessen, M.P. With 9 Illustrations, $\mathrm{Cr}, 8 \mathrm{vo}, 3 s, 6 d$.
Stories and Tales. By

Elizabeth M: Sewell. Cabinet Edition, in Ten Volumes, each contain. ing a complete Tale or Story :-

Amy Herbert, 2s. 6d. Gertrude, 2s. 6d. The Earl's Daughter, 25. $6 d$. The Experience of Life, 2s. $6 d$. Cleve Hall, 2s. 6d. Ivors, 2s. 6d. Katharine Ashton, 2s. 6d. Margaret Percival, 3s. 6d. Laneton Parsonage, 3s. $6 d$. Ursula, $3 s .6 d$.

\section{The IModern Novelist's}

Library. Each work complete in itself, price $2 s$. boards, or $2 s, 6 d$. cloth :-

By Lord BeAconsfieI.D.

Lothair.

Coningsby.

Sybil.

Tancred.

Venetia.

Henrietta Temple.

Contarini Fleming.

Alroy, Ixion, \&c.

The Young Duke, \&c. Vivian Grey.

By Anthony Trollope.

Barchester Towers.

The Warden, 
THE MODERN NOVELIST'S LIBRARY-continued.

By Major Whyte-Melville.

Digby Grand. General Bounce. Kate Coventry. The Gladiators. Queen's Maries.

Py the Author of 'The Rose Garden.'

Unawares.
By the Author of 'Mlle. Mori.'

The Atelier du Lys.

Mademoiselle Mori.

By Various IVriters.

Atherstone Priory.

The Burgomaster's Family.

Elsa and her Vulture.

The Six Sisters of the Valleys.

\section{The Novels and Tales of the Right Honourable} the Earl of Beaconsfield, K.G. Complete in Ten Volumes, crown 8vo, cloth extra, gilt edges, 30 s.

\section{POETRY and THE DRAMA.}

\section{Lays of Ancient Rome;} with Ivry and the Armada. By LORD MACAULAY. $16 \mathrm{mo} .3^{s, 6 d}$.

Horatii Opera. Library Edition, with English Notes, Marginal References \& various Readings. Edited by Rev. J. E. Yonge, M.A. 8vo. 2Is.

\section{Poetical Works of Jean} Ingelow. New Edition, reprinted, with Additional Matter, from the 23rd and 6th Editions of the two volumes respectively; with 2 Vignettes. 2 vols. fcp. 8vo. I $2 s$.

Poems by Jean Ingelow. First SERIES, with nearly Ioo Woodcut Illustrations. Fcp. 4to. 2 I $s$.

The Poem of the Cid: a Translation from the Spanish, with Introduction and Notes. By JoHn Ormsiy. Crown 8vo. 5s.
Festus, a Poem. By Philip James Bailey. Ioth Edition, enlarged \& revised. Crown 8vo, I2s. $6 \pi$.

The Iliad of Homer, Homometrically translated by C. B. Cayley. 8vo. 12s. 6d.

The 王neid of Virgil. Translated into English Verse. Py J. Conington, M.A. Crown 8vo. 9s.

\section{Bowdler's Family Shak-} speare. Genuine Edition, in 1 vol. medium 8vo. large type, with 36 Woorlcuts, I4s. or in 6 vols. fep. 8vo. 21 s.

\section{Southey's Poetical} Works, with the Author's last Cor= rections and Additions. Medium 8vo. with Portrait, 14s.

\section{RURAL SPORTS, HORSE and CATTLE MANAGEMENT, \&c.}

Annals of the Road; or, Notes on Mail and Stage-Coaching in Great Britain. By Captain MAiET. With 3 Woodcuts and ro Colouted Illustrations. Medium Sro, 2 Is.
Down the Road; or, Reminiscences of a Gentleman Coachman. By C. T. S. BIRCH REYNARDSON. Second Edition, with 12 Coloured Illustrations. Medium 8vo, 2 Is. 


\section{Blaine's Encyclopædia of} Rural Sports; Complete Accounts, Historical, Practical, and Descriptive, of Hunting, Shooting, Fishing, Racing, Sec. With 600 Woodcuts. 8vo. 2 Is.

A Book on Angling; or, Treatise on the Art of Fishing in every branch ; including full Illustrated Lists of Salmon Flies, By Francis Francis. Post 8vo. Portrait and Plates, I5s.

Wilcocks's Sea-Fisherman: comprising the Chief Methods of Hook and Line Fishing, a glance at Nets, and remarks on Boats and Boating. Post 8vo. Woodcuts, I2s. 6d.

The Fly-Fisher's Entomology. By ALFRED RONalds. With 20 Coloured Plates. 8vo. I4s.

Horses and Riding. By George Nevile, M.A. With 3I Illustrations. Crown 8 vo. $6 s$.

Youatt on the Horse. Revised and enlarged by W. WATSON, M.R.C.V.S. 8vo. Woodcuts, 12s. $6 d$.

\section{Youatt's Work on the} Dog. Revised and enlarged. 8vo. Woodcuts, 6 s.
The Dog in Health and Disease. By Stonehenge. Third Edition, with 78 Wood Engravings. Square crown 8vo, $7 s, 6 d$.

The Greyhound. By STONEHENGe. Revised Edition, with 25 Portraits of Greyhounds, \&c. Square crown 8vo. I5s.

Stables and Stable Fittings. By W. Mrles. Imp. 8vo. with 13 Plates, 15 s.

The Horse's Foot, and How to keep it Sound. By W. Miles. Imp. 8vo. Woodcuts, I2s. 6d.

A Plain Treatise on Horse-shoeing. By W. MILES. Post 8vo. Woodcuts, 2s. $6 d$.

Remarks on Horses' Teeth, addressed to Purchasers. By W. Miles. Post 8vo. Is. $6 d$.

The $\mathrm{Ox}$, his Diseases and their Treatment; with an Essay on Parturition in the Cow. By J. R. Dobson, M.R.C.V.S. Crown 8vo. Illustrations, $7 s .6 d$.

\section{WORKS of UTILITY and GENERAL INFORMATION.}

Maunder's Treasury of Knowledge and Library of Reference; comprising an English Dictionary and Grammar, Universal Gazetteer, Classical Dictionary, Chronology, Law Dictionary, Synopsis of the Peerage, Useful Tables, \&c. Fcp. 8vo. 6s.

\section{Maunder's Biographical} Treasury. Latest Edition, recon. structed and partly re-written, with above 1,600 additional Memoirs, by W. I. R. Cates, Ficp. 8vo. 6s.

\section{Maunder's Treasury of} Natural History; or, Popular Dictionary of Zoology. Revised and corrected Edition, Fcp. 8vo. with 900 Woodcuts, 6 s.
Maunder's Scientific and Literary Treasury; a Popular En. cyclopæedia of Science, Literature, and Art. Latest Edition, partly re-written, with above I, 000 New Articles, by J. Y. JoHnson, Fcp. 8vo, 6s.

Maunder's Treasury of Geography, Physical, Historical, Descriptive, and Political. Edited by W. Hughes, F.R.G.S. With 7 Maps and 16 Plates. Fcp. 8vo. 6 s.

\section{Maunder's Historical} Treasury; Introductory Outlines of Universal History, and Separate Histories of all Nations. Revised by the Rev. Sir G. W. Cox, Bart. M.A. Fcp, 8vo. 6s. 
The Treasury of Botany, or Popular Dictionary of the Vegetable Kingdom; with which is incorporated a Glossary of Botanical Terms. Edited by J. LINDLEY, F.R.S. and T. MOORE, F.L.S. With 274 Woodcuts and 20 Steel Plates. Two Parts, fcp. 8vo, I $2 s$.

\section{The Treasury of Bible} Knowledge ; being a Dictionary of the Books, Persons, Places, Events, and other Matters of which mention is made in Holy Scripture. By the Rev. J. Ayre, M. A. Maps, Plates \& Woodcuts. Fcp. 8vo. $6 s$.

\section{A Practical Treatise on} Brewing; with Formulie for Public Brewers \& Instructions for Private Families, By W. Black. 8vo. Ios. 6d.

\section{The Theory of the Mo-} dern Scientific Game of Whist. By W. Pole, F.R.S.' Tenth Edition. Fcp. 8vo. 2s. 6d.

\section{The Correct Card; or,} How to Play at Whist; a Whist Catechism. By Major A. CAMpbell. WALker, F.R.G.S. Latest Edition. Fcp. 8vo, 2s, 6d.

\section{The Cabinet Lawyer; a} Popular Digest of the Laws of England, Civil, Criminal, and Constitutional. Twenty-Fifth Edition, corrected and extended. Fcp. 8vo. 9s.

\section{Chess Openings. By F.W.} Longman, Balliol College, Oxford. New Edition. Fcp. 8vo. 2s. 6d.

\section{Pewtner's Compre-} hensive Specifier; a Guide to the Practical Specification of every kind of Building-Artificer's Work. Edited by W. Young. Crown 8vo. 6s.

\section{Modern Cookery for Pri-} vate Families, reduced to a System of Easy Practice in a Series of carefully. tested Receipts, By ElizA ActoN. With 8 Plates and 150 Woodcuts. Fcp. 8vo, 6s.
Food and Home Cookery.

A Course of Instruction in Practical Cookery and Cleaning, for Children in Elementary Schools. By Mrs. BuckTON. Woodcuts. Crown 8vo. 2s.

Hints to Mothers on the Management of their Health during the Period of Pregnancy and in the Lying-in Room. By Thomas Bulz, M.D. Fcp. 8vo. 2s, 6d.

The Maternal Management of Children in Health and Disease. By Thomas BuLL, M.D. Fcp. 8vo. 2s. 6d.

\section{The Farm Valuer. By} JoHn ScotT, Land Valuer. Crown 8 vo. 5s.

Rents and Purchases; or, the Valuation of Landed Property, Woods, Minerals, Buildings, \&c. By John SCOTT. Crown 8vo. $6 s$.

Economic Studies. By the late Walter Bagehot, M.A. Fellow of University College, London. Edited by Richard HOLT HutTon. 8vo. IOs. 6d.

\section{Economics for Beginners} By H. D. Macleod, M.A. Small crown 8vo, 2s. $6 d$.

\section{The Elements of Bank-} ing. By H. D. Macleod, M.A. Fourth Edition. Crown 8vo. 5 s.

The Theory and Practice of Banking. By H. D. MACLEOD, M.A. 2 vols. 8 vo. 26 s.

\section{The Resources of IMod-} em Countries; Essays towards an Estimate of the Economic Position of Nations and British Trade Prospects, By Alex. Wilson. 2 vols, 8yo, 24s.

\section{The Patentee's Manual;}

a Treatise on the Law and Practice of Letters Patent, for the use of Patentees and Inventors. By J. Johnson, Barrister-at-Law ; and J. H. JoHNSON, Assoc. Inst. C. E. Solicitor and Patent Agent, Lincoln's Inn Fields and Glasgow. Fourth Edition, enlarged. 8vo, price 10 s. $6 d_{\text {s }}$ 


\section{N D E X.}

Abbey \& Overton's English Church History I5 's Photography .............................. Acton's Modern Cookery.

Alpine Club Map of Switzerland

Alpine Guide ('The) .

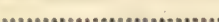

Amos's Jurisprudence

Primer of the Constitution..............

Fifty Years of the English Con-

stitution....

Anderson's Strength of Materials

Amestrong's Organic Chemistry .............

Amold's (Dr.) Lectures on Modern History Miscellaneous Works ....... Sermons ..........................

(T.) English Literature ............

Arnott's Elements of Physics.................

Atelier ('The) du Lys

Atherstone Priory.......................

Autumn Holidays of a Country Parson ...

Ayre's Treasury of Bible Knowledge ......

Bacon's Essays, by Whately

Life and Letters, by Spedding ... Works

Bagehot's Economic Studies Literary Studies

Bailey's Festus, a Poem ..........................

Bain's Mental and Moral Science.

on the Senses and Intellect

Emotions and Will.

Baker's Two Works on Ceylon

Balls Alpine Guides ............................

Barry on Railway Appliances ...............

Beaconsfield's (Lord) Novels and Tales I8 \&

Becker's Charicles and Gallus....................

Beesly's Gracchi, Marius, and Sulla ..........

Black's Treatise on Brewing ...................

Blackley's German-English Dictionary......

Blaine's Rural Sports ..............................

Bloxam's Metals ................................

Bolland and Lang's Aristotle's Politics......

Boultbee on 39 Articles.............................

- - History of the English Church...

Bourne's Works on the Steam Engine......

Bowdler's Family Shakespeare ................

Bramley-Moore's Six Sisters of the Valleys .

Brande's Dictionary of Science, Literature, and Art

Brassey's Sunshine and Storm in the East.

Voyage of the Sunbeam..............

Browne's Exposition of the 39 Articles......

Browuning's Modern England ...............

Buckle's History of Civilisation ................

Posthumous Remains .................

Buckton's Food and Home Cookery.......... Health in the House ................. Town and Window Gardening...

Bull's Hints to Mothers .........................

Maternal Management of Children.

Burgomaster's Family (The) ...................

Burke's Vicissitudes of Families................

Cabinet Lawyer.

Capes's Age of the Antonines....................

Carly Roman Empire ................

Cayley's Iliad of Homer ............................

Cetshwayo's Dutchman, translated by

Bishop Colenso
Changed Aspects of Unchanged Truths ...

Chesney's Indian Polity .............................. Waterloo Campaign ..................

Church's Beginning of the Middle Ages.... Colenso on Moabite Stone \&c. ............... I7 's Pentateuch and Book of Joshua. I7

Commonplace Philosopher..................... 7

Comte's Positive Polity ......................... 5

Conder's Handbook to the Bible ............ I5

Congreve's Politics of Aristotle ............... 6

Conington's Translation of Virgil's AEneid Ig

Miscellaneous Writings..........

Contanseau's Two French Dictionaries ...

Conybeare and Howson's St. Paul ............

Cooper's Tales from Euripides ................

Cordery's Struggle against Absolute Mon-

archy ...........................................

Cotta on Rocks, by Lawrence ............... 12

Counsel and Comfort from a City Pulpit... 7

Cox's (G. W.) Athenian Empire ........... 3

- Crusades ......................... 3

Greeks and Persians.........

Creighton's Age of Elizabeth ................... England a Continental Power Shilling History of England... Tudors and the Reformation

Cresy's Encyclopæedia of Civil Engineering

Critical Essays of a Country Parson..........

Crookes's Anthracen ................................. Chemical Analyses ..................... Dyeing and Calico-printing .......

Culley's Handbook of Telegraphy.............

Curteis's Macedonian Empire ...............

De Caisne and Le Maout's Botany .......... De Tocqueville's Democracy in America... Dixon's Rural Bird Life ..........................

Dobson on the Ox.

Dove's Law of Storms ................................

Doyle's (R.) Fairyland.............................. Drummond's Jewish Messiah .................. Eastlake's Hints on Household Taste...... Edwards's Nile..................................... Ellicott's Scripture Commentaries .......... Elsa and her Vulture .......................... Epochs of Ancient History........................ English History ................... Modern History .....................

Ewald's History of Israel ........................ Antiquities of Israel...................

Fairbairn's Applications of Iron ............ - Information for Engineers....... Mills and Millwork ................

Farrar's Language and Languages .......... Francis's Fishing Book .......................... Frobisher's Life by Fones........................ Froude's Cæar ................................... -English in Ireland

History of England ...................... Lectures on South Africa .......... Short Studies.

Gairdner's Houses of Lancaster and York Richard III. \& Perkin Warbeck

Ganot's Elementary Physics

Natural Philosophy

Gardiner's Buckingham and Charles .......

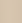

.

.


Gitrdiner's Personal Government of Charles I. 2 First Two Stuarts Thirty Years' War

German Home Life

Goodcue's Mechanics... Mechanism.

Gore's Art of Scientific Discovery.............. Electro-Metallurgy ....................

Gospel (The) for the Nineteenth Century.

Grant's Ethics of Aristotle....................

Graver Thoughts of a Country Parson......

Greville's Journal ...............................

Griffin's Algebra and Trigonometry.........

Griffth's A B C of Philosophy ................

Grove on Correlation of Physical Forces...

Gwilt's Encyclopæedia of Architecture.......

Hale's Fall of the Stuarts........................

Hartwig's Works on Natural History and

Popular Science

Hassall's Climate of San Remo................

Hawghton's Animal Mechanics ................

Hayruard's Selected Essays .................

Heer's Primeval World of Switzerland......

Heine's Life and Works, by Stigand ......

Helmholtz on Tone ..............................

Helmhollz's Scientific Lectures ................

Herschels Outlines of Astronomy ............

Hillebrand's Lectures on German Thought

Hobson's Amateur Mechanic ..................

Hopkins's Christ the Consoler

Hoskold's Engineer's Valuing Assistant ...

Hullah's History of Modern Music ......



Hume's Essays

Treatise on Human Nature...........

Ihne's Rome to its Capture by the Gauls...

History of Rome ...........................

\section{Ingelow's Poems}

Fameson's Sacred and Legendary Art.......

Memoirs by Macpherson ..........

Fenkin's Electricity and Magnetism.........

ferrold's Life of Napoleon....

Fohnson's Normans in Europe ................

Patentee's Manual ...................

Fohnston's Geographical Dictionary..........

Zukes's Types of Genesis

Fukes on Second Death

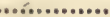

Kalisch's Bible Studies ........................

Commentary on the Bible.........

Keller's Lake Dwellings of Switzerland.....

Kerl's Metallurgy, by Crookes and Röhrig.

Kingzeti's Alkali Trade

Animal Chemistry

Kirby and Spence's Entomology

Kirby and Spence's Entomology ............

Klith's l'astor's Narrative ....................

Knatchbull-Hugessen's Fairy-Land .......... Landscapes, Churches, \&c......................

Lathamis English Dictionaries ..............

Handbook of English Language

Lecky's History of England....................... European Morals............ Rationalism

Leaders of Public Opinion..............

Leisure Hours in Town

Leslic's Essays in Poitical and Moral

Philosophy ........................................

Lessons of Middle Age ............................

Lewes's Biographical History of Philosophy

Lezvis on Authority ............................. Liddell and Scott's Greek-English Lexicons Lindley and Moore's Treasury of Botany ... Lloyd's Magnetism ................................

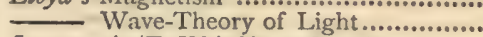
Longman's (F. W.) Chess Openings........... German Dictionary ... (W.) Edward the Third.......... Lectures on History of England Old and New St. Paul's

Loudon's Encyclopæedia of Agriculture ... Gardening......

Tubback's Plants............. Ludlow's American War of Independence Lyra Germanica ...................................

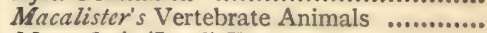
Macaulay's (Lord) Essays ...................... History of England ... Lays, Illustrated.......... Cheap Edition... Life and Letters......... Miscellaneous Writings Speeches .................. Works .....................

Writings, Selections from

McCulloch's Dictionary of Commerce ...... Macfarren on Musical Harmony ............. Macleod's Economical Philosophy.............. - Economics for Beginners .......... Theory and Practice of Banking

Macnamara's Himalayan Districts of British India

Mademoiselle Mori ................................ Mahaffy's Classical Greek Literature ....... Malet's Annals of the Road ................... Manning's Mission of the Holy Spirit ....... Marshman's Life of Havelock ................. Martineau's Christian Life....................... Hymns...................................

Maunder's Popular Treasuries........................

Maxwell s Theory of Heat .....................

May's History of Democracy .................... History of England ...

Melville's (Whyte) Novels and Tales ......

Mendelssohn's Letters ............................

Merivale's Early Church History .............. Fall of the Roman Republic .... General History of Rome ....... Roman Triumvirates................ Romans under the Empire.......

Merrifield's Arithmetic and Mensuration... Miles on Horse's Foot and Horse Shoeing on Horse's Teeth and Stables..........

Mill (J.) on the Mind

Mill (J. S.) Autobiograp...................... Dissertations \& Discussions Essays on Religion ............. Hamilton's Philosophy ...... Liberty Political Economy .............. Representative Government Subjection of Women......... System of J.ogic ............... Unsettled Questions ........... Utilitarianism 
M:llir's Elements of Chemistry ............ Inorganic Chemistry............... It

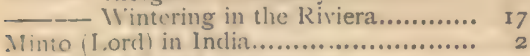
Mitchell s Manual of Assaying ............... 15 Molern Novelist's Library .............. I8 \& 19 Mensell's Spiritual Songs..................... 17 Morre's Irish Mlelodies, Iliustrated Edition I $_{3}$ Lalla Rookh, Illustrated Edition.. I3

Morill's I'hilosophical Fragments............ Merris's Age of Anne .........................

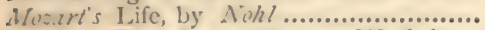
Ifiiller's Chips from a German Workshop. Hibbert Lectures on Religion ... Science of Language

Science of Religion

$\Lambda^{*}$ cison on the Moon....

Nezite's Horses and Riding

Necile's llorses and Riding ...................

Nicols's Puzzle of Life.

Noire's Miiller \& I thilosophy of Language Norlhcott's Lathes \& Turning ............... C)msly's Poem of the Cid ..................... Owen's Comparative Anatomy and Physiology of Vertebrate Animals ............

Packe's Guide to the Pyrenees .................

Pattison's Casaubon...

Payen's Industrial Chemistry................. Pcotner's Comprehensive Specifier ......... Phillips's Civil W'ar in Wales ............... Piesse's Art of Perfumery

Pole's Game of Whist ....

Powells Early England ........................

Precce \& Sivcwright's Telegraphy............

Present-Day Thoughts..............................

Proctor's Astronomical Works ............... Scientific Essays (Two Series) ...

Prothero's Life of Simon de Montfort .......

Public Schools Atlases

Rawlinson's Parthia.....

- Sassanians ........................

Recreations of a Country Parson .............

Reynardson's Down the Road .................

Kich's Dictionary of Antiquities ............

Rivers's Orchard House........................ Rose Amateur's Guide................

Rogers's Eclipse of Faith....................... Defence of Eclipse of Faith .......

Raget's English Thesaurus ....................

Ronald' I'ly-Fisher's Entomology .........

R'vilcy's Rise of the People ................... Settlement of the Constitution ...

Russia and England

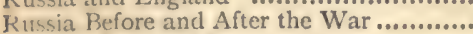

Rutley's Study of Rocks .......................

Sindars's Justinian's Institutes ...............

Sankcy's Sparta and Thebes

Surile on Apparitions

Schellen's Spectrum Analysis.

Seaside Musings

Scutt's Farm Valuer

Rents and Purchases.

Seebohm's Oxford Reformers of r.............

Seebohm's Protestant Revolution .............

Sewell's History of France
Siwell's Passing 'Thoughts on Religion ... 16 Preparation for Communion ...... 16

Stories and Tales ..................... 18

Thoughts for the Age ............... I6

Shelley's Workshop Appliances ................. Ix

Short's Church History ...................... I5

Smith's (Sydney) Wit and Wisdom ......... 6

(Dr. R. A.) Air and Rain ........... 9

(R. B.) Carthage \& the Carthaginians 3

Southey's Poetical Works..................... I9

Stanley's History of British Birds .......... I2

Stephen's Ecclesiastical Biography.......... 4

Stonehenge, Dog and Greyhound .......... 20

Stoney on Strains ................................. It

Stubbs's Early Plantagenets ................. 3

Sunday Afternoons, by A. K. H.B. ........ 7

Supernatural Religion ....................... if

Swinbourne's Picture Logic ................ 6

Tancock's England during the Wars, I778-r820 ...................................

Taylor's History of India ..................... Ancient and Modern History ... (Feremy) Works, edited by Eden

Text-Books of Science.

Thome's Botany

Thomson's Laws of Thought ... .................

Thorpe's Quantitative Analysis ..............

Thorpe and Muir's Qualitative Analysis ... Thudichum's Annals of Chemical Medicine Tilden's Chemical Philosoplyy ............... Practical Chemistry ..................... Todd on Parliamentary Government.......... Trench's Realities of Irish Life ................. Trollope's Warden and Barchester Towers Twiss's Law of Nations ....................... Tyndall's (Professor) Scientific Works ... Unawares ......................................... Unwin's Machine Design ........................ Ure's Arts, Manufactures, and Mines ....... Venn's Life, by Knight ....................... Ville on Artificial Manures..........................

Walker on Whist.................................

Walpole's History of England ....................

Warburton's Edward the Third .............

Watson's Geometery ............................

Watts's Dictionary of Chemistry ..............

$W c b b^{\prime}$ s Civil War in Herefordshire ..........

Weinhold's Experimental Physics..............

Wellington's Life, by Gleig ...................

Whately's English Synonymes ................. Logic

Rhetoric

White's Four Gospels in Greek................

- and Riddle's Latin Dictionaries.....

Wilcocks's Sea-Fisherman .....................

Williams's Aristotle's Ethics....................

Wilson's Resources of Modern Countries...

Wood's (J. G.) Popular Works on Natural

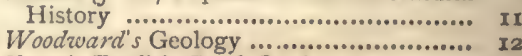

Yonge's English-Greek Lexicons ............ 8

Torace............................... I9

Youatt on the Dog .............................. 20

Zeller's Greek Philosophy ...................... 




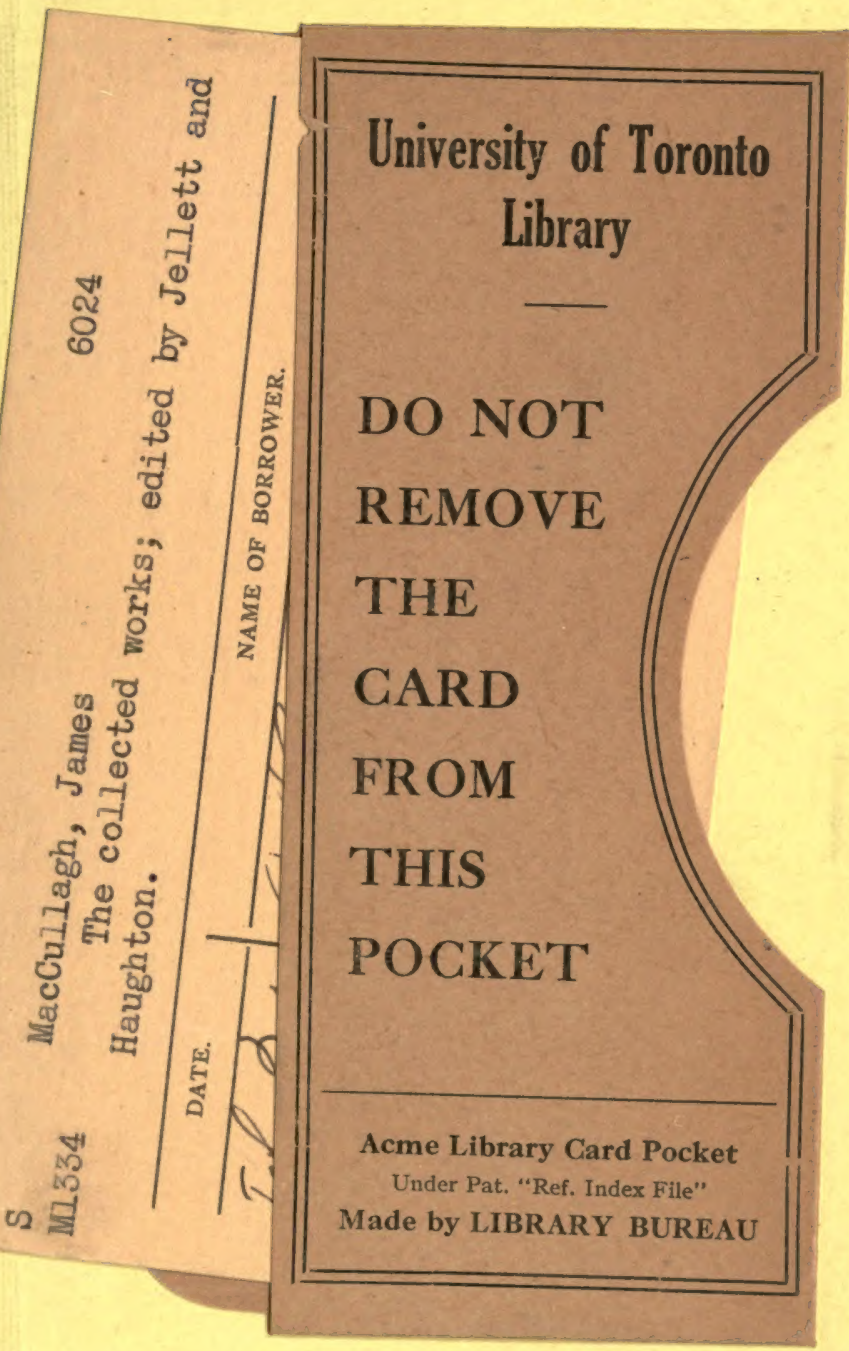




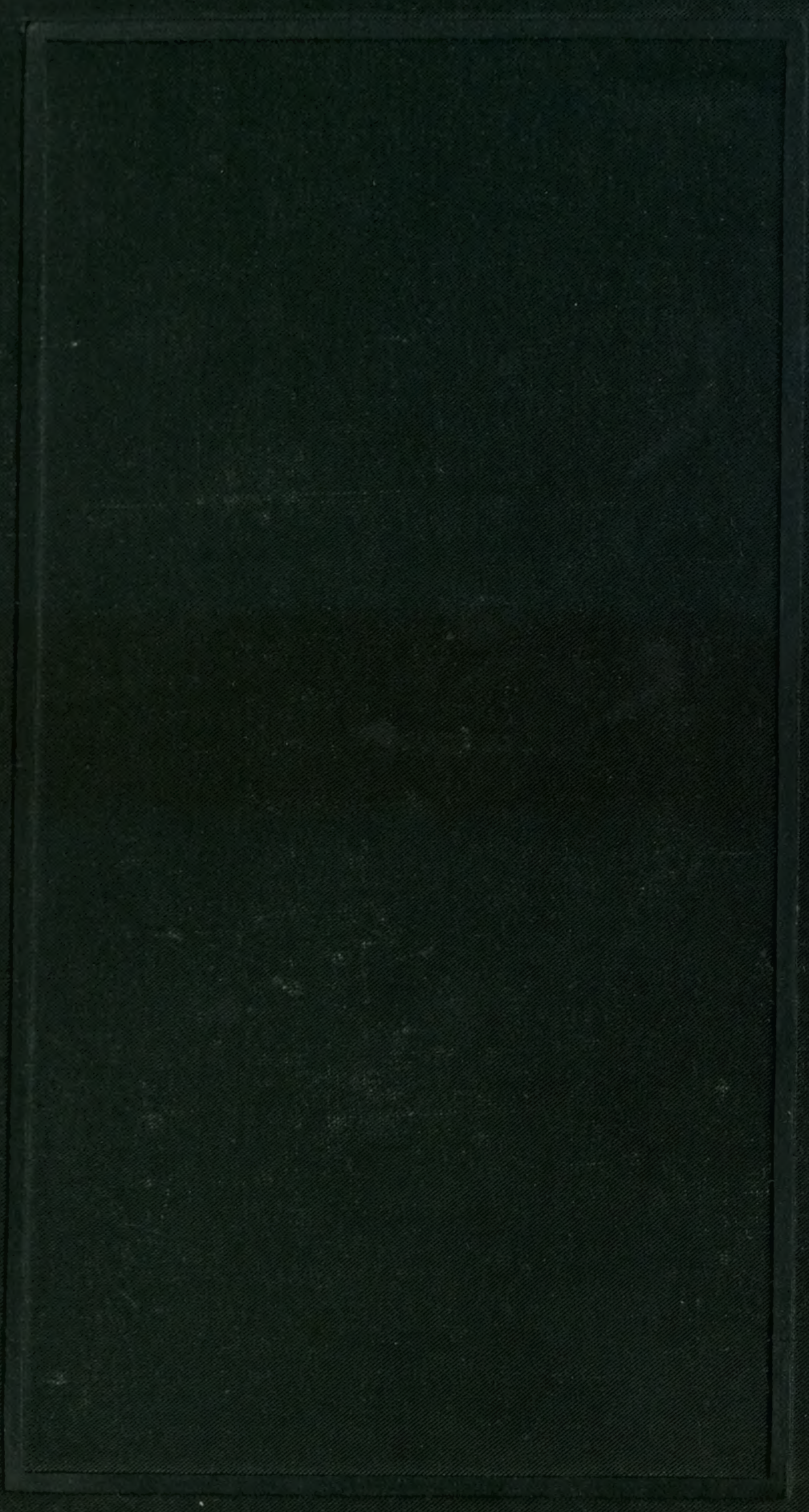

